\title{
Elucidating the anti-cancer mechanisms for transition-state structure inhibitors of nucleoside phosphorylases methylthioadenosine-DADMe-immucillin A and forodesine
}

By

Namal Vikum Carlo Coorey

A thesis submitted to the Victoria University of Wellington in fulfilment of the requirements for the degree of Doctor of Philosophy in Cell and Molecular Bioscience Victoria University of Wellington

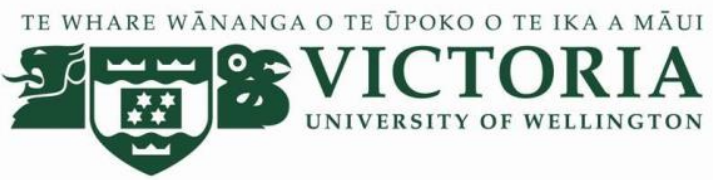




\section{Abstract}

Transition-state structure analogues are among the most powerful chemical inhibitors discovered to date with picomolar efficacy for enzymes. The nucleoside analogue methylthioadenosine-DADMe-immucillin A (MTDIA) is an inhibitor of the enzyme methylthioadenosine phosphorylase (MTAP) in polyamine biosynthesis. The recently approved forodesine (Mundesine ${ }^{\circledR}$ ) is an inhibitor of purine nucleoside phosphorylase (PNP) and purine synthesis. Although the targets of these drugs were known at the time of drug design, it is important to know the compendium of cellular perturbations resulting from use of these inhibitors. Several suspected mechanisms of MTDIA and forodesine in progression of apoptotic cell death have been identified but the underlying mechanisms initiating apoptosis remain elusive. We hypothesize that numerous cellular processes are affected in MTDIA and forodesine treatments given the importance of polyamine and purine synthesis in cancer cells. To elucidate the unsuspected mechanisms mediating anti-cancer activity, unbiased genomic analyses were employed using Saccharomyces cerevisiae. First, gene-gene interactions with MEU1 (the MTAP orthologue in yeast) were determined using Synthetic Genetic Array methodology followed by assessment of drug-gene interactions with MTDIA treatment under a MEU1 essential condition with MTA as the sole source of sulphur. Disruptions to suspected mechanisms of amino acid metabolism, carbohydrate metabolism, response to starvation, vesicle-mediated transport, vacuole fusion, lipid homeostasis, chromatin organisation, transcription, and translation were implicated well as unsuspected mechanisms of $\mathrm{NAD}^{+}$dependent cellular processes, multi-vesicular body formation, endosomal transport, ion homeostasis, mitochondrion organisation, and cell cycle progression. Induction of autophagy was subsequently confirmed with MTDIA to validate the disruptions to vesicle-mediated transport, response to starvation, multi-vesicular body formation and vacuolar fusion. Reduction in ergosterol levels and disruptions to ergosterol biosynthetic proteins were confirmed with MTDIA and meu1 $\Delta$ to validate disruptions to lipid homeostasis. To complement the genetic analyses, the abundance and localisation of proteins were evaluated in response to MTDIA or MEU1-deficiency. Disruptions to proteins 
implicated in carbohydrate metabolism, methionine salvage, transcription, translation, transmembrane transport, lipid homeostasis, cell cycle and DNA repair were identified with meu1 $\Delta$ and MTDIA. Key findings from the analysis of protein abundance and localization were the relocalisation of plasma membrane proteins and disruptions to vesicle mediated transport proteins consistent with the induction of autophagy and disruptions to proteins in homeostasis of all major lipid classes, further corroborating the findings of screening gene deletion mutants for elucidating drug mechanisms. To investigate the mechanisms of forodesine toxicity, genetic interactions with PNP1 (the PNP orthologue in yeast) were determined using Synthetic Genetic Array methodology. Disruptions to amino acid metabolism, starvation responsive genes, vacuolar organisation and vesicle mediated transport, carbohydrate metabolism, lipid homeostasis, chromatin organisation, chromosome segregation, transcription, and translation were identified in response to PNP1deficency. Despite the introduction of several human genes and supplementation of metabolites required for forodesine bioactivity in humans, forodesine was not sufficiently bioactive in yeast to evaluate sensitivity of gene deletion mutants to forodesine. Overall, chemical genomic analyses in yeast with transition-state structure analogues MTDIA and forodesine effectively highlight the vast number of cellular processes affected by inhibition of a single target. Moreover, genome-wide prescreening should be carried out in yeast to identify side-effects and secondary effects from drug target inhibition prior to assessing desired and undesired outcomes of highly specific drugs in human cells. 


\section{Acknowledgements}

There were a lot of people without whom this dissertation would not be possible. I owe particular debts of gratitude to my supervisors Dr Andrew Munkacsi and Prof Paul Atkinson, Dr Robert Keyzers, Dr Christine Stockum, Dr James Mathew, Dinidu Senanayake and lab members past and present. All of them have helped make this dissertation better in one way or another. I would also like to thank my wife Gabriella and my parents for all their encouragement, love and support. 


\begin{tabular}{|c|c|}
\hline A549 & Human alveolar basal epithelial cells \\
\hline AraC & Cytarabine \\
\hline Atg8 & Yeast orthologue of LC3 \\
\hline $\mathrm{CCL}$ & Chronic lymphatic leukaemia \\
\hline $\mathrm{CM}$ & Complete minimal \\
\hline CNT2 & Concentrative nucleoside transporter 2 \\
\hline Ctv & Cytosol to vacuole transport \\
\hline $\mathrm{dCK}$ & Deoxycytidine kinase \\
\hline dCyt & Deoxycytidine \\
\hline DFMO & L- $\alpha$-difluoromethylornithine \\
\hline dGuo & Deoxyguanosine \\
\hline DMSO & Dimethyl sulfoxide \\
\hline ENT1 & Equilibrative nucleoside transporter 1 \\
\hline EV & Empty vector \\
\hline FIC & Fractional inhibitory concentration \\
\hline Forodesine & Immucillin-H \\
\hline G418 & Geneticin \\
\hline GC-MS & Gas chromatography-mass spectrometry \\
\hline GFP & Green florescent protein \\
\hline GO & Gene ontology \\
\hline HSV-TK & Herpes simplex virus type- 1 thymidine kinase \\
\hline IC20 & $20 \%$ growth inhibitory concentration \\
\hline IMP & Inosine monophosphate \\
\hline kanR & Kanamycin resistance \\
\hline$K \mathrm{~d}$ & Dissociation constant \\
\hline Ki & Equilibrium constant \\
\hline KIE & Kinetic isotope effect \\
\hline MAU & Met/Ade/Ura \\
\hline MAUC & Met/Ade/Ura/Cys \\
\hline Meu1 & MTAP orthologue in yeast \\
\hline MIC & Minimum inhibitory concertation \\
\hline MTA & Methylthioadenosine \\
\hline MTAP & Methylthioadenosine phosphorylase \\
\hline MTDIA & Methylthioadenosine-DADMe-immucillin A \\
\hline NAT & ClonNAT \\
\hline natR & NAT resistance \\
\hline ODC & Ornithine decarboxylase \\
\hline ORF & Open reading frame \\
\hline $\mathrm{PI}$ & Phosphatidylinositol \\
\hline PI3K & Phosphoinositide 3-kinase \\
\hline PI3P & Phosphatidylinositol 3-phosphate \\
\hline PNP & Purine nucleoside phosphorylase \\
\hline SC & Synthetic complete \\
\hline SD & Synthetic dropout \\
\hline SD-N & Starvation media \\
\hline
\end{tabular}

SGA

SGD

SMS

SRM

SSAT

TOR

WT

$x x x \Delta$

YPD
Synthetic genetic array

Saccharomyces Genome Database

Spermine

Spermidine

Spermidine/spermine-N1-acetyltransfera

Target of rapamycin

Wild type

Non-essential gene deletion collection

Yeast/peptone/dextrose 


\section{Table of Contents}

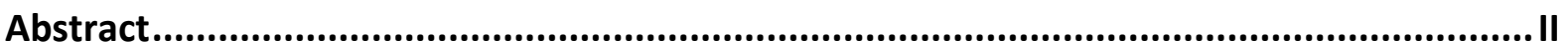

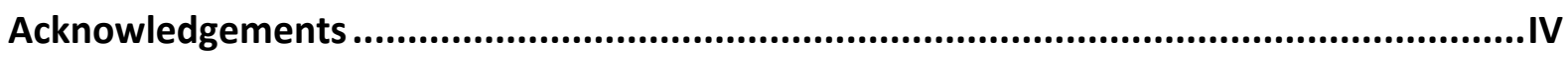

List of Abbreviations.......................................................................................................

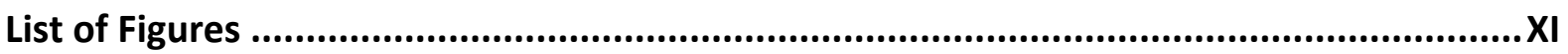

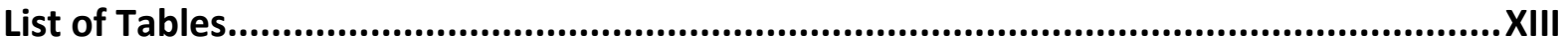

Chapter 1: Literature review....................................................................................... 1

1.1 Burden of cancer and current cancer therapies ................................................. 1

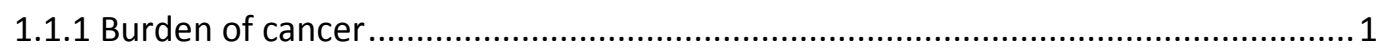

1.1.2 Current cancer therapies, undesired effects and resistance ................................. 2

1.1.3 Transition state structure analogues as antitumor therapeutics ............................ 4

1.2 Polyamine biosynthesis and MTAP inhibition as target for cancer therapy ............... 5

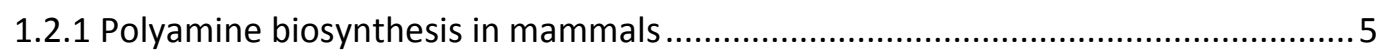

1.2.2 Polyamine biosynthesis as a target for cancer ..................................................... 6

1.2.3 MTAP in methionine salvage and synthesis of S-adenosyl methionine ...................

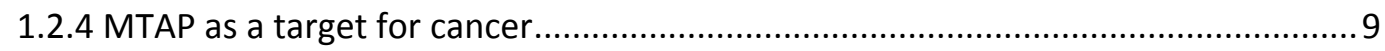

1.2.5 MTDIA: transition state analogue of MTA ……................................................ 10

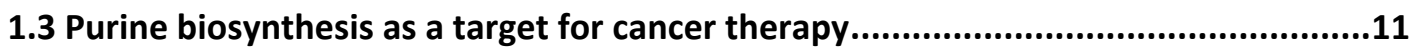

1.3.1 Structure and function of purine nucleoside phosphorylase (PNP) in humans .....11

1.3.2 PNP inhibitors and other deoxyguanosine analogues to treat T-cell activation ....12

1.3.3 High deoxyguanosine kinase, low 5' nucleosidase activity and nucleoside transport determines T-cell toxicity ................................................................................... 13

1.3.4 Proposed mechanisms for forodesine activity in T-cells ..................................... 15 
1.4.3 Genome-wide tools in yeast for elucidating gene function.

1.4.5 Purine nucleotide biosynthesis is highly conserved in yeast.

1.4.6 Structural conservation of MTAP and methionine salvage pathways........

Chapter 2 - Elucidating the anticancer mechanisms of nucleoside analogue MTDIA via with assessment of fitness for non-essential gene deletions

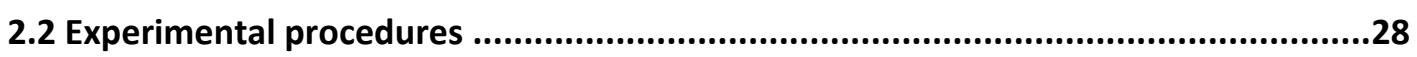

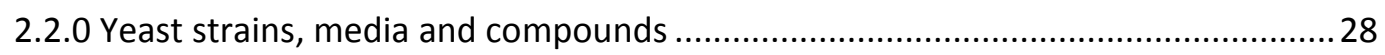

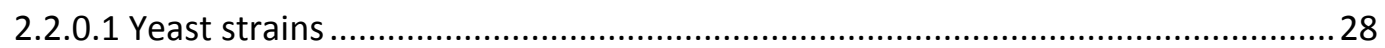

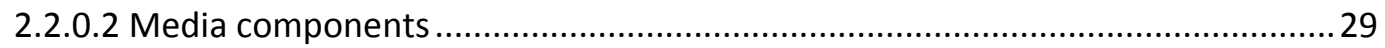

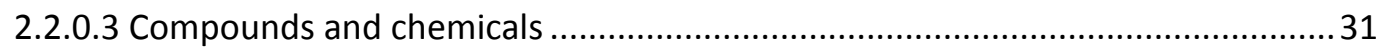

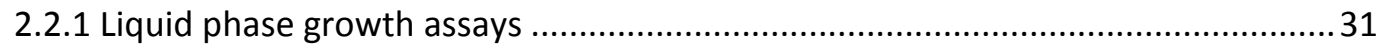

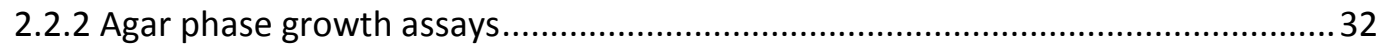

2.2.3 Quantification of genetic interactions with MEU1 …..............................................32

2.2.4 Validation of genetic interactions with MEU1 ....................................................... 32

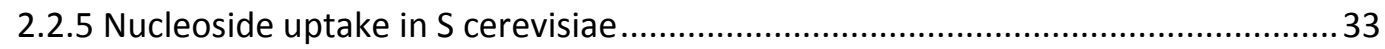

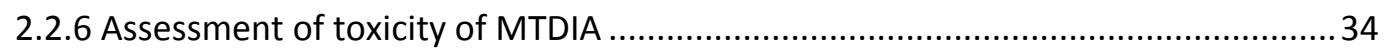

2.2.7 Construction of hENT1 expression yeast gene deletion library ...........................34

2.2.8 Screening the hENT1 yeast gene deletion library for sensitivity to MTDIA.............34

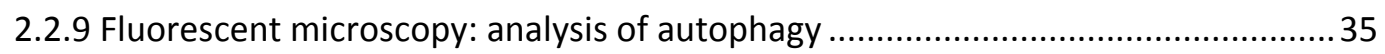




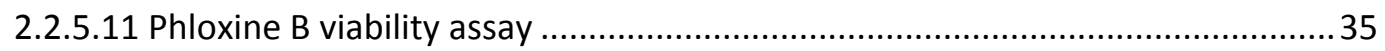

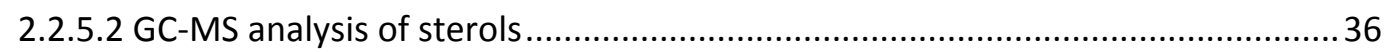

2.2.5.3 Calculation of synergy and fractional inhibitory concentration (FIC) index .........37

2.3 Results .37

2.3.1 SGA analysis identifies genetic interactions with the MEU1 target of MTDIA .......37

2.3.2 Heterologous expression of human gene results in nucleoside uptake in yeast ...43

2.3.3 Meu1 is essential when MTA is the only exogenous source of sulphur ................. 45

2.3.4 MTDIA inhibits growth by inhibiting MEU1 function and is bioactive in agar ........ 49

2.3.5 Genome-wide analysis identifies genetic interactions with MTDIA .....................52

2.3.6 Further evaluation of MTDIA and meu1 $\Delta$ mechanisms........................................59

2.3.6.1.0 Macroautophagy genes are sensitive to MTDIA treatment ............................59

2.3.6.1.1 MTDIA exacerbates the rapamycin induced growth arrest and induces

autophagic cell death .59

2.3.6.1.2 MTDIA regulates abundance and localisation of core autophagy proteins .....62

2.3.6.1.3 MTDIA induces autophagic trafficking of GFP-Atg8 independent of starvation

2.4 Discussion .71

Chapter 3 - Elucidating the anticancer mechanisms of nucleoside analogue MTDIA by

global analysis of protein localisation and abundance ................................................. 76

3.1 Introduction . .76

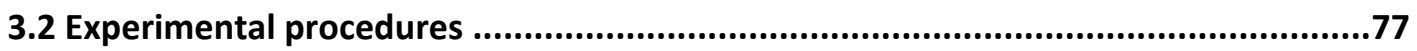

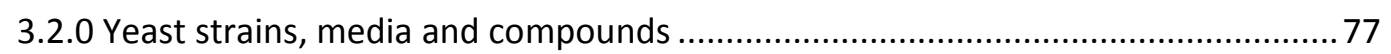

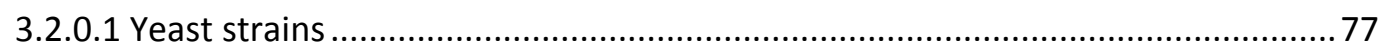


3.2.0.3 Compounds and chemicals

3.2.1 GFP library construction

3.3.2 Changes in protein abundance and localisation in response to MTDIA treatment and meu1 $1 \Delta$

3.3.2 MTDIA regulates proteins involved in glucose metabolism

3.3.3 Methionine salvage from glycine, cystine, choline and sulphate is regulated by MTDIA and meu1 $\Delta$

3.3.4 MTDIA regulates proteins involved in chromatin remodelling and transcription..95

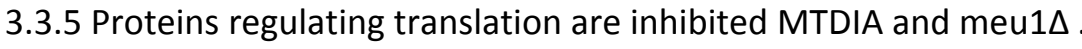
98

3.3.6 Cellular transport mechanisms disrupted by MTDIA and meu1 $\Delta$ are consistent with autophagy 102

3.3.7 Proteins involved in synthesis of cell wall, acetyl-CoA and homeostasis of sterols, glycerolipids and sphingolipids are regulated by MTDIA and meu1 $\Delta$ 108

3.3.8 Cell cycle and DNA repair proteins are regulated by MTDIA and meu1 $\triangle$

3.4 Discussion 114

\section{Chapter 4 - Elucidating the therapeutic mechanisms of PNP inhibitor forodesine via} assessment of fitness of non-essential gene deletions.............................................. 119

4.1 Introduction .119

4.2 Experimental procedures .120

4.2.1 Yeast strains, media components and compounds 120

4.2.2 PNP1 Synthetic Genetic Array (SGA) 
4.3.1 SGA analysis identifies genetic interactions with the yeast PNP1 target of forodesine 124

4.3.3 Minimal media identifies conditions where PNP1 is essential

4.3.4 PNP1 inhibitors are variably potent in yeast due to non-essential functionality of PNP1

4.3.5 Forodesine sensitivity is increased with deoxycytidine and deoxyguanosine

4.3.6 Mitochondrial accumulation of dGTP is insufficient to inhibit yeast growth 136

4.3.7 MTA exacerbates the forodesine growth defect 137

4.3.8 Nicotinic acid depletion does not exacerbate the growth defect of pnp1 $\Delta$ 139

4.3.9 Forodesine does not sufficiently inhibit yeast PNP1 142

4.4 Discussion 143

Chapter 5 - Implications and future directions 149

5.1 Summary 149

5.2 Implication for MTDIA as a cancer therapeutic 150

5.3 Implication for MTDIA in treating lysosomal storage disorders and other disease with defective autophagy 151

5.4 Implications of Vps34 in TORC1-dependent and independent autophagy 151

5.5 Implications of yeast ergosterol synthesis inhibition on sterol synthesis in mammals 
7.2.1 meu1 $\Delta$ mutant construction 180

7.2.2 Genetic interactions with MEU1 181

7.2.3 MTDIA screening of the gene deletion library 184

7.2.4 Drug-gene interactions and GO enrichment MTDIA 184

7.2.5 MTDIA treatment induces autophagy 190

7.3.1 yCG640 and yCG643 construction 191

7.3.2 GFP proteins localised to subcellular structures

7.3.3 Changes in either protein localisation or abundance with $100 \mathrm{nM}$ MTDIA or in meu1 $\Delta$ and Go enrichment

7.3.4 Go analysis of >2-fold upregulated or downregulated gene transcripts from Basu et al. 2011.

7.4.1 pnp1 $\Delta$ mutant construction. 223

7.4.2 Genetic interactions with PNP1 in complete and minimal media 224

\section{List of Figures}

Figure 1.1 - Polyamine biosynthesis in mammals

Figure $1.2-5^{\prime}$-methylthioadenosine phosphorylase in the salvage of methionine and polyamine synthesis.

Figure 1.3 - Transport and metabolism of nucleosides in humans

Figure 1.5 - Nucleoside metabolism is conserved from S. cerevisiae to humans

Figure 1.6 - Polyamine biosynthesis and methionine salvage pathways are conserved from yeast to humans. .25

Figure 2.3.1 - Growth of gene deletions with the MEU1 deletion..... .40 
Figure 2.3.2 - 52 negative and 2 positive genetic interactions with Meu1

Figure 2.3.3 - hENT1 permits the uptake of adenosine nucleosides and analogues

Figure 2.3.4 - MTDIA is a potent inhibitor of yeast MTAP and cytostatic when methionine salvage is essential .47

Figure 2.3.5 - MTDIA reduces growth by inhibiting Meu1 dependent salvage of methionine and is growth inhibitory in agar 52

Figure 2.3.6 - Gene deletion strains hypersensitive or resistant to 3 nM MTDIA..............................54

Figure 2.3.7a-61 gene deletion strains were hypertensive to MTDIA .57

Figure 2.3.7b - 29 gene deletion strains were resistant to MTDIA .58

Figure 2.3.8.1 - MTDIA treatment and meu1 $\Delta$ decreases chronological lifespan and exacerbates the growth defect induced by rapamycin

Figure 2.3.8.2 - Protein levels and localisation of core autophagy proteins in response to MTDIA treatment although the gene deletions do not show altered sensitivity 65

Figure 2.3.8.3 - MTDIA treatment or meu1s induces autophagy that is Atg1, Atg4 and Pep4 dependent. 68

Figure 3.3.1 - Global analysis of protein relocalisation and abundance in response to MTDIA and its genetic mimic meu1 $\Delta$ highlights mislocalisation of plasma membrane/ER proteins to vacuole. .85

Figure 3.3.2 - Proteins of central carbon metabolism that either increased or decreased in abundance or relocalised with MTDIA and meu1D .90

Figure 3.3.3 - Central carbon metabolism and proteins levels altered with meu1D or MTDIA. .92

Figure 3.3.4 - Methionine salvage was increased with MTDIA and meu1D. .93

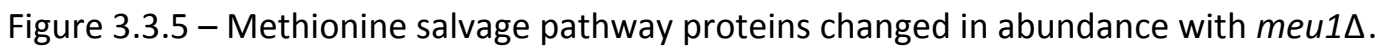
.95

Figure 3.3.6 - Proteins regulating gene expression, transcription and chromatin organisation changed in abundance with MTDIA and meu1 $\Delta$. .96

Figure 3.3.7 - Proteins regulating translation were downregulated in response to MTDIA or meu1 $\Delta$. 102

Figure. 3.3.8 - MTDIA and meu1 $\Delta$ deregulates membrane proteins 106

Figure. 3.3.9 - Vesicle-mediated transport proteins that were deregulated with MTDIA and meu1A.

Figure. 3.3.10 - MTDIA and meu1 $\Delta$ disrupts lipid homeostasis and cell wall synthesis. 
Figure. 3.3.11 - MTDIA disrupts cell cycle and DNA repair mechanisms

Figure 4.3.1a - Growth of gene deletion strains impacted by PNP1 deletion................................127

Fig. 4.3.1b- 53 negative and 5 positive genetic interactions with PNP1 .......................................128

Figure 4.3.2 - Tryptophan biosynthesis from chorismate is required for growth in the absence of PNP1 in minimal media

Figure 4.3.3 - Forodesine treatment or loss of PNP1 does not cause a growth defect in yeast.

Figure 4.3.4 - dCK expression improves growth inhibition by forodesine, and pnp1 $1 \Delta$ confers resistance.

Figure 4.3.5 - Expression of HSV-TK exacerbates the growth inhibition by deoxyguanosine and forodesine

Figure 4.3.6 - MTA-mediated growth exacerbation is not mediated through inhibition of Pnp1.....139

Figure 4.3.7 - The growth defect of pnp1s to dGuo is not further exacerbated in the absence of tryptophan and nicotinic acid

Figure 4.3.8 - Forodesine does not exacerbate the growth defect of gene deletions that are synthetic sick or synthetic lethal with pnp1 1. 143

Figure 7.2.1 - meu1 $\Delta$ construction and validation PCRs 180

Figure 7.2.2 - Absence of adenine improves sensitivity MTDIA. 184

Figure 7.2.3 - Vps34-GFP in WT and meu1 $\Delta$ in methionine replete complete minimal media 191

Figure 7.3.1 - YCG640 and YCG643 construction. 192

Figure 7.3.2 - GFP proteins localised to subcellular structures...... 193

Figure 7.3.3 - Relocalisation GIn1-GFP to cytoplasmic foci with MTDIA and in meu1 $\Delta$. 217

Figure 7.4.1 - pnp1 $\Delta$ construction and validation PCRs 223

\section{List of Tables}

Table 2.2.1 - Yeast strains used in this chapter 29

Table 3.2.1 - Yeast strains used in this chapter. .77

Table 3.2.2 - Expected phenotypes for WT (YCG640) in random spore analysis .81 
Table 4.2.1 - Yeast strains used in this chapter.

Table 7.2.1 - meu1 $\Delta$ construction and conformation primers.................................................. 180

Table 7.2.2a - 31 putative genetic interactions with MEU1 ........................................................ 181

Table 7.2.2b - 52 negative and 2 positive genetic interaction with Meu1 ...................................... 183

Table 7.2.3a - 52 putative drug-gene interactions with 3nM MTDIA .............................................185

Table 7.2.3b - 62 validated gene deletions hypertensive to 3 nM MTDIA .....................................187

Table 7.2.3c - 30 validated gene deletion strains were resistant to MTDIA ....................................189

Table 7.2.4 - GO enrichment of validated MTDIA hypersensitive strains.......................................189

Table 7.2.5 - Genes that were unviable in MTA as the only sulphur source. ..................................190

Table 7.2.6 - FIC index for rapamycin and MTDIA in combination ...............................................190

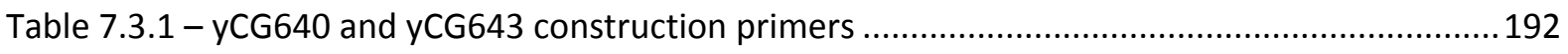

Table 7.3.2 - Proteins with change in either localisation or abundance with $100 \mathrm{nM}$ MTDIA or with

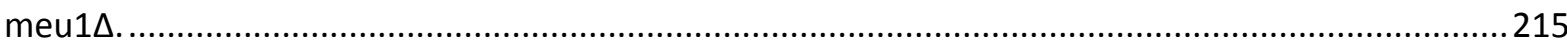

Table 7.3.3 - GO Enrichment for proteins affected by MTDIA treatment .......................................216

Table 7.3.4 - GO enrichment for proteins affected by meu1 $\Delta$.....................................................216

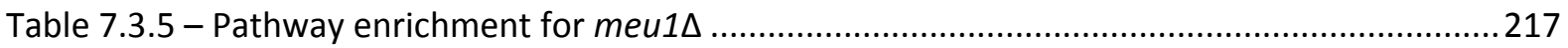

Table 7.3.6 - GO analysis of >2-fold upregulated or downregulated gene transcripts from Basu et al.

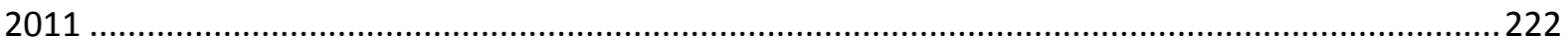

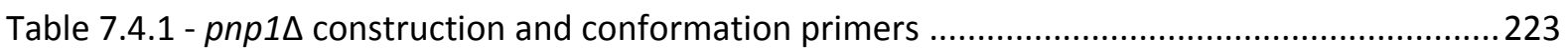

Table 7.4.2a -42 putative genetic interactions with PNP1 gene in synthetic complete media ........225

Table 7.4.2b - 53 negative and 5 positive genetic interactions with PNP1 ...................................227

Table 7.4.3 - 47 putative genetic interactions with PNP1 gene in minimal media ..........................228 


\section{Chapter 1: Literature review}

\subsection{Burden of cancer and current cancer therapies}

\subsubsection{Burden of cancer}

Cancer is the leading cause of death in both economically developed and developing countries (Jemal et al., 2011). In 2012, there were 14.1 million new cases of cancer world-wide which contributed to 8.2 million estimated deaths with lung, liver, stomach and colorectal cancers being the major causes of cancer related deaths (Torre et al., 2015). The number of new cases of cancer is expected to rise to 21.6 million and 13 million deaths annually by 2030 due to growth and increased lifespans of the current population (Ferlay et al., 2015). On average $\$ 5.6$ billion is spent every year on cancer research by National Institutes of Health, which is greater than the NIH budget for cardiovascular disease, infectious disease and Alzheimer's disease combined ("NIH Categorical Spending -NIH Research Portfolio Online Reporting Tools (RePORT)," 2015). The current consensus is that a large fraction of these deaths can be prevented by better management of cancer risk factors, early detection, physical activity and healthy dietary practises.

Cancer is the collective name given to a group of related diseases leading to development of an abnormal cell mass by uncontrollable cell division, replicative immortality and resistance to cell death (Perez-Herrero \& Fernandez-Medarde, 2015). It is a result of both genetic and environmental factors. The development of cancer is a multi-step process and is a result of gain of function mutations to proto-oncogenes that promote cell proliferation and differentiation as well as loss of function mutations in tumour suppressor genes that promote growth inhibition, arrest and cell death (Hanahan \& Weinberg, 2000). These changes are further accentuated by changes to cell energy metabolism and immune evasion (Hanahan \& Weinberg, 2011). Clonal expansion of these cells with unregulated cell division eventually leads to genetic instability and different evolutionary pressure from the tumour environments, which together result in multiple mutations and tumour heterogeneity (Marusyk et al., 2012). The access to vasculature spreads the cancerous cells throughout the body leading to development of new and diverse tumours away from the primary site (Hanahan \& Weinberg, 2011). 
The spread of cancer into vital organs leads to gradual loss of organ function and degeneration of health like other age-related pathologies resulting in death (Cooper, 2000).

\subsubsection{Current cancer therapies, undesired effects and resistance}

There are several approaches to manage and treat cancers. If diagnosed early, cancer is treatable either by surgical removal and radiotherapy that are often applied to localised tumours and are the most effective treatment for cancer (Perez-Herrero \& Fernandez-Medarde, 2015). Upon metastasis, the treatment of cancers requires the use of small molecular inhibitors or monoclonal antibodies capable of reaching every organ via the circulatory system (Perez-Herrero \& FernandezMedarde, 2015). The most readily used approach to treat metastatic cancers is chemotherapy and it often targets rapidly dividing cells. It uses cytotoxic compounds to inhibit processes in the cell division cycle such as DNA replication with either DNA alkylating agents (cisplastin, temozolomide), topoisomerase II with inhibitors (adriamycin, doxorubicin) and nucleoside analogues (gemcitabine, 5FU), and cell division machinery with inhibitors of microtubule dynamic (paclitaxol, nocodazole) (Zhao et al., 2013). Targeting these key cellular processes essential to all cells with chemotherapeutics leads to numerous undesired side effects by its toxic action on normal cells. As a consequence, cancer sufferers have to endure unwanted side effects of chemotherapy that can range from minor discomforts such as fatigue, vomiting and nausea to more severe side effects such as myelosuppression and organ damage which can be life threatening and a hindrance to continuation of chemotherapy (Attar et al., 2000). Chemotherapy can also cause permanent damage to nerves, lead to sterility and in rare occasions lead to development of other types cancers (Park, 2014). Some of these unwarranted side-effects are managed by administering cytoprotectants such as dexrazoxane and amifostine (Hensley et al., 2009). To reduce these side effects, novel approaches are being developed to specifically target cancerous cells and are known collectively as targeted therapy.

Molecularly targeted therapy from here on is referred to as targeted therapy, which unlike chemotherapy, exploits the differences in cancer cell metabolism. There are several targeted therapies available for cancer treatment including hormone therapies, signal transduction inhibitors 
(tyrosine kinase and mTOR inhibitors), gene expression modulators, apoptosis inducers, angiogenesis inhibitors, and toxin delivery molecules (https://www.cancer.gov/aboutcancer/treatment/types/targeted-therapies/targeted-therapies-fact-sheet, $\quad 2016$ ). Targeted therapies are deliberately designed based on interactions with a desired molecular target that is either differentially expressed or altered in the cancer cell. The fusion protein Bcr-Abl kinase or mutant allele BRAFV600E are examples of such targets that are expressed only in cancer cells (An et al., 2010; Cantwell-Dorris et al., 2011). Most targeted therapies modulate a cellular process that cancer cells depend on for cell proliferation such as polyamine and purine biosynthesis inhibitors investigated in this dissertation. Other promising alternatives to chemotherapy and targeted therapy include immunotherapy that uses engineered T-cell therapy, cancer vaccines (e.g. Sipuleucel-T (Anassi \& Ndefo, 2011)), oncolytic viruses (e.g. talimogene laherparepvec (Rehman et al., 2016)) and small molecule immune check-point inhibitors (anti-CTLA-4 Ipilimumab, anti-PD-1 nivolumab (Boutros et al., 2016)) to improve cancer cell recognition and boost the immune system to eliminate the cancer.

Kinase inhibitors are one of the most pursued small molecules for targeted cancer therapy in the recent decade. There are over 900 human kinases that catalyse the transfer of $\gamma$-phosphate group of ATP to target proteins to initiate signal transduction required for an array of cellular processes including cell proliferation, survival and differentiation (Wu et al., 2015). BCR-Alb kinase inhibitors (imatinib and dasatinib), ErbB inhibitors against EGFR and HER2 kinases (lapatinib) and multiple kinase inhibitor (vandetanib) against EGFR, VEGFR and RET, lipid kinase inhibitor (idelalisib) and serine/threonine kinase B-Raf inhibitors (vemurafenib and dabrafenib) are some of the commonly used kinase inhibitors (Wu et al., 2015). In addition to treatment of tumours, kinase inhibitors are also effective against inflammatory diseases, central nervous system disorders, cardiovascular diseases (Wu et al., 2015). From the success of tyrosine kinase inhibitors, several other small molecular inhibitors that modulate the activity of proteins in cancer have become the focus of attention. One such class of small molecules that has gained recent attention is transition state structure inhibitors. 


\subsubsection{Transition state structure analogues as antitumor therapeutics}

Transition state structure analogues are chemically stable structures that by design resemble an enzymatic transition state of a substrate that has a lifetime in femtoseconds (Schramm, 2013b). The enzymatic transition state structures are determined by kinetic isotope effects (KIEs) of labelled enzymes and reactants, combined with computational chemistry and the transition state structure analogues, are synthesised to closely match the Van der Waal's geometry and electrostatic features of transition state structures (Schramm, 2011; Schramm, 1999). By resembling a stable transition state structure, transition state analogues lower the activation barrier that is required for the reaction with the enzyme and bind more tightly than the substrate in the ground state (Wolfenden \& Radzicka, 1991). For some transition state structures, a single substituent is responsible for reaction with an enzyme and changing that one substituent can cause a severe decrease in binding affinity (Grubmeyer et al., 1989). Others may involve interactions with many substituents and these can be modified in the transition state analogue to further match the environment of the binding pocket in improve the binding affinity and specificity for the enzyme (Janes \& Schulz, 1990). Transition state analogues are among the most powerful chemical inhibitors of enzymes discovered to date (Schramm, 2011).

Formycin, an inhibitor of AMP nucleoside hydrolase, is a notable transition state structure analogue that was determined by analysis of KIEs (DeWolf et al., 1979). Several other transition state structure analogues have since been developed by analysis of KIEs. Notable of these are purine biosynthesis and polyamine biosynthesis inhibitors designed by the laboratory group of Professor Vern Schramm (Albert Einstein College of Medicine) and synthesised by members of the Ferrier Institute (Victoria University of Wellington) (Evans et al., 2004; Evans et al., 2003). Methylthio-DADMeImmucillin A (MTDIA) is an inhibitor of polyamine biosynthesis and a promising preclinical anticancer agent that is evaluated for therapeutic mechanisms in chapters 2 and 3. Immucillin $\mathrm{H}$ (forodesine) is an inhibitor of purine nucleoside biosynthesis for which therapeutic investigations were investigated in chapter 4 and was approved for treatment of refectory leukaemia in Japan during the writing of this dissertation. 


\subsection{Polyamine biosynthesis and MTAP inhibition as target for cancer therapy}

\subsubsection{Polyamine biosynthesis in mammals}

Putrescine, spermidine and spermine are polyamines that are nucleophilic polycationic molecules found in all eukaryotic cells, which cumulatively comprise $7-10 \%$ of all cellular content (Gugliucci, 2004). The structure and sheer volume of polyamines results in interactions with many negatively charged molecules including DNA, RNA and proteins (Minois et al., 2011), which associate polyamines with many important cellular processes. These include DNA replication, transcription and translation as well as affecting the activity of many kinases and phosphatases to modulate cell growth, proliferation and survival (Minois et al., 2011).

The levels of polyamines are tightly regulated through synthesis, catabolism and transport (Fig. 1.1) (Pegg, 2009). Ornithine decarboxylase (ODC) is the first enzyme in the polyamine biosynthesis pathway and it catalyses the conversion of ornithine to putrescine (Woster, 2006). ODC is rapidly degraded by ODC-antizyme and the $26 \mathrm{~S}$ proteasome to modulate the levels of putrescine (Murakami et al., 1992; Kahana et al., 2005). Putrescine is then converted to spermidine by aminopropyltransferase spermidine synthase, which is subsequently converted to spermine by another closely related aminopropyltransferase spermine synthase (Woster, 2006). 5'methylthioadenosine is produced as a by-product during spermine and spermidine synthesis from the co-substrate decarboxylated S-adenosyl methionine. $5^{\prime}$-methylthioadenosine is a potent inhibitor of the aminopropyl transfer process and must be rapidly hydrolysed by 5 'methylthioadenosine phosphorylase (MTAP) to maintain the forward reaction (Raina et al., 1984; Pajula et al., 1979b). Further control of polyamine levels are exerted through acetylation of spermidine and spermine by spermidine/spermine-N1-acetyltransferase (SSAT) and subsequent oxidative deamination by acetylpolyamine oxidase (PAO) (Woster, 2006). Spermine is also converted back to spermidine by spermine oxidase (SMO). SMO and SSAT/PAO provide interconversion of polyamines to maintain the appropriate levels of each of the polyamines (Pegg, 2009). Several polyamine transport mechanisms 
also exist and have been elucidated in several organisms (Abdulhussein \& Wallace, 2014).

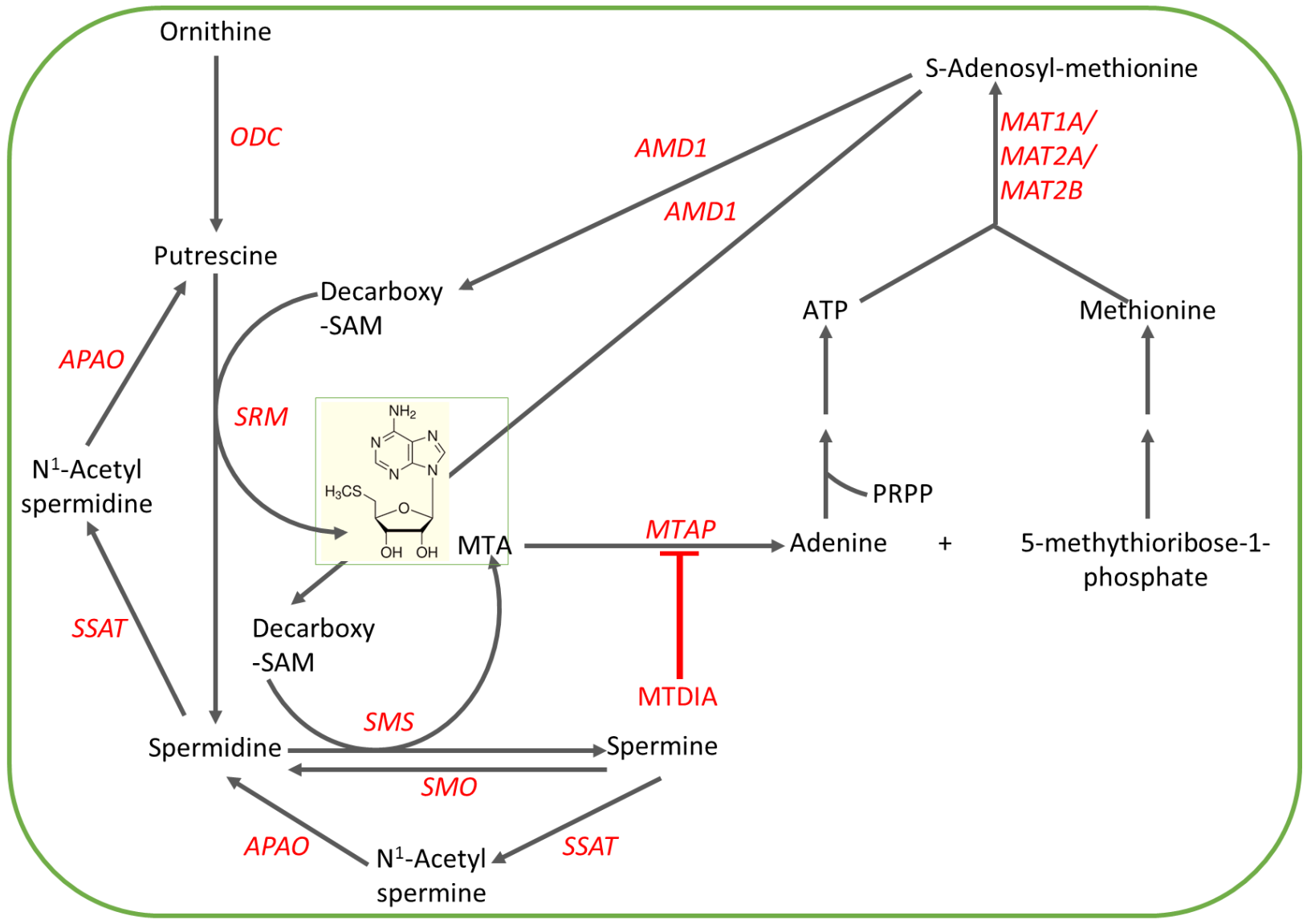

Figure 1.1 - Polyamine biosynthesis in mammals. ODC, ornithine decarboxylase; SRM, spermidine synthase; SMS, spermine synthase; SMO, spermine oxidase; APAO, acetylpolyamine oxidase; SSAT, spermidine/spermine-N1-acetyltransferase; AMD1, S-adenosylmethionine decarboxylase; MAT1A, MAT2A, MAT2B, S-adenosylmethionine synthetase; MTAP, 5'-methylthioadenosine phosphorylase; MTDIA, MT-DADMe-immucillin A.

\subsubsection{Polyamine biosynthesis as a target for cancer}

Although polyamine biosynthesis is highly characterised, the cellular mechanisms by which different polyamines exert their function have not been completely elucidated. It is well established that they are required for normal cellular homeostasis (Woster, 2006). The depletion of polyamine levels are observed with ageing and impressively, supplementation with putrescine and spermidine increases cellular survival (Vivó et al., 2001; Minois et al., 2011). In yeast, chronological ageing leads to an accumulation of acetic acid that induces apoptosis like phenotype, which can be alleviated by exogenous spermidine (Kaeberlein, 2009). Likewise, the polyamine system is highly upregulated in 
various human diseases such as cystic fibrosis, neurodegenerative disease including Parkinson's and Alzheimer's to cancers, carcinoma, melanoma and lymphoma, and depletion of polyamines induce quiescence and apoptosis (Wallace \& Fraser, 2004; Minois et al., 2011). Thus, presents a target for cancer therapy.

Several steps in polyamine biosynthesis, transport and metabolism have been targeted by inhibitors with varying therapeutic anti-cancer effects from quiescence to cytotoxicity. The catalyst of the first and rate-limiting step in polyamine biosynthesis (ODC) was the first to be targeted and $\alpha$ difluoromethylornithine (DFMO) has been the most effective and the only FDA approved inhibitor of ODC to date (Metcalf et al., 1978). The rapid turnover of ODC coupled with poor transport leads to a requirement of high DMFO concentrations to maintain ODC inhibition (Casero \& Marton, 2007; O'Shaughnessy et al., 1999). Subsequent synthesis of other symmetrically and unsymmetrically substituted alkylpolyamines capable of inhibiting the ODC such as AdoMet-DC (5'-\{[(Z)-4-amino-2butenyl]methylamino\}-5'-deoxyadenosine) as well as spermidine and spermine aminopropyltransferases (S-adenosyl-1,8-diamino-3-thiooctane and S-adenosyl-1,12-diamino-3-thio9-azadodecane respectively) have been generated (Tang et al., 1981; Woster et al., 1989). Some but not all alkylpolyamines induce cell-type specific induction of SSAT expression, in some cases more than a 1000-fold (Woster, 2006). The subsequent generation of hydrogen peroxide by deamination activity of PAO is thought be the mechanism by which these compounds induce cytotoxicity (Ha et al., 1997). The cause of toxicity in non-SSAT inducing alkylpolyamines is yet to be determined.

\subsubsection{MTAP in methionine salvage and synthesis of S-adenosyl methionine}

Methylthioadenosine phosphorylase (MTAP) encodes the initial enzyme in the methionine salvage pathway. It catalyses the conversion of $5^{\prime}$-methythioadenosine (MTA) that is generated as a byproduct of polyamine biosynthesis to adenine and methythioribose-1-phosphate (Avila et al., 2004). 5'-methythioribose-1-phosphate is further processed in subsequent reactions to salvage methionine (Backlund \& Smith, 1981; Finkelstein, 1990). Methionine is also re-synthesised from homocysteine by betaine-homocysteine methyltransferase and 5-methyltetrahydrofolatehomocysteine 
methyltransferase (Thomas et al., 2000). Homocysteine is generated primarily by metabolism of sadenosyl homocysteine but is also generated from various other amino acid biosynthetic processes (Fig. 1.2). Methionine and adenine generated during the polyamine biosynthesis pathway are further metabolised to generate S-adenosyl methionine, the primary methyl donor in methylation reactions. S-adenosyl methionine is the most widely used enzyme substrate after ATP and is a downstream product of MTA metabolism (Cantoni, 1975). In addition to serving as a source of polyamine for the synthesis of spermidine and spermine, S-adenosyl methionine also serves as an amino group for biotin

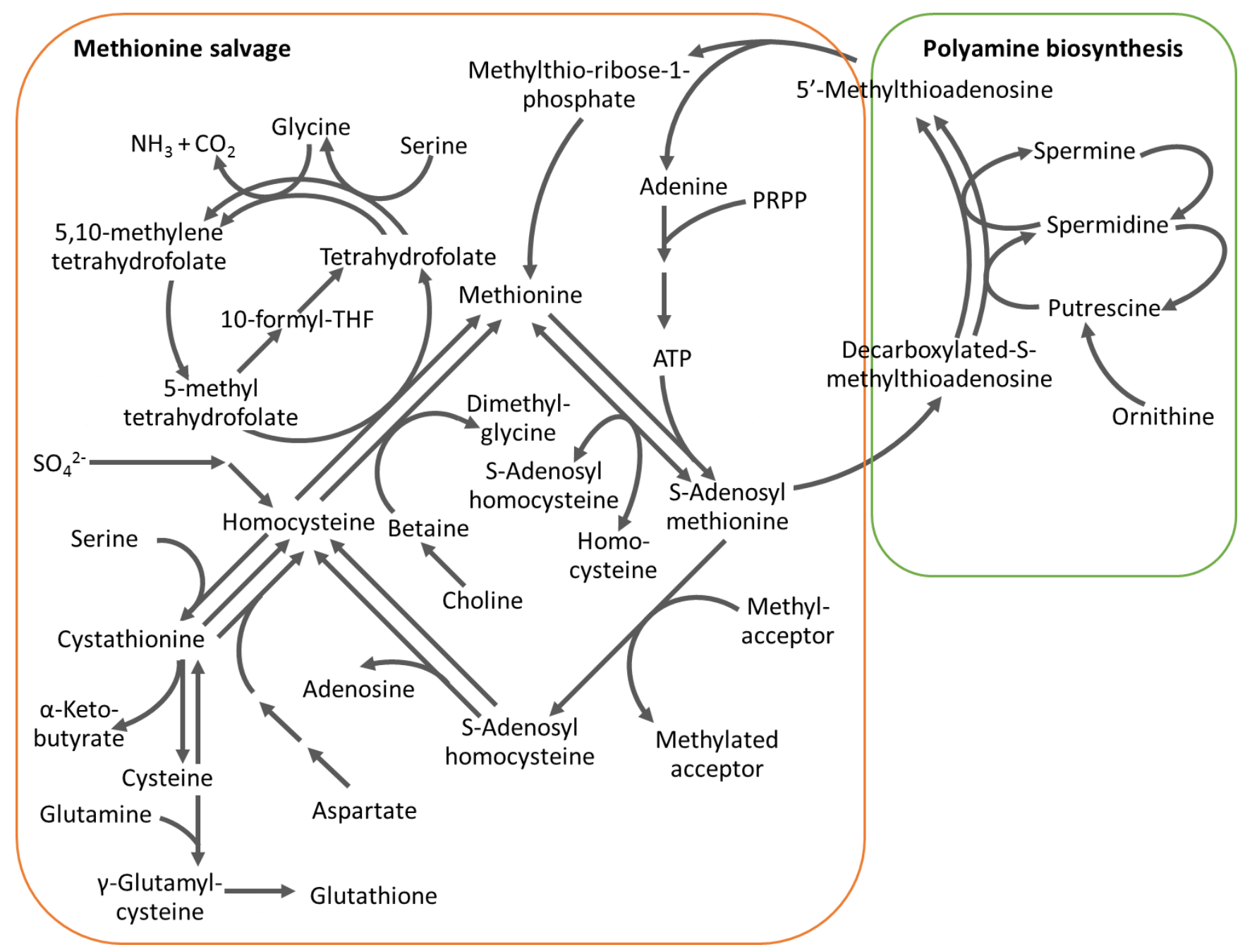

Figure $1.2-\mathbf{5}^{\prime}$-methylthioadenosine phosphorylase in the salvage of methionine and polyamine synthesis. Pathway is compiled from sources KEGG, SGD and Kanehisa et al. (Tripodi et al., 2015; Cherry et al., 2012; Kanehisa \& Goto, 2000). PRPP, 5-phosphoribosyl-1-pyrophosphate. 
synthesis (Stoner \& Eisenberg, 1975; Hall \& Dietrich, 2007), a ribosyl groups for post-translational modification of tRNA (Iwata-Reuyl, 2003), a methylene donor for synthesis of cyclopropane fatty acids (Taylor \& Cronan Jr, 1979), a source for re-synthesis methionine and glutathione formation (Lu, 2000) and a source for generation of $5^{\prime}$-deoxyrybosyl radicals from reaction with iron sulphur clusters containing metalloenzymes (Cheek \& Broderick, 2001). More importantly, 90\% of S-adenosyl methionine (AdoMet) is used in methyltransferase reactions where AdoMet acts as a methyl donor for an array of molecules including nucleic acids, proteins and lipids (Tehlivets et al., 2013). S-adenosyl methionine is therefore an important molecule generated by polyamine biosynthesis. During methylation, S-Adenosyl homocysteine is generated as an inhibitor of methyltransferase reactions involving S-adenosyl methionine and is therefore rapidly metabolised to adenosine and homocysteine (Finkelstein, 1990).

\subsubsection{MTAP as a target for cancer}

Although numerous inhibitors of polyamine biosynthetic pathway have been generated, the recycling of the toxic by-product 5'-methylthioadenosine by 5'-methythiodenosine phosphorylase (MTAP) has not been investigated as a target for therapeutic intervention until recently (Basu et al., 2007). MTAP is a tumour suppressor located in the chromosome 9p21-22 region (Carrera et al., 1984). Activity of MTAP is lost in numerous human cancers either through methylation of its promoter or is co-deleted with flanking tumour suppressors (e.g., CDKNA2 that encodes P15, p16 and p19) and interferon genes (Cairns et al., 1995; Chen et al., 1996). MTAP deficiency is common in numerous solid tumours including pancreatic, lung, endometrial, metastatic melanoma, oesophageal carcinoma, biliary tract cancer, gastrointestinal stromal tumours, soft tissue and osteosarcoma, gliomas, and in hematologic malignancies including adult T-cell leukemia, B-lineage ALL, T-cell acute leukemia, diffuse large cell lymphoma and mantle cell lymphoma (Bertino et al., 2011).

Ectopic MTAP expression in MTAP negative cancers led to inhibition of anchorageindependent growth as measured by colony formation on soft agar plates and suppression of tumour formation in SCID mice in both the fibrosarcoma cell line HT1080 and the breast adenocarcinoma cell 
line MCF-7 (Christopher et al., 2002; Tang et al., 2015). Decreases in levels of all three major polyamines and the ratio of putrescine to total polyamines were observed following MTAP reexpression for MCF-7 cells but not HT1080 cells. The ODC inhibitor DFMO inhibited anchorageindependent growth of MTAP negative MCF-7 cells but not MTAP negative HT1080 cell (Christopher et al., 2002; Tang et al., 2015). In contrast, expression of catalytically inactive MTAP in HT1080 cells inhibited anchorage-independent growth and promoted wound healing to similar levels of catalytically active MTAP but not in MCF-7 cells (Christopher et al., 2002; Tang et al., 2015). These results suggest that MTAP exerts its tumour suppressor activity via at least two independent mechanisms: the enzymatic mechanism that involves processing of MTA and the non-enzymatic mechanism that was observed in HT1080 cells.

\subsubsection{MTDIA: transition state analogue of MTA}

Methylthio-DADMe-Immucillin A (MTDIA) is a transition state structure analogue of $5^{\prime}$ methylthio adenosine (MTA) bound by the human enzyme 5'-methythio adenosine phosphorylase (MTAP). MTDIA is one of most potent inhibitors of MTAP generated by transition state structure analysis and is the most characterised (Singh et al., 2004). It is a $86 \mathrm{pM} K_{i}^{*}$ inhibitor of human MTAP in vitro (Schramm, 2011). It is currently preclinical with proven in vivo efficacy in mouse xenografts models for human lung, prostate, colon, cervical, head and neck, and triple negative breast cancers, as well as in vitro efficacy in various human cancer cell lines (Basu et al., 2007; Basu et al., 2011). MTAP inhibition by MTDIA leads to an accumulation of MTA, which inhibits polyamine biosynthesis (specifically the syntheses of putrescine, spermidine and spermine) and consequently the recycling of S-adenosyl methionine (Basu et al., 2011) (Fig. 1.1). One or more of these processes are responsible for subsequent cellular processes such as $\mathrm{G} 2 / \mathrm{M}$ arrest, loss of mitochondrial membrane integrity and cleavage of PARP and caspases resulting in apoptosis (Basu et al., 2011). The initiation of apoptosis however is cell type specific as normal fibroblast cells do not undergo apoptosis nor activate caspases, PARP or other cellular alterations observed with increased levels of MTA (Basu et al., 2007). The 
genetic mechanisms underlying the initiation of these processes are not understood and are the subject of this dissertation.

\subsection{Purine biosynthesis as a target for cancer therapy}

\subsubsection{Structure and function of purine nucleoside phosphorylase (PNP) in humans}

Purine nucleoside phosphorylase (PNP) is a ubiquitous protein belonging to the nucleoside phosphorylase-I family and is an enzyme of the purine salvage pathway (Parks Jr \& Agarwal, 1972). It is a $90 \mathrm{kDa}$ enzyme that exits as homotrimer in the cytosol for which the crystal structure has been solved (Toro et al., 2006). Each subunit consists of a disoriented $\beta$ structure flanked by seven $\alpha$ helices with the catalytic sites found between the subunit-subunit boundaries (Canduri et al., 2005). PNP in the $\beta$ structure configuration is specific for purine nucleosides. PNP is differentially expressed in tissues and is most abundant in peripheral lymphocytes and granulocytes (Toro et al., 2006; Carson et al., 1977). It reversibly metabolises purine nucleosides deoxyguanosine, deoxyinosine, guanosine, and inosine to their nucleobases and 2'-ribose (or deoxyribose) -1 -phosphate (Kim et al., 1968). Guanine and hypoxanthine are phosphorylated to GMP and IMP by hypoxanthine-guanine phosphoribosyltransferase (HGPRT) and subsequently to GTP and incorporated into RNA. Alternatively, the purine nucleobases can be metabolised by xanthine oxidase and excreted as uric acid (Toro et al., 2006).

A genetic deficiency of PNP was first identified in 1975 in a five year old child presenting with T-cell lymphopenia and erythrocytes lacking the PNP enzyme activity (Giblett et al., 1975). The mutation was later characterised as a consequence of a homozygous 265G >A mutation in exon 3 of the PNP gene resulting in a Glu89Lys amino acid substitution (Williams et al., 1987). The genetic deficiencies in the purine salvage pathway is one of the common causes of autosomal recessive Severe Combined ImmunoDeficiency (SCID) (Hirschhorn et al., 2013). In addition to the characteristic loss of T-cells with age, some SCID cases also present with neurological defects, bone marrow development abnormalities, poor humoral immunity and autoimmunity (Hirschhorn et al., 2013). 
Loss of PNP function leads to accumulation of the nucleoside substrates of PNP in all tissue types, body compartments, plasma and urine (Chantin et al., 1996; Hershfield \& Mitchell, 1995). Of these deoxyguanosine has an alternative fate where it is phosphorylated to dGMP by deoxynucleoside kinases and becomes trapped within the cell. dGMP is subsequently phosphorylated to dGTP by two non-rate limiting nucleotide kinases leading to pathologically elevated levels dGTP (Cohen et al., 1978). It is the accumulation of dGTP that is toxic to T-cells. The accumulation of dGTP leads to the disruption of the dNTP pool and inhibition of DNA synthesis leading to an induction in apoptosis (Balakrishnan et al., 2006). The loss of PNP activity also leads to depletion of GTP and uric acid levels (Wortmann et al., 1979). Conversely, the accumulated dGMP can be catabolised back to deoxyguanosine by 5'-nucleotidase. These enzymes provide important balance to the dGTP levels.

\subsubsection{PNP inhibitors and other deoxyguanosine analogues to treat T-cell activation}

The importance of PNP and deoxyguanosine (dGuo) in T-cell development from the literature just reviewed on T-cell lymphopenia reveals opportunities for regulation of diseases characterised by T-cell proliferation. Several PNP inhibitors and deoxyguanosine analogues that elevate dGuo in plasma have been assessed in vivo in animal models and in patents in clinical trial (Ravandi \& Gandhi, 2006). Well known PNP inhibitors allopurinol, 6-mercaptopurine, 6-methoxypurine and acyclovir showed poor affinity for human and bovine PNP and poor growth inhibition in cell lines (Krenitsky et al., 1968; Tuttle \& Krenitsky, 1984). From these early discovered inhibitors it was observed that $>95 \%$ continuous inhibition of PNP is required for sufficient dGTP accumulation to abrogate T-cell function and proliferation (Gandhi, 2009; Morris \& Montgomery, 1998). The C-8 substituted purine analogues (8-aminoguanosin and 8-iodoguanosine) and the dGuo analogue 8-amino-9-(2-thienylmethyl) guanine showed potent activity against T-cells in primary cell cultures but not in in animal models (Gilbertsen et al., 1987; Kazmers et al., 1981). The subsequent, N-7 substitutions of inosine and guanosine were then developed based on the crystal structure of PNP, which resulted in clinical trials of BCX-34 (peldesine) that demonstrated limited activity as it failed to achieve $>95 \%$ continuous inhibition of PNP (Viegas et al., 2000; Ravandi \& Gandhi, 2006). 
Following the partial success of these inhibitors, a series of 9-deazanucleotide analogues (termed Immucillins) were designed to mimic the transition state structure of nucleosides inosine and guanosine bound to PNP (Schramm, 2002). Immucillin-H (forodesine) (Kd $\sim 56 \mathrm{pM}$ ) and Immucillin-G ( $\mathrm{Kd} \sim 42 \mathrm{pM})$ are potent inhibitors of human PNP with dissociation constants in the picomolar range (Evans et al., 2003). Forodesine has undergone 14 phase I and II clinical trials to evaluate efficacy, safety, and pharmacokinetics in patients with lymphatic leukaemia and lymphoma (https://clinicaltrials.gov/ct2/results?term=bcx-1777). On 30 March 2017, forodesine was approved for treatment of recurrent/refractory peripheral T-cell lymphoma in Japan (http://www.mundipharma.com.sg/2017/03/30/mundipharma-wins-approval-antineoplastic-agentmundesine-treatment-relapsedrefractory-peripheral-t-cell-lymphoma-japan/, 2017). Nelarabine is another promising deoxyguanosine analogue that causes accumulation of dGTP in plasma. It is a PNPand deoxycytidine kinase (dCK)-resistant prodrug of arabinosyl guanine (AraG) that is converted to the active form by adenine deaminase (Lambe et al., 1995). It is phosphorylated exclusively by deoxyguanosine kinase to generate toxic AraG-GTP and it inhibits the growth of B-cells and monocytes as well as T-cells (Lambe et al., 1995). It has undergone extensive clinical trials and has been approved by the FDA for treatment of patients with T-cell acute lymphoblastic leukaemia and T-cell lymphoblastic lymphoma (Reilly \& Kisor, 2009; Ravandi \& Gandhi, 2006).

1.3.3 High deoxyguanosine kinase, low $5^{\prime}$ nucleosidase activity and nucleoside transport determines Tcell toxicity

Although the PNP activity is not dissimilar between the B and T lymphocytes, the activity of the PNP inhibitors differ drastically between these lymphocytes (Fridland \& Verhoef, 1985). This is attributed to the 2-3-fold greater deoxyguanosine kinase activity and reduced 5'-nucleotidase activity in T-cells leading to pathologically elevated dGTP formation in the presence of inhibitors (Osborne \& Scott, 1983; Madrid-Marina et al., 1993; Kazmers et al., 1981) (Fig. 1.4). The deoxyguanosine phosphorylation is conferred by the cytosolic/nuclear dCK and the mitochondrial deoxyguanosine kinase (dGK) leading to accumulation of dGTP in their respective organelles (Eriksson et al., 2002) (Fig. 
1.3). The dCK can phosphorylate all four deoxyribonucleotides with varying catalytic efficiency, while dGK phosphorylates only deoxyadenosine and deoxyguanosine nucleosides (Arnér \& Eriksson, 1995). dGK can also phosphorylate dCyt but only at $2 \%$ of its efficiency for dGuo. There is some contradiction as to whether it is the dGTP accumulation in the mitochondria or the cytosol that is responsible for the T-cell toxicity. Most nucleoside analogues phosphorylated by dGK are also phosphorylated by dCK

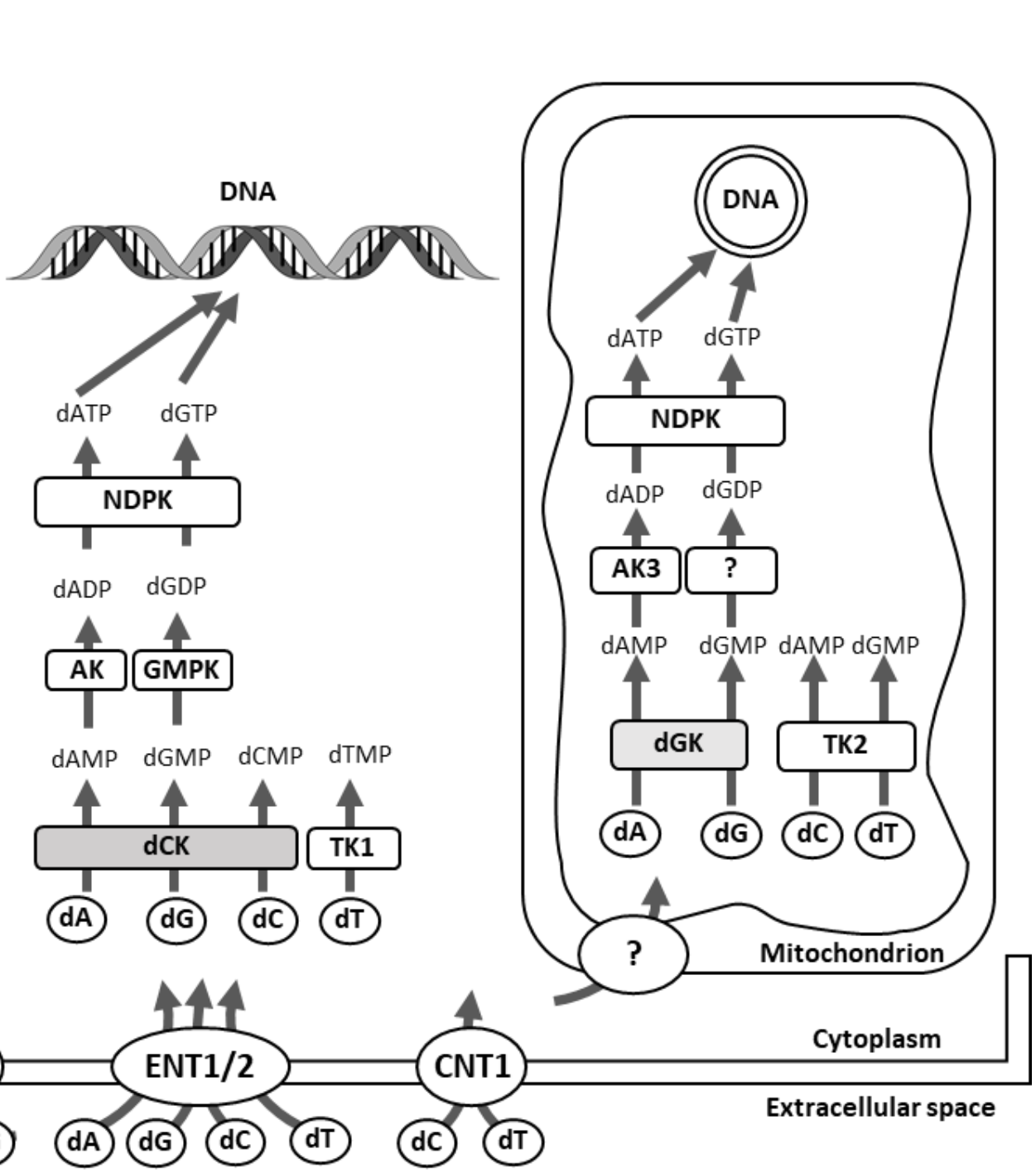

Figure 1.3 - Transport and metabolism of nucleosides in humans. CNTs, concentrative nucleoside transporters; ENTs, equilibrative nucleoside transporters; $d A$, deoxyadenosine; $d G$, deoxyguanosine; dC, deoxycytidine; dT, deoxythymidine; dCK, deoxycytidine kinase; dGK, deoxyguanosine kinase; TK1, thymidine kinase 1; TK2, thymidine kinase 2; AK, adenylate kinase; AK3, adenylate Kinase 3; GMPK, deoxyguanosine monophosphate kinase; NDPK, nucleotide diphosphate kinase. 
with greater efficiency except for AraG and 2-chloro-2'-deoxyadenosine (CdA). dCK is expressed at much higher levels than dGK in actively dividing cells and lymphocytes (Arnér \& Eriksson, 1995) and the mechanism of resistance to these compounds including AraG and CdA have been through downregulation of dCK and not dGK (Curbo et al., 2001). The resistance to deoxyguanosine in the absence of PNP where deoxyguanosine phosphorylase activity is concerned is due to down-regulation of dCK and not dGK (Ullman et al., 1979). In summary, dCK is predominantly responsible for the accumulation of deoxyguanosine monophosphate in T-cells in response to loss or inhibition of PNP activity. In addition to deoxyguanosine phosphorylation, deoxyguanosine transport and the transport of PNP inhibitors are equally important to T-cell toxicity and PNP deficient cells with defective nucleoside transport are resistant deoxyguanosine (Ullman et al., 1979). In T lymphoblast (CCRF-CEM) cells, forodesine and deoxyguanosine entry into the cell is carrier-mediated. The equilibrative nucleoside transporter 1 (ENT1) is predominantly responsible for forodesine transport and ENT2 to a lesser degree (Huang et al., 2008). Deoxyguanosine transport is predominantly mediated by the concentrative nucleoside transporters 2 (CNT2) but is also transported by ENT1 and ENT2 (Huang et al., 2008). In addition, ribonucleotide reductase (RR) resistance to feedback inhibition by dGTP have also been identified and confers resistance to deoxyguanosine (Ullman et al., 1979).

\subsubsection{Proposed mechanisms for forodesine activity in T-cells}

Forodesine (Immucillin H or BCX-1777) is a non-cleavable, transition state structure analogue of inosine bound human PNP and binds human PNP $7 \times 10^{5}$ fold more strongly that its native substrate inosine with a $56 \mathrm{pM} \mathrm{Kd}$ (Schramm, 2004). Forodesine induces apoptosis in activated T-cells at low nanomolar concentrations and lacks activity against resting T-cells and other cell types (Kicska et al., 2001). The proposed mechanism for forodesine mimics the loss of PNP activity. Forodesine and deoxyguanosine treatment of T-acute lymphoblastic leukemia (CEM-SS) cells lead to 154-fold increase in the accumulation of dGTP and an 8-fold increase in dATP accumulation (Bantia et al., 2003). Lamivudine ((-)- $\beta-L-2^{\prime}, 3^{\prime}$-dideoxy-3'-thiacytidine) mediated depletion of dGTP was able to partially rescue the forodesine toxicity in CEM-SS cells, although it led to a further increase in dATP (8-30 fold) 
(Bantia et al., 2003). This suggests that it is the accumulation of dGTP and not dATP that is responsible for the main mechanism of forodesine mediated toxicity. The proposed mechanisms for dGTPmediated toxicity is that it binds and allosterically facilitates the ribonucleotide reductase binding to ADP and conversion to dADP (Ahmad \& Dealwis, 2013). dADP is subsequently converted to dATP, which then binds and inhibits ribonucleotide reductase leading to the depletion of dCTD and dTTD pools and inhibition of DNA synthesis. However, no such disruptions were observed to pools of deoxycytidine and deoxythymidine following forodesine treatment, rather, it lead to an increase in deoxycytidine and deoxythymidine levels (Bantia et al., 2003). Marrow stromal cells treated with forodesine accumulate dGTP to levels similar to chronic lymphatic leukaemia (CCL) cells but without apoptosis (Balakrishnan et al., 2010b). Therefore, the accumulation of dGTP alone is also not sufficient to induce apoptosis and the exact mechanisms leading to apoptosis are not fully understood.

Following initiation of apoptosis, several mechanisms involved in the progression of mitochondria-dependent apoptotic cell death have been characterised in PNP-deficient human and mouse cells. Mitochondrial membrane dissipation and release of cytochrome $\mathrm{C}$ have been identified in CD4+ and CD8+ mouse thymocytes (Arpaia et al., 2000; Papinazath et al., 2011). Nuclear DNA fragmentation has also been identified in these cells. Several changes to proteins also involved in the intrinsic apoptotic pathway were identified such as stabilisation of p53 and activation of downstream p21, activation of caspases 3,8 and 9 , subsequent PARP cleavage and downregulation of antiapoptotic BCL-2 and MCL-1 and upregulation of proapoptotic BIM (Balakrishnan et al., 2006; Alonso et al., 2009). Forodesine-treated cells were also able to complete apoptosis when p53 function was compromised and this was via upregulation of $\mathrm{p73}$ and expression of proapoptotic genes FOXO1 and FOXO3A (Alonso et al., 2009).

In addition to induction of apoptosis, a positive correlation was observed in cells undergoing apoptosis and the phosphorylation of dCK in forodesine-treated CLL cells (Alonso et al., 2009). In addition to the increases in dGTP and dATP, significant decreases in ribonucleotide levels of ATP (20\%) and GTP (50\%) 


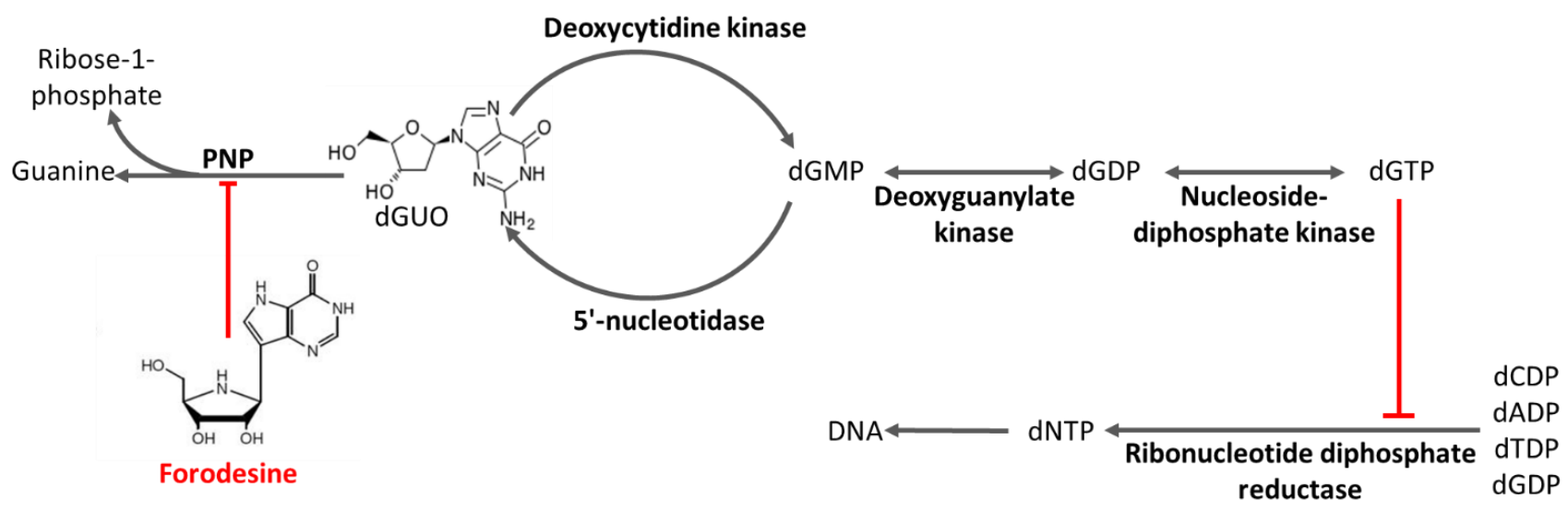

Figure 1.4 - Proposed mechanisms for forodesine and loss of PNP. dGuo, deoxyguanosine.

were observed for forodesine-treated CLL cells. Reduction in both RNA and protein synthesis was also observed with forodesine and dGuo in CCL cells (Balakrishnan et al., 2010b). The nuclear DNA fragmentation observed in PNP-deficient cells was also observed in treatment with a nucleoside analogue that accumulate dATP, deplete NAD+ and ATP pools, confer deficiency in DNA repair (Carson et al., 1986). However, the underlying genetic mechanisms and cellular processes that link dGTP accumulation and apoptosis are yet unknown.

\subsection{Yeast as a model for cell metabolism-targeted cancer therapeutics}

\subsubsection{Modifications to energy metabolism in cancer}

Modifications to energy metabolism are considered one of the hallmarks in development of cancer and are consequences of some of the same oncogenic changes driving tumour development (Hanahan \& Weinberg, 2011). Under aerobic conditions, cells use glycolysis to convert glucose to pyruvate and then to $\mathrm{CO}_{2}$ in the mitochondria by oxidative phosphorylation. In the majority of tumours, energy metabolism is reprogrammed to preferentially use lactate fermentation even in the presence of $\mathrm{O}_{2}$ and unimpaired mitochondria, which is termed the Warburg effect or aerobic glycolysis (Warburg, 1956; Ferreira, 2010). Under aerobic glycolysis, $85 \%$ of glucose in cancer cells is utilised for synthesis of lactate contributing to as high as 40 times the normal level of lactate in some cancers (Holm et al., 1995; Vander Heiden et al., 2009). Only 5\% is transported to the mitochondria as pyruvate 
for oxidative phosphorylation. The remaining $10 \%$ is diverted to biosynthetic pathways upstream of pyruvate production including glycolysis, nucleotide and fatty acid synthesis (DeBerardinis et al., 2007). To maintain a higher metabolic flux under hypoxic conditions, lactate is transported out of the cells along a concentration gradient. In oxygenated environments, lactate is recaptured by tumour cells via monocarboxylate transporter 1 and converted to pyruvate by lactate dehydrogenase $B$ and is used as an energy source instead of glucose (Corbet $\&$ Feron, 2017). The heterogeneity in the tumour microenvironment leads to different metabolic preferences of cells within a tumour and coexistence of cancer cells with normal levels of mitochondrial oxidation and cancer cells that preferentially generate lactate as an end product (Hensley et al., 2016).

In addition to aerobic glycolysis, proliferating cells at least partly rely on endogenous pathways for synthesis of amino acids, fatty acids and cholesterol, although these can also be supplied by the bloodstream (Beloribi-Djefaflia et al., 2016; Gaglio et al., 2011). The majority of metabolic flux in proliferating cells is dedicated to the synthesis of proteins and lipids with several enzymes in lipid synthesis crucial for proliferating cancer cells. Cholesterol is an essential sterol in the membranes of mammals that is required for membrane fluidity, localisation of membrane proteins and establishment of local asymmetry and membrane curvature. Cholesterol can be esterified and stored as triacylglycerol (TAG) in the lipid droplets to serve as a reserve for acetyl-CoA for use in energy production and synthesis of more complex lipids (Röhrig \& Schulze, 2016). Acetyl-CoA liberated from fatty acids also constitutes another source of TCA cycle intermediates and reducing equivalents such as NADH and $\mathrm{FADH}_{2}$. The uptake and conversion of fatty acids into acyl-CoA and subsequent conversion to acetyl-CoA by beta-oxidation in the mitochondria are upregulated in some cancers (Camarda et al., 2016).

Glutamine is another key molecule for cancers as it can provide both oxaloacetate and acetylCoA to maintain the TCA cycle independent of glucose and pyruvate as well as for synthesis of lipids (Corbet \& Feron, 2017). Glutamine is the obligate amide donor for at least three independent enzymatic reactions in purine biosynthesis and two reactions in pyrimidine biosynthesis where it is 
converted to glutamate (Wise $\&$ Thompson, 2010). Glutamate subsequently serves as an amine donor for $\alpha$-ketoacids for synthesis of non-essential amino acids by transamination. When synthesis exceeds demand, glutamate can also be exchanged for essential amino acids leading to activation of mTOR to promote protein synthesis and cell growth (Chen \& Cui, 2015). Both glutamine synthesis and transport of exogenous glutamine are required for survival of some cancers (Gross et al., 2014).

\subsubsection{Yeast as a model for cancer cell metabolism and human disease}

The exponentially growing Baker's yeast (Saccharomyces cerevisiae) share many metabolic similarities with proliferating cancer cells. These yeast cells possess the ability to adapt its metabolism to its environment and switch between respiratory and fermentative growth (the Crabtree effect). In the presence of high glucose, it preferentially uses fermentation as the main metabolic pathway and represses oxidative phosphorylation, similar to the aerobic glycolysis in tumour cells (Diaz-Ruiz et al., 2011). The central carbon metabolism in yeast is evolutionarily conserved and uses glucose and glutamate catabolism for growth and energy production (Natter \& Kohlwein, 2013). Sterol synthesis in yeast is also essential for viability (Bard et al., 1993), and along with glycerolipids and sphingolipids, are synthesised from acetyl-CoA generated by the central carbon metabolism and oxidation of fatty acids by conserved mechanisms (Natter \& Kohlwein, 2013).

Yeast has been used to study several other cancer relevant cellular processes including DNA replication (DiNardo et al., 1984), recombination and repair (Lisby et al., 2001), genome stability (Yuen et al., 2007; Stirling et al., 2011), cell division (Hartwell et al., 1974; Nurse, 1975), autophagy (Takeshige et al., 1992) and programmed cell death (Carmona-Gutierrez et al., 2010) as the cellular machinery for such basic processes are conserved from yeast to humans. Yeast has also been used to elucidate novel cancer biology where functional orthologs or entire pathways are absent. Heterologous expression of mammalian isoforms of PKC (Riedel et al., 1993), mammalian caspases (Lisa-SantamarÃ-a et al., 2009) and BCL2 (Khoury \& Greenwood, 2008) family proteins in yeast were used to identify suppressors of such pathways. Employing translational yeast genomics, several human tumour suppressor genes that were involved in cell cycle checkpoints, DNA replication/ repair, sister chromatid segregation have 
been identified (de Clare \& Oliver, 2013). The conservation of many higher eukaryotic features involved in cancer makes S. cerevisiae a powerful tool to unveil molecular mechanisms underlying basic human biology and a good model system for screening cancer therapeutics that target cell metabolism.

\subsubsection{Genome-wide tools in yeast for elucidating gene function}

The simple unicellular eukaryote $S$. cerevisiae is genetically tractable system, with a short 90minute generation time (Guthrie \& Fink, 2002) and has the most annotated genome of all organisms (Simon \& Bedalov, 2004; Forsburg, 2001). It has a small genome of 16 chromosomes comprised of 6000 open reading frames (ORFs), of which nearly 1,000 have orthologs that are implicated in human disease and can complement yeast loss of function mutations (Goffeau et al., 1996; Engel et al., 2014; Botstein \& Fink, 2011; Kachroo et al., 2015). S. cerevisiae has both haploid and diploid life cycles (Forsburg, 2001). Of the 6000 ORFs in S. cerevisiae only 1000 are indispensable for viability and the remaining $\sim 5000$ are non-essential genes that have been systematically inactivated to create comprehensive non-essential gene deletion libraries in haploid and diploid organisms (Tong et al., 2001; Ho et al., 2011; Winzeler et al., 1999; Boone et al., 2007). The haploid deletion library is readily utilised in Synthetic Genetic Array (SGA) experiments to generate double mutants by mass mating against a query gene deletion to identify gene-gene (genetic) interactions (Tong et al., 2001). Several other comprehensive mutant libraries are also available to investigate essential genes including the essential heterozygous diploid gene deletion libray, the decreased abundance by mRNA perturbation (DAmP) library (Schuldiner et al., 2005) and the TET-off library (Mnaimneh et al., 2004). Gene overexpression libraries are useful for complementing deletion mutant phenotypes and identifying new functions of genes (Douglas et al., 2012). GST- and HIS-tagged libraries are useful for investigating protein-protein interactions (Zhu et al., 2001). The green florescent protein (GFP) fusion library is used to visualise protein localisation and abundance (Tkach et al., 2012). The comprehensive genome-wide tools in yeast permit unbiased assessment of genes and small molecule function. 
The genetic interaction data available for $S$. cerevisiae are unmatched by other models organisms (Ho et al., 2011). Approximately $89 \%$ of the protein coding genes in S. cerevisiae have been annotated with a biological function (Jouhten et al., 2016). Other resources on S. cerevisiae include the Saccharomyces Genome Database (SGD; http://www.yeastgenome.org/) which provides extensive literature-based information on gene function for every gene that has been studied (Botstein \& Fink, 2011). In addition, a maintained three structured controlled vocabulary referred to as gene ontologies (GO categories) that describes gene/protein function in terms of biological processes, cellular components and molecular functions that are independent of organism, permits the transfer of findings from other organisms to yeast (Ashburner et al., 2000). The conservation of many higher eukaryotic features involved in cancer coupled with its genetically tractability, less complex, short life cycles and the most annotated genome of any organism makes $S$. cerevisiae a powerful tool to investigate cancer biology.

\subsubsection{Nucleoside transport in humans and yeast}

Nucleosides and their derivatives are hydrophobic molecules; hence the expression of membrane permeases determine their distribution in cells and presence in the extracellular environment. In humans, nucleoside transport is facilitated either by the $\mathrm{Na}^{+}$coupled symporter SCL28 family concentrative nucleoside transporters (CNT1-3) or by the energy dependent SCL29 family equilibrative nucleoside transporters (ENT1-4) (Young et al., 2013). These nucleoside transporters differ in their substrate specificity, subcellular localisation and expression in specific tissues leading to regulation of the pools of specific permeants (Young et al., 2013). In addition to transport of nucleosides, nucleobases and monoamines, nucleoside transporters also control the transport of various anticancer and antiviral drugs (Mackey et al., 1998; Pastor-Anglada et al., 1998). In addition to the CNTs and ENTs, SCL15 family peptide transporters and SLC22 family organic anion and cation transporters have also been implicated in transport of nucleoside drugs in humans (Cano - Soldado \& Pastor - Anglada, 2012; Ciarimboli, 2008). 
Both nucleoside transporter families are evolutionary conserved in wide range of eubacteria and eukaryotes. ENTs are distantly related to the major facilitator superfamily transporters found in S. cerevisiae. Yeast encodes a single ENT related nucleoside transporter (FUN26) and an unrelated putative nucleoside transporter (FUI1). Uptake is such a fundamental process that with only $18 \%$ amino acid identity between FUN26 and ENT1/ENT2, heterologous expression of FUN26 in X. laevis oocytes permits uptake of both purine and pyrimidine nucleosides and nucleobases (Vickers et al., 2000). FUN26 in yeast localises to the vacuolar membrane and functions as a high affinity nucleoside/nucleobase transporter. In contrast, FUI1 is a nucleobase:cation symporter of plasma membrane protein and it shares $69-70 \%$ amino acid similarity with uracil and allantoin permeases FUR4 and DAL4, respectively (Loewen et al., 2003; Vickers et al., 2000). FUI1 transports uridine, uracil, 5-fluorouracil, and thymine nucleobases and analogues but not nucleosides. There are also dipeptide transporters (PTR2 and DAL5) (Cai et al., 2007), oligo peptide transporters (OPT1 and OPT2) (Hauser et al., 2001), and organic anion/cation transporters (André, 1995) that transport nucleoside-derived small molecules in yeast. However, S. cerevisiae lack plasma membrane transporters that are capable of permitting cell entry to purine and pyrimidine nucleosides as well as the majority of nucleoside analogues which is a major limitation for the investigating the mechanisms of MTDIA and forodesine.

\subsubsection{Purine nucleotide biosynthesis is highly conserved in yeast}

The yeast purine nucleoside phosphorylase is encoded by PNP1. The catalytic and substrate binding residues of PNP are conserved in yeast with closer similarity to the human enzyme $(50 \%$ similarity) than to E. coli (<10\% identity) (Lecoq et al., 2001b). It is also more similar in structure and function to the human enzyme as it only metabolises guanosine and inosine nucleosides, while $E$. coli encodes a hexameric enzyme that can also process adenosine (Jensen \& Nygaard, 1975). The yeast PNP1 deletion $(p n p 1 \Delta)$ does not have any obvious growth defect while these cells accumulate guanosine and inosine (Lecoq et al., 2001b) similar to PNP-deficient mice (Arpaia et al., 2000). Yeast do not encode either a cytosolic deoxycytidine kinase or a mitochondrial deoxyguanosine kinase required for the conversion of deoxyguanosine to deoxyguanosine monophosphate (dGMP), nor do 
yeast express the $5^{\prime}$-nucleotidase responsible for catabolism of dGMP. However, the remainder of the nucleotide biosynthesis pathway is conserved in yeast (Fig. 1.5).

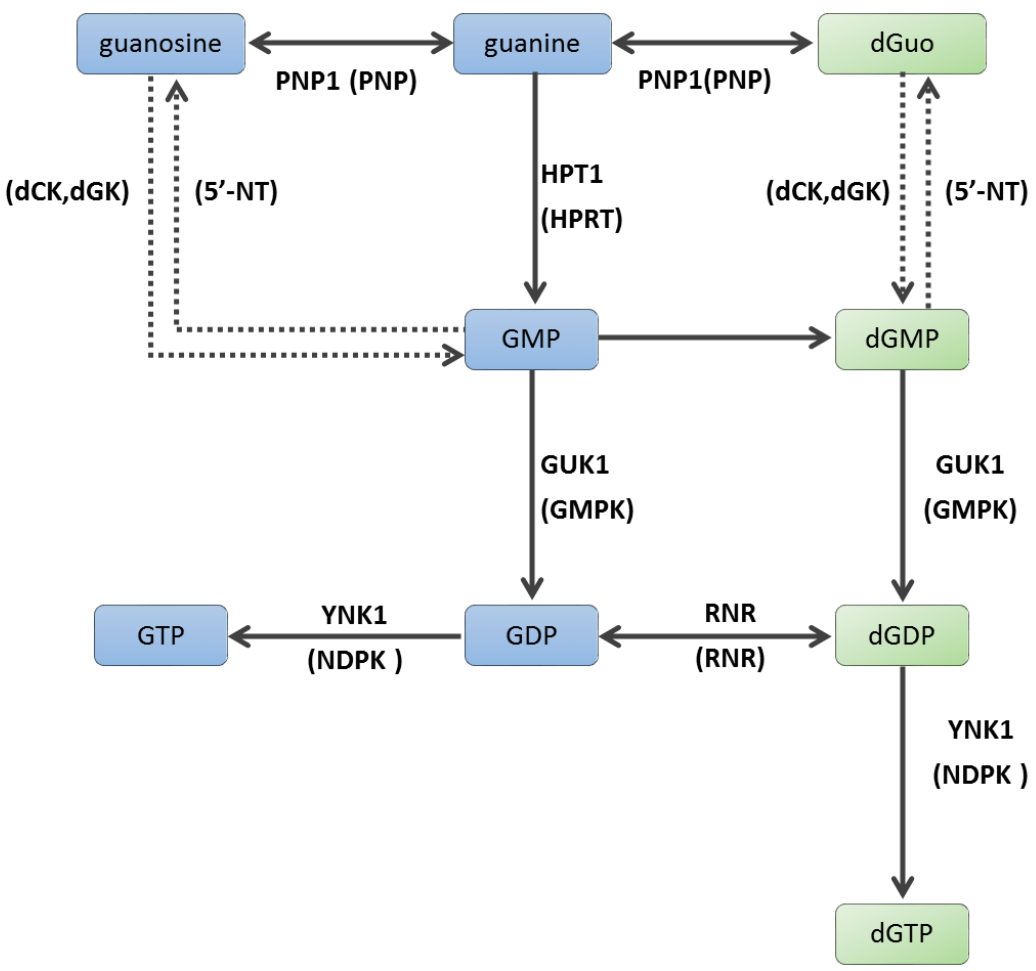

Figure 1.5 - Nucleoside metabolism is conserved from S. cerevisiae to humans. The dotted arrows indicate the pathways that are absent in yeast. The orthologue genes/complexes in humans are shown in parentheses. dCK, deoxycytidine kinase; dGK, deoxyguanosine kinase; 5'-NT, 5'-nucleotidase; GMPK, deoxyguanosine monophosphate kinase (GUK1); NDPK, nucleotide diphosphate kinase (NME1, NME2, NME3, NME4, NME6, NME7, NME1-NME2, AK9); HPT, hypoxanthine phosphoribosyltransferase (HPRT1); RR, ribonucleotides

\subsubsection{Structural conservation of MTAP and methionine salvage pathways}

The human methylthioadenosine phosphorylase (MTAP) is not conserved in bacteria and plants but is conserved in yeast. The MTAP activity in bacteria and plants are facilitated by a dual substrate specific enzyme 5'-methylthioadenosine/S-adenosylhomocysteine (MTA/AdoHcy) nucleosidase (MTAN) that reversibly cleaves MTA and AdoHcy to liberate adenine therefore shares little similarity with MTAP (Lee et al., 2004). MTAP in yeast is encoded by MEU1, an enzyme comprising 275 amino acids with 35\% identities and 53\% similarities with human MTAP (Subhi et al., 2003). The interactions involved in the active site for binding of MTA and the nucleoside analogue MTDIA are conserved in yeast (Lee et al., 2004; Singh et al., 2004) 
(https://blast.ncbi.nIm.nih.gov/Blast.cgi?CMD=Get\&RID=FD3YFTNJ113\#alnHdr Query 134717).

Moreover, the polyamine biosynthesis and methionine and adenine salvage pathways are conserved from yeast to humans (Pirkov et al., 2008).

Loss of MEU1 in yeast has no obvious growth defect when cultured in synthetic complete media. However, following mutations that permit the uptake of nucleosides, meu1 $\Delta$ cannot grow in the presence of MTA as the sole sulphur source and is required for salvage of methionine from MTA (Thomas et al., 2000). Loss of MEU1 leads to upregulation of ODC, leading to accumulation of both putrescine and spermidine and a depletion of spermine (Subhi et al., 2003). This is contrary to MTAP inhibition in mouse xenografts of human cancers that reduced gene expression of polyamine biosynthesis, ODC (ornithine decarboxylase), SSAT1 (spermine/spermidine N1-acetyltarnsferase 1), SRM (spermidine synthase) and SMS (spermine synthase) and a depletion of both spermidine and spermine (Basu et al., 2007; Basu et al., 2011). The differences at the transcriptional level may reflect a difference between knock-down and complete loss of MTAP function or a difference between loss of MTAP function in tumour only as compared to the whole organism. MTAP negative cancers also

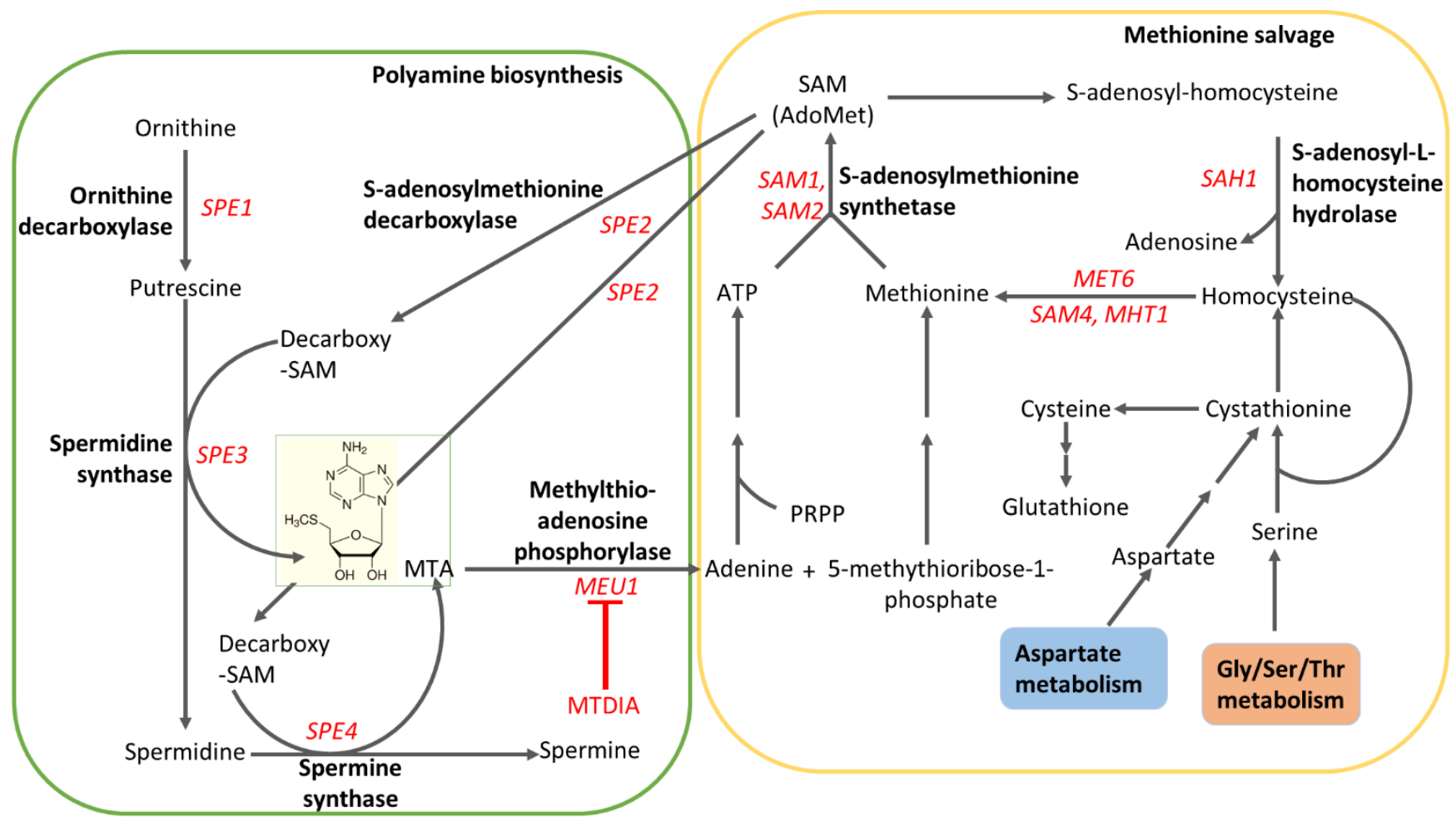


Figure 1.6 - Polyamine biosynthesis and methionine salvage pathways are conserved from yeast to humans.

show elevated ODC levels that are inhibited by MTAP re-expression but in cell type-specific manner (Christopher et al., 2002). This overexpression of ODC in the absence of MTAP/MEU1 is a consequence of 4-methylthio-2-oxobutanoic acid, an inhibitor of ODC in both mammals and yeast and a downstream product of MTAP (Subhi et al., 2003; Tang et al., 2006). An ODC antizyme is also present in higher eukaryotes that is generated by a frameshifting event that is stimulated by elevated ODC levels, yet these mechanisms are absent in yeast (Coffino, 2001). The conservation of polyamine biosynthesis at the transcription level and similar post-translational regulation mechanisms further corroborates the use of yeast as a model system to elucidate mechanisms MTAP inhibition (Fig. 1.6).

\subsubsection{Macroautophagy mechanisms are conserved in yeast}

Macroautophagy (hereafter referred to as autophagy) is a membrane transport pathway where cytosolic content such as longed lived proteins and damaged or surplus organelles are transported to the vacuole (lysosome in mammalian cells) and recycled to maintain cell homeostasis (Ohsumi, 2014). Autophagy is conserved across eukaryotes and it is induced in cells under nutrient deprivation or inhibition of TORC1 by rapamycin (Baba et al., 1994; Kamada et al., 2000). Following induction of autophagy, a cup shaped structure known as the phagophore forms in the cytosol and the leading edge fuses with the other to form a double-membraned vesicle known as the autophagosome that traps the cytosolic content within it (Suzuki \& Ohsumi, 2010). The autophagosomes subsequently fuse with the vacuolar membrane, and autophagic bodies are released within the vacuole/lysosome and degraded by the hydrolases within the vacuolar/lysosomal lumen. The resultant macromolecules are subsequently effluxed by vacuolar membrane permeases to the cytosol and reused in metabolic reactions. The process involves many genes that are collectively named autophagy related (ATG) genes (Klionsky et al., 2003). Although autophagy is non-selective, it shares a majority of molecular components with selective forms of autophagy such as cytosol to 
vacuole transport pathway, autophagy of peroxisomes (pexophagy), mitochondria (mitophagy) and ribosome (ribophagy) where cytosolic content is selectively degraded under nutrient rich conditions.

The autophagy pathway in yeast has been extensively studied and provided the basis for what is known about autophagy in mammalian cells (e.g., the 2016 Nobel Prize in Physiology/Medicine). Genes involved in each step of the autophagy pathway: autophagy induction, vesicle nucleation, expansion of the phagophore and autophagosome completion, docking and fusion of autophagosome with the vacuole, degradation of cargo and efflux of macromolecules have been thoroughly described (Yang \& Klionsky, 2009; Wen \& Klionsky, 2016). Briefly, nutrient deprivation or rapamycin inhibits TORC1 and phosphorylation of Atg13. Atg13 in turn promotes associations of Atg1 with Atg17-Atg31Atg29 (assembly of Atg1 kinase complex) and leading to induction of autophagy. Atg11 also associates with Atg1 under nutrient replete conditions for induction of selective forms of autophagy. During nucleation, Atg14-containing class III PtdIns3K complex (Vps34, Vps30/Atg6, Vps15, Atg14 and Atg38) is recruited to the PAS (phagosome assembly site) that requires Atg23, Atg27 and Atg11. The expansion and completion of the autophagosome requires two ubiquitin-like conjugation systems: Atg8-PE (requires Atg4, Atg3 and Atg7) and Atg12-Atg5-Atg16 (requires Atg7 and Atg10), and Atg12Atg5-Atg16 can subsequently act as a E3 like enzyme for lipidation of Atg8. Atg9, a membrane associated protein cycles between the peripheral site and the PAS carrying or directing membrane for autophagosome expansion. The trafficking of Atg9 from the peripheral site to the PAS requires Atg23, Atg27 and Atg11. Likewise, the trafficking from the PAS to the peripheral site requires Atg1-Atg13, Atg2-Atg18 and Atg14-containing class III PtdIns3K complex. Docking and fusion of the autophagosome with the vacuole requires SNARE proteins: Vam3, Vam7, Vti1, Ykt6, class C Vps/HOPS complex, Mon1-Ccz1 complex and the Rab GTPase, Ypt7. The degradation of vesicles within the vacuolar lumen requires hydrolases Pep4, Prb1 and Atg15 and the resulting macromolecules are effluxed by Atg22, Avt3 and Avt4 permeases. The evolutionary conservation of autophagy mechanisms permits evaluation of disruptions to autophagy in the well characterised yeast system to identify points of perturbation by mammalian active small molecules. 


\section{Chapter 2 - Elucidating the anticancer mechanisms of nucleoside analogue MTDIA via with assessment of fitness for non-essential gene deletions}

\subsection{Introduction}

Polyamine biosynthesis has been long proposed as a candidate for cancer therapy as levels of polyamines are elevated in proliferating cells (Wallace, 1996). Previous attempts at inhibiting the polyamine biosynthesis pathway by ornithine decarboxylase (ODC) inhibitors had limited success as consequence of poor transport, poor target affinity and presence of compensatory mechanisms (Casero \& Marton, 2007). 5'-methylthioadenosine phosphorylase (MTAP) is also an enzyme of the polyamine biosynthesis pathway and the only enzyme in humans that can catabolise $5^{\prime}$-methylthio adenosine (MTA) (Della Ragione et al., 1986). MTA is a potent inhibitor of the aminopropyl transferases spermidine and spermine synthases and must be rapidly hydrolysed to maintain the forward reaction (Pajula et al., 1979a; Raina et al., 1984). MTA is required for recycling of adenine, methionine and S-adenosyl methionine, the primary methyl donor for many methylation reactions (Backlund \& Smith, 1981; Finkelstein, 1990; Lu, 2000). Due to close association of MTAP with the polyamine biosynthesis pathway and recycling of S-adenosyl methionine, it has recently been proposed as a target for cancer therapy (Basu et al., 2007).

MTDIA is a potent picomolar inhibitor of the human enzyme $5^{\prime}$-methylthioadenosine phosphorylase (MTAP) and mimics the enzymatic transition state structure of MTA bound by MTAP

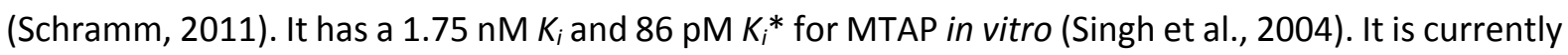
in preclinical stages, but has in vivo efficacy in mouse xenografts models for human lung, prostate, colon, cervical, head and neck, and triple negative breast cancers, as well as in vitro efficacy in various human cancer cell lines (Basu et al., 2007; Basu et al., 2011). MTDIA thus, mimics the loss of MTAP and leads to an accumulation of MTA and a reduction in AdoMet and polyamines (spermidine and spermine) levels (Basu et al., 2007; Basu et al., 2011). One or more of these processes are responsible for subsequent cellular processes mediating apoptosis such as $\mathrm{G} 2 / \mathrm{M}$ arrest, loss of mitochondrial membrane integrity, and cleavage of PARP and caspases (Basu et al., 2011). The initiation of apoptosis, 
however, is cell type specific as normal fibroblast cells do not undergo caspase or PARP-mediated apoptosis or other cellular alterations observed with increased levels of MTA (Basu et al., 2007). Therefore, genetic mechanisms underlying these processes are not fully understood and an additional level of complexity exists beyond MTA-initiated apoptosis.

To elucidate the genetic mechanisms of MTDIA that lead to initiation of cell death, a twopronged approached was employed that utilises the S. cerevisiae genetic model. First, the genetic (gene-gene) interactions surrounding loss of the MTDIA drug target in yeast were identified as genetic interactions with MEU1 (the yeast orthologue of MTAP). Second, the chemical genetic (drug-gene) interactions were investigated to provide a closer approximation of MTDIA action in humans. The mechanisms that were identified from these two approaches were investigated further to confirm these mechanisms as points of perturbation by MTDIA.

\subsection{Experimental procedures}

\subsubsection{Yeast strains, media and compounds}

\subsubsection{Yeast strains}

All S. cerevisiae strains used in this chapter were derived from the diploid strain BY4743 (Brachmann et al., 1998) and were constructed by PCR mediated disruption or heterologous expression of human equilibrative nucleoside transporter 1 (ENT1) gene from plasmid pYhENT1 ((Vickers et al., 1999); gift of Carol E. Cass) as follows:

\begin{tabular}{|c|c|c|}
\hline Strain & Genotype & Reference \\
\hline BY4743 & $\begin{array}{l}\text { MATa/a his } 3 \Delta 1 / \text { his3 } \Delta 1 \text { leu2 } \Delta 0 / \text { leu2 } \Delta 0 \text { LYS2/lys } 2 \Delta 0 \text { met } 15 \Delta 0 / M E T 15 \\
\text { ura3 } \Delta 0 / \text { ura } 3 \Delta 0\end{array}$ & $\begin{array}{l}\text { (Brachmann } \\
\text { et al., 1998) }\end{array}$ \\
\hline BY4742 & MATa his $3 \Delta 1$ leu2 $\Delta 0$ LYS2 met $15 \Delta 0$ ura3 $\Delta 0$ & $\begin{array}{l}\text { (Brachmann } \\
\text { et al., 1998) }\end{array}$ \\
\hline Y7092 (wild type) & 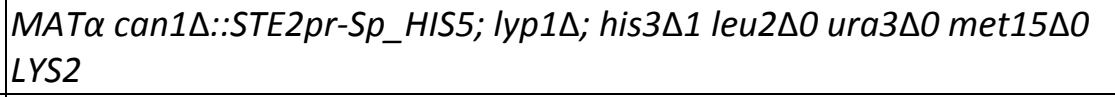 & \begin{tabular}{|lr} 
(Tong & $\&$ \\
Boone, 2007) \\
\end{tabular} \\
\hline 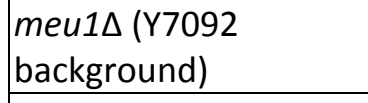 & 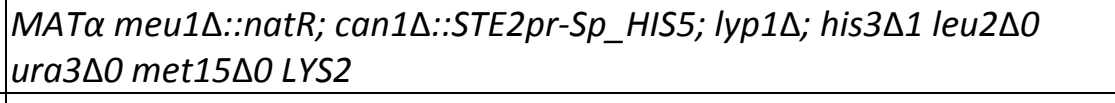 & This study \\
\hline WT(Y7092) + hENT1 & 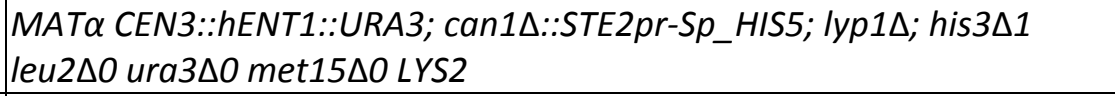 & This study \\
\hline $\begin{array}{l}\mathrm{WT}(\mathrm{Y} 7092)+h E N T 1+ \\
d C K\end{array}$ & 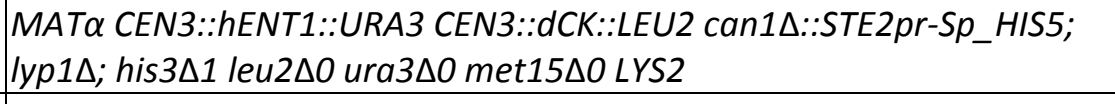 & This study \\
\hline $\operatorname{meu} 1 \Delta x x x \Delta$ & 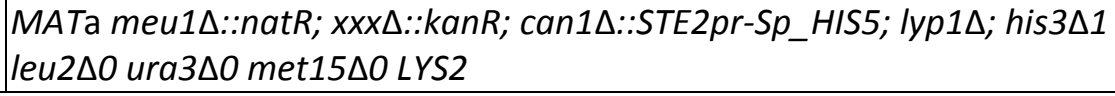 & This study \\
\hline
\end{tabular}




\begin{tabular}{|c|c|c|}
\hline$x x x \Delta$ & MATa $x x x \Delta:: k a n R$ his3 $\Delta 1$ leu $2 \Delta 0$ ura3 $\Delta 0$ met $15 \Delta O$ LYS2 & $\begin{array}{l}\text { (Tong et al., } \\
2001)\end{array}$ \\
\hline $\begin{array}{l}\text { Xxx-GFP (GFP library, } \\
\text { constructed in chapter } \\
\text { 3) }\end{array}$ & 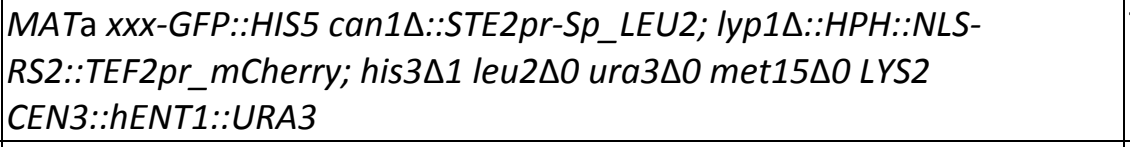 & This study \\
\hline $\begin{array}{l}\text { meu1 } 1 \mathrm{xxx}-\mathrm{GFP}(\mathrm{GFP} \\
\text { library, constructed in } \\
\text { chapter } 3 \text { ) }\end{array}$ & 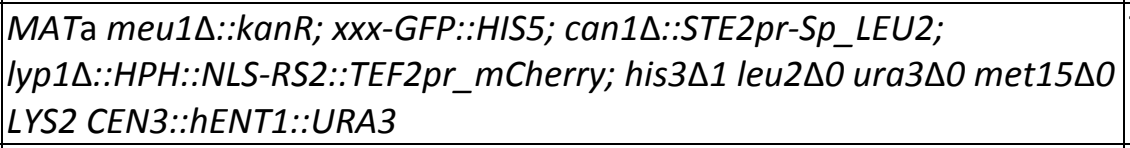 & This study \\
\hline $\begin{array}{l}\text { Y7092 + hENT1::natR } \\
\text { (yCG640 background) }\end{array}$ & 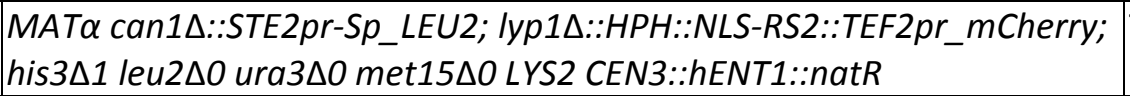 & This study \\
\hline GFP-Atg8 & 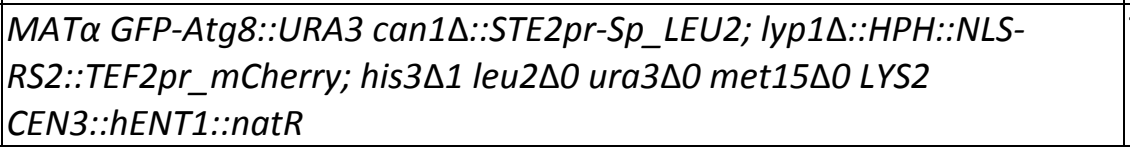 & This study \\
\hline $\begin{array}{l}\text { meu1 } 1:: \operatorname{kanR}+ \\
\text { hENT1::natR } \\
\text { (yCG643 background) }\end{array}$ & 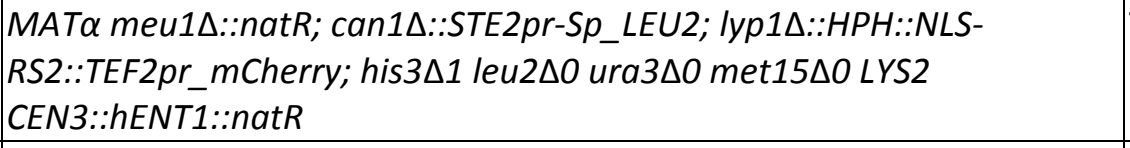 & This study \\
\hline meu1D GFP-Atg8 & 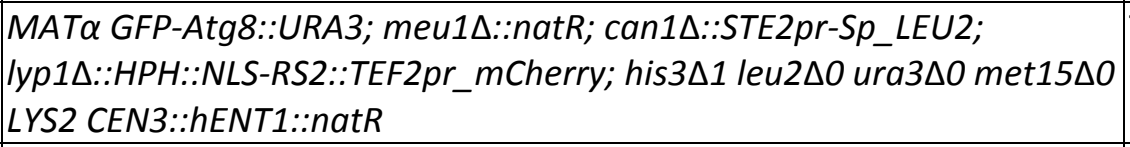 & This study \\
\hline $\begin{array}{l}x x x \Delta \text { GFP-Atg8 (pep4 } 4, \\
\operatorname{atg} 1 \Delta, \text { atg } 4 \Delta ; \mathrm{yCG} 640 \\
\text { background }\end{array}$ & 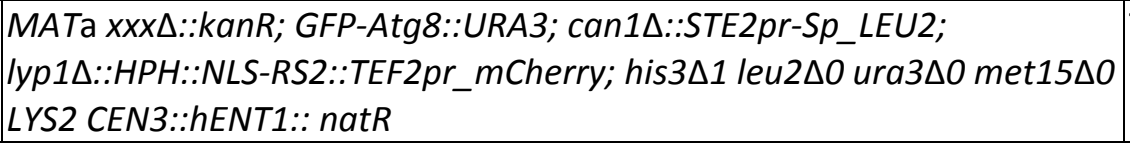 & udy \\
\hline
\end{tabular}

Table 2.2.1 - Yeast strains used in this chapter.

\subsubsection{Media components}

All S. cerevisiae strains were cultured in one of the following growth media supplemented with 100 $\mu \mathrm{g} / \mathrm{mL}$ ClonNAT (Werner bioAgents) and/or $200 \mu \mathrm{g} / \mathrm{mL}$ geneticin (G418). The amino acid analogues Lcanavanine (Sigma-Aldrich) and L-thialysine (Sigma-Aldrich) were used at $50 \mu \mathrm{g} / \mathrm{mL}$ in the absence of their amino acid analogues. All yeast growth media was autoclaved at $121^{\circ} \mathrm{C}$ and cooled to $65^{\circ} \mathrm{C}$ before adding $2 \%$ glucose and antibiotics unless otherwise stated. All E. coli strains were cultured in lysogeny broth (LB) supplemented with $100 \mu \mathrm{g} / \mathrm{mL}$ ampicillin (Sigma-Aldrich).

Yeast peptone dextrose (YPD) agar: 1\% (w/v) yeast extract (Becton, Dickinson and Company), 2\% $(\mathrm{w} / \mathrm{v})$ bacto-peptone (Becton, Dickinson and Company), 0.12\% (w/v) adenine (Sigma-Aldrich), 2\% (w/v) agar (Invitrogen) and 2\% (w/v) glucose (Sigma-Aldrich). YPD broth was prepared as above without the addition of agar.

Synthetic complete (SC) and synthetic dropout (SD) agar: $0.17 \%(\mathrm{w} / \mathrm{v})$ yeast nitrogen base without amino acids or ammonium sulphate (Becton, Dickinson and Company), $0.1 \%(\mathrm{w} / \mathrm{v})$ monosodium glutamate (Sigma-Aldrich), $0.2 \%(\mathrm{w} / \mathrm{v})$ amino acid mixture to suit (Sigma-Aldrich), $2 \%(\mathrm{w} / \mathrm{v})$ agar (Becton, Dickinson and Company) and 2\% (w/v) glucose (Sigma-Aldrich). SC broth was prepared as 
above without the addition of agar. SD media was made the same as SC, minus the appropriate amino acid supplement.

SD-Met/Ade/Ura/Cys (MAUC) $+2 \%$ raffinose $+2 \%$ galactose agar: $0.17 \%(w / v)$ yeast nitrogen base without amino acids or ammonium sulphate (Becton, Dickinson and Company), $0.1 \%(\mathrm{w} / \mathrm{v})$ monosodium glutamate (Sigma-Aldrich), 0.2\% (w/v) amino acid mixture to suit (Sigma-Aldrich), 2\% (w/v) agar (Becton, Dickinson and Company), 2\% (w/v) raffinose (Sigma-Aldrich) and $2 \%(\mathrm{w} / \mathrm{v})$ galactose (Sigma-Aldrich). Media was autoclaved at $121^{\circ} \mathrm{C}$ and cooled to $65^{\circ} \mathrm{C}$ prior to adding filter sterilised raffinose and galactose. SD broth was prepared as above without the addition of agar.

Complete minimal (CM)-Ura $+2 \%$ raffinose: $0.725(\mathrm{w} / \mathrm{v}) \% \mathrm{CM}$ amino acids and ammonium sulphate mixture to suit, $2 \%(\mathrm{w} / \mathrm{v})$ agar (Becton, Dickinson and Company) and $2 \%(\mathrm{w} / \mathrm{v})$ raffinose (Cabosynth). Media was autoclaved at $121^{\circ} \mathrm{C}$ and cooled to $65^{\circ} \mathrm{C}$ prior to adding filter sterilised raffinose. SD broth was prepared as above without the addition of agar.

$\mathrm{CM}$ - Ura + 2\% galactose agar: $0.725(\mathrm{w} / \mathrm{v}) \% \mathrm{CM}$ amino acids and ammonium sulphate mixture to suit, $2 \%(\mathrm{w} / \mathrm{v})$ agar (Becton, Dickinson and Company) and 2\% (w/v) galactose (Cabosynth). Media was autoclaved at $121^{\circ} \mathrm{C}$ and cooled to $65^{\circ} \mathrm{C}$ prior to adding filter sterilised galactose. SD broth was prepared as above without the addition of agar.

CM-Met/Ade/Ura (MAU) + 2\% galactose: $0.725(\mathrm{w} / \mathrm{v}) \% \mathrm{CM}$ amino acids and ammonium sulphate mixture to suit, $2 \%(\mathrm{w} / \mathrm{v})$ agar (Becton, Dickinson and Company) and $2 \%(\mathrm{w} / \mathrm{v})$ galactose (Cabosynth). Media was autoclaved at $121^{\circ} \mathrm{C}$ and cooled to $65^{\circ} \mathrm{C}$ prior to adding filter sterilised raffinose and galactose. SD broth was prepared as above without the addition of agar.

SD-N + 2\% galactose (starvation media) agar: $0.17 \%(\mathrm{w} / \mathrm{v})$ yeast nitrogen base without amino acids or ammonium sulphate (Becton, Dickinson and Company), 2\% (w/v) agar (Becton, Dickinson and Company) and $2 \%(\mathrm{w} / \mathrm{v})$ galactose (Cabosynth). Media was autoclaved at $121^{\circ} \mathrm{C}$ and cooled to $65^{\circ} \mathrm{C}$ prior to adding filter sterilised galactose. SD broth was prepared as above without the addition of agar. Enriched sporulation (SPO) agar: $1 \%(w / v)$ potassium acetate (Scharlau Chemie S.A.), $0.1 \%(w / v)$ yeast extract (Becton, Dickinson and Company), 0.05\% (w/v) glucose (Sigma-Aldrich), 0.2\% (w/v) amino acid 
mixture (Sigma-Aldrich), 2\% (w/v) agar (Becton, Dickinson and Company). Enriched sporulation broth was prepared as above without agar.

SC/SD amino acid mixtures: Amino acid mixture of SD media is a mixture of the following amino acids minus the appropriate amino acid supplement: $3 \mathrm{~g}$ adenine, $2 \mathrm{~g}$ uracil, $2 \mathrm{~g}$ inositol, $0.2 \mathrm{~g}$ paraaminobenzoic acid, $2 \mathrm{~g}$ alanine, $2 \mathrm{~g}$ asparagine, $2 \mathrm{~g}$ aspartic acid, $2 \mathrm{~g}$ cysteine, $2 \mathrm{~g}$ glutamic acid, $2 \mathrm{~g}$ glutamine, $2 \mathrm{~g}$ glycine, $2 \mathrm{~g}$ histidine, $2 \mathrm{~g}$ isoleucine, $10 \mathrm{~g}$ leucine, $2 \mathrm{~g}$ lysine, $2 \mathrm{~g}$ methionine, $2 \mathrm{~g}$ phenylalanine, $2 \mathrm{~g}$ proline, $2 \mathrm{~g}$ serine, $2 \mathrm{~g}$ threonine, $2 \mathrm{~g}$ tryptophan, $2 \mathrm{~g}$ tyrosine and $2 \mathrm{~g}$ valine. Amino acid mixture for enriched sporulation composed of $2 \mathrm{~g}$ histidine, $10 \mathrm{~g}$ leucine, $2 \mathrm{~g}$ lysine and $2 \mathrm{~g}$ uracil. CM amino acid and ammonium sulphate mixture: $100 \mathrm{~g}$ yeast nitrogen base without amino acids or ammonium sulphate, $293.11 \mathrm{~g}$ of ammonium sulphate, $1.2 \mathrm{~g}$ adenine, $1.2 \mathrm{~g}$ arginine, $1.2 \mathrm{~g}$ histidine, 1.8 isoleucine, $3.6 \mathrm{~g}$ leucine, 1.8 lysine, 1.2 methionine, $3 \mathrm{~g}$ phenylalanine, $1.2 \mathrm{~g}$ tryptophan, $1.8 \mathrm{~g}$ tyrosine, $1.2 \mathrm{~g}$ uracil, and $9 \mathrm{~g}$ valine.

Lysogeny broth (LB) Agar: $1 \%(w / v)$ bacto-tryptone (Becton, Dickinson and Company), $0.5 \%(w / v)$ yeast extract (Becton, Dickinson and Company), $1 \%(\mathrm{w} / \mathrm{v}) \mathrm{NaCl}$ (Scharlau Chemie S.A.) and adjusted to $\mathrm{pH} 7.5$ with $10 \mathrm{M} \mathrm{NaOH}$. The media was autoclaved at $121^{\circ} \mathrm{C}$ and cooled to $65^{\circ} \mathrm{C}$ before adding antibiotics unless otherwise stated. LB broth was prepared as above without addition of agar.

\subsubsection{Compounds and chemicals}

MTDIA was kindly provided by Prof Gary Evans (The Ferrier institute, Victoria University of Wellington) was dissolved in DMSO at a concentration of $10 \mathrm{mM}$ and stored at $-20^{\circ} \mathrm{C}$. MTA (AK Scientific) was dissolved in $\mathrm{ddH}_{2} \mathrm{O}$ at a concentration of $5 \mathrm{mM}$ and stored at $-20^{\circ} \mathrm{C}$.

\subsubsection{Liquid phase growth assays}

All liquid growth assays were performed in 96-well plates (TCP-000-096, JET BIOFIL) as previously described (Coorey et al., 2014). $5 \times 10^{5}$ cells $/ \mathrm{mL}$ were treated with either compound or vehicle control, and were incubated at $30^{\circ} \mathrm{C}$. The residual growth $\left(\mathrm{OD}_{600} \mathrm{Exp} / \mathrm{OD}_{600}\right.$ vehicle control $\mathrm{x}$ 100) was assessed in mid-log cultures $\left(0.3-0.5 \mathrm{OD}_{600}\right.$ units in the vehicle control) using Envision ${ }^{\mathrm{TM}}$ Xcite $^{\mathrm{TM}} 2102$ Multilabel Reader (Perkin-Elmer). 


\subsubsection{Agar phase growth assays}

All agar growth assays were performed in 24 well plates (JET BIOFIL) as previously described (Coorey et al., 2014). $1 \times 10^{5}, 1 \times 10^{3}$ and $1 \times 10^{1}$ cells were plated for each condition of compound or vehicle control. Growth reduction was visually inspected at $24 \mathrm{~h}$ and $48 \mathrm{~h}$ in SC + glucose media and $48 \mathrm{~h}$ and $96 \mathrm{~h}$ in SD-MAUC $+2 \%$ raffinose $+2 \%$ galactose media.

\subsubsection{Quantification of genetic interactions with MEU1}

To investigate the genetic interactions with MTAP, the meu1 $\Delta::$ natR strain was constructed in Y7092 background (Tong \& Boone, 2007) by PCR mediated disruption of the MEU1 gene with the natMX4 selectable marker from plasmid p4339 (Goldstein \& McCusker, 1999) (Appendix Fig. 7.2.1; Appendix Table 7.2.1). The non-essential haploid yeast gene deletion library (Boone et al., 2007) was maintained across 14 plates at 1536 colony format with each deletion strain in quadruplicate. This gene deletion library was mated en masse against the meu1 $\Delta:$ :natR deletion strain using the RoToR HDA replicator (Singer Instrument Co. Ltd, Somerset, UK) and SGA methodology (Tong et al., 2001), which generated haploid double gene deletion mutants that were assessed for epistasis (based on colony size) using Gitter tools (Wagih \& Parts, 2014) on RStudio v0.99.896 (Racine, 2012) and ScreenMill (Dittmar et al., 2010). Double gene deletion strains that showed a growth defect (growth $<40 \%, p<0.05$ ) or growth improvement (growth $>40 \%, p<0.05$ ) compared to the parental haploid non-essential gene deletion library in at least two out of three SGAs were considered as putative epistatic interaction of meu1 and were independently validated.

\subsubsection{Validation of genetic interactions with MEU1}

To validate the putative epistatic interactions, the meu1 $x x x x \Delta$ double mutants were reconstructed by performing a mini SGA comprised of 154 deletion strains (31 putative genetic interactions and 123 functionally related strains). A control SGA was performed using ura3 $\triangle::$ nat $R$ as a query strain to exclude mating- and sporulation-deficient deletion strains. The double mutants were assessed for growth defects in liquid 96-well plate growth assays using the parental single deletion and Y7092 (wild type) as control strains. The yeast strains were cultured in 96 -well plates for $2 \mathrm{~d}$ at 
room temperature and inoculated into fresh SC media in 96-well plates using the 96-long pin RePads $\subset$ on the RoToR HDA replicator (Singer Instrument Co. Ltd). Plates were incubated at $30^{\circ} \mathrm{C}$ and growth was evaluated by measuring $\mathrm{OD}_{600}$ from $14-24 \mathrm{~h}$ at $2 \mathrm{~h}$ intervals as previously described (Section 2.2.1). The epistatic growth defect of the double mutants was calculated relative to the growth defect of the meu1 $\Delta$ single mutant $((\operatorname{meu} 1 \Delta x x x \Delta /$ meu1 $\Delta) /(x x x \Delta / \mathrm{WT}))$, modified from the calculation used for assessment of drug sensitivity. The relative growth of the double mutants to the single mutants was normalised against the meu1 $\Delta$ his $3 \Delta$ double gene deletion strain, which is phenotypically identical to meu1 $\Delta$ single gene deletion strain that contains a partial deletion of the HIS3 gene. Stringent cutoffs of growth reduction $(<40 \%$ treated compared to untreated with $p<0.05)$ and growth improvement $(>40 \%$ treated compared to untreated with $p<0.05)$ were used to determine significant changes in growth. The double gene deletion mutants with extreme growth defects $(<5 \%$ treated compared to untreated with $p<0.05)$ were considered synthetic lethal with the meu1 $\Delta$ strain.

The validated genes were assigned to the three major hierarchical gene ontology (GO) categories: biological process, molecular function and cellular component using the haploid nonessential gene deletion library as a reference set on YeastMine (Gene Ontology Consortium, 2004; Ashburner et al., 2000; Balakrishnan et al., 2012) with $p$-values corrected for multiple testing using the Benjamin and Hochberg false discovery rate of 0.05 (Benjamini \& Hochberg, 1995). In addition, genes were assigned to functional categories according to the simplified GO category biological process on GO slim mapper on SGD tools and the gene descriptions on YeastMine (Cherry et al., 2012) (Balakrishnan et al., 2012).

\subsubsection{Nucleoside uptake in S cerevisiae}

To assess the uptake of nucleosides in S. cerevisiae, yeast strains: Y7092 (wild type, methionine auxotroph) and ade2 $\Delta$ ade $3 \Delta$ (adenine and methionine auxotroph) were transformed with either the human equilibrative nucleoside transporter (hENT1) containing yeast expression plasmid pYhENT1(Vickers et al., 1999) or empty vector pYES2 as previously described (Gietz \& Schiestl, 2007). The ade $2 \Delta$ ade $3 \Delta$ double mutant was cultured in media supplemented with MTA or adenosine in the 
absence of methionine or adenine, respectively. Bioactivity of known toxic nucleoside analogues 3'deoxyadenosine (cordycepin) and 7-deaza-7-cyanoadenosine (toyocamycin) was evaluated using growth assays in liquid as previously described (Section 2.2.1).

\subsubsection{Assessment of toxicity of MTDIA}

To assess if MTDIA was cytotoxic or cytostatic in S. cerevisiae, two approaches were employed. In the first approach, WT+hENT1 cells in $99 \mu$ Lolumes SD-Met/Ade/Ura/Cys + MTA + 2\%raffinose + $2 \%$ galactose (SD-MAUC+MTA+RG) of liquid media were treated with inhibitory concentrations of MTDIA and incubated at $30^{\circ} \mathrm{C}$ as previously described (Section 2.2.1). At $0,6,12,24$ and $48 \mathrm{~h}$ intervals, $1 \mu \mathrm{L}$ of cells was removed and plated onto SD-MAUC+RG agar media or SD-Ura agar media. The plates were incubated at $30^{\circ} \mathrm{C}$ and photographed at $12 \mathrm{~h}$ and $48 \mathrm{~h}$ using a digital camera (EOS 600D, Canon). Alternatively, WT+hENT1 cells were treated with MTDIA in a liquid growth assay for $48 \mathrm{~h}$ and growth was assessed at $\mathrm{OD}_{600}$ as previously described (Section 2.2.1). The cells were centrifuged at $2400 \mathrm{rpm}$ for 5 min, washed twice with MAUC+MTA+RG media, and suspended in fresh MAUC+MTA+RG media. The cells were then incubated for $48 \mathrm{~h}$ at $30^{\circ} \mathrm{C}$ and assessed for growth at $\mathrm{OD}_{600}$.

\subsubsection{Construction of hENT1 expression yeast gene deletion library}

To construct the haploid yeast gene deletion library expressing hENT1 ( $x x x \Delta+h E N T 1)$, the WT+hENT1 strain was crossed with the non-essential haploid yeast gene deletion library (gift of Charles Boone) using SGA methodology (Tong et al., 2001).

\subsubsection{Screening the hENT1 yeast gene deletion library for sensitivity to MTDIA}

To assess deletion strains for sensitivity to MTDIA, the haploid $x x x \Delta+h E N T 1$ gene deletion library was prepared on agar in 1536 format with each strain in quadruplicate and 384 strains per plate. The deletion strains were freshly pinned in 1536 format on SD-Ura $+4 \%$ raffinose agar plates and incubated at $30^{\circ} \mathrm{C}$ for $24 \mathrm{~h}$. Colonies were then replicated on SD-MAUC+MTA+RG agar plates with and without $3 \mathrm{nM} \mathrm{MTDIA}$, incubated for $48 \mathrm{~h}$ at $30^{\circ} \mathrm{C}$, and photographed using a digital camera (EOS 600D, Cannon). All replications of treated and untreated strains were performed in triplicate, colony 
sizes were measured using Gitter tools (Wagih \& Parts, 2014) in RStudio v0.99.896 (Racine, 2012) and subsequently compared using ScreenMill (Dittmar et al., 2010).

\subsubsection{Fluorescent microscopy: analysis of autophagy}

To monitor autophagy, cells were transformed with GFP-Atg8 (Suzuki et al., 2001) using lithium acetate/single-stranded carrier DNA/PEG as previously described (Gietz \& Schiestl, 2007). Transformed cells were cultured overnight $\left(\mathrm{OD}_{660}=0.2\right)$ in SD-Ura $+2 \%$ raffinose, washed twice in $\mathrm{dH}_{2} \mathrm{O}$, and resuspended in fresh media (YPD $+2 \%$ glucose, $S D-N+2 \%$ galactose, $S D-U r a+2 \%$ galactose, SD-MAU $+2 \%$ galactose $+250 \mu \mathrm{M}$ MTA \pm MTDIA), and cultured at $30^{\circ} \mathrm{C}$ for $3 \mathrm{~h}$. Cells expressing GFPAtg8 were visualised under 100x magnification using a fluorescent microscope (BX63, Olympus) equipped with camera (Dp70, Olympus) using DIC and filters suitable for FITC and Texas Red.

\subsubsection{Automated fluorescent microscopy}

Cells cultured for $20 \mathrm{~h}$ in SD-Ura+2\% raffinose agar media were inoculated into black-walled, clear bottom 384 well plates (Perkin Elmer Cell Carrier) containing SD-MAU+ MTA + 2\% galactose liquid media with and without MTDIA to an $\mathrm{OD}_{660}$ of 0.3 , using the 384 long pin RePads@ on the RoToR HDA replicator (Singer Instrument Co. Ltd) as previously described (Bircham et al., 2011). Plates were incubated at $30^{\circ} \mathrm{C}$ for $6 \mathrm{~h}$ and the fluorescent signal was detected at $488 \mathrm{~nm}$ (GFP) and $561 \mathrm{~nm}$ (nuclear localisation signal fused to RedStar2 and cytosolic mCherry) using the 60x water immersion lens (NA 1.2) in the high-throughput spinning disk confocal microscope (Evo Tec OPERA, Perkin Elmer). Three visual fields were imaged for each condition for two channels at a height of $0.4 \mu \mathrm{m}$ as previously described (Bircham et al., 2011).

\subsubsection{Phloxine B viability assay}

To assay viability, cells $\left(\mathrm{OD}_{660}=0.2\right.$ for WT, 0.6 for meu1 $\Delta$ and $10 \mathrm{nM}$ MTDIA) cultured overnight in SD-Ura + 2\% raffinose were harvested, washed twice with $10 \mathrm{~mL} C M-M A U C+R G+M T A$, resuspended in $50 \mathrm{~mL}$ of CM-MAUC + RG + MTA, and cultured for $10 \mathrm{~d}$ at $30^{\circ} \mathrm{C}$. At $0,2,4,6,8$ and 10 d, $1 \mathrm{~mL}$ of culture was transferred into a microfuge tube, stained with $2 \mu \mathrm{g} / \mathrm{mL}$ phloxine B (Sigma- 
Aldrich) as previously described (Noda, 2008). Cells were visualised under 60x magnification using a fluorescent microscope (BX63, Olympus) equipped with camera (Dp70, Olympus). Percent viability (unlabelled cells/GFP labelled cells + unlabelled cells) $\times 100$ was determined from a minimum of 1000 cells across five visual fields for each condition.

\subsubsection{GC-MS analysis of sterols}

To quantify sterols, yeast cells from an overnight culture were inoculated to an $\mathrm{OD}_{660}$ of $\mathrm{O} .05$ in $15 \mathrm{~mL}$ of SD-MAUC $+250 \mu \mathrm{M}$ MTA $+2 \%$ galactose media with and without MTDIA, and then incubated at $30^{\circ} \mathrm{C}$ until an $\mathrm{OD}_{660}$ of 1.0 was reached. For meu1 $\Delta$ and $10 \mathrm{nM}$ MTDIA, 0.2 OD units were inoculated into $15 \mathrm{~mL}$ volumes to account for the slow growth rate. Then 5.0 OD units of cells were harvested, washed twice with $\mathrm{dH}_{2} \mathrm{O}$, stored at $-80^{\circ} \mathrm{C}$, and the lipids were extracted as previously described (Munkacsi et al., 2011). In brief, $150 \mu \mathrm{L}$ of glass beads and $900 \mu \mathrm{L}$ of choloroform:methanol (1:2) were added to the cell pellet, vortexed for $10 \mathrm{~min}$ at $4^{\circ} \mathrm{C}$, and incubated overnight at $4^{\circ} \mathrm{C}$ at 1100 rpm using a thermomixer (Eppendorf, Hamburg, Germany). Then $300 \mu \mathrm{L}$ chloroform and $300 \mu \mathrm{L}$ water were added, vortexed for $30 \mathrm{~s}$, and incubated on ice for $2 \mathrm{~min}$. The samples were centrifuged at 9000 rpm for $5 \mathrm{~min}$ and the lower organic phase $(\sim 850 \mu \mathrm{L})$ was collected. The extraction was repeated with $600 \mu \mathrm{L}$ of chloroform and the two organic extracts were combined and dried using a Centrivap Concentrator (Labconco). The lipid film was stored at $-80^{\circ} \mathrm{C}$. To derivatise, $1.2 \mathrm{mg} / \mathrm{ml}$ methoxamine $\mathrm{HCl}$ in pyridine was added to the lipid film, vortexed for $15 \mathrm{~min}$, and incubated at room temperature for $16 \mathrm{~h}$. Samples were silylated with $30 \mu \mathrm{L}$ of N-Methyl-N-(trimethylsilyl) trifluoroacetamide with $1 \%$ trimethylchlorosilane, vortexed for $1 \mathrm{~min}$, incubated at $70^{\circ} \mathrm{C}$ for $30 \mathrm{~min}$, and vortexed for an additional $1 \mathrm{~min}$. The sterols were diluted in hexane to a final volume of $200 \mu \mathrm{L}$ and $100 \mu \mathrm{L}$ was transferred into $2 \mathrm{~mL}$ glass vials with microvolume insert (Agilent). Gas chromatograph-mass spectrometer (GC-MS) data acquisition and post run analysis was performed using Shimadzu GCMS solution v2.72 (Shimadzu, Kyoto) as previously described (Alzeer et al., 2016; Wu et al., 2012). Briefly, the samples were analysed with a Shimadzu QP2010-Plus GC-MS (Shimadzu) with an RXI - 5SilMS column (30 m x $0.25 \mathrm{~mm}$ ID with $0.25 \mu \mathrm{m}$ film thickness, Restek) attached to an electron impact mass spectrometer operating at 
$70 \mathrm{eV}$ in positive ion mode, scanning from $\mathrm{m} / \mathrm{z} 42-600$ every $0.3 \mathrm{~s} .1 \mu \mathrm{L}$ of samples were introduced using a an AOC-20i auto-sampler with a split injection (20:1) at an injector temperature of $250^{\circ} \mathrm{C}$ using He as the carrier gas at a linear velocity of $43.4 \mathrm{~cm} / \mathrm{s}(1.37 \mathrm{~mL} / \mathrm{min})$ at constant flow. Each sample was injected at an initial oven temperature of $60^{\circ} \mathrm{C}$ for $1 \mathrm{~min}$, followed by a ramp at $10^{\circ} \mathrm{C} / \mathrm{min}$ to $325^{\circ} \mathrm{C}$ with a final hold for $3.5 \mathrm{~min}$. Samples were carried to the mass spectrometer at an interface temperature of $330^{\circ} \mathrm{C}$. Sterols were identified by comparison of the detected fragmentation patterns and retention times with those of authentic standards for ergosterol, lanosterol and squalene (Avanti Polar Lipids).

\subsubsection{Calculation of synergy and fractional inhibitory concentration (FIC) index}

To investigate if MTDIA treatment induces autophagy, cells treated with rapamycin (RPM), a known inducer of autophagy, were co-treated with MTDIA and the growth inhibition was assessed in a liquid phase growth assay as previously described (Section 2.2.1). The growth defect by rapamycin and MTDIA in combination relative to either drug alone (MTDIA+RPM/MTDIA)/(RPM/DMSO)x100 was determined using a modified drug sensitivity calculation (Best et al., 2013). In addition, fractional inhibitory concentration FIC index was calculated to quantify drug-drug interaction as the following: synergistic (FIC $\leq 0.5)$, additive $(0.5<\mathrm{FIC}<1)$, indifferent $(1<\mathrm{FIC}>4)$, and antagonistic $(\mathrm{FIC} \geq 4)$ where FIC is defined as (drug A in combination/drug A alone) + (drug B in combination/ drug B alone) (Hall et al., 1983; Odds, 2003; McElhaney-Feser et al., 1998).

\subsection{Results}

\subsubsection{SGA analysis identifies genetic interactions with the MEU1 target of MTDIA}

The assessment of gene-gene (genetic) interactions provides insight into the buffering mechanisms for a pathway or cellular process of interest and can be used to discern novel biology. To investigate the genetic interactions with the target of MTDIA, MEU1 (the yeast orthologue of MTAP) was deleted in a query strain as previously described (Section 2.2.3). Haploid meu1 $\Delta x x x \Delta$ double gene deletion mutants were generated by crossing the haploid non-essential gene deletion library en masse against the query strain by SGA methodology as previously described (Tong et al., 2001). A significant 
reduction in growth of meu1 $\Delta$ was conferred with the addition of 31 single gene deletions (Fig. 2.3.1; Appendix Table 7.2.2a). To validate the putative MEU1 genetic interaction, double mutants were reconstructed in a mini SGA comprised of 123 gene deletion strains (31 putative genetic interactions and 123 functionally related strains). The resulting double mutants were evaluated for reduction in growth phenotype relative to the single gene deletion parents and wild type. Validations were performed in triplicate in liquid media as the growth can be accurately quantified by measuring turbidity at $\mathrm{OD}_{600}$. Using a stringent cut-off of growth reduction $>40 \%$ growth inhibition in the double gene deletion mutant relative to the corresponding single gene deletion mutants (i.e., aah1 1 pnp1 $1 \Delta / p n p 1 \Delta$ vs. aah1 $1 / \mathrm{WT}), 52$ gene deletion strains (11/31 from putative genetic interactions and $41 / 123$ selected based on shared function) were determined to have a growth defect when co-deleted for meu1 $\Delta$ (Fig. 2.3.2; Appendix Table 7.2.2b). These genes were assigned to cellular processes using the GO slim mapping tool in SGD (Cherry et al., 2012) and were involved in amino acid metabolism, response to starvation, vacuole fusion and vesicle-mediated transport, carbohydrate metabolism, lipid homeostasis, chromatin organisation, chromosome segregation, transcription and translation.

Synthetic lethality of meu1 $\Delta$ with gene deletions involved in amino acid metabolism and transport (SPE2, TAT2, AMD1 and AAH1) are likely the consequence of MEU1 function in amino acid salvage and NAD+ biosynthesis. SPE2 catalyses the conversion of S-adenosyl-L-methionine (AdoMet) to S-adenosylmethioninamine and is required for the syntheses of spermidine and spermine as well as the recycling of AdoMet back to MTA. Loss of SPE2 would lead to depletion of MTA and hence the growth defect. This is consistent with the inability of the meu1 $\Delta s p e 2 \Delta$ double deletion mutant to accumulate MTA (Chattopadhyay et al., 2006). TAT2 encodes a high affinity tryptophan transporter. Tryptophan is used as a precursor for synthesis of nicotinic acid mononucleotide (NaMN) in NAD ${ }^{+}$ synthesis and it bypasses the enzymatic step catalysed by MEU1 (nicotinamide ribose to nicotinamide) (Belenky et al., 2007). As TAT2 is also mislocalised to the vacuole in response to ergosterol biosynthesis inhibition (Gaber et al., 1989), the exacerbated growth defect in meu1 $\Delta$ tat2 $\Delta$ may reflect a further 
reduction of $\mathrm{NAD}^{+}$and a link between $\mathrm{NAD}^{+}$and ergosterol. $A M D 1$ and $A A H 1$ are required for synthesis of inosine monophosphate (IMP) from adenine and deletion of MEU1 prevents the recycling of adenine from MTA. The double deletions meu1 $\Delta a m d 1 \Delta$ and meu1 1 aah $1 \Delta$ must rely solely on adenine and inosine in the media and other functionally redundant enzymes (encoded by APT1, PNP1 and HPT1) for synthesis of IMP. It is likely that reduced IMP in these double mutants is responsible for

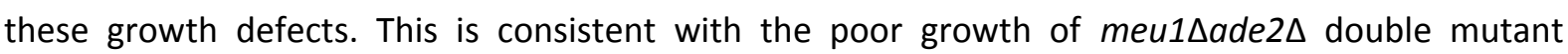
compared with the ade $2 \Delta$ and meu1 $\Delta$ single mutants in the absence of adenine (Subhi et al., 2003).

As MTA inhibits DNA methyl transferases in bacteria and humans (Woodcock et al., 1983; Lautenberger \& Linn, 1972), the changes in chromatin organisation likely underlie growth defects in meu1D caused by deletions in YLR184W (adjacent to TOS4/YLR183C), HIR2, EAF1, IES5, UME6, PAT1 and HTZ1. Likewise, changes in transcription also underlie growth defects in meu1A caused by deletions in SIP3, MED1, DAL81, KNS1 and YDR290W (overlaps RTT103). Changes in DNA methylation patterns were also observed in human cell lines following MTAP inhibition with MTDIA (Basu et al., 2011). Interestingly, yeast do not encode DNA methyltransferases (Tehlivets et al., 2013). Therefore, the mechanisms by which MEU1 affect chromatin organisation are independent of Sadenosylmethionine dependent reactions. The depletion of polyamine in yeast by deletion of SPE1 (ornithine decarboxylase, the rate limiting step in polyamine biosynthesis) was previously shown to modulate chromatin remodelling and transcriptional regulation by bypassing a core histone deacetylase (GCN5) (Pollard et al., 1999). Furthermore, SPE3 (spermidine synthase) gene deletion alleviated mitotic repression of sporulation specific genes (Friesen et al., 1998). Therefore, altered chromatin modulation in response to loss of MEU1 is likely a result of polyamine depletion. The overexpression of ornithine decarboxylase in mice lead to upregulation of both histone acetyl transferases and histone deacetylase (Hobbs \& Gilmour, 2000; Hobbs et al., 2002), thus disruptions to chromatin remodelling and transcription in meu1 $\Delta$ were expected results. Disruptions to translation such as deletions in RPL9A, YPR126C (overlaps YLH47), RPL22A, RPS6A, RPS19A and RPS10A were also expected. For example, L- $\alpha$-difluoromethylornithine (the ODC inhibitor DFMO) or GC7 (spermidine 
synthesis inhibitor) inhibited translation initiation by stimulating the phosphorylation of translation initiation factor elF2 $\alpha$ and 4E-BP1 (elF4E repressor) as well as translation elongation by inhibiting the hypusination of translation elongation factor elF5A by spermidine (Landau et al., 2010). Disruptions of genes involved in lipid homeostasis (OPI3, CSG2, ETR1, INO2, SHR5, CEM1), vacuole fusion and vesicle-mediated transport (VAM3, VPS41, ROM2, MTC5, GGA2, CCZ1, GOS1, SRO9, VAM6 and RIM1) and response to starvation (RVS167, PHO5, IRS4 and RVS161) were observed with loss of MEU1 and are novel findings in this thesis.

a
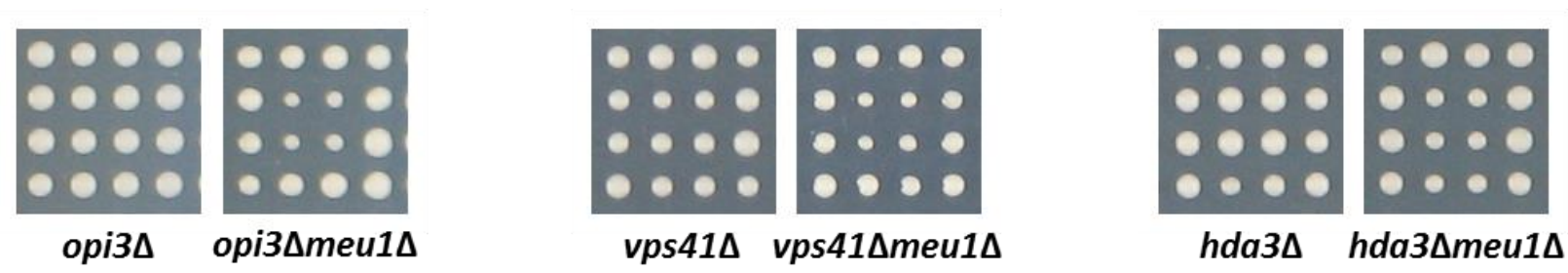

b

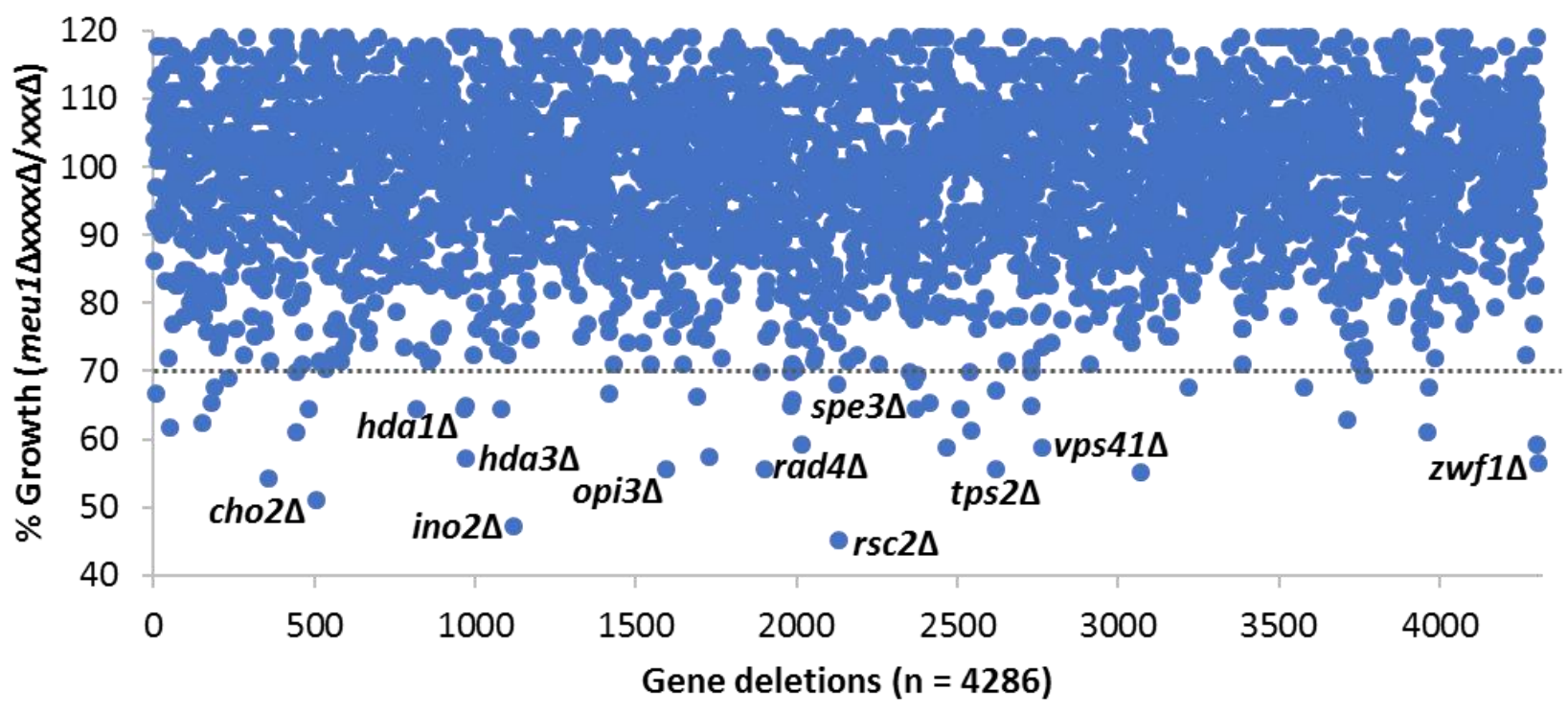

Figure 2.3.1 - Growth of gene deletions with the MEU1 deletion. (a) Growth of exemplar double $x x x \Delta$ meu $1 \Delta$ and single $x x x \Delta$ mutants in technical quadruplicate assessed on agar. (b) Percent growth of 4,286 $x x x \Delta$ meu1 $\Delta$ deletion mutants relative to $x x x \Delta$ from biological triplicate of technical quadruplicate experiments. Three independent SGAs were performed using meu1 $\Delta$ query strain agar using the RoToR HAD (Singer Instrument Co. Ltd) and mated against the haploid single gene deletion library en masse using SGA methodology (Tong et al., 2001). The haploid meu1 $\Delta x x x \Delta$ double mutant meiotic progeny were assessed for epistatic colony size relative to the haploid parental mutants using 
Gitter tools (Wagih \& Parts, 2014) in RStudio v0.99.896 (Racine, 2012) and ScreenMill (Dittmar et al., 2010). 

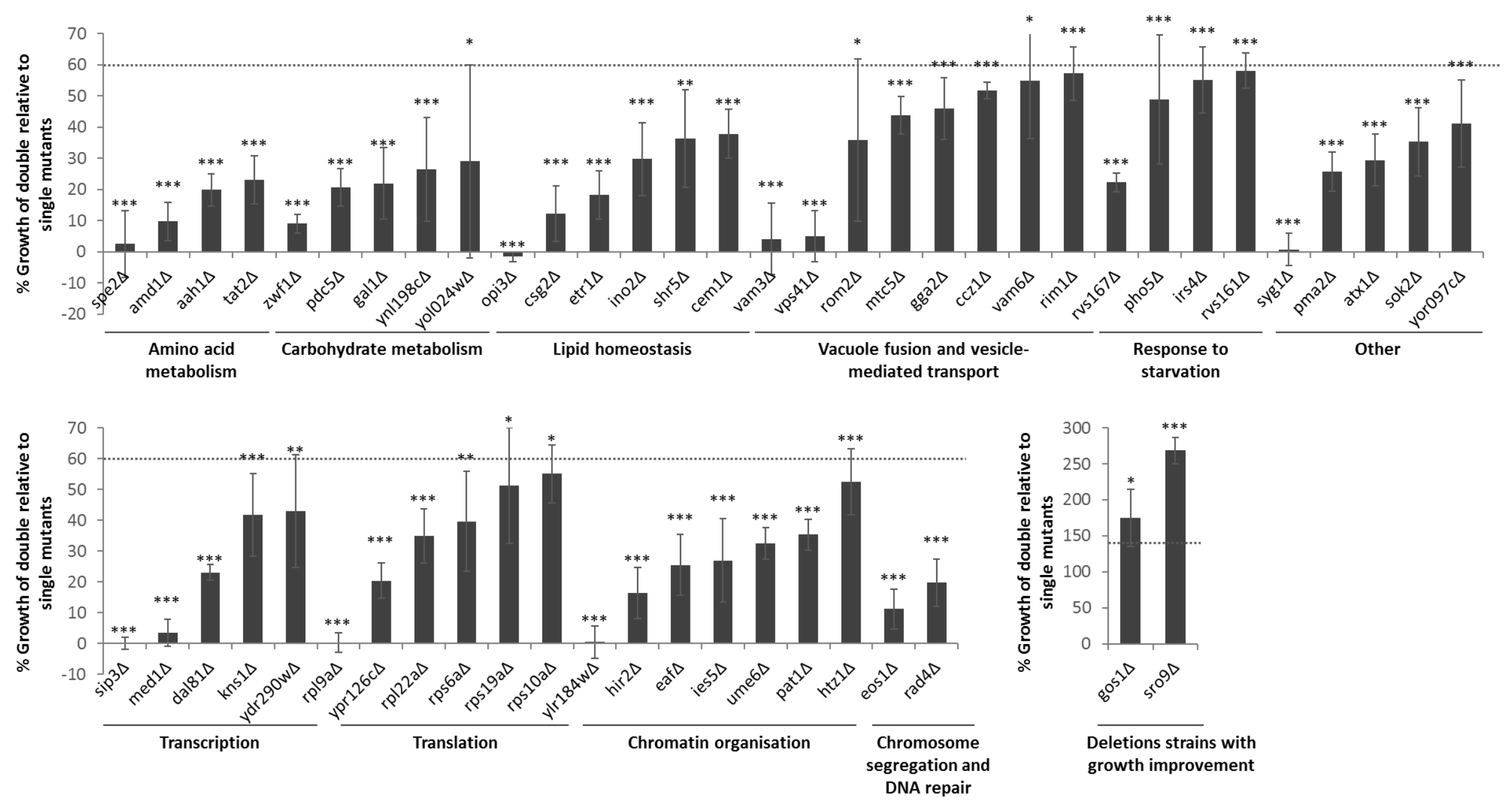

Figure 2.3.2 - 52 negative and 2 positive genetic interactions with Meu1. The growth of the double mutants relative to the single haploid parental strains $($ meu1 $\Delta x x x \Delta /$ meu1 $\Delta) /(x x x \Delta /$ WT) $x 100$ was determined in liquid media at OD600 when the single mutant control ( $x x x \Delta)$ was 0.3-0.5. The growth of the

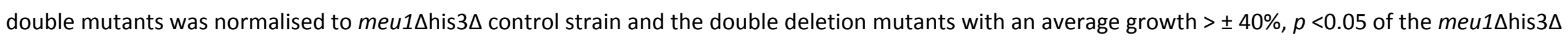
strain were considered a growth defect or a growth improvement. 
2.3.2 Heterologous expression of human gene results in nucleoside uptake in yeast

S. cerevisiae lack the orthologous plasma membrane transporters present in humans that permit the uptake of exogenous purines, pyrimidines and derivatives, including MTDIA and MTA (Young et al., 2013). The heterologous expression of recombinant human equilibrative nucleoside transporter 1 (hENT1) in S. cerevisiae (Vickers et al., 1999; Griffiths et al., 1997) permitted the uptake of pyrimidines, thymidine and uridine and derivatives $3^{\prime}$-deoxy-3'-fluorothymidine and 5bromodeoxyuridine (Paproski et al., 2008; Vernis et al., 2003) and in humans also transports purine and purine derivatives (Young et al., 2013). To achieve the uptake MTDIA, hENT1 was expressed in S. cerevisiae and the uptake of purine nucleosides and derivatives were assessed. The adenine auxotroph ade2 2 ade $3 \Delta$ double mutant was assessed for growth in the presence of nucleosides adenosine and 5'methylthioadenosine (MTA) in the absence of exogenous adenine. The adenine auxotroph expressing the empty vector (ade2 $\Delta$ ade $3 \Delta+\mathrm{EV}$ ) exhibited normal growth in the absence of ENT1 when cultured in media supplemented with adenine and no growth was observed with adenosine or MTA to concentration as high as $2 \mathrm{mM}$ (Fig. 2.3.3a). The growth in adenosine and MTA in the absence of adenine as expected required the expression of hENT1 (Fig. 2.3.3b-c). In addition to permitting transport dependent growth on nucleosides, the expression of hENT1 also improves growth on adenine compared to expression of EV, and is a consequence of hENT1 permitting the uptake of several nucleobases including adenine (Yao et al., 2011). Expression of hENT1 permitted growth of ade2 $\Delta$ ade $3 \Delta$ mutant following supplementation with adenosine and MTA with optimum growth with supplementation of $250 \mu \mathrm{M}$ adenosine and $500 \mu \mathrm{M}$ MTA. Less than optimal growth was observed for at 2 mM MTA, but not adenosine. This is consistent with MTA-mediated inhibition of cell proliferation in humans (Andreu-Pérez et al., 2010; Pajula et al., 1979b). As wild type yeast do not uptake MTA, this is the first demonstration of MTA mediating growth inhibition in yeast.

In addition, the WT strain was assessed for growth inhibition by toxic nucleosides toyocamycin and cordycepin. The treatment of WT with toyocamycin showed no growth inhibition at $100 \mu \mathrm{M}$, the 
highest concentration tested (Fig. 2.3.3d). Upon expression of hENT1, toyocamycin was growth
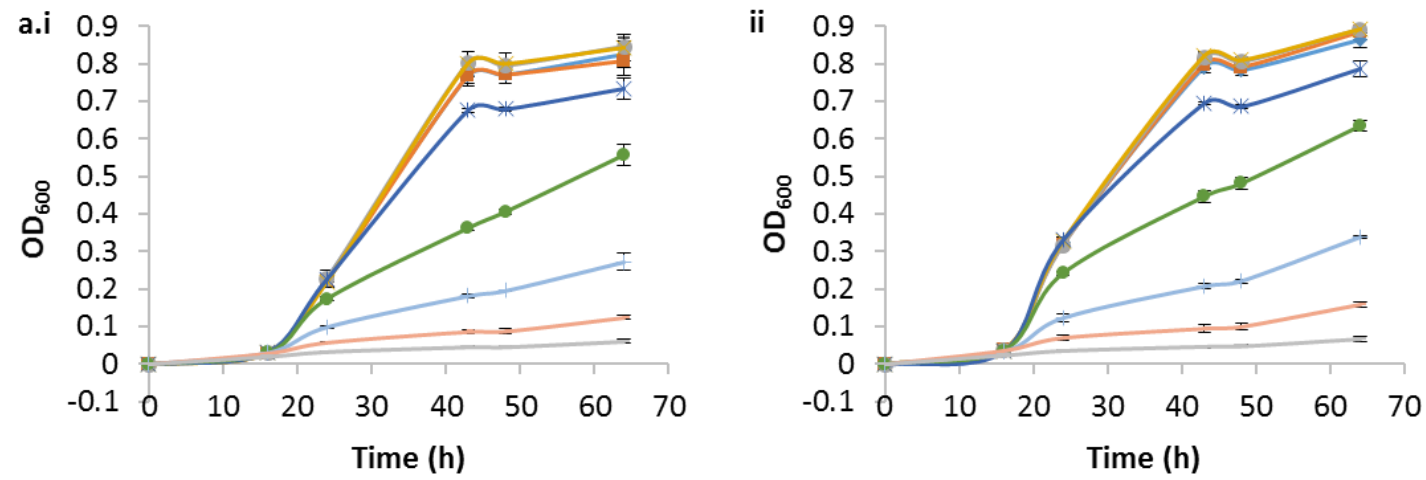

Adenine [mM
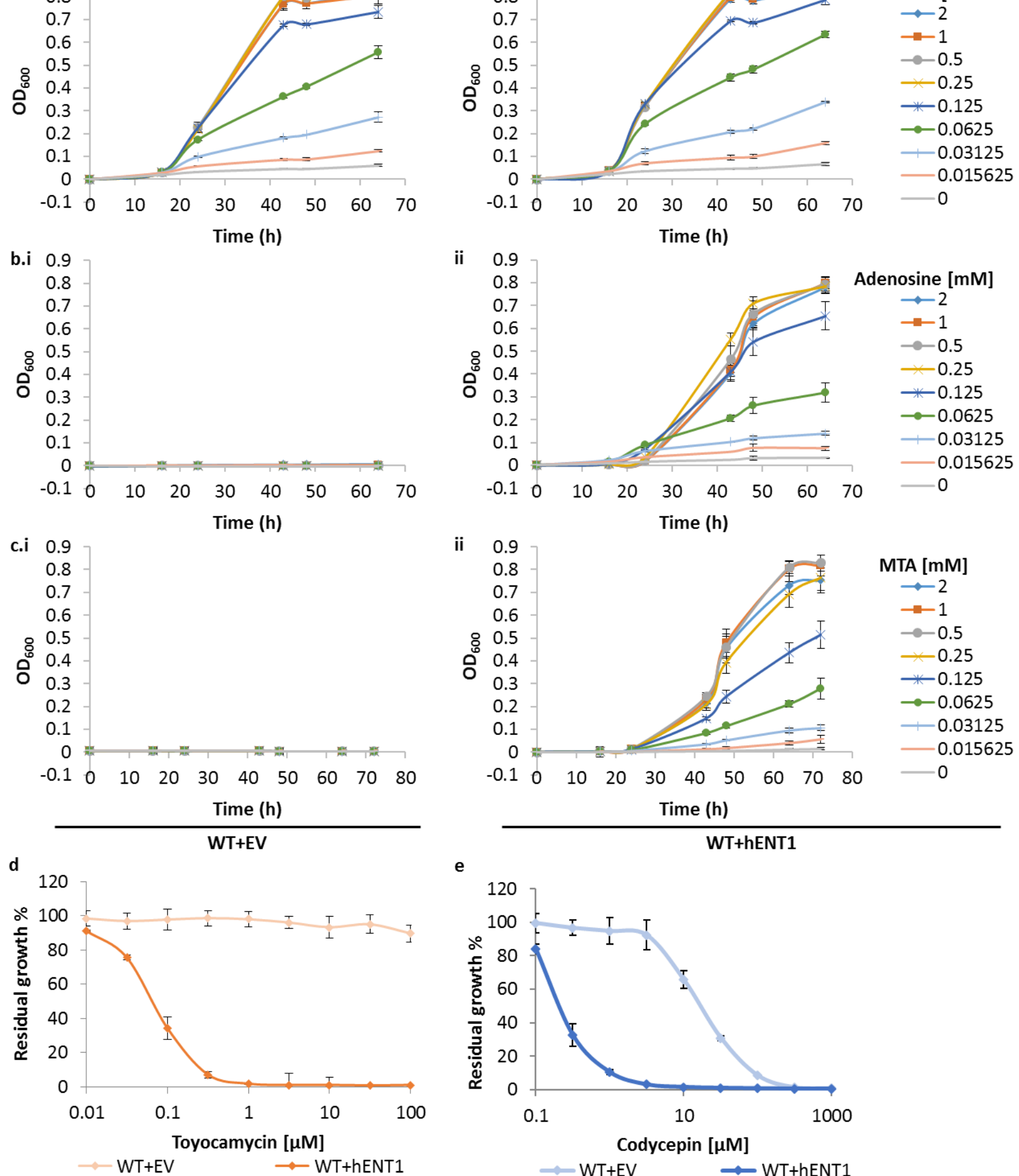

Figure 2.3.3 - hENT1 permits the uptake of adenosine nucleosides and analogues. (a.i-ii) ade $2 \Delta a d e 3 \Delta+\mathrm{EV}$ and ade2$\Delta a d e 3 \Delta+\mathrm{hENT1}$ supplemented with adenine. (b.i-ii) adenosine. (c.i-ii) 5'methylthioadeonisine (MTA). (d) WT+EV and WT+hENT1 treated with toyocamycin. (e) Cordycepin. Cells cultured overnight in SD-U+R media were seeded in SD-U+RG (for WT, methionine auxotroph) or 
SD-UA+RG (for ade2 $\Delta$ ade $3 \Delta$, adenine and methionine auxotroph) to $5 \times 10^{5}$ cells $/ \mathrm{mL}$, treated with nucleosides or nucleobases and were incubated at $30^{\circ} \mathrm{C} . \mathrm{OD}_{600}$ was determined using Envision ${ }^{\mathrm{TM}} \mathrm{Xcite}^{\mathrm{TM}}$ 2102 Multilabel Reader (Perkin-Elmer). Where appropriate, percent growth $\left(\mathrm{OD}_{600} \mathrm{Exp}_{\mathrm{O}} / \mathrm{OD}_{600}\right.$ vehicle control $x$ 100) was determined in mid-log cultures $\left(0.3-0.5 O_{600}\right.$ units in the vehicle control). SD-U+R, SD-Uracil $+2 \%$ raffinose; SD-U+RG, SD-Uracil $+2 \%$ raffinose $+2 \%$ galactose; SD-UA+RG, SDUracil/adenine $+2 \%$ raffinose $+2 \%$ galactose; $\mathrm{EV}$, empty vector.

inhibitory at low nanomolar concentrations $\left(\mathrm{IC}_{50} \sim 6.6 \mathrm{nM}\right)$. Although, the drug target of toyocamycin is conserved in yeast (Kiburu \& LaRonde-LeBlanc, 2012), this is the first instance where bioactivity of toyocamycin has been demonstrated in vivo due to the absence of plasma membrane nucleoside transporters in S. cerevisiae. In contrast, cordycepin, a natural product that has been previously analysed in chemogenomic analyses of yeast (Holbein et al., 2009), was bioactive in WT (IC $50 \sim 17 \mu \mathrm{M})$ and the toxicity was improved $\sim 85$ fold with the expression of hENT1 (IC ${ }_{50} \sim 2$ nM) (Fig. 2.3.3e). This suggests that hENT1 is not the sole nucleoside transporter of cordycepin and the permease(s) responsible for uptake have not been characterised.

\subsubsection{Meu1 is essential when MTA is the only exogenous source of sulphur}

The $\mathrm{IC}_{10-20}$ of compounds in WT cells are required for screening of gene deletion mutants for hypersensitivity and growth inhibition requires a perturbation of an essential target or essential macromolecule in cells (Parsons et al., 2004; Cong et al., 2012). MEU1 in yeast encodes a non-essential protein and, we sought to make a MEU1 conditionally essential by screening the methionine auxotroph yeast strains WT+EV and WT+hENT1 with Meu1 inhibitors MT-DADMe-immucillin A (MTDIA) or DADMe-immucillin A (DIA) in different media conditions. The growth inhibition was assessed in cells cultured with or without the supplementation of MTA (native substrate of MEU1) in media with or without methionine and cysteine. WT expressing empty vector (WT+EV) showed slight growth inhibition with MTDIA at high micromolar concentration $\left(\mathrm{IC}_{20}=0.32-1 \mathrm{mM}\right)$ in SC media and the growth inhibition was unchanged by the expression of hENT1 (Fig. 2.3.4a). WT+EV and WT+hENT1 strains also showed similar levels of growth inhibition to DIA at micromolar concentrations $\left(\mathrm{IC}_{20}>320\right.$ 
$\mu \mathrm{M})$ (Fig. 2.3.4b). This slight growth inhibition at high micromolar concentrations are likely a result of induction of a general stress mechanism (Petrenko et al., 2013) or nucleoside analogues interfering with the uptake of other nutrients (Yao et al., 2011).

In contrast, the treatment of WT+hENT1 in MTA with MTDIA lead to significant growth inhibition at low nanomolar concentrations (IC $20 \sim 15 \mathrm{nM})$ (Fig. 2.3.4c). The growth inhibition plateaued at $42 \%$ and was not further inhibited at concentrations $>1 \mu \mathrm{M}$ MTDIA. In the absence of methionine/adenine/cysteine, the growth of WT+hENT1 treated MTDIA and $250 \mu \mathrm{M}$ MTA was inhibited by $93 \%$ at the lowest concentration tested of $10 \mathrm{nM}$ and the MIC was $31.6 \mathrm{nM}$. Similarly, DIA+ $250 \mu \mathrm{M}$ MTA inhibited the growth of WT+hENT1 at nanomolar concentrations $\left(\mathrm{IC}_{20} \sim 125 \mathrm{nM}\right)$ in SC but plateaued at $68 \%$ growth at $31.6 \mu \mathrm{M}$ and in the absence of methionine/adenine/cysteine the growth the was inhibited by $33 \%$ at $10 \mathrm{nM}$. These results indicate an essential condition that include the heterologous expression of hENT1, MTA as the sole sulphur source in the absence of amino acids methionine/cysteine are required to render MEU1 as conditionally essential.

In the absence of methionine and cysteine, MTA is processed by Meu1 to generate adenine and 5-methythioribose-1-phosphate that are further processed in subsequent steps to salvage methionine (Pirkov et al., 2008). Inhibition of this salvage pathway by MTDIA/DIA-facilitated inhibition of MEU1 was likely responsible for the observed growth inhibition. The absence of adenine enhanced growth inhibition but was not required to achieve complete inhibition (Appendix Fig. 7.2.2). This was likely a consequence of reduced adenine available for synthesis of s-adenosyl methionine from which methionine is salvaged (Thomas et al., 2000). From here forth, MTDIA was used as the MEU1 inhibitor as it was more potent than DIA and media lacking amino acids methionine/adenine/uracil/cysteine supplemented with $250 \mu \mathrm{M}$ MTA and $2 \%$ galactose as the carbon source (SD-MAUC+MTA+2\% Gal) was used as the condition where MEU1 is essential.

Following identification of the MEU1 essential condition, the nature of growth inhibition by MTDIA (i.e., cytostasis or cytotoxicity) was assessed. Two approaches were employed for this 
assessment. In the first approach, WT+hENT1 cells were treated with MTDIA (as previously described in section 2.2.1), and at set intervals $(0,6,12,24$ and $48 \mathrm{~h})$, a subset of cells were removed and
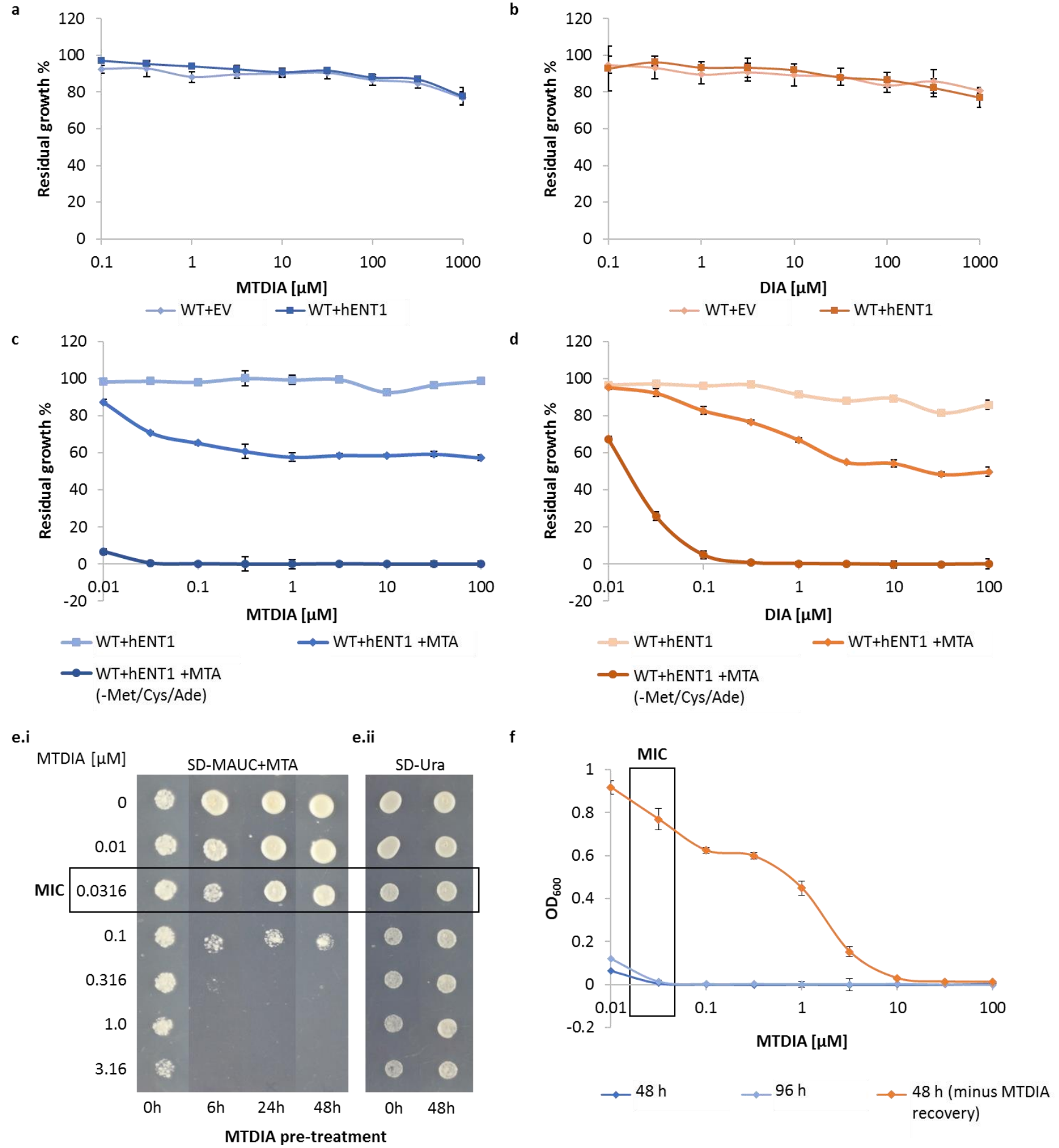

Figure 2.3.4 - MTDIA is a potent inhibitor of yeast MTAP and cytostatic when methionine salvage is essential. (a) WT+EV and WT+hENT1 treated with MT-DADMe-immucillin A (MTDIA). (b) DADMeimmucillin A (DIA). (c) WT+hENT1 treated with MTDIA in SD-URA+RG, SD-URA+MTA+RG and SDMAUC+MTA+RG. (d) DIA. (e.i) Recovery WT+hENT1 pre-treated with MTDIA for 0, 6, 12, 24, and $48 \mathrm{~h}$ 
in SD-MAUC+MTA+RG agar (e.ii) Recovery of MTDIA pre-treated WT+hENT1 in SD-URA+RG agar. (f) Recovery WT+hENT1 cells pre-treated with MTDIA for $48 \mathrm{~h}$, following $48 \mathrm{~h}$ in SD-MAUC+MTA+RG or SD-MAUC+MTA+RG+ MTDIA[ $\mu M]$. WT cells (expressing EV or hENT1) were cultured overnight in SD$U+R$ media were seeded in fresh SD-U+RG or SD-MAUC+RG media supplemented $\pm 250 \mu M$ MTA to $5 \times 10^{5}$ cells $/ \mathrm{mL}$, treated with MTDAI or DIA and were incubated at $30^{\circ} \mathrm{C} . \mathrm{OD}_{600}$ was determined using Envision $^{\text {TM }}$ Xcite $^{\mathrm{TM}} 2102$ Multilabel Reader (Perkin-Elmer) and where appropriate, \% growth $\left(\mathrm{OD}_{600} \operatorname{Exp} / \mathrm{OD}_{600}\right.$ vehicle control $\left.x 100\right)$ was determined in mid-log cultures $\left(0.3-0.5 \mathrm{OD}_{600}\right.$ units in the vehicle control). The MTDIA cells were recovered by either by plating on agar media or resuspending in media without MTDIA following MTDIA treatment. The recovery was measured either by visual inspection on agar or measuring $\mathrm{OD}_{600}$ in liquid media. SD-U+R, SD-Uracil $+2 \%$ raffinose; SD-U+RG, SDUracil $+2 \%$ raffinose $+2 \%$ galactose; SD-MAUC+MTA+RG, SD-Methionine/Adenine/Uracil/Cystine + $250 \mu \mathrm{M} 5$ '-methythiodenosine $+2 \%$ raffinose $+2 \%$ galactose; EV, empty vector.

incubated for growth on SD-MAUC+MTA+RG agar media and SD-Ura agar media (Fig. 2.3.4e.i). The growth of cells immediately following MTDIA treatment was generally comparable to untreated cells for all MTDIA concentrations with only slight growth inhibition observed for the highest concentration (3.16 $\mu \mathrm{M}$ MTDIA). In contrast, cells pre-treated with MTDIA for more than $6 \mathrm{~h}$ were unable to recover beyond MTDIA concentrations $\geq 316 \mathrm{nM}$. This was however, an order of magnitude greater than the MIC of MTDIA. When the cells were plated in SD-Ura media (without MTA), cells were able to recover independent of the time or concentration of the MTDIA treatment (Fig. 2.3.4e.ii). Together, these results suggest that MTDIA is cytostatic and not cytotoxic. MTDIA only inhibits growth as long as MEU1 remains essential (i.e., in the absence of methionine and cysteine with supplemented MTA). The growth inhibition is thus a consequence of the inability to salvage methionine.

In the second approach, WT+hENT1 cells were treated with MTDIA for $48 \mathrm{~h}$, washed in fresh SD-MAUC+MTA+RG media, incubated at $30^{\circ} \mathrm{C}$ for $48 \mathrm{~h}$, and growth was measured at $\mathrm{OD}_{600}$ (Fig. 2.3.4f). After treatment with MTDIA, cells were able to recover at concentrations two orders of magnitude beyond the MIC of MTDIA. The recovery however was incomplete as cells pre-treated with higher concentrations $(>10 \mu \mathrm{M})$ of MTDIA were unable to recover. The recovery was absent in the presence of MTDIA even after $96 \mathrm{~h}$ from the initial MTDIA treatment. Together, this shows that following entry 
into the cell, MTDIA is a potent inhibitor of yeast MEU1. This is consistent with the high degree of conservation of the MTAP active site from yeast to humans (Subhi et al., 2003; Lee et al., 2004)

(https://blast.ncbi.nIm.nih.gov/Blast.cgi?CMD=Get\&RID=FD3YFTNJ113\#alnHdr Query 134717,

2017) and low nM IC 50 of MTDIA (10-100 nM MTDIA with $20 \mu \mathrm{M}$ MTA) for human head and neck and lung cancer cell lines (Basu et al., 2007; Basu et al., 2011).

2.3.4 MTDIA inhibits growth by inhibiting MEU1 function and is bioactive in agar

To confirm that MTDIA toxicity in S. cerevisiae is mediated through MEU1, WT and meu1A deletion strains were screened for sensitivity to MTDIA or MTA alone. The growth of the meu1 + hENT1 strain was mostly unaffected by MTDIA treatment alone and was similar to WT+hENT1 (Fig. 2.3.5a). Only a small reduction in growth (90\% growth at $100 \mu \mathrm{M})$ was observed in both strains at the highest concentration tested and was consistent with previous experiments. This is likely a result of MTDIA saturating the hENT1 transporter and interfering with the uptake of other nucleobases (Yao et al., 2011).

The growth of WT+hENT1 in the absence of methionine/adenine/cystine was dependent on MTA concentration as previously observed (Fig. 2.3.3). Maximum growth was achieved between 100$316 \mu \mathrm{M}$ and was slightly growth inhibitory at $1 \mathrm{mM}$ (Fig. 2.3.5b). In contrast, the growth of meu1 + hENT1 was significantly impaired (15\% of WT at $100 \mathrm{nM}$ MTA) but not abolished in the presence of MTA in the absence of methionine/adenine/cysteine. These results and the near lethal combination of MTA and MTDIA (Fig. 2.3.4c) validates MEU1 as the target of MTDIA in yeast. The slight growth observed for meu1 $\Delta\left(\mathrm{OD}_{600}=0.07\right.$ at $\left.100 \mathrm{nM}\right)$ reflects the presence of other methionine salvage pathways in yeast (Thomas et al., 2000; Finkelstein, 1990).

Following the validation of Meu1 as the target of MTDIA, the conditions for screening the $x x x \Delta+h E N T 1$ deletion library were optimised in agar. The his $3 \Delta+h E N T 1$ strain, that is phenotypically equivalent to WT that carries a partial deletion of the HIS3 gene was screened for sensitivity to MTDIA in SD-MAUC $+250 \mu \mathrm{M}$ MTA + RG agar media by spotting $5 \times 10^{5}, 5 \times 10^{3}$ and $5 \times 10^{1}$ cells from an overnight culture. Plates were incubated at $30^{\circ} \mathrm{C}$ and were visually inspected for growth reduction in comparison 
to the vehicle control at 24, 48, 72 and $96 \mathrm{~h}$ (Fig. 2.3.5c). A reduction in growth was observed at MTDIA concentrations $\geq 3.125 \mathrm{nM}$ for all three cell densities dependent on the incubation time. The 


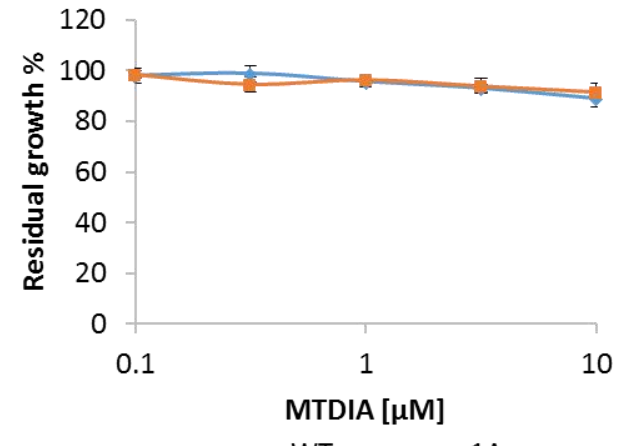

b

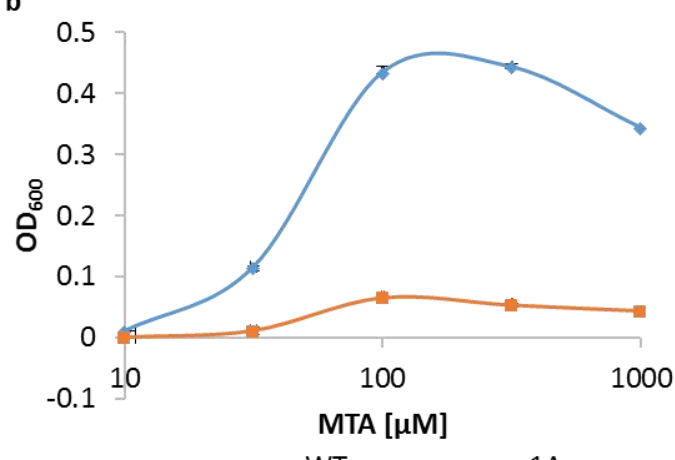

$\because$ WT $\quad \rightarrow$ meu1 $\Delta$

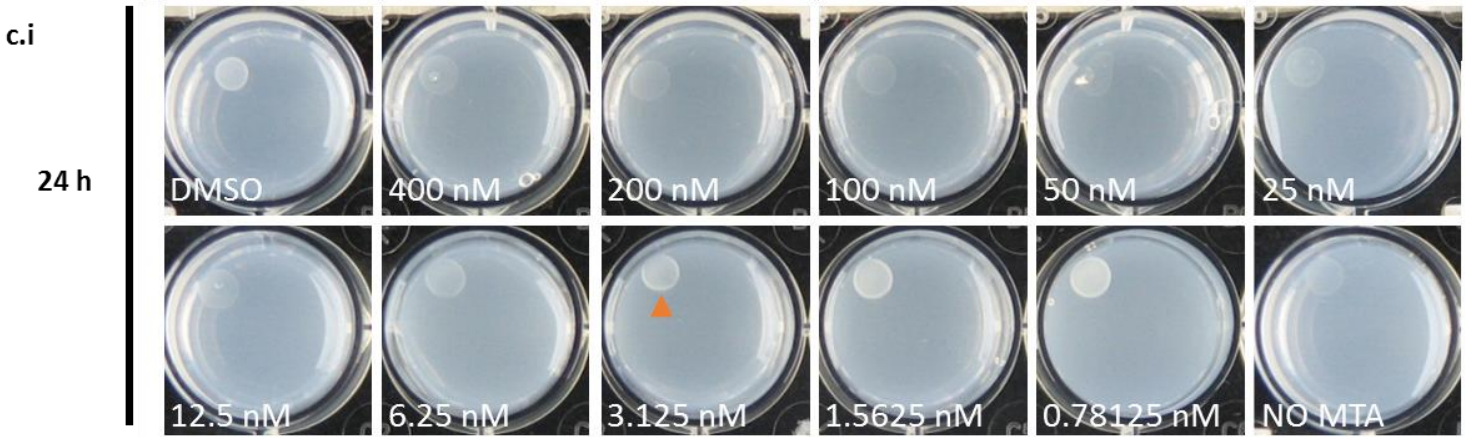

c.ii
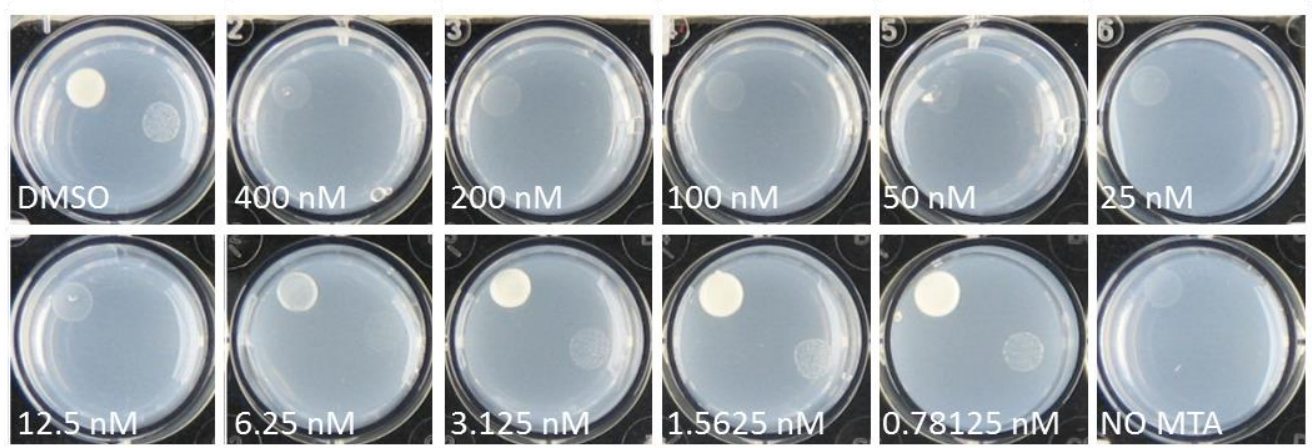

c.iii
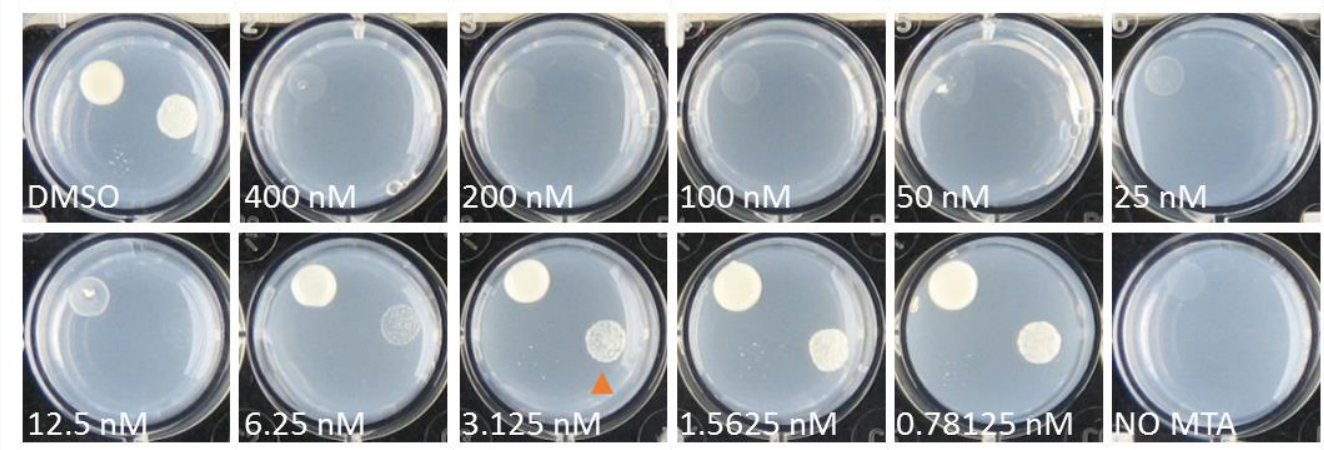

c.iv
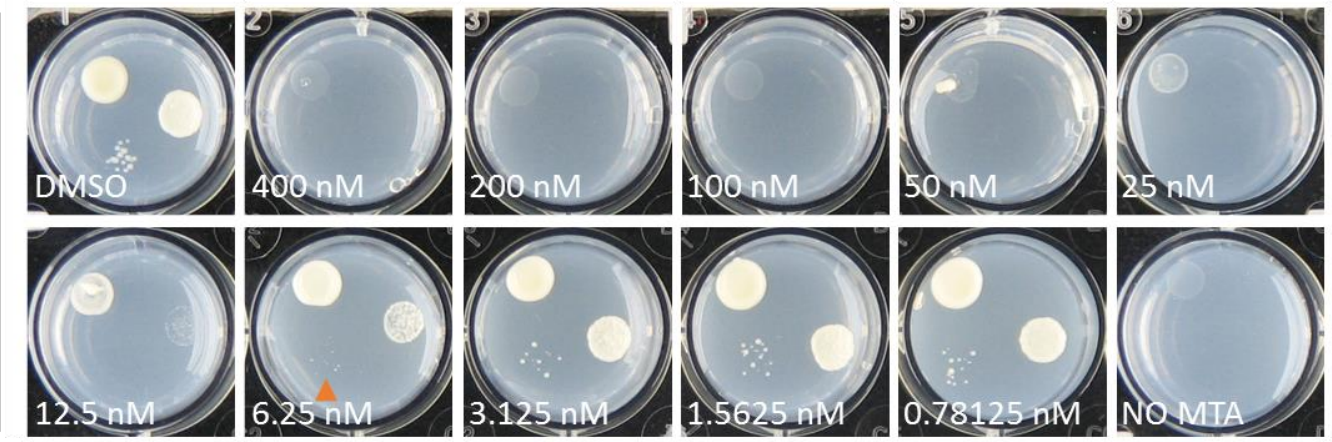

$250 \mu \mathrm{M}$ MTA+ MTDIA [nM] 
Figure 2.3.5 - MTDIA reduces growth by inhibiting Meu1 dependent salvage of methionine and is growth inhibitory in agar. (a) WT+hENT1 and meu1 $1+h E N T 1$ treated with MTDIA. (b) WT+hENT1 and meu1 $1+$ hENT1 treated with MTA. (c) $5 \times 10^{5}, 5 \times 10^{3}$ and $5 \times 10^{1}$ his $3 \Delta+$ hENT1 (WT equivalent) cells treated with MTDIA and $250 \mu \mathrm{M}$ MTA in agar. Growth of WT+hENT1 and meu1 + hENT1 treated with MTDIA or MTA alone in SD-U+RG or SD-MAUC+RG were determined at $\mathrm{OD}_{600}$ was using Envision ${ }^{\mathrm{TM}}$ Xcite $^{\text {TM }} 2102$ Multilabel Reader (Perkin-Elmer) and where appropriate, percent growth $\left(\mathrm{OD}_{600} \mathrm{Exp} / \mathrm{OD}_{600}\right.$ vehicle control $\left.\times 100\right)$ was determined in mid-log cultures $\left(0.3-0.5 \mathrm{OD}_{600}\right.$ units in the vehicle control). In the agar screen, $5 \times 10^{5}, 5 \times 10^{3}$ and $5 \times 10^{1}$ cells from an overnight culture in SD-U+R was spotted onto SD-MAUC+250 $\mu \mathrm{M} \mathrm{MTA}+R G$ agar media, incubated at $30^{\circ} \mathrm{C}$ and imaged at 24 (ci), 48 (cii), 72 (ciii) and $96 \mathrm{~h}$ (civ). The arrow heads indicate the lowest concertation that shows a notable growth reduction for each cell density. SD-U+R, SD-Uracil $+2 \%$ raffinose; SD-U+RG, SD-Uracil $+2 \%$ raffinose $+2 \%$ galactose; SD-MAUC+MTA+RG, SD-Methionine/Adenine/Uracil/Cysteine $+250 \mu \mathrm{M} \mathrm{5}$ methythiodenosine $+2 \%$ raffinose $+2 \%$ galactose .

his $3 \Delta+$ hENT1 strain as previously shown was unable to grow in the absence of MTA. These results show that MTDIA was not inactivated by adding to $65^{\circ} \mathrm{C}$ agar media and therefore the chemogenomic analysis can be performed in agar. Further, MTDIA is growth inhibitory at MTDIA concentrations as low as $3.125 \mathrm{nM}$ in agar media.

\subsubsection{Genome-wide analysis identifies genetic interactions with MTDIA}

The $\mathrm{IC}_{10-20}$ of compounds in WT cells are used for screening of gene deletions mutants for hypersensitivity (Lee et al., 2014; Hoepfner et al., 2014). The $I C_{20}$ was used for screening yeast gene deletion libraries as it identifies sufficient number of drug hypersensitive strains and permits sufficient growth to distinguish sensitive from hypersensitive sensitive strains. To optimise screening conditions to evaluate sensitivity of the $x x x \Delta+h E N T 1$ deletion library to MTDIA, we sought to determine the $\sim \mathrm{C}_{20}$ in 1536 colony format, the format to be used for the genome-wide analysis. A single plate from the $x x x \Delta+h E N T 1$ library was screened against semi-inhibitory concentrations of MTDIA previously determined in agar. Growth of plate 12 of the $x x x \Delta+h E N T 1$ deletion library was quantified at $3 n M, 6$ $\mathrm{nM}$, and $12 \mathrm{nM}$ MTDIA relative to a vehicle control. The median colony size of plate 12 was reduced 19, 26 and $33 \%$ compared to the vehicle control with treatments of $3 \mathrm{nM}, 6 \mathrm{nM}$ and $12 \mathrm{nM}$ MTDIA, 
respectively (Fig. 2.3.6a). In addition to reduction of the plate-wide median colony size, several gene deletion strains also showed a growth defect in comparison to the plate median, such as hir $2 \Delta$ that encodes a subunit of the nucleosome assembly complex required for regulation of transcription (Prochasson et al., 2005). From the three semi-inhibitory MTDIA concentrations assessed in 1536 colony format, $3 \mathrm{nM}$ was chosen as the screening for the $x x x \Delta+h E N T 1$ deletion library as this concentration inhibited a panel of 384 strains by $18 \%$ compared to untreated cells (i.e., within the desired $I C_{10-20}$ level of inhibition).

For assessment of MTDIA drug-gene interactions, the $x x x \Delta+h E N T 1$ library comprising 4310 unique gene deletion strains was treated with $3 \mathrm{nM}$ MTDIA or vehicle control in SD-MAUC+MTA+RG media in agar in three biological replicates and 12 technical replicates. Colony sizes of treated and untreated cells were quantified using Gitter (Wagih \& Parts, 2014) and compared using ScreenMill (Dittmar et al., 2010). Relative to the vehicle control in at least two of the three biological replicates, 39 deletion strains exhibited a significant growth defect based on a growth ratio $<0.8$ with a $p<0.05$. In contrast, 13 deletion strains exhibited a significant growth improvement based on a growth ratio $>120 \%$ with a $p<0.05$ (Fig. 2.3.7b; Appendix Table 7.2.3a). To validate the putative drug-gene interactions with MTDIA, growth of selected $x x x \Delta+h E N T 1$ gene deletions strains (52 putative genetic interactions with MTDIA and 558 additional deletion strains that were either genetic interactors with MEU1 or had shared function with the putative interactions with MTDIA) was quantified in the presence and absence of $2 \mathrm{nM}$ MTDIA, the $\mathrm{IC}_{20}$ concentration in liquid media. The validations were performed in triplicate and in liquid media as it is more quantitative than comparing colony size in agar. Stringent cut-offs were applied to determine MTDIA hypersensitivity (growth<75\%, $p<0.05$ ) or resistance (growth>125\%, $p<0.05$ ) and were assigned to GO categories using the haploid non-essential gene deletion library as a reference set on YeastMine (Balakrishnan et al., 2012).

The growth defect of 61 gene deletion strains (20/52 putative and 41/558 related) was significantly exacerbated (growth<75\%, $p 0.05$ ) in response to MTDIA treatment and were 
a

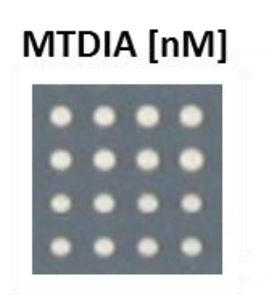

0

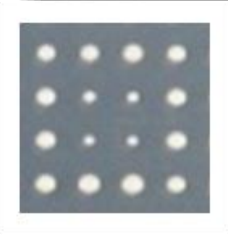

$3(18.7 \%)$

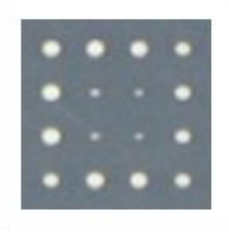

$6(26.2 \%)$

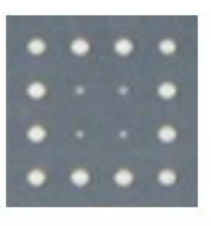

$12(33.3 \%)$

hir2 $\Delta+h E N T 1$

b

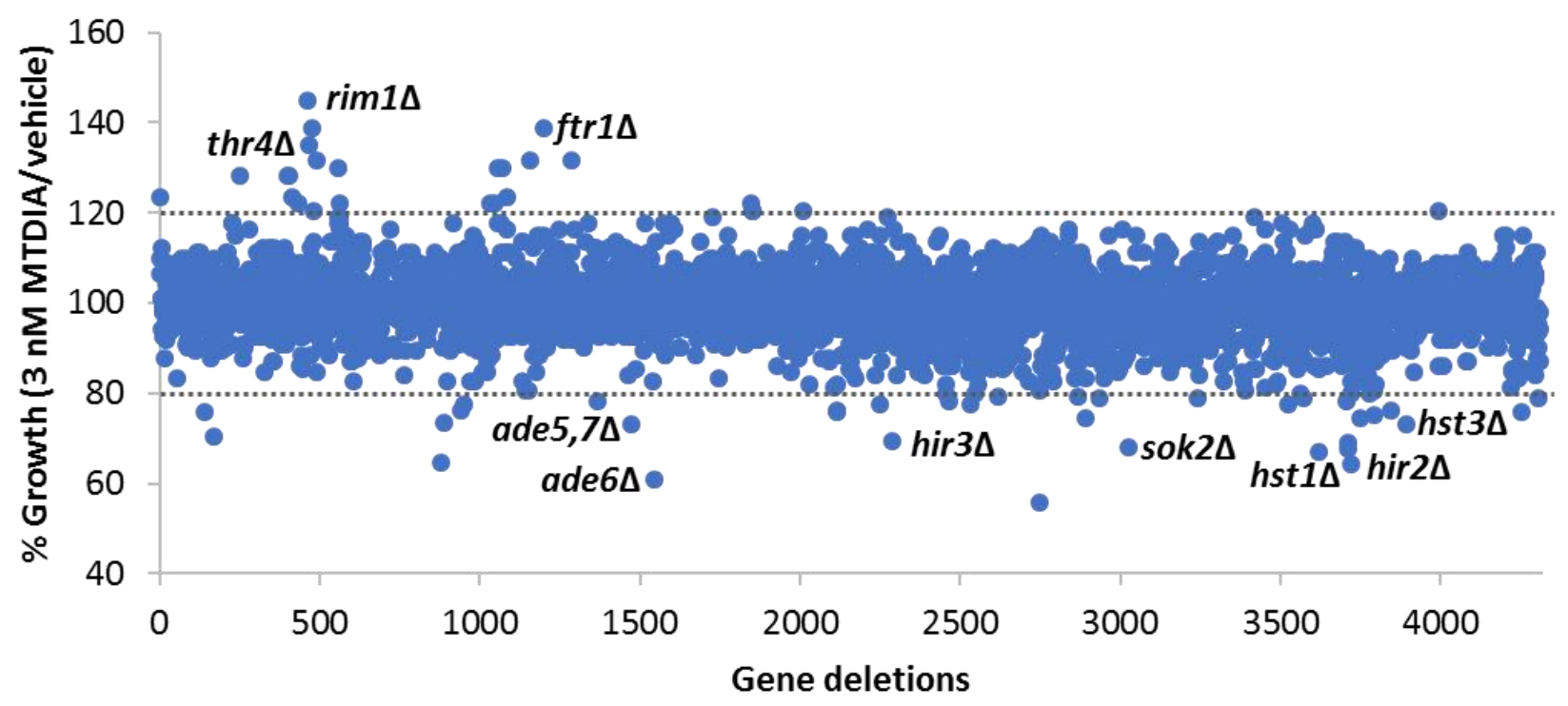

Figure 2.3.6 - Gene deletion strains hypersensitive or resistant to 3 nM MTDIA. (a) The plate-wide reduction in median colony size with 3, 6 and $12 \mathrm{nM}$ MTDIA treatment and hir2 $\Delta+h E N T 1$ showing dose dependent sensitivity to MTDIA. (b) $x x x \Delta+h E N T 1$ library screened against 3nM MTDIA in agar. A single $x x x \Delta+h E N T 1$ library plate was screened against semi-inhibitory concentrations of MTDIA in SDMAUC+MTA+RG agar media. The plates were imaged and the median colony size was determined using Gitter. Percent reduction in plate-wide median colony size was determined by comparing against the vehicle control and are presented in parenthesis. For assessment of MTDIA drug-gene interactions, the $4310 x x x \Delta+h E N T 1$ gene deletion strains were treated with $3 \mathrm{nM}$ MTDIA or vehicle control in SDMAUC+MTA+RG media in agar in triplicate experiments. The $x x x \Delta+h E N T 1$ strains with a growth defect or growth improvement (growth $<80 \%$ or growth $>120 \%, p<0.05$ ) in at least two out of three SGAs were considered as hypersensitive or resistant to MTDIA.

considered hypersensitive to MTDIA (Fig. 2.3.7a; Appendix Table 7.2.3b). These genes were significantly enriched for the major biological processes of regulation of gene expression $(p=5.97 \mathrm{E}-$ 04) and 'de novo' IMP biosynthetic process ( $p=1.45 \mathrm{E}-04)$ following Benjamin-Hochberg correction for 
multiple testing $(p<0.05)$. Several sub-categories within these biological processes were also significantly enriched including negative regulation of gene expression $(p=1.18 \mathrm{E}-04)$, histone deacetylation ( $p=1.39 \mathrm{E}-02)$ chromatin organization $(p=2.80 \mathrm{E}-02)$, transcription from RNA polymerase III promoter $(p=3.81 \mathrm{E}-02)$ and negative regulation of transcription during mitotic cell cycle $(p=4.67 \mathrm{E}-02)$ (Appendix Table 7.2.4). In contrast, 30 gene deletions (4/52 putative and $26 / 558)$ conferred a significant growth improvement (growth $>130 \%, p<0.05$ ) in response to MTDIA treatment and were considered resistant to MTDIA (Fig. 2.3.7b; Appendix Table 7.2.3c). These 30 genes did not show enrichment for GO categories when corrected for multiple testing.

In addition to MTDIA-sensitive and MTDIA-resistant strains, several gene deletion strains failed to grow when MTA was provided as the only sulphur source (Appendix Table 7.2.5). Several of these were noteworthy such as MEU1, MRI1, MDE1, UTR4 and ADI1 that encode enzymes involved in salvage of methionine from MTA (Pirkov et al., 2008). Genes required for endocytosis (RSV167 and RSV161) (Youn et al., 2010), guanine nucleotide exchange factor (CCZ1), and iron transport (FTR1 and FET3) were inviable in MTA and also showed an exacerbated growth defect with meu1 $\Delta$.

There is strong evidence that the MTDIA screen is targeting MEU1 based on overlap with the meu1D SGA results. The MTDIA hypersensitive genes included gene deletion strains defective in adenine metabolism ( $A D E 1, A D E 4, A D E 5,7, A D E 6, A D E 8)$ and involved in inosine monophosphate (IMP) biosynthesis. Interestingly, these genes sensitive to MTDIA were involved in de novo synthesis of IMP from PARP(Rébora et al., 2001; Jones \& Fink, 1982), while the meu1 $\Delta x x x \Delta$ strains with exacerbated growth defects were involved in the salvage of IMP from adenine (Pirkov et al., 2008). This was perhaps a consequence of performing the meu1 $\Delta$ screen in adenine-replete conditions and the MTDIA screen in adenine-deficient media supplemented with MTA. Loss of either pathway in conjunction with MEU1 inhibition with MTDIA or with deletion of MEU1 further reduces availability of adenine for purine nucleoside biosynthesis. This is consistent with inhibition of purine synthesis by MTA in MTAP ${ }^{+/+}$ human lymphoblasts and the increased sensitivity of MTAP negative cancers to purine synthesis inhibitors (Bertino et al., 2011; Gordon et al., 1987; Gordon \& Emmerson, 1986). Gene deletions 
sensitive to MTDIA that also were synthetic lethal with meu1 $\Delta$ also included genes involved in gene expression at the levels of chromatin organisation (BRE1, DPB4, HDA3, HIR2, HIR3, HOS4, HST1, HST3, IES1, NPT1, PAT1, RFM1, RXT2, SIR4, SNF5, SUM1), transcription (SOK2, YLR236C, YLR235C, TDA5, RPN4, PSP2, CKA2, DHH1, YGR054W, KNS1, CTH1, DPB4, GTR2, YORO24W), and translation (RPL9A, YPR126C (overlaps YLH47), RPL22A, RPS6A, RPS19A and RPS10A). These were likely consequences of depletion in polyamine levels that resulted in negatively regulated gene repression (Hobbs \& Gilmour, 2000; Hobbs et al., 2002) as well as inhibition of both translation initiation and elongation (Landau et al., 2010).

In addition, several novel cellular processes were identified in the MTDIA screen that were not previously identified with MTAP inhibition or the meu1 SGA. First, zinc homeostasis was suggested by hypersensitivity of the zinc ion homeostasis genes (ICE2 and ZRT1) to MTDIA. In response to zinc depletion, CDP-DAG pathway (CHO1, OPI3, CHO2) is down regulated (Iwanyshyn et al., 2004) and phosphatidylinositol synthase in upregulated (Henry et al., 2012). The already depleted phospholipid biosynthesis pathway by MTAP inhibition may be further downregulated by low zinc conditions generated by ZRT1 and ICE2 loss. Second, pH homeostasis was implicated by genes involved in the RIM101 pathway (RIM20, DFG16, RIM21) that were sensitive to MTDIA treatment. These genes along with RIM9 forms a protein complex that regulate RIM101, a transcriptional repressor of alkaline $\mathrm{pH}$ responsive genes (Xu \& Mitchell, 2001) and RIM101 pathway that acts in parallel to PKC pathway in responding to cell wall damage (Castrejon et al., 2006). This pathway is constitutively activated by a deletion of VPS4 (Hayashi et al., 2005), a gene deletion that was resistant to MTDIA. The poor cell wall composition in cells cultured in galactose (Aguilar - Uscanga \& Francois, 2003) and disruptions to sterol homeostasis by MTDIA would be exacerbated by the deletions in genes required for cell synthesis, thus, leading to the growth defect as these pathways are interconnected. 

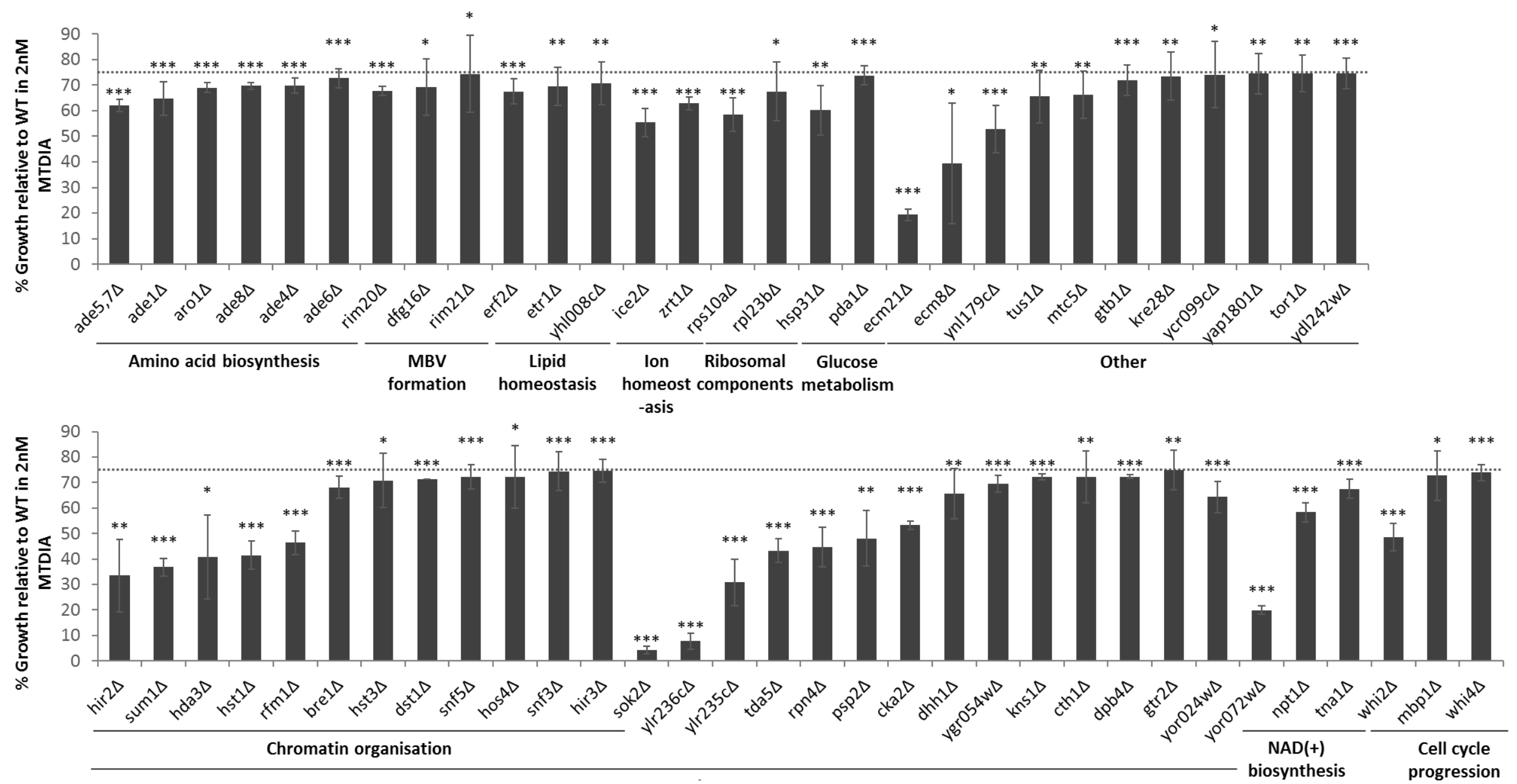

Figure 2.3.7a-61 gene deletion strains were hypertensive to MTDIA. The growth of the single gene deletion mutants treated with MTDIA relative to WT treated with MTDIA ( $x x x \Delta+$ MTDIA/ $x x x \Delta+$ +carrier)/ (WT+MTDIA/ WT+carrier) $x 100$ was determined in liquid media at $\mathrm{OD}_{600}$ when the carrier control was $0.3-0.5$. The his3 $\Delta$ deletion strain was used as the WT control. The single mutants with average growth $<75 \%, p<0.05$ from three replicates compared to the WT control were considered hypertensive to MTDIA. These were assigned to simplified GO categories using GO slim mapping tool on SGD and were involved in amino acid biosynthesis, multivesicular body formation, Sterol biosynthesis, ion homeostasis, ribosome biogenesis, glucose metabolism, gene expression, NAD+ biosynthesis, and cell cycle progression. 


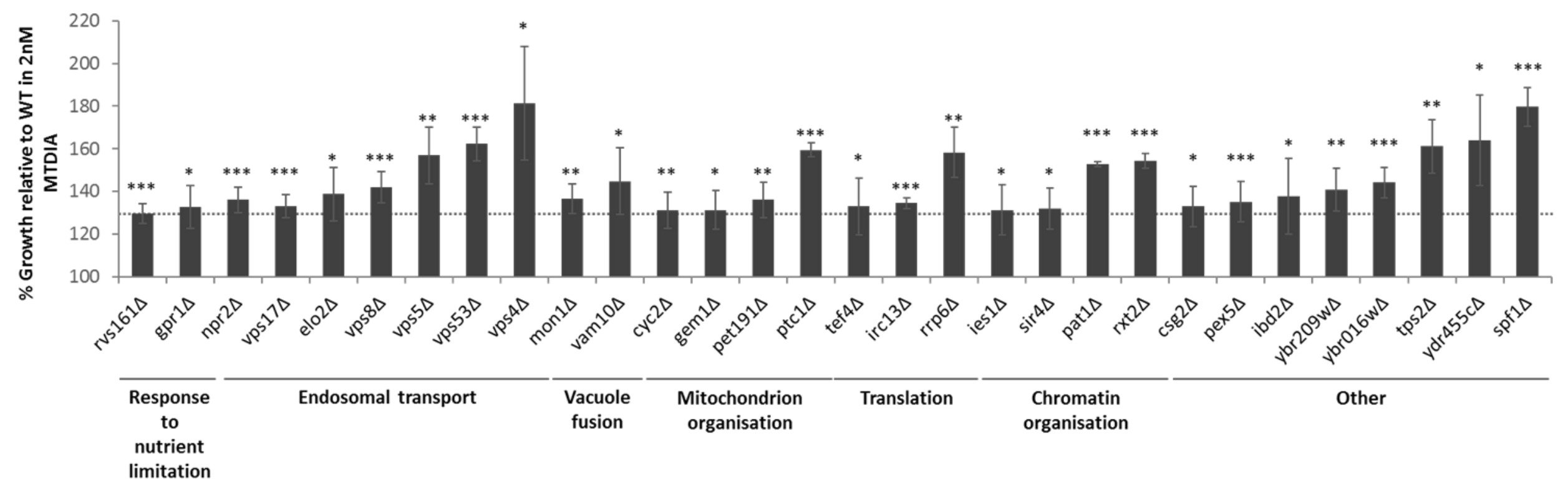

Figure 2.3.7b-29 gene deletion strains were resistant to MTDIA. The growth of the single gene deletion mutants treated with MTDIA relative to WT treated with MTDIA ( $x x x \Delta+$ MTDIA/ $x x x \Delta+$ carrier)/ (WT+MTDIA/ WT+carrier) $x 100$ was determined in liquid media at $0_{600}$ when the carrier control was $0.3-0.5$. The his $3 \Delta$ deletion strain was used as the WT control. The single mutants with average growth $>130 \%, p<0.05$ from three replicates compared to the WT control were considered resistant to MTDIA. These were assigned to simplified GO categories using GO slim mapping tool on SGD and were involved in endosomal transport, vacuole fusion, mitochondrion organization, translation, and chromatin organisation. 


\subsubsection{Further evaluation of MTDIA and meu1D mechanisms}

\subsection{Macroautophagy genes are sensitive to MTDIA treatment}

A subset of the gene deletions that were resistant to MTDIA were involved in fusion of vesicles and autophagosomes with the vacuole (ELO2, VPS17, VPS4, VPS5, VPS53, YDR455C/overlaps VPS44, VPS8, VAM10 and MON1) (Klionsky, 2005), pexophagy (GPR1(Budovskaya et al., 2004) and PEX5) and negative regulation of TORC1 (NPR2). Another subset of gene deletions hypersensitive to MTDIA were involved in negative regulation of autophagy (TOR1 and SOK2). Interestingly, some of the same deletion that were resistant to MTDIA showed a growth defect in the meu1 $\triangle \mathrm{SGA}$, including genes involved in vesicle fusion with the vacuole (CCZ1, VAM3, VAM6, VPS41, ROM2, MTC5, GGA2 and RIM1) and response to starvation (PHO5, IRS4, RVS161, RVS167). This disparity between the two experiments might be a reflection of the condition in which the screens were performed where mild autophagy may promote survival while induction excessive autophagy may be detrimental to viability (Tsujimoto \& Shimizu, 2005). To determine if MTDIA disrupts the autophagy process in yeast, several assays were employed to measure responses to MTDIA treatment and meu1 $\Delta$ are investigated below. We thus hypothesize that autophagy is a novel mechanism of MTDIA toxicity.

\subsection{MTDIA exacerbates the rapamycin induced growth arrest and induces autophagic cell death}

To determine if MTDIA treatment disrupts the autophagic flux, cells were co-treated with MTDIA and rapamycin, an inhibitor of the TOR nutrient sensing pathway and a hallmark inducer of autophagy (Kamada et al., 2000). The growth of WT cells treated with semi-inhibitory concentrations of MTDIA and its genetic mimic meu1 $\Delta$ were assessed following rapamycin treatment (Fig. 2.3.8.1a) and the fractional inhibitory concentration (FIC) index (Hall et al., 1983; Odds, 2003) was assessed to determine synergy (Appendix Table 7.2.6). A concentration dependent growth reduction of WT was observed following rapamycin treatment $(6.5,12.5$ and $25 \mathrm{nM})$ that was exacerbated by 1,3 , and 5 nM MTDIA treatment. FIC $<0.5$ was observed for co-treatment of rapamycin and MTDIA at concentrations lower than $\mathrm{IC}_{50}$ of each compound. This increased sensitivity to rapamycin was also

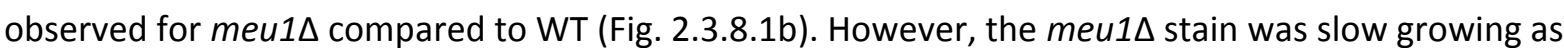


a

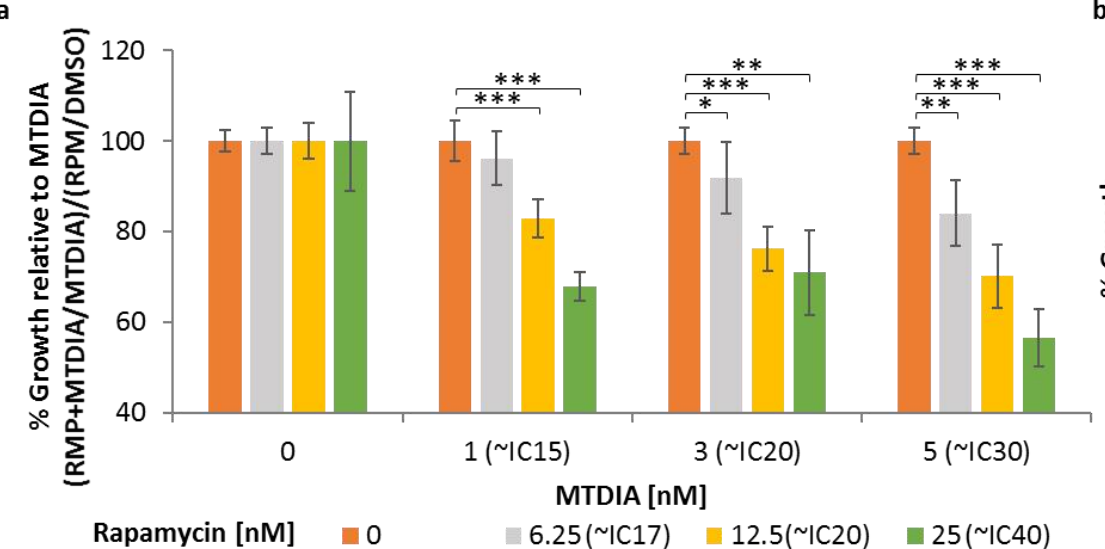

b

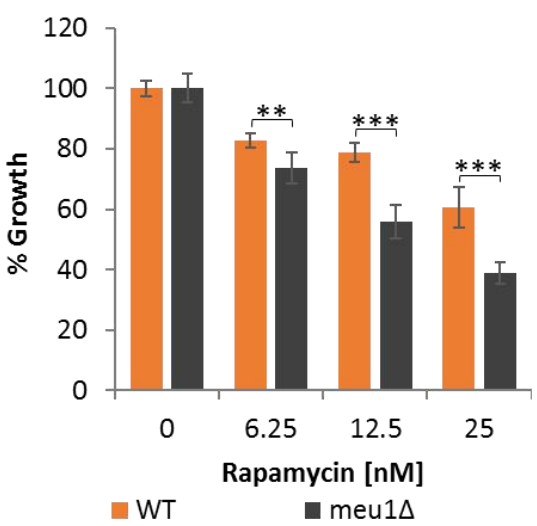

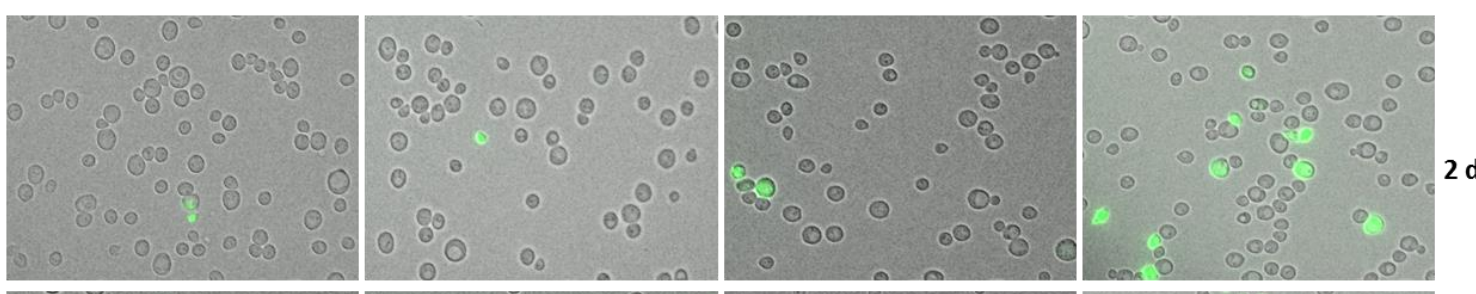
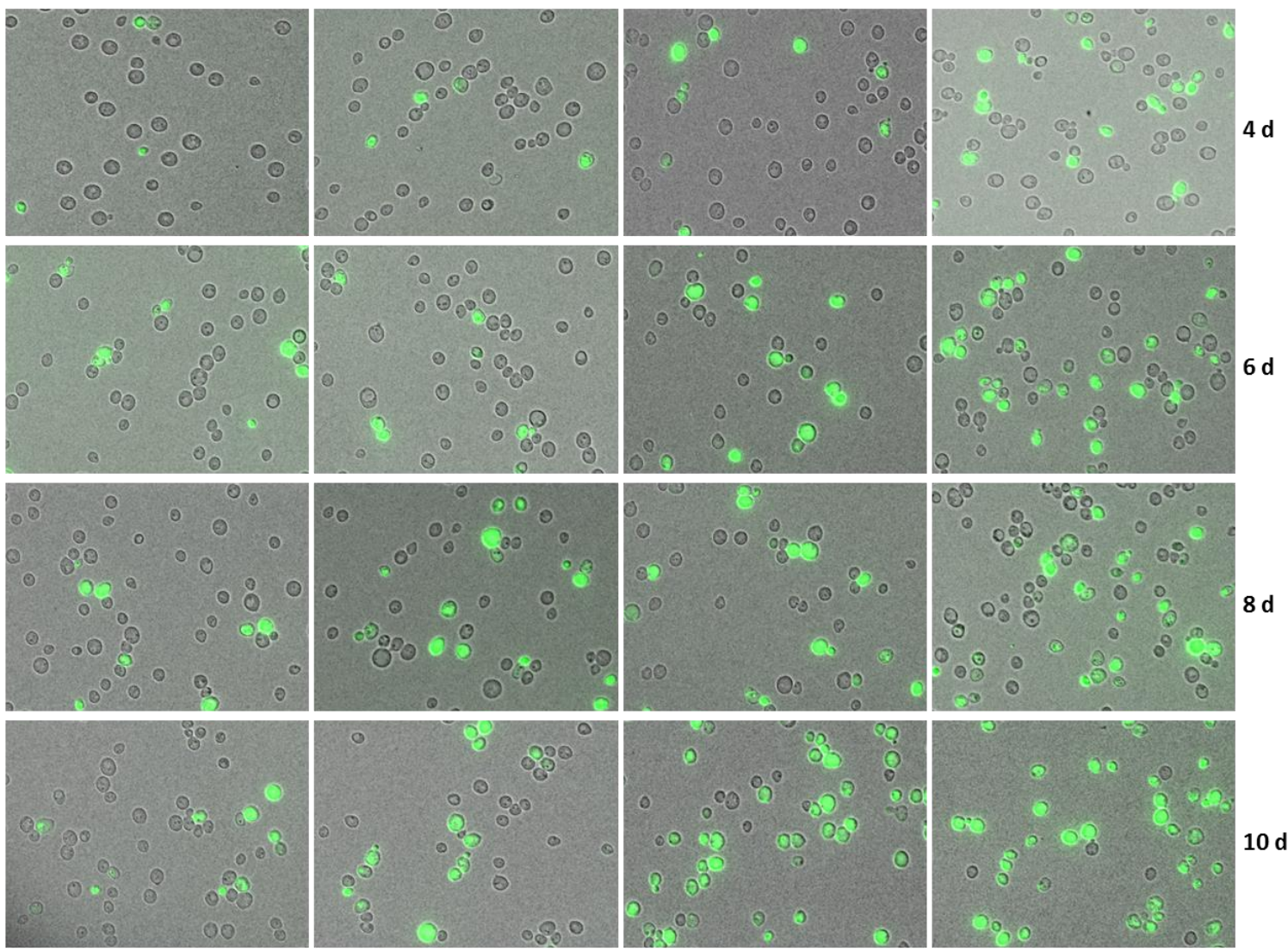

0 nM MTDIA

1 nM MTDIA

3 nM MTDIA

meu1s

d

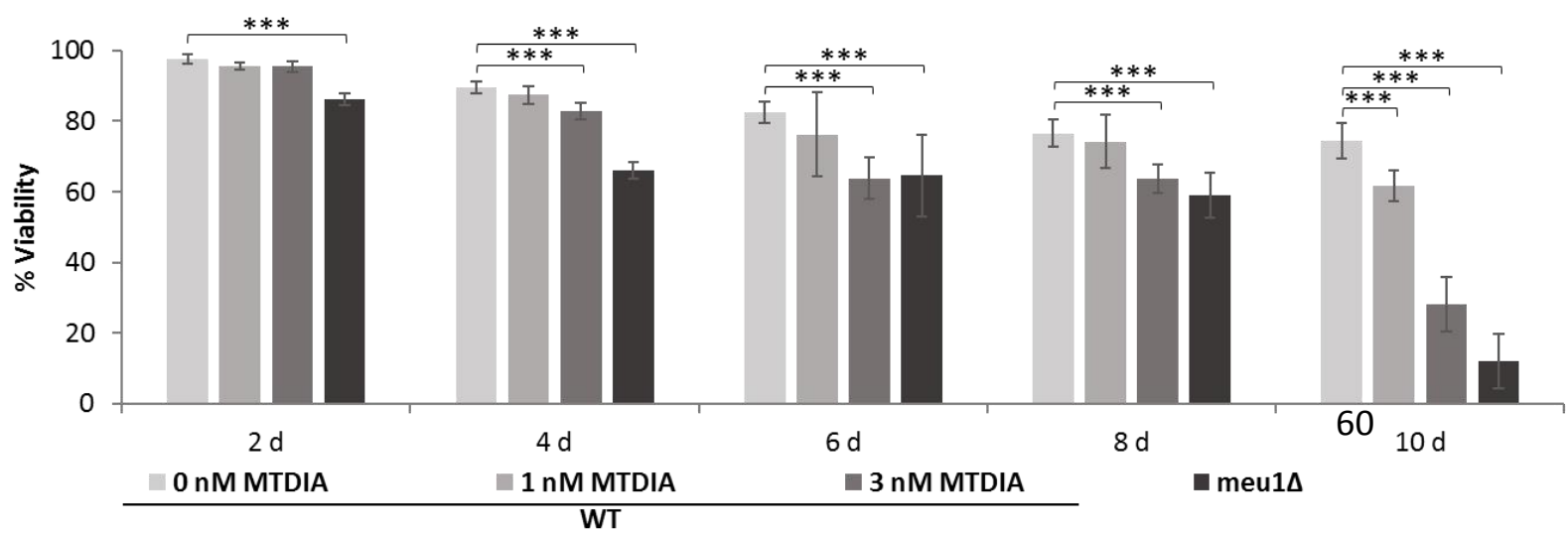


Figure 2.3.8.1 - MTDIA treatment and meu1 $\Delta$ decreases chronological lifespan and exacerbates the growth defect induced by rapamycin. (a) Growth of Rapamycin and MTDIA co-treated cells relative to MTDIA treatment alone. (c) WT and meu1D treated with rapamycin. (c-d) Viability of WT cells treated MTDIA and meu1D assessed with phloxine B at $2 \mathrm{~d}$ intervals. MTDIA and rapamycin cotreatments were performed in liquid media $30^{\circ} \mathrm{C}$ for $48 \mathrm{~h}$. Percent growth was determined in liquid from the growth in the MTDIA + rapamycin co-treatment relative to MTDIA and rapamycin alone (RPM+MTDA/MTDIA)/(RPM/DMSO)×100 at $\mathrm{OD}_{600}$ from six biological replicates. Fractional inhibitory concentration (FIC) index was calculated to distinguish synergy from additive (Appendix Table 7.2.6). Viability assays was performed by culturing yeast strains in $50 \mathrm{~mL}$ volumes of SD-MAUC $+250 \mu \mathrm{M}$ MTA $+2 \%$ raffinose and $2 \%$ galactose media at $30^{\circ} \mathrm{C}$ for $10 \mathrm{~d}$. At $2 \mathrm{~d}$ intervals, $1 \mathrm{~mL}$ of cells were strained with $2 \mu \mathrm{g} / \mathrm{mL}$ phloxine B and were visualised under 60x magnification under DIC and $488 \mathrm{~nm}$ using Upright Automated Fluorescence Microscope (BX63, Olympus) equipped with camera (Dp70, Olympus). Percent viability (unlabelled cells/GFP labelled cells + unlabelled cells)×100 was determined from 1000-1300 cells across five visual fields for each condition and the standard deviation from the five different visual fields are presented.

it is unable to utilise MTA and relied on other methionine salvage pathways for growth. These results are also consistent with the TOR1 gene deletion showing increased sensitivity to MTDIA compared to WT (in the MTDIA chemical screen, Fig. 2.3.7). These results suggest that the exacerbated rapamycininduced growth arrest by MTDIA and meu1s was either due to further induction of autophagy or exacerbation of some other mechanism of rapamycin. The synergistic interaction between rapamycin and MTDIA suggests a TORC1 independent pathway for induction of autophagy.

Defects in autophagy can result in loss of viability (Liu \& Levine, 2015). Measure of cell survival is one approach to monitoring the impact of autophagy and this can be assayed with phloxine $B$, which stains the cytosol of dead cells with disrupted plasma membranes (Noda, 2008). To investigate autophagic cell death, the WT cells treated with MTDIA and meu1A were assessed for viability with phloxine B. The WT cells treated with MTDIA showed a reduction in viability starting at $4 \mathrm{~d}(82.9 \%$ for 3nM MTDIA ( $\left.\sim \mathrm{IC}_{20}\right)$ compared with $89.6 \%$ for WT untreated) (Fig. 2.3.8.1c-d). This defect was further enhanced at $10 \mathrm{~d}$ resulting in a concentration dependent reduction in viability with MTDIA $(74.5 \%$ for WT untreated, $61.7 \%$ for $1 \mathrm{nM}$ MTDIA( $\left.\sim \mathrm{IC}_{10}\right), 28.2 \%$ for $3 \mathrm{nM}$ MTDIA). The decrease in cell viability was 
further exacerbated in the meu1 $\Delta$ gene deletion strain in the absence of MTDIA (66.2\% viability at $4 \mathrm{~d}$ and $13.0 \%$ viability at $10 \mathrm{~d}$ ). This shows that MTAP inhibition maintained over a period of days is cytotoxic and contrary to the cytostatic phenotype observed in section 2.3.3. Together, the exacerbation of rapamycin-mediated growth arrest by MTDIA and increase in autophagic cell death suggests that MTDIA treatment may induce toxic levels of autophagy in yeast. Given that some genes required for autophagy were dispensable in response to MTDIA treatment while others were not, suggesting that some autophagy is needed for survival following MTDIA treatment while too much autophagy is detrimental.

\subsection{MTDIA regulates abundance and localisation of core autophagy proteins}

The autophagy pathway in yeast have been extensively studied and genes involved in each step of autophagy: induction, recognition of cargo and packaging, vesicle nucleation, expansion of the phagophore and autophagosome completion, Atg protein cycling, autophagosome fusion with the vacuole, brake down of cargo and recycling of macromolecules have been identified (Yang \& Klionsky, 2009). To further investigate the potential induction of autophagy in response to MTDIA treatment, the deletion of genes that are essential for both non-selective (macroautophagy) and selective forms of autophagy (cytosol to vacuole transport pathway, pexophagy, mitophagy and ribophagy) were assessed for sensitivity to MTDIA. The genes deletions of core-autophagy proteins did not present sensitive or resistant to MTDIA compared to WT (i.e. $\pm 25 \%$ growth difference) (Fig. 2.3.8.2a). It is possible that although these deletions do not show altered sensitivity, that their function may be altered at the protein level. To investigate if the functions of core-autophagy proteins were affected by MTDIA, the GFP fusion proteins of the core-autophagy genes (from the GFP/dual RFP+hENT1 libraries constructed in Chapter 4) were assessed for change in localisation and abundance in response to MTDIA treatment and loss of meu1 $\Delta$ by Acapella automated image analysis software (Perkin Elmer). The autophagy proteins Atg1 (Atg1 kinase complex), Vps34 (phosphoinositide 3-kinase), Atg3 and Atg4 (Atg8 conjugation system), Atg2 and Atg18 (ATG9 recycling), and Atg20 (cytosol to vacuole targeting 
pathway) showed changes in localisation or change in protein abundance or both either in response to MTDIA treatment or in response to loss of MEU1. Atg1-GFP relocalised from endosomes to the vacuole, and similarly Atg2-GFP relocalised from the cytosol to endosomes (Fig. 2.3.8.2b) following MTDIA treatment, results that were consistent with rapamycin-induced autophagy phenotypes (Nakatogawa et al., 2012; Suzuki et al., 2007). The meu1s in these strains showed phenotypes consistent with MTDIA treatment. The Atg18-GFP was excluded from the vacuole(Suzuki et al., 2007), Atg3-GFP increase in abundance (Huh et al., 2003) and Atg20-GFP relocalised cytosol and endosomes to the mitochondria (Koh et al., 2015) showed nutrient starvation consistent phenotypes in the meu1 $\Delta$ deletion but not with MTDIA treatment under the conditions investigated. The changes in distribution of core-autophagy protein with meu1s in the absence of changes in WT treated with MTDIA may reflect the difference in knock out and knock down of the drug target and a higher concentration of MTDIA or longer treatment regime may be need to observe these phenotypes with MTDIA treatment.

In contrast, Vps34-GFP showed a change in localisation from the vacuole and vacuolar membrane to cytoplasmic foci and ER in meu1D but not with MTDIA treatment. Interestingly, the mislocalisation of Vps34 in meu1 $\Delta$ was also observed in complete minimal (methionine replete) media compared to WT (Appendix Fig. 7.2.3). Therefore, it may present a likely point of perturbation for autophagy induction. All the other observed changes in localisation and abundance were also confirmed by assessment of GFP abundance and localisation in complete minimal (methionine replete) and starvation media. In addition, significant changes in GFP localisation or abundance within the cell was also detected for Atg11, Atg27 and Atg31 with MTDIA treatment in the meu1 $\Delta$ by Acapella. However, these were indistinguishable from WT untreated upon visual inspection but may reflect actual changes and could be validated by western blot analysis. The changes in abundance and localisation of autophagy proteins to MTDIA treatment or loss of its drug target (with the exception of Vps34) are consistent with starvation media and rapamycin treatment in published literature and confirms an induction of starvation induced autophagy although the deletions of their genes did not show altered sensitivity from WT. The change in Vps34 in complete media may reflect some additional 


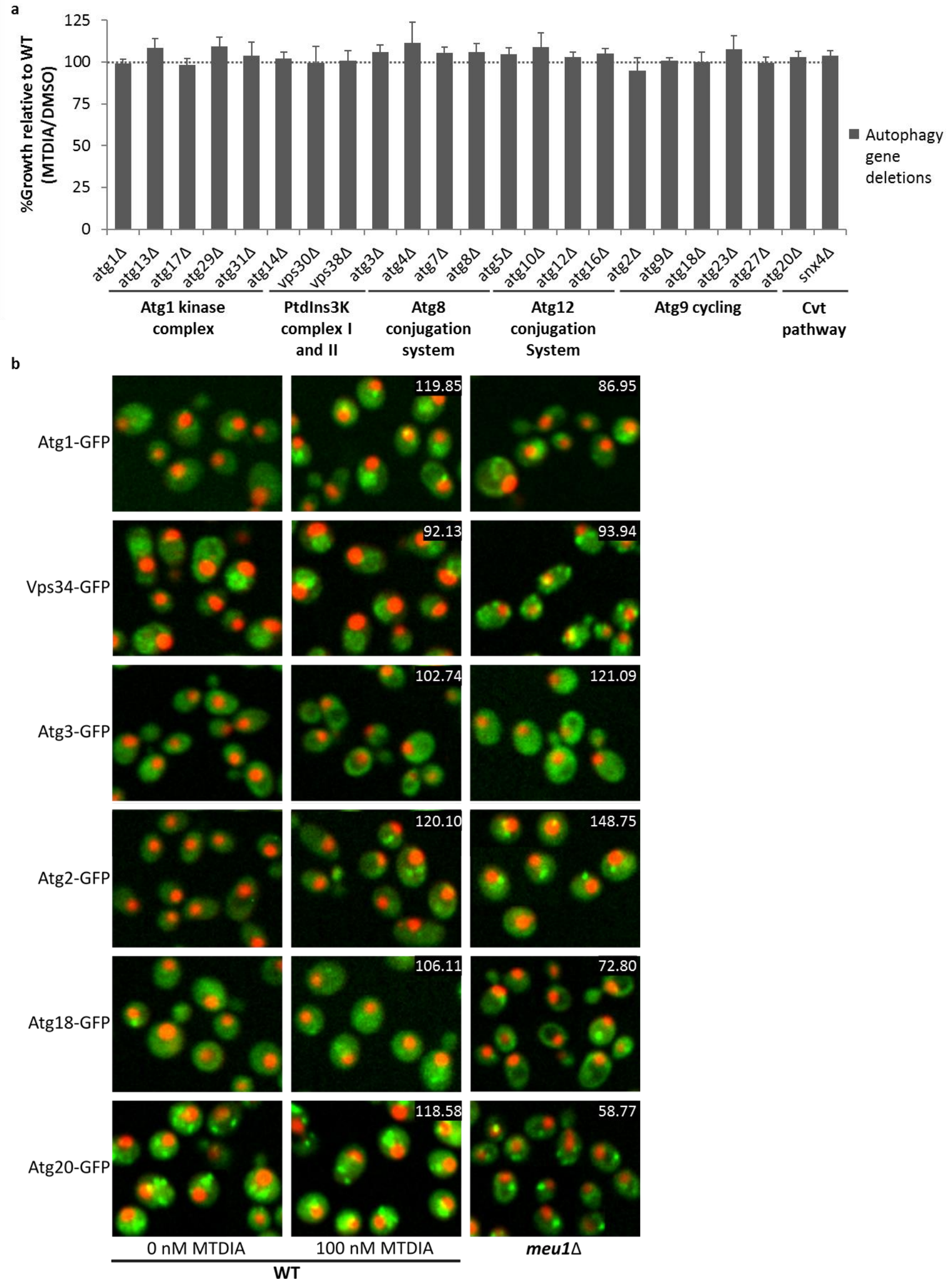


Figure 2.3.8.2 - Protein levels and localisation of core autophagy proteins in response to MTDIA treatment although the gene deletions do not show altered sensitivity. (a) Deletions strains defective in core-autophagy genes treated with $3 \mathrm{nM}$ MTDIA $\left(\sim \mathrm{IC}_{30}\right)$. (b) Abundance and localisation of coreautophagy proteins fused to GFP in WT, WT with $100 \mathrm{nM}$ MTDIA and in meu1A. The gene deletion strains were treated with $2 \mathrm{nM}$ MTDIA in SD-MAUC $+2 \%$ galactose $+250 \mu \mathrm{M}$ MTA at $30^{\circ} \mathrm{C}$ for $48 \mathrm{~h}$. The growth of the mutants relative to WT in MTDIA and carrier (MTDIA $x x x \Delta / D M S O x x x \Delta$ )/ (MTDIA WT /DMSO WT) $\times 100$ were determined at $\mathrm{OD}_{600}$. Mutants with a growth defect or growth improvement $> \pm 25 \%, p<0.05$ relative to WT were considered a growth defect or a growth improvement. The coreautophagy GFP strains were cultured in SD-MAU $+2 \%$ galactose $+250 \mu \mathrm{M} \mathrm{MTA}$ media for $6 \mathrm{~h}$ at $30^{\circ} \mathrm{C}$. Florescent signals were detected at $488 \mathrm{~nm}$ (GFP) and 561 (nuclear localisation signal fused to RS2 and cytosolic mCherry) using the high-throughput spinning disk confocal microscope (Evo Tec OPERA, Perkin Elmer) using the 60x water immersion lens (NA 1.2). The GFP abundance and localisation was quantified by automated image analysis (Acapella, Perkin Elmer)(Bircham et al., 2011). Numbers inset in each image are the percent changes in GFP abundance with either MTDIA or meu1 $\Delta$ relative to WT untreated. Phosphoinositide 3-kinase (PtdIns3K); cytosol to vacuole transport (cvt).

inducer of autophagy besides amino acid starvation. Several potential induces of autophagy that are amino acid starvation independent were identified in the meu1D and MTDIA screens notable of these were disruptions to ergosterol biosynthesis and phospholipid biosynthesis both of which can induce autophagy (Cheng et al., 2006).

\subsection{MTDIA induces autophagic trafficking of GFP-Atg8 independent of starvation}

To further confirm an induction of autophagy following MTDIA treatment, the localization of GFP-Atg8, the yeast orthologue of the mammalian autophagosome marker LC3, was evaluated. LC3/Atg8 is one of two proteins that remain associated with the completed vesicle throughout the autophagy pathway and is frequently used as a marker for monitoring autophagic activity in yeast and mammalian cells (Merkulova et al., 2014; Klionsky et al., 2007; Strømhaug et al., 2004). Following induction of autophagy in starvation media or following rapamycin treatment, Atg8 localises from cytosolic foci (autophagosomes) to the vacuole (Suzuki et al., 2001; Kim et al., 2001). The yeast strains YCG640 (WT) and YCG643 (meu1A) with hENT1::URA3 (constructed in Chapter 4) were transformed 
with natMX4 selectable marker from p4339 plasmid (Goldstein \& McCusker, 1999) (to replace URA3 with natR) followed by GFP-ATG8::Cen::URA3 plasmid (Suzuki et al., 2001) to generate GFP-Atg8 expressing strains in the dual RFPs background (to observe GFP-Atg8 fluorescence relative to RFP fluorescence of cytosol and nucleus in each cell). The localisation of GFP-Atg8 in WT and meu1D were assessed by florescence microscopy after $3 \mathrm{~h}$ incubation in SD-MAU+ $2 \%$ galactose $+250 \mu \mathrm{M}$ MTA media with and without MTDIA. In the absence of MTDIA treatment, WT cells showed nearly equal amounts of vacuolar and cytosolic localisation of GFP-Atg8 (47.2\% and $52.9 \%$ respectively) (Fig. 2.3.8.3a-b). Following treatment of $10 \mathrm{nM}$ MTDIA, $72.8 \%$ of cells exhibited vacuolar localisation of GFP-Atg8. In the genetic mimic meu1 $\Delta$, this autophagy phenotype was also apparent with $79.43 \%$ of the cells showing vacuolar localisation of GFP-Atg8.

To reduce the background contribution of starvation-induced autophagy, cells were cultured in media supplemented with $1 \mathrm{mM}$ MTA (Fig. 2.3.8.3c-d) and were treated with $100 \mathrm{nM}$ MTDIA to account for higher levels of MTA and the short incubation time. Under these conditions $88 \%$ of the WT cells showed cytosolic location for GFP-Atg8 in the absence of MTDIA. Following 100 nM MTDIA treatment, a direct inverse of this was observed where nearly $90 \%$ of the cells showed vacuolar localisation of GFP-Atg8. This phenotype was conserved in the meu1 $\Delta$ strain in the absence of MTDIA wherein vacuolar localisation of GFP-Atg8 was observed.

To further investigate the induction of autophagy by MTDIA treatment, the autophagy pathway was disrupted with gene deletions that halted the autophagy process at various steps. The conjugation of Atg8 to the phosphatidylethanolamine (PE) on the autophagasomal membrane and deconjugation of ATG8 from the PE requires the cysteine protease, Atg4 (Yu et al., 2012). The dissociation of Atg proteins from the phagophore assembly site (PAS) requires Atg1 kinase (Cheong et al., 2008). Following delivery of autophagic bodies to the vacuolar lumen, intra vacuolar breakdown requires the vacuolar hydrolase, Pep4 (Xie et al., 2008). The deletions of genes ATG1, ATG4 and PEP4 were used for assessment of GFP-Atg8 localisation following induction of autophagy. As expected, WT cells showed mostly cytosolic localisation of GFP-Atg8 without treatment and mostly vacuolar 


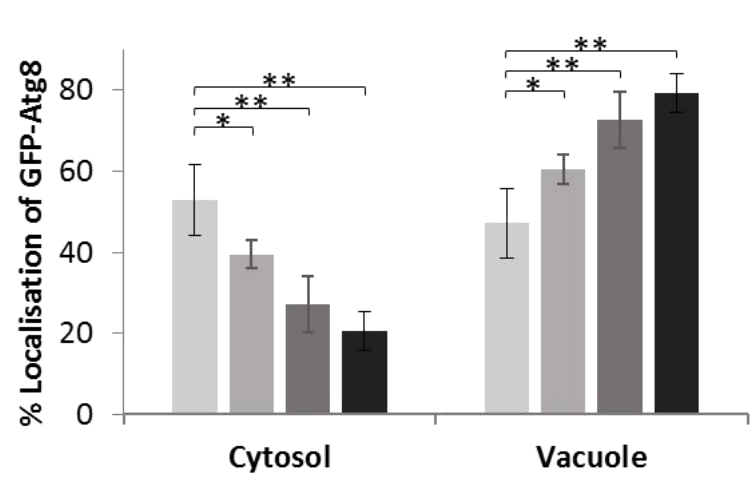

WT OnM MTDIA WT 10nM MTDIA

WT 3nM MTDIA

meu1 $1 \Delta$

C

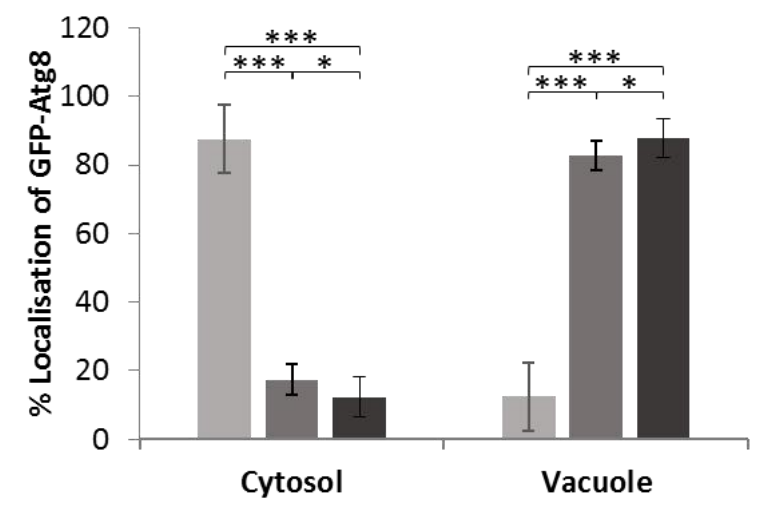

WT+1 mM MTA

- WT+1mM MTA +100nM MTDIA

- meu1 $1 \Delta+1 \mathrm{mM}$ MTA

e

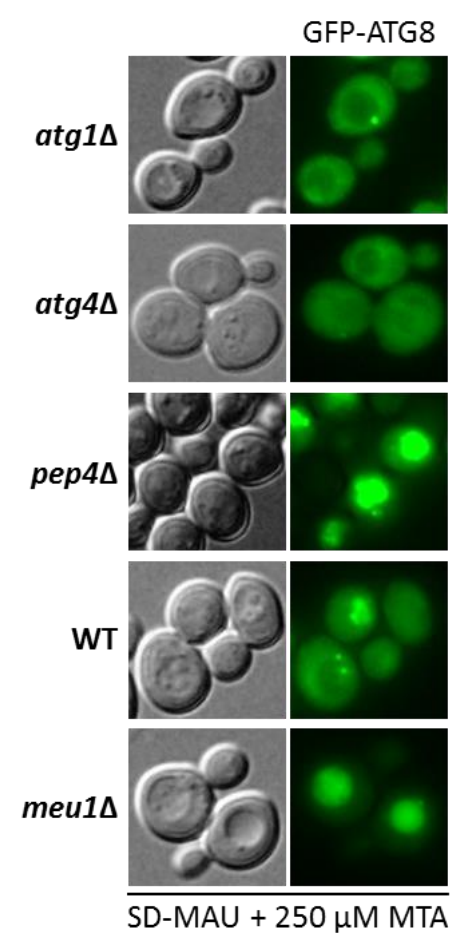

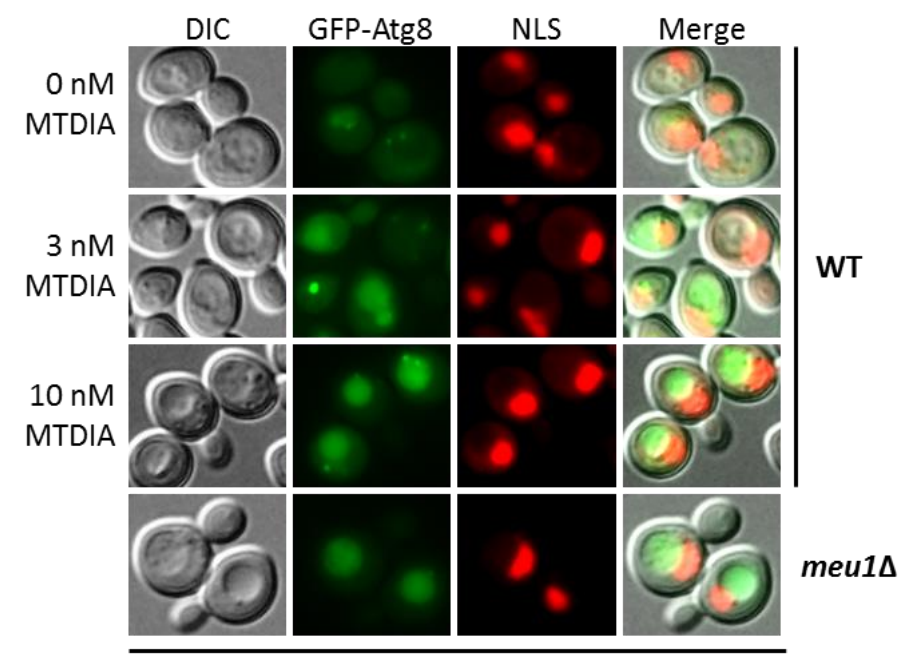

SD-MAU+ $250 \mu \mathrm{M}$ MTA

d

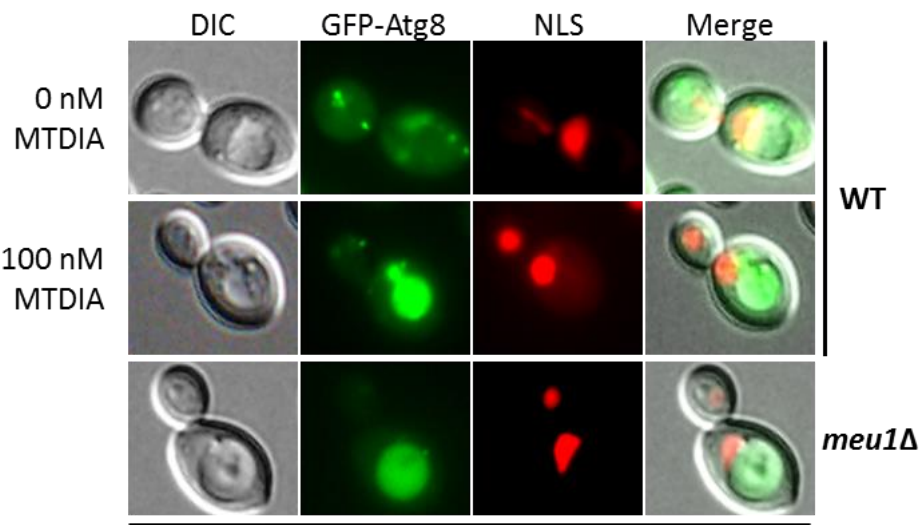

SD-MAU+ 1 mM MTA
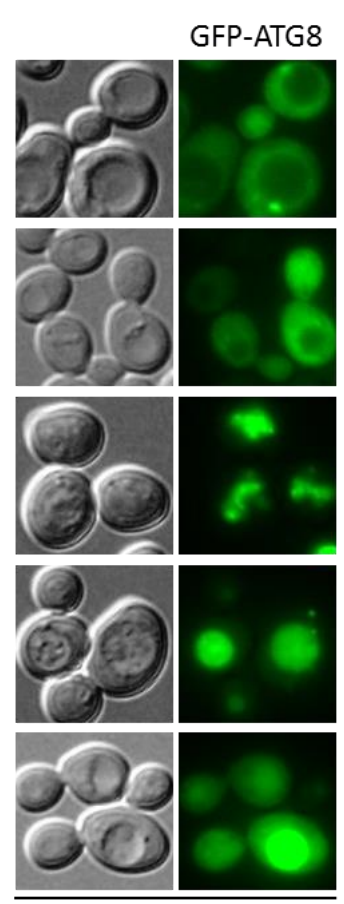

SD-MAU + $250 \mu \mathrm{M}$ MTA $+10 \mathrm{nM}$ MTDIA
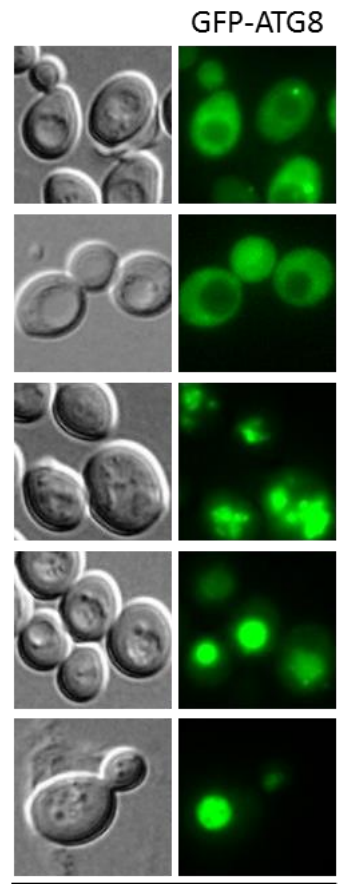

SD-N + $2 \%$ Gal
GFP-ATG8
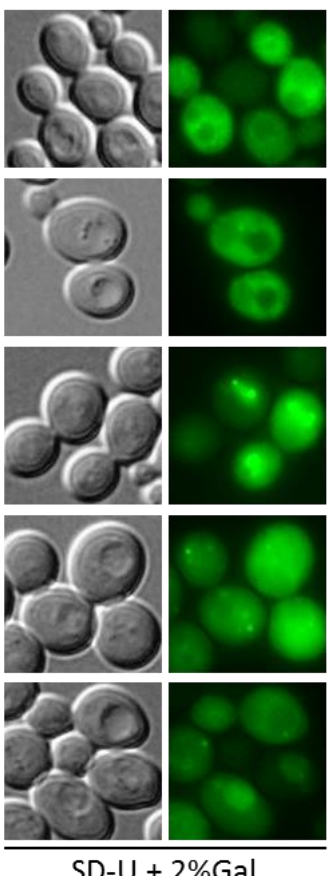

SD-U $+2 \%$ Gal 
Figure 2.3.8.3 - MTDIA treatment or meu1D induces autophagy that is Atg1, Atg4 and Pep4 dependent. The localisation of GFP-ATG8 was visualised in cells treated with MTDIA (a-b) WT and

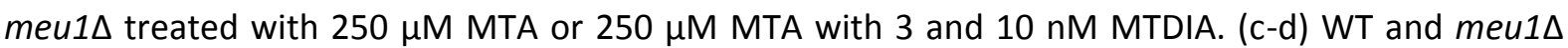
treated with $1 \mathrm{mM}$ MTA or $1 \mathrm{mM}$ MTA with $100 \mathrm{nM}$ MTDIA. (e) atg1 subcultured in SD-N, SD-Ura or SD-MAU media with $250 \mu \mathrm{M}$ MTA or $250 \mu \mathrm{M}$ MTA + 10 nM MTDIA. GFP-ATG8 expressing strains were subcultured with 250 or 1 mM MTA and appropriate concentrations of MTDIA in SD-MAU+2\% galactose media for $3 \mathrm{~h}$ (or SD-N $+2 \%$ galactose, SD-Ura+2\% galactose). The fluorescent signals were detected at $488 \mathrm{~nm}$ (GFP-ATG8) and 589 (nuclear localisation signal fused to RS2) using Upright Automated Fluorescence Microscope (BX63, Olympus) equipped with camera (Dp70, Olympus). Where appropriate, the localisation of GFP-ATG8 is presented as an average from five visual fields with $300-400$ cells counted per condition.

localisation following MTDIA treatment. In contrast, the $\operatorname{atg} 1 \Delta$ and $\operatorname{atg} 4 \Delta$ deletions prevented the localisation of GFP-Atg8 to the vacuole and showed predominantly cytosolic location in both untreated and MTDIA treated cells (Fig. 2.3.8.3e). The pep4 $\Delta$ prevented the degradation of GFP-Atg8 at the vacuolar lumen with localisation to vesicles in the vacuole in both MTDIA-treated and untreated cells. The GFP-Atg8 localisations observed for $\operatorname{atg} 1 \Delta$, atg $4 \Delta$ and pep4 $\Delta$ in MTDIA treated and untreated cells were unchanged from phenotypes observed in starvation-induced autophagy (Yeh et al., 2010; Kim et al., 2001), suggesting that MTDIA is inducing a stable and conserved form of autophagy. Overall, the increased localisation of GFP-Atg8 to the vacuole by MTDIA treatment that is dependent on a functional autophagic pathway confirms an induction of autophagy as a mechanism of MTDIA toxicity.

\subsection{MTDIA inhibits ergosterol biosynthesis}

Gene deletions involved in sterol homeostasis were sensitive (ERF2, ETR1, YHLOO8C) or resistant (CGS2) to MTDIA and showed an exacerbated growth phenotype with meu1D (OPI3, CSG2, ETR1, INO2, SHR5, CEM1). We hypothesize that sterol biosynthesis is not directly affected by loss of MEU1 activity but rather by depletion of the downstream product S-adenosyl methionine, a coenzyme required for the delta(24)-sterol C-methyltransferase activity facilitated by ERG6 (Hardwick \& 
Pelham, 1994) and phospholipid methyl transferases activity by CHO2 and OPI3 (Kodaki \& Yamashita, 1987). In contrast higher eukaryotes, the yeast plasma membrane consists of mainly ergosterol and minor amounts of zymosterol. The conversion of fecosterol to zymosterol is facilitated by Erg6 (Heidepriem et al., 1992; Lees et al., 1995). Although Erg6 function is non-essential for yeast cell division, it is required for normal membrane permeability and fluidity as reflected by altered sensitivity and resistance to xenobiotics and the permeability of nutrients (Gaber et al., 1989). Notable is Tat2, a high-affinity tryptophan transporter of the plasma membrane that is missorted to vacuole in response to loss of Erg6 function (Umebayashi \& Nakano, 2003) showed an exacerbated growth defect with meu1 1 . Phospholipid composition of the membrane regulated by $\mathrm{OPI} 3$ and $\mathrm{CHO} 2$ are also required for membrane fluidity (de Kroon et al., 2013). The de novo biosynthesis of phosphatidylcholine from phosphatidylethanolamine by S-adenosyl methionine dependent phospholipid methyl transferases is the major consumer of S-adenosyl methionine in mice (Noga et al., 2003), in humans (Stead et al., 2006), and in yeast cultured in the absence of exogenous choline and ethanolamine (Tehlivets, 2011). It is possible that decreased S-adenosyl methionine is leading to a reduction in Erg6, Cho2 and Opi3 activity and consequently, a downregulation in ergosterol and phospholipid biosynthetic pathways.

To investigate disruptions to sterol synthesis, the abundance and localisation of four GFP fusion proteins in middle and late stages of the ergosterol biosynthesis pathway were assessed in response to MTDIA treatment of WT cells or in the meu1s strain by confocal microscopy. The localisation of three proteins was altered in response to MTDIA treatment as well as MEU1 deletion. Erg24 was relocalised from the ER to cytosol and vacuole, Erg6 from ER and lipid droplets to lipid droplets, and Erg3 from ER to the vacuole (Fig. 2.3.8.4a). In addition, the levels of Erg1 was significantly decreased with MTDIA. These results suggest that MTDIA treatment leads to a disruption in the sterol biosynthesis pathway. To further characterise disruptions to sterol biosynthesis pathway, the levels of ergosterol and ergosterol precursors (squalene and lanosterol) were quantified by GC-MS (Fig. 2.3.8.4c). Reduced levels of ergosterol coincident with increased levels of squalene and lanosterol were observed in MTDIA-treated WT cells or untreated meu1 $\Delta$ cells. These results are consistent with 
a
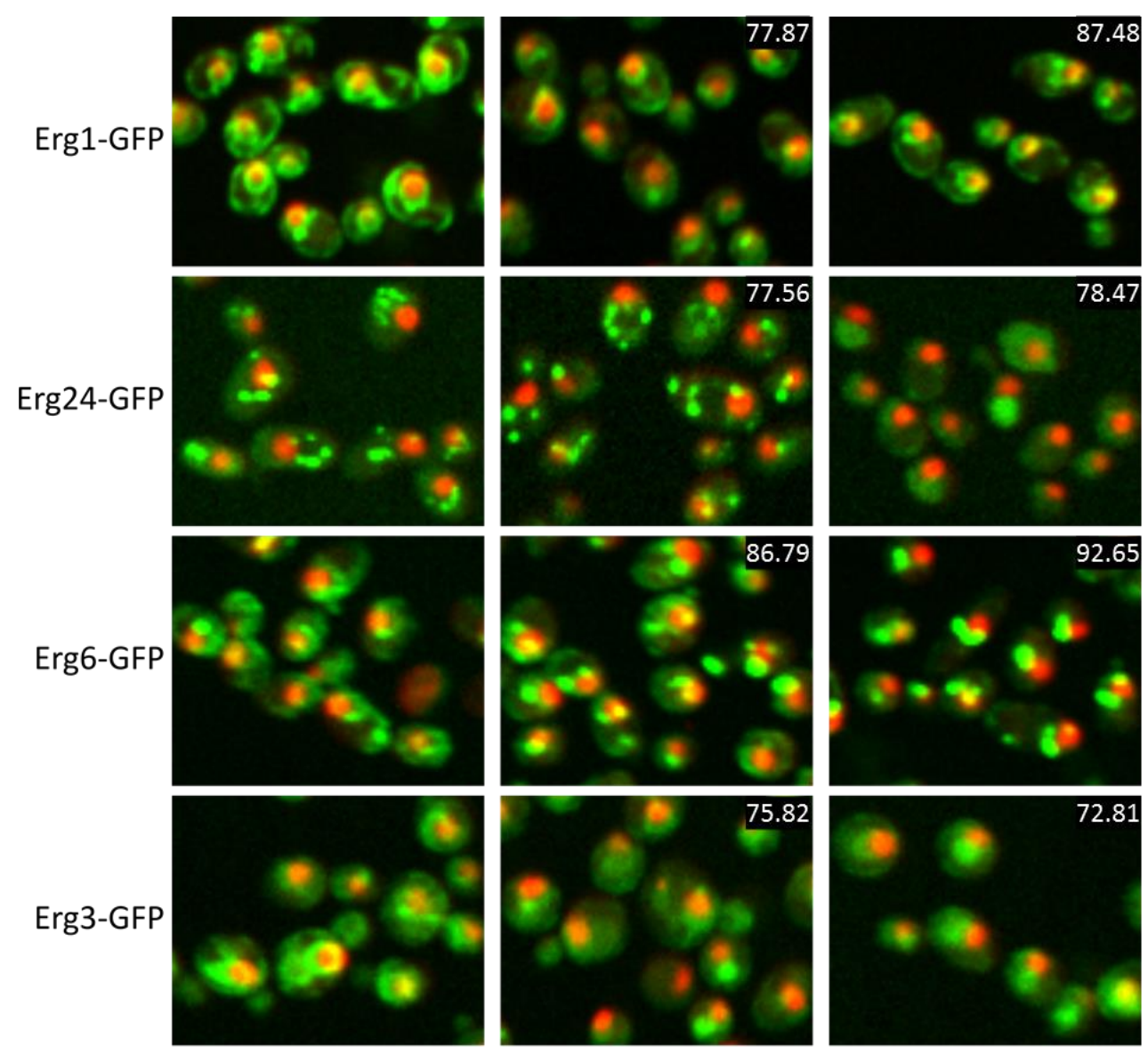

0 nM MTDIA

100 nM MTDIA

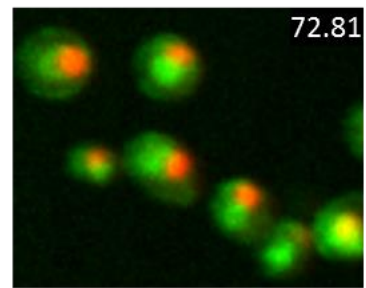

meu1s

c Acetyl-CoA $\underset{\text { (9 steps) }}{\longrightarrow}$ Squalene $\underset{\text { Erg1 }}{\longrightarrow}$ (S)-2,3-Epoxysqualene

WT

Erg7 Lanosterol

$\operatorname{Erg11}$

Erg25, Erg26, Erg27 (10 Steps)

Erg24

$\downarrow$

Zymosterol $\underset{\text { Erg6 }}{\longrightarrow}$ Fecosterol $\underset{\text { Erg2 }}{\longrightarrow}$ Episterol $\underset{\text { Erg3 }}{\longrightarrow} \begin{aligned} & \text { E,7,24(28)- } \\ & \text { Ergostatrienol }\end{aligned} \underset{\text { Erg5 }}{\longrightarrow} \underset{\text { Erg4 }}{\longrightarrow}$ Ergosterol

d

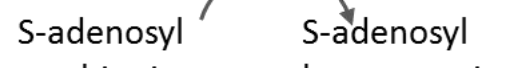

methionine homocysteine

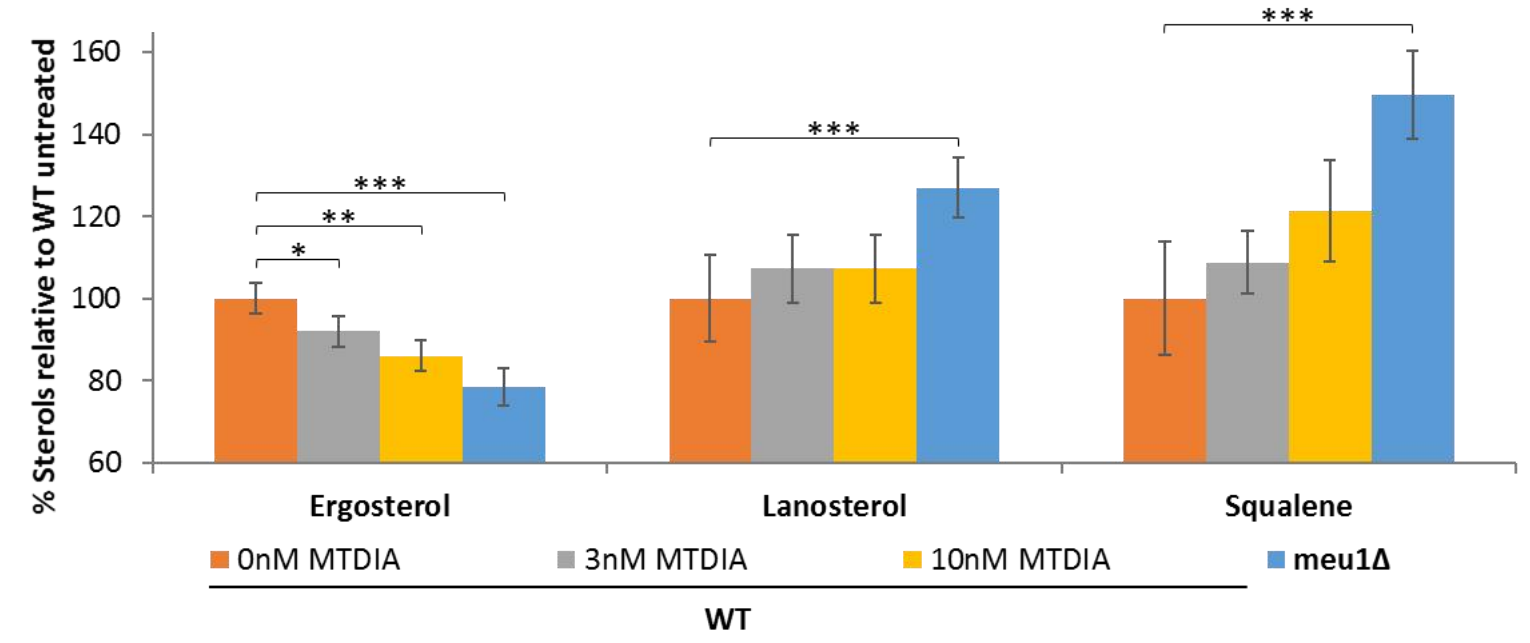


Figure 2.3.8.4 - MTDIA treatment and meu1s inhibits ergosterol biosynthesis. (a) WT expressing ergosterol biosynthesis proteins fused to GFP treated with $100 \mathrm{nM}$ MTDIA and meu1 $\Delta$. (b) Simplified ergosterol biosynthesis pathway showing sterol intermediates and genes of relevance. (c) Quantitation of sterol intermediates in WT and meu1A by GC-MS. GFP fusion strains within ergosterol biosynthesis were cultured in SD-MAU $+2 \%$ galactose $+250 \mu \mathrm{M}$ MTA media for $6 \mathrm{~h}$ at $30^{\circ} \mathrm{C}$. Florescent signal was detected at $488 \mathrm{~nm}$ (GFP) and 561 (nuclear localisation signal fused to RS2 and cytosolic mCherry) using the high-throughput spinning disk confocal microscope (Evo Tec OPERA, Perkin Elmer) using the 60x water immersion lens (NA 1.2). Numbers inset in each image are the percent changes in GFP abundance with either MTDIA or meu1 1 relative to WT untreated. Sterols were extracted from OD600 5.0 units using chloroform-methanol. The lipid pellets were dried, derivatised, diluted in hexane and run on a GC-MS (QP2010-Plus, Shimadzu). The identity of the sterols were confirmed using authentic standards. The percent of each sterol relative to the total sterols (i.e., Ergosterol /(Squalene+Lanosterol+Ergosterol)x100) from six biological replicates experiments are presented.

reduced protein levels of Erg1 and Erg24 (Fig. 2.3.8.4a) and overall confirm that MTDIA and loss of MEU1 inhibits the ergosterol biosynthesis in yeast.

\subsection{Discussion}

Screening of S. cerevisiae gene deletion mutants for gene-drug and gene-gene interactions permits an unbiased assessment of drug and drug target functions. In this chapter, we employed these methodologies for assessment of mechanisms in MT-DADMe-immucillin A (MTDIA), a transition state structure inhibitor of human MTAP. In this two-pronged approach, the non-essential nature of MEU1 (yeast orthologue of MTAP) was exploited for assessment of gene-gene interactions with the drug target of MTDIA. However, the non-essentiality of Meu1 also prevented the bioactivity of MTDIA coupled with the lack of nucleoside uptake in yeast. To achieve bioactivity, MEU1 was made essential by providing 5'-methylthioadenosine (MTA) as the only sulphur source in the met17 $\Delta$ background that requires the function of MEU1 for synthesis of methionine from MTA, and the uptake of nucleoside MTA and MTDIA was permitted by expression of the human ENT1 nucleoside transporter. The druggene interaction screen that followed identified the same cellular processes as the gene-gene interaction screen, thus corroborating the use of the drug target deletion as a proxy for MTDIA. These 
cellular processes included both expected and novel mechanisms for MTDIA. The cellular processes chromatin organisation, transcription and translation were expected and are consistent with findings in mammalian cell line and murine models (Pollard et al., 1999; Mandal et al., 2013). The novel findings included disruptions to amino acid metabolism, response to starvation, vacuole fusion and vesiclemediated transport, carbohydrate metabolism, lipid homeostasis, and $\mathrm{NAD}^{+}$biosynthesis. From these, disruptions to autophagy and ergosterol biosynthesis were further investigated in follow-up experiments.

Autophagy is an intracellular degradation pathway where cytosolic content is degraded via the vacuole and recycled to compensate for nutrient deprivation (Suzuki \& Ohsumi, 2010). Autophagy was investigated as a possible mechanism of MTDIA mediated growth inhibition in yeast and was shown to induce TOR1C-independent autophagic cell death. MTDIA and meu1D exacerbated the rapamycin induced growth arrest and reduced replicative lifespan. The protein levels and localisation of several core-autophagy genes changed in response to MTDIA treatment and meu1 $\Delta$ including Atg8 (yeast orthologue of LC3), a well characterised marker for autophagy. The change in localisation of Atg8 was prevented by deletion of essential autophagy pathway genes such as ATG1, ATG4 and PEP4. These results confirmed induction of autophagy by MTAP inhibition or loss in yeast. Of these core autophagy proteins, Vps34 (PI3PK) distribution was altered from the vacuolar lumen/vacuolar membrane to cytoplasmic foci in meu1 $\Delta$. Interestingly, Vps34 inhibitors (e.g. alpelisib and buparlisib) are promising drugs currently in clinical trials (Massacesi et al., 2016).

Sterol biosynthesis is an essential process required for viability under both aerobic and anaerobic conditions (Bard et al., 1993). Ergosterol is the major sterol in yeast membranes and is synthesised from acetyl-CoA in a pathway that is highly conserved from yeast to humans (Nielsen, 2009; Nes, 2011). Following MTDIA treatment or in the meu1 $\Delta$ strain, the localisation and abundance of several ergosterol biosynthetic proteins were altered. In addition, there was a reduction in ergosterol levels coincident with increases in two sterol intermediates (lanosterol and squalene), which was consistent with the downregulation of Erg1 and Erg24 proteins that process these sterol 
intermediates. Interestingly, Erg1 and Erg6 were downregulated with MTDIA and meu1 $\Delta$ which, based on the upregulation of these proteins in response to amino acid deprivation (Huh et al., 2003), suggests that the sterol changes with MTDIA and meu1D are independent of starvation. Therefore, inhibition of ergosterol biosynthesis is a novel mechanism of Meu1 inhibition. The inhibition of ergosterol biosynthesis is likely mediated through inhibition of Meu1 leading to an accumulation of decarboxylated S-adenosyl methionine, an inhibitor of S-adenosylmethionine dependent methyltransferase activity of Erg6 (McCammon et al., 1984; Frostesjo et al., 1997). This prediction would be consistent with our observation of reduced Erg6 levels with MTDIA treatment or loss of Meu1 and localisation. We also observed mislocalisation of Erg3 from the ER to the vacuole, which may be due to degradation of sterols and lipid droplets by autophagy (lipophagy) to compensate for the reduced ergosterol levels resulting from Meu1 inhibition (Liu \& Czaja, 2013). Although the Erg6 mechanism suggested for MTDIA-mediated sterol biosynthesis is not conserved from yeast to humans (i.e., the conversion of zymosterol to fecosterol catalysed by s-adenosyl methionine dependent Erg6 is not conserved (Umate, 2015)), polyamine depletion in mammals reduces cholesterol levels and may still present a viable mechanism of MTAP inhibition and an inducer of autophagy (Kee et al., 2004). Compellingly, MTDIA treatment of A549 tumours in mice lead to $>3$-fold change in expression of genes required for cholesterol homeostasis (Basu et al., 2011).

Lipids are critical in the regulation of many biochemical processes including the membrane remodelling that underlies autophagosome biogenesis and autophagy process (Jaishy \& Abel, 2016). In humans, lipids and lipid metabolising enzymes mediate the autophagy process (Dall'Armi et al., 2013). For example, mammalian phosphoinositide 3-kinase class I (PI3K-I), when activated phosphorylates $\mathrm{PI}(4,5) \mathrm{P} 2$ to $\mathrm{PI}(3,4,5) \mathrm{P} 3$ on the plasma membrane that recruits and activates $\mathrm{AKT}$, which in turn promotes mTORC1 signalling pathway and inhibits autophagy (Zoncu et al., 2011). Although, PI3K-I kinases are absent in yeast, the pathway downstream of PI3K-I kinase is conserved from yeast to humans including TOR and regulators of TOR (Vivanco \& Sawyers, 2002). Phosphatidylinositol 3-phosphate (PI3P) synthesised by PI3Kinase class III (PI3K-III, yeast Vps34 
orthologue) acts as a membrane-bound signal for assembly of protein scaffolds at the membranecytosol interface and is required for autophagosome formation, maturation and transport (Knævelsrud \& Simonsen, 2012). Also related to autophagy, phosphatidylethanolamine (PE) is required for lipidation of LC3/Atg8 to LC3/Atg8-PE (Kabeya et al., 2004). Lipids also directly affect physiochemical properties of the lipid bilayer itself (e.g., curvature and fluidity) (Haucke \& Di Paolo, 2007). In addition, non-canonical mTOR independent forms of autophagy have been identified in mammals that are mediated by small molecules and unesterified fatty acids that can bypass the requirement of majority of Atg proteins (Codogno et al., 2012; Niso - Santano et al., 2015). The roles of Vps34/PI3K-III and lipids highlighted here reflects the importance of Vps34 and lipids in regulation of autophagy in mammals.

In yeast, there are two Vps34 (PI3K-III) complexes that differ in their subcellular localisation and function. Vps34 complex II (Vps34, Vps30/Atg6, Vps15, Vps38) is localised to endosomes and involved in carboxypeptidase Y (CPY) sorting to the vacuole. Vps34 complex II in humans also plays a role in autophagy mediated through BECN1 (Vps30/Atg6 orthologue) interactions with UVRAG (Vps38 orthologue) (Simonsen \& Tooze, 2009). Vps34 complex I (Vps34, Vps30/Atg6, Vps15, Atg14) is localised to the PAS (phagosome assembly site) and required for induction of autophagy. In higher eukaryotes, vesicles containing Vps34 complex I are targeted to subdomains of ER during starvation where PI3P is synthesised and autophagy is induced (Axe et al., 2008). The recruitment of Vps34 complex I to the subdomains of ER in response to starvation is well characterised in mammals, but has not been observed in yeast previously. Based on the mislocalisation of Vps34 observed with Meu1 loss in yeast, we predict a similar role for Vps34 complex I to PI3K-III complex I. The increased levels of PI3P produced by mislocalised Vps34 at subdomains of the ER/sites of autophagosome formation is responsible for autophagy observed with Meu1 loss and presents novel mechanism for induction of autophagy by MTDIA in yeast. The depletion of cholesterol is also known to induce autophagy in mammalian cells (Cheng et al., 2006), thus it will be interesting to investigate any link between the ergosterol reduction by MTDIA and autophagy induction by MTDIA. Polyamine depletion by 
upregulation of SSAT (spermidine/spermine $\mathrm{N}(1)$-acetyltransferases) reduces the plasma cholesterol levels (Pirinen et al., 2010), therefore the cholesterol biosynthesis pathway might yet be modulated by MTAP inhibition. Moreover, changes in membrane curvature by depletion membrane sterol may be responsible for mislocalisation of $\mathrm{Vps} 34$, and thus would be interesting to investigate.

Overall, the results of this chapter provided a global survey of the cellular effects of MTAP inhibition, either via MTDIA treatment or MEU1 deletion. Suspected pathways were identified that justified the use of yeast, and notable among the unsuspected pathways was the induction of autophagy via a rapamycin-independent and Vps34/PI3P-dependent mechanism. As phospholipid synthesis (methyltransferase dependent) and phosphoinosiditde-3-phosphate synthesis (Vps34) are conserved from yeast to humans, MTAP inhibition may also induce autophagy in humans and might be a mechanism for MTDIA mediated cell death in humans. Given that small molecules modulating autophagy in a myriad of human disorders including cancer, heart disease, obesity and neurodegeneration are currently the focus of intensive investigation (Jiang \& Mizushima, 2014; Kovsan et al., 2010; Levine \& Kroemer, 2008), the results of this chapter suggest that MTDIA can be considered among these autophagy modulators. 


\section{Chapter 3 - Elucidating the anticancer mechanisms of nucleoside analogue MTDIA by global analysis of protein localisation and abundance}

\subsection{Introduction}

MTDIA is a potent inhibitor of the human enzyme $5^{\prime}$-methylthioadenosine phosphorylase (MTAP) in vitro $(K d=86 \mathrm{pM})$ that mimics the enzymatic transition state structure of MTA bound by MTAP (Schramm, 2011). Its efficacy has been demonstrated in vivo in mouse xenograft models for human lung, prostate, colon, cervical, head and neck, and triple negative breast cancers, as well as various human cancer cell lines (Basu et al., 2007; Basu et al., 2011). MTDIA mimics the loss of MTAP, resulting in an accumulation of MTA, a reduction in S-adenosyl methionine, and a reduction in polyamine levels (spermidine and spermine). The mechanisms responsible for activation of apoptotic pathways leading to G2/M arrest, loss of mitochondrial membrane integrity, cleavage of PARP and caspases are yet to be characterised (Basu et al., 2011). Since the initiation of apoptosis is cell type specific (Basu et al., 2007), an additional level of complexity exists beyond the accumulation of MTA initiating apoptosis and the genetic mechanisms underlying these processes are not characterised.

In Chapter 2, several potential mechanisms for MTDIA mediated growth inhibition were identified including induction of autophagy and inhibition of ergosterol biosynthesis. To complement the gene-gene and gene-drug interactions that were identified with genomic libraries of gene deletion strains, this chapter investigated MTDIA mechanisms via measurements of the abundance and localisation of normal (not mutated) proteins. Specifically, experiments were conducted with a GFP library expressing human ENT1 (4,900 strains) where each strain contains a different protein fused at the C-terminus to GFP. Protein localisation and abundance were investigated in meu1 $\Delta$ (genetic mimic of MTDIA treatment) and MTDIA-treated cells. This unbiased approach both complements the growthspecific phenotypes in deletion library screens as well as provides new insight into changes at the protein level that may not necessarily alter the growth phenotype. The GFP library also permits the assessment of proteins for essential genes that were absent in the gene deletion libraries screen with meu1D and MTDIA in Chapter 2. 


\subsection{Experimental procedures}

3.2.0 Yeast strains, media and compounds

\subsubsection{Yeast strains}

All S. cerevisiae strains used in this chapter were derived from yeast strains BY4741 and BY4742 (Brachmann et al., 1998) and were constructed by heterologous expression of human equilibrative nucleoside transporter 1 (ENT1) gene from plasmid pYhENT1 ((Vickers et al., 1999); gift of Carol E. Cass) (Table 3.2.1).

\begin{tabular}{|c|c|c|}
\hline Strain & Genotype & Reference \\
\hline BY4741 & MATa his3 $\Delta 1$ leu2 $\triangle 0$ lys $2 \Delta 0 \mathrm{MET15}$ ura3 $\Delta 0$ & $\begin{array}{l}\text { (Brachmann et } \\
\text { al., 1998) }\end{array}$ \\
\hline BY4742 & MATa his3 $\Delta 1$ leu $2 \Delta 0$ LYS2 met15 $\Delta$ ura3 $\Delta 0$ & $\begin{array}{l}\text { (Brachmann et } \\
\text { al., 1998) }\end{array}$ \\
\hline YCG386 & 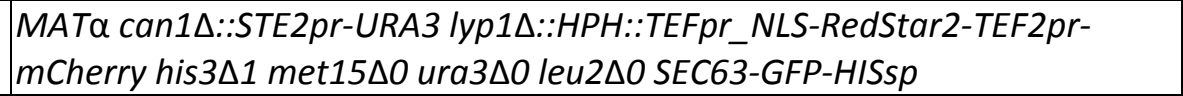 & (Low, 2013) \\
\hline Y7039 & 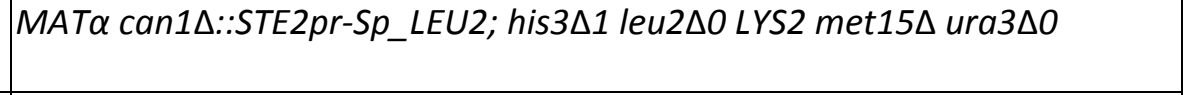 & $\begin{array}{l}\text { (Tong \& Boone, } \\
2007)\end{array}$ \\
\hline WT (diploid) & 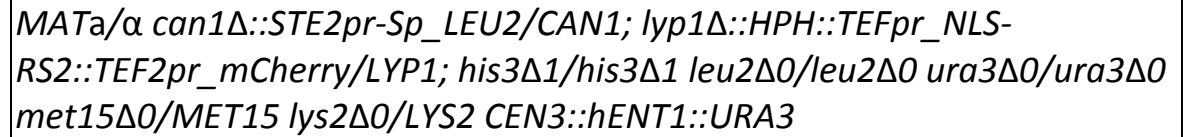 & This study \\
\hline WT (YCG640) & 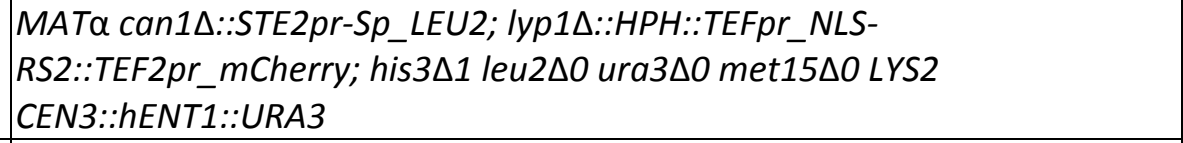 & This study \\
\hline $\begin{array}{l}\operatorname{meu1\Delta } \\
(\text { YCG640) }\end{array}$ & 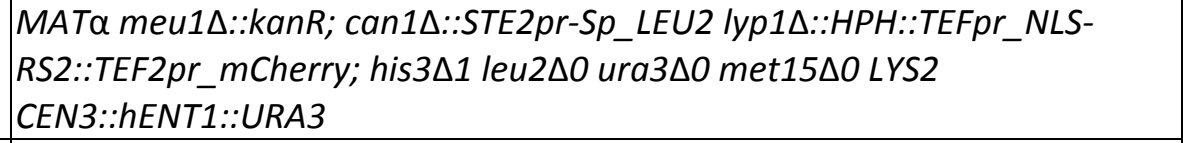 & This study \\
\hline $\begin{array}{l}\text { Xxx-GFP (GFP } \\
\text { library) }\end{array}$ & 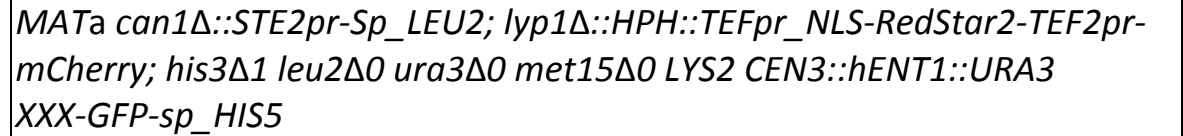 & This study \\
\hline $\begin{array}{l}\operatorname{meu} 1 \Delta \mathrm{xxx}- \\
\text { GFP (GFP- } \\
\text { library) }\end{array}$ & 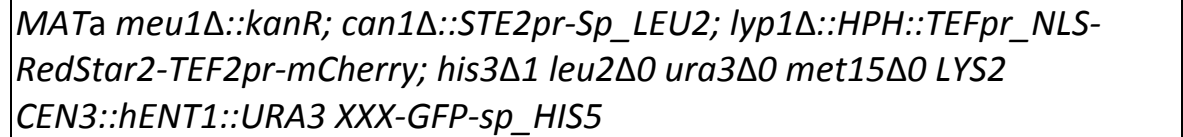 & This study \\
\hline
\end{tabular}

Table 3.2.1 - Yeast strains used in this chapter.

\subsubsection{Media}

All S. cerevisiae strains were cultured in one of the following growth media supplemented with the appropriate antibiotics: $100 \mu \mathrm{g} / \mathrm{mL}$ ClonNAT (NAT; Werner BioAgents), $200 \mu \mathrm{g} / \mathrm{mL}$ geneticin (G418; Carbosynth) and $300 \mu \mathrm{g} / \mathrm{mL}$ hygromycin B (HPH; Formedium). The amino acid analogues canavanine (Sigma-Aldrich) and thialysine (Sigma-Aldrich) were used at $50 \mu \mathrm{g} / \mathrm{mL}$ in the absence of 
their amino acid analogues. All yeast growth media was autoclaved at $121^{\circ} \mathrm{C}$ and cooled to $65^{\circ} \mathrm{C}$, before adding $2 \%$ glucose and antibiotics unless otherwise stated. All E. coli strains were cultured in lysogeny broth (LB) supplemented with $100 \mu \mathrm{g} / \mathrm{mL}$ ampicillin (Sigma-Aldrich).

Yeast peptone dextrose (YPD) agar: 1\% (w/v) yeast extract (Becton, Dickinson and Company), 2\% $(\mathrm{w} / \mathrm{v})$ bacto-peptone (Becton, Dickinson and Company), 0.12\% (w/v) adenine (Sigma-Aldrich), 2\% (w/v) agar (Invitrogen) and 2\% (w/v) glucose (Sigma-Aldrich). YPD broth was prepared as above without the addition of agar.

Synthetic complete (SC) and synthetic dropout (SD) agar: $0.17 \%(\mathrm{w} / \mathrm{v})$ yeast nitrogen base without amino acids or ammonium sulphate (Becton, Dickinson and Company), $0.1 \%(\mathrm{w} / \mathrm{v}$ ) monosodium glutamate (Sigma-Aldrich), $0.2 \%(\mathrm{w} / \mathrm{v})$ amino acid mixture to suit (Sigma-Aldrich), $2 \%(\mathrm{w} / \mathrm{v})$ agar (Becton, Dickinson and Company) and 2\% (w/v) glucose (Sigma-Aldrich). SC broth was prepared as above without the addition of agar. SD media was made same as SC minus the appropriate amino acid supplement.

$\mathrm{CM}$ (complete minimum)-Ura $+2 \%$ galactose agar: $0.725 \%(\mathrm{w} / \mathrm{v}) \mathrm{CM}$ amino acids/ammonium sulphate mixture to suit, $2 \%(\mathrm{w} / \mathrm{v})$ agar (Becton, Dickinson and Company) and $2 \%(\mathrm{w} / \mathrm{v})$ galactose (Carbosynth). Media was autoclaved at $121^{\circ} \mathrm{C}$ and cooled to $65^{\circ} \mathrm{C}$ prior to adding filter sterilised galactose. SD broth was prepared as above without the addition of agar.

$\mathrm{CM}-$ Ura $+2 \%$ raffinose agar: $0.725 \%(\mathrm{w} / \mathrm{v}) \mathrm{CM}$ amino acids/ammonium sulphate mixture to suit, $2 \%$ (w/v) agar (Becton, Dickinson and Company) and $2 \%(w / v)$ raffinose (Carbosynth). Media was autoclaved at $121^{\circ} \mathrm{C}$ and cooled to $65^{\circ} \mathrm{C}$ prior to adding filter sterilised galactose. SD broth was prepared as above without the addition of agar.

CM-Met/Ade/Ura (MAU) + 2\% galactose: $0.725 \%(w / v) ~ C M$ amino acids/ammonium sulphate mixture to suit, $2 \%(\mathrm{w} / \mathrm{v})$ agar (Becton, Dickinson and Company) and 2\% (w/v) galactose (Carbosynth). Media was autoclaved at $121^{\circ} \mathrm{C}$ and cooled to $65^{\circ} \mathrm{C}$ prior to adding filter sterilised raffinose and galactose. SD broth was prepared as above without the addition of agar. 
Enriched sporulation (SPO) agar: $1 \%$ (w/v) potassium acetate (Scharlau Chemie S.A.), $0.1 \%$ (w/v) yeast extract (Becton, Dickinson and Company), 0.05\% (w/v) glucose (Sigma-Aldrich), 0.2\% (w/v) amino acid mixture to suit (Sigma-Aldrich), 2\% (w/v) agar (Becton, Dickinson and Company). Enriched sporulation broth was prepared as above without agar.

Amino acid mixture for SC media: $3 \mathrm{~g}$ adenine, $2 \mathrm{~g}$ uracil, $2 \mathrm{~g}$ inositol, $0.2 \mathrm{~g}$ para-aminobenzoic acid, 2 g alanine, 2 g asparagine, 2 g aspartic acid, 2 g cysteine, 2 g glutamic acid, 2 g glutamine, 2 g glycine, 2 $\mathrm{g}$ histidine, $2 \mathrm{~g}$ isoleucine, $10 \mathrm{~g}$ leucine, $2 \mathrm{~g}$ lysine, $2 \mathrm{~g}$ methionine, $2 \mathrm{~g}$ phenylalanine, $2 \mathrm{~g}$ proline, $2 \mathrm{~g}$ serine, $2 \mathrm{~g}$ threonine, $2 \mathrm{~g}$ tryptophan, $2 \mathrm{~g}$ tyrosine, and $2 \mathrm{~g}$ valine. Amino acid mixture of SD media is a mixture of the amino acids (Sigma-Aldrich) above minus the appropriate amino acid supplement. Amino acid mixture for enriched sporulation composed of $2 \mathrm{~g}$ histidine, $10 \mathrm{~g}$ leucine, $2 \mathrm{~g}$ lysine, and 2 g uracil.

Amino acid/ammonium sulphate mixture for $\mathrm{CM}$ ): $100 \mathrm{~g}$ yeast nitrogen base without amino acids or ammonium sulphate, $293.11 \mathrm{~g}$ of ammonium sulphate, $1.2 \mathrm{~g}$ adenine, $1.2 \mathrm{~g}$ arginine, $1.2 \mathrm{~g}$ histidine, $1.8 \mathrm{~g}$ isoleucine, $3.6 \mathrm{~g}$ leucine, 1.8 lysine, 1.2 methionine, $3 \mathrm{~g}$ phenylalanine, $1.2 \mathrm{~g}$ tryptophan, $1.8 \mathrm{~g}$ tyrosine, $1.2 \mathrm{~g}$ uracil, and $9 \mathrm{~g}$ valine.

\subsubsection{Compounds and chemicals}

MTDIA was kindly provided by Prof Gary Evans (Ferrier Institute, Victoria University of Wellington), dissolved in DMSO at a concentration of $10 \mathrm{mM}$, and stored at $-20^{\circ} \mathrm{C} .5^{\prime}$-methylthio adenosine (MTA) (W1114, AK Scientific) was dissolved in $\mathrm{ddH}_{2} \mathrm{O}$ at a concentration of $5 \mathrm{mM}$ and stored at $-20^{\circ} \mathrm{C}$.

\subsubsection{GFP library construction}

A significant proportion of the GFP library labelled with the dual RFP markers available in my laboratory showed a significant vacuolar morphology defect. This was inherent to the query strain (YCG256) used in construction of the GFP/dual RFP library. Therefore, the GFP library was reconstructed by crossing the GFP library (MATa) with a MATa query strain expressing hENT1. The 
new query strain was constructed via a single selectable marker for the dual RFPs (LEU2) and another selectable marker for hENT1 expression (URA3). PCR-mediated disruption was used to engineer BY4741, specifically replace the replace the CAN1 and LYP1 loci with STE2pr-Sp_LEU2 from y7039 (gift from C. Boone)(Tong \& Boone, 2007) and integrate the HPH::TEFpr_NLS-RS2::TEF2pr_mCherry construct from YCG383 (Low, 2013). BY4742 was transformed with pYhENT1 (CEN3::hENT1::URA3) (Vickers et al., 1999) using primers in Appendix Table 7.3.1 (Appendix Fig. 7.3.1) (Janke et al., 2004;

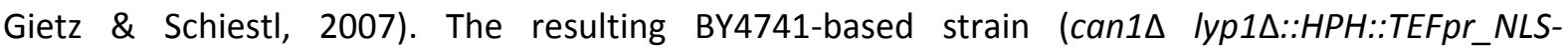
RS2::TEF2pr_mCherry) and the BY4742 + hENT1::URA3 transformants were mated to generate a diploid strain. Briefly, the diploid strain was cultured overnight in SD-Ura $+\mathrm{HPH}+2 \%$ glucose liquid media, washed with water, resuspended in sporulation media, incubated at $30^{\circ} \mathrm{C}$ for $7 \mathrm{~d}$, plated on SD-Arg/Leu/Ura + Can/Thia $+2 \%$ glucose agar, and incubated for $2 \mathrm{~d}$ at $30^{\circ} \mathrm{C}$. The resulting colonies were inoculated into SD-Ura media in a 96-well plate (one colony per well) and incubated at $30^{\circ} \mathrm{C}$ overnight. The random spore analysis was performed as previously described (Tong \& Boone, 2006) in liquid media, where $\sim 1 \mu \mathrm{L}$ of spores in sporulation media were cultured in SD-Ura were transferred into SD-Leu, SD-Met/Cys, SD-His, SD-Ura, and SD-MAUC + 2\% galactose liquid media in 96 well plates using the RoToR HDA replicator (Singer Instrument Co.) to assess for genotypes in Table 3.2.2. Concomitantly, MATa (his3::kanR) and Mata (pdr1::natR) mating type strains with selectable markers were cultured overnight, resuspended in YPD to an OD of 0.3 in $90 \mu \mathrm{L}$ of either MATa or MAT $\alpha$ were transferred into wells of a 96 well plate followed by $10 \mu \mathrm{L}$ SD-Ura cultured spores and incubated at $30^{\circ} \mathrm{C}$ for $1 \mathrm{~d}$. $10 \mu \mathrm{L}$ of cells (mated with MATa or MAT $\alpha$ ) were inoculated into SD-Ura+G418 or SDUra+NAT were incubated at $30^{\circ} \mathrm{C}$ for $2 \mathrm{~d}$. Eight spores were acquired by random spore analysis that showed phenotypes consistent with WT YCG640 genotype (MATa can1A::STE2pr-Sp_LEU2;

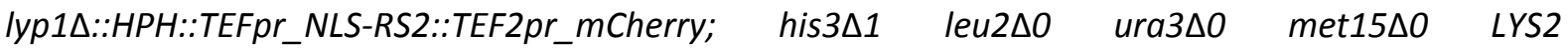
CEN3::hENT1::URA3) and were assessed for normal (unfragmented) vacuolar morphology by light microscopy and florescence microscopy utilising the nuclear (NLS-RedStar2) and cytoplasmic (TEF2pr_mCherry) RFP markers. Cells derived from five spores showed normal morphology, three 
were frozen down and one was used as the query strain in the construction of the GFP/dual RFP + hENT1 library by synthetic genetic array (SGA) methodology as previously described (Tong et al., 2001) in Chapter 2 (section 2.2.3). MEU1 was replaced by PCR mediated disruption with pFA6-kanMX4 (Goldstein \& McCusker, 1999) with primers (Appendix Table 7.3.1) to generate an isogenic

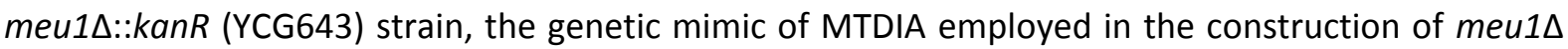
GFP/dual RFP + hENT1 library by SGA methodology as previously described (Tong et al., 2001).

\begin{tabular}{|c|c|c|}
\hline Media & $\begin{array}{l}\text { Expected } \\
\text { viability }\end{array}$ & Construct confirmed \\
\hline SD-Leu & - & leu2 $\Delta 0$ \\
\hline SD-Ura & + & CEN3::hENT1::URA3 \\
\hline SD-Met/Cys & - & $\operatorname{met} 15 \Delta 0$ \\
\hline SD-MAUC+MTA+2\% galactose & + & CEN3::hENT1::URA3 \\
\hline SD-His & - & his $3 \Delta 1$ \\
\hline SD-Arg/Lys +Can/Thia+HPH & + & 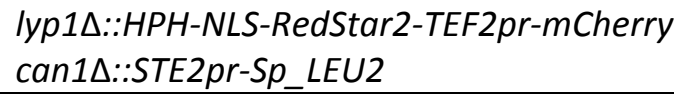 \\
\hline SD-Ura+G418 & + & MATa mating type \\
\hline SD-Ura+NAT & - & MATa mating type \\
\hline
\end{tabular}

Table 3.2.2 - Expected phenotypes for WT (YCG640) in random spore analysis. Spores were inoculated into appropriate amino acid dropout media supplemented with $2 \%$ glucose and were incubated at $30^{\circ} \mathrm{C}$ overnight unless specified otherwise. Spores cultured in SD-MAUC+MTA+2\% galactose media were grown for $2 \mathrm{~d}$ to obtain sufficient growth. +/- indicates growth/no growth. MAUC, Met/Ade/Ura/Cys; MTA, 5'-methylthoadenosine.

\subsubsection{Automated fluorescence microscopy}

Cells expressing the GFP-tagged protein and the dual RFPs maintained on agar plates in 384 colony format were pinned on SD-Ura+2\% raffinose agar using RoToR HDA replicator (Singer Instrument $\mathrm{Co}$ ), cultured at $30^{\circ} \mathrm{C}$ for $20 \mathrm{~h}$, inoculated into a black-walled clear bottom 384 well cell carrier plate (Perkin Elmer) to an $\mathrm{OD}_{660}$ of 0.3 in $50 \mu \mathrm{L}$ volumes of SD-MAU+ MTA+ $2 \%$ galactose liquid media with and without MTDIA and were cultured at $30^{\circ} \mathrm{C}$ for $6 \mathrm{~h}$. Cells were visualised using a highthroughput spinning disk confocal microscope (Evo Tec OPERA, Perkin Elmer) as previously described in Chapter 2 (section 2.2.10). The change in GFP intensity (>20\% increase or decrease compared to WT untreated) was quantified using Acapella automated image analysis software (Perkin Elmer), and 
change in localisation was confirmed by visual inspection and were validated by repeat evaluation and analysis. A cut-off $>20 \%$ change in abundance was used as significant change, as changes in abundance $>18 \%$ were consistently detectable by visual inspection.

\subsection{Results}

\subsubsection{Construction of the GFP/dual RFPs + hENT1 libraries}

A new GFP library comprised of $\sim 4,900$ unique GFP-tagged fusion proteins in the dual nuclear and cytosolic RFPs with hENT1 heterologous expression was constructed by mating the query strain yCG640 with the GFP library (Open Biosystems) as previously described (Tong et al., 2001). MEU1 was deleted from YCG640 to create the genetic mimic of MTDIA (YCG643) and was mated against the GFP library to create an isogeneic meu1 $\triangle$ GFP/dual RFP + hENT1 library.

\subsubsection{Changes in protein abundance and localisation in response to MTDIA treatment and meu1 $\Delta$}

To further investigate the cellular processes that were perturbed by MTDIA treatment (genedrug interactions) or loss of MEU1 (gene-gene interactions), the newly constructed genome-wide GFP libraries were assayed using high throughput confocal microscopy. The localisation and abundance of individual proteins were assessed in vivo in response to MTDIA treatment and meu1D using Acapella automated image analysis software (Perkin Elmer) and by visual inspection. Out of $\sim 4,900$ GFP strains grown in three conditions (untreated, MTDIA treated, and in the meu1 $\Delta$ condition; Fig. 3.3.1a), 762 proteins were identified by Acapella to show a change in abundance and 124 strains to show a change in localisation with visual inspection with either meu1s or with MTDIA treatment. Of 886 that exhibited a change in localisation/abundance, 336 GFPs were altered with meu1 $\Delta$ and 147 GFPs were altered with MTDIA (with 135 overlapping) based on reproducibility in an independent assay. Specifically, 242 showed a change only in abundance (median GFP $> \pm 20 \%$ of WT untreated control), 47 showed a change only in localisation, and 59 proteins showed changes in both localisation and abundance compared to WT untreated (Fig. 3.3.1b-c).

The 201 localisation/abundance changes for meu1s that were not observed with MTDIA mostly showed responses that were similar to untreated WT, indicating a genetic dosage effect. The 
12 changes with MTDIA that were different from WT untreated and meu1A included only changes in abundance of mitochondrial, cytoplasmic and nuclear proteins with MTAP inhibition (Fig. 3.3.1b). An additional 17 proteins that relocalised meu1D showed intermediary responses with MTDIA. Fourteen proteins were downregulated and three proteins were upregulated with MTDIA without clear relocalisation observed in meu1 $\Delta$. Overall, there were 118 of 147 changes in abundance/localisation with MTDIA that were in agreement with meu1 $\Delta$.

The 348 proteins that showed a change in either localisation/abundance with MTDIA and meu1s were in various organelles within cells (Fig. 3.3.1d-e) (Appendix Fig. 7.3.2; Appendix Table 7.3.2). The majority of the 242 proteins that showed a change in abundance alone were localised to cytoplasm, nucleus and mitochondria. The majority of the 106 proteins that showed a change in localisation were relocalised from cytoplasm to nucleus, from nucleus to cytoplasm, from ER/plasma membrane to vacuole, and from ER/cytoplasmic foci to cytoplasmic foci with either MTDIA or meu1A (Fig. 3.3.1f-g). In addition, five bud neck proteins relocalised to the plasma membrane and three microtubule associated proteins relocalised to nuclear foci with meu1 1 but not with MTDIA with the exception of the bud neck protein Sec8 that relocalised to the plasma membrane.

These 348 proteins that changed in localization/abundance were assigned into the three major hierarchical categories. Most informative was the biological process based on enrichment. The proteins that changed in abundance/localisation with MTDIA were significantly enriched for transport $(p=1.27 \mathrm{E}-05)$ within subcategories of transmembrane transport $(p=4.39 \mathrm{E}-07)$, organic acid transport ( $p=5.67 \mathrm{E}-07)$, carboxylic acid transmembrane transport $(p=1.38 \mathrm{E}-06)$ and establishment of 
a

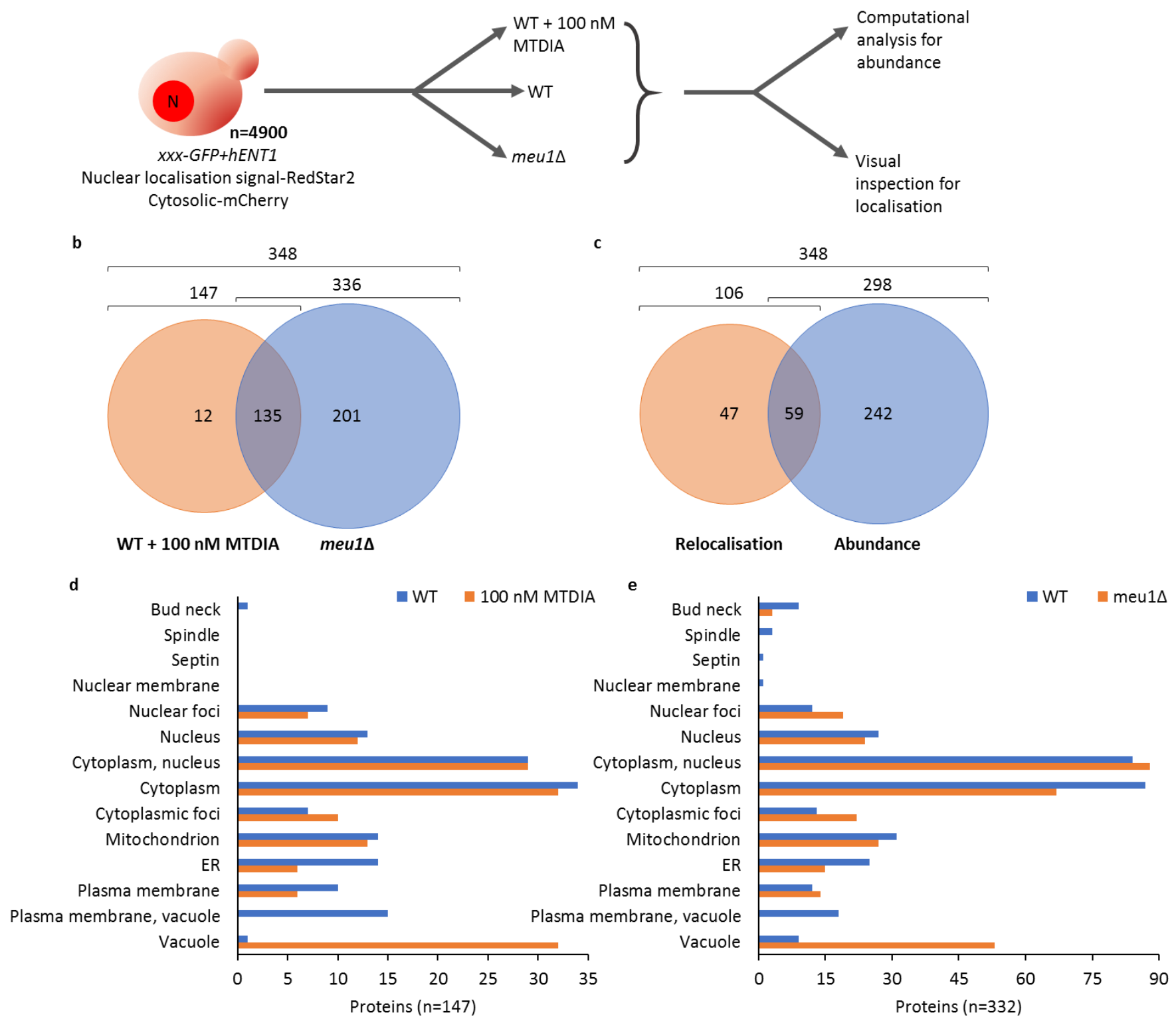



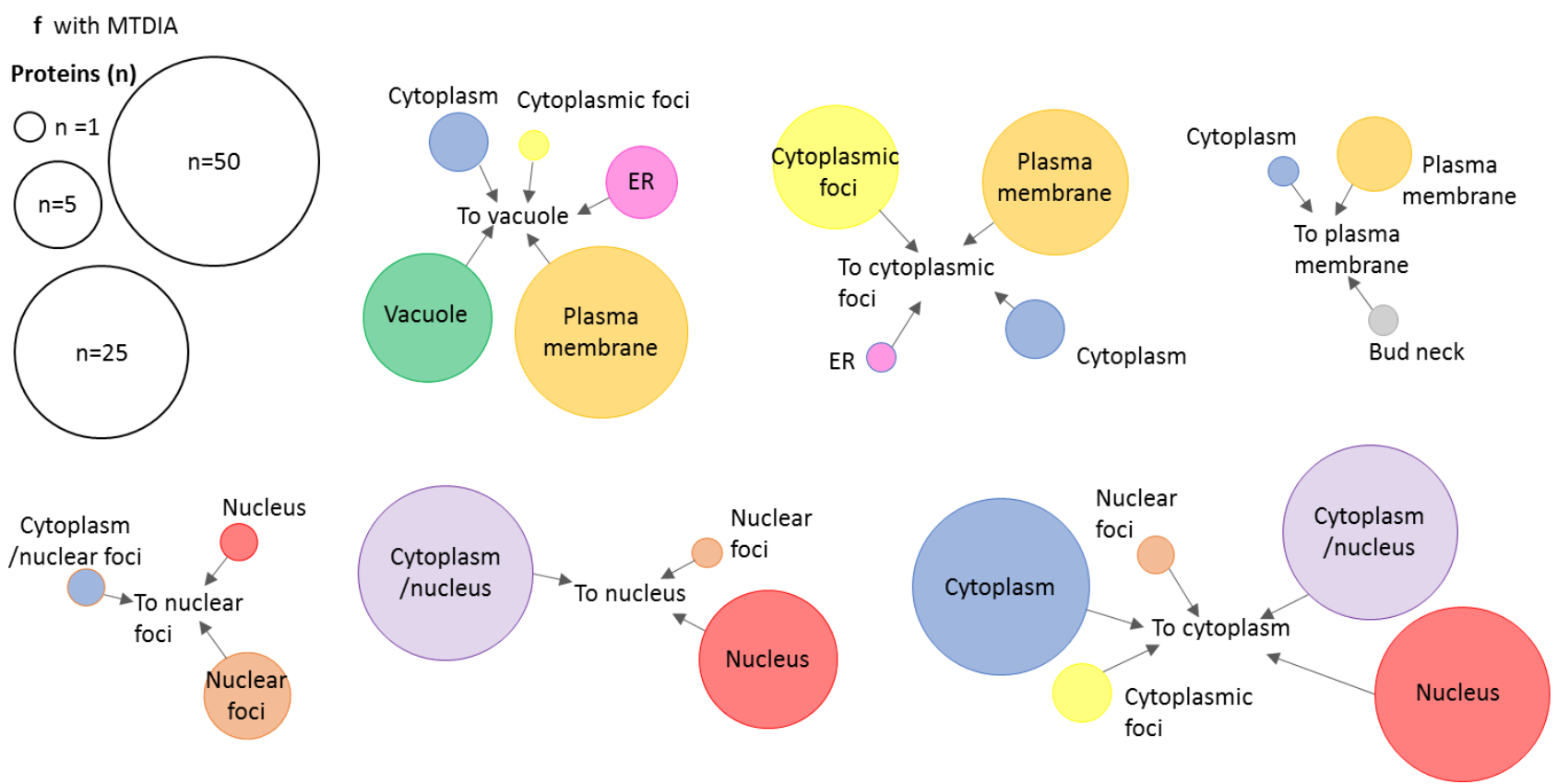

g with meu1s

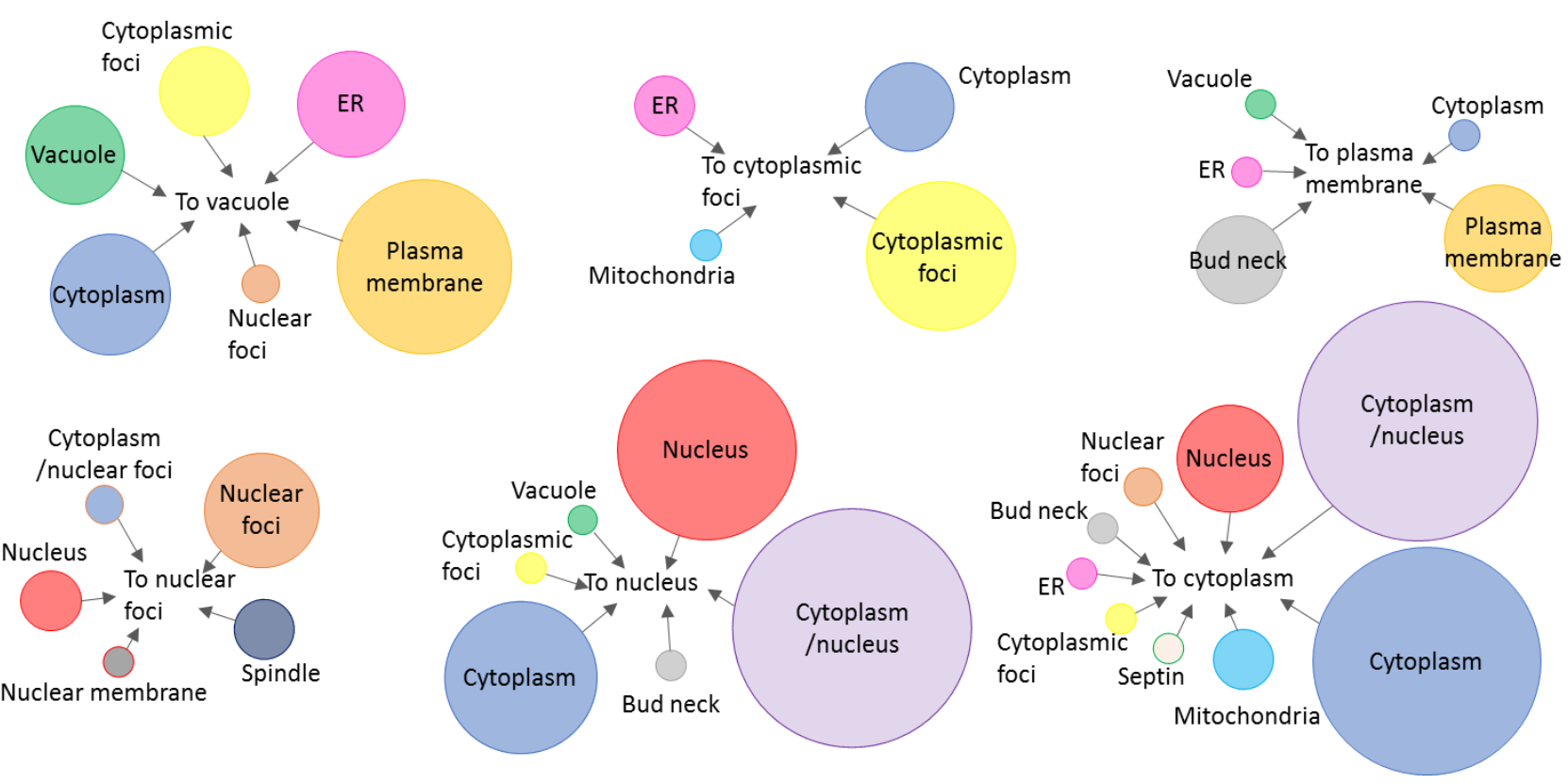

Figure 3.3.1 - Global analysis of protein relocalisation and abundance in response to MTDIA and its

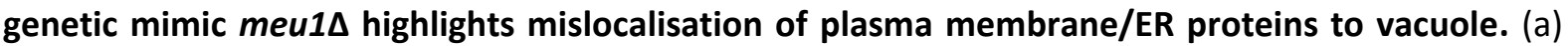
Schematic representation of the screening and quantification approach used in analysis of $\sim 4900 \mathrm{GFP}$ fusion proteins. (b) Proteins that changed in localisation/abundance with MTDIA vs. meu1A. (c) Changes in localisation vs. abundance. (d) Localisation of proteins in WT vs. WT with MTDIA. (e) Localisation of proteins in WT vs. meu1A. (f) Relocalisations with MTDIA treatment. (g) Relocalisation with meu1s. 4,900 GFP fusion proteins in the dual RFP (NLS-RS2 and mCherry) background were screened against MTDIA and meu1A using high-throughput spinning disk confocal microscope (Evo Tec OPERA, Perkin Elmer) and change in abundance (>20\% increase or decrease) was quantified with automated image analysis software (Acapella, Perkin Elmer) (Bircham et al., 2011) and relocalisation 
by visual inspection. Shown here are changes in localisation and abundance reproducible from the high-throughput screen.

localization ( $p=3.63 \mathrm{E}-05)$ (Appendix Table 7.3.3). The notable significant enrichments with meu1 $\Delta$ were for pyruvate metabolic process $(p=1.70 \mathrm{E}-10)$, glycolytic process $(p=5.62 \mathrm{E}-08)$, generation of precursor metabolites and energy $(p=2.96 \mathrm{E}-08)$, acyl-CoA metabolic process $(p=5.12 \mathrm{E}-06)$, cytoplasmic translation ( $p=4.34 \mathrm{E}-09)$, nicotinamide nucleotide metabolic process $(p=2.02 \mathrm{E}-09)$ and nucleotide phosphorylation ( $p=2.74 \mathrm{E}-08$ ) (Appendix Table 7.3.4). This shows that loss of MTAP/MEU1 affects cellular processes involving synthesis of metabolites, while MTDIA treatment affects transport of metabolites. This is likely a difference between downregulation of the drug target (MTDIA treatment) and the complete loss of gene function (meu1 $\Delta)$. Metabolite transport is relied upon under downregulation of MTAP to compensate for the reduced metabolites, while loss of MTAP requires upregulation of synthesis since upregulation of transport cannot compensate. Alternatively, such differences may be a reflection of recently determined non-enzymatic activities of MTAP that are still present during MTAP inhibition yet absent in meu1 (Tang et al., 2015).

\subsubsection{MTDIA regulates proteins involved in glucose metabolism}

In addition to GO enrichment, the proteins showing changes in localisation/abundance also showed enrichment for pathways of central carbon metabolism, such as glycolysis $(p=1.83 \mathrm{E}-04)$, glucose fermentation ( $p=1.71 \mathrm{E}-07)$, gluconeogenesis $(p=3.21 \mathrm{E}-04)$ and pyruvate dehydrogenase complex $(p=2.11 \mathrm{E}-02)$ with meu1 $($ Appendix Table 7.3.5). These results were consistent with deletions of glucose metabolism genes (e.g., pyruvate dehydrogenase complex E1 alpha subunit PDA1 and methylglyoxalase HSP31) showed a growth defect with meu1D in chapter 2 and Pda1 and Hsp31

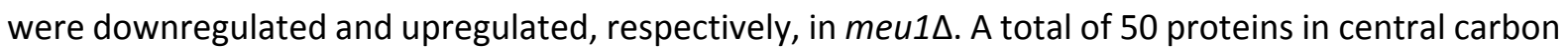
metabolism were deregulated with MTDIA or meu1 $\Delta$ and were assigned to respective pathways of glycolysis/gluconeogenesis, trehalose metabolism, pentose phosphate pathway, glycogen metabolism, acetoin and butanediol synthesis, glycerol biosynthesis, ethanol fermentation, TCA cycle, 
glyoxylate cycle, and long chain fatty acid biosynthesis (Fig. 3.3.3). The enzymes that catalyse the rate limiting step in glycolysis (Hxk1, Hxk2, Glk1) were increased whilst Cdc19 that catalyses the final step in pyruvate synthesis was reduced with MTDIA and meu1 $\Delta$. The enzymes that catalyse the intervening steps of glycolysis/gluconeogenesis are bidirectional and showed either an increase (Pfk2, Thd1, Thd2, Pgk1, Gpm1, Gpm2, Eno1) or decrease (Pgi1, Thd3) in protein levels (Fig. 3.3.2a). There was a reduction in Pgm2, which catalyses the initial step glycogen metabolism and shunts glucose-6-phosphate away from glycolysis, whilst proteins that catalyse synthesis of the final products (e.g., glycogen synthase Gsy2 and transketolase Tkl2 of pentose phosphate pathway) were increased compared to control (Fig. 3.3.2c). Similarly, MTDIA and meu1 1 resulted in upregulation of the enzyme Tps1 that salvages UDPglucose from glycogen metabolism for subsequent synthesis of glucose by Nth1 (Fig. 3.3.2b) and as well as the enzymes Tps1 and TkI2 that salvage glyceraldehyde-3-phosphate from pentose phosphate pathway and glycerol metabolism (Fig. 3.3.2b,d), respectively, for the utilisation in glycolysis/gluconeogenesis. The proteins Gpd1 and Gpd2 that synthesise glycerol-3-phosphate were also upregulated while Gut2 that catalyses the reverse reaction in glycerol metabolism pathway was downregulated with MTDIA and meu1s, which may also reflect the requirement for glycerol-3phosphate in the synthesis of glycerophospholipids (Fig. 3.3.2e).

Pyruvate is synthesised by pyruvate kinase Cdc19 from glycolysis or by malate dehydrogenase Mae1 from the TCA cycle, both of which were downregulated with meu1D. Likewise, pyruvate decarboxylases that metabolise pyruvate for ethanol fermentation and acetoin/butanediol biosynthesis (Pdc1, Pdc6) and for the TCA cycle (Pyc1) were increased with both MTDIA and meu1 $\Delta$ (Fig. 3.3.2g). In contrast, there was downregulation in pyruvate dehydrogenases Pda1 and Lat1 that synthesise acetyl CoA from pyruvate and acetyl-coA transferase Ach 1 as well as acetyl-coA synthetases Acs1 and Acs2 that synthesise acetyl-CoA from acetate (Fig.3.3.10a). Citrate synthases (Cit1, Cit2) that catalyse the rate limiting step in the TCA cycle and acetyl-CoA carboxylase Acc1 from long chain fatty acid synthesis that utilises acetyl-CoA as a reactant were increased whilst the protein levels of Cit3 (a 
a

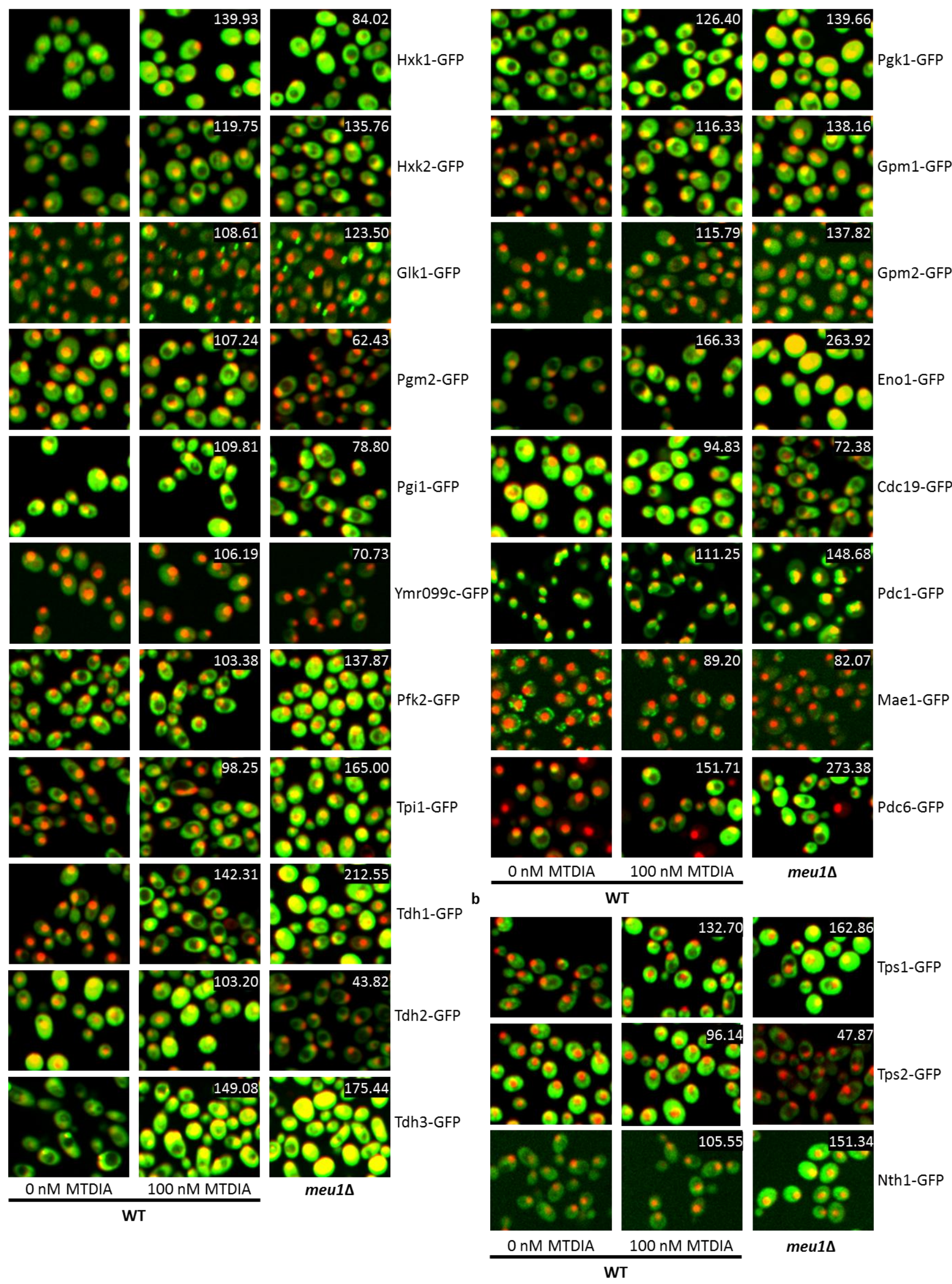



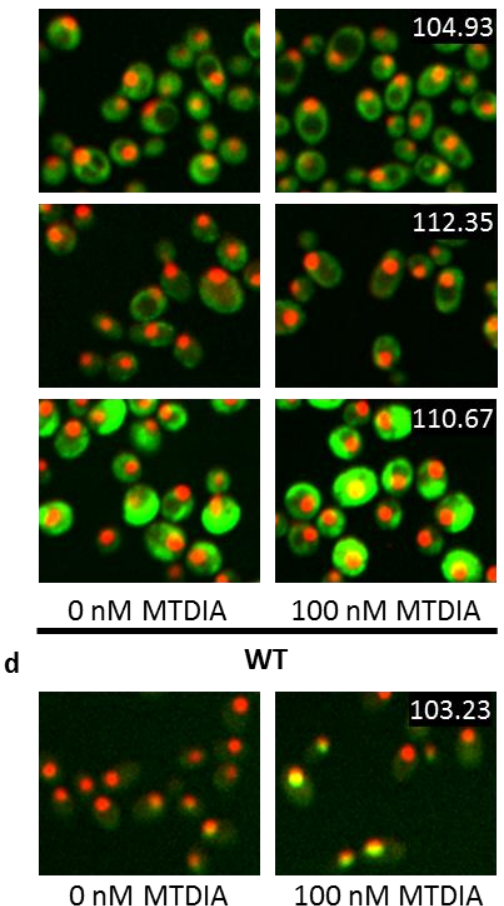

WT

e
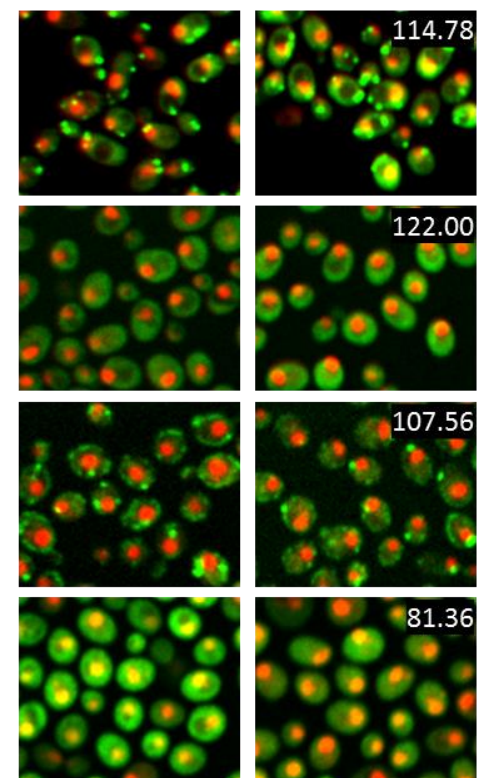

0 nM MTDIA
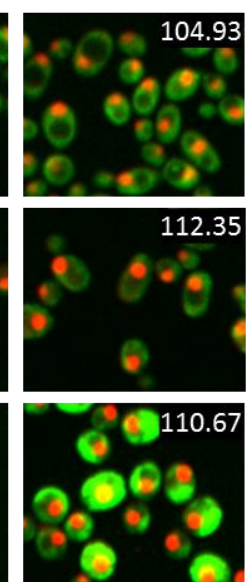

100 nM MTDIA
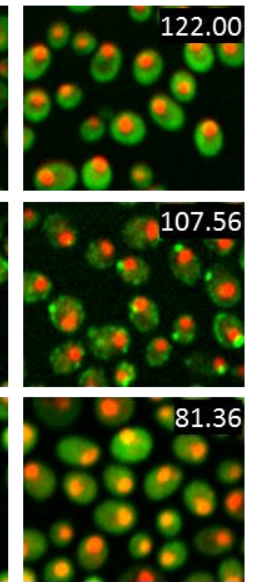

100 nM MTDIA
WT

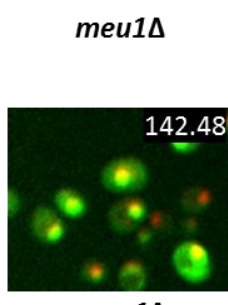

meu1s

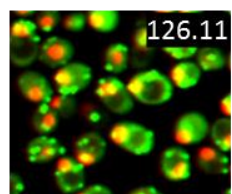

Gdp1-GFP

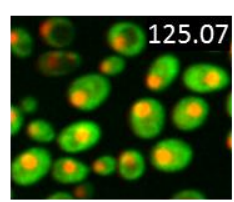

Ugp1-GFP

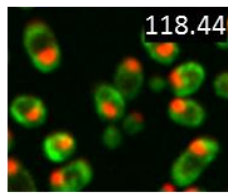

Gsy2-GFP

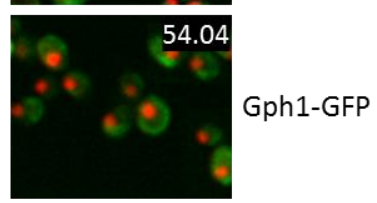

Tkl2-GFP

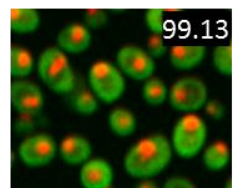

Gdp2-GFP

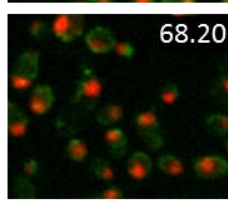

Gut2-GFP

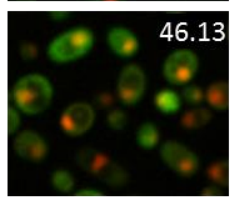

meu1s
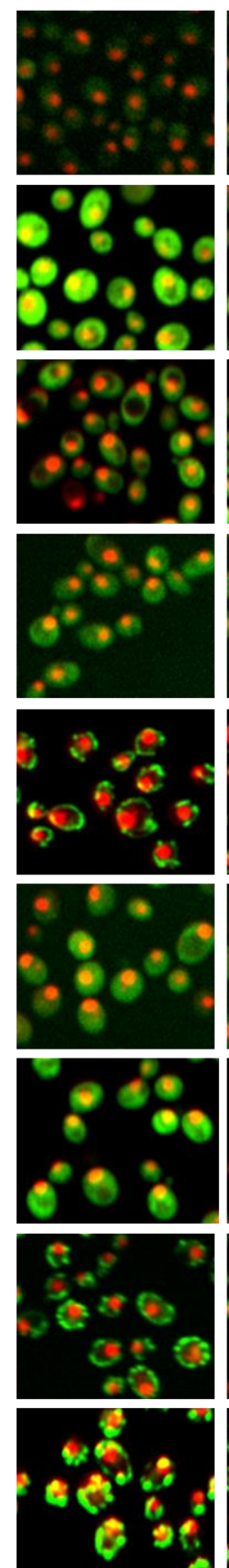

0 nM MTDIA
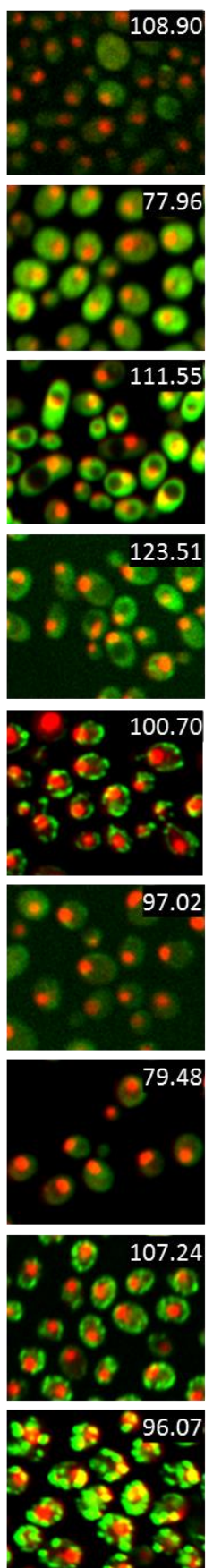

100 nM MTDIA

WT
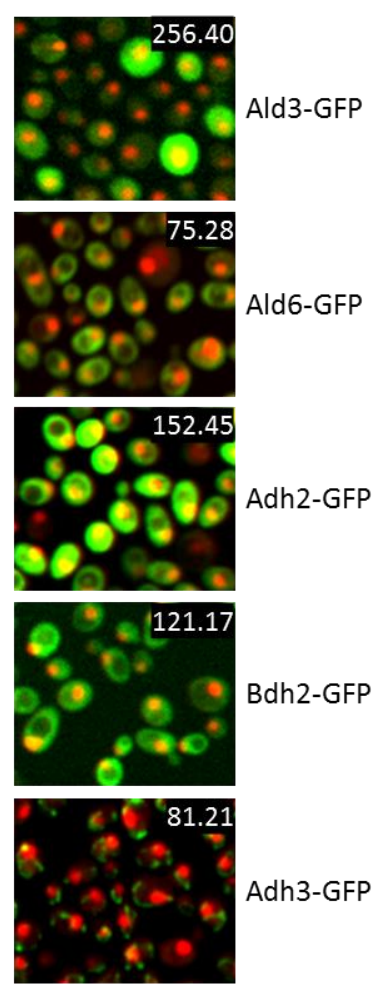

Adh3-GFP

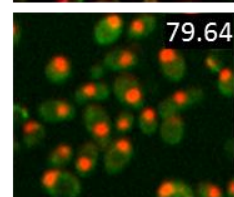

Adh4-GFP

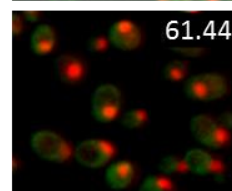

Adh6-GFP
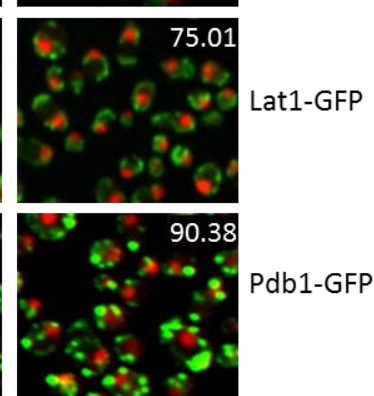

meu1s

dual specific citrate/methylcitrate synthase that utilises propionyl-CoA for synthesis of methylcitrate and acetyl-CoA for citrate synthesis) were reduced. Protein levels of three alcohol dehydrogenases (Adh3, Adh4, Adh6) that metabolise acetaldehyde to ethanol and the aldehyde dehydrogenase Ald6 that metabolises acetaldehyde to acetate were reduced (Fig.3.3.2f). Interestingly, the levels of alcohol 
g
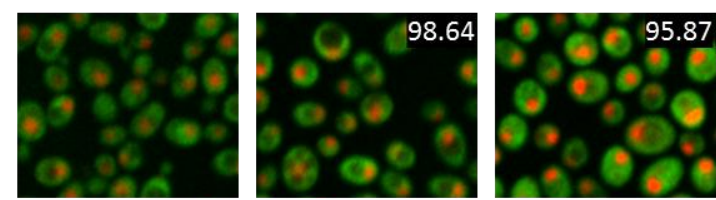

Pyc2-GFP
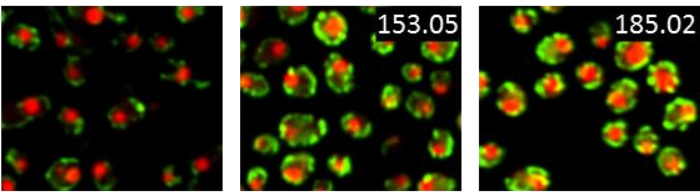

Cit1-GFP
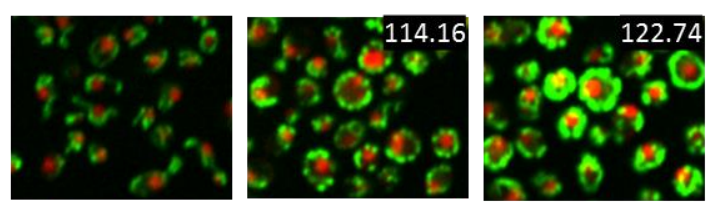

Kgd2-GFP
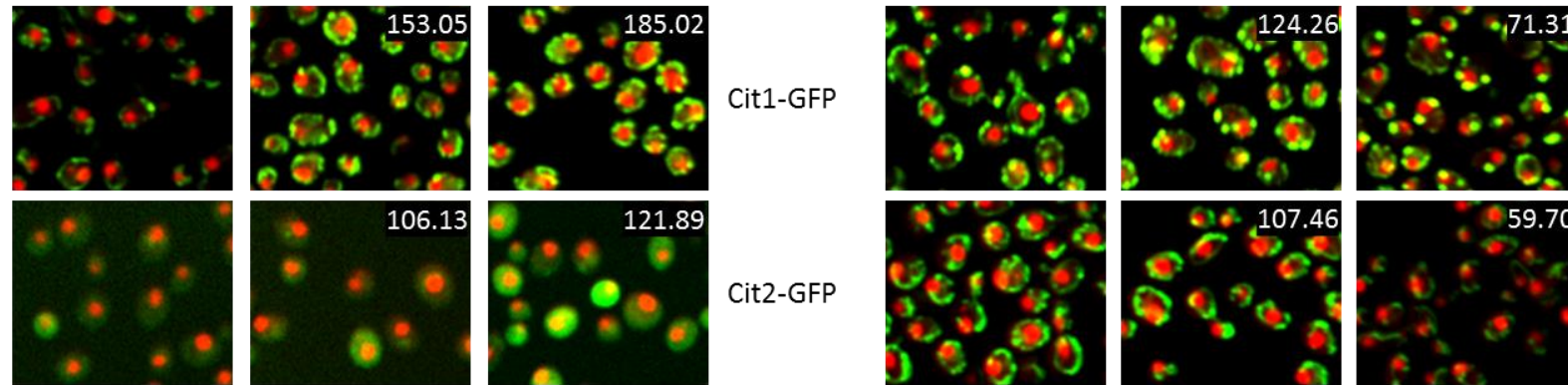

Cit2-GFP
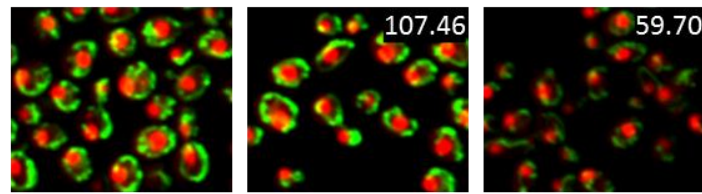

Sdh4-GFP
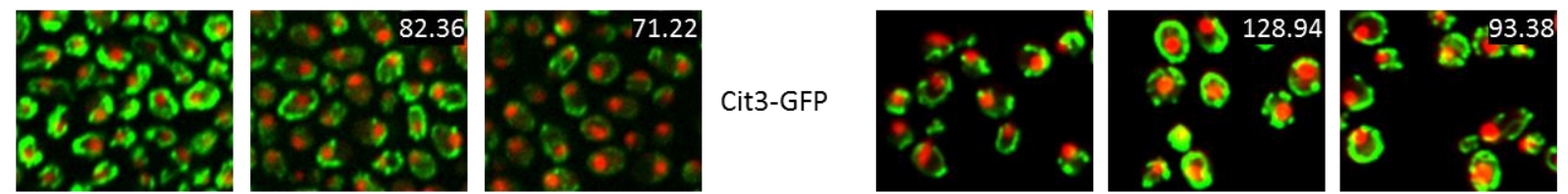

Lpd1-GFP

Cit3-GFP

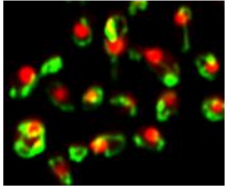

0 nM MTDIA

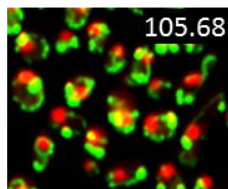

100 nM MTDIA

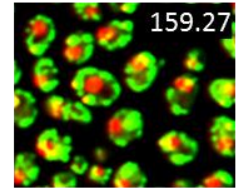

meu1s

WT

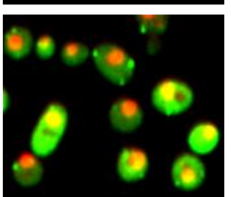

$0 \mathrm{nM}$ MTDIA

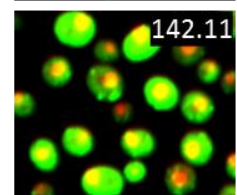

100 nM MTDIA

WT

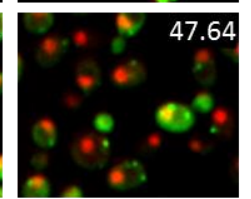

meu1s

Figure 3.3.2 - Proteins of central carbon metabolism that either increased or decreased in abundance or relocalised with MTDIA and meu1s. (a) Glycolysis/gluconeogenesis. (b) Trehalose metabolism. (c) Glycogen metabolism. (d) Pentose phosphate pathway. (e) Glycerol biosynthesis. (f) Ethanol fermentation. (g) TCA cycle. GFP fusion proteins were visualised using the high-throughput spinning disk confocal microscope (Evo Tec OPERA, Perkin) using the 60x water immersion lens (NA 1.2). The GFP abundance was quantified by automated image analysis (Acapella, Perkin Elmer)(Bircham et al., 2011) and relocalisation was determined by visual inspection. Numbers inset in each image are the percent changes in GFP abundance with either MTDIA or meu1 $\Delta$ relative to WT untreated.

dehydrogenases Adh2 that catalyse the reverse reaction and aldehyde dehydrogenase Ald 3 that also synthesises $\beta$-alanine from spermine metabolism were increased. Protein levels of ketoglutarate dehydrogenase complex KGD2 were increased while levels of ketoglutarate dehydrogenase Lpd1 that also function in the glycine cleavage complex were increased with MTDIA but relocalised to mitochondrial foci with meu1 $\Delta$. Similarly, malate dehydrogenase Mdh2 levels increased with MTDIA 
Upregulated (GFP abundance $>20 \%$ )

Down regulated (GFP abundance $<20 \%$ )

Unchanged

Absent in the GFP library

* Opposing responses with MTDIA and meu1A
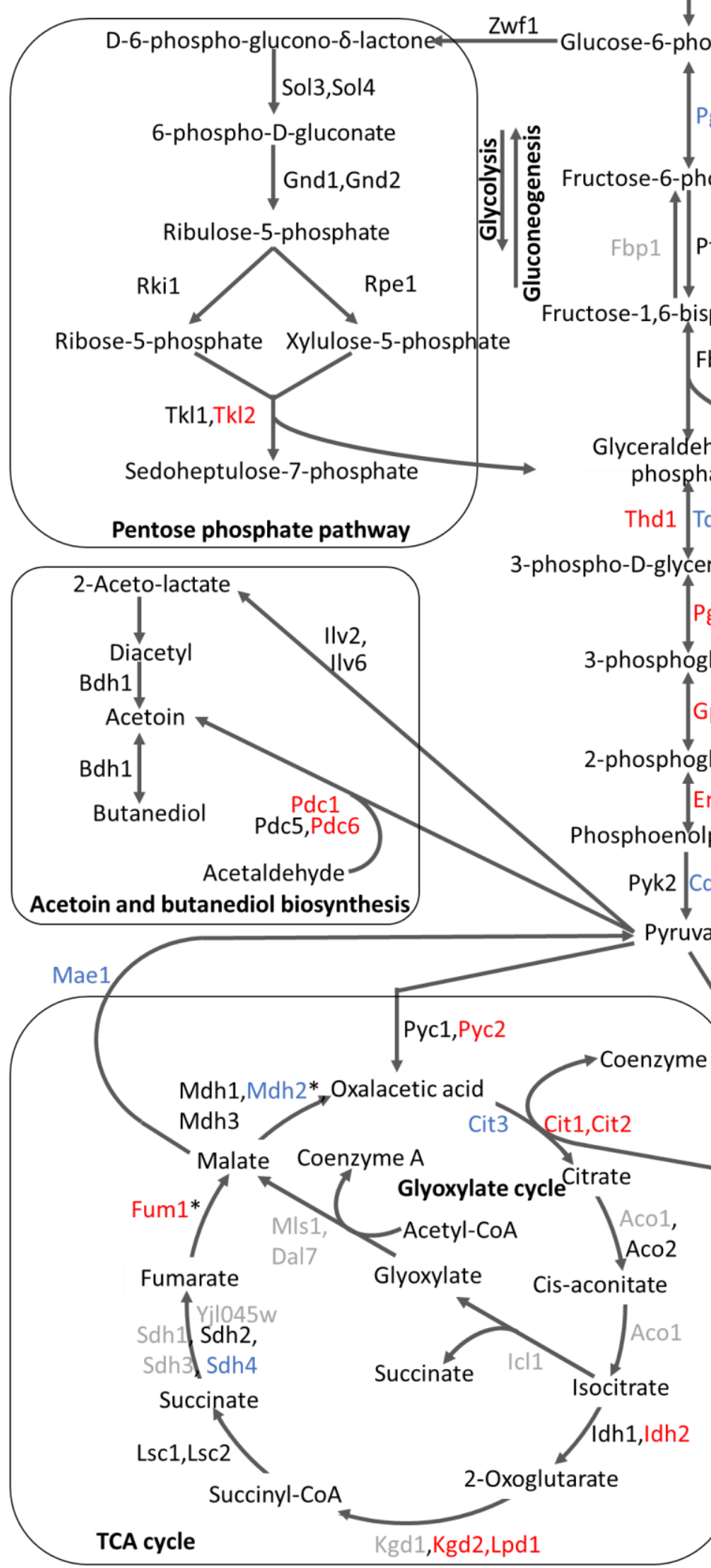

$\beta$-D-glucose

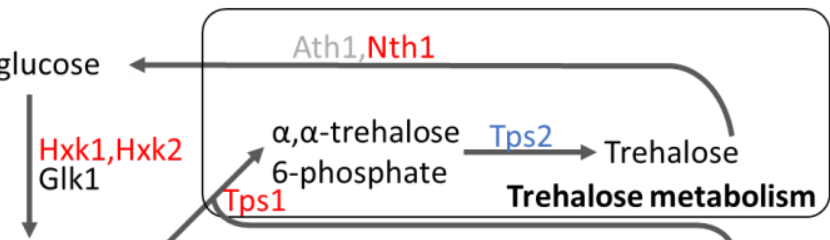

Ath1,Nth1

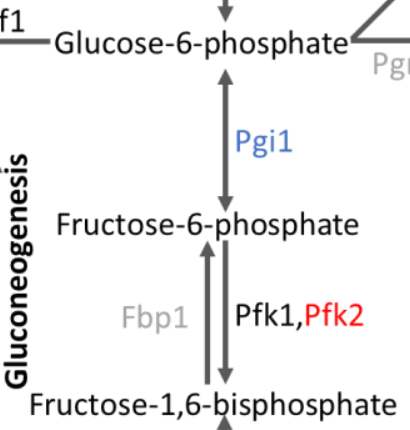

Fructose-1,6-bisphosphate
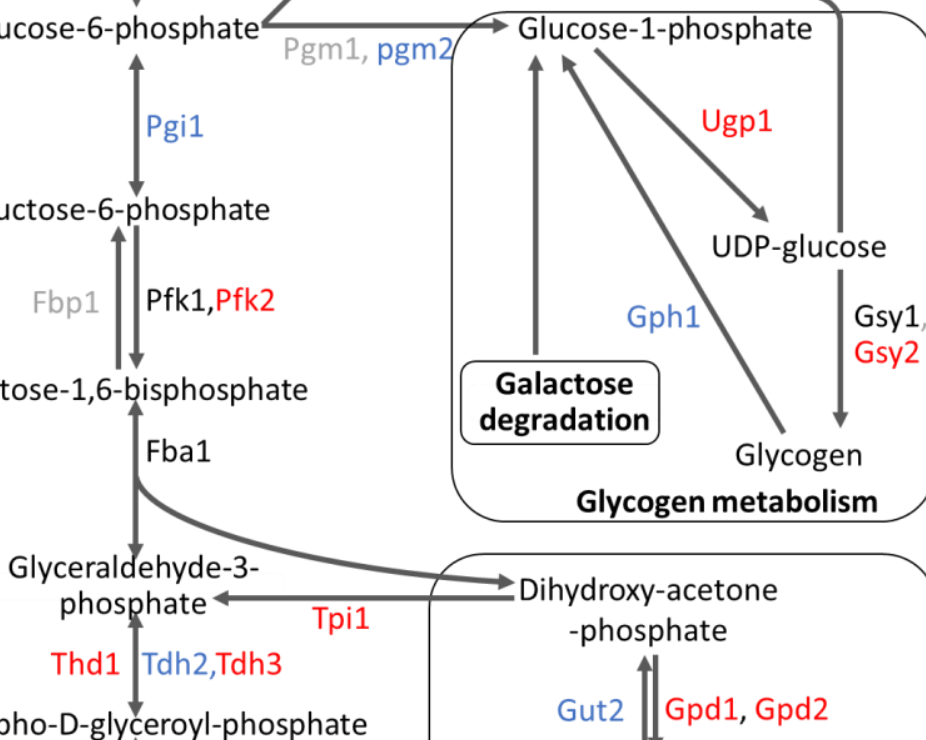

Glycogen metabolism

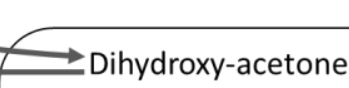

-phosphate

Gut2 Gpd1, Gpd2

Glycerol-3-phosphate

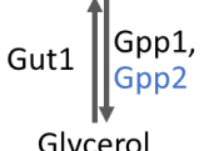

Glycerol

Glycerol biosynthesis

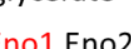
Adh4,Adh5,Adh6

$\underset{\text { Pdc5,pdc6 }}{A}$ Acetaldehyde $\underset{\text { Adh2 }}{\rightleftharpoons}$ Ethano Pda1,Pdb1 Ald4,Ald5,Ald6
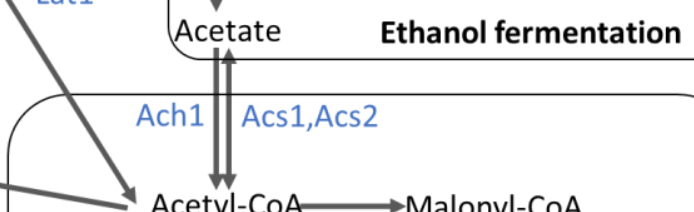

Acs1,Acs2
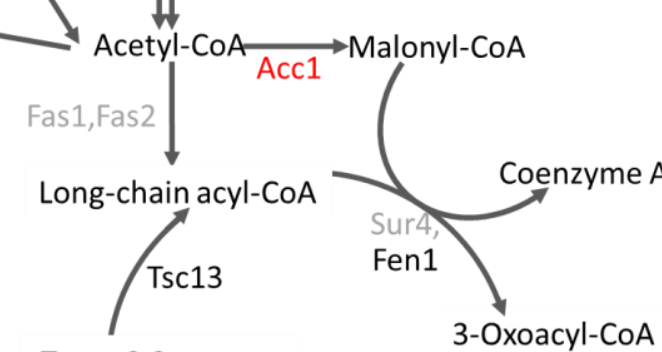

Trans-2,3-

dehydroacyl-CoA
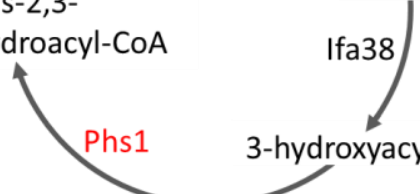

3-hydroxyacyl-CoA 
Figure 3.3.3 - Central carbon metabolism and proteins levels altered with meu1D or MTDIA. Pathway is compiled from sources KEGG, SGD and Tripodi et al. (Cherry et al., 2012; Kanehisa \& Goto, 2000; Tripodi et al., 2015).

but decreased with meu1 $\Delta$. In addition, fumarate hydrolase Fum1 was increased with MTDIA treatment. The membrane anchor of succinate dehydrogenase complex (Sdh3) was also reduced with meu1A. Since glycolysis serves as a sink for acetyl-CoA synthesis, the regulation of central carbon metabolism processes may be a result of disruptions to lipid homeostasis. Several glycolysis genes were also upregulated or downregulated by $>2$-fold with MTDIA in A549 lung cancer cells but this was not further investigated (Basu et al., 2011). For example, the deletion of Meu1 here resulted in upregulated levels of alcohol dehydrogenase Adh2, which was concordant with the 2.37-fold upregulation of the human orthologue of Adh2 with MTDIA in A549 lung cancer cells (Basu et al., 2011).

3.3.3 Methionine salvage from glycine, cystine, choline and sulphate is regulated by MTDIA and meu1 $\Delta$ In the absence of methionine and cysteine, MTA is processed by Meu1/MTAP to generate adenine and 5-methythioribose-1-phosphate that is subsequently processed to methionine (Pirkov et al., 2008). Deletion of genes required for salvage of methionine from MTA (MRI1, MDE1, UTR4, ADI1 and MEU1) were inviable in MTA as the sole source of methionine in Chapter 2. Proteins required for salvage of methionine by pathways independent of MTA metabolism were increased in abundance with either MTDIA or meu1s (Fig. 3.3.5), which was consistent with increased requirement for

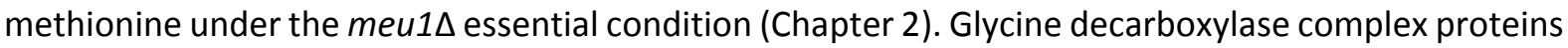
(Gcv1, Gcv2 Gcv3 and Lpd1) were enriched for the glycine cleavage complex pathway ( $p=2.11 \mathrm{E}-02)$, which catalyses the cleavage of glycine to $\mathrm{NH}_{3}$ and $\mathrm{CO}_{2}$ and concomitant synthesis 5,10-methylene tetrahydrofolate from tetrahydrofolate for methionine salvage. The levels of Gcv1, Gcv2 and Gcv3 were increased at the mitochondria and the levels of Lpd1 mitochondrial foci were increased with meu1D (Fig. 3.3.4a). Related, MEU1-deficiency resulted in the upregulation of the cytosolic serine 

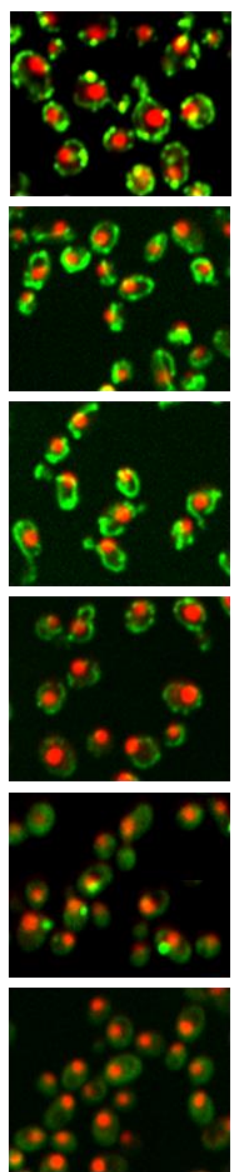

0 nM MTDIA WT

b
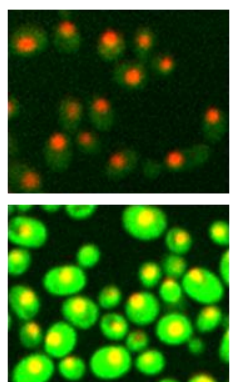

O nM MTDIA 100 nM MTDIA
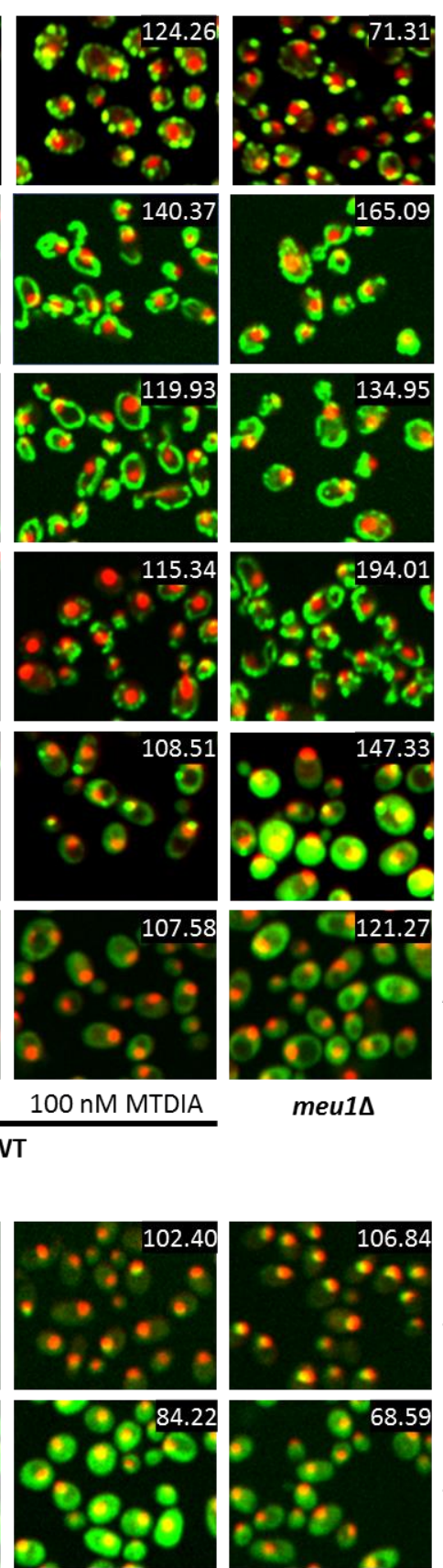

100 nM MTDIA

WT meu1s
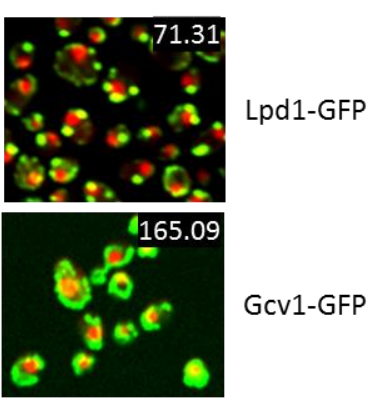

Gcv1-GFP
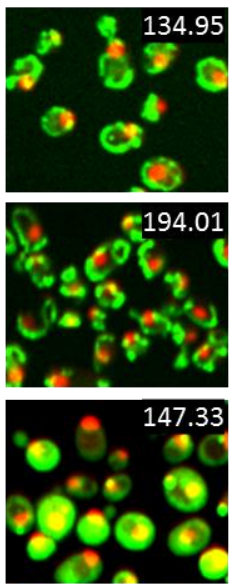

Shm2-GFP

Ade17-GFP

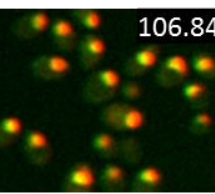

Str2-GFP

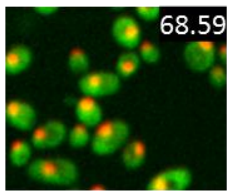

meu1s

Gcv2-GFP

Gcv3-GFP

Spe3-GFP
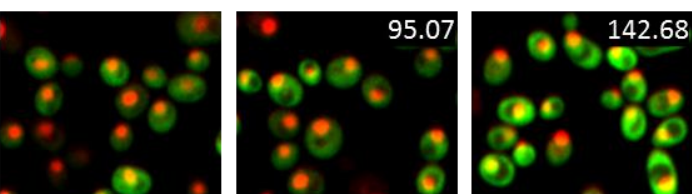

Met14-GFP
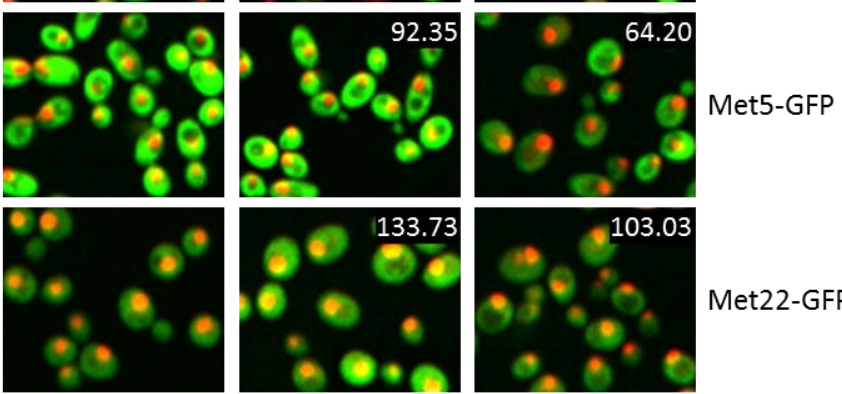

Met22-GFP

0 nM MTDIA 100 nM MTDIA WT
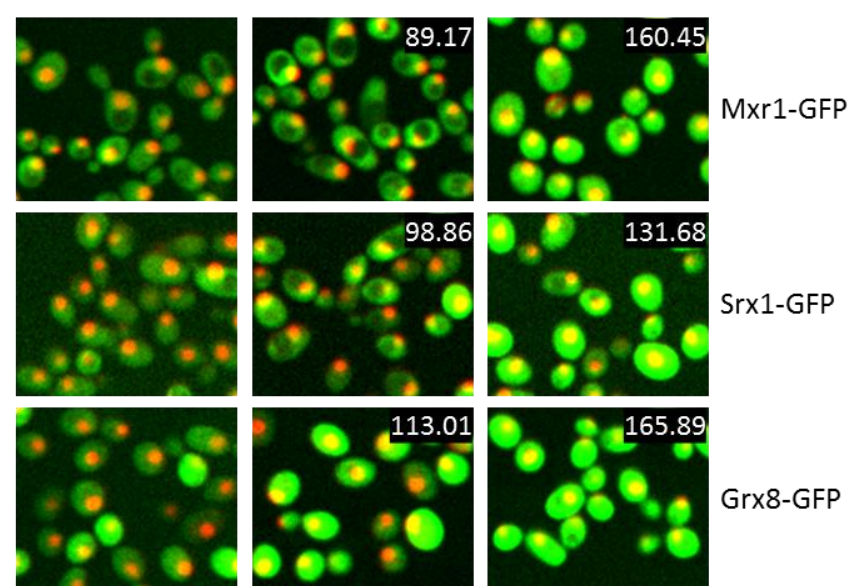

Grx8-GFP
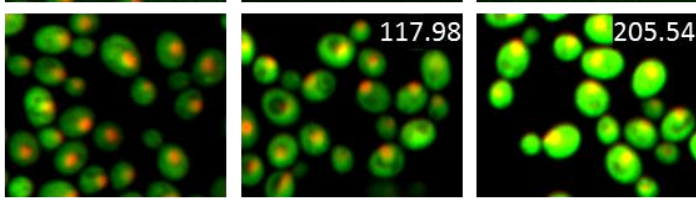

Ahp1-GFP
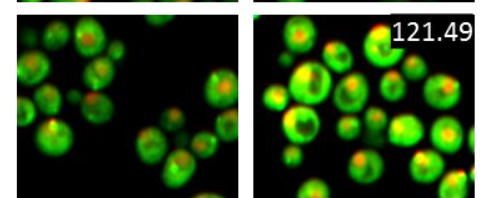

O nM MTDIA meu1s

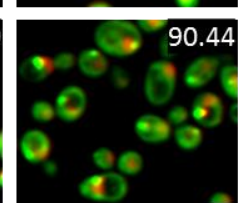

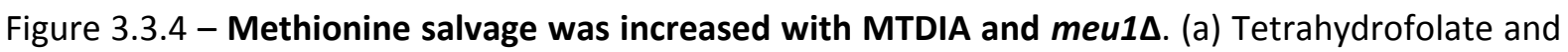
10-formyl- tetrahydrofolate assimilation. (b) Methionine salvage from ccysteine and polyamine biosynthesis (c) Sulphate assimilation. (d) methionine sulfoxide reduction. GFP fusion proteins were visualised using the high-throughput spinning disk confocal microscope (Evo Tec OPERA, Perkin) using the 60x water immersion lens (NA 1.2). The GFP abundance was quantified by automated image analysis (Acapella, Perkin Elmer) (Bircham et al., 2011) and relocalisation was determined by visual inspection. Numbers inset in each image are the percent changes in GFP abundance with either MTDIA

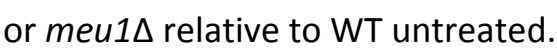


hydroxymethyltransferase Shm2 that catalyses the synthesis of glycine from serine and concomitant synthesis of 5,10-methylene tetrahydrofolate, which is subsequently converted to 5-methyl tetrahydrofolate and then methionine (Fig. 3.3.4a). Also related in meu1 $\Delta$ was the upregulation of Ade17 that catalyses for synthesis of the tetrahydrofolate from 10-formyl-THF. Cystathionine gammasynthase Str2 required for synthesis of cystathionine from cysteine, which is subsequently converted to homocysteine and then methionine was also upregulated in meu1s (Fig. 3.3.4b). In contrast, spermidine synthase Spe3 required for methionine salvage from decarboxylated adenosyl methionine in a pathway inhibited by meu1A and MTDIA was downregulated, possibly because of product inhibition by MTA (Pajula et al., 1979b). Met14 and Met5 are required for salvage of homocysteine and subsequently methionine from sulphate assimilation. Met14 catalyses the second step in sulphate

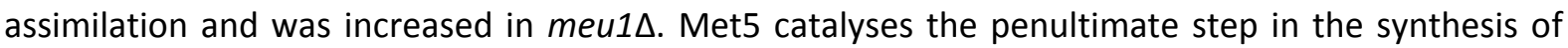
homocysteine and was decreased in meu1 $\Delta$. This is likely due to homocysteine serving as a reactant in the synthesis of S-adenosyl homocysteine, a potent inhibitor of methyltransferase reactions requiring S-adenosyl methionine. Related, Met22 catalyses metabolism of sulphate assimilation pathway product adenosine $3^{\prime}, 5^{\prime}$-bisphosphate to AMP and phosphate to maintain the forward reaction and was increased in meu1 $\Delta$ (Fig. 3.3.4c).

Thioredoxins are required for cellular oxidant detoxification reactions and the reduction of methionine sulphoxide to methionine. Levels of several thioredoxins (methionine-S-sulfoxide reductase Mrx1, sulfiredoxin Srx1, glutaredoxin Grx8 and alkyl hydroperoxide reductase Ahp1) were increased in meu1 $\Delta$, whilst levels of thioredoxin peroxidase Tsa1 were increased with MTDIA and accumulation increased at cytosolic foci in meu1s (Fig. 3.3.4d). In addition, permeases required for uptake of precursors for methionine salvage such as cystine (Bpa2, Bpa3, Yct1), cystine/methionine (Mup1), adenine (Fcy2), choline (Hnm1) and polyamines (Top4) relocalised from the plasma membrane to the vacuole with MTDIA treatment and in meu1 $\Delta$. 


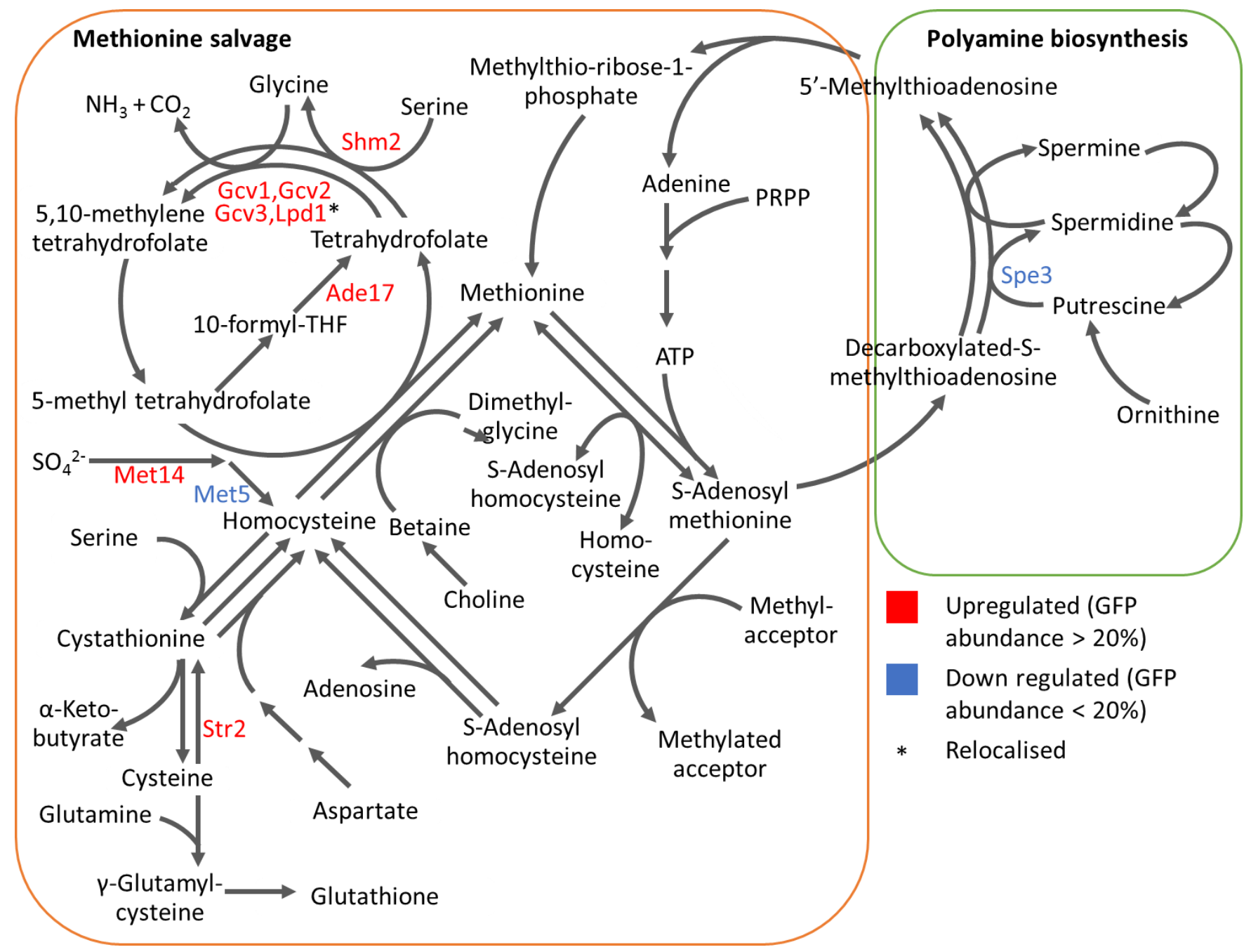

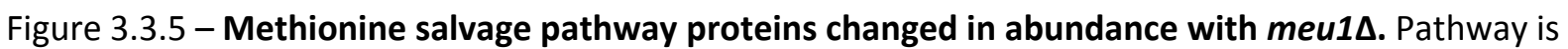
compiled from sources KEGG, SGD and Kanehisa and Goto (Tripodi et al., 2015; Cherry et al., 2012; Kanehisa \& Goto, 2000).

\subsubsection{MTDIA regulates proteins involved in chromatin remodelling and transcription}

Gene deletions of transcription and chromatin remodelling genes (HIR2, EAF1, IES5, UME6, PAT1, HTZ1, SIP3, MED1, DAL81, KNS1, YLR184W (adjacent to TOS4/YLR183C), YDR290W) showed a growth defect in meu1A, while these deletions (BRE1, DPB4, HDA3, HIR2, HIR3, HOS4, HST1, HST3, IES1, NPT1, PAT1, RFM1, RXT2, SIR4, SNF5, SUM1, SOK2, YLR236C, YLR235C, TDA5, RPN4, PSP2, CKA2, DHH1, YGR054W, KNS1, CTH1, DPB4, GTR2, YOR024W) showed a growth defect with MTDIA treatment (Chapter 2). Likewise, several proteins involved in overlapping cellular processes of transcription, chromatin organisation and gene expression showed altered abundance with MTDIA 


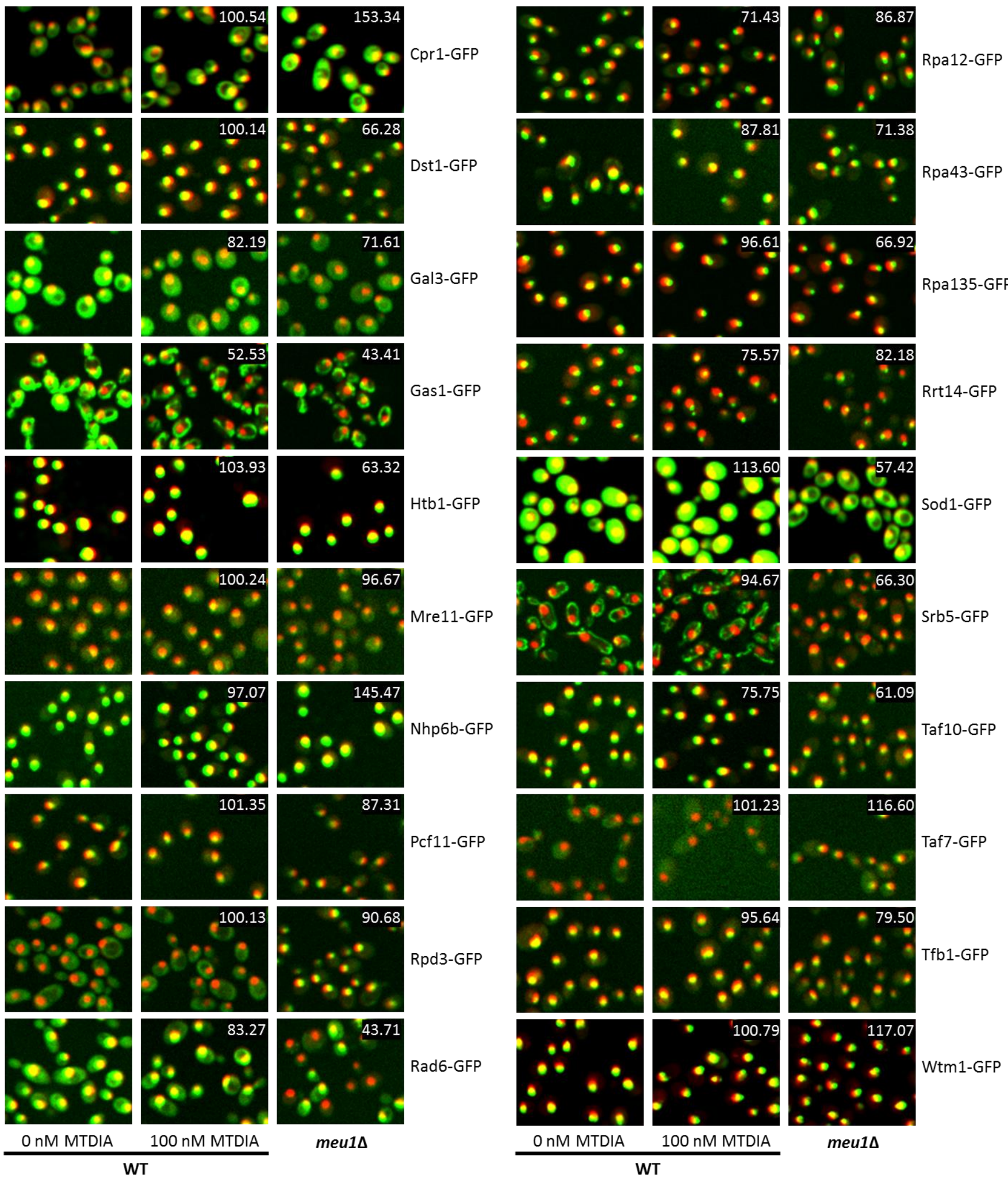

Figure 3.3.6 - Proteins regulating gene expression, transcription and chromatin organisation changed in abundance with MTDIA and meu1A. GFP fusion proteins in the dual RFP (NLS-RS2 and mCherry) background were screened against MTDIA and meu1 $\Delta$ using a high-throughput spinning disk confocal microscope (Evo Tec OPERA, Perkin Elmer). Change in abundance ( $>20 \%$ increase or 
decrease) was quantified with automated image analysis software (Acapella, Perkin Elmer) as previously described (Bircham et al., 2011). Numbers inset in each image are the percent changes in GFP abundance with either MTDIA or meu1A relative to WT untreated.

treatment and loss of Meu1. Transcription proteins downregulated with MTDIA or meu1 1 include RNA polymerase constituents (Rpa12, Rpa43, Rpa135 and Rpc10), RNA polymerase II mediator complex subunit Srb5, TFIID subunit Taf10, TFIIH subunit Tfb1, galactose responsive genes Gal3, superoxide dismutase Sod1, histone H2B Htb1, histone deacetylase of Rpd3S and Rpd3L complexes RPD3, DNA double standard break repair proteins Mre11 and Rad6, transcription elongation factor TFIIS Dst1, and transcription termination factor Pcf11(Fig. 3.3.6). In contrast, nucleosome remodelling protein required for genes transcription initiation of tRNA genes Nhp6b, TFIID subunit Taf7 and cytoplasmic peptidyl-prolyl cis-trans isomerase Cpr1 were upregulated with MTDIA and meu1A (Fig. 3.3.6). Disruptions to levels of proteins in chromatin organisation, transcription and gene expression observed were consistent with polyamine regulated changes in expression of both histone deacetylases and histone acetyltransferases (Pollard et al., 1999; Christopher et al., 2002). The histone modification in response to polyamine depletion would alter the expression of many of genes and are concordant with our observation of the deregulation of proteins involved in chromatin organisation, transcription and gene expression, as well as the altered expression of hundreds of genes in MTDIAtreated A549 lung cancer cells and with expression of MTAP in MTAP negative MCF-7 breast cancer cells (Basu et al., 2011; Christopher et al., 2002). 


\subsubsection{Proteins regulating translation are inhibited MTDIA and meu1 $\Delta$}

Polyamine depletion by ornithine decarboxylase and spermidine synthase inhibition inhibits both translation initiation and elongation (Landau et al., 2010). Consistent with this proposed role of polyamines in translation, deletions of translation genes RPL9A, YPR126C (overlaps mitochondrial ribosomal associated protein YLH47), RPL22A, RPS6A, RPS19A and RPS10A showed a growth defect with meu1 1 and deletion of RPS10A RPL23B showed a growth defect with MTDIA treatment (Chapter 2). GFP fluorescence of 72 proteins required for translation changed in abundance in response to MTDIA treatment or meu1 $\Delta$. These proteins were involved in different stages of translation (Fig. 3.3.7a-e). Out of 72 proteins, 65 were down regulated with MTDIA and meu1 $\Delta$ and were involved in pre-ribosomal RNA processing (C/D box snoRNA proteins Bcd1 and Bud21, C/D snoRNA interacting protein Slx9, rRNA and ribosome biosynthesis regulon protein Fcf2, DExD/H box RNA helicase Dbp3, H/ACA snoRNP proteins Gar1, Nhp2, RNA polymerase II C-terminal domain binding protein Hrr25, RNA recognition motif containing protein Nop6, RNA polymerase I subunits Rpa12, Rpa43,Rpa135, 27SA3 pre-rRNA processing proteins Rpl17a, Rpl7b, Rpl8a, Rpl8b, 20S pre-rRNA processing Rps14b, RNA metabolism protein of unknown function Rtc3) (Fig. 3.3.7a), constituents of the 60S ribosomal subunit (Rpl1a, Rpl1b, Rpl3, Rpl6b, Rpl9a, Rpl9b, Rpl11a, Rpl12b, Rpl15b, Rpl16a, Rpl21a, Rpl21b, Rpl22a, Rpl23a, Rpl24b, Rpl33b, Rpl34a, Rpl36a, Rpl36b, Rpl40a, Rpl40b), constituents of the 40S ribosomal subunit (Rps6a, Rps10a, Rps18b, Rps22b, Rps23b, Rps24a, Rps24b, Rps26b, Rps30a) (Fig. 3.3.7b), proteins requires for nuclear export of pre-60s ribosomal subunit (Alb1, Arx1, Bud20 Ecm1 Nog2 Rrp12 Rpp1a) (Fig. 3.3.7c), proteins that regulate translation (mRNA decapping protein Edc2, translational activator of mRNAs with internal ribosome entry sites Gis2, glutamine tRNA synthetase Gln4, P-bodydependent granule assembly protein $\mathrm{Pbp} 1$, and translation machinery associated protein Tma22) (Fig. 3.3.7d). Translation elongation related proteins (Eft1, Eft2, Yef3, Tma7, ribosomal stalk protein P1 alpha Rpp1a, and cytoplasmic RNA-binding protein Sro9 were also reduced in response to MTDIA and

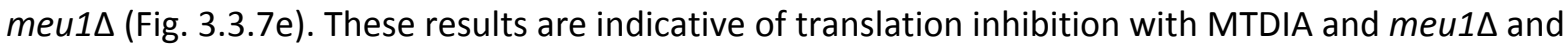




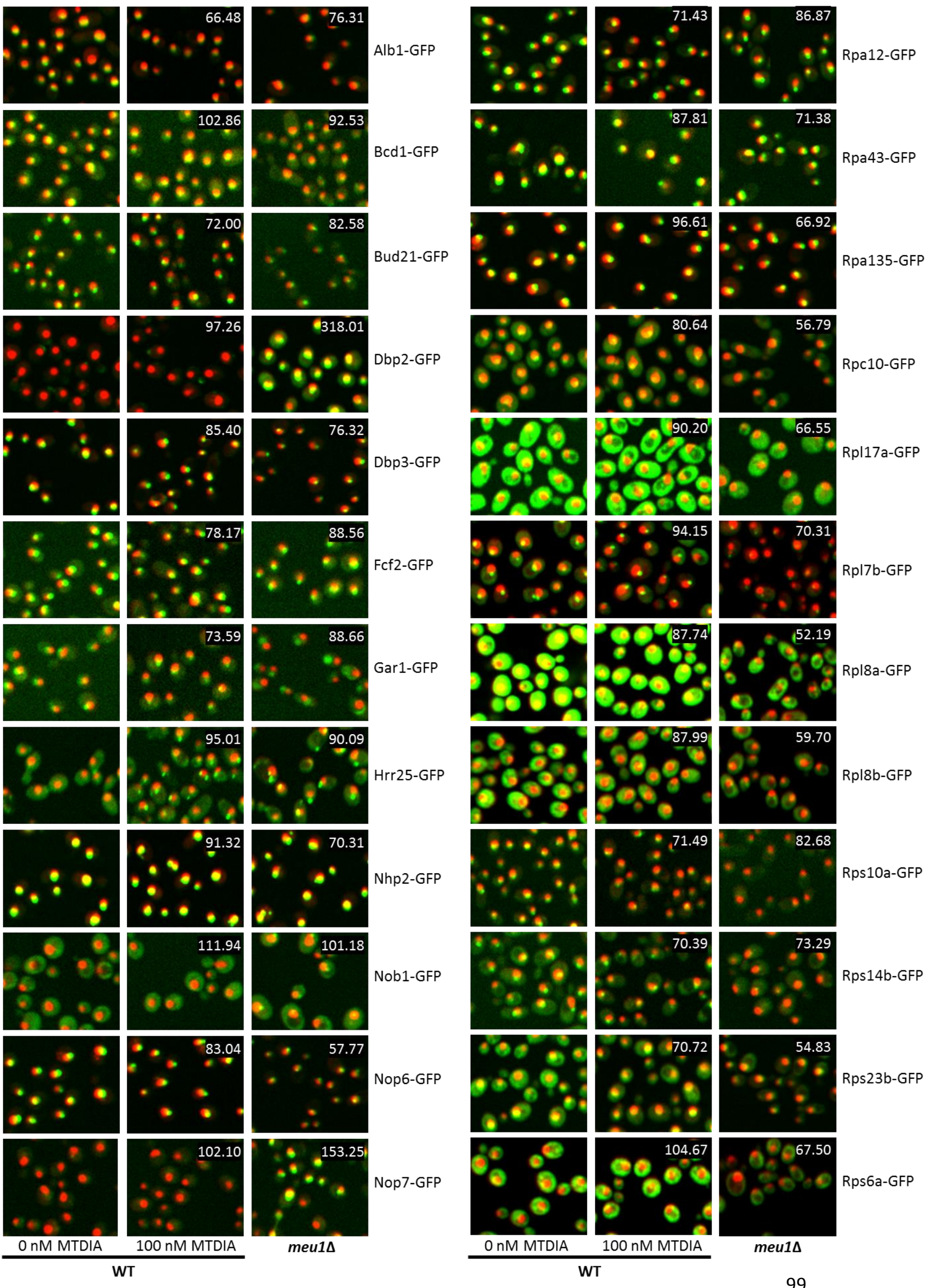

WT
WT $\begin{array}{lll}* & & 66.92 \\ * & 0 & \text { Rpa135-GFP }\end{array}$

Rps10a-GFP 

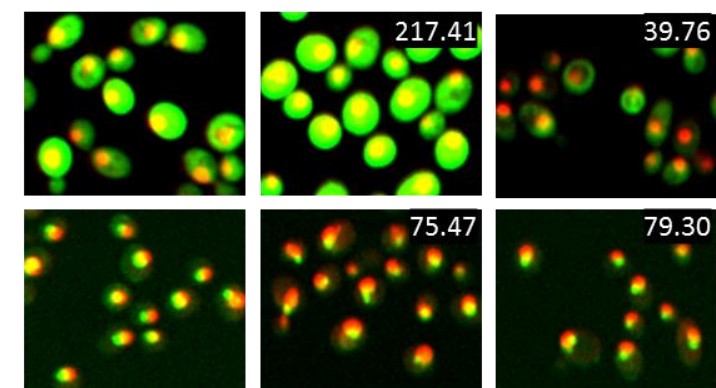

*, 75.47

0 nM MTDIA

100 nM MTDIA

b
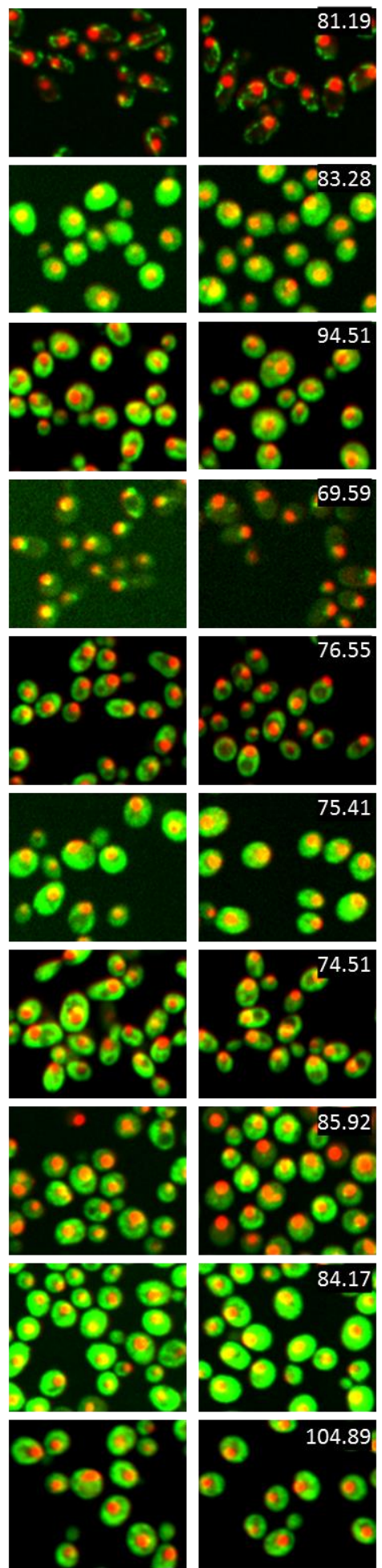

O nM MTDIA

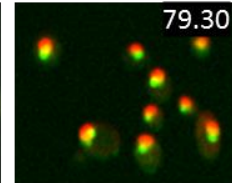

meu1వ
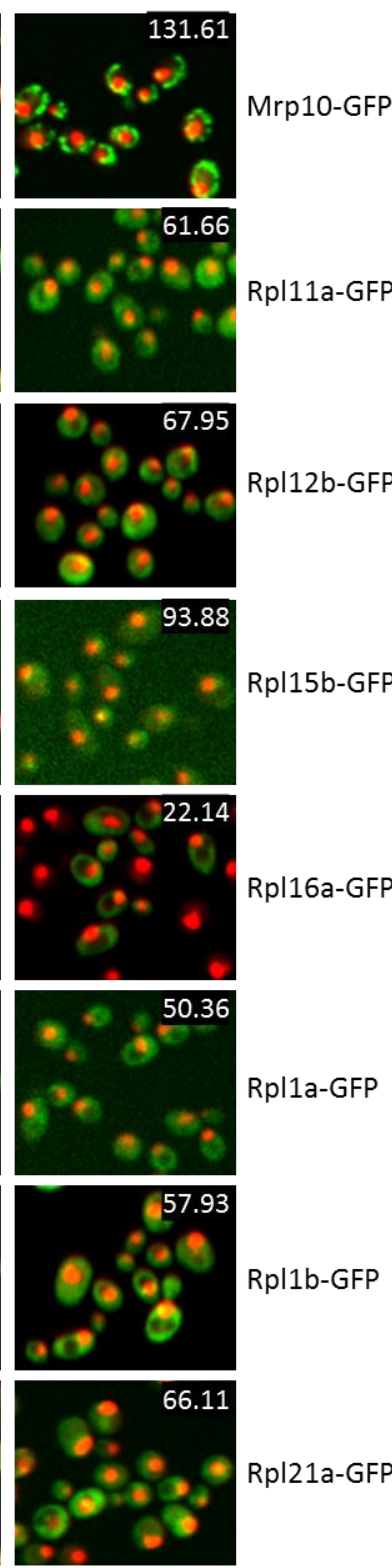

Rpl15b-GFP

Rpl16a-GFP

Rpl1a-GFP

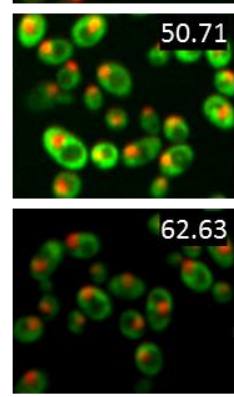

meu1s

Rpl11a-GFP

Rpl21a-GFP

Rpl21b-GFP

Rpl22a-GFP
Rtc3-GFP
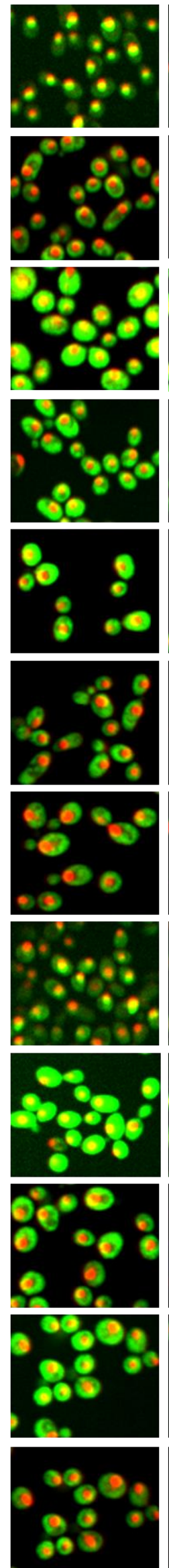

0 nM MTDIA
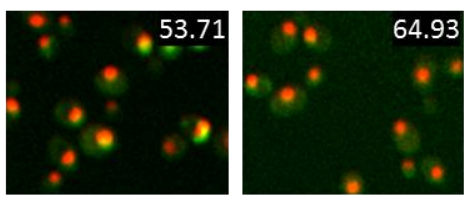

Rpl23a-GFP
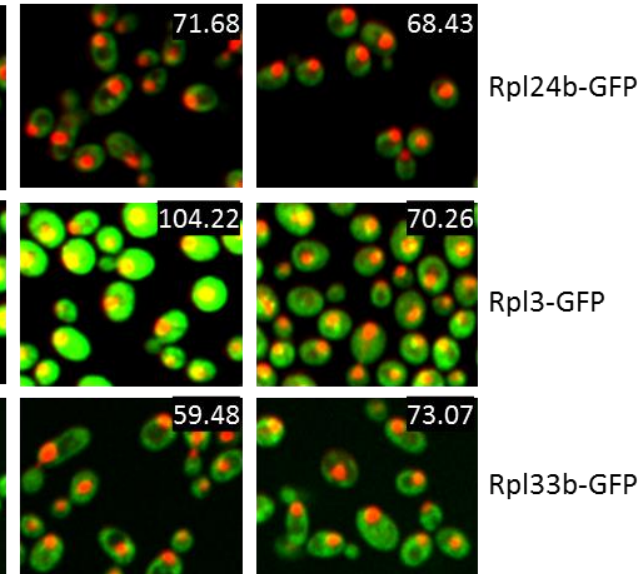

Rpl3-GFP

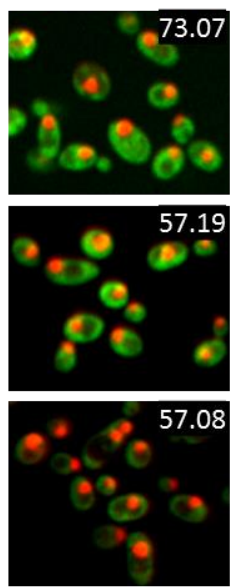

Rpl33b-GFP
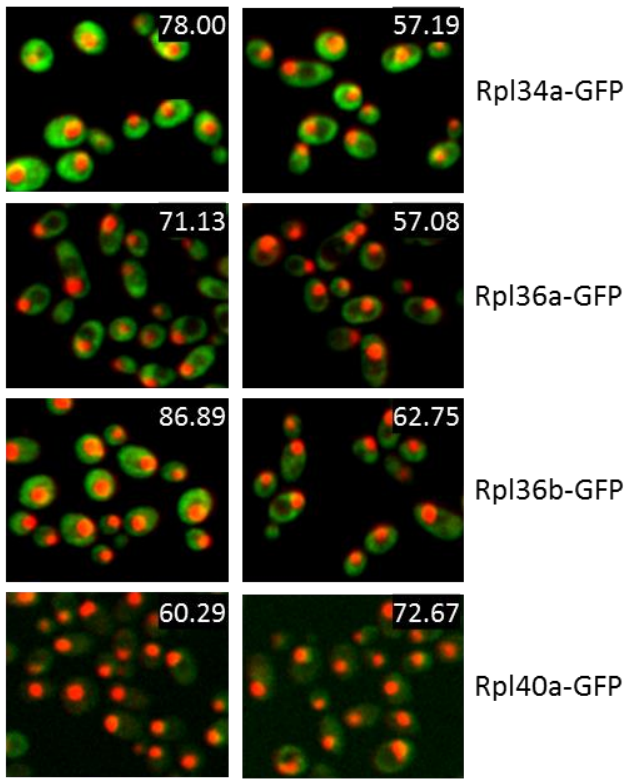

Rp|40a-GFP
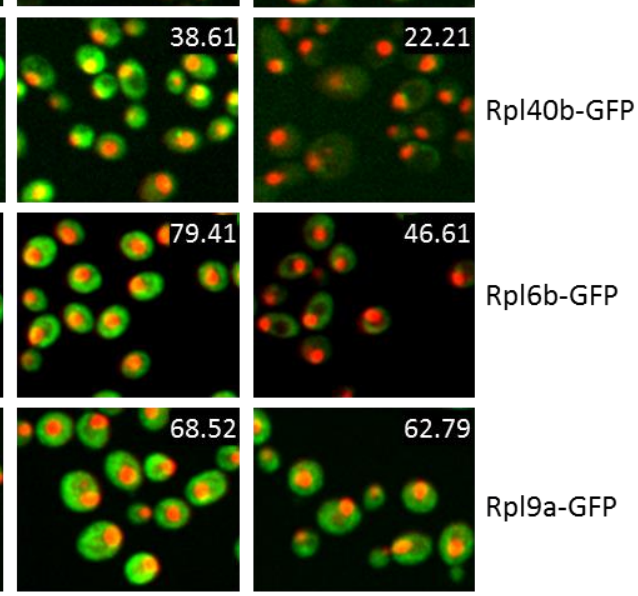

Rpl6b-GFP
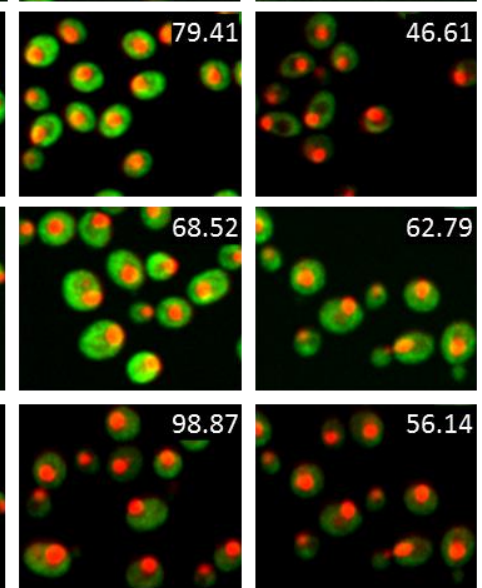

100 nM MTDIA

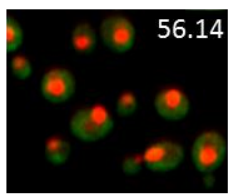

Rpl9a-GFP

WT 
d

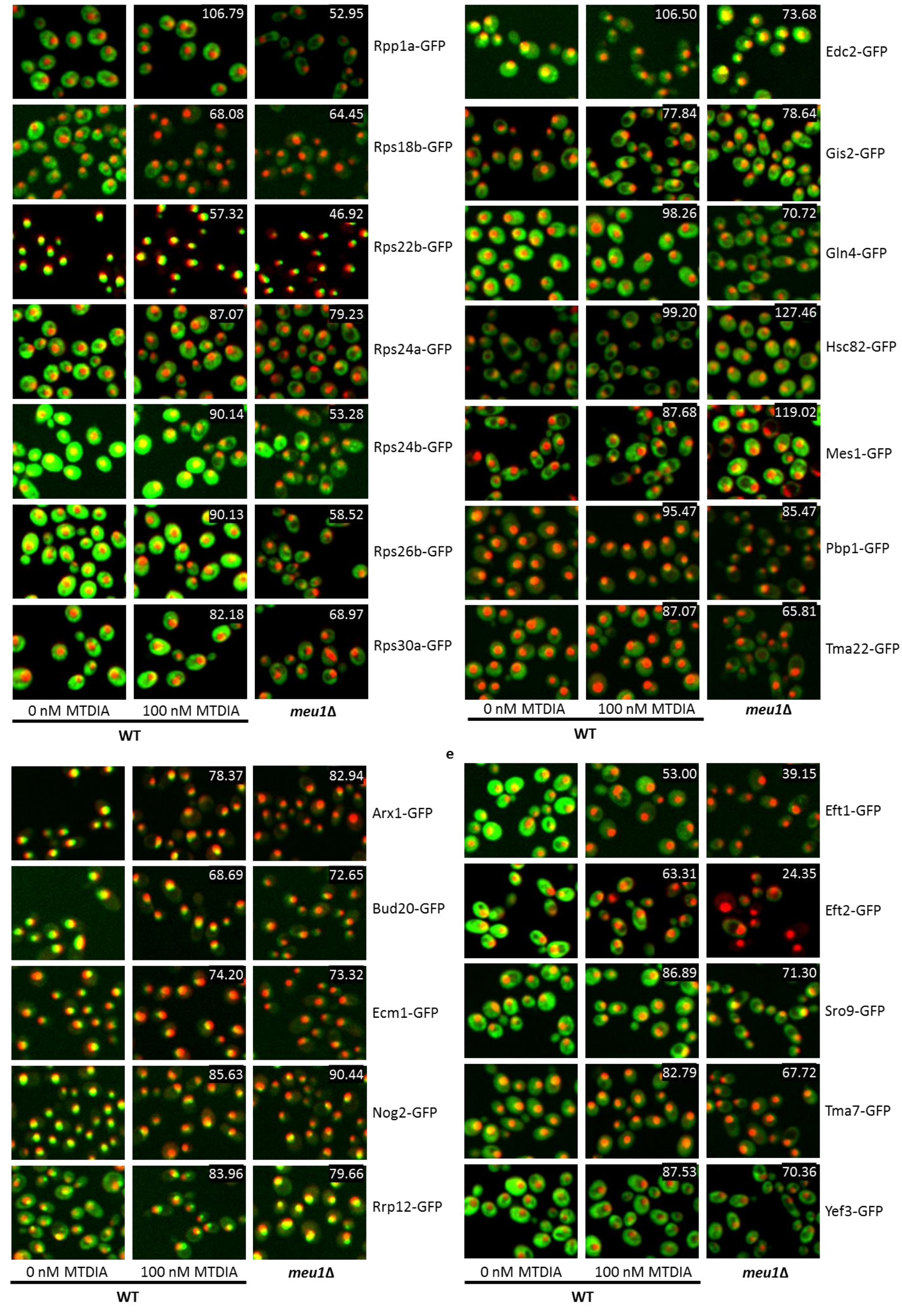




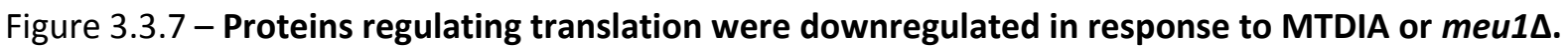
(a) RNA processing and ribosome biogenesis. (b) Ribosomal constituents and assembly. (c) Ribosomal subunit and rRNA export from the nucleus. (d) Translation regulation. (e) Translation elongation. GFP fusion proteins were visualised using the high-throughput spinning disk confocal microscope (Evo Tec OPERA, Perkin) using the 60x water immersion lens (NA 1.2). The GFP abundance was quantified by automated image analysis (Acapella, Perkin Elmer)(Bircham et al., 2011) and relocalisation was determined by visual inspection. Numbers inset in each image are the percent changes in GFP abundance with either MTDIA or meu1A relative to WT untreated.

are consistent with growth defects in non-essential gene deletion of translation related genes in Chapter 2.

In contrast, two proteins, methionyl-tRNA synthetase Mes1 and mitochondrial ribosomal subunit Mrp10 were upregulated with MTDIA and meu1 $\Delta$. Three proteins changed localisation were the ATP-dependent RNA helicase Dbp2 and the pre-ribosomal particle component Nop7 that relocalised to the nucleus as well as the 20S pre-rRNA processing protein Nob1 that relocalised to nuclear foci (Fig. 3.3.7a-e). Growth defects in translation genes and mostly deregulation of translation proteins are concordant with the role of polyamines in other systems where polyamines stimulate ribosomal RNA and aminoacyl-tRNA synthesis as well as improve translation fidelity by stabilising ribosomal subunits and ribosomal RNA, improving discrimination of aminoacyl-tRNA binding to the ribosome and subsequent proof reading, and inhibiting of polypeptide chain termination (Thompson et al., 1981; Sperrazza \& Spremulli, 1983; Igarashi et al., 1982; Tabor \& Tabor, 1984).

3.3.6 Cellular transport mechanisms disrupted by MTDIA and meu1 $\Delta$ are consistent with autophagy Disruptions to vesicle-mediated transport was a novel mechanism of MTDIA and MTAP loss identified in this thesis represented by altered growth observed for deletions of vesicle-mediated transport genes (ELO2, VPS17, VPS4, VPS5, VPS53, YDR455C/overlaps VPS44, VPS8, VAM10 and MON1) observed with MTDIA and deletions of CCZ1, VAM3, VAM6, VPS41, ROM2, MTC5, GGA2 and RIM1 observed with meu1s (Chapter 2). Consistent with these findings were changes in proteins involved in transmembrane transport either in intensity or localisation with MTDIA and meu1 $\Delta$. Amino 
acid permeases of the plasma membrane such as the plasma membrane regulator of polyamine and carnitine transport Apg2, branched amino acid permeases Bap2 and Bap3, 7-keto 8-aminopelargonic acid permease Bio5, high affinity glutamine permease Gnp1 that also transports other amino acids (Leu, Ser, Thr, Cys, Met and Asn), high-affinity histidine permease Hip1, choline, ethanolamine, and carnitine permease Hnm1, high affinity methionine permease Mup1 relocalised from the plasma membrane to the vacuole in response to MTDIA or meu1A (Fig. 3.3.8a). Likewise, plasma membrane ion transport proteins (high-affinity copper transporter Ctr1, ferro-O2-oxidoreductase Fet3, high affinity iron permease Ftr1, iron siderophore Arn1, negative regulator of Pma1 $\mathrm{H}^{+}$-ATPase HSP30, highaffinity cysteine permease Yct1, and acetic acid responsive plasma membrane protein Yro2 relocalised from the plasma membrane to the vacuole (Fig. 3.3.8b). Plasma membrane xenobiotic efflux transporters such as ATP-binding cassette (ABC) transporters (Pdr5, Snq2, Yor1 and Pdr12), and major facilitator superfamily proteins ( $\mathrm{Hol} 1$ and Tpo4) also relocalised from the plasma membrane to the vacuole (Fig. 3.3.8c). Several other plasma membrane permeases (high-affinity $\mathrm{H}+$ /biotin (vitamin $\mathrm{H}$ ) symporter Vht1, ammonium transporter Ato3, purine-cytosine permease Fcy2, high affinity uridine permease Fui1, and myo-inositol transporters Itr1 and Itr2) also relocalised from the plasma membrane to the vacuole (Fig. 3.3.8d). Several ER membrane transport proteins (the ER high affinity $\mathrm{Ca}^{2+} / \mathrm{Mn}^{2+}$ permease Pmr1 and the protein translocon complex subunit Ssh1) increased in abundance,

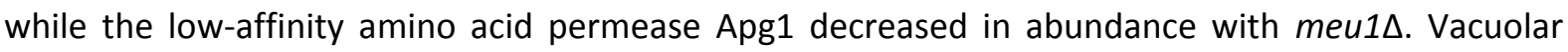
membrane proteins such as the zinc transporter Zrc1, vacuolar $\mathrm{H}(+)$-ATPase subunit Vma1 were down regulated with meu1 (Fig. 3.3.8a-d).

Mitochondrial proteins were also regulated by MTDIA and meu1 $\Delta$. Mitochondrial F1F0 ATP synthase subunits Atp7 and Atp15, subunit IV of cytochrome c oxidase Cox4, ubiquinol cytochrome-c reductase complex subunit Qcr6, inner membrane citrate transporter Ctp1, phosphate carrier Mir1, citrate and oxoglutarate transporter Yhm2, as well as mitochondrial translocase of outer membrane (TOM) complex subunit Tom7 were downregulated with MTDIA or meu1A (Fig. 3.3.8e). Only eight mitochondrial proteins are encoded and synthesised in the mitochondria itself while the remainder 
a
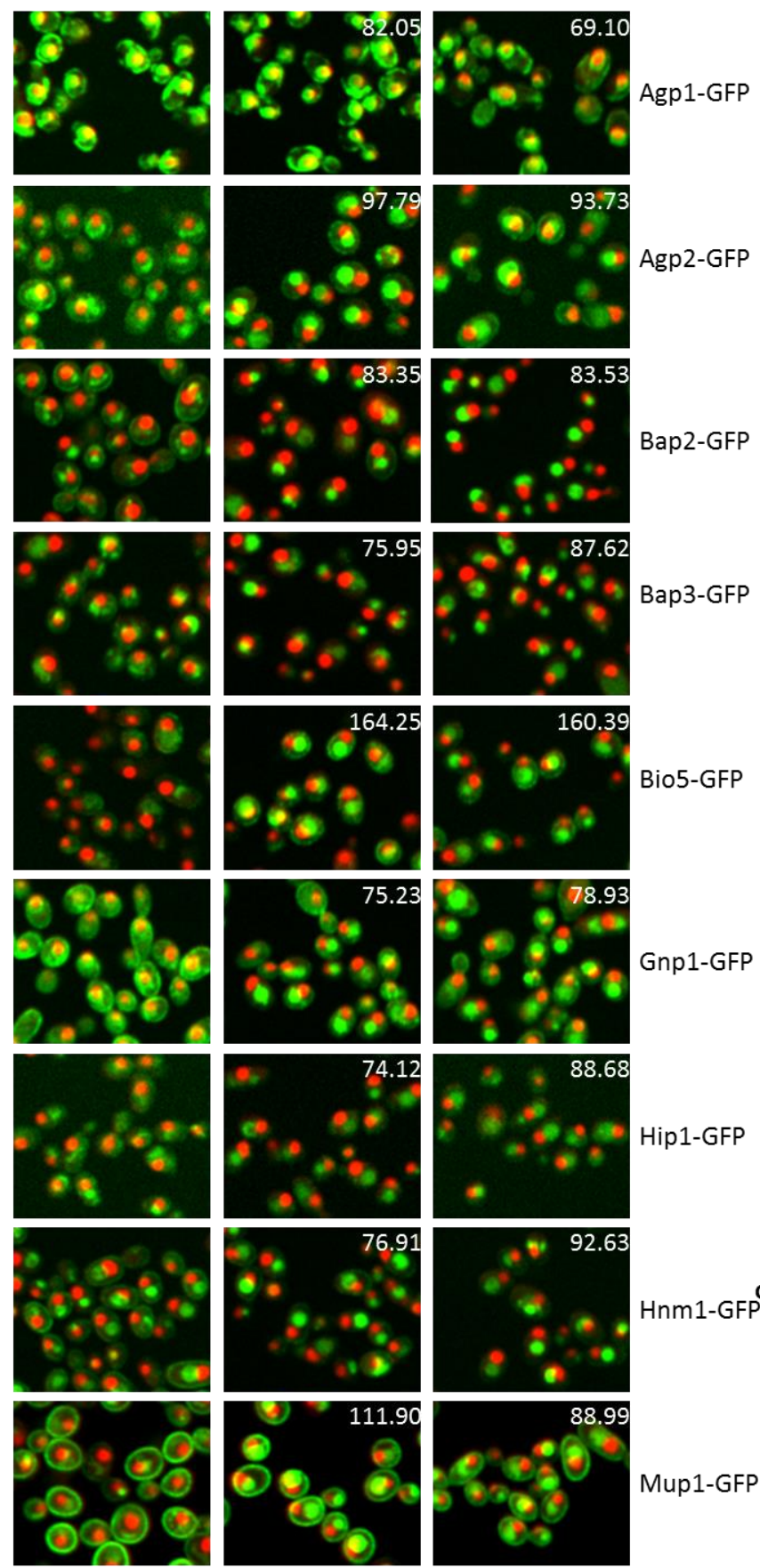

0 nM MTDIA

b

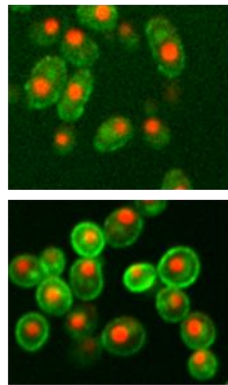

0 nM MTDIA
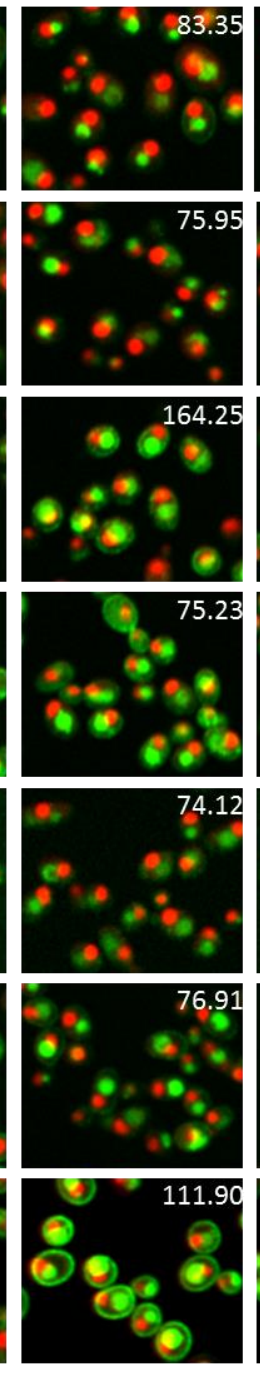

100 nM MTDIA WT
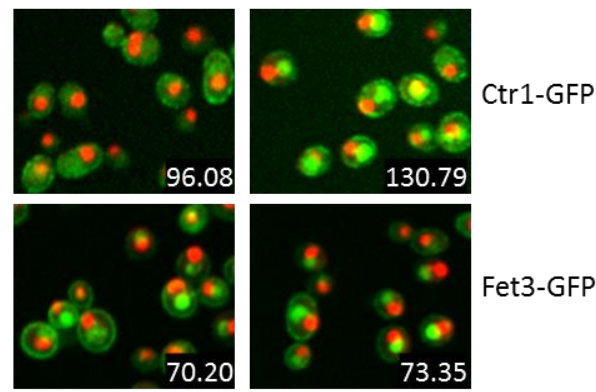

meu1s

Bap2-GFP

Bap3-GFP

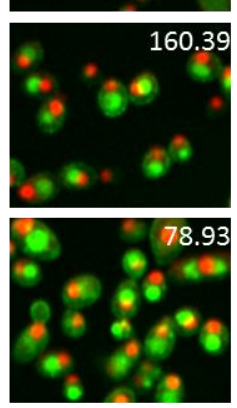

Gnp1-GFP
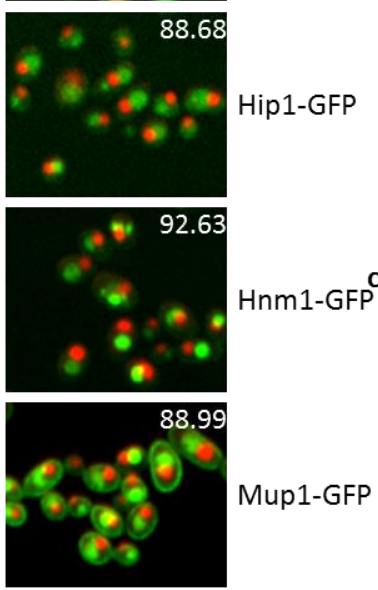

Mup1-GFP

Fet3-GFP

100 nM MTDIA WT
Agp2-GFP
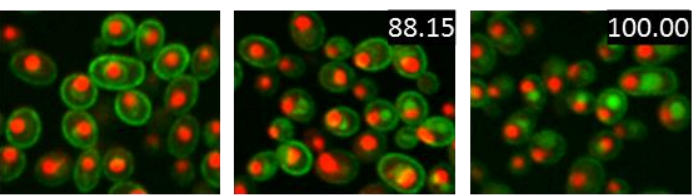

Ftr1-GFP
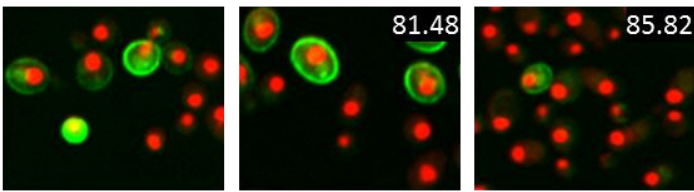

Hsp30-GFP
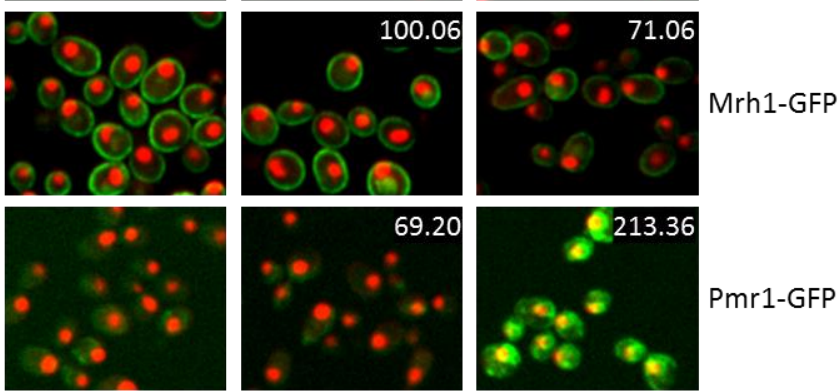

Pmr1-GFP
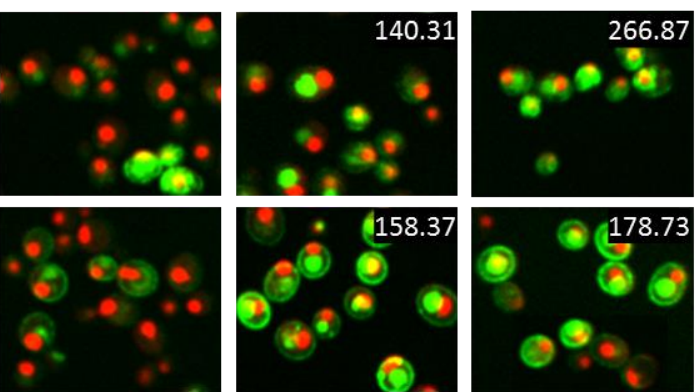

Yct1-GFP
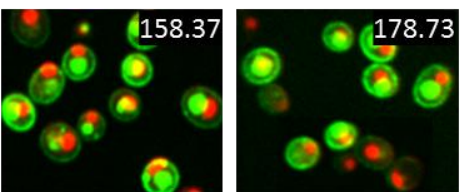

Yro2-GFP
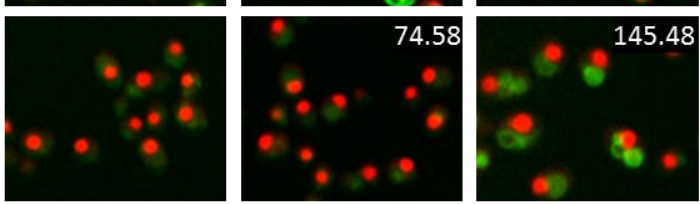

Zrc1-GFP

0 nM MTDIA

100 nM MTDIA WT
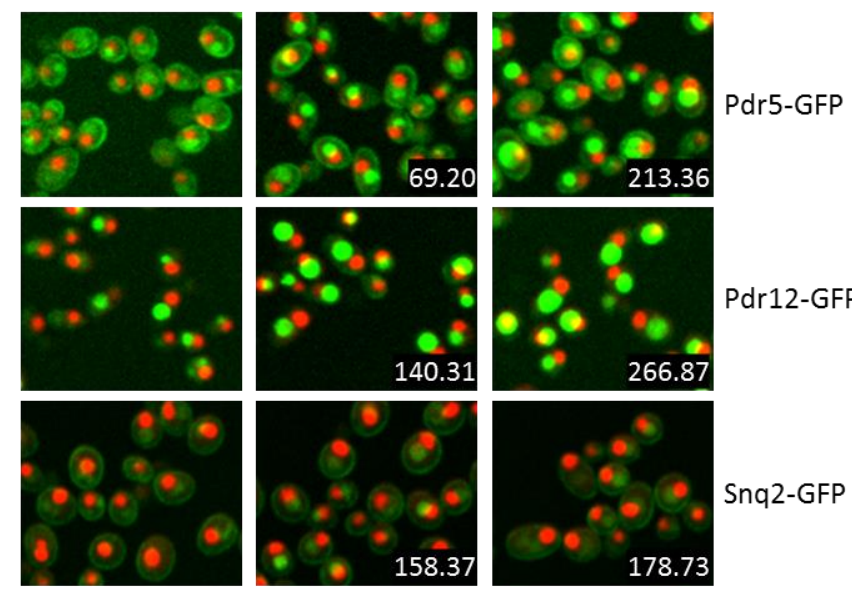

Q 213.36

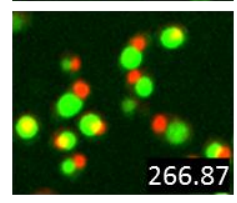

Pdr12-GFP
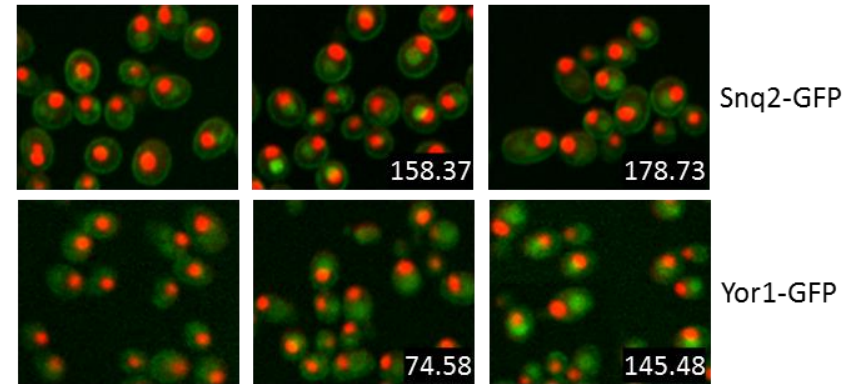

O nM MTDIA WT

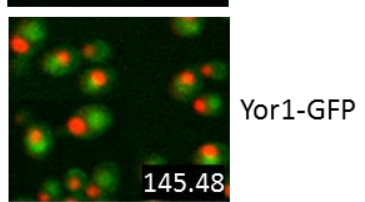

meu1s 
d
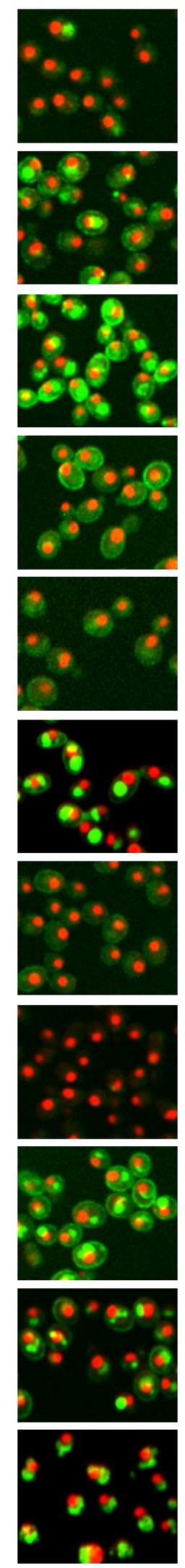

0 nM MTDIA
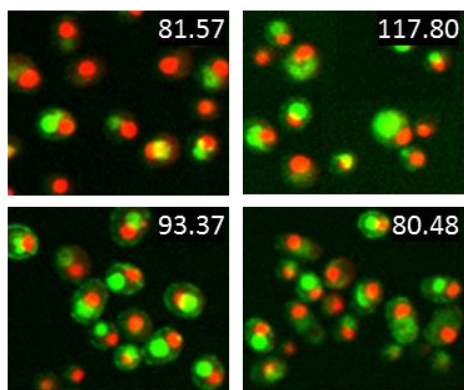

Arn1-GFP
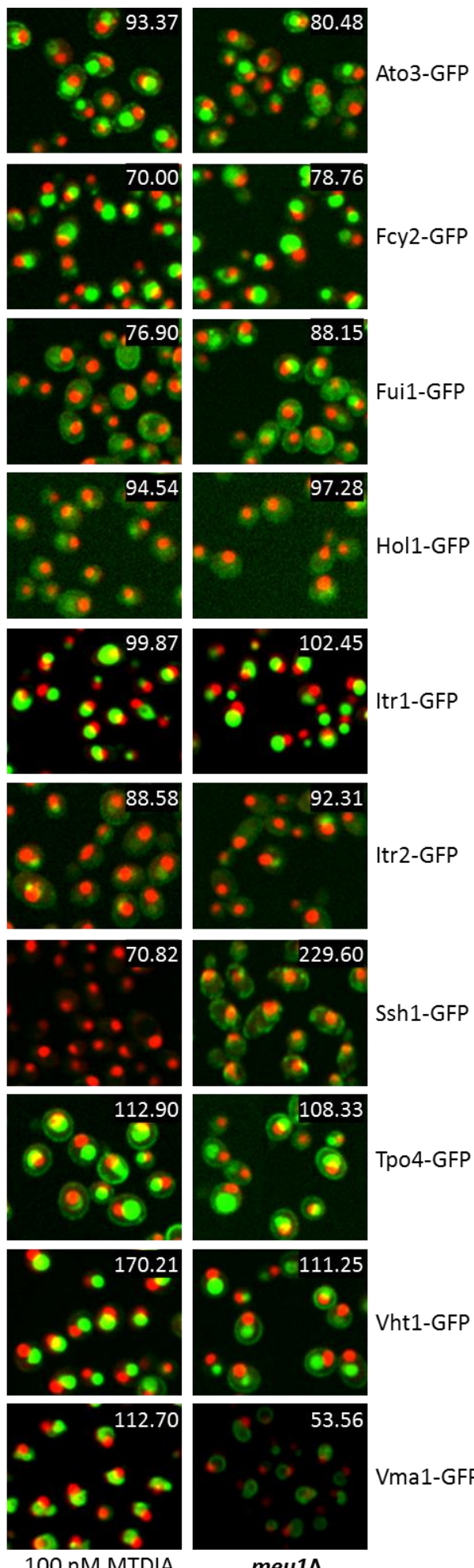

100 nM MTDIA

WT
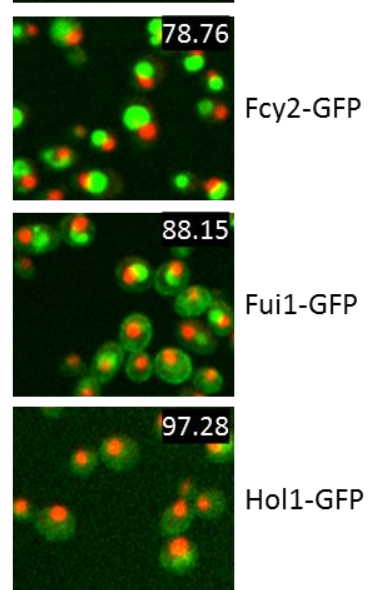

Hol1-GFP
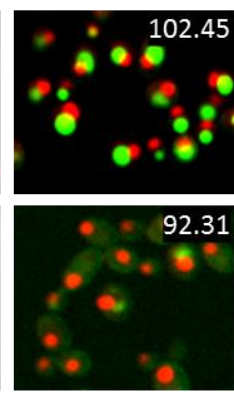

Itr2-GFP
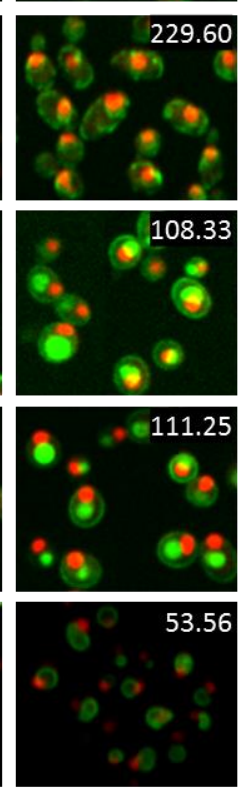

meu1s

Tpo4-GFP

Vma1-GFP
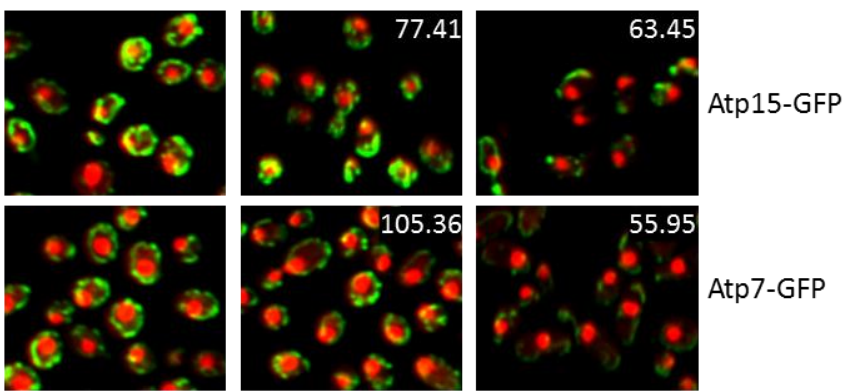

Atp7-GFP
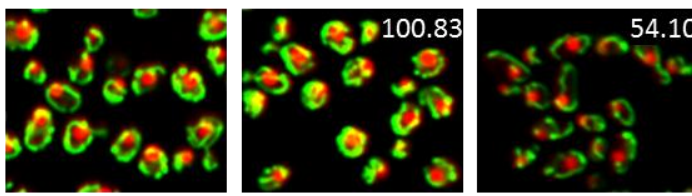

Cox4-GFP
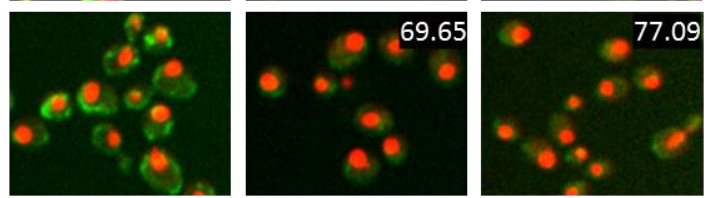

Ctp1-GFP
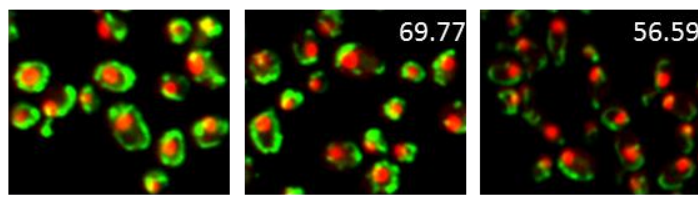

Mir1-GFP
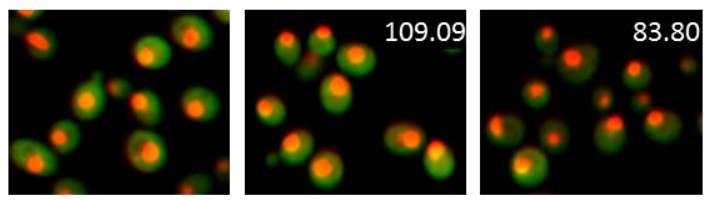

Qcr6-GFP
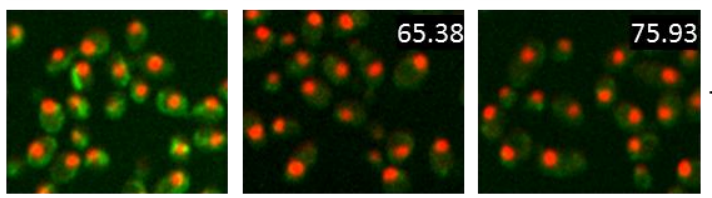

Tom7-GFP
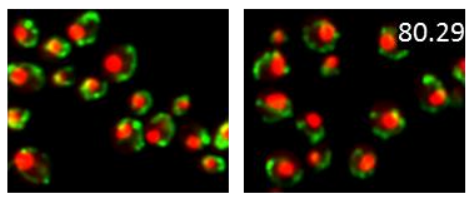

100 nM MTDIA

0 nM MTDIA
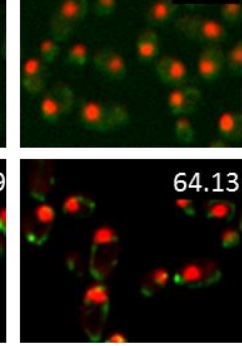

Yhm2-GFP

$$
\text { WT }
$$

meu1s 

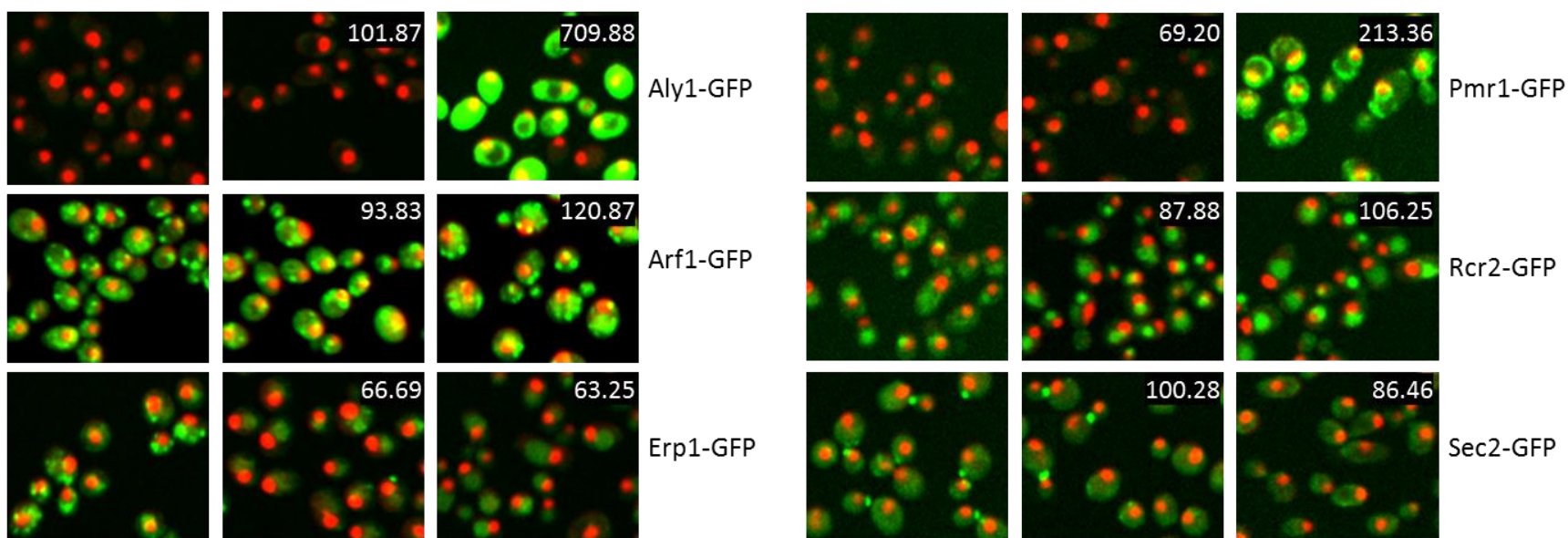

Sec2-GFP
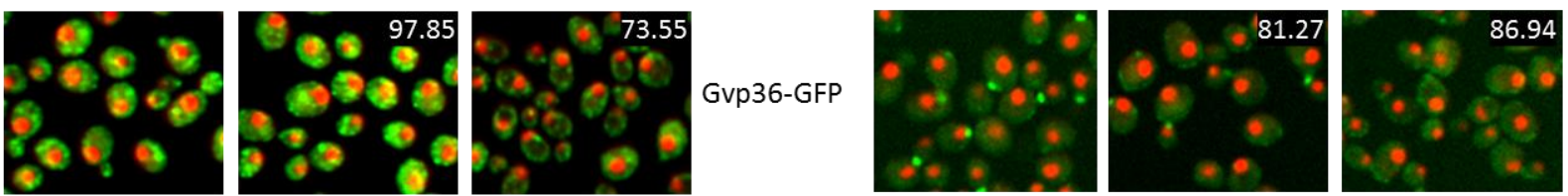

Sec8-GFP
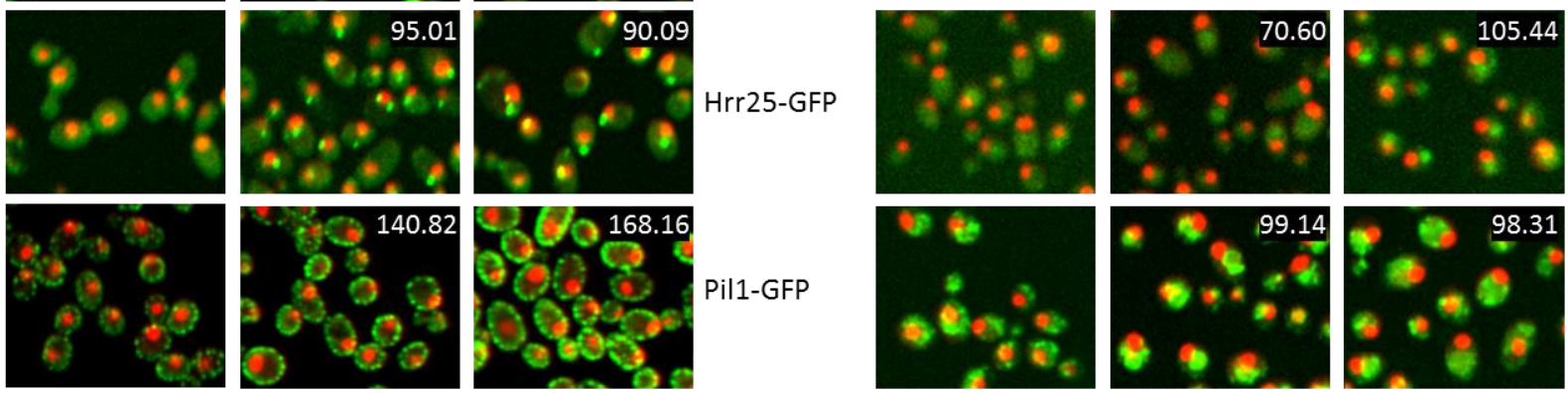

SsO2-GFP

O nM MTDIA

WT

meu1s

$0 \mathrm{nM}$ MTDIA

100 nM MTDIA

meu1s

Figure. 3.3.8 - MTDIA and meu1s deregulates membrane proteins. (a) Amino acid permeases. (b) Ion transporters. (c) Xenobiotic efflux transporters. (d) Mitochondria targeted transport. (e) Other transport mechanisms. (f) Vesicle-mediated transport proteins. GFP fusion proteins were visualised using the high-throughput spinning disk confocal microscope (Evo Tec OPERA, Perkin). The GFP abundance was quantified by automated image analysis (Acapella, Perkin Elmer)(Bircham et al., 2011) and relocalisation was determined by visual inspection. Numbers inset in each image are the percent changes in GFP abundance with either MTDIA or meu1A relative to WT untreated.

are encoded by nuclear genes and synthesised by the cytosolic ribosomes and are translocated to the mitochondria with the aid of translocase of outer mitochondrial membrane (TOM) complex and complexes of the translocase of the inner membrane (TIM) (Lithgow, 2000; Koehler, 2000). Therefore, the localisation of nuclear genome encoded mitochondrial proteins are dependent on vesicle- 
mediated transport and are sensitive to secretory pathway and vesicle-mediated transport disruptions.

Consistent with vacuolar localisation of plasma membrane proteins, several proteins required for vesicle-mediated transport were also localised to the vacuole (Fig. 3.3.8f). These included the ER to Golgi transport protein Erp1, the Kar9 localisation determinant protein Tvp38, the endosomalvacuolar trafficking pathway protein Rcr2 and the plasma membrane t-SNARE Sso2. Whilst others such as casein kinase HRR25 relocalised from cytoplasm to cytoplasmic foci and ADP-ribosylation factor

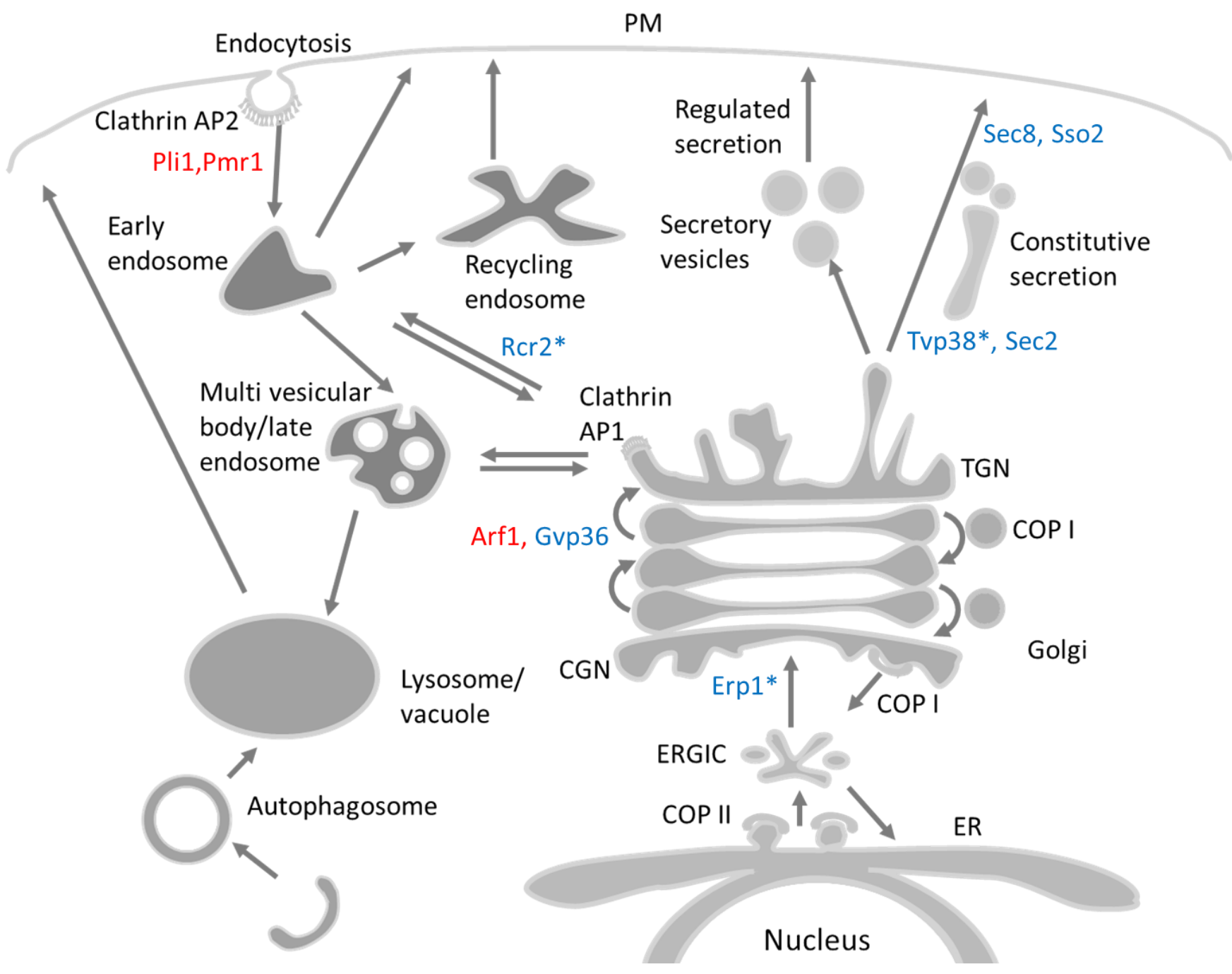

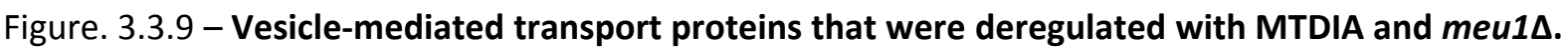

Proteins levels that were upregulated (red) downregulated (blue) and relocalised to the vacuole $(*)$. The assignments of genes in the pathway are based on SGD (Cherry et al., 2012). 
Arf1 increased in the number of cytoplasmic foci, levels of other proteins were reduced (the endocytosis protein Gvp36, ER/Golgi inheritance protein Sec8, guanyl-nucleotide exchange factor Sec2) or increased (high affinity $\mathrm{Ca}^{2+} / \mathrm{Mn}^{2+}$ P-type ATPase eisosome core component Pil1, calcineurin substrate Aly1) in response to MTDIA and meu1 (Fig. 3.3.8f; Fig.3.3.9). These disruptions to vesiclemediated transport are likely responsible for the mislocalisation of plasma membrane proteins to the vacuole and would be consistent with the induction of autophagy as a mechanism of MTDIA described in chapter 2.

3.3.7 Proteins involved in synthesis of cell wall, acetyl-CoA and homeostasis of sterols, glycerolipids and sphingolipids are regulated by MTDIA and meu1ડ

Ergosterol and zymosterol are the major sterols in yeast, and with fatty acids, glycerophospholipids and sphingolipids, comprise the lipid constituents of membranes. The synthesis of ergosterol is co-regulated with the synthesis of other lipids. The inhibition of HMG-CoA of ergosterol synthesis also leads to downregulation of sphingolipid synthesis and synthetic lethality of erg2 $\operatorname{erg} 24 \Delta$ is suppressed by deletion of fatty acid elongases ELO2 or ELO3 critical to sphingolipid synthesis (Storey et al., 1998; Bhuiyan et al., 2007). Deletion of ERG26 also changes the phospholipid composition of membranes, and inhibition of Erg11 inhibits fatty acid synthesis by inhibiting the transcription of ELO1, FAS1 and OLE1 (Baudry et al., 2001; Bammert \& Fostel, 2000). In chapter 2, deletions of genes involved in lipid homeostasis OPI3, CSG2, ETR1, INO2, SHR5, CEM1 showed a growth defect with meu1 $\triangle$ and deletion of ERF2, ETR1, YHLOO8C genes showed a growth defect with MTDIA, while deletion of fatty acid elongase ELO2 was resistant to MTDIA. Likewise, deletions of genes required for cell wall synthesis and Rim101 pathway RIM20, DFG16, RIM21 also showed a growth defect with MTDIA and vps4 4 that constitutively activate RIM101 improved growth. Herein we show disruptions to proteins involved in acetyl-CoA metabolism, sphingolipid metabolism, phospholipid metabolism and cell wall synthesis following MTDIA treatment or with meu1 (Fig. 3.3.10a-e).

Acetyl-CoA synthetases Acs1, Acs2 and Ccw14 as well as CoA transferase Ach1 were downregulated, fatty acid uptake protein Fat3 was upregulated, and acetyl-CoA carboxylase Acc1 
mislocalised from the cytoplasm to cytoplasmic foci (Fig. 3.3.10a). Ergosterol biosynthetic proteins such as squalene epoxidase Erg1 were downregulated, while two others (C-5 sterol desaturase Erg3 and C-14 sterol reductase Erg24) relocalised from ER and cytoplasmic foci to the vacuole. Delta(24)sterol C-methyltransferase Erg6 was relocalised from ER to the cytoplasmic foci as previously reported in Chapter 2 (section 2.3.6.2 and Fig. 3.3.10b). Proteins required for sphingolipid metabolism were also affected such as long chain fatty acyl-CoA synthetase Faa4 that relocalised from the ER to cytoplasmic foci, essential 3-hydroxyacyl-CoA dehydratase Phs1 and phosphatidylinositol phosphate (PtdlnsP) phosphatase Sac1 that relocalised from the ER to vacuole, glycerol 3phosphate/dihydroxyacetone phosphate sn-1 acyltransferase Sct1 that decreased in expression levels at the ER, and levels of integral plasma membrane proteins required for sphingolipid homeostasis (Fmp45 and Ynl194c) were upregulated with MTDIA and meu1s (Fig. 3.3.10c). Phospholipid synthesis proteins were also affected by MTDIA and meu1A such as the downregulation of cholinephosphate cytidylyltransferase Pct1, major CTP synthase isozyme Ura7, Phosphatidylethanolamine methyltransferase Cho2, and phospholipid metabolism regulator Scs2. ER methylene-fatty-acylphospholipid synthase Opi3, plasma membrane transporter for choline/ethanolamine/carnitine Hnm1, and ER phospholipid translocase Neo1 relocalised to the vacuole. Inter-organellar calcium and phospholipid exchange protein Mmm1 localised to cytoplasmic foci that are likely peroxisomes (Fig. 3.3.10d). Glycerol-3-phosphate dehydrogenases (Gpd1 and Gpd2) required for synthesis of glycerol3-phosphate (a precursor for glycerophospholipid synthesis) were also upregulated with MTDIA and in meu1 $\Delta$ (Fig. 3.3.2e). These disruptions to phospholipid synthesis proteins were expected since Opi3 and Cho2 that catalyse the penultimate and final steps in phospholipid synthesis are s-adenosyl methionine dependent methyl transferases inhibited by accumulation of MTA as consequence of MTAP inhibition (Kodaki \& Yamashita, 1987; Williams-Ashman et al., 1982). 

$3^{\circ} 0^{\circ}$

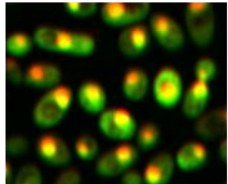

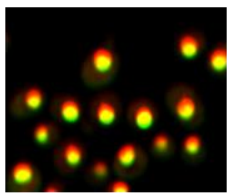

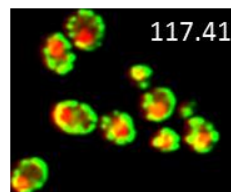
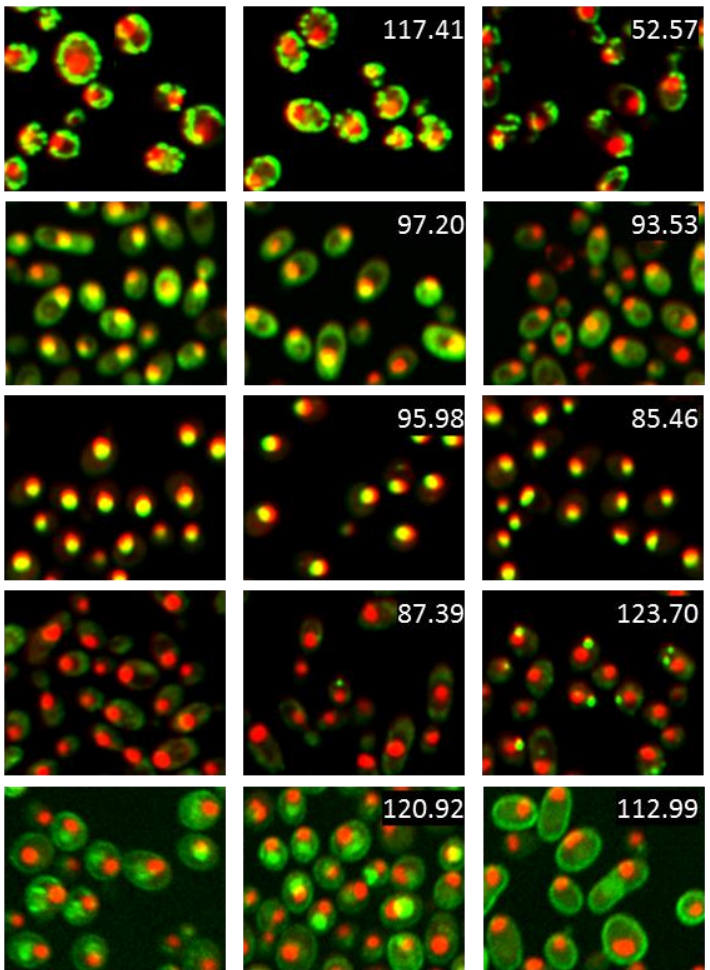

Acc1-GFP

Acs2-GFP

O nM MTDIA

100 nM MTDIA

b WT
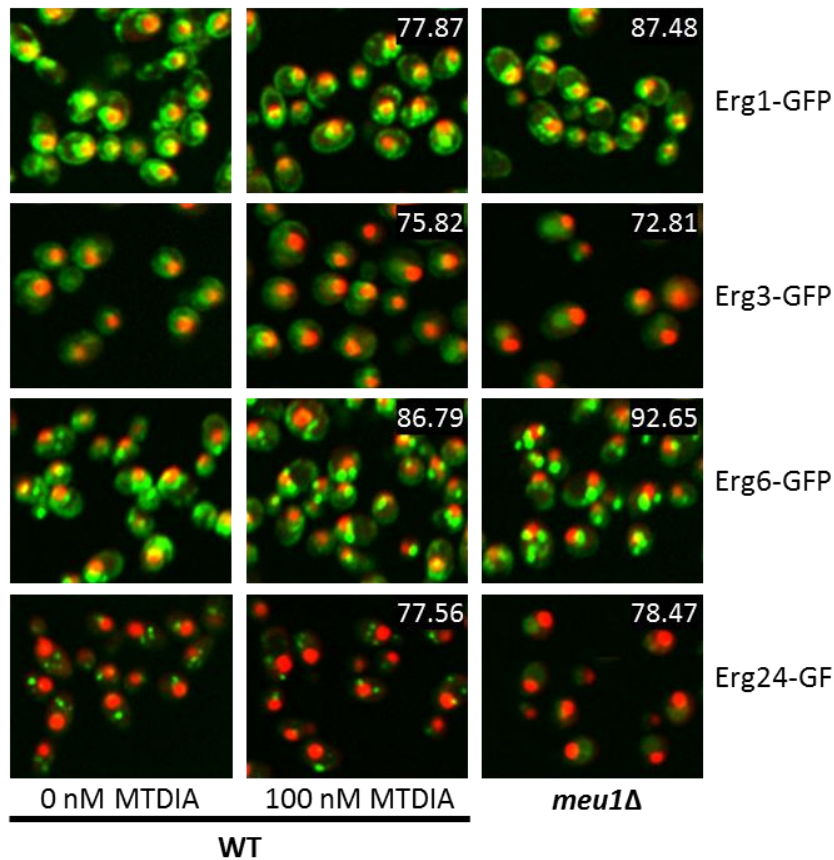

meu1s

c
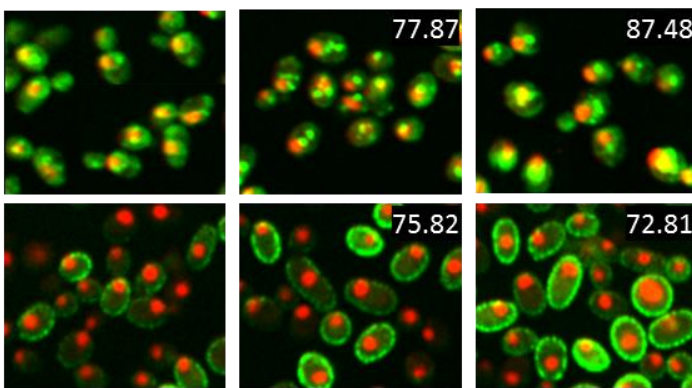

Erg6-GFP

Erg24-GFP
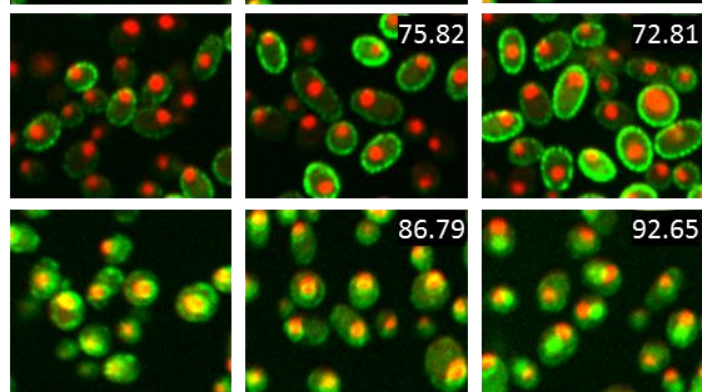

0 nM MTDIA
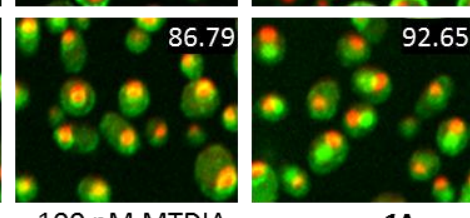

meu1s

Fmp45-GFP
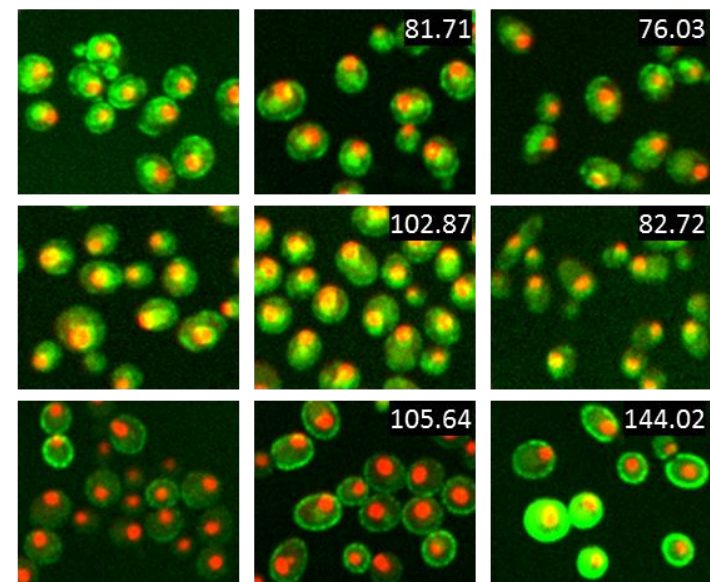

Sac1-GFP
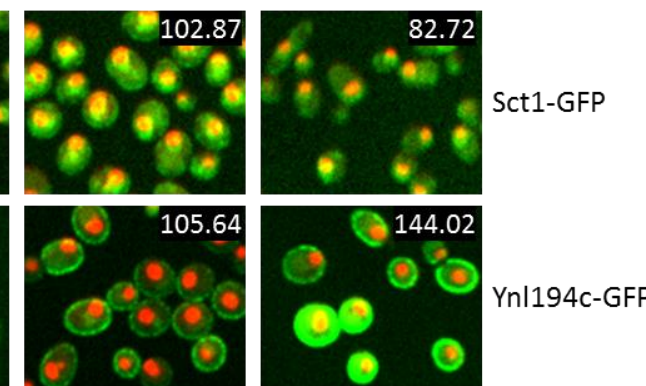

0 nM MTDIA WT

d
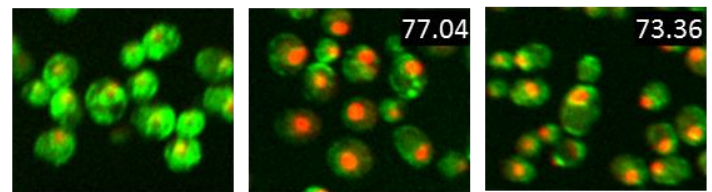

Cho2-GFP
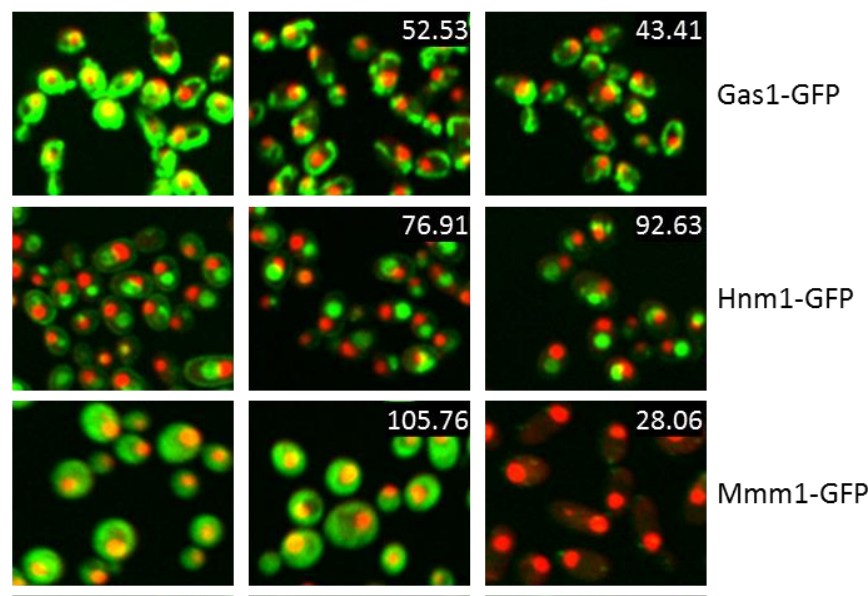

Mmm1-GFP
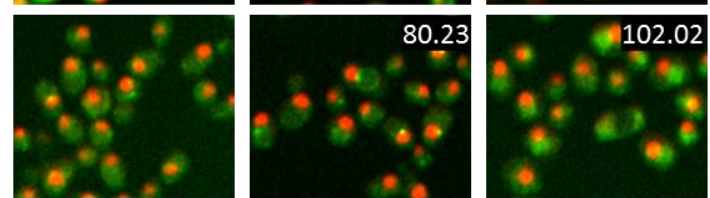

Neo1-GFP
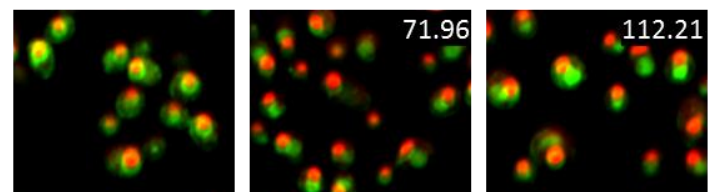

Opi3-GFP
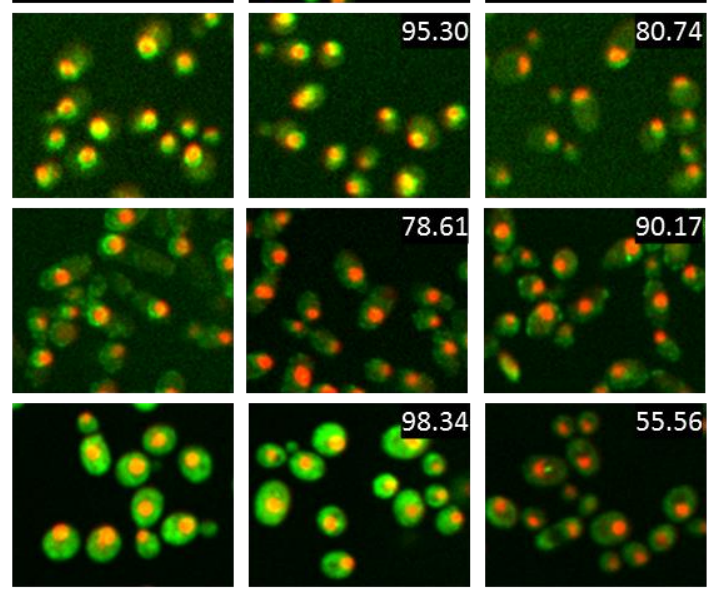

Pct1-GFP

0 nM MTDIA 
e
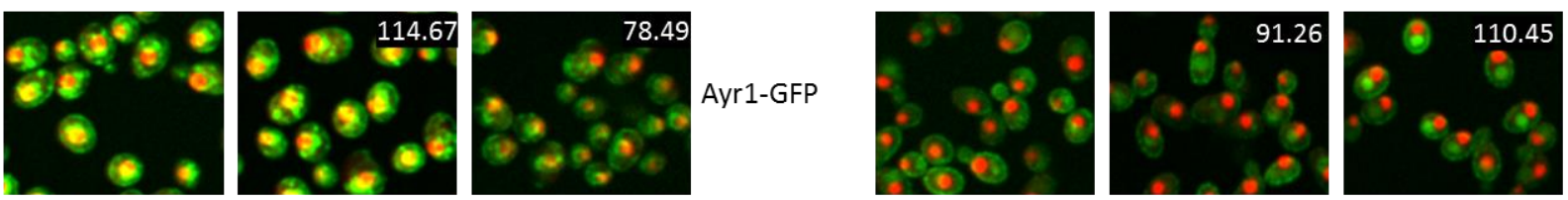

Pun1-GFP
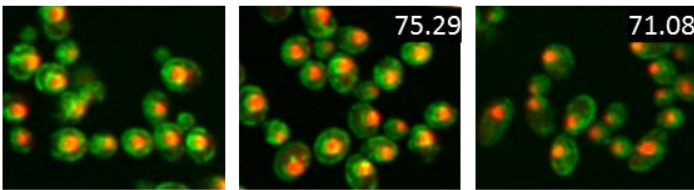

Ccw14-GFP
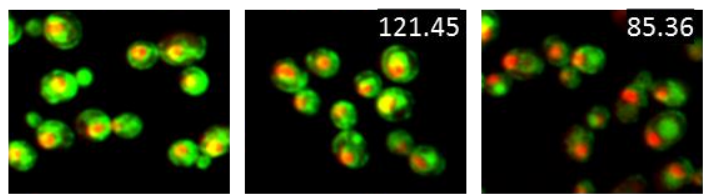

Sed1-GFP
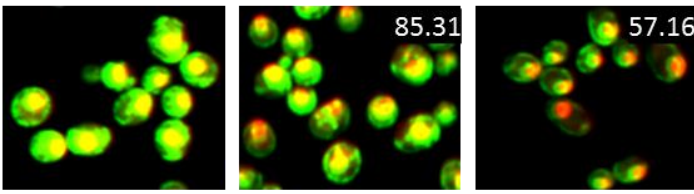

Cwp2-GFP
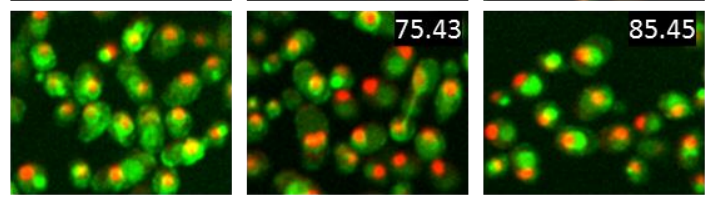

Tos6-GFP
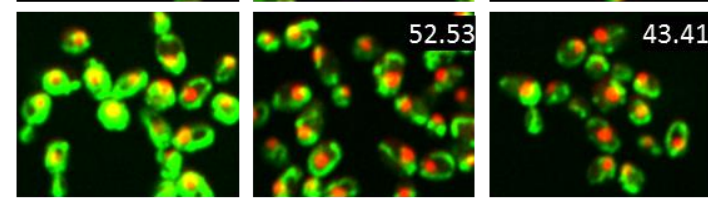

0 nM MTDIA

100 nM MTDIA

meu1s

Gas1-GFP

WT

0 nM MTDIA

100 nM MTDIA

WT

meu1s

Figure. 3.3.10 - MTDIA and meu1 $\Delta$ disrupts lipid homeostasis and cell wall synthesis. (a) Acetyl-CoA metabolism. (b) Ergosterol biosynthesis. (c) Sphingolipid metabolism (d) Phospholipid metabolism. (e) Cell wall synthesis. GFP fusion proteins were visualised using the high-throughput spinning disk confocal microscope (Evo Tec OPERA, Perkin). The GFP abundance was quantified by automated image analysis (Acapella, Perkin Elmer)(Bircham et al., 2011) and relocalisation was determined by visual inspection. Numbers inset in each image are the percent changes in GFP abundance with either MTDIA or meu1 $1 \Delta$ relative to WT untreated.

In addition to lipid homeostasis several cell wall synthesis and cell wall associated proteins were also perturbed by MTDIA and meu1D. The structure and biosynthesis of a fungal cell wall is unique to fungi being comprised of chitin, glucans, mannans and glycoproteins crosslinked together (Bowman \& Free, 2006). The glycoproteins in particular are synthesised at the ER where a glycosylphosphatidylinositol (GPI) anchor is attached that consists of ceramides attached to phosphatidylinositol linked to a oligosaccharide chain ending with a phosphatidylethanolamine moiety to which the glycosylated protein is attached (Bowman \& Free, 2006). The disruptions to vesicle-mediated transport or lipid homeostasis would therefore impact on glycoprotein composition of the cell wall. Reduction in levels of several cell wall synthesis and cell wall associated proteins were 
observed with MTDIA and meu1s (e.g., the bifunctional triacylglycerol lipase and 1-acyl DHAP reductase Ayr1, covalently linked cell wall glycoproteins (Ccw14 and Cwp2) and mitochondrial beta1,3-glucanosyltransferase Gas1 required for cell wall assembly) whilst others relocalised to the vacuole (plasma membrane associated protein cell wall integrity protein Pun1, GPI-cell wall glycoprotein Sed1 and glycosylphosphatidylinositol-dependent cell wall protein Tod6) (Fig. 3.3.10e).

\subsubsection{Cell cycle and DNA repair proteins are regulated by MTDIA and meu1D}

Deletions of cell cycle progression genes MBP1,WHI2, WHI4 and CLB3 and the nucleotide excision repair gene RAD4 showed a growth defect with MTDIA or meu1 (Chapter 2). Consistent with these findings, several cell cycle related proteins and DNA repair proteins were deregulated in response to MTDIA and meu1A. Bud neck associated septin ring components (Cdc10, Cdc11 and Shs1) and bud-site selection protein Bud4 showed reduced accumulation at the bud neck with meu1s (Fig. 3.3.12). Likewise, microtubule associated essential subunits of the Dam1/DASH complex (Ask1, Dad2, Duo1) also showed reduced spindle formation following meu1 $\Delta$. In addition, nuclear localised bud-site selection protein Bud20, cohesin complex subunit Irr1, and MIND kinetochore complex protein Mtw1 were downregulated. Cytosolic foci protein required for asymmetric localization of Kar9p during mitosis Tvp38 was localised to the vacuole with meu1A. DNA double-strand break repair proteins (Mre11, Rad6, Rdh54, Tfb1) were downregulated with meu1s. The dysregulation of cell cycle associated mRNAs were previously observed with MTDIA treatment of A549 lung cancer cell line but the dysregulation of DNA repair mRNAs was not detected. The depletion of polyamines by inhibition of ornithine decarboxylase however, impaired the repair of DNA double standard breaks generated during the repair of thymine dimers generated UV and was partially reversed by polyamine supplementation (Snyder \& Sunkara, 1990). Therefore, the downregulation of DNA repair proteins is likely a result of polyamine depletion. 

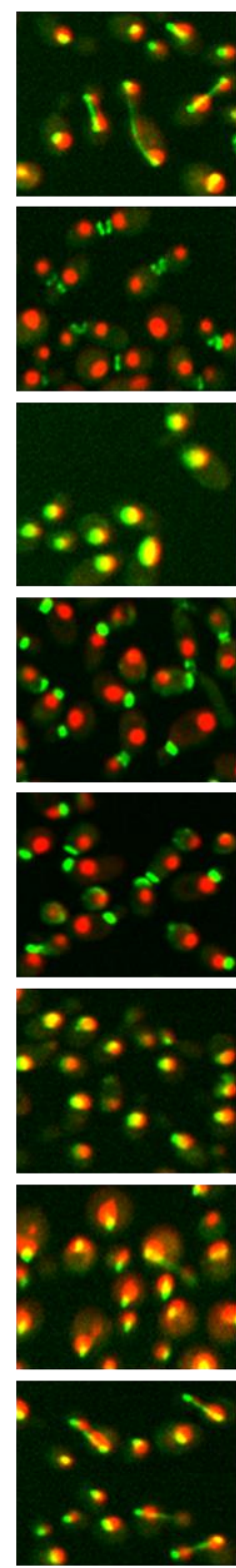

0 nM MTDIA
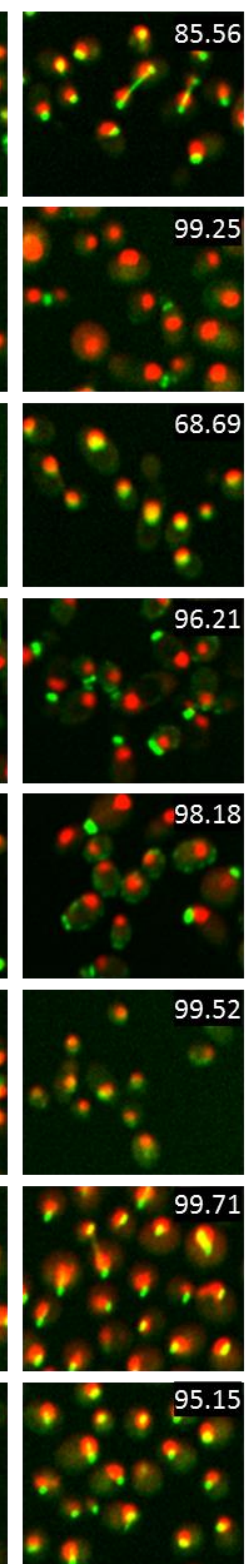

100 nM MTDIA WT
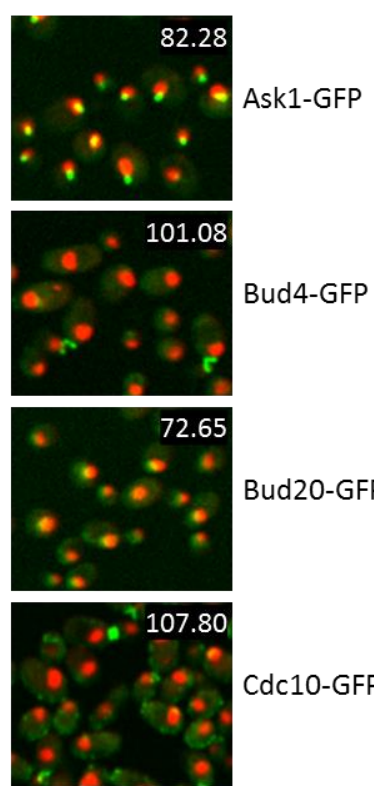

Cdc10-GFP

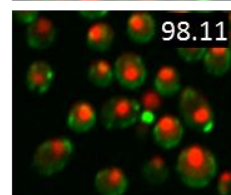

Cdc11-GFP

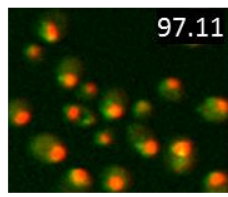

Irr1-GFP

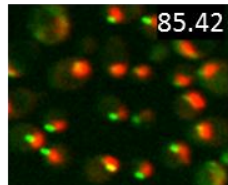

Duo1-GFP

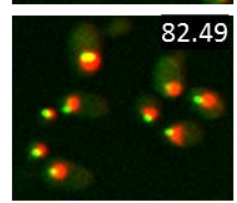

meu1s
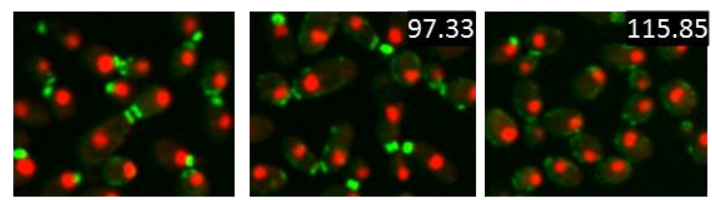

Shs1-GFP
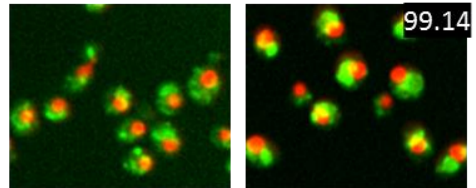

100 nM MTDIA

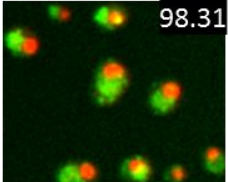

WT
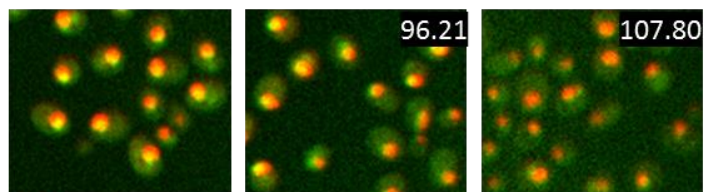

Mre11-GFP
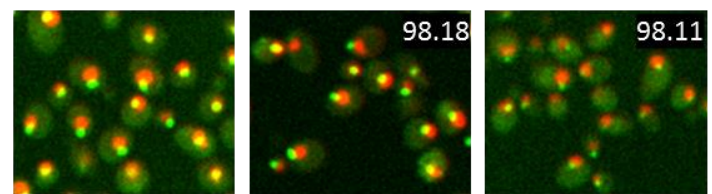

Mtw1-GFP
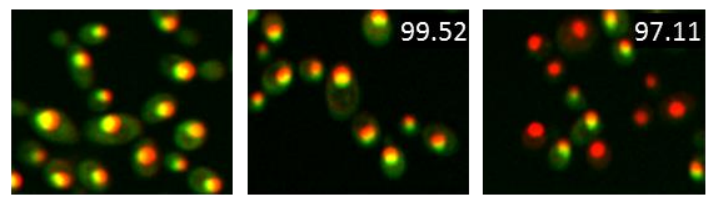

Rad6-GFP
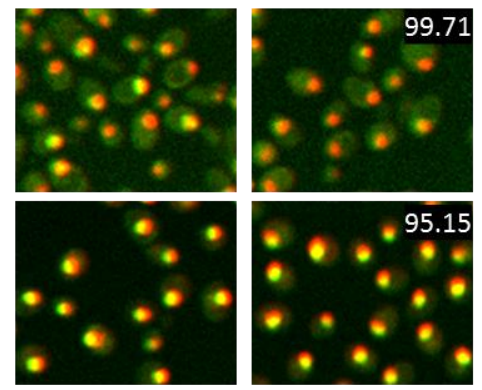

0 nM MTDIA

100 nM MTDIA

WT

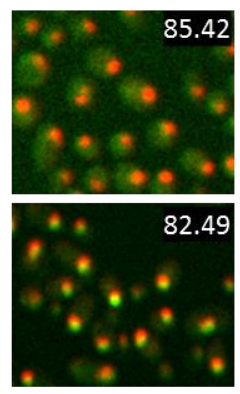

Rdh54-GFP

fb1-GFP

Figure. 3.3.11 - MTDIA disrupts cell cycle and DNA repair mechanisms. (a) cell cycle associated proteins. (b) DNA repair proteins. GFP fusion proteins were visualised using the high-throughput spinning disk confocal microscope (Evo Tec OPERA, Perkin). The GFP abundance was quantified by automated image analysis (Acapella, Perkin Elmer)(Bircham et al., 2011) and relocalisation was determined by visual inspection. Numbers inset in each image are the percent changes in GFP abundance with either MTDIA or meu1 $\Delta$ relative to WT untreated. 


\subsection{Discussion}

The global analysis of proteins in vivo provides unparalleled information on changes to both abundance and localisation that is not discernible to transcriptomic and proteomic methods that measure only abundance. In this chapter, the Saccharomyces cerevisiae GFP fusion collection comprising $\sim 4,900$ strains was screened with the transition state structure inhibitor of $5^{\prime}$ methylthioadenosine phosphorylase (MTAP) MTDIA and the genetic mimic in yeast meu1A. These experiments complemented the mechanisms identified in chapter 2 using non-essential gene deletions. Of the 4,900 GFP-fusion proteins screened against MTDIA, 147 showed altered protein abundance/localisation compared to WT untreated and as expected nearly twice as many (336 proteins) showed altered abundance/localisation with complete loss of MEU1 compared to WT, demonstrating dose-dependent response. As a consequence, there were 135 overlaps between MTDIA treatment and meu1s. Out of 348 total proteins, the majority (242/348) increased in abundance and under a third (106/348) showed change in localisation. Approximately half of the mislocalised proteins (59/106) exhibited a significant change in abundance. The remaining 47 proteins showed a change in localisation without a significant change in protein abundance and that would be undetectable to transcriptomic or proteomic methods. Proteins that increased in abundance showed mostly cytoplasmic, nuclear or cytoplasmic foci localisation and proteins that changed in localisation relocalised from cytoplasm to nucleus or plasma membrane/ER to the vacuole for both MTDIA

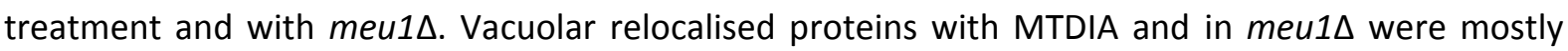
transmembrane proteins (amino acid permeases, ion transporters, drug efflux transporters and transporters of other solutes of the plasma membrane). These proteins are synthesised in the ER and are secreted to the plasma membrane via Golgi apparatus by vesicle-mediated transport (Van der Rest et al., 1995). The vesicle-mediated transport proteins themselves were either downregulated or vacuolar localised with MTDIA and meu1s.

Several cellular processes directly related the synthesis of polyamine were perturbed with MTDIA and meu1D. MTDIA is an inhibitor of polyamine synthesis and MTAP/MEU1, which processes 
5'-methylthioadenosine (MTA) generated as a by-product and inhibitor of spermidine and spermine synthesis to ribose-1-phosphate and adenine to salvage methionine (Avila et al., 2004). Previous studies have shown that inhibition of meu1D induces ODC activity by $\sim 8$-fold leading to 20 -fold elevation of putrescine and $>2$-fold elevation of spermidine levels that was attributed to a reduction in 4-methylthio-2-oxobutanoic acid, a downstream product of MTAP and inhibitor ODC (Subhi et al., 2003). These findings were consistent with the role of MTAP as a tumour suppressor where expression of MTAP in MTAP negative breast cancer cell line (MCF-7) lead to 6-fold decrease in putrescine levels and $\sim 2$-fold reduction in spermidine and spermine levels (Christopher et al., 2002). However, a followup study into MTAP expression in MTAP negative fibrosarcoma cell line (HT1080) showed no change in ODC or polyamine levels (Tang et al., 2015). Other studies have shown that MTA accumulation in MTAP positive cells is pro-apoptotic in human hepatocytes and cytotoxic in melanocytes (Ansorena et al., 2002; Andreu-Pérez et al., 2010). MTAP inhibition by MTDIA and MTA co-treatment induced apoptosis of MTAP positive cell lines of lung, head and neck, and mouse xenografts models for both MTAP positive and negative lung, prostate, colon, cervical and triple negative breast cancers, and the polyamine levels were depleted in MTDIA treated cancers (Basu et al., 2007; Basu et al., 2011; Schramm, 2013a). In the current work, the upregulation of ornithine decarboxylase Spe1 and spermidine synthase Spe3 was not observed with either MTDIA or meu1A and the spermidine synthase was downregulated $84 \%$ and $69 \%$ of untreated WT with MTDIA and meu1 1 , respectively. These results were consistent with spermidine synthase inhibition by MTA observed in bovine brain tissue and reduction in spermidine levels with MTDIA dependent inhibition of MTAP in lung cancer and head and neck cancer cells (Pajula et al., 1979b; Basu et al., 2007; Basu et al., 2011). Furthermore, the processing of MTA by MTAP/MEU1 is necessary for salvage of methionine (Pirkov et al., 2008), therefore, proteins required for salvage methionine from glycine, serine, cysteine, choline and sulphate and reduction of methionine sulfoxide were all upregulated with meu1 $\Delta$ and MTDIA. These results functionally validate the MTAP inhibition by MTDIA in yeast. 
Several mechanisms relating to polyamine depletion were detected in the screening of the GFP-fusion proteins with MTDIA and in meu1A. Proteins in overlapping cellular processes of transcription, chromatin organisation and gene expression were downregulated with MTDIA and meu1 1 and disruptions to these proteins were consistent with polyamine depletion (Pollard et al., 1999). The role of acetyl-CoA in histone modification is likely a contributor for these disruptions, given that yeast do not encode DNA methyl transferase present in mammals (Tehlivets et al., 2013). Notable was the upregulation in meu1 $\mathrm{A}$ of the Nhp6 architectural transcription factor Nhp6b that functions in a parallel pathway to the histone acetyltransferase Gcn5 that is bypassed by polyamine depletion (Yu et al., 2000; Pollard et al., 1999). Many proteins required for translation were also downregulated with MTDIA and meu1s, consistent with the positive regulatory role of polyamines in translation and subsequent polyamine depletion with MTAP inhibition. Several proteins involved in resolving DNA interstrand cross-links were also downregulated with MTDIA and in meu1D and was a consequence of polyamine depletion (Snyder \& Sunkara, 1990). These mechanisms were not detected with MTDIA treatment of A549 tumours (Basu et al., 2011). Given that some of the commonly used chemotherapeutics are used for the sole purpose of inducing DNA strand cross-links (Dronkert \& Kanaar, 2001), MTDIA in combination with these chemotherapeutic agents may improve the effectiveness preventing the repair of DNA strand cross-linking.

A comparison of the proteins altered with MTDIA and meu1s in this study with the gene expression changes detected in the MTDIA treated A549 mouse xenograft tumours showed overlaps in perturbed cellular processes. $\mathrm{GO}$ analysis of $>2$-fold upregulated or downregulated gene transcripts from Basu et al. enriched for biological processes including processes regulating steroid biosynthesis $(p=1.92 \mathrm{E}-04)$, response to lipids ( $p=4.69 \mathrm{E}-03)$ vesicle-mediated transport $(p=1.68 \mathrm{E}-03)$, and gene expression ( $p=9.99 \mathrm{E}-05)$ (Appendix Table 7.3.6), albeit only regulation of cell cycle and apoptotic processes were reported (Basu et al., 2011). In our study, we observed disruptions to proteins that regulate all three lipids classes, vesicle-mediated transport, gene expression and cell cycle consistent 
with these disruptions in A549 tumours (Basu et al., 2011). The overlaps in the genetic screens confirm the use of yeast as a valid model for elucidation of drug mechanisms affecting human diseases.

The vacuolar relocalisation of the plasma membrane/ER proteins and disruptions to vesiclemeditated transport are consistent with induction of autophagy mechanisms with MTDIA and meu1 $\Delta$ (Chapter 2). Notable was aldehyde dehydrogenase Ald6 that is preferentially degraded by starvation induced autophagy and in response was downregulated (Onodera \& Ohsumi, 2004). Another example is the multifunctional casein kinase Hrr25 that phosphorylates and promotes association of Atg19 with Atg11 a core autophagy protein required for cytosol to vacuole targeting pathway (Tanaka et al., 2014). Hrr25 also phosphorylates proteins Atg36 to promote pexophagy and Atg32 to promote mitophagy (Nakatogawa, 2015). In response to MTDIA treatment and meu1s, Hrr25 accumulated in cytoplasmic foci. Loss of Hrr25 does not affect nitrogen starvation induced non-selective autophagy (Tanaka et al., 2014); therefore, induction of Hrr25 with MTDIA and meu1D may reflect induction of other selective forms of autophagy such as lipid droplet autophagy (lipophagy) in response to disrupted lipid homeostasis. Lipid droplet autophagy was only recently described (Singh et al., 2009), thus the proteins involved in lipophagy are less well characterised than that of other forms of autophagy and the role of Hrr25 in lipophagy is yet to be investigated. Long chain fatty acyl-CoA synthetase Faa4, localises to the ER/lipid droplets and following induction of lipophagy increases in accumulation at lipid droplets with some localised to the vacuole (van Zutphen et al., 2014). Here we observed increased localisation of Faa4 at lipid droplets with MTDIA and further increase in accumulation at the lipid droplets as well as what appears to be vacuolar localisation of Faa4 with meu1 $\Delta$. A similar relocalisation was also observed for the ER-localised Erg6-GFP, which would also be consistent with induction of lipid droplet autophagy. Consistent with the degradation of lipid droplets for fatty acid synthesis, the bifunctional triacylglycerol lipase Ayr1 was also upregulated with meu1 $\Delta$. Cellular processes affected by regulation of acetyl-CoA were also disrupted with MTDIA and meu1s. These included proteins involved in central carbon metabolism, cell wall synthesis and lipid homeostasis. Acetyl-CoA is an intermediate of central carbon metabolism and a precursor common to 
the synthesis of all lipid classes (Carman et al., 2008; Schug et al., 2015). Acetyl-CoA levels are elevated in various cancers by upregulation of enzymes that metabolise acetate such as acetyl-coA synthetases (Comerford et al., 2014). The acetyl-CoA synthesis in our yeast model would be expected to be increased with the demand for synthesis of ergosterol and glycerophospholipids, given that MTDIA/meu1 $\Delta$ inhibits methyl transferase activities of Erg6 in ergosterol synthesis (Chapter 2) and Opi3/Cho2 in glycerophospholipid synthesis (in this chapter). However, acetyl-CoA synthetases Acs1 and Acs2, and acetyl-coA transferase Ach1 levels were downregulated in meu1D, possibly due to inhibition by Erg6/Opi3/Cho2. Acetyl-CoA transferase ACAT1 was also downregulated in A549 tumours following MTDIA treatment (Basu et al., 2011). The downregulation of acetyl-CoA synthetases/transferases may play a role in the anti-tumour activity of MTDIA, given that loss of acetyl-CoA synthetase enzyme, ACSS2 reduce the tumour burden in models of hepatocellular carcinoma (Comerford et al., 2014).

In summary, previously suspected mechanisms of MTDIA detected in Chapter 2 in screening of non-essential gene deletion mutants with MTDIA and meu1 (disruption to amino acid metabolism, carbohydrate metabolism, transcription, translation, chromatin organisation, gene expression, vesicle-mediated transport, lipid homeostasis) were validated with perturbations to GFP-fusion proteins involved in these processes. Several additional cellular perturbations were also identified in disruptions to essential cell cycle and DNA repair proteins, yet were not evident in the results obtained with the non-essential deletion collection and were not detected previously. Furthermore, this chapter identified vacuolar localisation of numerous plasma membrane proteins, further suggesting induction of autophagy as a mechanism of MTDIA. The increased accumulation of lipid homeostasis proteins to lipid droplets suggests lipid droplet autophagy as an additional mechanism of MTDIA. Overall, the screening of the GFP library in this chapter both complemented and validated the screening of gene deletions in chapter 2. In addition, screening the GFP collection with MTDIA and the genetic mimic meu1 $\Delta$ showed a dose response of MTAP inhibition, which may give insight into cellular mechanisms resulting from moderate and extended treatments with MTDIA. 


\section{Chapter 4 - Elucidating the therapeutic mechanisms of PNP inhibitor forodesine via assessment of fitness of non-essential gene deletions}

\subsection{Introduction}

Purine nucleoside phosphorylase (PNP) is an enzyme of the purine salvage pathway that is required for T-cell proliferation (Parks Jr \& Agarwal, 1972). Loss of PNP leads to T-cell selective suppression and reveals opportunities for regulation of diseases characterised by T-cell proliferation (Bantia \& Kilpatrick, 2004). Previous attempts at inhibiting the purine salvage pathway had limited success due to lack of target affinity and the absence of $95 \%$ continuous inhibition of PNP required to abrogate T-cell function and proliferation (Gandhi, 2009; Morris \& Montgomery, 1998). Forodesine is a potent inhibitor of the human PNP enzyme in vitro (e.g., IC $\mathrm{C}_{50}=56 \mathrm{pM} \mathrm{Kd}$ in vitro) (Evans et al., 2003). Between 2001-2017, forodesine has undergone 14 phase I and II clinical studies to evaluate efficacy, safety and pharmacokinetics in patients with lymphatic leukaemia and lymphoma (https://clinicaltrials.gov/ct2/results?term=bcx-1777). In April 2017, forodesine was approved in Japan to treat recurrent and refectory peripheral T-cell Lymphoma (http://www.mundipharma.com.sg/2017/03/30/mundipharma-wins-approval-antineoplastic-agentmundesine-treatment-relapsedrefractory-peripheral-t-cell-lymphoma-japan/, 2017). Forodesine mimics the transition state structure of the PNP substrate inosine bound to the enzyme PNP; therefore, $\sim 7 \times 10^{5}$ fold more strongly to PNP than inosine (Schramm, 2011). Thus, forodesine inhibits PNP with previously unparalleled efficacy.

The proposed mechanism for forodesine-mediated toxicity essentially mimics the genetic deficiency of PNP. The inhibition of PNP1 causes an accumulation of its substrates (deoxyguanosine, deoxyinosine, guanosine, and inosine) within cells (Kim et al., 1968; Fox et al., 1980; Wortmann et al., 1979; Chantin et al., 1996). Of these, deoxyguanosine has an alternate fate where it is phosphorylated to dGTP by elevated dCK activity in T-cells (Osborne \& Scott, 1983). As dGTP inhibits the ribonucleotide diphosphate reductase (RR) and disrupts the dNTP pool, forodesine inhibits DNA synthesis leading to apoptosis of T-cells (Tattersall et al., 1975; Arpaia et al., 2000). The mechanisms by which PNP 
inhibition results in apoptosis have been investigated in T-cells wherein the progression of apoptosis is mediated by changes in mitochondrial membrane potential, P53 stabilisation, caspase activation and cleavage of PARP (Balakrishnan et al., 2006; Balakrishnan et al., 2010a). However, the underlying mechanisms that link dGTP accumulation to initiation of apoptosis have not been characterised. Furthermore, not all cells with elevated dGTP in response to forodesine treatment succumb to apoptosis (Balakrishnan et al., 2006; Balakrishnan et al., 2010a).

Purine nucleoside phosphorylase activity in S. cerevisiae is encoded by PNP1, which is a structural and functional homologue of human PNP (Lecoq et al., 2001b). Yeast PNP1 shares 50\% sequence identity with the human enzyme and the catalytic and substrate binding residues of PNP are conserved (Lecoq et al., 2001b). This chapter uses yeast genetic tools not available in human cell cultures to elucidate the mechanisms responsible for forodesine mediated toxicity to better understand its human cell toxicity. To elucidate the therapeutic mechanisms of forodesine, the genetic (gene-gene) interactions surrounding loss of the yeast drug target PNP1 were identified as functional interactions underlying forodesine toxicity. In addition, conditions required to conduct chemical genetic (gene-drug) screening were also investigated.

\subsection{Experimental procedures}

\subsubsection{Yeast strains, media components and compounds}

All S. cerevisiae strains used in this chapter were derived either from the haploid strains BY4742 (Brachmann et al., 1998) or Y7092 (Tong \& Boone, 2007) and were constructed by PCR mediated disruption or heterologous expression of human genes equilibrative nucleoside transporter 1 (ENT1) ((Vickers et al., 1999); gift of Carol E. Cass), deoxycytidine kinase (dCK) ((O’Konek et al., 2009); gift of Donna S. Shewach) and herpes simplex virus type-1 thymidine kinase (HSV-TK) ((O'Konek et al., 2009); gift of Donna S. Shewach) in plasmids. The haploid non-essential gene deletion mutant library ((Tong et al., 2001); gift of Charles Boone) was mated in en masse in the construction of genome-wide deletion libraries. All S. cerevisiae and E. coli strains were cultured in growth media as stated in Chapter 
2 unless otherwise specified. The PNP inhibitors forodesine, DADMe-immucillin G and immucillin H were kindly provided by Prof Gary Evans (Ferrier Institute, Victoria University of Wellington) and Cytarabine (AraC) (Sigma-Aldrich) were dissolved in DMSO at a concentration of $10 \mathrm{mM}$ and stored at $-20^{\circ} \mathrm{C}$.

\begin{tabular}{|c|c|c|}
\hline Strain & Genotype & Reference \\
\hline BY4743 & $\begin{array}{l}\text { MATa/a his } 3 \Delta 1 / \text { his } 3 \Delta 1 \text { leu } 2 \Delta 0 / \text { leu2 } \Delta 0 \\
\text { LYS2/lys } 2 \Delta 0 \text { met } 15 \Delta 0 / M E T 15 \\
\text { ura3 } \Delta 0 / \text { /ura3 } \Delta 0\end{array}$ & (Brachmann et al., 1998) \\
\hline Y7092 (wild-type) & 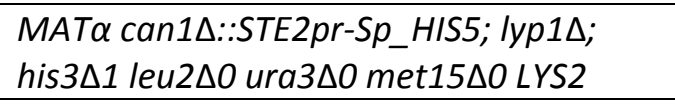 & (Tong \& Boone, 2007) \\
\hline BY4742 & $\begin{array}{l}\text { MATa his3 } \Delta 1 \text { leu2 } \Delta O \text { LYS2 met } 15 \Delta O \\
\text { ura3 } \Delta O\end{array}$ & (Brachmann et al., 1998) \\
\hline $\begin{array}{l}\text { pnp1 } \Delta \text { (Y7092 } \\
\text { background) }\end{array}$ & 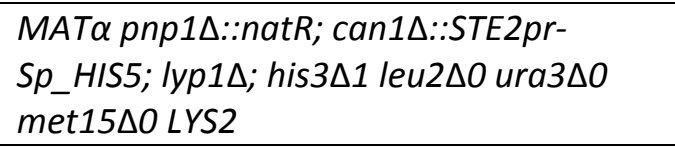 & This study \\
\hline $\begin{array}{l}\text { Y7092 + hENT1 + } \\
\text { dCK }\end{array}$ & 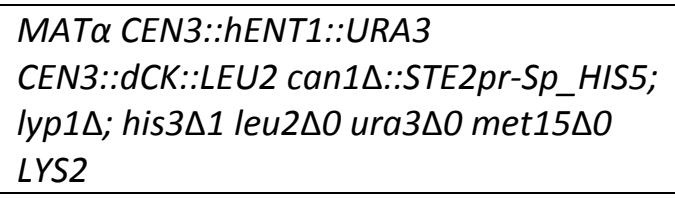 & This study \\
\hline $\begin{array}{l}\text { Y7092 + hENT1 + } \\
\text { HSV-TK }\end{array}$ & 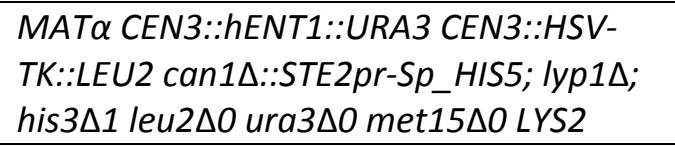 & This study \\
\hline $\begin{array}{l}\text { pnp1 } 1 \Delta+\mathrm{hENT} 1+ \\
\mathrm{dCK}\end{array}$ & 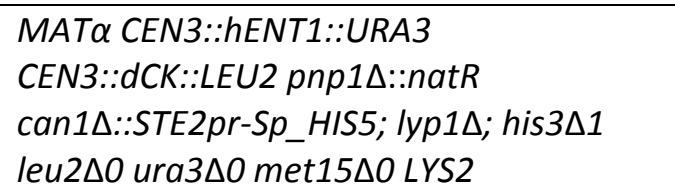 & This study \\
\hline $\begin{array}{l}\text { ade } 2 \Delta \text { ade } 2 \Delta+ \\
\text { hENT1 }+\mathrm{dCK}\end{array}$ & 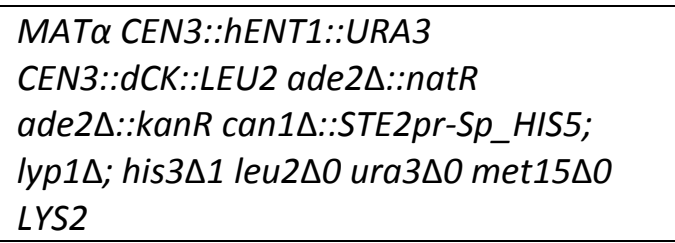 & This study \\
\hline $\begin{array}{l}x x x \Delta \text { (haploid } \\
\text { nonessential gene } \\
\text { deletion library) } \\
\end{array}$ & $\begin{array}{l}\text { MATa } x x x \Delta:: k a n R \text { his } 3 \Delta 1 \text { leu } 2 \Delta O \text { LYS2 } \\
\text { met15 } \Delta 0 \text { ura } \Delta \Delta O\end{array}$ & (Tong et al., 2001) \\
\hline $\begin{array}{l}\text { pnp1 } 1 \Delta x x \Delta \text { (double } \\
\text { mutants generated } \\
\text { in the } p n p 1 \Delta S G A)\end{array}$ & 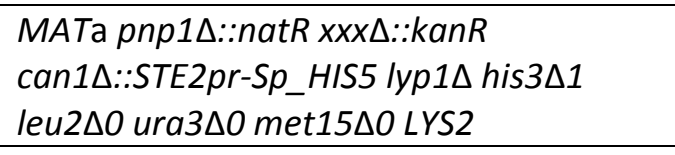 & This study \\
\hline $\begin{array}{l}\text { ura3 } \Delta \text { (Y7092 } \\
\text { background SGA } \\
\text { control strain) }\end{array}$ & 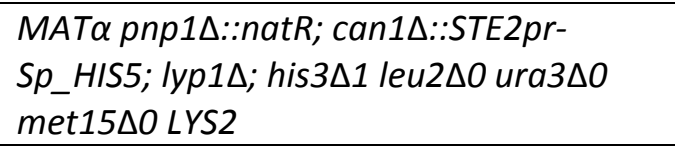 & (Bircham et al., 2011) \\
\hline $\begin{array}{l}\text { ura } 3 \Delta x x \Delta \text { (double } \\
\text { mutants generated } \\
\text { in the control mini } \\
\text { SGA) }\end{array}$ & 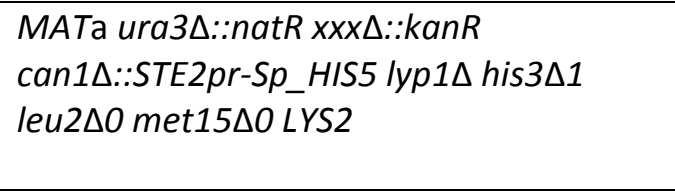 & This study \\
\hline $\begin{array}{l}\text { Y7092 + pBY011 } \\
\text { (CEN::URA3 control } \\
\text { strain) }\end{array}$ & 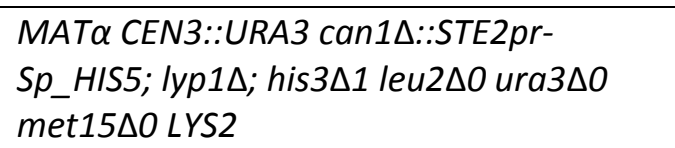 & This study \\
\hline
\end{tabular}




\begin{tabular}{|c|c|c|}
\hline $\begin{array}{l}x x x \Delta+p B Y 011 \\
\text { (control gene } \\
\text { deletion library used } \\
\text { in the minimal } \\
\text { media experiment) }\end{array}$ & 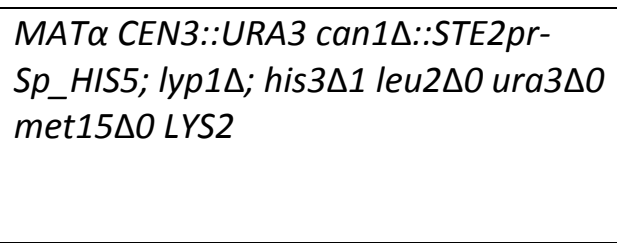 & This study \\
\hline
\end{tabular}

Table 4.2.1 - Yeast strains used in this chapter.

\subsubsection{PNP1 Synthetic Genetic Array (SGA)}

To investigate the genetic interactions with PNP, the pnp1 1 ::natR was constructed in yeast by PCR mediated disruption of the PNP1 gene with natMX4 selectable marker from p4339 plasmid (Goldstein \& McCusker, 1999) (Appendix Fig. 7.4.1; Appendix Table 7.4.1). The haploid yeast gene deletion library (Boone et al., 2007) was maintained in 1536 colony format on agar with each deletion strain in quadruplicate with the entire non-essential gene deletion library occupying 14 plates. This gene deletion library was mated en masse against the pnp1::natR deletion strain using the RoToR HDA replicator (Singer Instrument Co. Ltd.) according to SGA methodology (Tong et al., 2001). The haploid resulting were analysed with Gitter (Wagih \& Parts, 2014) and ScreenMill (Dittmar et al., 2010) previously described (section 2.2.3). Double gene deletion strains that showed a growth defect (growth $<30 \%, p<0.05$ ) compared to the parental haploid non-essential gene deletion library in at least two out of three SGAs were considered as putative epistatic interactions of PNP1 and were independently validated.

\subsubsection{Screening of the pnp1 $\Delta x x x \Delta$ deletion strains in minimal media}

To identify a media condition where $p n p 1 \Delta$ is essential, the $p n p 1 \Delta x x x \Delta$ double mutants were pinned onto minimal media supplemented with three essential amino acids (SD-N + Met/Ura/Leu + $2 \%$ glucose) and the ability to utilise de novo amino acid biosynthesis was assessed. The plates were incubated for $5 \mathrm{~d}$ at $30^{\circ} \mathrm{C}$ and colony sizes were assessed using Gitter tools (Wagih \& Parts, 2014) on RStudio v0.99.896 (Racine, 2012) and ScreenMill (Dittmar et al., 2010) against the WT control (Cen::URA3) SGA screened under the same conditions. Genes responsible for uracil biosynthesis were excluded from this analysis as these were only present in the Cen::URA3 control SGA. 


\subsubsection{Validation of PNP1 interactions}

To validate the putative epistatic interactions, the pnp1 $\Delta x x x \Delta$ double mutants were reconstructed by performing a mini SGA comprised of specific deletion strains. A control mini SGA was performed using ura3 $\Delta:: n a t R$ as a query strain to filter out mating and sporulation deficient deletion strains. The double mutants were assessed for growth defects in liquid 96-well plate growth assays as previously described in Chapter 2 (Section 2.2.4) using the parental haploid strains and Y7092 wildtype (WT) as controls. The epistatic growth defect of the double mutants was calculated relative to the growth defect of the pnp1 $\Delta$ single mutant $((p n p 1 \Delta x x x \Delta / p n p 1 \Delta) /(x x x \Delta / \mathrm{WT}))$ modified from the calculation used for assessment of drug sensitivity (Best et al., 2013). The relative growth of the double mutants to the single mutants was normalised against the control pnp1 $1 \Delta$ his $3 \Delta$ double deletion strain, which is phenotypically identical to pnp $1 \Delta$ single gene deletion strain that carries a partial deletion of the HIS3 gene. Stringent cut-offs of growth reduction ( $>40 \%$ growth in double mutant compared to single mutants, $p<0.05$ ) and growth improvement ( $>40 \%$ growth in double mutant compared to single mutants, $p<0.05)$ were used to determine significant changes in growth. The double gene deletion mutants with extreme growth defects $(<5 \%$ growth in double mutant compared to single mutants, $p$ $<0.05)$ were considered synthetic lethal interactions.

\subsubsection{Optimisation for forodesine chemical genetic analysis}

To achieve forodesine bioactivity (growth inhibition) in yeast, heterologous expression of human genes responsible for the uptake and metabolism of nucleosides was conducted. Plasmids expressing human genes hENT1 (pYhENT1), dCK (pTW382) and herpes simplex gene HSV-TK (pTW383) expression plasmids, and were introduced into the yeast strains using lithium acetate/single-stranded carrier DNA/PEG as previously described (Gietz \& Schiestl, 2007). The activity of hENT1 was validated with uptake of adenosine and 5'-methylthioadenosine in Chapter 2 (Section 2.3.2). The dCK and HSVTK activity was assessed in yeast liquid growth assays against cytarabine (AraC) in SD-Uracil $+2 \%$ raffinose(Raf) $+2 \%$ galactose(Gal) media. To identify a condition where PNP1 is conditionally essential, yeast strains WT+hENT1+hdCK, pnp1 $1+\mathrm{hENT} 1+\mathrm{hdCK}$, and ade2 $\triangle$ ade $3 \Delta+\mathrm{hENT} 1+\mathrm{hdCK}$ strains were 
assessed for growth in a range of synthetic dropout media conditions. Unless otherwise stated, all growth assays were performed in liquid in appropriate media supplemented with $2 \%$ galactose and $2 \%$ raffinose to induce the expression of hENT1 under the Gal1-10 prompter.

\subsection{Results}

\subsubsection{SGA analysis identifies genetic interactions with the yeast PNP1 target of forodesine}

Forodesine is an inhibitor of purine nucleoside phosphorylase (PNP) that was recently approved to treat recurrent and refectory peripheral T-cell lymphoma (http://www.mundipharma.com.sg/2017/03/30/mundipharma-wins-approval-antineoplastic-agentmundesine-treatment-relapsedrefractory-peripheral-t-cell-lymphoma-japan/, 2017). However, the mechanisms of action are not fully understood. Some mechanisms have been identified and these are the suspected mechanisms already previously associated with apoptosis (Balakrishnan et al., 2006; Balakrishnan et al., 2010a). To identify unsuspected mechanisms of action of forodesine, PNP1 (the yeast orthologue of human PNP) was used as a genetic mimic of forodesine treatment. Genome-wide representatives of $p n p 1 \Delta x x x \Delta$ double deletion meiotic progeny generated using $p n p 1 \Delta$ as a query against the nonessential gene deletion library by SGA methodology and evaluated for epistatic growth defect. Based on (growth $<30 \%, p<0.05$ ), 43 gene deletions conferred a significant reduction in growth of pnp1S (Fig 4.3.1a; Appendix Table 7.4.2a). To validate the putative PNP1 genetic interactions identified from the PNP1 SGA, the double mutants were reconstructed in a mini SGA comprised of 42 putatively interacting gene deletion strains and 112 additional functionally related gene deletion strains. The resulting 154 double mutants were evaluated for reduction in growth phenotype relative to the single deletion mutant parents and wildtype. Validations were performed in triplicate and in liquid media as the growth of a specific number of cells can be accurately measured by measuring turbidity in a spectrophotometer.

Using a stringent cut-off of $>40 \%$ growth reduction in double mutant compared to parental single mutants and WT $(p<0.05), 53$ gene deletion strains $(18 / 42$ from putative genetic interactions and $35 / 112$ functionally related genes) were considered to exacerbate the growth defect of pnp1 $\Delta$ 
(Fig. 4.3.2; Appendix Table 7.4.2b). These genes were assigned to cellular processes using the GO Slim mapping tool on SGD (Cherry et al., 2012) and were involved in amino acid metabolism, response to starvation, vacuole fusion, vesicle-mediated transport, carbohydrate metabolism, lipid homeostasis, chromatin organisation chromosome segregation, transcription, translation and other cellular processes. As proof-of-principle, the genes defective in amino acid metabolism (AMD1, AAH1 and $A D E 5,7)$ were all involved in purine salvage, and BAS1, a transcriptional regulator of genes involved in purine metabolism, helps explain molecular mechanisms of purine deficiency caused by PNP1 deletion (Daignan-Fornier \& Fink, 1992). PDC5, although involved in pyruvate degradation, also catalyses the catabolism of several amino acids including tryptophan (Dickinson et al., 2003). INO2 is required for growth in the absence of inosine and regulates the expression of genes in synthesis of phosphatidylcholine from phosphocholine including OPI3 (Kersting et al., 2004). VPS41/VAM2, VAM3, MON1 and CCZ1 are GTPase activating enzymes involved in regulation of YPT/Rab GTPase YPT7 that functions in endosome transport and autophagy (De Antoni et al., 2002; Plemel et al., 2011; Brett et al., 2008; Chen et al., 2014). Vps29 forms the cargo sorting complex with Vps26 and Vps35 which sorts cargo from endosome to trans-Golgi and is an effector of YPT7 (Liu et al., 2012).Several other genes involved in autophagy (YDR128W/MTC5, PHO5, IRS4 and ATG13) also showed a growth defect when PNP1 was co-deleted and are consistent with reduced autophagic activity observed for pnp1 1 in nitrogen starvation and with rapamycin treatment (Dengjel et al., 2012).

Deletion of PNP1 also exacerbated the growth defect of numerous genes that regulate gene expression in response to nutrient limitation at the levels of transcription and translation. DAL81 positively regulates nitrogen utilisation and promotes the expression of genes involved in catabolism of poor nitrogen sources such as leucine, $\gamma$-aminobutyric acid and allantoin and also the expression genes involved in amino acid uptake in nitrogen limited conditions (Palavecino et al., 2015). GCR2 (overlaps YNL198Cis required for transcriptional initiation of glycolytic genes (Uemura \& Fraenkel, 1990). KNS1 is a protein kinase that negatively regulates RNA Pol III and an effector of the TOR pathway (Lee et al., 2012). RTT103 (overlaps YDR290W) regulates transcription termination from RNA Pol II 
(Kim et al., 2004). YKE2 is a subunit of the prefoldin complex that is recruited to chromatin and facilitates transcriptional elongation by RNA Pol II (Millan-Zambrano et al., 2013). In addition, translation elongation factor TEF4 also showed an exacerbated growth defect with the pnp1 $\Delta$ deletion. SNF5 is subunit of the SWI/SNF chromatin remodelling complex that is involved in transcriptional regulation. Other genes were also involved in ribosomal function (RPS19A, RPL22A, $R P S 6 A, R P L 9 A, B U D 21)$, chromatin remodelling including histone acetyl transferases (EAF1, UME6 and TOS4 (adjacent YLR184W), nucleosome and histone assembly (HIR2 and HTZ1 and SNF5), DNA repair (CST9, RAD4), mitochondrial maintenance and inheritance (RIM1 and PTC1) and ATPases (VPH1, PMA2).

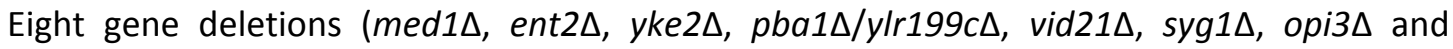
vam $3 \Delta)$ conferred extreme growth defects in pnp1 $\Delta(<5 \%$ growth in double mutant compared to parental single mutants, $p<0.05$ ) and were identified as synthetic lethal interactions (Appendix Table 7.4.2b). In addition, 21 additional double deletion strains were inviable (11/53 putative genetic interactions) but were also inviable in the control SGA and were thus excluded as these were deficient in either the mating or sporulation steps of the SGA procedure. Five gene deletion strains (ymr085w $\Delta$, $\operatorname{rim} 13 \Delta, y n / 296 w \Delta, y 0 r 082 c \Delta$ and $h s t 3 \Delta$ ) conferred a growth improvement in pnp1 $\Delta$ (>40\% growth in double mutant compared to parental single mutants, $p<0.05$ ). Of these MON2 (overlaps YNL296W) negatively regulates another GTPase $A R L 1$, involved in membrane trafficking and trans-Golgi organisation. HST3 encoded $\mathrm{NAD}^{+}$-dependent protein deacetylases involved in gene silencing. WHI5 (overlaps YOR082) that encodes G1/S phase transcription repressor. YMR084W-YMR085W fused open reading frame encode a paralogue to GFA1 that involved in in the initial step of chitin synthesis. RIM13 involved in proteolytic activation of Rim101 in response to alkaline $\mathrm{pH}$. 
a

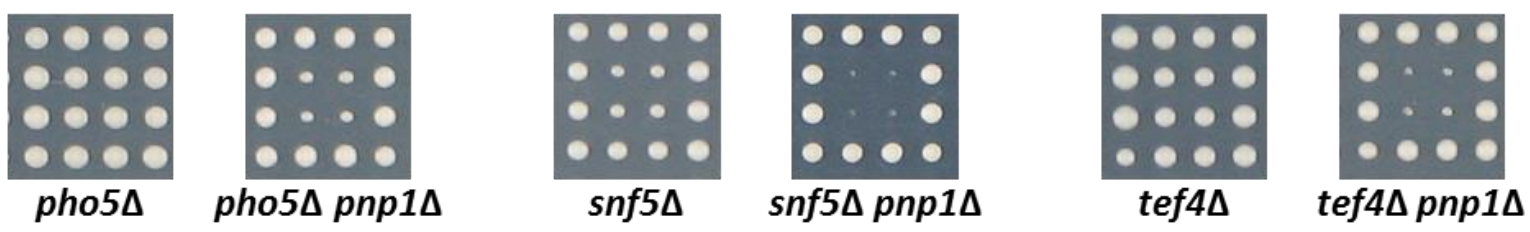

b

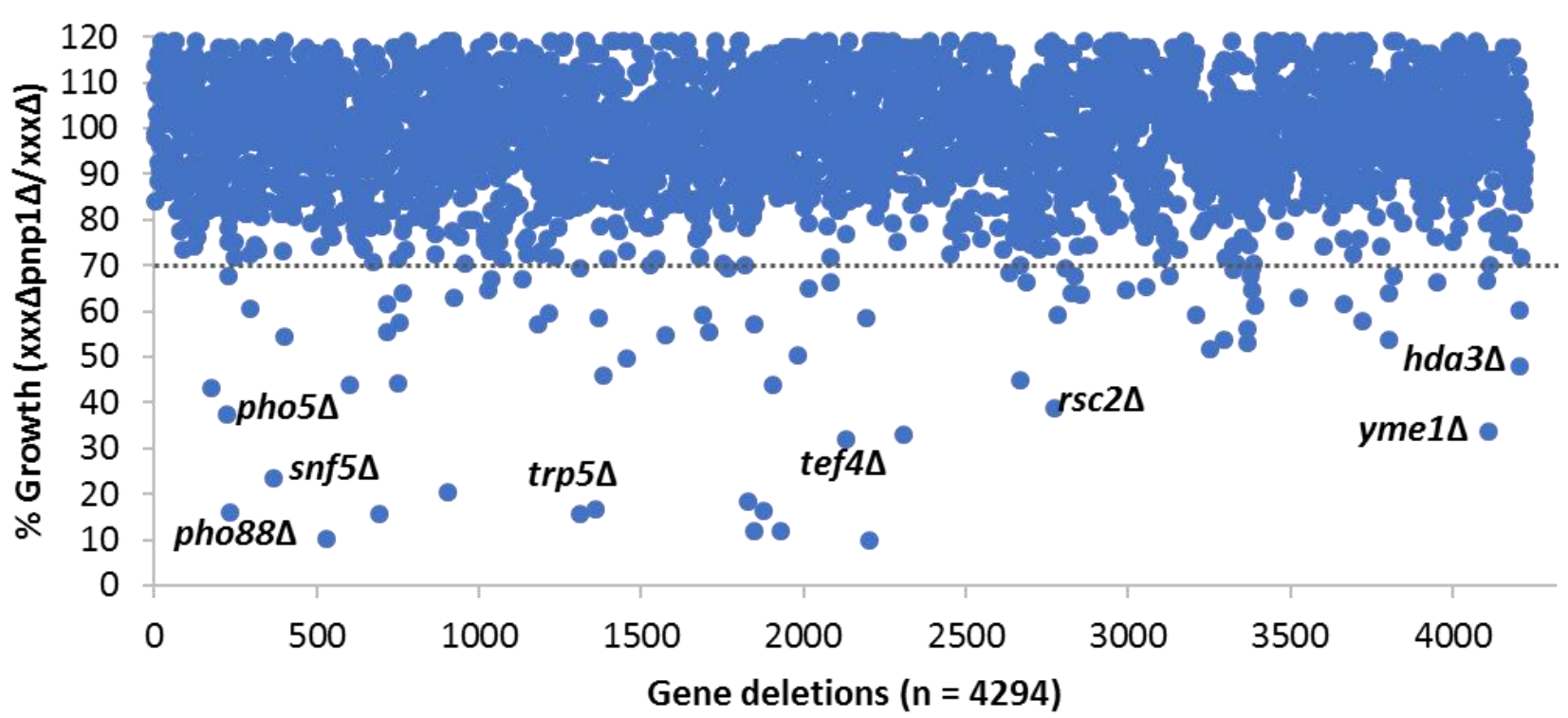

Figure 4.3.1a - Growth of gene deletion strains impacted by PNP1 deletion. (a) Growth of double $x x x \Delta p n p 1 \Delta$ and single $x x x \Delta$ in technical quadruplicate assessed on agar. (b) Percent growth of 4,294 $x x x \Delta$ meu1 $\Delta$ deletion mutants relative to $x x x \Delta$ from biological triplicate of technical quadruplicate experiments. Three independent SGAs were performed using pnp1 $\Delta$ query strain agar using the RoToR HDA (Singer Instrument Co. Ltd) and mated against the haploid single gene deletion library en masse to according to SGA methodology (Tong et al., 2001). The haploid pnp1 $\Delta x x x \Delta$ double mutant meiotic progeny were assessed for epistatic colony size relative to the haploid parental mutants using Gitter tools (Wagih \& Parts, 2014) in RStudio v0.99.896 (Racine, 2012) and ScreenMill (Dittmar et al., 2010). 


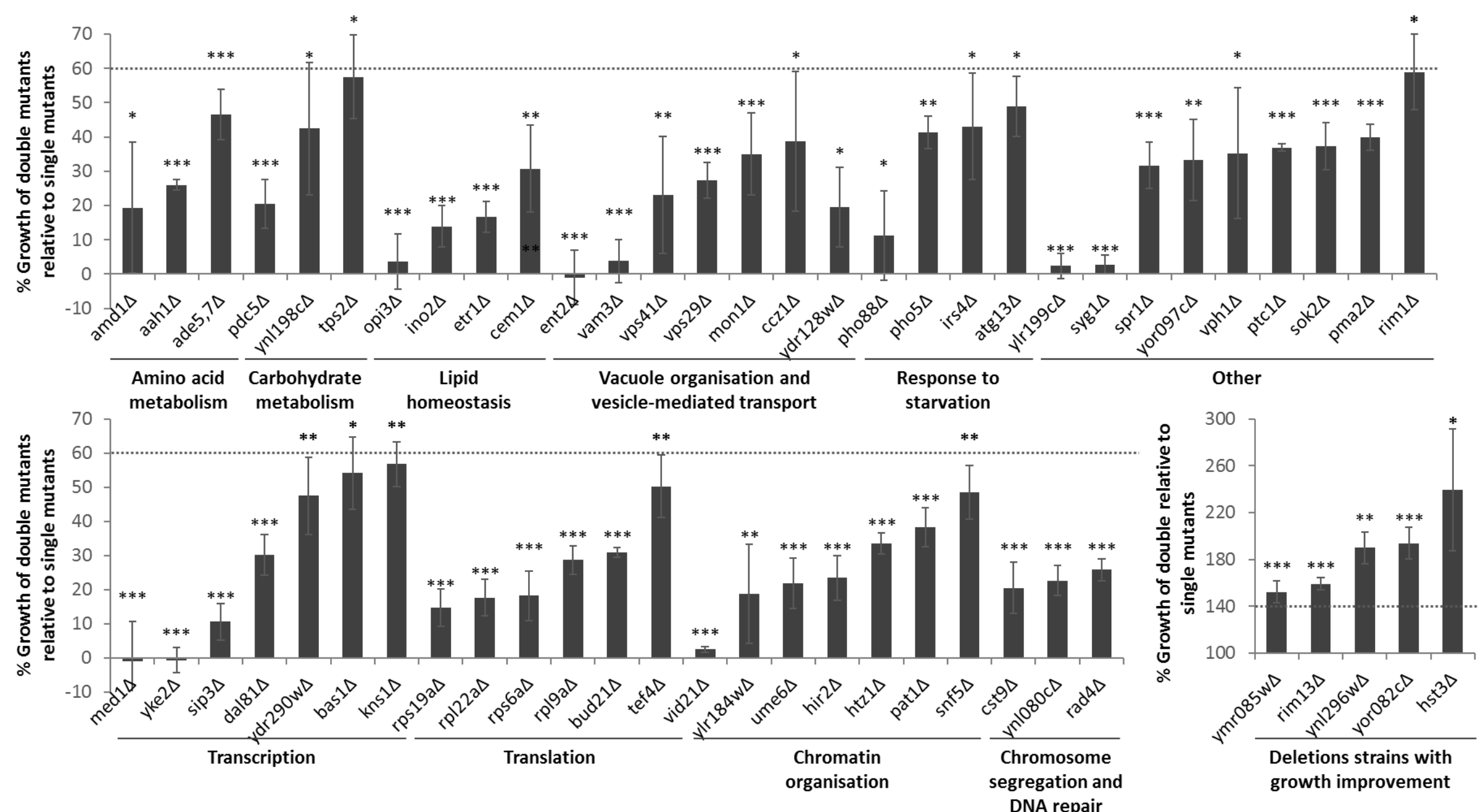

Fig. 4.3.1b- 53 negative and 5 positive genetic interactions with PNP1. The growth of the double mutants relative to the single haploid parental strains ( $p n p 1 \Delta x x x \Delta$ /pnp1 $\Delta) /(x x x \Delta /$ WT $) \times 100$ was determined in liquid media at $O D^{600}$ between 0.3-0.5 for single mutant $(x x x \Delta)$ control. The growth of the double mutants was normalised to $p n p 1 \Delta h i s 3 \Delta$ control strain and the double deletion mutants with an average growth $> \pm 40 \%, p<0.05$ of the pnp1 $\Delta h i s 3 \Delta$ strain were considered a growth defect or a growth improvement. 


\subsubsection{Minimal media identifies conditions where PNP1 is essential}

The $\mathrm{IC}_{10-20}$ of compounds in WT cells are required for screening of gene deletions mutants for hypersensitivity and growth inhibition requires a perturbation of an essential target or essential macromolecule in cells (Parsons et al., 2004; Cong et al., 2012). PNP1 in yeast encodes a non-essential protein and, we sought to make a PNP1 conditionally essential by screening the $\sim 4200$ haploid pnp1 $1 x x x \Delta$ double deletion strains generated by SGA methodology on minimal media supplemented with the essential amino acids leucine, histidine, and uracil. Based on (growth $> \pm 30 \%$ treated compared to untreated, $p<0.05)$ criteria, the deletion of 47 genes significantly altered the growth of pnp1 $\Delta$ relative to the $x x x \Delta$ single gene deletions strains and included 24 growth defects and 23 improvements (Fig. 4.3.2a-b; Appendix Table 7.4.3). The deletion of genes involved in chorismite metabolism (ARO7, PHA2, TRP1, TRP2), inosine monophosphate metabolism (ADE2, ADE4, ADE8) and threonine biosynthesis (THR1) were affected by deletion of pnp1 $\Delta$ and included growth defects and improvements. Chorismite is a precursor for the syntheses of aromatic amino acids and the branch point in the shikimate pathway that precedes synthesis of phenylalanine, tyrosine, and tryptophan (Braus, 1991). Likewise, the deletion of genes required for the synthesis of phenylalanine and tyrosine show a growth improvement in the pnp1s deletion strain while deletion of genes in tryptophan

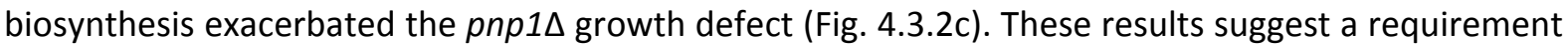
of tryptophan in the absence of PNP1. In contrast, deletions of genes involved in inosine monophosphate metabolism were non-informative as their growth was severely impaired in the absence of adenine independent of PNP1 while only THR1 of the threonine biosynthesis pathway

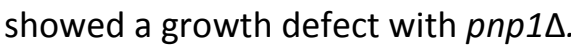


a
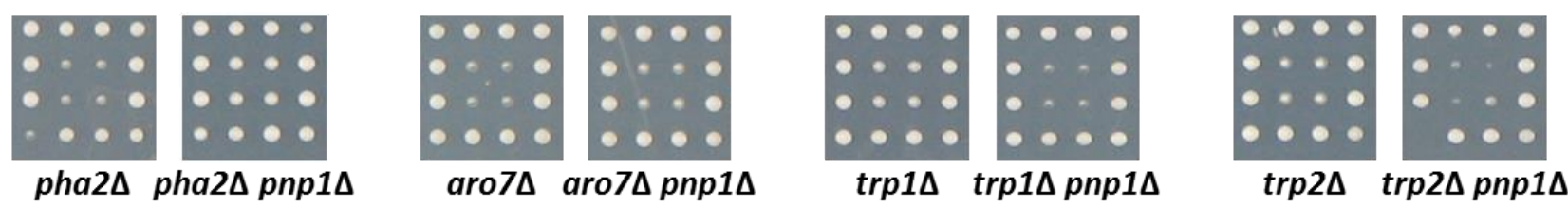

b
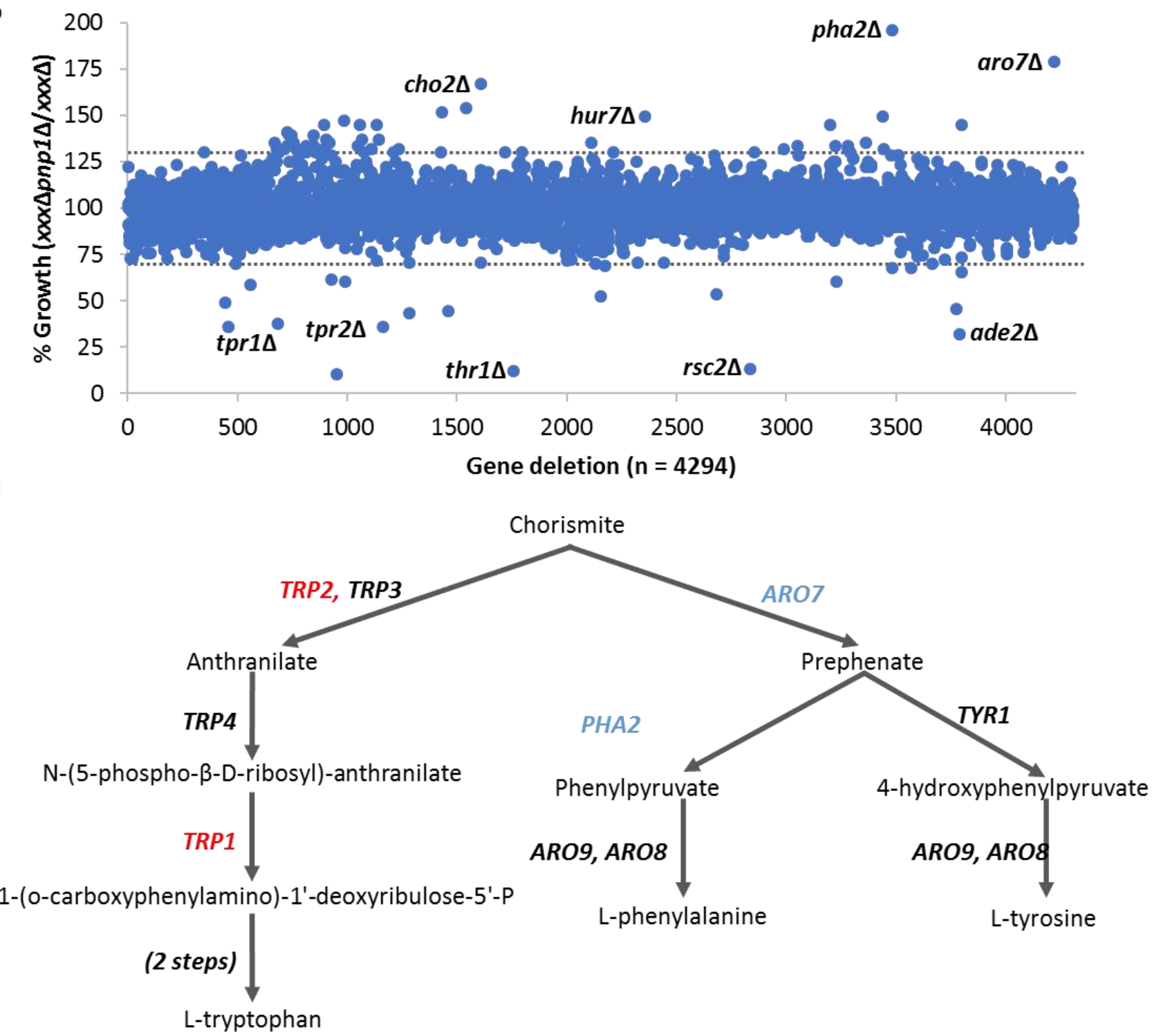

Figure 4.3.2 - Tryptophan biosynthesis from chorismate is required for growth in the absence of PNP1 in minimal media. (a) Growth of double $x x x \Delta p n p 1 \Delta$ and single $x x x \Delta$ in technical quadruplicate assessed on agar. (b) Percent growth of 4,286 $x x x \Delta p n p 1 \Delta$ deletion mutants relative to $x x x \Delta$ from technical quadruplicate experiments. (c) Simplified shikimate pathway. The pnp1 $x x x x \Delta$ double mutants and the $x x x \Delta$ parental haploid mutants were pinned onto minimal media supplemented with the essential amino acids methionine, leucine and uracil using the RoToR HDA (Singer Instrument Co. 
Ltd) and were incubated for $5 \mathrm{~d}$ at $30^{\circ} \mathrm{C}$. Deletions of genes labelled red in the simplified shikimate pathway reduced growth of $p n p 1 \Delta$ and deletion of genes labelled in blue improved the growth $p n p 1 \Delta$. 4.3.4 PNP1 inhibitors are variably potent in yeast due to non-essential functionality of PNP1

To evaluate the bioactivity of PNP1 inhibitors, forodesine (inosine analogue) and DADMeImmucillin G (DIG) (guanosine analogue) were screened against the WT+hENT1 strain. Forodesine was inactive in yeast at concentrations up to $1 \mathrm{mM}$ (the highest concentration tested) (Fig. 4.3.3a). DIG conferred $13 \%$ growth defect at $316 \mu \mathrm{M}$ and a $68 \%$ growth defect at $1 \mathrm{mM}$, As these compounds are low nanomolar inhibitors of human T cell leukemia cell lines MOLT-4 and CCRF-CEM (forodesine IC 50 $=0.1-10 \mathrm{nM}$ with $20 \mu \mathrm{M}$ deoxyguanosine (Kicska et al., 2001), the potent bioactivity at $1 \mathrm{mM}$ only with DIG was likely due to secondary target effects. Under the same conditions, the loss of PNP1 is nonessential (Fig. 4.3.3b), indicating that the activity of DIG is not mediated through PNP1 inhibition. A minute but significant growth defect was observed for pnp1 $\Delta$ at saturation (>36 hours) compared with WT. Therefore, DIG was excluded from consideration as a PNP1 inhibitor in subsequent experiments.

To determine whether absence of growth inhibition by forodesine was due to lack of forodesine transport, the ability of forodesine to competitively inhibit the uptake of nucleosides adenosine and cordycepin via hENT1 was assessed. Both compounds are transported by hENT1 as previously shown in Chapter 2 section 2.3.2. The growth of ade2 $\Delta$ ade3 $\Delta+\mathrm{hENT1}$ with adenosine in the absence of exogenous adenine and the growth WT+hENT1 under cordycepin treatment was assessed following forodesine co-treatment. A forodesine concentration-dependent decrease in growth $\left(\mathrm{IC}_{20}=\right.$ $0.8 \mu \mathrm{M}$ and $\mathrm{IC}_{40}=100 \mu \mathrm{M}$ ) was observed in the ade2 $\Delta$ ade $3 \Delta+\mathrm{hENT} 1$ strain cultured in $250 \mu \mathrm{M}$ adenosine supplemented media. In contrast, the ade2 $\Delta$ ade $3 \Delta+\mathrm{hENT1}$ strain in $250 \mu \mathrm{M}$ adenine supplemented media showed no growth inhibition with forodesine (Fig. 4.3.3c) and was similar to the WT+hENT1 strain treated with forodesine in synthetic complete media (Fig. 4.3.3a). The WT+hENT1 strain treated with semi-inhibitory concentrations of cordycepin showed a growth improvement when co-treated with forodesine (Fig. 4.3.3d). As expected, growth improvement was more pronounced at 

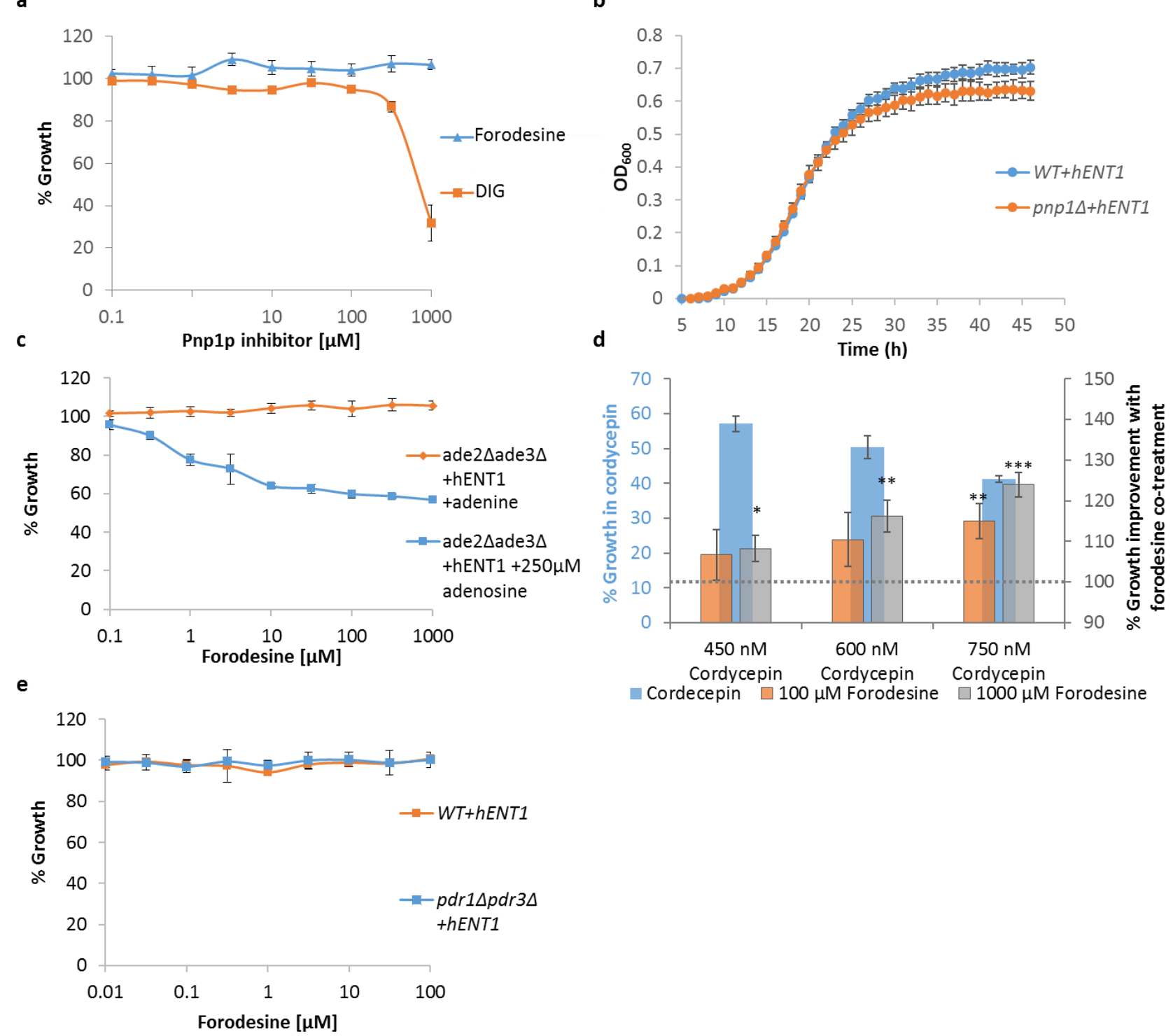

Figure 4.3.3 - Forodesine treatment or loss of PNP1 does not cause a growth defect in yeast. (a) Bioactivity of DADMe-Immucillin G (DIG) and forodesine evaluated in the WT+hENT1 strain. Percent growth $\left(\mathrm{OD}_{600} \mathrm{Exp} / \mathrm{OD}_{600}\right.$ vehicle control $\left.\mathrm{x} 100\right)$ was determined in mid log cultures $\left(0.3-0.5 \mathrm{OD}_{600}\right.$ units

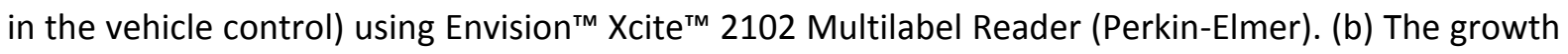
of the WT+hENT1 and pnp1 $1+\mathrm{hENT1}$. (c) ade2 $\triangle$ ade $3 \Delta+\mathrm{hENT} 1$ strain treated with forodesine and 250 $\mu \mathrm{M}$ adenine or $250 \mu \mathrm{M}$ adenosine. (d) WT+hENT1 strain co-treated with toxic nucleoside analogue cordycepin and 100 or $1000 \mu \mathrm{M}$ forodesine. The percent growth inhibition by cordycepin in the absence of forodesine is shown in blue. The percent growth improvement $\left(\mathrm{OD}_{600} \mathrm{Cor}+\mathrm{For} / \mathrm{OD}_{600} \mathrm{Cor}\right.$ control $\times 100)$ with forodesine treatment shown in orange (100 $\mu \mathrm{M}$ forodesine) and grey (1000 $\mu \mathrm{M}$ forodesine). (e) WT+hENT1 and pdr1 1 pdr3 $3+\mathrm{hENT1}$ with forodesine. 
higher concentrations of both cordycepin and forodesine due to saturation of the hENT1 transporter. This is also consistent with the $\sim 85$-fold increase in $\mathrm{IC}_{50}$ of cordycepin with expression of the hENT1 in WT (Chapter 2 section 2.3.2). Together, the results with adenosine and cordycepin confirm that forodesine enters the cells via the hENT1 transporter, although forodesine is not growth inhibitory.

To determine if the secondary effects observed with DIG are also present for forodesine but masked by the highly-regulated drug efflux mechanisms in yeast, a pleotropic drug response (PDR) attenuated strain (Coorey et al., 2015) expressing hENT1 ( $p d r 1 \Delta p d r 3 \Delta+\mathrm{hENT1}$ ) was evaluated for growth inhibition by forodesine. No significant difference in bioactivity was observed with various forodesine concentrations in the pdr1 $1 p d r 3 \Delta+h E N T 1$ strain compared to the WT+hENT1 strain (Fig. 4.3.3e). This indicates that the lack of growth inhibition is not due to PDR-network mediated efflux of forodesine, and moreover that secondary target effects observed for DIG is absent for forodesine. The mechanisms of forodesine are likely mediated through the non-essential PNP1 in yeast that needs to be made conditionally essential in order to investigate its therapeutic mechanism.

\subsubsection{Forodesine sensitivity is increased with deoxycytidine and deoxyguanosine}

Increased expression of dCK is necessary for PNP inhibition mediated T-cell toxicity and dCK is absent in lysates of forodesine-resistant human leukaemia cells (Ullman et al., 1979; Osborne \& Scott, 1983; Huang et al., 2008). To achieve PNP1 inhibition-facilitated growth inhibition in yeast, WT+hENT1 and pnp1 $1 \Delta+h E N T 1$ strains were transformed with the yeast expression plasmid pTW382 (O'Konek et al., 2009) expressing the human dCK gene. Cytarabine, a toxic analogue of 2 'deoxycytidine, is converted to cytosine arabinoside triphosphate by dCK inducing cytotoxicity (Verschuur et al., 2001). The dCK activity was thus assessed by screening against cytarabine. Cytarabine conferred $19 \%$ growth inhibition in WT+hENT1+dCK at $100 \mathrm{nM}$ (the lowest concentration tested), and completely inhibitory at concentrations $\geq 3.1 \mu \mathrm{M}$ (Fig. 4.3.4a). In contrast, cytarabine was inactive in the absence of dCK at all concentrations between $100 \mathrm{nM}-1 \mathrm{mM}$ in both WT and WT+hENT1. 
Following the conformation of dCK function in yeast, the mechanism by which forodesine causes T-cell toxicity was investigated in yeast. The bioactivity of PNP1 inhibitor forodesine and nucleosides deoxyguanosine and deoxyadenosine were assed in WT, WT+hENT1 and WT+hENT1+dCK strains. As expected forodesine was inactive in the WT in the absence of the hENT1 required for forodesine transport (Fig. 4.3.4b). A small (7\%) reduction in growth was observed with $10 \mu \mathrm{M}$ forodesine in the presence of the hENT1 transporter and it was further exacerbated (16\%) with expression of human dCK. Similarly, deoxyguanosine was inactive in WT in the absence of hENT1 from 0.063-1 $\mathrm{mM}$ and active and inhibiting growth when hENT1 was expressed $\left(\mathrm{IC}_{20} \sim 656.25 \mu \mathrm{M}\right)$ (Fig. 4.3.4c). The growth inhibition by deoxyguanosine was significantly increased in the WT+hENT1+dCK strain $\left(\mathrm{IC}_{20} \sim 93.75 \mu \mathrm{M}\right)$ but only reached $\mathrm{IC}_{50}$ at $1 \mathrm{mM}$. The growth inhibition by both forodesine and deoxyguanosine observed in the absence of human dCK expression is therefore likely mediated through a yet uncharacterised deoxyguanosine kinase in S. cerevisiae with a lower catalytic efficiency than the human dCK. The closest fungal orthologs of human $\mathrm{dCK}$ is encoded by a hypothetical protein GLOINDRAFT_331615 in Rhizophagus irregulars (23\% identities and 46\% similarities) and CCR4-NOT transcription complex subunit 3 of Cryptococcus neoformans (26\% identities and $47 \%$ similarities), likewise, the hypothetical protein GLOINDRAFT_331615 (28\% identities and $46 \%$ similarities) is also the closest fungal orthologue of human dGK and are distant orthologue (Balakrishnan et al., 2005). GLOINDRAFT_331615 shares minor homology (similarities 21-23\%) with 50 proteins in S. cerevisiae with unshared function that do not possess kinase activity.

The yeast strains WT+hENT1+dCK and pnp1 $+\mathrm{hENT1+dCK}$ were assessed for growth inhibition by deoxyguanosine and forodesine. Upon deletion of the drug target of forodesine, the pnp1 $\Delta$ deletion mutant showed only slight increase in sensitivity to deoxyguanosine $\left(\mathrm{IC}_{20} \sim 93.75 \mu \mathrm{M}, \mathrm{IC}_{50} \sim 593.75 \mu \mathrm{M}\right)$ compared to WT $\left(\mathrm{IC}_{20} \sim 93.75, \mathrm{IC}_{50}>1000 \mu \mathrm{M}\right)$ (Fig. 4.3.4d). The growth defect of WT+hENT1+dCK in forodesine was exacerbated when co-treated with $250 \mu \mathrm{M}$ deoxyguanosine $\left(\mathrm{IC}_{20} \sim 5 \mu \mathrm{M}\right)$ and this growth defect was negated when PNP1 was deleted (Fig. 4.3.4e). A slight improvement in growth was 

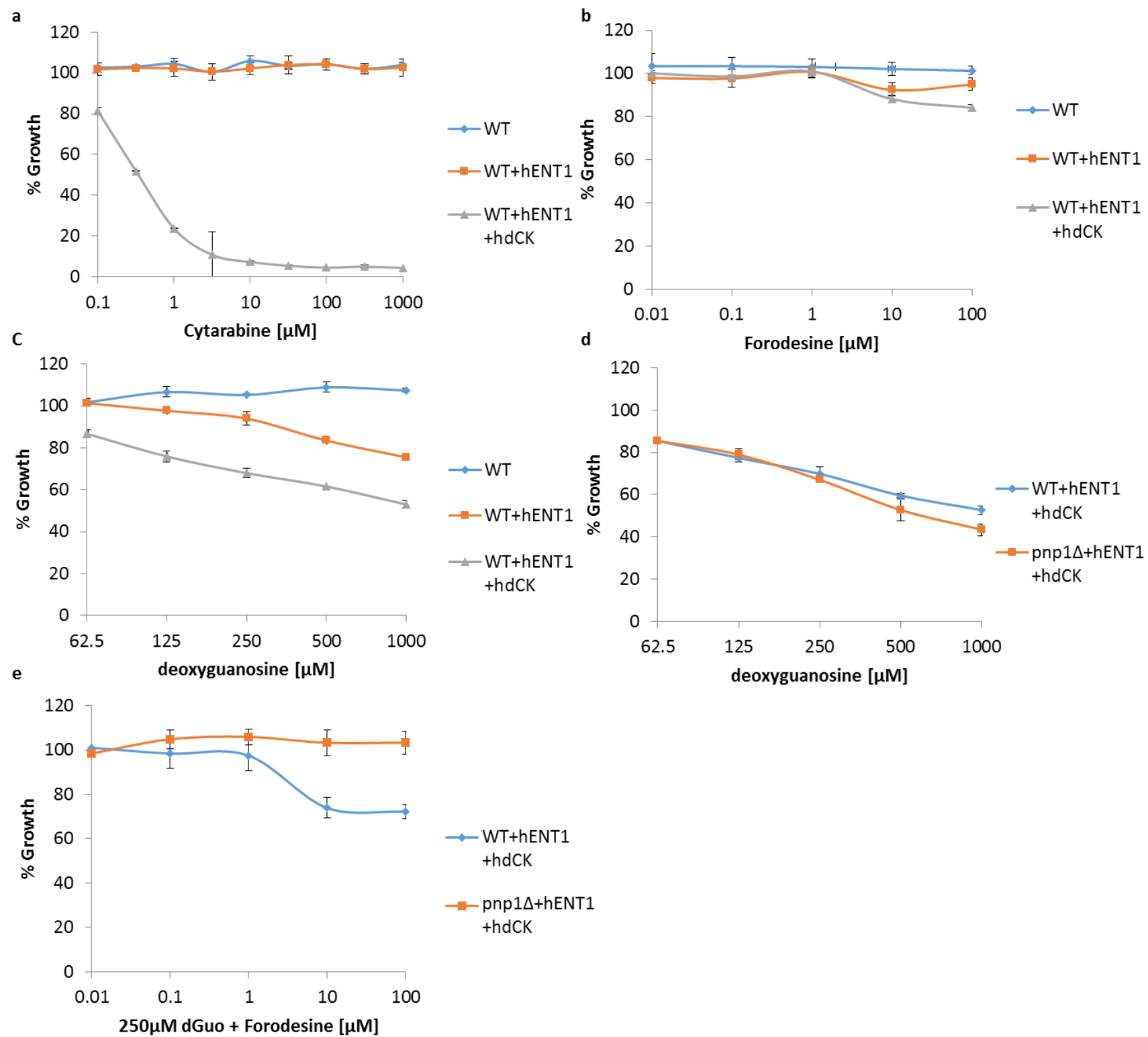

Figure 4.3.4 - dCK expression improves growth inhibition by forodesine, and pnp1 $\Delta$ confers resistance. Growth assays demonstrating the bioactivity of cytarabine, forodesine and 2'deoxyguanosine (dGuo) in yeast strains WT and pnp1L, expressing the human equilibrative nucleoside transporter (hENT1) and the human deoxycytidine kinase (hdCK). (a) Cytarabine in WT, WT+hENT1, WT+hENT1+dCK. (b) Forodesine. (c) dGuo. (d) dGuo in WT+hENT1+hdCK and pnp1D hENT1+hdCK strains. (f) $250 \mu \mathrm{M}$ dGuo+ forodesine. \% growth $\left(\mathrm{OD}_{600} \mathrm{Exp} / \mathrm{OD}_{600}\right.$ Vehical control $\left.\mathrm{x} 100\right)$ was determined in mid-log cultures $\left(0.3-0.5 \mathrm{OD}_{600}\right.$ units in the vehicle control) using Envision ${ }^{\mathrm{TM}} \mathrm{Xcite}^{\mathrm{TM}} 2102$ Multilabel Reader (Perkin-Elmer). Standard deviation from triplicate experiments are presented. 
observed at the higher doses of forodesine for $p n p 1 \Delta$, albeit this is likely due to saturation of the hENT1 transporter by forodesine leading to a reduction in transport of deoxyguanosine. Overall, these results suggest that the growth inhibition by forodesine is mediated through PNP1.

\subsubsection{Mitochondrial accumulation of dGTP is insufficient to inhibit yeast growth}

To further improve on the toxicity induced by PNP1 inhibition or deletion, the herpes simplex virus type-1 thymidine kinase (HSV-TK) was expressed in the WT+hENT1 strain in the absence of dCK. HSV-TK is a human TK-2 related kinase with broad substrate specificity and phosphorylates various guanosine analogues including ganciclovir (GCV) (O'Konek et al., 2009). Given that heterologous expression of HSV-TK in mice liver accumulates phosphorylated GCV in the mitochondria (van der Eb et al., 2003) (Herraiz et al., 2003) and that deoxyguanosine was previously shown to be a substrate HSV-TK (Fyfe et al., 1978), we sought to evaluate the contribution of the mitochondrial deoxyguanosine kinase activity in response to a treatment of forodesine and $250 \mu \mathrm{M}$ deoxyguanosine (Fig. 4.3.5a\&b). The expression of HSV-TK was confirmed by phosphorylation cytarabine to toxic AraC triphosphate by HSV-TK. WT expressing HSV-TK showed growth inhibition (7\% at $100 \mu \mathrm{M}$ and $53 \%$ at $1 \mathrm{mM}$ ) to cytarabine whilst, WT and WT+hENT1 strains showed on growth inhibition to cytarabine treatment from 100 nM- 1 mM. (Fig. 4.3.5a). The expression of HSV-TK in WT+hENT1 strain resulted in increased sensitivity to deoxyguanosine with $100 \mathrm{nM}$ forodesine (21\% growth inhibition) compared to basal inhibition in the WT+hENT1 strain (13\% growth inhibition) (Fig. 4.3.5b). The treatment of WT+HSV-TK with deoxyguanosine alone showed similar growth inhibition to WT $10 \%$ in WT+hENT1+HSV-TK and $9 \%$ in WT+hENT1). However, dCK in WT showed more sensitivity to deoxyguanosine with $100 \mathrm{nM}$ forodesine (37\% inhibition) and deoxyguanosine alone (inhibition $28 \%$ than the expression of cytosolic dCK. (Fig. 4.3.5b). From these results, it cannot be excluded that mitochondrial accumulation of dGMP is not a significant contributor to the growth inhibition by forodesine. 


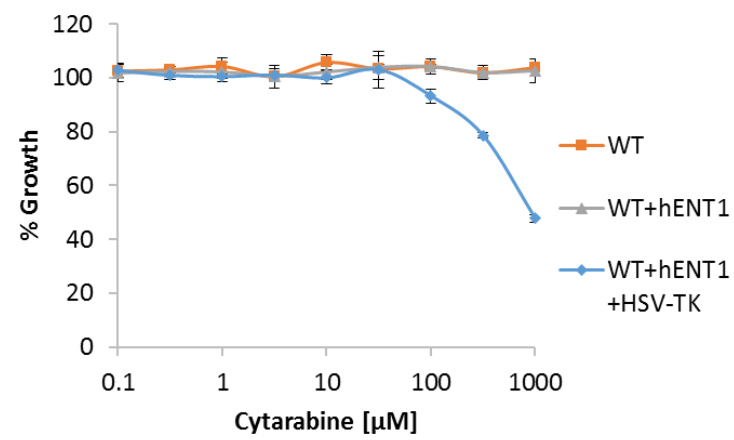

b

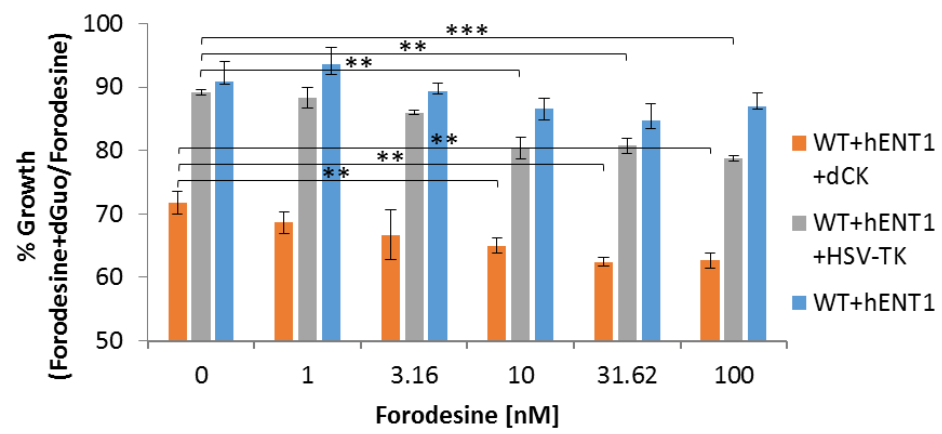

Figure 4.3.5 - Expression of HSV-TK exacerbates the growth inhibition by deoxyguanosine and forodesine. (a) WT, WT+hENT1 and WT+hENT1+HSV-TK against cytarabine. (b) WT+hENT1, WT+hENT1+dCK and WT+hENT1+HSV-TK against $500 \mu \mathrm{M}$ dGuo and forodesine relative to forodesine alone. Triplicate growth assays were performed in liquid media at $30^{\circ} \mathrm{C}$ for WT cells expressing human equilibrative nucleoside transporter (hENT1) and human deoxycytidine kinase (dCK) or herpes simplex viral thymidine kinase (HSV-TK). Percent Growth $\left(\mathrm{OD}_{600} \mathrm{Exp} / \mathrm{OD}_{600}\right.$ Vehical control $\left.\mathrm{x} 100\right)$ was determined in mid-log cultures (0.3-0.5 $\mathrm{OD}_{600}$ units in the vehicle control) using Envision ${ }^{\text {TM }}$ Xcite $^{\text {TM }} 2102$ Multilabel Reader (Perkin-Elmer).

\subsubsection{MTA exacerbates the forodesine growth defect}

Forodesine and deoxyguanosine treatment in T-cells lead to increased accumulation of dGTP (154-fold) and dATP (8-fold) (Bantia et al., 2003) although phosphorylation of adenosine and deoxyadenosine is catalysed by adenosine kinase an enzyme conserved in yeast (Lecoq et al., 2001a). As the growth inhibition by forodesine and deoxyguanosine was reversed by deoxycytidine without a reduction in dATP accumulation (Bantia et al., 2003), the disruptions to the adenine salvage pathways

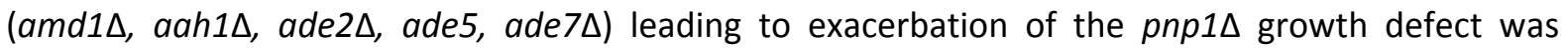
unexpected. To investigate this further and attempt to make PNP1 essential, the growth defect of WT and pnp1s was assessed against deoxyadenosine or 5'-methylthioadenosine (MTA) with deoxyguanosine and forodesine. Treatment of WT+hENT1+dCK with deoxyadenosine showed sensitivities similar to those previously observed for deoxyguanosine $\left(\sim C_{50} 900 \mu \mathrm{M}\right)$ and deletion of PNP1 showed only slight exacerbation in sensitivity $\left(\sim \mathrm{IC}_{50} 500 \mu \mathrm{M}\right)$ to deoxyadenosine (Fig. 4.3.6a). In contrast, the ade2 $\Delta a d e 3 \Delta$ double mutant showed resistance to deoxyadenosine (39\% growth 
inhibition at $1 \mathrm{mM}$ ), which is likely a consequence of adenosine and adenine being generated from deoxyadenosine catabolism.

The treatment of WT with deoxyguanosine plus forodesine with MTA, in the absence of exogenous adenine or methionine, showed $1 \%, 68 \%$ and $94 \%$ growth inhibition $1 \mu \mathrm{M} 10 \mu \mathrm{M}$ and 100 $\mu \mathrm{M}$, respectively (Fig. 4.3.6b). This, however, was at micromolar ( $\left.{ }^{\prime} C_{50} 6 \mu \mathrm{M}\right)$ concentrations of forodesine compared with MTDIA (in Chapter 2 section 2.3.3) which was inhibitory at sub pico/low

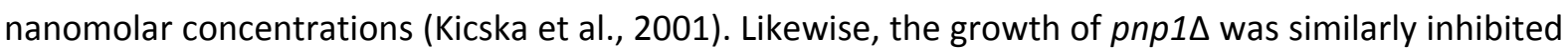
although it was less sensitive to forodesine with deoxyguanosine than WT (Fig. 4.3.6b), which was not expected as the deletion of PNP1 was expected to reverse the growth defect induced by forodesine with deoxyguanosine and MTA. The sensitivity of pnp1D to forodesine suggests that the growth inhibition observed under these conditions was not solely mediated by PNP1 inhibition. WT+hENT1 treated with forodesine with MTA in the absence of methionine and cystine resulted in growth inhibition (in the absence of dCK or deoxyguanosine) due to decreased the MTA uptake (Fig. 4.3.6c), and is consistent with the pnp1 $1 \Delta$ inhibition observed for deoxyguanosine with forodesine with MTA. These observations suggest that forodesine-mediated PNP1 inhibition achieved with MTA is a consequence of hENT1 saturation and the inability to transport MTA. Therefore, MTA was no longer used in subsequent experiments in combination with forodesine.

The growth inhibition by forodesine with deoxyguanosine was also assessed in adenosine supplemented media in the absence of exogenous adenine (Fig. 4.3.6c). WT and pnp1 $\Delta$ were resistant to forodesine with deoxyguanosine when treated with adenosine (WT+hENT1+dCK, while ade $2 \Delta$ ade $3 \Delta$ exhibited minor sensitivity to forodesine with deoxyguanosine ( $18 \%$ inhibition with 100 $\mu \mathrm{M}$ forodesine and $250 \mu \mathrm{M}$ deoxyguanosine) (Fig. 4.3.6d). The resistance to deoxyguanosine in WT and $p n p 1 \Delta$ was likely a result of adenosine competing with the deoxyguanosine kinase activity by $\mathrm{dCK}$ and the uptake of forodesine and deoxyguanosine by hENT1. The growth reduction in ade2 $\triangle$ ade $3 \Delta$ at 
higher concentrations of forodesine is likely a result of hENT1 saturation, which results in reduced uptake of adenosine required by the ade2 $\Delta a d e 3 \Delta$ (adenine auxotroph).
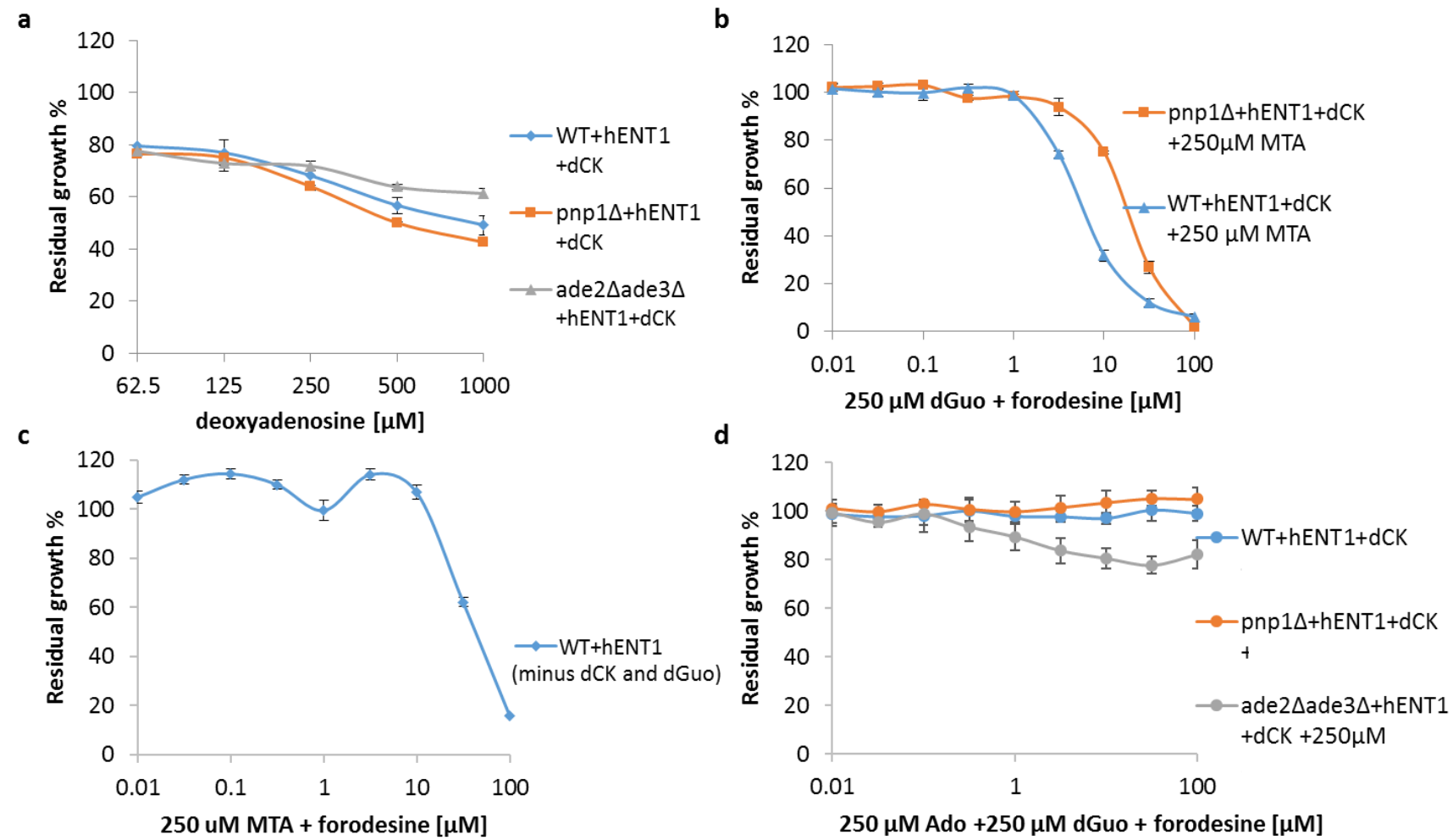

Figure 4.3.6 - MTA-mediated growth exacerbation is not mediated through inhibition of Pnp1p. Growth assays evaluating WT+hENT1+dCK, ade2 $\Delta a d e 3 \Delta+h E N T 1+d C K$ and pnp $1 \Delta+h E N T 1+d C K$ strains for growth inhibition by $250 \mu \mathrm{M}$ dAdo and $250 \mu \mathrm{M}$ dGuo plus forodesine in $250 \mu \mathrm{M}$ MTA supplemented media in absence of exogenous adenine and methionine. (a) WT, ade2 $\Delta$ ade $3 \Delta$ and

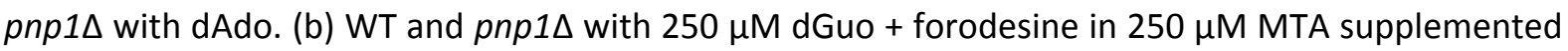
media. (c) WT + hENT1 with forodesine in $250 \mu \mathrm{M}$ MTA supplemented media. (d) WT and pnp1 $1 \Delta$ with $250 \mu \mathrm{M}$ dGuo + forodesine in $250 \mu \mathrm{M}$ adenosine supplemented media. Percent growth (OD600Exp/OD600Vehical control $\times 100$ ) was determined in mid-log cultures using Envision ${ }^{\text {TM }}$ Xcite $^{\text {TM }}$ 2102 Multilabel Reader (Perkin-Elmer). dAdo, deoxyadenosine; dGuo, deoxyguanosine; Ado, adenosine; MTA, methylthioadenosine.

4.3.8 Nicotinic acid depletion does not exacerbate the growth defect of pnp1 $\Delta$

PNP1 is involved in NAD+ biosynthesis, wherein with URH1 and MEU1, constitutes alternative pathways in salvage of nicotinic acid from nicotinamide riboside (via PNP1, URH1, Meu1) and nicotinate riboside (via PNP1, URH1) that is subsequently converted to nicotinamide mononucleotide 
(Belenky et al., 2007; Belenky et al., 2009). Likewise, tryptophan is also used as a precursor for synthesis of nicotinamide mononucleotide (Bedalov et al., 2003). Since there is a requirement of tryptophan and nicotinic acid either through transport or synthesis to maintain appropriate $\mathrm{NAD}^{+}$ levels required for replicative lifespan and possibly general fitness, we sought to investigate the requirement of $P N P 1$ in nicotinamide mononucleotide and NAD biosynthesis. Growth inhibition by deoxyguanosine in the absence of tryptophan, nicotinic acid (NA) and other nutrients was evaluated in pnp1S and WT.

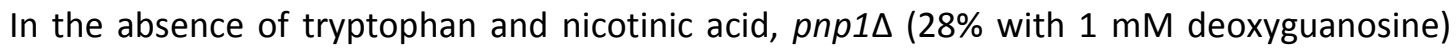
showed more growth inhibition to deoxyguanosine than WT (19\% with $1 \mathrm{mM}$ deoxyguanosine) (Fig. 4.3.7a), but the inhibition of both strains occurred at higher concentrations than previously observed

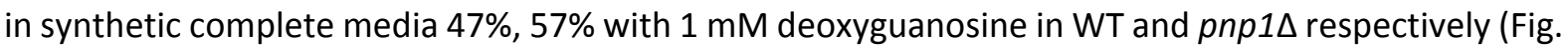
4.3.4d). In the absence of non-essential amino acids, the growth defect was observed for WT and pnp1 $\Delta$ with lower concentrations of deoxyguanosine $(62.5-250 \mu \mathrm{M}$ and $62.5 \mu \mathrm{M}$ deoxyguanosine with nitrogen sources MSG or ammonium sulphate respectively). (Fig. 4.3.7b). However, no growth difference was observed between WT and pnp1 $\Delta$ at higher concentrations of $0.5 \mathrm{mM}$ and $1 \mathrm{mM}$ deoxyguanosine whether the nitrogen source was MSG (Fig. 4.3.7b) or ammonium sulphate (Fig. 4.3.7c). The deoxyguanosine-facilitated growth defect in WT and pnp1D with minimal amino acids (cysteine and glutamine to permit the synthesis of methionine and tryptophan, respectively) in the absence of MSG, ammonium sulphate or nicotinic acid was assessed. Relative to poor growth in the absence of nitrogen in the form of MSG or ammonium sulphate $\left(\mathrm{OD}_{600}=0.06\right.$ for WT at $5 \mathrm{~d}$ with $1.7 \mathrm{~g} / \mathrm{L}$ yeast nitrogen base), incremental increases in the concentration of yeast nitrogen base improved growth (e.g., $\mathrm{OD}_{600} 0.16$ and 0.23 for WT at $5 \mathrm{~d}$ with 3.4 and $6.8 \mathrm{~g} / \mathrm{L}$ yeast nitrogen base) but also alleviated the growth inhibition of pnp1s and WT to deoxyguanosine (Fig. 4.3.7d-e). In summary, although the absence of nicotinic acid decreases replicative lifespan in the pnp1 $\Delta$ strain, it does not impact viability. 
a
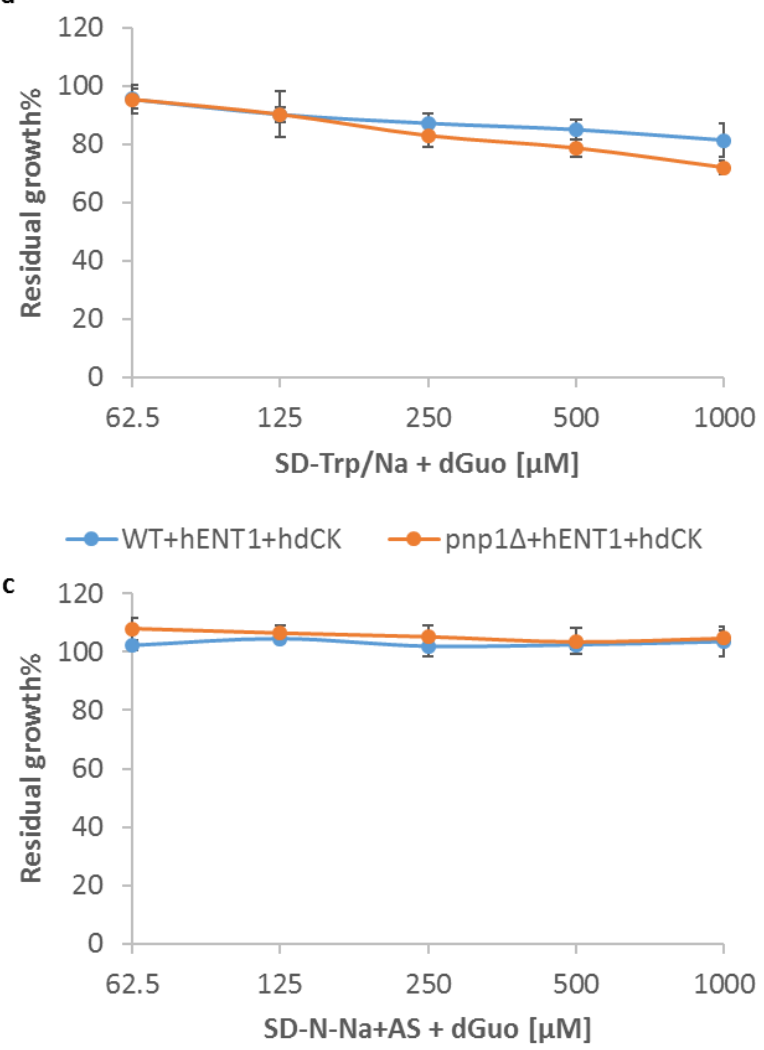

$\multimap$ WT+hENT1+hdCK $\rightarrow$ pnp1 $1+$ hENT1+hdCK

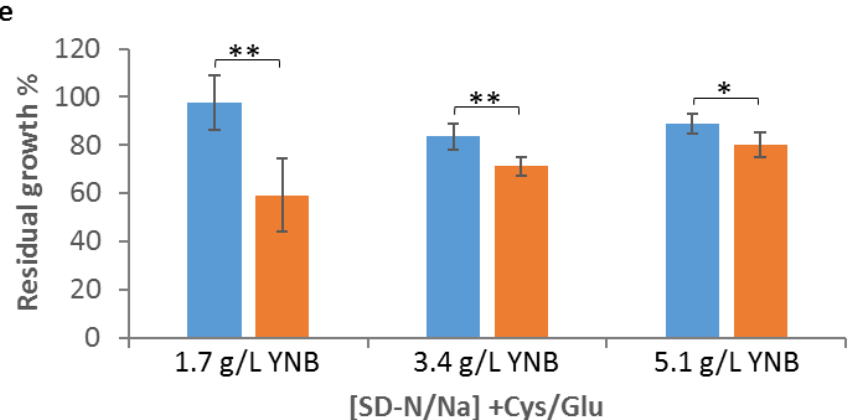

b
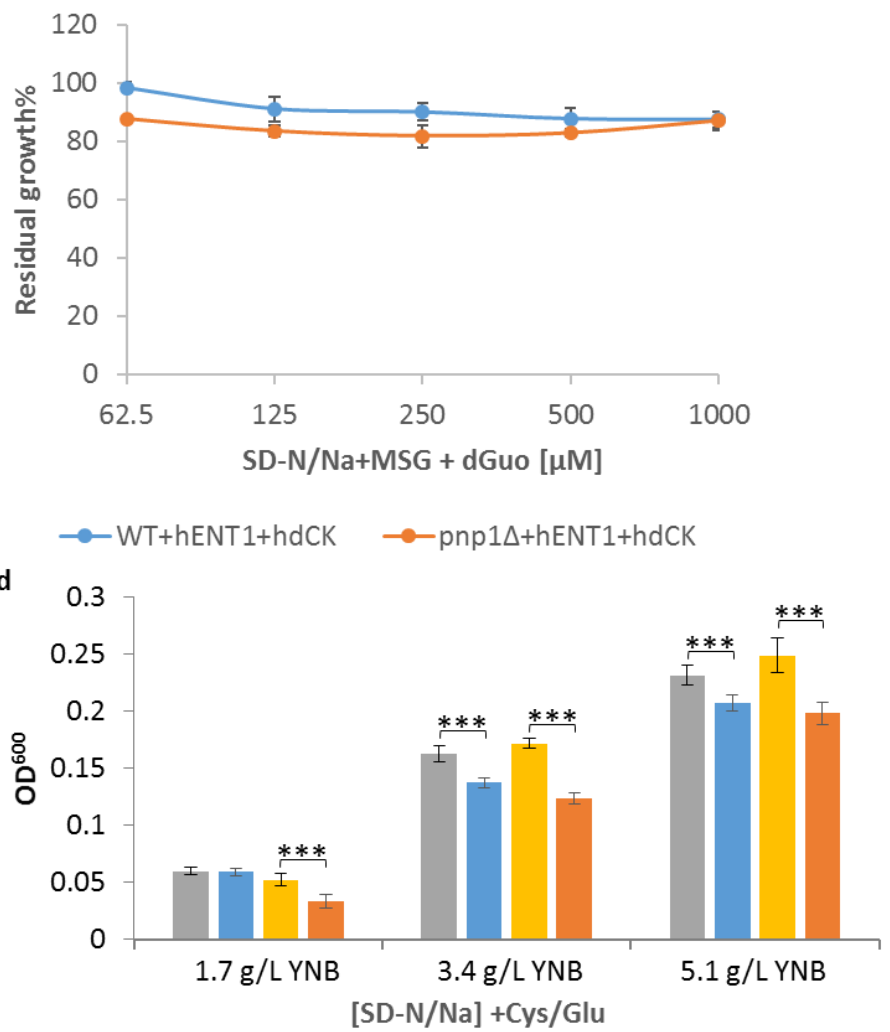

WT+hENT1+dCK

pnp $1 \Delta+\mathrm{hENT} 1+\mathrm{dCK}$
WT+hENT1+dCK $+250 \mu \mathrm{M}$ dGuo

pnp1 $\Delta+$ hENT1+dCK $+250 \mu \mathrm{M}$ dGuo

- WT+hENT1+dCK +250 $\mu \mathrm{M}$ dGuo $n$ pnp1 $\Delta+\mathrm{hENT1+dCK}+250 \mu \mathrm{M}$ dGuo

Figure 4.3.7 - The growth defect of pnp1 $1 \Delta$ to dGuo is not further exacerbated in the absence of

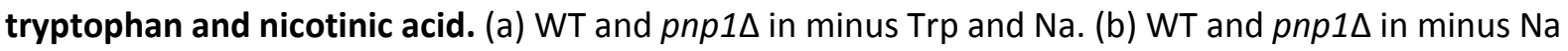
and all non-essential amino acids. (c) WT and pnp1 1 in minus $\mathrm{Na}, \mathrm{AA}$ and non-essential amino acids. (d) WT and pnp1s in Cys and Gly and increasing concentration of yeast nitrogen base without $\mathrm{Na}$. (e) Relative growth defect of WT and pnp1 $1 \Delta$ to $250 \mu \mathrm{M}$ deoxyguanosine in Cys and Gly and increasing concentration of yeast nitrogen base without $\mathrm{Na}$. Yeast strains WT+hENT1+dCK and pnp1 $1+\mathrm{hENT1}+\mathrm{dCK}$ were screened against deoxyguanosine in SD-Trp/Na, SD-MSG, SD-AA plus essential amino acids and SD-N+Cys/Glu. The liquid growth assays were performed in $2 \%$ raffinose and 
2\% galactose in triplicate with the exception of Fig. $4.3 .7 \mathrm{~d}$-e for which six replicates were performed for each condition. \% Growth $\left(\mathrm{OD}_{600} \mathrm{Exp} / \mathrm{OD}_{600}\right.$ Vehical control $\left.\times 100\right)$ was determined in mid-log cultures $\left(\mathrm{OD}_{600}\right.$ 0.3-0.5 unless otherwise stated) using Envision ${ }^{\mathrm{TM}} \mathrm{Xcit}^{\mathrm{TM}} 2102$ Multilabel Reader (Perkin-Elmer). Na, nicotinic acid; AA, ammonium acetate, MSG, monosodium glutamate; Trp, tryptophan; Cys, cysteine; Glu, glutamine.

\subsubsection{Forodesine does not sufficiently inhibit yeast PNP1}

To assess if forodesine sufficiently inhibits PNP1 function in yeast, the yeast strains WT, $n r k 1 \Delta$,

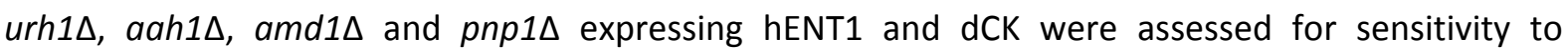
forodesine and deoxyguanosine. The yeast strains aah1 $1 \Delta$ and $a m d 1 \Delta$ have growth defects with loss of PNP1 (Section 4.3.1). NRK1 and URH1 are genes involved in NAD+ synthesis, and the deletions of each of these genes have previously been identified as inviable with pnp1s in the absence of tryptophan and nicotinic acid (Tempel et al., 2007). In the current studies, $n r k 1 \Delta$ and urh1 $1 \Delta$ deletions did not have a growth defect with the deletion of pnp1 $\Delta$ in synthetic complete media (Section 4.3.1), therefore these were assessed in synthetic complete media in the absence of nicotinic acid and tryptophan. The WT strain showed sensitivity to both deoxyguanosine and deoxyguanosine and forodesine in both complete and Trp/Nicotinic acid drop out media and the growth defect in each media was slightly exacerbated (12\% in SC and $15 \%$ in $\operatorname{Trp} / \mathrm{Na}$ ) with forodesine treatment. The pnp1 $\Delta$ strain, as previously observed showed sensitivity to deoxyguanosine (Fig. 4.3.8c), and did not exacerbate growth inhibition with forodesine treatment in both synthetic complete and Trp/Nicotinic acid dropout media (Fig. 4.3.8a-b). The growth defects of $a a h 1 \Delta$ and $a m d 1 \Delta$ in synthetic complete media as well as $n r k 1 \Delta$ and urh1 1 in Trp/Nicotinic acid dropout media were exacerbated with co-treatment of forodesine and deoxyguanosine. However, the growth defect was not different from WT for these deletion strains (Fig. 4.3.8a-b). Interestingly, urh1 $\Delta$ showed resistance to forodesine compared with WT. This was unexpected as these deletions had a defect in chronological lifespan when the PNP1 drug target of forodesine was deleted in the background (Belenky et al., 2007). This is likely the difference between knockout of the PNP1 gene in the double mutant compared to the knockdown PNP1 in the single 
mutant treated with forodesine. Interestingly, deoxyguanosine sensitivity in both WT and pnp1 $1 \Delta$ was reduced in the absence of tryptophan and nicotinic acid compared to synthetic complete media. The lack of growth reduction in genes deletion strains that previously demonstrated a growth defect with complete abolition of PNP1 function suggest that forodesine does not sufficiently inhibit PNP1 in yeast.
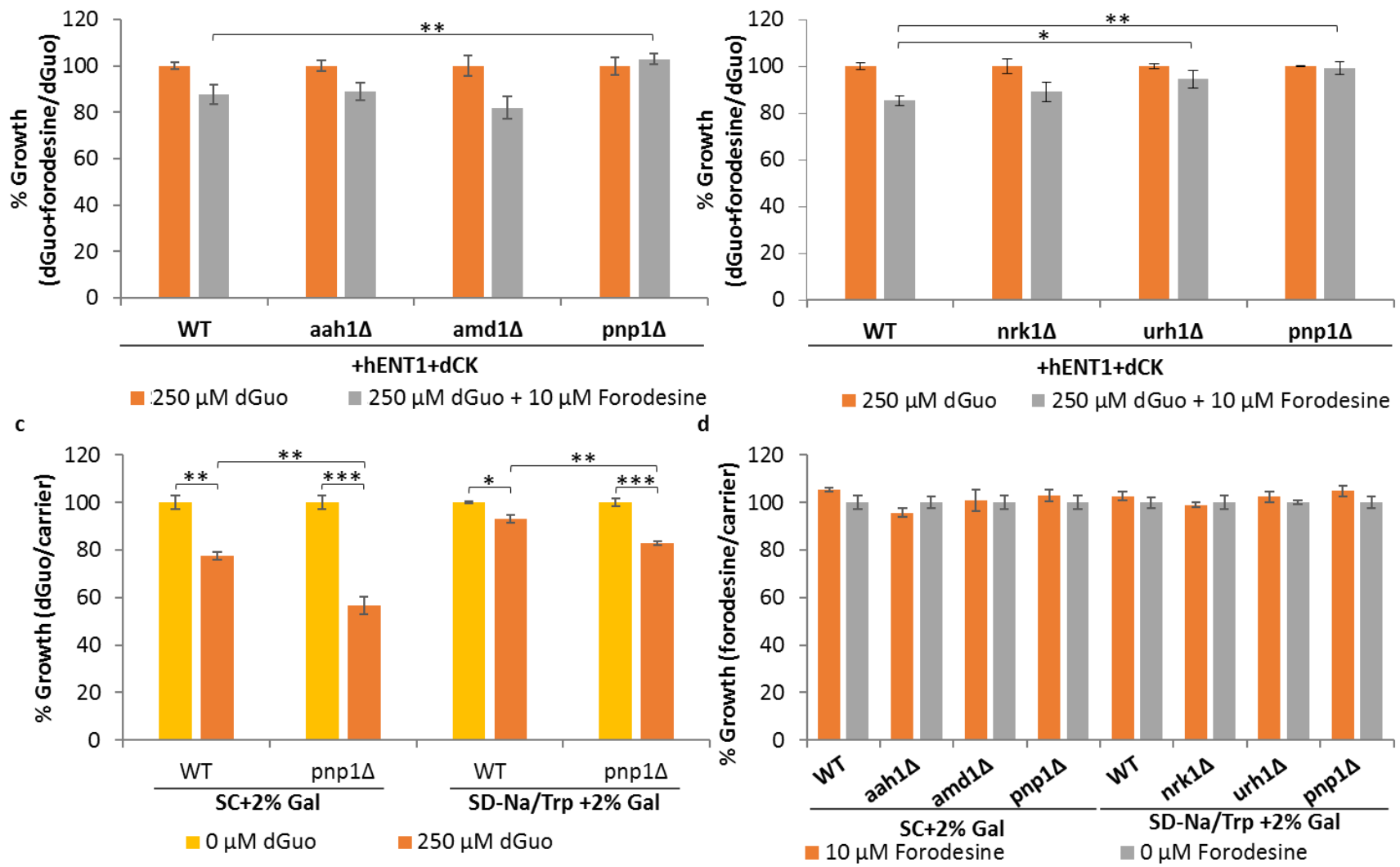

Figure 4.3.8-Forodesine does not exacerbate the growth defect of gene deletions that are synthetic

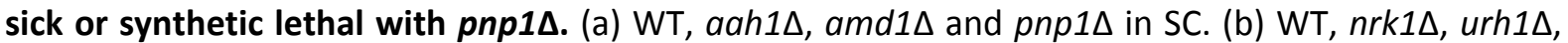

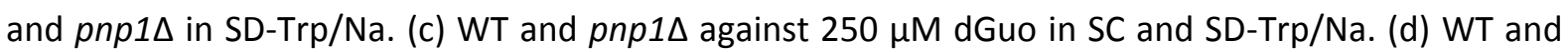
pnp1 $\Delta$ against $10 \mu \mathrm{M}$ forodesine in SC and SD-Trp/Na. Percent growth $\left(\mathrm{OD}_{600}\right.$ Exp/OD ${ }_{600}$ Vehical control $x$ 100) was determined in mid-log cultures using Envision ${ }^{\mathrm{TM}} \mathrm{Xcite}^{\mathrm{TM}} 2102$ Multilabel Reader (PerkinElmer).

\subsection{Discussion}

To investigate the therapeutic mechanism of PNP inhibition, we attempted a two-pronged approach in S. cerevisiae. The first identified the gene-gene interactions with the drug target. These 
interactions were then used to optimise media conditions to make PNP essential in yeast so the effect of the PNP inhibitor forodesine could be better discerned. The second approach was to identify genedrug interactions under the PNP1 essential condition in order to follow up on these findings in biologically relevant experiments. To these ends, a null deletion of the $S$. cerevisiae PNP1 was constructed and genetic interactions were investigated among 4400 pairwise deletions of pnp1 $\Delta$ and non-essential genes. Of these, 53 negative genetic interactions (growth reduction) and five positive genetic interactions (growth improvement) were identified. These genes were classified into two main categories: 1) genes that are modulated in response to nutrient limitation such as amino acid metabolism, response to starvation, vacuole organisation, vesicle-mediated transport, carbohydrate metabolism, and lipid homeostasis; and 2) genes that modulate gene expression such as chromatin organisation, chromosome segregation and repair, transcription and translation.

Of these, the amino acid metabolism genes (AMD1, AAH1 and ADE5,7) and PNP1 were involved exclusively in the salvage of purine nucleotides via three different pathways each ending with synthesis of IMP. IMP is synthesised either from phosphorylation of inosine to hypoxanthine by PNP1 and subsequent of phosphorylation of hypoxanthine by HPT1 or deamination of adenine to AMP by $A A H 1$ and subsequent deamination of AMP by $A M D 1$ or hydrolysis of 5'-phosphoribosyl-5-formamido4-imidazole carboxamide by ADE16 and ADE17 (Walther et al., 2010; Tibbetts \& Appling, 1997). The first of such pathways is compromised by deletion of PNP1. Second, by the deletion of AMD1 and $A A H 1$ genes and third, by the deletion of $A D E 5,7$. Loss of these otherwise buffering pathways for

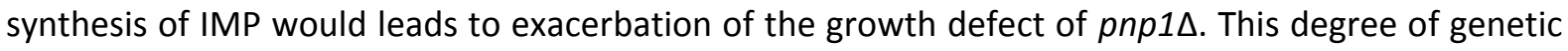
and pathway redundancy is conserved from yeast to humans (Stepchenkova et al., 2005; Simoni et al., 2007) and demonstrates the importance of purine ribonucleotide salvage and the dispensability of PNP1.

Furthermore, the majority of the genes involved in vacuolar and vesical transport are GTPases (VPS41/VAM2, VAM3, MON1, CCZ1 and ARL1 (negatively regulated by MON2); and these cellular 
processes may be compromised indirectly by the loss of PNP1 as a result of GTP depletion (Balakrishnan et al., 2010b). The disruptions to transcription and translation (DAL81, GCR2 (overlaps YNL198C), KNS1, RTT103 (overlaps YDR290W), YKE2, TEF4, SNF5, RPS19A, RPL22A, RPS6A, RPL9A, BUD21) observed is also a reflection of the reduced ATP and GTP levels in the absence of PNP1. This is consistent with the reduction in incorporation of radioactively labelled uridine to mRNA and leucine to proteins observed in forodesine treated CCL cells (Balakrishnan et al., 2010b) as well as depletion of purine ribonucleotides ATP (20\%) and GTP (50\%) observed in forodesine-treated CLL cells (Balakrishnan et al., 2010b).

The PNP1-interacting genes that are involved in chromatin organisation and gene expression are also involved in mitigating the depletion of purines nucleotides. EAF1/VID21 encodes NuA4 histone acetyltransferase complex specific subunit and directly interacts with BAS1 and PHO2 transcription factors (Cheng et al., 2015). Bas1 regulates the expression of genes involved in purine synthesis and is constitutively bound to the promoters of ADE genes. During adenine starvation, Bas1 undergoes a conformation change that recruits histone variant H2A.Z (encoded by HTZ1), SAGA histone acetyltransferase complex and SWI/SNF chromatin re-modelers leading to nucleosome disassembly and transcription initiation (Cheng et al., 2015). The loss of EAF1 also leads to reduced ADE gene expression. The expression of $\mathrm{PHO5}$, a nucleotide phosphatase, is also regulated by recruitment HTZ1 by NuA4 and is also impaired by the deletion of EAF1 (Auger et al., 2008). Under phosphate starvation, $\mathrm{PHO} 5$ is positively regulated by both Ino2/Ino4 transcription factors (He et al., 2012). The carbohydrate metabolism was also impaired in the absence of PNP1, but this is likely an indirect effect of reduced levels of both nicotinate riboside salvage in NAD+ biosynthesis. Ribose-1phosphate molecules generated by PNP1 during purine nucleoside phosphorylation are converted further to ribose-5-phosphate and used as precursors in the non-oxidative pentose phosphate pathway (Xu et al., 2013). PNP1 with URH1 and MEU1 constitute alternative pathways synthesis of precursors for NAD+ biosynthesis that is independent of NRK1 (Belenky et al., 2007; Belenky et al., 


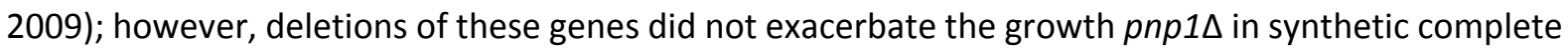
media or synthetic complete minus Trp/nicotinic acid media.

From the cellular processes identified herein that buffer the loss of PNP1 in S. cerevisiae, it is evident that PNP1 is required for homeostasis of ribonucleotides. This however, differs from the main proposed mechanism of dGTP accumulation in CCL cells treated with PNP inhibitors (Bantia et al., 2003). This is due to the absence of deoxyguanosine kinase activity in yeast required for conversion of accumulated deoxyguanosine to deoxyguanosine monophosphate. Although the remainder of the pathway is conserved, S. cerevisiae cannot accumulate dGTP to the extent required to inhibit growth. Therefore, PNP1 loss in S. cerevisiae does not completely mirror the PNP loss in mammals, at least with the situation in T-cells.

To replicate the mechanisms by which PNP1 inhibitors induce T cell toxicity several modifications to the S. cerevisiae genome were required. The $S$. cerevisiae genome lacks both ENT and CNT families of plasma membrane nucleoside transporters required for transport of most nucleosides (Young et al., 2013) (Vickers et al., 2000) (including forodesine and deoxyguanosine(Huang et al., 2008)) as well as lacks deoxyguanosine kinase activity (Arnér \& Eriksson, 1995). Therefore, the human equilibrative nucleoside transporter (ENT1) and the human deoxycytidine kinase were expressed in S. cerevisiae. This modified strain was treated with forodesine in combination with $250 \mu \mathrm{M}$ deoxyguanosine to achieve growth inhibition. The forodesine-facilitated growth defect in yeast $\left(\mathrm{IC}_{20}\right.$ $\sim 5.0 \mu \mathrm{M}, \mathrm{IC}_{50}>100 \mu \mathrm{M}$ ) however paled in comparison to picomolar/low nanomolar activity in human cells (Kicska et al., 2001).

To improve the toxicity in yeast, media conditions were manipulated to target pathways in which PNP1 functions. The nicotinamide mononucleotide salvage of $\mathrm{NAD}^{+}$biosynthesis was targeted with forodesine or pnp1s in the absence of precursors nicotinic acid and tryptophan (Belenky et al., 2009). Purine salvage (Walther et al., 2010; Tibbetts \& Appling, 1997) was targeted with adenosine, natural analogues of adenosine (MTA and dAdo), and different variants of minimal and synthetic 
media were also assessed but all were unsuccessful in improving the toxicity of forodesine plus deoxyguanosine. When exploring the minimal media conditions, supplementation with some amino acids was necessary as for viability of the genetic background of yeast strains, maintenance of plasmids, and for the SGA manipulations used for construction of the haploid hENT1 + dCK gene deletion library. It is possible these may have prevented toxicity of forodesine.

In addition to the aforementioned cytosolic dCK, deoxyguanosine phosphorylation at the mitochondria was assessed by expression of broad specificity nucleoside kinase HSV-TK. However, less inhibition was observed with HSV-TK expression than with dCK expression. It is likely that PNP1 was not inhibited due to the genetic redundancies unique to $S$. cerevisiae. Examples of such redundancies include URH1 that functions in the salvage of nicotinamide riboside to nicotinate (Belenky et al., 2007) and PNC1 that catalyses the subsequent step in NAD+ synthesis (Ghislain et al., 2002) and do not have mammalian orthologues but are conserved across fungi (Wood, 2006; Byrne \& Wolfe, 2005). In addition to presence of genetic redundancy in yeast, the lack of gene conservation required for further processing of metabolites may also be a reason for lack of forodesine toxicity, which was the case with deoxycytidine and deoxyguanosine kinases. The absence of other pathways that are yet to be characterised may prevent the mechanisms required for forodesine toxicity in yeast. It is also possible that forodesine does not achieve the $95 \%$ continuous inhibition of PNP1 required to accumulate sufficient dGTP to get similar growth inhibition in yeast as observed for T-cells.

In summary, findings from yeast herein present novel targets to improve the efficacy of forodesine. The synthetic lethal and synthetic sick interactions with pnp1s in yeast show the requirement for inosine monophosphate (IMP) salvage, tryptophan, inosine and phospholipid biosynthesis for growth in the absence of PNP1 activity. Immucillin-HP and Immucillin-GP are transition state structure inhibitors of hypoxanthine guanine phosphoribosyl transferase (HGPRT) (Li et al., 1999) and the AMP deaminase (AMD) inhibitor 3-[2-(3-carboxy-4-bromo-5,6,7,8tetrahydronaphthyl)ethyl]-3,6,7,8-tetrahydroimidazo[4,5-d][1,3]diazepin-8-ol (24b) (Kasibhatla et al., 
2001), and these are two parallel pathways required for salvage of IMP (Walther et al., 2010; Tibbetts \& Appling, 1997). Inhibitors of either pathway in conjunction with forodesine would improve the toxicity of forodesine. The inhibition of HPRT (or AMD) would deplete the cells of GMP, IMP, and AMP (also by AMD inhibition) as well as the allosteric regulation of ribonucleotide reductase by dGTP (Ahmad \& Dealwis, 2013) that converts the already depleted ADP to dADP thereby inhibiting transcription. The elevated dADP would thus inhibit dCTP and dTTP synthesis by ribonucleotide reductase, leading to disruption of nucleotide pools and forodesine-mediated growth inhibition. 


\section{Chapter 5-Implications and future directions}

\subsection{Summary}

Herein elucidated are the mechanisms for transition state structure analogues methylthioadenosine-DADMe-immucillin A (MTDIA) and the recently approved forodesine (Mundesine ${ }^{\circledR}$ ). It is necessary to know the compendium of cellular perturbations resulting from use of these inhibitors, given the importance of polyamine and purine synthesis in cancer. Perturbation to glucose metabolism, translation, transcription, vesicle-mediated transport, lipid homeostasis, cell wall synthesis and cell cycle progression were discerned with MTDIA and its genetic mimic meu1A in the evaluation of non-essential gene deletions. Disruptions to these cellular processes were also revealed in the global analysis of protein abundance and localisation, confirming disruptions to these processes as mechanisms of MTAP inhibition or MTAP loss. Many plasma membrane proteins were relocalised to the vacuole consistent with autophagy as a mechanism of MTDIA. The disruptions to lipid homeostasis were further evaluated by quantification of sterols and significant reduction in ergosterol levels coincident with accumulation of ergosterol precursors were confirmed. This dissertation highlights the importance of genome-wide studies in discerning drug mechanisms and understanding gene function, given that initiating apoptotic pathways (Basu et al., 2007) and changing DNA methylation patterns (Basu et al., 2011) were the only known mechanisms of MTDIA prior to this dissertation. The novel cellular processes perturbed by MTAP inhibition and MTDIA provide additional insight into possible combinatorial solutions for treating cancers such as in combination with DNA interstrand cross-links inducers (e.g., cisplatin and melphalan). The assessment of the drug target of forodesine in yeast, Pnp1, for genetic interactions identified similar cellular processes to MTDIA and loss of MTAP. However, despite the introduction of several human genes and supplementation of metabolites required for bioactivity in humans, forodesine lacked efficacy in yeast, thus a condition where forodesine was sufficiently bioactive for genomic analyses was not determined. Findings with transition state structure analogues herein illustrate that even highly target-specific inhibitors affect 
vast numbers of cellular processes. Thus, genome-wide pre-screening of drugs could efficiently be carried out in yeast prior to assessing mechanisms in human cells.

\subsection{Implication for MTDIA as a cancer therapeutic}

Remodelling of cell energy metabolism is one of the driving causes of cancer development. These changes include altered glycolysis, lipid metabolism, glutamine metabolism and autophagy to meet the demands for cell proliferation (Zhao et al., 2013). Targeting these changes are one such way to specially target the growth of cancer cells. MTDIA is an inhibitor of methylthioadenosine phosphorylase (MTAP), an enzyme that processes MTA, the toxic byproduct of polyamine synthesis, to maintain the forward reaction and salvage methionine and adenine (Schramm, 2011; Pajula et al., 1979b). Polyamines are important molecules in cancer that promote cell proliferation by facilitating transcription (Friesen et al., 1998), translation (Landau et al., 2010), DNA repair (Snyder \& Sunkara, 1990) and maintaining basal levels of autophagy (Eisenberg et al., 2009). Exogenously added polyamines such as spermidine induce autophagy in yeast and mammalian cells, and likewise, depletion of spermidine has previously been shown to inhibit autophagy (Morselli et al., 2011; Vanrell et al., 2013). The depletion of polyamines leading to induction of autophagy has not been detected previously. The induction of excessive autophagy leading to cell death by MTAP inhibition identified herein in yeast would be a novel means to treat cancers if conserved in mammals. It is possible that the differences in programmed cell death between yeast and humans may lead to different outcomes from excessive induction of autophagy. For example, autophagy may not be a key mechanism in mammalian cells if apoptosis is efficient.

MTDIA may potentially regulate cell energy metabolism and immune evasion in cancer cells at several different stages. MTDIA treatment or loss of MTAP in yeast downregulated pyruvate synthesis, oxidation of acetaldehyde, and the synthesis of acetyl-CoA. Other changes to cell energy metabolism with MTDIA/meu1 included disrupted synthesis of all major lipids, as well as glutamine metabolism where glutamine synthases $(\mathrm{G} \ln 1)$ was mislocalised to cytoplasmic foci (Appendix Fig. 
7.3.3). In addition, a product of polyamine synthesis, S-adenosyl methionine (downstream of MTAP) regulates innate immunity through modulation of methyltransferase reactions (Ding et al., 2015). Although the immune processes are beyond investigations carried out in yeast, the GO analysis of gene expression changes with MTDIA in A549 tumours was enriched for myeloid leukocyte mediated immunity $(p=2.13 \mathrm{E}-02)$ and neutrophil mediated immunity ( $p=4.93 \mathrm{E}-02)$ (Appendix Table 7.3.6). These changes along with induction of autophagy may be responsible for the induction of proapoptotic responses observed in cancers.

5.3 Implication for MTDIA in treating lysosomal storage disorders and other disease with defective autophagy

Autophagy is an essential catabolic process that degrades cytoplasmic protein aggregates, lipids, and damaged organelles via the lysosome/vacuole to maintain cellular homeostasis. Large numbers of human diseases result from deregulated autophagy including cancer, heart disease, obesity and neurodegenerative diseases (Cheng et al., 2013; Choi et al., 2013). The findings from this dissertation add MTDIA to the to the existing arsenal of autophagy regulators (Li et al., 2015), suggesting MTDIA can be effective modulating diseases other than cancer. Particularly, yeast orthologues of proteins that drive development of neurodegenerative diseases such as late-onset Parkinson disease (GLUD2, ATXN2, PARK7, GSK3B), Alzheimer's disease (HSD11B1, DLST, SOD1, GSK3B) and Huntington's disease (CS, GAPDH, ATXN2, SGTA, UBB, ABCB1, ASL, HSPB1, GSK3B) (Hamosh et al., 2005) were regulated by MTDIA.

\subsection{Implications of Vps34 in TORC1-dependent and independent autophagy}

Several mechanisms responsible for induction of autophagy in yeast and mammals have been identified, the most characterised being the TOR1/mTOR-dependent autophagy in which40 different genes are required (Sarkar, 2013; Feng et al., 2014). TOR1/mTOR is regulated by extracellular signals such as amino acids and growth factors, availability of ATP, and $\mathrm{O}_{2}$ levels (Burman \& Ktistakis, 2010). Phosphoinositide 3-kinase Vps34 converts phosphoinositide to phosphoinositide 3-kinase, is required 
for autophagosome maturation, and is an upstream regulator of TOR1/mTOR (Dall'Armi et al., 2013). Vps34 activates TOR1/mTOR in the presence but not the absence of glutamine and therefore plays a role in amino acid starvation-induced autophagy (Tanigawa \& Maeda, 2017). Given that Vps34 relocalises from vacuole/lysosome to subdomains of the ER in response to starvation (Burman \& Ktistakis, 2010) and that exogenously added phosphoinositide 3-kinase induces autophagy in human colon cancer cells (Petiot et al., 2000), our observation that Vps34 relocalises from the vacuole to what appears to be subdomain of the ER with meu1A under methionine replete conditions suggests that Vps34 is likely the inducer of autophagy, and if so, it is not yet certain if it is TOR-dependent or TORindependent. Therefore, the role of $\mathrm{Vps} 34$ should be further investigated in the induction of autophagy with MTDIA. Comparisons of MTDIA and the Vps34 inhibitor wortmannin (Blommaart et al., 1997) would provide valuable insight into the role of Vps34 in MTDIA mediated induction of autophagy. The mislocalisation of Vps34 as the cause of autophagy induction by MTDIA would be confirmed if wortmannin prevents the relocalisation of Vps34 to sub-domains of ER in meu1A. It is also yet to be determined if the autophagic vesicles accumulated within the vacuole are degraded following induction of autophagy with MTDIA. The GFP-Atg8 used herein for microscopy-based detection of autophagy induction is also used in a Western blot analysis to detect degradation of autophagic vesicles at the vacuole. Atg8 is rapidly degraded in the vacuolar lumen by vacuolar/lysosome proteases, resulting in free GFP. Thus the ratio of GFP-ATG8 to free GFP will give an indication of rate of degradation of autophagic vesicles (autophagic flux) (Klionsky, 2011).

\subsection{Implications of yeast ergosterol synthesis inhibition on sterol synthesis in mammals}

In addition to induction of autophagy, MTAP inhibition/loss in yeast also disrupted lipid homeostasis. Deletions of lipid homeostasis genes OPI3, SIP3, and SYG1 were synthetically lethal with MEU1 deletion and localisation/abundance of these proteins required for homeostasis of all major classes of lipids were altered with meu1s or MTDIA. The synthesis of sterols in yeast involves methyltransferase reactions by delta(24)-sterol C-methyltransferase Erg6. Although the 
methyltransferases are absent in sterol synthesis in humans, polyamine depletion via MTAP inhibition of A549 tumours in mice lead to $>3$-fold change in expression of cholesterol homeostasis genes (Kee et al., 2004; Basu et al., 2011), suggesting that non-methyltransferase reactions of sterol synthesis are affected by MTAP inhibition. The role of other methyltransferases in lipid homeostasis are also conserved and worthy of further investigation. The yeast glycerophospholipid methyltransferases Cho2 and Opi3 required for conversion of PE to PC are encoded by a single methyltransferase PEMT in humans, and therefore prone to product inhibition by MTA (Gaynor et al., 1991; Vance \& Ridgway, 1988). The observed MTDIA-mediated disruptions to fatty acid synthesis and sphingolipid synthesis likely do not involve methyltransferases. The downregulation of acetyl-CoA synthetases and acetylCoA transferases by MTAP inhibition in yeast (in this study) and in humans (Basu et al., 2011) suggest that it maybe causal rather than consequence of lipid homeostasis disruption given that acetyl-CoA is used for as precursor for synthesis of all major classes of lipids (Natter \& Kohlwein, 2013). The disruption of MTDIA on the synthesis of lipids other than sterols needs to be quantified in yeast and the translation of these findings to A459 or FaDu cells would give a better indication of the conservation of lipid homeostasis disruptions as a mechanism in head and neck and lung cancers treated with MTDIA.

MTDIA as a consequence of sterol synthesis inhibition may induce lipid droplet autophagy. Consistent with induction of lipid droplet autophagy were increased accumulation of Erg6 and Faa4 at the lipid droplets (Bouchez et al., 2015) with MTDIA and meu1A. Another protein of interest, the multifunctional casein kinase Hrr25, was induced by MTDIA and meu1A. Hrr25 has roles in several nonselective forms of autophagy but not in starvation induced autophagy, and its role in lipophagy has not been investigated. Therefore, the uncharacteristic mislocalisation of Hrr25 from cytoplasm to cytoplasmic foci with MTDIA and meu1 $\Delta$ should be investigated to confirm the role in lipophagy with other lipophagy inducers in yeast such as oleate (Niso - Santano et al., 2015). If Hrr25 is not 
mislocalised with induction of lipophagy, it may reflect activation of other selective forms of autophagy by MTDIA or yet uncharacterised role of Hrr25 in starvation induced autophagy.

Sterol synthesis inhibition is a promising anti-cancer mechanism, given the requirement of lipids for cell division and changes in cell energy metabolism in cancer cells. Sterol synthesis inhibitors HMG-CoA (Statins) are some of the most heavily prescribed drugs worldwide for cardiovascular diseases, and results from clinical trials show reduced incidence of cancer with statins as chemopreventatives (Boudreau et al., 2010; Demierre et al., 2005). Statins are currently undergoing or have recently completed nearly 150 clinical trials for its efficacy as a cancer therapeutic against various cancers including lung, liver, breast and prostate cancers (https://clinicaltrials.gov/ct2/results?cond=cancer\&term=statin\&cntry1=\&state1=\&Search=Search\# wrapper). Interestingly, statins also induce autophagy and are at least in part responsible for the antimetastatic effects of statins (Parikh et al., 2010). Given the mechanisms of MTDIA as an inhibitor of sterol synthesis and an inducer of autophagy, it is possible that anti-cancer mechanisms of MTDIA are mediated through disruptions to lipid homeostasis in a similar manner to statins. Thus, MTDIA in combination with statins may improve the potency of both compounds as chemotherapeutics.

\subsection{Implications of toxicity in the inhibition of yeast Pnp1}

Loss of Pnp1 in yeast illustrated the role of PNP in purine synthesis and the subsequent impacts on many cellular processes (e.g., amino acid metabolism, response to starvation, vacuole fusion, vesicle-mediated transport, carbohydrate metabolism, lipid metabolic processes, chromatin organisation chromosome segregation, transcription, and translation) were similar to what was observed with MTAP inhibition and suggests a similar stress response. As PNP inhibition/loss mediated toxicity in mammals is a combination of dGTP accumulation (proposed mechanisms(Osborne \& Scott, 1983)) and GTP depletion (Balakrishnan et al., 2010b), the assessment of the pnp1 $\Delta x x x \Delta$ double deletions for genetic interactions did not determine cellular process perturbed by dGTP accumulation but rather was a result of GTP depletion. Yeast lack deoxyguanosine kinase activity (dCK/dGK 
orthologues) required for conversion of the Pnp1 product deoxyguanosine to dGMP, enzymes crucial for the proposed toxicity in humans. Yeast also lack the nucleoside transporters required for entry of deoxyguanosine (Arnér \& Eriksson, 1995). Therefore, the genetic screen of pnp1 $x x x x \Delta+\mathrm{hENT} 1+\mathrm{dCK}$ library against $250 \mu \mathrm{M}$ deoxyguanosine should be performed (following construction of the pnp1 $x x x \Delta+h E N T 1+d C K$ gene deletion library) to identify the complete Pnp1 interactome affected by both GTP depletion and dGTP accumulation. Under these conditions, pnp1 $1 \Delta$ is growth inhibitory and non-lethal therefore permitting the assessment of genetic interactions with PNP1. This experiment was not performed in this dissertation due to time constraints and a concerted effort investigating the mechanisms of MTDIA; however, this dissertation provided insight into dGTP independent mechanism of forodesine via PNP1 deficiency.

The poor efficacy of forodesine for yeast Pnp1 was also a contributor to lack of bioactivity, given that gene deletions sensitive to pnp1s were insensitive to forodesine. Therefore, better transport mechanisms should be investigated such as combinations of the expression of the CNT2 major transporter for deoxyguanosine (Huang et al., 2008), expression of the major forodesine transporter hENT1, and dGTP accumulation should be measured. Alternatively, the expression of human mitochondrial deoxyguanosine kinase (Arpaia et al., 2000) or Drosophila melanogaster multifunctional nuclear Dm-dNK (Solaroli et al., 2007; Munch-Petersen et al., 1998) with hENT1 may also improve the accumulation of dGTP. The lack of specificity for yeast Pnp1 by forodesine can possibly be overcome by replacing the yeast Pnp1 with the human PNP. Removing redundant pathways in yeast may increase the reliance on PNP1 and present conditions where PNP is conditionally essential. To that end, PNP1 loss herein rendered yeast sensitive to disruptions of inosine monophosphate salvage. The deletion of hypoxanthine guanine phosphoribosyl transferase (HPT1/HGPRT) or inhibition by transition state structure inhibitors of HGPRT by immucillin-HP and immucillin-GP (Li et al., 1999), would render yeast more sensitive to inhibition by forodesine. These and other inhibitors of IMP synthesis (e.g., the AMP deaminase inhibitor 3-[2-(3-carboxy-4-bromo- 
5,6,7,8-tetrahydronaphthyl)ethyl]-3,6,7,8-tetrahydroimidazo[4,5-d][1,3]diazepin-8-ol (Kasibhatla et al., 2001)) would also render lymphatic leukemia cells more sensitive to the action of forodesine and would improve the toxicity in treating lymphatic leukemia.

\subsection{Implications on transition state structure analogues}

Transition state structure analogues are structures chemically tailored to inhibit the function of a protein of interest and are some of the most potent inhibitors discovered to date (Schramm, 2011). Forodesine was the first of these transition state analogues designed base on the kinetic isotope effects and computational chemistry to be approved for human use (http://www.mundipharma.com.sg/2017/03/30/mundipharma-wins-approval-antineoplastic-agentmundesine-treatment-relapsedrefractory-peripheral-t-cell-lymphoma-japan/, 2017) and is likely the first of many given the efficacy and potency of MTDIA as an anti-cancer inhibitor (Basu et al., 2007; Basu et al., 2011) and the efficacy of other immucillins against infectious pathogens (Ting et al., 2005; Singh et al., 2006). In the next few years, the genome-wide studies performed in this thesis can be carried out human cells with application of CRISPR/Cas9 based gene editing to reveal the complete genetic interactions resulting from inhibition of MTAP (MTDIA target) or PNP (forodesine target) as well as pairwise synthetic lethal interactions with specific cancer alleles. These types of analyses have recently been reported for subsets of the human genome (e.g., 73 pairwise interactions of tumour suppressor genes with relevant drug targets (Shen et al., 2017)). With time, these studies could also be carried out in situ in cancer cells. The synthetic lethal (oncogene expression/loss of tumour suppressor-geneX) interactions detected by CRISPR/Cas9 gene editing would identify novel drug targets, that can be drugged with the application of kinetic isotope effects and computational chemistry in designing novel transition state structure inhibitors. Synthetic lethality based transition state structure inhibitor design would not be limited to treatment of cancers but also in the treatment of other human disorders. 


\section{6 - Bibliography}

Abdulhussein, A. A., \& Wallace, H. M. (2014). Polyamines and membrane transporters. Amino Acids, 46(3), 655-660. doi:10.1007/s00726-013-1553-6

Aguilar - Uscanga, B., \& Francois, J. (2003). A study of the yeast cell wall composition and structure in response to growth conditions and mode of cultivation. Letters in applied microbiology, 37(3), 268-274.

Ahmad, M. F., \& Dealwis, C. G. (2013). The structural basis for the allosteric regulation of ribonucleotide reductase. Prog Mol Biol Transl Sci, 117, 389-410. doi:10.1016/b978-0-12386931-9.00014-3

Alonso, R., López-Guerra, M., Upshaw, R., Bantia, S., Smal, C., Bontemps, F., Manz, C., Mehrling, T., Villamor, N., Campo, E., Montserrat, E., \& Colomer, D. (2009). Forodesine has high antitumor activity in chronic lymphocytic leukemia and activates p53-independent mitochondrial apoptosis by induction of p73 and BIM. Blood, 114(8), 1563.

Alzeer, M. I., MacKenzie, K. J., \& Keyzers, R. A. (2016). Porous aluminosilicate inorganic polymers (geopolymers): a new class of environmentally benign heterogeneous solid acid catalysts. Applied Catalysis A: General, 524, 173-181.

An, X., Tiwari, A. K., Sun, Y., Ding, P. R., Ashby, C. R., Jr., \& Chen, Z. S. (2010). BCR-ABL tyrosine kinase inhibitors in the treatment of Philadelphia chromosome positive chronic myeloid leukemia: a review. Leuk Res, 34(10), 1255-1268. doi:10.1016/j.leukres.2010.04.016

Anassi, E., \& Ndefo, U. A. (2011). Sipuleucel-T (Provenge) Injection: The First Immunotherapy Agent (Vaccine) For Hormone-Refractory Prostate Cancer. Pharmacy and Therapeutics, 36(4), 197202.

André, B. (1995). An overview of membrane transport proteins in Saccharomyces cerevisiae. Yeast, 11(16), 1575-1611.

Andreu-Pérez, P., Hernandez-Losa, J., Moliné, T., Gil, R., Grueso, J., Pujol, A., Cortés, J., Avila, M. A., \& Recio, J. A. (2010). Methylthioadenosine (MTA) inhibits melanoma cell proliferation and in vivo tumor growth. BMC cancer, 10(1), 265.

Ansorena, E., García - Trevijano, E. R., Martínez - Chantar, M. L., Huang, Z. Z., Chen, L., Mato, J. M., Iraburu, M., Lu, S. C., \& Avila, M. A. (2002). S - adenosylmethionine and methylthioadenosine are antiapoptotic in cultured rat hepatocytes but proapoptotic in human hepatoma cells. Hepatology, 35(2), 274-280.

Arnér, E. S., \& Eriksson, S. (1995). Mammalian deoxyribonucleoside kinases. Pharmacology \& therapeutics, 67(2), 155-186.

Arpaia, E., Benveniste, P., Di Cristofano, A., Gu, Y., Dalal, I., Kelly, S., Hershfield, M., Pandolfi, P. P., Roifman, C. M., \& Cohen, A. (2000). Mitochondrial basis for immune deficiency. Evidence from purine nucleoside phosphorylase-deficient mice. J Exp Med, 191(12), 2197-2208.

Ashburner, M., Ball, C. A., Blake, J. A., Botstein, D., Butler, H., Cherry, J. M., Davis, A. P., Dolinski, K., Dwight, S. S., Eppig, J. T., Harris, M. A., Hill, D. P., Issel-Tarver, L., Kasarskis, A., Lewis, S., Matese, J. C., Richardson, J. E., Ringwald, M., Rubin, G. M., \& Sherlock, G. (2000). Gene Ontology: tool for the unification of biology. Nat Genet, 25(1), 25-29.

Attar, E. C., Ervin, T., Janicek, M., Deykin, A., \& Godleski, J. (2000). Side Effects of Chemotherapy. Journal of Clinical Oncology, 18(3), 697-697. doi:10.1200/JCO.2000.18.3.697

Auger, A., Galarneau, L., Altaf, M., Nourani, A., Doyon, Y., Utley, R. T., Cronier, D., Allard, S., \& Côté, J. (2008). Eaf1 Is the Platform for NuA4 Molecular Assembly That Evolutionarily Links Chromatin Acetylation to ATP-Dependent Exchange of Histone H2A Variants. Molecular and Cellular Biology, 28(7), 2257-2270. doi:10.1128/mcb.01755-07

Avila, M. a. A., García-Trevijano, E. R., Lu, S. C., Corrales, F. J., \& Mato, J. M. (2004). Methylthioadenosine. The international journal of biochemistry \& cell biology, 36(11), 21252130. 
Axe, E. L., Walker, S. A., Manifava, M., Chandra, P., Roderick, H. L., Habermann, A., Griffiths, G., \& Ktistakis, N. T. (2008). Autophagosome formation from membrane compartments enriched in phosphatidylinositol 3-phosphate and dynamically connected to the endoplasmic reticulum. The Journal of Cell Biology, 182(4), 685-701.

Baba, M., Takeshige, K., Baba, N., \& Ohsumi, Y. (1994). Ultrastructural analysis of the autophagic process in yeast: detection of autophagosomes and their characterization. The Journal of Cell Biology, 124(6), 903-913.

Backlund, P., \& Smith, R. (1981). Methionine synthesis from 5'-methylthioadenosine in rat liver. Journal of Biological Chemistry, 256(4), 1533-1535.

Balakrishnan, K., Burger, J. A., Quiroga, M. P., Henneberg, M., Ayres, M. L., Wierda, W. G., \& Gandhi, V. (2010a). Influence of bone marrow stromal microenvironment on forodesine-induced responses in CLL primary cells. Blood, 116(7), 1083-1091.

Balakrishnan, K., Burger, J. A., Quiroga, M. P., Henneberg, M., Ayres, M. L., Wierda, W. G., \& Gandhi, V. (2010b). Influence of bone marrow stromal microenvironment on forodesine-induced responses in CLL primary cells. Blood, 116(7), 1083.

Balakrishnan, K., Nimmanapalli, R., Ravandi, F., Keating, M. J., \& Gandhi, V. (2006). Forodesine, an inhibitor of purine nucleoside phosphorylase, induces apoptosis in chronic lymphocytic leukemia cells. Blood, 108(7), 2392-2398. doi:10.1182/blood-2006-03-007468

Balakrishnan, R., Christie, K. R., Costanzo, M. C., Dolinski, K., Dwight, S. S., Engel, S. R., Fisk, D. G., Hirschman, J. E., Hong, E. L., Nash, R., Oughtred, R., Skrzypek, M., Theesfeld, C. L., Binkley, G., Dong, Q., Lane, C., Sethuraman, A., Weng, S., Botstein, D., \& Cherry, J. M. (2005). Fungal BLAST and Model Organism BLASTP Best Hits: new comparison resources at the Saccharomyces Genome Database (SGD). Nucleic Acids Res, 33(Database issue), D374-377. doi:10.1093/nar/gki023

Balakrishnan, R., Park, J., Karra, K., Hitz, B. C., Binkley, G., Hong, E. L., Sullivan, J., Micklem, G., \& Cherry, J. M. (2012). YeastMine--an integrated data warehouse for Saccharomyces cerevisiae data as a multipurpose tool-kit. Database (Oxford), 2012, bar062. doi:10.1093/database/bar062

Bammert, G. F., \& Fostel, J. M. (2000). Genome-wide expression patterns inSaccharomyces cerevisiae: comparison of drug treatments and genetic alterations affecting biosynthesis of ergosterol. Antimicrobial Agents and Chemotherapy, 44(5), 1255-1265.

Bantia, S., Ananth, S. L., Parker, C. D., Horn, L. L., \& Upshaw, R. (2003). Mechanism of inhibition of Tacute lymphoblastic leukemia cells by PNP inhibitor--BCX-1777. Int Immunopharmacol, 3(6), 879-887. doi:10.1016/s1567-5769(03)00076-6

Bantia, S., \& Kilpatrick, J. M. (2004). Purine nucleoside phosphorylase inhibitors in T-cell malignancies. Curr Opin Drug Discov Devel, 7(2), 243-247.

Bard, M., Lees, N., Turi, T., Craft, D., Cofrin, L., Barbuch, R., Koegel, C., \& Loper, J. (1993). Sterol synthesis and viability oferg11 (cytochrome P450 lanosterol demethylase) mutations inSaccharomyces cerevisiae andCandida albicans. Lipids, 28(11), 963-967.

Basu, I., Cordovano, G., Das, I., Belbin, T. J., Guha, C., \& Schramm, V. L. (2007). A Transition State Analogue of 5' -Methylthioadenosine Phosphorylase Induces Apoptosis in Head and Neck Cancers. Journal of Biological Chemistry, 282(29), 21477-21486. doi:10.1074/jbc.M702287200

Basu, I., Locker, J., Cassera, M. B., Belbin, T. J., Merino, E. F., Dong, X., Hemeon, I., Evans, G. B., Guha, C., \& Schramm, V. L. (2011). Growth and metastases of human lung cancer are inhibited in mouse xenografts by a transition state analogue of 5 '-methylthioadenosine phosphorylase. $J$ Biol Chem, 286(6), 4902-4911. doi:10.1074/jbc.M110.198374

Baudry, K., Swain, E., Rahier, A., Germann, M., Batta, A., Rondet, S., Mandala, S., Henry, K., Tint, G. S., \& Edlind, T. (2001). The effect of the erg26-1 mutation on the regulation of lipid metabolism in Saccharomyces cerevisiae. Journal of Biological Chemistry, 276(16), 12702-12711. 
Bedalov, A., Hirao, M., Posakony, J., Nelson, M., \& Simon, J. A. (2003). NAD+-dependent deacetylase Hst1p controls biosynthesis and cellular NAD+ levels in Saccharomyces cerevisiae. Molecular and Cellular Biology, 23(19), 7044-7054.

Belenky, P., Christensen, K. C., Gazzaniga, F., Pletnev, A. A., \& Brenner, C. (2009). Nicotinamide riboside and nicotinic acid riboside salvage in fungi and mammals quantitative basis for urh1 and purine nucleoside phosphorylase function in NAD+ metabolism. Journal of Biological Chemistry, 284(1), 158-164.

Belenky, P., Racette, F., Bogan, K., McClure, J., Smith, J., \& Brenner, C. (2007). Nicotinamide Riboside Promotes Sir2 Silencing and Extends Lifespan via Nrk and Urh1/Pnp1/Meu1 Pathways to NAD(+). Cell, 129(3), 473 - 484.

Beloribi-Djefaflia, S., Vasseur, S., \& Guillaumond, F. (2016). Lipid metabolic reprogramming in cancer cells. Oncogenesis, 5(1), e189.

Benjamini, Y., \& Hochberg, Y. (1995). Controlling the false discovery rate: a practical and powerful approach to multiple testing. Journal of the royal statistical society. Series B (Methodological), 289-300.

Bertino, J. R., Waud, W. R., Parker, W. B., \& Lubin, M. (2011). Targeting tumors that lack methylthioadenosine phosphorylase (MTAP) activity: current strategies. Cancer Biol Ther, 11(7), 627-632.

Best, H. A., Matthews, J. H., Heathcott, R. W., Hanna, R., Leahy, D. C., Coorey, N. V. C., Bellows, D. S., Atkinson, P. H., \& Miller, J. H. (2013). Laulimalide and peloruside A inhibit mitosis of Saccharomyces cerevisiae by preventing microtubule depolymerisation-dependent steps in chromosome separation and nuclear positioning. Molecular BioSystems, 9(11), 2842-2852. doi:10.1039/c3mb70211a

Bhuiyan, M. S. A., Eckstein, J., Barbuch, R., \& Bard, M. (2007). Synthetically lethal interactions involving loss of the yeast erg24: the sterol c-14 reductase gene. Lipids, 42(1), 69-76.

Bircham, P. W., Maass, D. R., Roberts, C. A., Kiew, P. Y., Low, Y. S., Yegambaram, M., Matthews, J., Jack, C. A., \& Atkinson, P. H. (2011). Secretory pathway genes assessed by high-throughput microscopy and synthetic genetic array analysis. Molecular BioSystems, 7(9), 2589-2598.

Blommaart, E. F., Krause, U., Schellens, J. P., Vreeling - Sindelárová, H., \& Meijer, A. J. (1997). The phosphatidylinositol 3 - kinase inhibitors wortmannin and LY294002 inhibit autophagy in isolated rat hepatocytes. Eur J Biochem, 243(1 - 2), 240-246.

Boone, C., Bussey, H., \& Andrews, B. J. (2007). Exploring genetic interactions and networks with yeast. Nature Reviews Genetics, 8(6), 437-449. doi:10.1038/nrg2085

Botstein, D., \& Fink, G. R. (2011). Yeast: An Experimental Organism for 21st Century Biology. Genetics, 189(3), 695-704. doi:10.1534/genetics.111.130765

Bouchez, I., Pouteaux, M., Canonge, M., Genet, M., Chardot, T., Guillot, A., \& Froissard, M. (2015). Regulation of lipid droplet dynamics in Saccharomyces cerevisiae depends on the Rab7-like Ypt7p, HOPS complex and V1-ATPase. Biology open, 4(7), 764-775.

Boudreau, D. M., Yu, O., \& Johnson, J. (2010). Statin use and cancer risk: a comprehensive review. Expert opinion on drug safety, 9(4), 603-621.

Boutros, C., Tarhini, A., Routier, E., Lambotte, O., Ladurie, F. L., Carbonnel, F., Izzeddine, H., Marabelle, A., Champiat, S., Berdelou, A., Lanoy, E., Texier, M., Libenciuc, C., Eggermont, A. M. M., Soria, J.-C., Mateus, C., \& Robert, C. (2016). Safety profiles of anti-CTLA-4 and anti-PD-1 antibodies alone and in combination. Nat Rev Clin Oncol, 13(8), 473-486. doi:10.1038/nrclinonc.2016.58

http://www.nature.com/nrclinonc/journal/v13/n8/abs/nrclinonc.2016.58.html\#supplementaryinformation

Bowman, S. M., \& Free, S. J. (2006). The structure and synthesis of the fungal cell wall. Bioessays, 28(8), 799-808.

Brachmann, B. C., Davies, A., Cost, G. J., Caputo, E., Li, J., Hieter, P., \& Boeke, J. D. (1998). Designer deletion strains derived from Saccharomyces cerevisiae S288C: A useful set of strains and 
plasmids for PCR-mediated gene disruption and other applications. Yeast, 14(2), 115-132. doi:10.1002/(sici)1097-0061(19980130)14:2<115::aid-yea204>3.0.co;2-2

Braus, G. H. (1991). Aromatic amino acid biosynthesis in the yeast Saccharomyces cerevisiae: a model system for the regulation of a eukaryotic biosynthetic pathway. Microbiological Reviews, 55(3), 349-370.

Brett, C. L., Plemel, R. L., Lobingier, B. T., Vignali, M., Fields, S., \& Merz, A. J. (2008). Efficient termination of vacuolar Rab GTPase signaling requires coordinated action by a GAP and a protein kinase. J Cell Biol, 182(6), 1141-1151. doi:10.1083/jcb.200801001

Budovskaya, Y. V., Stephan, J. S., Reggiori, F., Klionsky, D. J., \& Herman, P. K. (2004). The Ras/cAMPdependent protein kinase signaling pathway regulates an early step of the autophagy process in Saccharomyces cerevisiae. J Biol Chem, 279(20), 20663-20671. doi:10.1074/jbc.M400272200

Burman, C., \& Ktistakis, N. T. (2010). Regulation of autophagy by phosphatidylinositol 3 - phosphate. Febs Letters, 584(7), 1302-1312.

Byrne, K. P., \& Wolfe, K. H. (2005). The Yeast Gene Order Browser: combining curated homology and syntenic context reveals gene fate in polyploid species. Genome Research, 15(10), 1456-1461.

Cai, H., Hauser, M., Naider, F., \& Becker, J. M. (2007). Differential regulation and substrate preferences in two peptide transporters of Saccharomyces cerevisiae. Eukaryotic Cell, 6(10), 1805-1813.

Cairns, P., Polascik, T. J., Eby, Y., Tokino, K., Califano, J., Merlo, A., Mao, L., Herath, J., Jenkins, R., \& Westra, W. (1995). Frequency of homozygous deletion at p16/CDKN2 in primary human tumours. Nature genetics, 11(2), 210-212.

Camarda, R., Zhou, A. Y., Kohnz, R. A., Balakrishnan, S., Mahieu, C., Anderton, B., Eyob, H., Kajimura, S., Tward, A., \& Krings, G. (2016). Inhibition of fatty acid oxidation as a therapy for MYCoverexpressing triple-negative breast cancer. Nature medicine, 22(4), 427-432.

Canduri, F., Silva, R. G., dos Santos, D. M., Palma, M. S., Basso, L. A., Santos, D. S., \& de Azevedo, W. F., Jr. (2005). Structure of human PNP complexed with ligands. Acta Crystallogr D Biol Crystallogr, 61(Pt 7), 856-862. doi:10.1107/s0907444905005421

Cano - Soldado, P., \& Pastor - Anglada, M. (2012). Transporters that translocate nucleosides and structural similar drugs: structural requirements for substrate recognition. Medicinal research reviews, 32(2), 428-457.

Cantoni, G. L. (1975). Biological methylation: selected aspects. Annual Review of Biochemistry, 44(1), 435-451.

Cantwell-Dorris, E. R., Leary, J. J., \& Sheils, O. M. (2011). BRAF\&lt;sup\&gt;V600E\&lt;/sup\&gt;: Implications for Carcinogenesis and Molecular Therapy. Molecular Cancer Therapeutics, 10(3), 385.

Carman, A. J., Vylkova, S., \& Lorenz, M. C. (2008). Role of acetyl coenzyme A synthesis and breakdown in alternative carbon source utilization in Candida albicans. Eukaryotic Cell, 7(10), 1733-1741.

Carmona-Gutierrez, D., Eisenberg, T., Buttner, S., Meisinger, C., Kroemer, G., \& Madeo, F. (2010). Apoptosis in yeast: triggers, pathways, subroutines. Cell Death Differ, 17(5), 763-773.

Carrera, C. J., Eddy, R. L., Shows, T. B., \& Carson, D. A. (1984). Assignment of the gene for methylthioadenosine phosphorylase to human chromosome 9 by mouse-human somatic cell hybridization. Proceedings of the National Academy of Sciences, 81(9), 2665-2668.

Carson, D. A., Kaye, J., \& Seegmiller, J. E. (1977). Lymphospecific toxicity in adenosine deaminase deficiency and purine nucleoside phosphorylase deficiency: possible role of nucleoside kinase(s). Proc Natl Acad Sci U S A, 74(12), 5677-5681.

Carson, D. A., Seto, S., Wasson, D. B., \& Carrera, C. J. (1986). DNA strand breaks, NAD metabolism, and programmed cell death. Exp Cell Res, 164(2), 273-281.

Casero, R. A., \& Marton, L. J. (2007). Targeting polyamine metabolism and function in cancer and other hyperproliferative diseases. Nature reviews Drug discovery, 6(5), 373-390. 
Castrejon, F., Gomez, A., Sanz, M., Duran, A., \& Roncero, C. (2006). The RIM101 pathway contributes to yeast cell wall assembly and its function becomes essential in the absence of mitogenactivated protein kinase Slt2p. Eukaryotic Cell, 5(3), 507-517.

Chantin, C., Bonin, B., Boulieu, R., \& Bory, C. (1996). Liquid-chromatographic study of purine metabolism abnormalities in purine nucleoside phosphorylase deficiency. Clinical Chemistry, 42(2), 326.

Chattopadhyay, M. K., Tabor, C. W., \& Tabor, H. (2006). Methylthioadenosine and polyamine biosynthesis in a Saccharomyces cerevisiae meu1s mutant. Biochemical and Biophysical Research Communications, 343(1), 203-207.

Cheek, J., \& Broderick, J. B. (2001). Adenosylmethionine-dependent iron-sulfur enzymes: versatile clusters in a radical new role. Journal of Biological Inorganic Chemistry, 6(3), 209-226.

Chen, L., \& Cui, H. (2015). Targeting glutamine induces apoptosis: a cancer therapy approach. International journal of molecular sciences, 16(9), 22830-22855.

Chen, Y., Zhou, F., Zou, S., Yu, S., Li, S., Li, D., Song, J., Li, H., He, Z., Hu, B., Bjorn, L. O., Lipatova, Z., Liang, Y., Xie, Z., \& Segev, N. (2014). A Vps21 endocytic module regulates autophagy. Mol Biol Cell, 25(20), 3166-3177. doi:10.1091/mbc.E14-04-0917

Chen, Z.-H., Zhang, H., \& Savarese, T. M. (1996). Gene Deletion Chemoselectivity: Codeletion of the Genes for p16INK4, Methylthioadenosine Phosphorylase, and the $\alpha$-and $\beta$-Interferons in Human Pancreatic Cell Carcinoma Lines and Its Implications for Chemotherapy. Cancer research, 56(5), 1083-1090.

Cheng, J., Ohsaki, Y., Tauchi-Sato, K., Fujita, A., \& Fujimoto, T. (2006). Cholesterol depletion induces autophagy. Biochemical and Biophysical Research Communications, 351(1), 246-252.

Cheng, X., Auger, A., Altaf, M., Drouin, S., Paquet, E., Utley, R. T., Robert, F., \& Côté, J. (2015). Eaf1 links the NuA4 histone acetyltransferase complex to Htz1 incorporation and regulation of purine biosynthesis. Eukaryotic Cell, 14(6), 535-544.

Cheng, Y., Ren, X., Hait, W. N., \& Yang, J.-M. (2013). Therapeutic targeting of autophagy in disease: biology and pharmacology. Pharmacological Reviews, 65(4), 1162-1197.

Cheong, H., Nair, U., Geng, J., \& Klionsky, D. J. (2008). The Atg1 kinase complex is involved in the regulation of protein recruitment to initiate sequestering vesicle formation for nonspecific autophagy in Saccharomyces cerevisiae. Molecular Biology of the Cell, 19(2), 668-681.

Cherry, J. M., Hong, E. L., Amundsen, C., Balakrishnan, R., Binkley, G., Chan, E. T., Christie, K. R., Costanzo, M. C., Dwight, S. S., Engel, S. R., Fisk, D. G., Hirschman, J. E., Hitz, B. C., Karra, K., Krieger, C. J., Miyasato, S. R., Nash, R. S., Park, J., Skrzypek, M. S., Simison, M., Weng, S., \& Wong, E. D. (2012). Saccharomyces Genome Database: the genomics resource of budding yeast. Nucleic Acids Research, 40(D1), D700-D705. doi:10.1093/nar/gkr1029

Choi, A. M., Ryter, S. W., \& Levine, B. (2013). Autophagy in human health and disease. New England Journal of Medicine, 368(7), 651-662.

Christopher, S. A., Diegelman, P., Porter, C. W., \& Kruger, W. D. (2002). Methylthioadenosine phosphorylase, a gene frequently codeleted with p16cdkN2a/ARF, acts as a tumor suppressor in a breast cancer cell line. Cancer research, 62(22), 6639-6644.

Ciarimboli, G. (2008). Organic cation transporters. Xenobiotica, 38(7-8), 936-971.

Codogno, P., Mehrpour, M., \& Proikas-Cezanne, T. (2012). Canonical and non-canonical autophagy: variations on a common theme of self-eating? Nature reviews Molecular cell biology, 13(1), 712.

Coffino, P. (2001). Regulation of cellular polyamines by antizyme. Nat Rev Mol Cell Biol, 2(3), 188-194.

Cohen, A., Gudas, L. J., Ammann, A. J., Staal, G. E. J., \& Martin, D. W. (1978). Deoxyguanosine Triphosphate as a Possible Toxic Metabolite in the Immunodeficiency Associated with Purine Nucleoside Phosphorylase Deficiency. Journal of Clinical Investigation, 61(5), 1405-1409.

Comerford, S. A., Huang, Z., Du, X., Wang, Y., Cai, L., Witkiewicz, A. K., Walters, H., Tantawy, M. N., Fu, A., \& Manning, H. C. (2014). Acetate dependence of tumors. Cell, 159(7), 1591-1602. 
Cong, F., Cheung, A. K., \& Huang, S. M. (2012). Chemical genetics-based target identification in drug discovery. Annu Rev Pharmacol Toxicol, 52, 57-78. doi:10.1146/annurev-pharmtox-010611134639

Cooper, G. M. (2000). The development and causes of cancer. The cell: A molecular approach.

Coorey, N. C., Sampson, L. P., Barber, J., \& Bellows, D. (2014). Chemical Genetic and Chemogenomic Analysis in Yeast. In J. S. Smith \& D. J. Burke (Eds.), Yeast Genetics (Vol. 1205, pp. 169-186): Springer New York.

Coorey, N. V. C., Matthews, J. H., Bellows, D. S., \& Atkinson, P. H. (2015). Pleiotropic drug-resistance attenuated genomic library identified more drug target specific genetic interactions than the commercially available genomic libraries. Molecular BioSystems. doi:10.1039/C5MB00406C

Corbet, C., \& Feron, O. (2017). Cancer cell metabolism and mitochondria: nutrient plasticity for TCA cycle fueling. Biochimica et Biophysica Acta (BBA)-Reviews on Cancer.

Curbo, S., Zhu, C., Johansson, M., Balzarini, J., \& Karlsson, A. (2001). Dual Mechanisms of 9- $\beta$-dArabinofuranosylguanine Resistance in CEM T-Lymphoblast Leukemia Cells. Biochemical and Biophysical Research Communications, 285(1), 40-45. doi:http://dx.doi.org/10.1006/bbrc.2001.5124

Daignan-Fornier, B., \& Fink, G. (1992). Coregulation of purine and histidine biosynthesis by the transcriptional activators BAS1 and BAS2. Proceedings of the National Academy of Sciences, 89(15), 6746-6750.

Dall'Armi, C., Devereaux, K. A., \& Di Paolo, G. (2013). The role of lipids in the control of autophagy. Current Biology, 23(1), R33-R45.

De Antoni, A., Schmitzová, J., Trepte, H.-H., Gallwitz, D., \& Albert, Š. (2002). Significance of GTP Hydrolysis in Ypt1p-regulated Endoplasmic Reticulum to Golgi Transport Revealed by the Analysis of Two Novel Ypt1-GAPs. Journal of Biological Chemistry, 277(43), 41023-41031. doi:10.1074/jbc.M205783200

de Clare, M., \& Oliver, S. (2013). Copy-number variation of cancer-gene orthologs is sufficient to induce cancer-like symptoms in Saccharomyces cerevisiae. BMC Biology, 11(1), 1-15. doi:10.1186/1741-7007-11-24

de Kroon, A. I., Rijken, P. J., \& De Smet, C. H. (2013). Checks and balances in membrane phospholipid class and acyl chain homeostasis, the yeast perspective. Progress in lipid research, 52(4), 374394.

DeBerardinis, R. J., Mancuso, A., Daikhin, E., Nissim, I., Yudkoff, M., Wehrli, S., \& Thompson, C. B. (2007). Beyond aerobic glycolysis: transformed cells can engage in glutamine metabolism that exceeds the requirement for protein and nucleotide synthesis. Proc Natl Acad Sci U S A, 104(49), 19345-19350. doi:10.1073/pnas.0709747104

Della Ragione, F., Carteni-Farina, M., Gragnaniello, V., Schettino, M. I., \& Zappia, V. (1986). Purification and characterization of 5 '-deoxy-5'-methylthioadenosine phosphorylase from human placenta. Journal of Biological Chemistry, 261(26), 12324-12329.

Demierre, M.-F., Higgins, P. D., Gruber, S. B., Hawk, E., \& Lippman, S. M. (2005). Statins and cancer prevention. Nature Reviews Cancer, 5(12), 930-942.

Dengjel, J., Hoyer-Hansen, M., Nielsen, M. O., Eisenberg, T., Harder, L. M., Schandorff, S., Farkas, T., Kirkegaard, T., Becker, A. C., Schroeder, S., Vanselow, K., Lundberg, E., Nielsen, M. M., Kristensen, A. R., Akimov, V., Bunkenborg, J., Madeo, F., Jaattela, M., \& Andersen, J. S. (2012). Identification of autophagosome-associated proteins and regulators by quantitative proteomic analysis and genetic screens. Mol Cell Proteomics, 11(3), M111.014035. doi:10.1074/mcp.M111.014035

DeWolf, W., Fullin, F. A., \& Schramm, V. (1979). The catalytic site of AMP nucleosidase. Substrate specificity and $\mathrm{pH}$ effects with AMP and formycin 5'-PO4. Journal of Biological Chemistry, 254(21), 10868-10875. 
Diaz-Ruiz, R., Rigoulet, M., \& Devin, A. (2011). The Warburg and Crabtree effects: on the origin of cancer cell energy metabolism and of yeast glucose repression. Biochimica et Biophysica Acta (BBA)-Bioenergetics, 1807(6), 568-576.

Dickinson, J. R., Salgado, L. E. J., \& Hewlins, M. J. (2003). The catabolism of amino acids to long chain and complex alcohols in Saccharomyces cerevisiae. Journal of Biological Chemistry, 278(10), 8028-8034.

DiNardo, S., Voelkel, K., \& Sternglanz, R. (1984). DNA topoisomerase II mutant of Saccharomyces cerevisiae: topoisomerase II is required for segregation of daughter molecules at the termination of DNA replication. Proceedings of the National Academy of Sciences, 81(9), 26162620.

Ding, W., Smulan, L. J., Hou, N. S., Taubert, S., Watts, J. L., \& Walker, A. K. (2015). sAdenosylmethionine levels govern innate immunity through distinct methylation-dependent pathways. Cell metabolism, 22(4), 633-645.

Dittmar, J. C., Reid, R. J., \& Rothstein, R. (2010). ScreenMill: A freely available software suite for growth measurement, analysis and visualization of high-throughput screen data. BMC Bioinformatics, 11(1), 1-11. doi:10.1186/1471-2105-11-353

Douglas, A. C., Smith, A. M., Sharifpoor, S., Yan, Z., Durbic, T., Heisler, L. E., Lee, A. Y., Ryan, O., Göttert, H., \& Surendra, A. (2012). Functional analysis with a barcoder yeast gene overexpression system. G3: Genes/ Genomes/ Genetics, 2(10), 1279-1289.

Dronkert, M. L., \& Kanaar, R. (2001). Repair of DNA interstrand cross-links. Mutation Research/DNA Repair, 486(4), 217-247.

Eisenberg, T., Knauer, H., Schauer, A., Büttner, S., Ruckenstuhl, C., Carmona-Gutierrez, D., Ring, J., Schroeder, S., Magnes, C., \& Antonacci, L. (2009). Induction of autophagy by spermidine promotes longevity. Nature Cell Biology, 11(11), 1305-1314.

Engel, S. R., Dietrich, F. S., Fisk, D. G., Binkley, G., Balakrishnan, R., Costanzo, M. C., Dwight, S. S., Hitz, B. C., Karra, K., Nash, R. S., Weng, S., Wong, E. D., Lloyd, P., Skrzypek, M. S., Miyasato, S. R., Simison, M., \& Cherry, J. M. (2014). The Reference Genome Sequence of Saccharomyces cerevisiae: Then and Now. G3: Genes/Genomes/Genetics, 4(3), 389-398. doi:10.1534/g3.113.008995

Eriksson, S., Munch-Petersen, B., Johansson, K., \& Eklund, H. (2002). Structure and function of cellular deoxyribonucleoside kinases. Cell Mol Life Sci, 59(8), 1327-1346.

Evans, G. B., Furneaux, R. H., Lewandowicz, A., Schramm, V. L., \& Tyler, P. C. (2003). Exploring Structure-Activity Relationships of Transition State Analogues of Human Purine Nucleoside Phosphorylase. Journal of Medicinal Chemistry, 46(15), 3412-3423. doi:10.1021/jm030145r

Evans, G. B., Furneaux, R. H., Schramm, V. L., Singh, V., \& Tyler, P. C. (2004). Targeting the Polyamine Pathway with Transition-State Analogue Inhibitors of 5'-Methylthioadenosine Phosphorylase. Journal of Medicinal Chemistry, 47(12), 3275-3281. doi:10.1021/jm0306475

Feng, Y., He, D., Yao, Z., \& Klionsky, D. J. (2014). The machinery of macroautophagy. Cell research, 24(1), 24-41.

Ferlay, J., Soerjomataram, I., Dikshit, R., Eser, S., Mathers, C., Rebelo, M., Parkin, D. M., Forman, D., \& Bray, F. (2015). Cancer incidence and mortality worldwide: sources, methods and major patterns in GLOBOCAN 2012. International journal of cancer, 136(5), E359-E386.

Ferreira, L. M. (2010). Cancer metabolism: the Warburg effect today. Experimental and molecular pathology, 89(3), 372-380.

Finkelstein, J. D. (1990). Methionine metabolism in mammals. The Journal of nutritional biochemistry, $1(5), 228-237$.

Forsburg, S. L. (2001). The art and design of genetic screens: yeast. Nat Rev Genet, 2(9), 659-668.

Fox, I. H., Kaminska, J., Edwards, N. L., Gelfand, E., Rich, K. C., \& Arnold, W. N. (1980). Altered purine and pyrimidine metabolism in erythrocytes with purine nucleoside phosphorylase deficiency. Biochemical genetics, 18(3-4), 221-234. 
Fridland, A., \& Verhoef, V. (1985). Metabolism and selectivity of arabinonucleoside in human lymphoid cells. Proc Soc Exp Biol Med, 179(4), 456-462.

Friesen, H., Tanny, J. C., \& Segall, J. (1998). SPE3, which encodes spermidine synthase, is required for full repression through NREDIT in Saccharomyces cerevisiae. Genetics, 150(1), 59-73.

Frostesjo, L., Holm, I., Grahn, B., Page, A. W., Bestor, T. H., \& Heby, O. (1997). Interference with DNA methyltransferase activity and genome methylation during F9 teratocarcinoma stem cell differentiation induced by polyamine depletion. J Biol Chem, 272(7), 4359-4366.

Fyfe, J., Keller, P., Furman, P., Miller, R., \& Elion, G. (1978). Thymidine kinase from herpes simplex virus phosphorylates the new antiviral compound, 9-(2-hydroxyethoxymethyl) guanine. Journal of Biological Chemistry, 253(24), 8721-8727.

Gaber, R., Copple, D., Kennedy, B., Vidal, M., \& Bard, M. (1989). The yeast gene ERG6 is required for normal membrane function but is not essential for biosynthesis of the cell-cycle-sparking sterol. Molecular and Cellular Biology, 9(8), 3447-3456.

Gaglio, D., Metallo, C. M., Gameiro, P. A., Hiller, K., Danna, L. S., Balestrieri, C., Alberghina, L., Stephanopoulos, G., \& Chiaradonna, F. (2011). Oncogenic K-Ras decouples glucose and glutamine metabolism to support cancer cell growth. Mol Syst Biol, 7, 523. doi:10.1038/msb.2011.56

Gandhi, V. (2009). Forodesine-Preclinical Studies. Paper presented at the Hematology Meeting Reports (formerly Haematologica Reports).

Gaynor, P. M., Gill, T., Toutenhoofd, S., Summers, E. F., McGraw, P., Homann, M. J., Henry, S. A., \& Carman, G. M. (1991). Regulation of phosphatidylethanolamine methyltransferase and phospholipid methyltransferase by phospholipid precursors in Saccharomyces cerevisiae. Biochimica et Biophysica Acta (BBA)-Gene Structure and Expression, 1090(3), 326-332.

Gene Ontology Consortium. (2004). The Gene Ontology (GO) database and informatics resource. Nucl. Acids Res., 32(suppl_1), D258-261. doi:10.1093/nar/gkh036

Ghislain, M., Talla, E., \& François, J. M. (2002). Identification and functional analysis of the Saccharomyces cerevisiae nicotinamidase gene, PNC1. Yeast, 19(3), 215-224.

Giblett, E., Ammann, A., Sandman, R., Wara, D., \& Diamond, L. (1975). Nucleoside-phosphorylase deficiency in a child with severely defective T-cell immunity and normal B-cell immunity. The Lancet, 305(7914), 1010-1013. doi:http://dx.doi.org/10.1016/S0140-6736(75)91950-9

Gietz, R. D., \& Schiestl, R. H. (2007). High-efficiency yeast transformation using the LiAc/SS carrier DNA/PEG method. Nat. Protocols, 2(1), 31-34.

Gilbertsen, R. B., Scott, M. E., Dong, M. K., Kossarek, L. M., Bennett, M. K., Schrier, D. J., \& Sircar, J. C. (1987). Preliminary report on 8-amino-9-(2-thienylmethyl)guanine (PD 119,229), a novel and potent purine nucleoside phosphorylase inhibitor. Agents Actions, 21(3-4), 272-274.

Goffeau, A., Barrell, B. G., Bussey, H., Davis, R. W., Dujon, B., Feldmann, H., Galibert, F., Hoheisel, J. D., Jacq, C., Johnston, M., Louis, E. J., Mewes, H. W., Murakami, Y., Philippsen, P., Tettelin, H., \& Oliver, S. G. (1996). Life with 6000 genes. Science, 274(5287), 546-\&. doi:10.1126/science.274.5287.546

Goldstein, A. L., \& McCusker, J. H. (1999). Three new dominant drug resistance cassettes for gene disruption in Saccharomyces cerevisiae. Yeast, 15(14), 1541-1553. doi:10.1002/(sici)10970061(199910)15:14<1541::aid-yea476>3.0.co;2-k

Gordon, R. B., Blackwell, K., \& Emmerson, B. T. (1987). Synthesis of purines in human lymphoblast cells deficient in methylthioadenosine phosphorylase activity. Biochimica et Biophysica Acta (BBA)Molecular Cell Research, 927(1), 1-7.

Gordon, R. B., \& Emmerson, B. T. (1986). Inhibition of De Novo Purine Synthesis by Methylthioadenosine Purine and Pyrimidine Metabolism in Man V (pp. 659-662): Springer.

Griffiths, M., Beaumont, N., Yao, S. Y., Sundaram, M., Boumah, C. E., Davies, A., Kwong, F. Y., Coe, I., Cass, C. E., \& Young, J. D. (1997). Cloning of a human nucleoside transporter implicated in the cellular uptake of adenosine and chemotherapeutic drugs. Nature medicine, 3(1), 89-93. 
Gross, M. I., Demo, S. D., Dennison, J. B., Chen, L., Chernov-Rogan, T., Goyal, B., Janes, J. R., Laidig, G. J., Lewis, E. R., Li, J., Mackinnon, A. L., Parlati, F., Rodriguez, M. L., Shwonek, P. J., Sjogren, E. B., Stanton, T. F., Wang, T., Yang, J., Zhao, F., \& Bennett, M. K. (2014). Antitumor activity of the glutaminase inhibitor CB-839 in triple-negative breast cancer. Mol Cancer Ther, 13(4), 890901. doi:10.1158/1535-7163.mct-13-0870

Grubmeyer, C. T., Insinga, S., Bhatia, M., \& Moazami, N. (1989). Salmonella typhimurium histidinol dehydrogenase: complete reaction stereochemistry and active site mapping. Biochemistry, 28(20), 8174-8180.

Gugliucci, A. (2004). Polyamines as clinical laboratory tools. Clin Chim Acta, 344(1-2), 23-35. doi:10.1016/j.cccn.2004.02.022

Guthrie, C., \& Fink, G. R. (2002). Guide to yeast genetics and molecular and cell biology: Gulf Professional Publishing.

Ha, H. C., Woster, P. M., Yager, J. D., \& Casero, R. A. (1997). The role of polyamine catabolism in polyamine analogue-induced programmed cell death. Proceedings of the National Academy of Sciences of the United States of America, 94(21), 11557-11562.

Hall, C., \& Dietrich, F. S. (2007). The reacquisition of biotin prototrophy in Saccharomyces cerevisiae involved horizontal gene transfer, gene duplication and gene clustering. Genetics, 177(4), 2293-2307.

Hall, M. J., Middleton, R. F., \& Westmacott, D. (1983). The fractional inhibitory concentration (FIC) index as a measure of synergy. Journal of Antimicrobial Chemotherapy, 11(5), 427-433. doi:10.1093/jac/11.5.427

Hamosh, A., Scott, A. F., Amberger, J. S., Bocchini, C. A., \& McKusick, V. A. (2005). Online Mendelian Inheritance in Man (OMIM), a knowledgebase of human genes and genetic disorders. Nucleic Acids Research, 33(suppl 1), D514-D517.

Hanahan, D., \& Weinberg, R. A. (2000). The Hallmarks of Cancer. Cell, 100(1), 57-70. doi:http://dx.doi.org/10.1016/S0092-8674(00)81683-9

Hanahan, D., \& Weinberg, R. A. (2011). Hallmarks of cancer: the next generation. Cell, 144(5), 646674.

Hardwick, K. G., \& Pelham, H. R. B. (1994). XIII. Yeast sequencing reports. SED6 is identical to ERG6, and encodes a putative methyltransferase required for ergosterol synthesis. Yeast, 10(2), 265269. doi:10.1002/yea.320100213

Hartwell, L. H., Culotti, J., Pringle, J. R., \& Reid, B. J. (1974). Genetic controlof the cell division cycle in yeast. Science (Wash. DC), 183, 46-51.10.

Haucke, V., \& Di Paolo, G. (2007). Lipids and lipid modifications in the regulation of membrane traffic. Current Opinion in Cell Biology, 19(4), 426-435.

Hauser, M., Narita, V., Donhardt, A. M., Naider, F., \& Becker, J. M. (2001). Multiplicity and regulation of genes encoding peptide transporters in Saccharomyces cerevisiae. Molecular membrane biology, 18(1), 105-112.

Hayashi, M., Fukuzawa, T., Sorimachi, H., \& Maeda, T. (2005). Constitutive activation of the pHresponsive Rim101 pathway in yeast mutants defective in late steps of the MVB/ESCRT pathway. Molecular and Cellular Biology, 25(21), 9478-9490.

He, Y., Swaminathan, A., \& Lopes, J. M. (2012). Transcription regulation of the Saccharomyces cerevisiae PHO5 gene by the Ino2p and Ino4p basic helix-loop-helix proteins. Mol Microbiol, 83(2), 395-407. doi:10.1111/j.1365-2958.2011.07941.x

Heidepriem, R. W., Livant, P. D., Parish, E. J., Barbuch, R. J., Broaddus, M. G., \& Bard, M. (1992). A simple method for the isolation of zymosterol from a sterol mutant of Saccharomyces cerevisiae. The Journal of steroid biochemistry and molecular biology, 43(7), 741-743.

Henry, S. A., Kohlwein, S. D., \& Carman, G. M. (2012). Metabolism and regulation of glycerolipids in the yeast Saccharomyces cerevisiae. Genetics, 190(2), 317-349. 
Hensley, C. T., Faubert, B., Yuan, Q., Lev-Cohain, N., Jin, E., Kim, J., Jiang, L., Ko, B., Skelton, R., Loudat, L., Wodzak, M., Klimko, C., McMillan, E., Butt, Y., Ni, M., Oliver, D., Torrealba, J., Malloy, C. R., Kernstine, K., Lenkinski, R. E., \& DeBerardinis, R. J. (2016). Metabolic Heterogeneity in Human Lung Tumors. Cell, 164(4), 681-694. doi:10.1016/j.cell.2015.12.034

Hensley, M. L., Hagerty, K. L., Kewalramani, T., Green, D. M., Meropol, N. J., Wasserman, T. H., Cohen, G. I., Emami, B., Gradishar, W. J., Mitchell, R. B., Thigpen, J. T., Trotti, A., von Hoff, D., \& Schuchter, L. M. (2009). American Society of Clinical Oncology 2008 Clinical Practice Guideline Update: Use of Chemotherapy and Radiation Therapy Protectants. Journal of Clinical Oncology, 27(1), 127-145. doi:10.1200/JCO.2008.17.2627

Herraiz, M., Beraza, N., Solano, A., Sangro, B., Montoya, J., Qian, C., Prieto, J., \& Bustos, M. (2003). Liver failure caused by herpes simplex virus thymidine kinase plus ganciclovir therapy is associated with mitochondrial dysfunction and mitochondrial DNA depletion. Hum Gene Ther, 14(5), 463-472. doi:10.1089/104303403321467225

Hershfield, M., \& Mitchell, B. (1995). Immunodeficiency diseases caused by adenosine deaminase deficiency and purine nucleoside phosphorylase deficiency. The metabolic and molecular bases of inherited disease, 2, 1725-1768.

Hirschhorn, R., Grunebaum, E., Roifman, C., \& Candotti, F. (2013). Immunodeficiency due to defects of purine metabolism. Primary immunodeficiency diseases: a molecular and genetic approach, 188-230.

Ho, C. H., Piotrowski, J., Dixon, S. J., Baryshnikova, A., Costanzo, M., \& Boone, C. (2011). Combining functional genomics and chemical biology to identify targets of bioactive compounds. Current Opinion in Chemical Biology, 15(1), 66-78. doi:10.1016/j.cbpa.2010.10.023

Hobbs, C. A., \& Gilmour, S. K. (2000). High levels of intracellular polyamines promote histone acetyltransferase activity resulting in chromatin hyperacetylation. Journal of cellular biochemistry, 77(3), 345-360.

Hobbs, C. A., Paul, B. A., \& Gilmour, S. K. (2002). Deregulation of polyamine biosynthesis alters intrinsic histone acetyltransferase and deacetylase activities in murine skin and tumors. Cancer research, 62(1), 67-74.

Hoepfner, D., Helliwell, S. B., Sadlish, H., Schuierer, S., Filipuzzi, I., Brachat, S., Bhullar, B., Plikat, U., Abraham, Y., \& Altorfer, M. (2014). High-resolution chemical dissection of a model eukaryote reveals targets, pathways and gene functions. Microbiological research, 169(2), 107-120.

Holbein, S., Wengi, A., Decourty, L., Freimoser, F. M., Jacquier, A., \& Dichtl, B. (2009). Cordycepin interferes with $3^{\prime}$ end formation in yeast independently of its potential to terminate RNA chain elongation. RNA, 15(5), 837-849. doi:10.1261/rna.1458909

Holm, E., Hagmuller, E., Staedt, U., Schlickeiser, G., Gunther, H. J., Leweling, H., Tokus, M., \& Kollmar, H. B. (1995). Substrate balances across colonic carcinomas in humans. Cancer Res, 55(6), 13731378.

Huang, M., Wang, Y., Gu, J., Yang, J., Noel, K., Mitchell, B. S., Schramm, V. L., \& Graves, L. M. (2008). Determinants of sensitivity of human T-cell leukemia CCRF-CEM cells to immucillin-H. Leukemia Research, 32(8), 1268-1278. doi:10.1016/j.leukres.2007.12.015

Huh, W.-K., Falvo, J. V., Gerke, L. C., Carroll, A. S., Howson, R. W., Weissman, J. S., \& O'shea, E. K. (2003). Global analysis of protein localization in budding yeast. Nature, 425(6959), 686-691.

Igarashi, K., HASHIMOTO, S., MIYAKE, A., KASHIWAGI, K., \& HIROSE, S. (1982). Increase of fidelity of polypeptide synthesis by spermidine in eukaryotic cell - free systems. Eur J Biochem, 128(2 3), 597-604.

Iwanyshyn, W. M., Han, G.-S., \& Carman, G. M. (2004). Regulation of phospholipid synthesis in Saccharomyces cerevisiae by zinc. Journal of Biological Chemistry, 279(21), 21976-21983.

Iwata-Reuyl, D. (2003). Biosynthesis of the 7-deazaguanosine hypermodified nucleosides of transfer RNA. Bioorganic chemistry, 31(1), 24-43. 
Jaishy, B., \& Abel, E. D. (2016). Lipids, lysosomes, and autophagy. Journal of Lipid Research, 57(9), 1619-1635.

Janes, W., \& Schulz, G. E. (1990). Role of the charged groups of glutathione disulfide in the catalysis of glutathione reductase: crystallographic and kinetic studies with synthetic analogues. Biochemistry, 29(16), 4022-4030.

Janke, C., Magiera, M. M., Rathfelder, N., Taxis, C., Reber, S., Maekawa, H., Moreno-Borchart, A., Doenges, G., Schwob, E., Schiebel, E., \& Knop, M. (2004). A versatile toolbox for PCR-based tagging of yeast genes: new fluorescent proteins, more markers and promoter substitution cassettes. Yeast, 21(11), 947-962. doi:10.1002/yea.1142

Jemal, A., Bray, F., Center, M. M., Ferlay, J., Ward, E., \& Forman, D. (2011). Global cancer statistics. CA: A Cancer Journal for Clinicians, 61(2), 69-90. doi:10.3322/caac.20107

Jensen, K. F., \& Nygaard, P. (1975). Purine nucleoside phosphorylase from Escherichia coli and Salmonella typhimurium. Purification and some properties. Eur J Biochem, 51(1), 253-265.

Jiang, P., \& Mizushima, N. (2014). Autophagy and human diseases. Cell research, 24(1), 69-79.

Jones, E. W., \& Fink, G. R. (1982). Regulation of amino acid and nucleotide biosynthesis in yeast. Cold Spring Harbor Monograph Archive, 11, 181-299.

Jouhten, P., Ponomarova, O., Gonzalez, R., \& Patil, K. R. (2016). Saccharomyces cerevisiae metabolism in ecological context. FEMS Yeast Research, 16(7), fow080. doi:10.1093/femsyr/fow080

Kabeya, Y., Mizushima, N., Yamamoto, A., Oshitani-Okamoto, S., Ohsumi, Y., \& Yoshimori, T. (2004). LC3, GABARAP and GATE16 localize to autophagosomal membrane depending on form-II formation. J Cell Sci, 117(Pt 13), 2805-2812. doi:10.1242/jcs.01131

Kachroo, A. H., Laurent, J. M., Yellman, C. M., Meyer, A. G., Wilke, C. O., \& Marcotte, E. M. (2015). Systematic humanization of yeast genes reveals conserved functions and genetic modularity. Science, 348(6237), 921-925.

Kaeberlein, M. (2009). Spermidine surprise for a long life. Nature Cell Biology, 11(11), 1277-1278.

Kahana, C., Asher, G., \& Shaul, Y. (2005). Mechanisms of protein degradation: an odyssey with ODC. Cell Cycle, 4(11), 1461-1464. doi:10.4161/cc.4.11.2115

Kamada, Y., Funakoshi, T., Shintani, T., Nagano, K., Ohsumi, M., \& Ohsumi, Y. (2000). Tor-mediated induction of autophagy via an Apg1 protein kinase complex. The Journal of Cell Biology, 150(6), 1507-1513.

Kanehisa, M., \& Goto, S. (2000). KEGG: kyoto encyclopedia of genes and genomes. Nucleic Acids Research, 28(1), 27-30.

Kasibhatla, S. R., Bookser, B. C., Xiao, W., \& Erion, M. D. (2001). AMP deaminase inhibitors. 5. Design, synthesis, and SAR of a highly potent inhibitor series. J Med Chem, 44(4), 613-618.

Kazmers, I. S., Mitchell, B. S., Dadonna, P. E., Wotring, L. L., Townsend, L. B., \& Kelley, W. N. (1981). Inhibition of purine nucleoside phosphorylase by 8-aminoguanosine: selective toxicity for $\mathrm{T}$ lymphoblasts. Science, 214(4525), 1137-1139.

Kee, K., Foster, B. A., Merali, S., Kramer, D. L., Hensen, M. L., Diegelman, P., Kisiel, N., Vujcic, S., Mazurchuk, R. V., \& Porter, C. W. (2004). Activated polyamine catabolism depletes acetyl-CoA pools and suppresses prostate tumor growth in TRAMP mice. Journal of Biological Chemistry, 279(38), 40076-40083.

Kersting, M. C., Choi, H.-S., \& Carman, G. M. (2004). Regulation of the yeast EKI1-encoded ethanolamine kinase by inositol and choline. Journal of Biological Chemistry, 279(34), 3535335359.

Khoury, C. M., \& Greenwood, M. T. (2008). The pleiotropic effects of heterologous Bax expression in yeast. Biochimica et Biophysica Acta (BBA) - Molecular Cell Research, 1783(7), 1449-1465. doi:http://dx.doi.org/10.1016/j.bbamcr.2007.12.013

Kiburu, I. N., \& LaRonde-LeBlanc, N. (2012). Interaction of Rio1 kinase with toyocamycin reveals a conformational switch that controls oligomeric state and catalytic activity. Plos One, 7(5), e37371. 
Kicska, G. A., Long, L., Hörig, H., Fairchild, C., Tyler, P. C., Furneaux, R. H., Schramm, V. L., \& Kaufman, H. L. (2001). Immucillin H, a powerful transition-state analog inhibitor of purine nucleoside phosphorylase, selectively inhibits human T lymphocytes. Proceedings of the National Academy of Sciences, 98(8), 4593-4598.

Kim, B. K., Cha, S., \& Parks, R. E. (1968). Purine Nucleoside Phosphorylase from Human Erythrocytes: II. kinetic analysis and substrate-binding studies. Journal of Biological Chemistry, 243(8), 17711776.

Kim, J., Huang, W.-P., \& Klionsky, D. J. (2001). Membrane recruitment of Aut7p in the autophagy and cytoplasm to vacuole targeting pathways requires Aut1p, Aut $2 p$, and the autophagy conjugation complex. The Journal of Cell Biology, 152(1), 51-64.

Kim, M., Krogan, N. J., Vasiljeva, L., Rando, O. J., Nedea, E., Greenblatt, J. F., \& Buratowski, S. (2004). The yeast Rat1 exonuclease promotes transcription termination by RNA polymerase II. Nature, 432(7016), 517-522.

Klionsky, D. J. (2005). The molecular machinery of autophagy: unanswered questions. J Cell Sci, 118(1), 7-18.

Klionsky, D. J. (2011). For the last time, it is GFP-Atg8, not Atg8-GFP (and the same goes for LC3): Taylor \& Francis.

Klionsky, D. J., Cregg, J. M., Dunn Jr, W. A., Emr, S. D., Sakai, Y., Sandoval, I. V., Sibirny, A., Subramani, S., Thumm, M., \& Veenhuis, M. (2003). A Unified Nomenclature for. Developmental Cell, 5, 539-545.

Klionsky, D. J., Cuervo, A. M., \& Seglen, P. O. (2007). Methods for monitoring autophagy from yeast to human. Autophagy, 3(3), 181-206.

Knævelsrud, H., \& Simonsen, A. (2012). Lipids in autophagy: constituents, signaling molecules and cargo with relevance to disease. Biochimica et Biophysica Acta (BBA)-Molecular and Cell Biology of Lipids, 1821(8), 1133-1145.

Kodaki, T., \& Yamashita, S. (1987). Yeast phosphatidylethanolamine methylation pathway. Cloning and characterization of two distinct methyltransferase genes. Journal of Biological Chemistry, 262(32), 15428-15435.

Koehler, C. M. (2000). Protein translocation pathways of the mitochondrion. Febs Letters, 476(1-2), 27-31.

Koh, J. L., Chong, Y. T., Friesen, H., Moses, A., Boone, C., Andrews, B. J., \& Moffat, J. (2015). CYCLoPs: A comprehensive database constructed from automated analysis of protein abundance and subcellular localization patterns in Saccharomyces cerevisiae. G3: Genes / Genomes / Genetics, 5(6), 1223-1232.

Kovsan, J., Blüher, M., Tarnovscki, T., Klöting, N., Kirshtein, B., Madar, L., Shai, I., Golan, R., HarmanBoehm, I., \& Schön, M. R. (2010). Altered autophagy in human adipose tissues in obesity. The Journal of Clinical Endocrinology \& Metabolism, 96(2), E268-E277.

Krenitsky, T. A., Elion, G. B., Henderson, A. M., \& Hitchings, G. H. (1968). Inhibition of Human Purine Nucleoside Phosphorylase: Studies with intact erythrocytes and the purified enzyme. Journal of Biological Chemistry, 243(11), 2876-2881.

Lambe, C. U., Averett, D. R., Paff, M. T., Reardon, J. E., Wilson, J. G., \& Krenitsky, T. A. (1995). 2-Amino6-methoxypurine Arabinoside: An Agent for T-Cell Malignancies. Cancer research, 55(15), 3352-3356.

Landau, G., Bercovich, Z., Park, M. H., \& Kahana, C. (2010). The role of polyamines in supporting growth of mammalian cells is mediated through their requirement for translation initiation and elongation. Journal of Biological Chemistry, 285(17), 12474-12481.

Lautenberger, J. A., \& Linn, S. (1972). The Deoxyribonucleic Acid Modification and Restriction Enzymes of Escherichia coli B I. PURIFICATION, SUBUNIT STRUCTURE, AND CATALYTIC PROPERTIES OF THE MODIFICATION METHYLASE. Journal of Biological Chemistry, 247(19), 6176-6182. 
Lecoq, K., Belloc, I., Desgranges, C., \& Daignan-Fornier, B. (2001a). Role of adenosine kinase in Saccharomyces cerevisiae: identification of the ADO1 gene and study of the mutant phenotypes. Yeast, 18(4), 335-342. doi:10.1002/1097-0061(20010315)18:4<335::aidyea674>3.0.co;2-x

Lecoq, K., Belloc, I., Desgranges, C., Konrad, M., \& Daignan-Fornier, B. (2001b). YLR209c Encodes Saccharomyces cerevisiae Purine Nucleoside Phosphorylase. Journal of Bacteriology, 183(16), 4910-4913. doi:10.1128/JB.183.16.4910-4913.2001

Lee, A. Y., Onge, R. P. S., Proctor, M. J., Wallace, I. M., Nile, A. H., Spagnuolo, P. A., Jitkova, Y., Gronda, M., Wu, Y., \& Kim, M. K. (2014). Mapping the cellular response to small molecules using chemogenomic fitness signatures. Science, 344(6180), 208-211.

Lee, J., Moir, R. D., McIntosh, K. B., \& Willis, I. M. (2012). TOR signaling regulates ribosome and tRNA synthesis via LAMMER/Clk and GSK-3 family kinases. Molecular Cell, 45(6), 836-843.

Lee, J. E., Settembre, E. C., Cornell, K. A., Riscoe, M. K., Sufrin, J. R., Ealick, S. E., \& Howell, P. L. (2004). Structural comparison of MTA phosphorylase and MTA/AdoHcy nucleosidase explains substrate preferences and identifies regions exploitable for inhibitor design. Biochemistry, 43(18), 5159-5169.

Lees, N., Skaggs, B., Kirsch, D., \& Bard, M. (1995). Cloning of the late genes in the ergosterol biosynthetic pathway ofSaccharomyces cerevisiae-A review. Lipids, 30(3), 221-226.

Levine, B., \& Kroemer, G. (2008). Autophagy in the pathogenesis of disease. Cell, 132(1), 27-42.

Li, C. M., Tyler, P. C., Furneaux, R. H., Kicska, G., Xu, Y., Grubmeyer, C., Girvin, M. E., \& Schramm, V. L. (1999). Transition-state analogs as inhibitors of human and malarial hypoxanthine-guanine phosphoribosyltransferases. Nature Structural \& Molecular Biology, 6(6), 582-587.

Li, S., Wang, L., Hu, Y., \& Sheng, R. (2015). Autophagy regulators as potential cancer therapeutic agents: a review. Current topics in medicinal chemistry, 15(8), 720-744.

Lisa-SantamarÃ-a, P., Neiman, A. M., Cuesta-MarbÃin, Ã. I., Mollinedo, F., Revuelta, J. L., \& JimÃ @nez, A. (2009). Human initiator caspases trigger apoptotic and autophagic phenotypes in Saccharomyces cerevisiae. Biochimica et Biophysica Acta (BBA) - Molecular Cell Research, 1793(3), 561-571. doi:http://dx.doi.org/10.1016/j.bbamcr.2008.12.016

Lisby, M., Rothstein, R., \& Mortensen, U. H. (2001). Rad52 forms DNA repair and recombination centers during $S$ phase. Proceedings of the National Academy of Sciences, 98(15), 8276-8282. doi:10.1073/pnas.121006298

Lithgow, T. (2000). Targeting of proteins to mitochondria. Febs Letters, 476(1-2), 22-26.

Liu, K., \& Czaja, M. (2013). Regulation of lipid stores and metabolism by lipophagy. Cell Death \& Differentiation, 20(1), 3-11.

Liu, T.-T., Gomez, T. S., Sackey, B. K., Billadeau, D. D., \& Burd, C. G. (2012). Rab GTPase regulation of retromer-mediated cargo export during endosome maturation. Molecular Biology of the Cell, 23(13), 2505-2515. doi:10.1091/mbc.E11-11-0915

Liu, Y., \& Levine, B. (2015). Autosis and autophagic cell death: the dark side of autophagy. Cell Death \& Differentiation, 22(3), 367-376.

Loewen, S. K., Ng, A. M., Mohabir, N. N., Baldwin, S. A., Cass, C. E., \& Young, J. D. (2003). Functional characterization of a $\mathrm{H}+$ /nucleoside co - transporter (CaCNT) from Candida albicans, a fungal member of the concentrative nucleoside transporter (CNT) family of membrane proteins. Yeast, 20(8), 661-675.

Low, Y. S. (2013). Kinetochores are Required to Fully Activate Secretory Pathway in Elevated ER Stress. $(\mathrm{PhD})$, Victoria University of Wellington.

Lu, S. C. (2000). S-adenosylmethionine. The international journal of biochemistry \& cell biology, 32(4), 391-395.

Mackey, J. R., Baldwin, S. A., Young, J. D., \& Cass, C. E. (1998). Nucleoside transport and its significance for anticancer drug resistance. Drug Resistance Updates, 1(5), 310-324. 
Madrid-Marina, V., Lestan, B., Nowak, P. J., Fox, I. H., \& Spychala, J. (1993). Altered properties of human $\mathrm{t}$-lymphoblast soluble low $\mathrm{K} \mathrm{m} \mathrm{5'}$-nucleotidase: Comparison with B-lymphoblast enzyme. Leukemia Research, 17(3), 231-240.

Mandal, S., Mandal, A., Johansson, H. E., Orjalo, A. V., \& Park, M. H. (2013). Depletion of cellular polyamines, spermidine and spermine, causes a total arrest in translation and growth in mammalian cells. Proceedings of the National Academy of Sciences, 110(6), 2169-2174.

Marusyk, A., Almendro, V., \& Polyak, K. (2012). Intra-tumour heterogeneity: a looking glass for cancer? Nature Reviews Cancer, 12(5), 323-334.

Massacesi, C., Di Tomaso, E., Urban, P., Germa, C., Quadt, C., Trandafir, L., Aimone, P., Fretault, N., Dharan, B., \& Tavorath, R. (2016). PI3K inhibitors as new cancer therapeutics: implications for clinical trial design. OncoTargets and therapy, 9, 203.

McCammon, M., Hartmann, M., Bottema, C., \& Parks, L. (1984). Sterol methylation in Saccharomyces cerevisiae. Journal of Bacteriology, 157(2), 475-483.

McElhaney-Feser, G. E., Raulli, R. E., \& Cihlar, R. L. (1998). Synergy of Nitric Oxide and Azoles againstCandida Species In Vitro. Antimicrobial Agents and Chemotherapy, 42(9), 2342-2346.

Merkulova, E. A., Guiboileau, A., Naya, L., Masclaux-Daubresse, C., \& Yoshimoto, K. (2014). Assessment and optimization of autophagy monitoring methods in Arabidopsis roots indicate direct fusion of autophagosomes with vacuoles. Plant and Cell Physiology, pcu041.

Metcalf, B. W., Bey, P., Danzin, C., Jung, M. J., Casara, P., \& Vevert, J. P. (1978). Catalytic irreversible inhibition of mammalian ornithine decarboxylase (E.C.4.1.1.17) by substrate and product analogs. Journal of the American Chemical Society, 100(8), 2551-2553. doi:10.1021/ja00476a050

Mi, H., Huang, X., Muruganujan, A., Tang, H., Mills, C., Kang, D., \& Thomas, P. D. (2017). PANTHER version 11: expanded annotation data from Gene Ontology and Reactome pathways, and data analysis tool enhancements. Nucleic Acids Research, 45(D1), D183-D189.

Millan-Zambrano, G., Rodriguez-Gil, A., Penate, X., de Miguel-Jimenez, L., Morillo-Huesca, M., Krogan, N., \& Chavez, S. (2013). The prefoldin complex regulates chromatin dynamics during transcription elongation. PLoS Genet, 9(9), e1003776. doi:10.1371/journal.pgen.1003776

Minois, N., Carmona-Gutierrez, D., \& Madeo, F. (2011). Polyamines in aging and disease. Aging (Albany NY), 3(8), 716-732.

Mnaimneh, S., Davierwala, A. P., Haynes, J., Moffat, J., Peng, W. T., Zhang, W., Yang, X., Pootoolal, J., Chua, G., Lopez, A., Trochesset, M., Morse, D., Krogan, N. J., Hiley, S. L., Li, Z., Morris, Q., Grigull, J., Mitsakakis, N., Roberts, C. J., Greenblatt, J. F., Boone, C., Kaiser, C. A., Andrews, B. J., \& Hughes, T. R. (2004). Exploration of essential gene functions via titratable promoter alleles. Cell, 118(1), 31-44. doi:10.1016/j.cell.2004.06.013

Morris, J., Philip E, \& Montgomery, J. A. (1998). Inhibitors of the enzyme purine nucleoside phosphorylase. Expert Opinion on Therapeutic Patents, 8(3), 283-299.

Morselli, E., Mariño, G., Bennetzen, M. V., Eisenberg, T., Megalou, E., Schroeder, S., Cabrera, S., Bénit, P., Rustin, P., \& Criollo, A. (2011). Spermidine and resveratrol induce autophagy by distinct pathways converging on the acetylproteome. The Journal of Cell Biology, 192(4), 615-629.

Munch-Petersen, B., Piskur, J., \& Søndergaard, L. (1998). Four deoxynucleoside kinase activities from Drosophila melanogaster are contained within a single monomeric enzyme, a new multifunctional deoxynucleoside kinase. Journal of Biological Chemistry, 273(7), 3926-3931.

Munkacsi, A. B., Chen, F. W., Brinkman, M. A., Higaki, K., Gutierrez, G. D., Chaudhari, J., Layer, J. V., Tong, A., Bard, M., Boone, C., loannou, Y. A., \& Sturley, S. L. (2011). An "exacerbate-reverse" strategy in yeast identifies histone deacetylase inhibition as a correction for cholesterol and sphingolipid transport defects in human Niemann-Pick type C disease. J Biol Chem, 286(27), 23842-23851. doi:10.1074/jbc.M111.227645 
Murakami, Y., Matsufuji, S., Kameji, T., Hayashi, S., Igarashi, K., Tamura, T., Tanaka, K., \& Ichihara, A. (1992). Ornithine decarboxylase is degraded by the $26 \mathrm{~S}$ proteasome without ubiquitination. Nature, 360(6404), 597-599. doi:10.1038/360597a0

Nakatogawa, H. (2015). Hrr25: An emerging major player in selective autophagy regulation in Saccharomyces cerevisiae. Autophagy, 11(2), 432-433.

Nakatogawa, H., Ohbayashi, S., Sakoh-Nakatogawa, M., Kakuta, S., Suzuki, S. W., Kirisako, H., KondoKakuta, C., Noda, N. N., Yamamoto, H., \& Ohsumi, Y. (2012). The autophagy-related protein kinase Atg1 interacts with the ubiquitin-like protein Atg8 via the Atg8 family interacting motif to facilitate autophagosome formation. Journal of Biological Chemistry, 287(34), 2850328507.

Natter, K., \& Kohlwein, S. D. (2013). Yeast and cancer cells-common principles in lipid metabolism. Biochimica et Biophysica Acta (BBA)-Molecular and Cell Biology of Lipids, 1831(2), 314-326.

Nes, W. D. (2011). Biosynthesis of cholesterol and other sterols. Chemical Reviews, 111(10), 64236451.

Nielsen, J. (2009). Systems biology of lipid metabolism: from yeast to human. Febs Letters, 583(24), 3905-3913.

NIH Categorical Spending -NIH Research Portfolio Online Reporting Tools (RePORT). (2015, 10-042013). Retrieved from http://report.nih.gov/categorical spending.aspx

Niso - Santano, M., Malik, S. A., Pietrocola, F., Bravo - San Pedro, J. M., Mariño, G., Cianfanelli, V., Ben - Younès, A., Troncoso, R., Markaki, M., \& Sica, V. (2015). Unsaturated fatty acids induce non - canonical autophagy. The EMBO journal, e201489363.

Noda, T. (2008). Chapter 2 Viability Assays to Monitor Yeast Autophagy Methods in Enzymology (Vol. Volume 451, pp. 27-32): Academic Press.

Noga, A. A., Stead, L. M., Zhao, Y., Brosnan, M. E., Brosnan, J. T., \& Vance, D. E. (2003). Plasma homocysteine is regulated by phospholipid methylation. Journal of Biological Chemistry, 278(8), 5952-5955.

Nurse, P. (1975). Genetic control of cell size at cell division in yeast. Nature, 256, 547-551.

O'Konek, J. J., Boucher, P. D., lacco, A. A., Wilson, T. E., \& Shewach, D. S. (2009). MLH1 deficiency enhances tumor cell sensitivity to ganciclovir. Cancer gene therapy, 16(9), 683-692.

O'Konek, J. J., Boucher, P. D., lacco, A. A., Wilson, T. E., \& Shewach, D. S. (2009). MLH1 Deficiency Enhances Tumor Cell Sensitivity to Ganciclovir. Cancer gene therapy, 16(9), 683-692. doi:10.1038/cgt.2009.16

O'Shaughnessy, J. A., Demers, L. M., Jones, S. E., Arseneau, J., Khandelwal, P., George, T., Gersh, R., Mauger, D., \& Manni, A. (1999). $\alpha$-Difluoromethylornithine as treatment for metastatic breast cancer patients. Clinical cancer research, 5(11), 3438-3444.

Odds, F. C. (2003). Synergy, antagonism, and what the chequerboard puts between them. Journal of Antimicrobial Chemotherapy, 52(1), 1-1. doi:10.1093/jac/dkg301

Ohsumi, Y. (2014). Historical landmarks of autophagy research. Cell research, 24(1), 9-23.

Onodera, J., \& Ohsumi, Y. (2004). Ald6p is a preferred target for autophagy in yeast, Saccharomyces cerevisiae. J Biol Chem, 279(16), 16071-16076. doi:10.1074/jbc.M312706200

Osborne, W. R., \& Scott, C. R. (1983). The metabolism of deoxyguanosine and guanosine in human B and T lymphoblasts. A role for deoxyguanosine kinase activity in the selective T-cell defect associated with purine nucleoside phosphorylase deficiency. Biochem J, 214(3), 711-718.

Pajula, R. L., Raina, A., \& Eloranta, T. (1979a). Polyamine synthesis in mammalian tissues. The FEBS Journal, 101(2), 619-626.

Pajula, R. L., Raina, A., \& Eloranta, T. (1979b). Polyamine synthesis in mammalian tissues. Isolation and characterization of spermine synthase from bovine brain. Eur J Biochem, 101(2), 619-626.

Palavecino, M. D., Correa-Garc, \#xed, a, S. R., Berm, \#xfa, \& dez-Moretti, M. (2015). Genes of Different Catabolic Pathways Are Coordinately Regulated by Dal 81 in Saccharomyces cerevisiae. Journal of Amino Acids, 2015, 8. doi:10.1155/2015/484702 
Papinazath, T., Min, W., Sujiththa, S., Cohen, A., Ackerley, C., Roifman, C. M., \& Grunebaum, E. (2011). Effects of purine nucleoside phosphorylase deficiency on thymocyte development. Journal of Allergy and Clinical Immunology, 128(4), 854-863. e851.

Paproski, R. J., Ng, A. M., Yao, S. Y., Graham, K., Young, J. D., \& Cass, C. E. (2008). The role of human nucleoside transporters in uptake of $3^{\prime}$-deoxy-3' -fluorothymidine. Molecular pharmacology, 74(5), 1372-1380.

Parikh, A., Childress, C., Deitrick, K., Lin, Q., Rukstalis, D., \& Yang, W. (2010). Statin - induced autophagy by inhibition of geranylgeranyl biosynthesis in prostate cancer PC3 cells. The Prostate, 70(9), 971-981.

Park, H. J. (2014). Chemotherapy induced peripheral neuropathic pain. Korean Journal of Anesthesiology, 67(1), 4-7. doi:10.4097/kjae.2014.67.1.4

Parks Jr, R. E., \& Agarwal, R. P. (1972). 16 Purine Nucleoside Phosphorylase. In D. B. Paul (Ed.), The Enzymes (Vol. Volume 7, pp. 483-514): Academic Press.

Parsons, A. B., Brost, R. L., Ding, H. M., Li, Z. J., Zhang, C. Y., Sheikh, B., Brown, G. W., Kane, P. M., Hughes, T. R., \& Boone, C. (2004). Integration of chemical-genetic and genetic interaction data links bioactive compounds to cellular target pathways. Nature Biotechnology, 22(1), 62-69. doi:10.1038/nbt919

Pastor-Anglada, M., Felipe, A., \& Casado, F. J. (1998). Transport and mode of action of nucleoside derivatives used in chemical and antiviral therapies. Trends in Pharmacological Sciences, 19(10), 424-430.

Pegg, A. E. (2009). Mammalian Polyamine Metabolism and Function. IUBMB life, 61(9), 880-894. doi:10.1002/iub.230

Perez-Herrero, E., \& Fernandez-Medarde, A. (2015). Advanced targeted therapies in cancer: Drug nanocarriers, the future of chemotherapy. Eur J Pharm Biopharm, 93, 52-79. doi:10.1016/j.ejpb.2015.03.018

Petiot, A., Ogier-Denis, E., Blommaart, E. F., Meijer, A. J., \& Codogno, P. (2000). Distinct classes of phosphatidylinositol $3^{\prime}$-kinases are involved in signaling pathways that control macroautophagy in HT-29 cells. Journal of Biological Chemistry, 275(2), 992-998.

Petrenko, N., Chereji, R. z. V., McClean, M. N., Morozov, A. V., \& Broach, J. R. (2013). Noise and interlocking signaling pathways promote distinct transcription factor dynamics in response to different stresses. Molecular Biology of the Cell, 24(12), 2045-2057.

Pirinen, E., Gylling, H., Itkonen, P., Yaluri, N., Heikkinen, S., Pietilä, M., Kuulasmaa, T., Tusa, M., Cerrada-Gimenez, M., \& Pihlajamäki, J. (2010). Activated polyamine catabolism leads to low cholesterol levels by enhancing bile acid synthesis. Amino Acids, 38(2), 549-560.

Pirkov, I., Norbeck, J., Gustafsson, L., \& Albers, E. (2008). A complete inventory of all enzymes in the eukaryotic methionine salvage pathway. FEBS Journal, 275(16), 4111-4120.

Plemel, R. L., Lobingier, B. T., Brett, C. L., Angers, C. G., Nickerson, D. P., Paulsel, A., Sprague, D., \& Merz, A. J. (2011). Subunit organization and Rab interactions of Vps-C protein complexes that control endolysosomal membrane traffic. Mol Biol Cell, 22(8), 1353-1363. doi:10.1091/mbc.E10-03-0260

Pollard, K. J., Samuels, M. L., Crowley, K. A., Hansen, J. C., \& Peterson, C. L. (1999). Functional interaction between GCN5 and polyamines: a new role for core histone acetylation. The EMBO journal, 18(20), 5622-5633.

Prochasson, P., Florens, L., Swanson, S. K., Washburn, M. P., \& Workman, J. L. (2005). The HIR corepressor complex binds to nucleosomes generating a distinct protein/DNA complex resistant to remodeling by SWI/SNF. Genes Dev, 19(21), 2534-2539. doi:10.1101/gad.1341105

Racine, J. S. (2012). RStudio: A Platform-Independent IDE for R and Sweave. Journal of Applied Econometrics, 27(1), 167-172. doi:10.1002/jae.1278 
Raina, A., Hyvönen, T., Eloranta, T., Voutilainen, M., Samejima, K., \& Yamanoha, B. (1984). Polyamine synthesis in mammalian tissues. Isolation and characterization of spermidine synthase from bovine brain. Biochemical Journal, 219(3), 991-1000.

Ravandi, F., \& Gandhi, V. (2006). Novel purine nucleoside analogues for T-cell-lineage acute lymphoblastic leukaemia and lymphoma. Expert opinion on investigational drugs, 15(12), 1601-1613.

Rébora, K., Desmoucelles, C., Borne, F., Pinson, B. t., \& Daignan-Fornier, B. (2001). Yeast AMP pathway genes respond to adenine through regulated synthesis of a metabolic intermediate. Molecular and Cellular Biology, 21(23), 7901-7912.

Rehman, H., Silk, A. W., Kane, M. P., \& Kaufman, H. L. (2016). Into the clinic: Talimogene laherparepvec (T-VEC), a first-in-class intratumoral oncolytic viral therapy. Journal for Immunotherapy of Cancer, 4, 53. doi:10.1186/s40425-016-0158-5

Reilly, K. M., \& Kisor, D. F. (2009). Profile of nelarabine: use in the treatment of T-cell acute lymphoblastic leukemia. Onco Targets Ther, 2, 219-228.

Riedel, H., Su, L., \& Hansen, H. (1993). Yeast phenotype classifies mammalian protein kinase C cDNA mutants. Molecular and Cellular Biology, 13(8), 4728-4735. doi:10.1128/mcb.13.8.4728

Röhrig, F., \& Schulze, A. (2016). The multifaceted roles of fatty acid synthesis in cancer. Nature Reviews Cancer.

Sarkar, S. (2013). Regulation of autophagy by mTOR-dependent and mTOR-independent pathways: autophagy dysfunction in neurodegenerative diseases and therapeutic application of autophagy enhancers: Portland Press Limited.

Schramm, V. L. (1999). Enzymatic transition-state analysis and transition-state analogs. Methods in Enzymology, 308, 301-355.

Schramm, V. L. (2002). Development of transition state analogues of purine nucleoside phosphorylase as anti-T-cell agents. Biochimica et Biophysica Acta (BBA)-Molecular Basis of Disease, 1587(2), 107-117.

Schramm, V. L. (2004). Immucillins as Antibiotics for T - Cell Proliferation and Malaria. Nucleosides, Nucleotides and Nucleic Acids, 23(8-9), 1305-1311.

Schramm, V. L. (2011). Enzymatic transition states, transition-state analogs, dynamics, thermodynamics, and lifetimes. Annual Review of Biochemistry, 80, 703-732.

Schramm, V. L. (2013a). [MTAP inhibitors as potent, non - toxic anti-cancer therapeutics].

Schramm, V. L. (2013b). Transition states, analogues, and drug development. ACS Chemical Biology, $8(1), 71-81$.

Schug, Z. T., Peck, B., Jones, D. T., Zhang, Q., Grosskurth, S., Alam, I. S., Goodwin, L. M., Smethurst, E., Mason, S., \& Blyth, K. (2015). Acetyl-CoA synthetase 2 promotes acetate utilization and maintains cancer cell growth under metabolic stress. Cancer Cell, 27(1), 57-71.

Schuldiner, M., Collins, S. R., Thompson, N. J., Denic, V., Bhamidipati, A., Punna, T., Ihmels, J., Andrews, B., Boone, C., Greenblatt, J. F., Weissman, J. S., \& Krogan, N. J. (2005). Exploration of the function and organization of the yeast early secretory pathway through an epistatic miniarray profile. Cell, 123(3), 507-519. doi:10.1016/j.cell.2005.08.031

Shen, J. P., Zhao, D., Sasik, R., Luebeck, J., Birmingham, A., Bojorquez-Gomez, A., Licon, K., Klepper, K., Pekin, D., Beckett, A. N., Sanchez, K. S., Thomas, A., Kuo, C. C., Du, D., Roguev, A., Lewis, N. E., Chang, A. N., Kreisberg, J. F., Krogan, N., Qi, L., Ideker, T., \& Mali, P. (2017). Combinatorial CRISPR-Cas9 screens for de novo mapping of genetic interactions. Nat Methods, 14(6), 573576. doi:10.1038/nmeth.4225

Simes, R. J. (1986). An improved Bonferroni procedure for multiple tests of significance. Biometrika, 73(3), 751-754.

Simon, J. A., \& Bedalov, A. (2004). Opinion - Yeast as a model system for anticancer drug discovery. Nature Reviews Cancer, 4(6), 481-488. doi:10.1038/nrc1372 
Simoni, R., Ferreira Gomes, L. N., Scalco, F., Oliveira, C. P., Aquino Neto, F., \& Costa de Oliveira, M. (2007). Uric acid changes in urine and plasma: an effective tool in screening for purine inborn errors of metabolism and other pathological conditions. Journal of inherited metabolic disease, 30(3), 295-309.

Simonsen, A., \& Tooze, S. A. (2009). Coordination of membrane events during autophagy by multiple class III PI3-kinase complexes. The Journal of Cell Biology, 186(6), 773-782.

Singh, R., Kaushik, S., Wang, Y., Xiang, Y., Novak, I., Komatsu, M., Tanaka, K., Cuervo, A. M., \& Czaja, M. J. (2009). Autophagy regulates lipid metabolism. Nature, 458(7242), 1131-1135.

Singh, V., Shi, W., Almo, S. C., Evans, G. B., Furneaux, R. H., Tyler, P. C., Painter, G. F., Lenz, D. H., Mee, S., \& Zheng, R. (2006). Structure and inhibition of a quorum sensing target from Streptococcus pneumoniae. Biochemistry, 45(43), 12929-12941.

Singh, V., Shi, W., Evans, G. B., Tyler, P. C., Furneaux, R. H., Almo, S. C., \& Schramm, V. L. (2004). Picomolar transition state analogue inhibitors of human 5 '-methylthioadenosine phosphorylase and X-ray structure with MT-Immucillin-A. Biochemistry, 43(1), 9-18.

Snyder, R. D., \& Sunkara, P. S. (1990). Effect of polyamine depletion on DNA damage and repair following UV irradiation of HeLa cells. Photochemistry and photobiology, 52(3), 525-532.

Solaroli, N., Zheng, X., Johansson, M., Balzarini, J., \& Karlsson, A. (2007). Mitochondrial expression of the Drosophila melanogaster multisubstrate deoxyribonucleoside kinase. Molecular pharmacology, 72(6), 1593-1598.

Sperrazza, J. M., \& Spremulli, L. L. (1983). Quantitation of cation binding to wheat germ ribosomes: Influences on submit association equilibria and ribosome activity. Nucleic Acids Research, 11(9), 2665-2679.

Stead, L. M., Brosnan, J. T., Brosnan, M. E., Vance, D. E., \& Jacobs, R. L. (2006). Is it time to reevaluate methyl balance in humans? The American Journal of Clinical Nutrition, 83(1), 5-10.

Stepchenkova, E. I., Kozmin, S. G., Alenin, V. V., \& Pavlov, Y. I. (2005). Genome-wide screening for genes whose deletions confer sensitivity to mutagenic purine base analogs in yeast. $B M C$ genetics, 6(1), 31.

Stirling, P. C., Bloom, M. S., Solanki-Patil, T., Smith, S., Sipahimalani, P., Li, Z., Kofoed, M., Ben-Aroya, S., Myung, K., \& Hieter, P. (2011). The complete spectrum of yeast chromosome instability genes identifies candidate $\mathrm{CIN}$ cancer genes and functional roles for ASTRA complex components. PLoS Genet, 7(4), e1002057.

Stoner, G., \& Eisenberg, M. (1975). Biosynthesis of 7, 8-diaminopelargonic acid from 7-keto-8aminopelargonic acid and S-adenosyl-L-methionine. The kinetics of the reaction. Journal of Biological Chemistry, 250(11), 4037-4043.

Storey, M. K., Byers, D. M., \& Ridgway, N. D. (1998). Cholesterol regulates oxysterol binding protein (OSBP) phosphorylation and Golgi localization in Chinese hamster ovary cells: correlation with stimulation of sphingomyelin synthesis by 25 -hydroxycholesterol. Biochemical Journal, 336(1), 247-256.

Strømhaug, P. E., Reggiori, F., Guan, J., Wang, C.-W., \& Klionsky, D. J. (2004). Atg21 is a phosphoinositide binding protein required for efficient lipidation and localization of Atg8 during uptake of aminopeptidase I by selective autophagy. Molecular Biology of the Cell, 15(8), 3553-3566.

Subhi, A. L., Diegelman, P., Porter, C. W., Tang, B., Lu, Z. J., Markham, G. D., \& Kruger, W. D. (2003). Methylthioadenosine Phosphorylase Regulates Ornithine Decarboxylase by Production of Downstream Metabolites. Journal of Biological Chemistry, 278(50), 49868-49873. doi:10.1074/jbc.M308451200

Suzuki, K., Kirisako, T., Kamada, Y., Mizushima, N., Noda, T., \& Ohsumi, Y. (2001). The pre autophagosomal structure organized by concerted functions of APG genes is essential for autophagosome formation. The EMBO journal, 20(21), 5971-5981. 
Suzuki, K., Kubota, Y., Sekito, T., \& Ohsumi, Y. (2007). Hierarchy of Atg proteins in pre autophagosomal structure organization. Genes to Cells, 12(2), 209-218.

Suzuki, K., \& Ohsumi, Y. (2010). Current knowledge of the pre - autophagosomal structure (PAS). Febs Letters, 584(7), 1280-1286.

Tabor, C. W., \& Tabor, H. (1984). Polyamines. Annual Review of Biochemistry, 53(1), 749-790.

Takeshige, K., Baba, M., Tsuboi, S., Noda, T., \& Ohsumi, Y. (1992). Autophagy in yeast demonstrated with proteinase-deficient mutants and conditions for its induction. J Cell Biol, 119(2), 301-311.

Tanaka, C., Tan, L.-J., Mochida, K., Kirisako, H., Koizumi, M., Asai, E., Sakoh-Nakatogawa, M., Ohsumi, Y., \& Nakatogawa, H. (2014). Hrr25 triggers selective autophagy-related pathways by phosphorylating receptor proteins. J Cell Biol, jcb. 201402128.

Tang, B., Kadariya, Y., Chen, Y., Slifker, M., \& Kruger, W. D. (2015). Expression of MTAP inhibits tumorrelated phenotypes in HT1080 cells via a mechanism unrelated to its enzymatic function. G3: Genes/ Genomes/ Genetics, 5(1), 35-44.

Tang, B., Kadariya, Y., Murphy, M. E., \& Kruger, W. D. (2006). The methionine salvage pathway compound 4-methylthio-2-oxobutanate causes apoptosis independent of down-regulation of ornithine decarboxylase. Biochemical Pharmacology, 72(7), 806-815.

Tang, K.-C., Mariuza, R., \& Coward, J. K. (1981). Synthesis and evaluation of some stable multisubstrate adducts as specific inhibitors of spermidine synthase. Journal of Medicinal Chemistry, 24(11), 1277-1284.

Tanigawa, M., \& Maeda, T. (2017). An in vitro TORC1 kinase assay that recapitulates the Gtrindependent glutamine-responsive TORC1 activation mechanism on yeast vacuoles. Molecular and Cellular Biology, MCB. 00075-00017.

Tattersall, M. H. N., Ganeshaguru, K., \& Hoffbrand, A. V. (1975). The effect of external deoxyribonucleosides on deoxyribonucleoside triphosphate concentrations in human lymphocytes. Biochemical Pharmacology, 24(16), 1495-1498. doi:http://dx.doi.org/10.1016/0006-2952(75)90025-8

Taylor, F. R., \& Cronan Jr, J. E. (1979). Cyclopropane Fatty Acid Synthase of Escherichia coli. Stabilization, Purification, and Interaction with Phospholipid Vesiclest. Urbana, 51, 61801.

Tehlivets, O. (2011). Homocysteine as a risk factor for atherosclerosis: is its conversion to s-adenosylL-homocysteine the key to deregulated lipid metabolism? Journal of lipids, 2011.

Tehlivets, O., Malanovic, N., Visram, M., Pavkov-Keller, T., \& Keller, W. (2013). S-adenosyl-Lhomocysteine hydrolase and methylation disorders: yeast as a model system. Biochimica et Biophysica Acta (BBA)-Molecular Basis of Disease, 1832(1), 204-215.

Tempel, W., Rabeh, W. M., Bogan, K. L., Belenky, P., Wojcik, M., Seidle, H. F., Nedyalkova, L., Yang, T., Sauve, A. A., \& Park, H.-W. (2007). Nicotinamide riboside kinase structures reveal new pathways to NAD+. PLoS Biol, 5(10), e263.

Thomas, D., Becker, A., \& Surdin-Kerjan, Y. (2000). Reverse methionine biosynthesis fromsadenosylmethionine in eukaryotic cells. Journal of Biological Chemistry, 275(52), 4071840724.

Thompson, R. C., Dix, D. B., Gerson, R. B., \& Karim, A. (1981). Effect of Mg2+ concentration, polyamines, streptomycin, and mutations in ribosomal proteins on the accuracy of the twostep selection of aminoacyl-tRNAs in protein biosynthesis. Journal of Biological Chemistry, 256(13), 6676-6681.

Tibbetts, A. S., \& Appling, D. R. (1997). Saccharomyces cerevisiae expresses two genes encoding isozymes of 5-aminoimidazole-4-carboxamide ribonucleotide transformylase. Arch Biochem Biophys, 340(2), 195-200. doi:10.1006/abbi.1997.9919

Ting, L.-M., Shi, W., Lewandowicz, A., Singh, V., Mwakingwe, A., Birck, M. R., Ringia, E. A. T., Bench, G., Madrid, D. C., \& Tyler, P. C. (2005). Targeting a novel Plasmodium falciparum purine recycling pathway with specific immucillins. Journal of Biological Chemistry, 280(10), 9547-9554. 
Tkach, J. M., Yimit, A., Lee, A. Y., Riffle, M., Costanzo, M., Jaschob, D., Hendry, J. A., Ou, J., Moffat, J., \& Boone, C. (2012). Dissecting DNA damage response pathways by analysing protein localization and abundance changes during DNA replication stress. Nature Cell Biology, 14(9), 966-976.

Tong, A. H., \& Boone, C. (2006). Synthetic genetic array analysis in Saccharomyces cerevisiae. Methods Mol Biol, 313, 171-192.

Tong, A. H. Y., \& Boone, C. (2007). 16 High-Throughput Strain Construction and Systematic Synthetic Lethal Screening in. Methods in Microbiology, 36, 369-707.

Tong, A. H. Y., Evangelista, M., Parsons, A. B., Xu, H., Bader, G. D., Pagé, N., Robinson, M., Raghibizadeh, S., Hogue, C. W., \& Bussey, H. (2001). Systematic genetic analysis with ordered arrays of yeast deletion mutants. Science, 294(5550), 2364-2368.

Toro, A., Paiva, M., Ackerley, C., \& Grunebaum, E. (2006). Intracellular delivery of purine nucleoside phosphorylase (PNP) fused to protein transduction domain corrects PNP deficiency in vitro. Cell Immunol, 240(2), 107-115. doi:10.1016/j.cellimm.2006.07.003

Torre, L. A., Bray, F., Siegel, R. L., Ferlay, J., Lortet-Tieulent, J., \& Jemal, A. (2015). Global cancer statistics, 2012. CA: A Cancer Journal for Clinicians, 65(2), 87-108. doi:10.3322/caac.21262

Tripodi, F., Nicastro, R., Reghellin, V., \& Coccetti, P. (2015). Post-translational modifications on yeast carbon metabolism: Regulatory mechanisms beyond transcriptional control. Biochimica et Biophysica Acta (BBA)-General Subjects, 1850(4), 620-627.

Tsujimoto, Y., \& Shimizu, S. (2005). Another way to die: autophagic programmed cell death. Cell Death \& Differentiation, 12, 1528-1534.

Tuttle, J. V., \& Krenitsky, T. A. (1984). Effects of acyclovir and its metabolites on purine nucleoside phosphorylase. J Biol Chem, 259(7), 4065-4069.

Uemura, H., \& Fraenkel, D. (1990). gcr2, a new mutation affecting glycolytic gene expression in Saccharomyces cerevisiae. Molecular and Cellular Biology, 10(12), 6389-6396.

Ullman, B., Gudas, L. J., Clift, S. M., \& Martin, D. W., Jr. (1979). Isolation and characterization of purinenucleoside phosphorylase-deficient T-lymphoma cells and secondary mutants with altered ribonucleotide reductase: genetic model for immunodeficiency disease. Proc Natl Acad Sci U $S A, 76(3), 1074-1078$.

Umate, P. (2015). Comparison of Genes Encoding Enzymes of Sterol Biosynthesis from Plants to Orthologs in Yeast. Rice Research: Open Access.

Umebayashi, K., \& Nakano, A. (2003). Ergosterol is required for targeting of tryptophan permease to the yeast plasma membrane. The Journal of Cell Biology, 161(6), 1117-1131.

van der Eb, M. M., Geutskens, S. B., van Kuilenburg, A. B., van Lenthe, H., van Dierendonck, J. H., Kuppen, P. J., van Ormondt, H., van de Velde, C. J., Wanders, R. J., van Gennip, A. H., \& Hoeben, R. C. (2003). Ganciclovir nucleotides accumulate in mitochondria of rat liver cells expressing the herpes simplex virus thymidine kinase gene. J Gene Med, 5(12), 1018-1027. doi:10.1002/jgm.450

Van der Rest, M., Kamminga, A. H., Nakano, A., Anraku, Y., Poolman, B., \& Konings, W. N. (1995). The plasma membrane of Saccharomyces cerevisiae: structure, function, and biogenesis. Microbiological Reviews, 59(2), 304-322.

van Zutphen, T., Todde, V., de Boer, R., Kreim, M., Hofbauer, H. F., Wolinski, H., Veenhuis, M., van der Klei, I. J., \& Kohlwein, S. D. (2014). Lipid droplet autophagy in the yeast Saccharomyces cerevisiae. Molecular Biology of the Cell, 25(2), 290-301.

Vance, D. E., \& Ridgway, N. D. (1988). The methylation of phosphatidylethanolamine. Progress in lipid research, 27(1), 61-79.

Vander Heiden, M. G., Cantley, L. C., \& Thompson, C. B. (2009). Understanding the Warburg effect: the metabolic requirements of cell proliferation. Science, 324(5930), 1029-1033. 
Vanrell, M. C., Cueto, J. A., Barclay, J. J., Carrillo, C., Colombo, M. I., Gottlieb, R. A., \& Romano, P. S. (2013). Polyamine depletion inhibits the autophagic response modulating Trypanosoma cruzi infectivity. Autophagy, 9(7), 1080-1093.

Vernis, L., Piskur, J., \& Diffley, J. F. (2003). Reconstitution of an efficient thymidine salvage pathway in Saccharomyces cerevisiae. Nucleic Acids Research, 31(19), e120-e120.

Verschuur, A., Brinkman, J., Van Gennip, A., Leen, R., Vet, R., Evers, L., Voute, P., \& Van Kuilenburg, A. (2001). Cyclopentenyl cytosine induces apoptosis and increases cytarabine-induced apoptosis in a T-lymphoblastic leukemic cell-line. Leukemia Research, 25(10), 891-900.

Vickers, M. F., Mani, R. S., Sundaram, M., Hogue, D. L., Young, J. D., Baldwin, S. A., \& Cass, C. E. (1999). Functional production and reconstitution of the human equilibrative nucleoside transporter (hENT1) in Saccharomyces cerevisiae. Interaction of inhibitors of nucleoside transport with recombinant hENT1 and a glycosylation-defective derivative (hENT1/N48Q). Biochem. J., 339(1), 21-32.

Vickers, M. F., Yao, S. Y., Baldwin, S. A., Young, J. D., \& Cass, C. E. (2000). Nucleoside Transporter Proteins of Saccharomyces cerevisiae. Demonstration of a transporter (FUI1) with high uridine selectivity in plasma membranes and a transporter (FUN26) with broad nucleoside selectivity in intracellular membranes. Journal of Biological Chemistry, 275(34), 25931-25938.

Viegas, T. X., Omura, G. A., Stoltz, R. R., \& Kisicki, J. (2000). Pharmacokinetics and pharmacodynamics of peldesine (BCX-34), a purine nucleoside phosphorylase inhibitor, following single and multiple oral doses in healthy volunteers. J Clin Pharmacol, 40(4), 410-420.

Vivanco, I., \& Sawyers, C. L. (2002). The phosphatidylinositol 3-kinase-AKT pathway in human cancer. Nature Reviews Cancer, 2(7), 489-501.

Vivó, M., de Vera, N., Cortés, R., Mengod, G., Camón, L. s., \& Martínez, E. (2001). Polyamines in the basal ganglia of human brain. Influence of aging and degenerative movement disorders. Neuroscience letters, 304(1), 107-111.

Wagih, O., \& Parts, L. (2014). gitter: a robust and accurate method for quantification of colony sizes from plate images. G3 (Bethesda), 4(3), 547-552. doi:10.1534/g3.113.009431

Wallace, H., \& Fraser, A. (2004). Inhibitors of polyamine metabolism: review article. Amino Acids, 26(4), 353-365.

Wallace, H. M. (1996). Polyamines in human health. Proceedings of the Nutrition Society, 55(1B), 419431.

Walther, T., Novo, M., Rössger, K., Létisse, F., Loret, M.-O., Portais, J.-C., \& François, J.-M. (2010). Control of ATP homeostasis during the respiro-fermentative transition in yeast. Molecular Systems Biology, 6, 344-344. doi:10.1038/msb.2009.100

Warburg, O. (1956). On the origin of cancer cells. Science, 123(3191), 309-314.

Wen, X., \& Klionsky, D. J. (2016). An overview of macroautophagy in yeast. Journal of Molecular Biology, 428(9), 1681-1699.

Williams-Ashman, H. G., Seidenfeld, J., \& Galletti, P. (1982). Trends in the biochemical pharmacology of 5' -deoxy-5' -methylthioadenosine. Biochemical Pharmacology, 31(3), 277-288.

Williams, S. R., Gekeler, V., Mclvor, R. S., \& Martin, D. W., Jr. (1987). A human purine nucleoside phosphorylase deficiency caused by a single base change. J Biol Chem, 262(5), 2332-2338.

Winzeler, E. A., Shoemaker, D. D., Astromoff, A., Liang, H., Anderson, K., Andre, B., Bangham, R., Benito, R., Boeke, J. D., Bussey, H., Chu, A. M., Connelly, C., Davis, K., Dietrich, F., Dow, S. W., El Bakkoury, M., Foury, F., ccedil, oise, Friend, S. H., Gentalen, E., Giaever, G., Hegemann, J. H., Jones, T., Laub, M., Liao, H., Liebundguth, N., Lockhart, D. J., Lucau-Danila, A., Lussier, M., M'Rabet, N., Menard, P., Mittmann, M., Pai, C., Rebischung, C., Revuelta, J. L., Riles, L., Roberts, C. J., Ross-MacDonald, P., Scherens, B., Snyder, M., Sookhai-Mahadeo, S., Storms, R. K., eacute, ronneau, S., Voet, M., Volckaert, G., Ward, T. R., Wysocki, R., Yen, G. S., Yu, K., Zimmermann, K., Philippsen, P., Johnston, M., \& Davis, R. W. (1999). Functional 
Characterization of the S.\&nbsp;cerevisiae Genome by Gene Deletion and Parallel Analysis. Science, 285(5429), 901-906. doi:10.1126/science.285.5429.901

Wise, D. R., \& Thompson, C. B. (2010). Glutamine addiction: a new therapeutic target in cancer. Trends in Biochemical Sciences, 35(8), 427-433.

Wolfenden, R., \& Radzicka, A. (1991). Transition-state analogues. Current Opinion in Structural Biology, 1(5), 780-787.

Wood, V. (2006). Schizosaccharomyces pombe comparative genomics; from sequence to systems Comparative genomics (pp. 233-285): Springer.

Woodcock, D., Adams, J., Allan, R., \& Cooper, I. (1983). Effect of several inhibitors of enzymatic DNA methylation on the in vivo methylation of different classes of DNA sequences in a cultured human cell line. Nucleic Acids Research, 11(2), 489-499.

Wortmann, R. L., Andres, C., Kaminska, J., Mejias, E., Gelfand, E., Arnold, W., Rich, K., \& Fox, I. H. (1979). Purine Nucleoside Phosphorylase Deficiency. Arthritis \& Rheumatism, 22(5), 524-531. doi:10.1002/art.1780220513

Woster, P. M. (2006). Polyamine structure and synthetic analogs Polyamine cell signaling (pp. 3-24): Springer.

Woster, P. M., Black, A. Y., Duff, K. J., Coward, J. K., \& Pegg, A. E. (1989). Synthesis and biological evaluation of S-adenosyl-1, 12-diamino-3-thio-9-azadodecane, a multisubstrate adduct inhibitor of spermine synthase. Journal of Medicinal Chemistry, 32(6), 1300-1307.

Wu, M., St. Onge, R., Suresh, S., Davis, R., Peltz, G., Aronova, S., \& Baumann, S. (2012). Metabolic profiling of yeast sterols using the Agilent 7200 series GC/Q-TOF System. Retrieved from www.agilent.com/cs/library/applications/5991-0571EN.pdf

Wu, P., Nielsen, T. E., \& Clausen, M. H. (2015). FDA-approved small-molecule kinase inhibitors. Trends in Pharmacological Sciences, 36(7), 422-439. doi:10.1016/j.tips.2015.04.005

Xie, Z., Nair, U., \& Klionsky, D. J. (2008). Atg8 controls phagophore expansion during autophagosome formation. Molecular Biology of the Cell, 19(8), 3290-3298.

Xu, W., \& Mitchell, A. P. (2001). Yeast PalA/AIP1/Alix homolog Rim20p associates with a PEST-like region and is required for its proteolytic cleavage. Journal of Bacteriology, 183(23), 6917-6923.

Xu, Y. F., Letisse, F., Absalan, F., Lu, W., Kuznetsova, E., Brown, G., Caudy, A. A., Yakunin, A. F., Broach, J. R., \& Rabinowitz, J. D. (2013). Nucleotide degradation and ribose salvage in yeast. Mol Syst Biol, 9, 665. doi:10.1038/msb.2013.21

Yang, Z., \& Klionsky, D. J. (2009). An overview of the molecular mechanism of autophagy Autophagy in infection and immunity (pp. 1-32): Springer.

Yao, S. Y., Ng, A. M., Cass, C. E., Baldwin, S. A., \& Young, J. D. (2011). Nucleobase transport by human equilibrative nucleoside transporter 1 (hENT1). Journal of Biological Chemistry, 286(37), 32552-32562.

Yeh, Y.-Y., Wrasman, K., \& Herman, P. K. (2010). Autophosphorylation within the Atg1 activation loop is required for both kinase activity and the induction of autophagy in Saccharomyces cerevisiae. Genetics, 185(3), 871-882.

Youn, J. Y., Friesen, H., Kishimoto, T., Henne, W. M., Kurat, C. F., Ye, W., Ceccarelli, D. F., Sicheri, F., Kohlwein, S. D., McMahon, H. T., \& Andrews, B. J. (2010). Dissecting BAR domain function in the yeast Amphiphysins Rvs161 and Rvs167 during endocytosis. Mol Biol Cell, 21(17), 30543069. doi:10.1091/mbc.E10-03-0181

Young, J. D., Yao, S. Y. M., Baldwin, J. M., Cass, C. E., \& Baldwin, S. A. (2013). The human concentrative and equilibrative nucleoside transporter families, SLC28 and SLC29. Molecular Aspects of Medicine, 34(2-3), 529-547. doi:http://dx.doi.org/10.1016/j.mam.2012.05.007

Yu, Y., Eriksson, P., \& Stillman, D. J. (2000). Architectural transcription factors and the SAGA complex function in parallel pathways to activate transcription. Mol Cell Biol, 20(7), 2350-2357. 
Yu, Z.-Q., Ni, T., Hong, B., Wang, H.-Y., Jiang, F.-J., Zou, S., Chen, Y., Zheng, X.-L., Klionsky, D. J., \& Liang, Y. (2012). Dual roles of Atg8- PE deconjugation by Atg4 in autophagy. Autophagy, 8(6), 883892.

Yuen, K. W., Warren, C. D., Chen, O., Kwok, T., Hieter, P., \& Spencer, F. A. (2007). Systematic genome instability screens in yeast and their potential relevance to cancer. Proceedings of the National Academy of Sciences, 104(10), 3925-3930.

Zhao, Y., Butler, E. B., \& Tan, M. (2013). Targeting cellular metabolism to improve cancer therapeutics. Cell Death \& Disease, 4. doi:10.1038/cddis.2013.60

Zhu, H., Bilgin, M., Bangham, R., Hall, D., Casamayor, A., Bertone, P., Lan, N., Jansen, R., Bidlingmaier, S., Houfek, T., Mitchell, T., Miller, P., Dean, R. A., Gerstein, M., \& Snyder, M. (2001). Global analysis of protein activities using proteome chips. Science, 293(5537), 2101-2105. doi:10.1126/science.1062191

Zoncu, R., Efeyan, A., \& Sabatini, D. M. (2011). mTOR: from growth signal integration to cancer, diabetes and ageing. Nat Rev Mol Cell Biol, 12(1), 21-35. doi:10.1038/nrm3025 
7- Appendix

7.2.1 meu1 $\Delta$ mutant construction

a

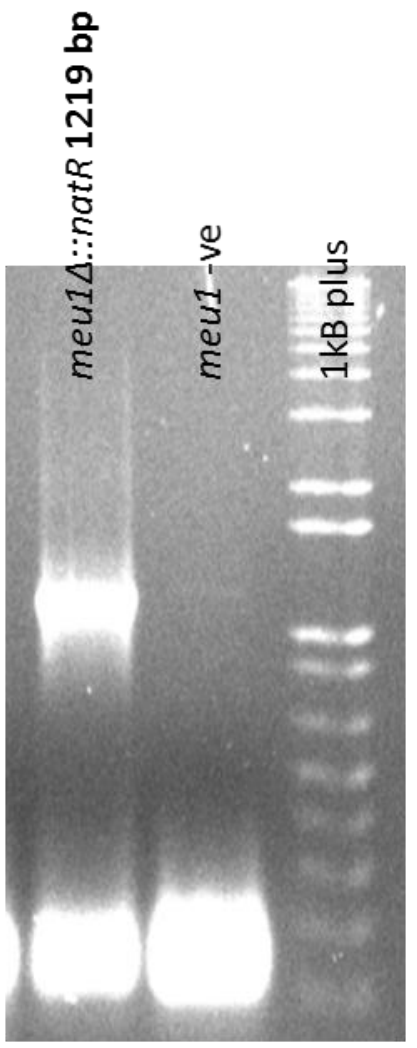

b

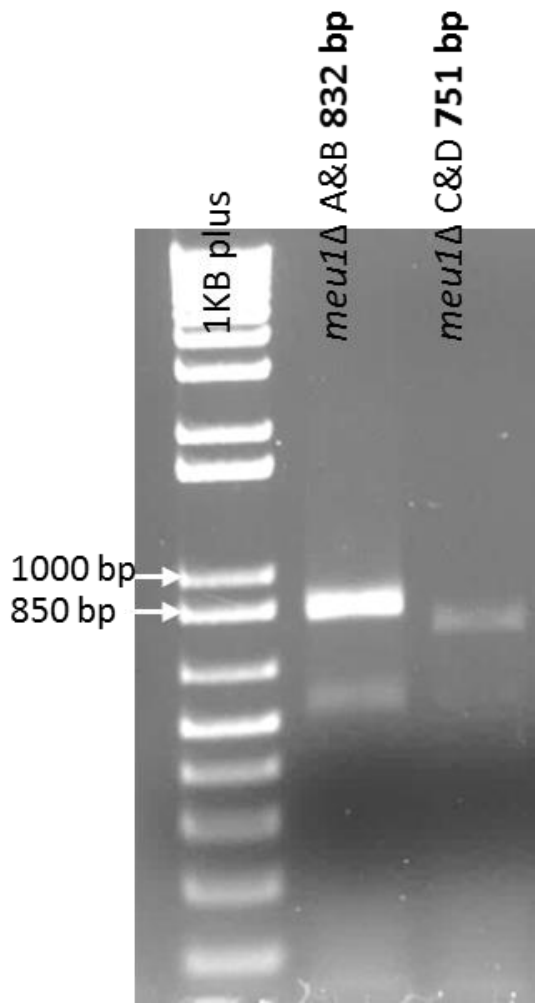

Figure 7.2.1 - meu1 $\Delta$ construction and validation PCRs. PCR products were run on a $1 \%$ agarose gel with $1 \mathrm{~KB}$ plus ladder at $100 \mathrm{mV}$. (a) meu1A::natR deletion construction PCR. (b) PCR validation of MEU1 knockout.

\begin{tabular}{|l|l|}
\hline Primer & Sequence \\
\hline $\begin{array}{l}\text { Fw primer } \\
(383)\end{array}$ & AGATGAACGTGTGAATGAAAAAAAACTATTTCGAGATGAGATGAGATTAAACATGGA \\
\hline $\begin{array}{l}\text { Rev primer } \\
(384)\end{array}$ & ATATATGTTTTCTCTCCTATTTATATTTTACATATGATTAGCGGCAACCACAGTATAGCG \\
\hline $\begin{array}{l}\text { Conformation } \\
\text { primer A (3) }\end{array}$ & CATGTCTATACGCAAATG \\
\hline $\begin{array}{l}\text { Conformation } \\
\text { primer B (388) }\end{array}$ & TACGAGACGACCACGAAGC \\
\hline $\begin{array}{l}\text { Conformation } \\
\text { primer C (389) }\end{array}$ & CATGTCTATACGCAAATG \\
\hline $\begin{array}{l}\text { Conformation } \\
\text { primer D (4) }\end{array}$ & TTATACGTTACTGTACAT \\
\hline
\end{tabular}

Table 7.2.1 - meu1s construction and conformation primers. Primers were designed according to Janke et al. 2006 (Janke et al., 2004). 


\subsubsection{Genetic interactions with MEU1}

\begin{tabular}{|c|c|c|c|c|c|}
\hline $\begin{array}{l}\text { ORF } \\
\text { name }\end{array}$ & $\begin{array}{l}\text { Gene } \\
\text { name }\end{array}$ & Function & $p$-value & $\begin{array}{l}- \\
\text { Score }\end{array}$ & $\begin{array}{l}\% \\
\text { Growt } \\
h\end{array}$ \\
\hline YCL025C & AGP1 & Low-affinity amino acid permease with broad substrate range & $3.04 \mathrm{E}-02$ & 2.17 & 61.73 \\
\hline YOR141C & ARP8 & Nuclear actin-related protein involved in chromatin remodelling & $3.62 \mathrm{E}-02$ & 2.09 & 62.50 \\
\hline YGR157W & $\mathrm{CHO} 2$ & Phosphatidylethanolamine methyltransferase (PEMT) & $7.03 E-03$ & 2.70 & 54.35 \\
\hline YBR036C & CSG2 & Endoplasmic reticulum membrane protein & $3.35 \mathrm{E}-02$ & 2.13 & 60.98 \\
\hline YNL286W & CUS2 & Putative checkpoint factor in transcription & 3.87E-02 & 2.07 & 64.52 \\
\hline YIR023W & DAL81 & $\begin{array}{l}\text { Positive regulator of genes in multiple nitrogen degradation } \\
\text { pathways }\end{array}$ & 2.93E-03 & 2.98 & 51.02 \\
\hline YDL160C & DHH1 & Cytoplasmic DEAD-box helicase, stimulates mRNA decapping & $3.10 \mathrm{E}-29$ & 11.22 & 7.52 \\
\hline YBL016W & FUS3 & $\begin{array}{l}\text { Mitogen-activated serine/threonine protein kinase involved in } \\
\text { mating }\end{array}$ & $4.66 \mathrm{E}-02$ & 1.99 & 64.52 \\
\hline YNL281W & $\mathrm{HCH} 1$ & Heat shock protein regulator & $4.13 \mathrm{E}-02$ & 2.04 & 64.52 \\
\hline YPR179C & HDA3 & Subunit of the HDA1 histone deacetylase complex & $1.30 \mathrm{E}-02$ & 2.48 & 57.14 \\
\hline YIL090W & ICE2 & $\begin{array}{l}\text { Integral ER membrane protein with type-III transmembrane } \\
\text { domains }\end{array}$ & $3.99 \mathrm{E}-02$ & 2.05 & 64.52 \\
\hline YDR123C & INO2 & Transcription factor & 1.37E-03 & 3.20 & 47.17 \\
\hline YJR073C & OPI3 & Methylene-fatty-acyl-phospholipid synthase & $9.09 \mathrm{E}-03$ & 2.61 & 55.56 \\
\hline YBR093C & $\mathrm{PHO5}$ & Repressible acid phosphatase & $1.42 \mathrm{E}-02$ & 2.45 & 57.47 \\
\hline YER162C & RAD4 & $\begin{array}{l}\text { Protein that recognizes and binds damaged DNA (with Rad23p) } \\
\text { during NER }\end{array}$ & $6.56 \mathrm{E}-03$ & 2.72 & 55.56 \\
\hline YMR154C & RIM13 & Calpain-like cysteine protease & $4.16 \mathrm{E}-02$ & 2.04 & 64.94 \\
\hline YLR371W & ROM2 & Guanine nucleotide exchange factor (GEF) for Rho1p and Rho2p & $1.98 \mathrm{E}-02$ & 2.33 & 59.17 \\
\hline YGL147C & RPL9A & Ribosomal 60S subunit protein L9A & $9.05 \mathrm{E}-24$ & 10.05 & 9.88 \\
\hline YLR357W & RSC2 & Component of the RSC chromatin remodelling complex & $4.83 \mathrm{E}-04$ & 3.49 & 45.25 \\
\hline YPR069C & SPE3 & Spermidine synthase & $4.88 \mathrm{E}-02$ & 1.97 & 64.52 \\
\hline YNL179C & SRF6 & Dubious open reading frame next to $\mathrm{RHO} 5$ & $4.46 \mathrm{E}-02$ & 2.01 & 65.36 \\
\hline YCL032W & STE50 & Adaptor protein for various signalling pathways & $1.33 \mathrm{E}-02$ & 2.48 & 58.82 \\
\hline YDR146C & SWI5 & $\begin{array}{l}\text { Transcription factor that recruits Mediator and Swi/Snf } \\
\text { complexes }\end{array}$ & $4.99 \mathrm{E}-02$ & 1.96 & 64.52 \\
\hline YKL081W & TEF4 & Gamma subunit of translational elongation factor eEF1B & $2.94 \mathrm{E}-02$ & 2.18 & 61.35 \\
\hline YDR074W & TPS2 & $\begin{array}{l}\text { Phosphatase subunit of the trehalose-6-P } \\
\text { synthase/phosphatase complex }\end{array}$ & $1.15 \mathrm{E}-02$ & 2.53 & 55.56 \\
\hline YDR080W & VPS41 & Subunit of the HOPS endocytic tethering complex & $2.23 \mathrm{E}-02$ & 2.29 & 58.82 \\
\hline YDR128W & YDR128W & Subunit of SEACAT, a subcomplex of the SEA complex & $1.08 \mathrm{E}-02$ & 2.55 & 55.25 \\
\hline YLR184W & YLR184W & Dubious open reading frame next to TOS4 & $4.42 \mathrm{E}-02$ & 2.01 & 62.89 \\
\hline YNL285W & YNL285W & $\begin{array}{l}\text { Dubious open reading frame that overlaps tRNA-Gly and } \\
\text { putative ASR1409 }\end{array}$ & $2.79 \mathrm{E}-02$ & 2.20 & 60.98 \\
\hline YGL255W & ZRT1 & High-affinity zinc transporter of the plasma membrane & $2.33 \mathrm{E}-02$ & 2.27 & 59.17 \\
\hline YNL241C & ZWF1 & Glucose-6-phosphate dehydrogenase (G6PD) & $1.39 \mathrm{E}-02$ & 2.46 & 56.50 \\
\hline
\end{tabular}

Table 7.2.2a - 31 putative genetic interactions with MEU1. The putative interactions with an epistatic

growth $((\operatorname{meu} 1 \Delta x x x \Delta / x x x \Delta)<70 \%, p<0.05)$ in at least two out of the triplicate SGAs are presented. 
Three independent SGAs were performed on agar using the RoToR HAD (Singer Instrument Co. Ltd). meu1 $1 \Delta$ query strain was mated against the haploid single gene deletion library en masse to according to SGA methodology (Tong et al., 2001). The haploid meu1 $x x x x \Delta$ double mutant meiotic progeny were assessed for epistatic colony size relative to the haploid parental mutants using Gitter tools (Wagih \& Parts, 2014) in RStudio v0.99.896 (Racine, 2012) and ScreenMill (Dittmar et al., 2010).

\begin{tabular}{|c|c|c|c|c|c|}
\hline $\begin{array}{l}\text { ORF } \\
\text { name }\end{array}$ & $\begin{array}{l}\text { Gene } \\
\text { name }\end{array}$ & Function & $p$-value & $\begin{array}{l}\text { Standard } \\
\text { deviation }\end{array}$ & $\begin{array}{l}\text { Growth } \\
\%\end{array}$ \\
\hline YNL141W & AAH1 & Adenine deaminase (adenine aminohydrolase) & $2.63 \mathrm{E}-15$ & 5.09 & 19.84 \\
\hline YML035C & AMD1 & AMP deaminase & 0.000146 & 6.20 & 9.74 \\
\hline YNL259C & ATX1 & Cytosolic copper metallochaperone & 1.69E-08 & 8.34 & 29.43 \\
\hline YBR131W & CCZ1 & $\begin{array}{l}\text { Subunit of a heterodimeric guanine nucleotide } \\
\text { exchange factor (GEF) }\end{array}$ & $1.24 \mathrm{E}-10$ & 2.66 & 51.81 \\
\hline YER061C & CEM1 & Mitochondrial beta-keto-acyl synthase & 6.74E-08 & 7.84 & 37.86 \\
\hline YBR036C & CSG2 & Endoplasmic reticulum membrane protein & 7.86E-09 & 8.89 & 12.23 \\
\hline YIR023W & DAL81 & $\begin{array}{l}\text { Positive regulator of genes in multiple nitrogen } \\
\text { degradation pathways }\end{array}$ & $6.15 \mathrm{E}-18$ & 2.54 & 23.01 \\
\hline YDR359C & EAF1 & $\begin{array}{l}\text { Component of the NuA4 histone acetyltransferase } \\
\text { complex }\end{array}$ & $6.21 \mathrm{E}-07$ & 9.97 & 25.51 \\
\hline YNL080C & EOS1 & Protein involved in $\mathrm{N}$-glycosylation & $4.51 \mathrm{E}-13$ & 6.47 & 11.17 \\
\hline YBR026C & ETR1 & 2-enoyl thioester reductase & $2.15 \mathrm{E}-10$ & 7.75 & 18.24 \\
\hline YBR020W & GAL1 & Galactokinase & 1.39E-05 & 11.45 & 21.91 \\
\hline YHR108W & GGA2 & $\begin{array}{l}\text { Protein that regulates Arf1p, Arf2p to facilitate Golgi } \\
\text { trafficking }\end{array}$ & $2.02 \mathrm{E}-05$ & 9.89 & 45.94 \\
\hline YHL031C & GOS1 & V-SNARE protein involved in Golgi transport & 0.04846 & 39.77 & 175.05 \\
\hline YOR038C & HIR2 & Subunit of HIR nucleosome assembly complex & $1.48 \mathrm{E}-09$ & 8.23 & 16.36 \\
\hline YOL012C & HTZ1 & Histone variant $\mathrm{H} 2 \mathrm{AZ}$ & 5.7E-05 & 10.78 & 52.43 \\
\hline YER092W & IES5 & $\begin{array}{l}\text { Non-essential INO80 chromatin remodelling complex } \\
\text { subunit }\end{array}$ & 0.000237 & 13.49 & 26.97 \\
\hline YDR123C & INO2 & Transcription factor & 1.39E-05 & 11.71 & 29.72 \\
\hline YKR019C & IRS4 & EH domain-containing protein & 0.000404 & 10.59 & 55.22 \\
\hline YLL019C & KNS1 & $\begin{array}{l}\text { Protein kinase involved in negative regulation of PollII } \\
\text { transcription }\end{array}$ & 0.000385 & 13.45 & 41.74 \\
\hline YPR070W & MED1 & Subunit of the RNA polymerase II mediator complex & 4.3E-08 & 4.32 & 3.46 \\
\hline YDR128W & MTC5 & Subunit of SEACAT, a subcomplex of the SEA complex & $4.24 \mathrm{E}-10$ & 6.05 & 43.92 \\
\hline YJR073C & OPI3 & Methylene-fatty-acyl-phospholipid synthase & $3.58 \mathrm{E}-24$ & 1.57 & -1.61 \\
\hline YCR077C & PAT1 & Deadenylation-dependent mRNA-decapping factor & $3.49 \mathrm{E}-12$ & 4.99 & 35.39 \\
\hline YLR134W & PDC5 & Minor isoform of pyruvate decarboxylase & $1.42 \mathrm{E}-13$ & 6.05 & 20.75 \\
\hline YBR093C & PHO5 & Repressible acid phosphatase & 0.010172 & 20.69 & 48.93 \\
\hline YPL036W & PMA2 & Plasma membrane $\mathrm{H}+$-ATPase & $4.43 \mathrm{E}-12$ & 6.35 & 25.73 \\
\hline YER162C & RAD4 & $\begin{array}{l}\text { Protein that recognizes and binds damaged DNA (with } \\
\text { Rad23p) during NER }\end{array}$ & $4.23 \mathrm{E}-10$ & 7.70 & 19.72 \\
\hline $\begin{array}{l}\text { YCR028C- } \\
\mathrm{A}\end{array}$ & RIM1 & $\begin{array}{l}\text { ssDNA-binding protein essential for mitochondrial } \\
\text { genome maintenance }\end{array}$ & $4.42 \mathrm{E}-05$ & 8.57 & 57.26 \\
\hline
\end{tabular}




\begin{tabular}{|c|c|c|c|c|c|}
\hline YLR371W & ROM2 & $\begin{array}{l}\text { Guanine nucleotide exchange factor (GEF) for Rho1p } \\
\text { and Rho2p }\end{array}$ & 0.025124 & 26.01 & 35.89 \\
\hline YLR061W & RPL22A & Ribosomal 60S subunit protein L22A & $3.73 \mathrm{E}-07$ & 8.88 & 34.92 \\
\hline YGL147C & RPL9A & Ribosomal 60S subunit protein L9A & 3.09E-21 & 3.16 & 0.34 \\
\hline YOR293W & RPS10A & $\begin{array}{l}\text { Protein component of the small (40S) ribosomal } \\
\text { subunit }\end{array}$ & 0.013664 & 9.38 & 55.11 \\
\hline YOL121C & RPS19A & $\begin{array}{l}\text { Protein component of the small (40S) ribosomal } \\
\text { subunit }\end{array}$ & 0.021084 & 18.73 & 51.26 \\
\hline YPL090C & RPS6A & $\begin{array}{l}\text { Protein component of the small (40S) ribosomal } \\
\text { subunit }\end{array}$ & 0.001387 & 16.24 & 39.66 \\
\hline YCR009C & RVS161 & Amphiphysin-like lipid raft protein & $8.19 \mathrm{E}-08$ & 5.65 & 58.15 \\
\hline YDR388W & RVS167 & Calmodulin-binding actin-associated protein & $6.98 \mathrm{E}-18$ & 3.05 & 22.29 \\
\hline YOL110W & SHR5 & Palmitoyltransferase subunit & 0.001225 & 15.63 & 36.34 \\
\hline YNL257C & SIP3 & Putative sterol transfer protein & $1.74 \mathrm{E}-12$ & 2.03 & 0.00 \\
\hline YMR016C & SOK2 & $\begin{array}{l}\text { Nuclear protein that negatively regulates } \\
\text { pseudohyphal differentiation }\end{array}$ & $1.28 \mathrm{E}-05$ & 10.86 & 35.27 \\
\hline YOL052C & SPE2 & S-adenosylmethionine decarboxylase & $6.11 \mathrm{E}-07$ & 10.74 & 2.55 \\
\hline YCL037C & SRO9 & Cytoplasmic RNA-binding protein & 0.000207 & 18.38 & 268.71 \\
\hline YIL047C & SYG1 & Plasma membrane protein of unknown function & $3.96 \mathrm{E}-18$ & 5.20 & 0.80 \\
\hline YOL020W & TAT2 & High affinity tryptophan and tyrosine permease & $5.6 \mathrm{E}-10$ & 7.73 & 23.01 \\
\hline YDR207C & UME6 & Rpd3L histone deacetylase complex subunit & 4.09E-05 & 5.13 & 32.41 \\
\hline YOR106W & VAM3 & Syntaxin-like vacuolar t-SNARE & $2.27 \mathrm{E}-06$ & 11.45 & 4.09 \\
\hline YDL077C & VAM6 & $\begin{array}{l}\text { Guanine nucleotide exchange factor for the GTPase } \\
\text { Gtr1p }\end{array}$ & 0.016927 & 18.40 & 54.79 \\
\hline YDR080W & VPS41 & Subunit of the HOPS endocytic tethering complex & $1.69 \mathrm{E}-10$ & 8.13 & 5.05 \\
\hline YDR290W & YDR290W & Dubious open reading frame overlaps RTT103 & 0.007849 & 18.21 & 42.96 \\
\hline YLR184W & YLR184W & Dubious open reading frame adjacent to TOS4 & $4.29 \mathrm{E}-18$ & 5.18 & 0.40 \\
\hline YNL198C & YNL198C & Dubious open reading frame overlaps GCR2 & 0.001642 & 16.59 & 26.43 \\
\hline YOL024W & YOL024W & Putative protein overlaps ARS1511 & 0.042438 & 30.98 & 29.10 \\
\hline YOR097C & YOR097C & Putative protein of unknown function & 0.000405 & 13.97 & 41.22 \\
\hline YPR126C & YPR126C & Dubious open reading frame overlaps YLH47 & $5.23 \mathrm{E}-14$ & 5.70 & 20.38 \\
\hline YNL241C & ZWF1 & Glucose-6-phosphate dehydrogenase (G6PD) & 1.96E-06 & 3.04 & 9.00 \\
\hline
\end{tabular}

Table 7.2.2b-52 negative and 2 positive genetic interaction with Meu1. The growth of the double mutants relative to the single haploid parental strains (meu1 $\Delta x x x \Delta /$ meu1 $\Delta) /(x x x \Delta /$ WT) $x 100$ was determined in liquid media at $16 \mathrm{~h}$ and $\mathrm{OD}_{600}$ for all cultures with the exception $x x x \Delta$ slow growers where the $\mathrm{OD}_{600}$ was measured at later time points. The growth of the double mutants was normalised to meu1 $\Delta$ his $3 \Delta$ control strain and the double deletion mutants with an average growth $> \pm 40 \%, p$ $<0.05$ of the meu1 $\Delta$ his $3 \Delta$ strain were considered a growth defect or a growth improvement. 
7.2.3 MTDIA screening of the gene deletion library

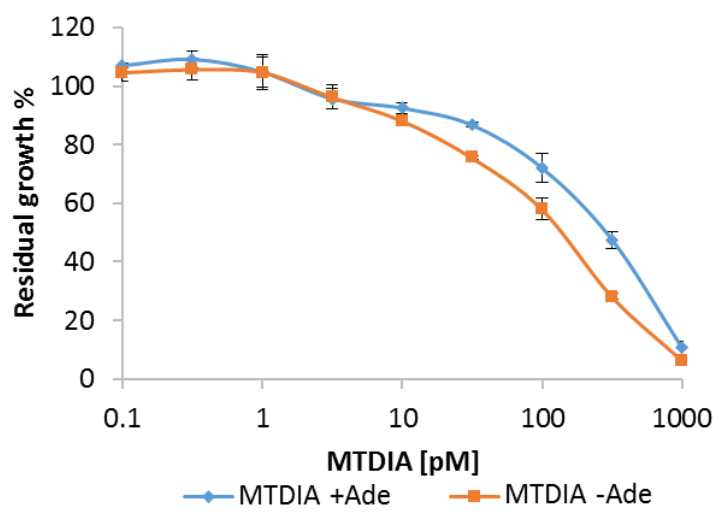

Figure 7.2.2 - Absence of adenine improves sensitivity MTDIA. Wt+hENT1 treated with MTDIA with and without adenine. The growth assays were performed as previously described in SDMAUC+MTA+RG media.

7.2.4 Drug-gene interactions and GO enrichment MTDIA

\begin{tabular}{|l|l|l|l|l|l|}
\hline $\begin{array}{l}\text { ORF } \\
\text { name }\end{array}$ & $\begin{array}{l}\text { Gene } \\
\text { name }\end{array}$ & Function & p-value & $\begin{array}{l}\text { Z- } \\
\text { Score }\end{array}$ & $\begin{array}{l}\% \\
\text { Growth }\end{array}$ \\
\hline YOR128C & ADE2 & Phosphoribosylaminoimidazole carboxylase & $4.22 \mathrm{E}-02$ & 2.03 & 75.19 \\
\hline YMR300C & ADE4 & Phosphoribosylpyrophosphate amidotransferase (PRPPAT) & $5.64 \mathrm{E}-02$ & 1.91 & 78.74 \\
\hline YGL234W & ADE5,7 & $\begin{array}{l}\text { Enzyme of the 'de novo' purine nucleotide biosynthetic } \\
\text { pathway }\end{array}$ & $9.46 \mathrm{E}-03$ & 2.60 & 72.99 \\
\hline YGR061C & ADE6 & Formylglycinamidine-ribonucleotide (FGAM)-synthetase & $3.68 \mathrm{E}-04$ & 3.56 & 60.98 \\
\hline YDR408C & ADE8 & Phosphoribosyl-glycinamide transformylase & $4.04 \mathrm{E}-02$ & 2.05 & 77.52 \\
\hline YCL025C & AGP1 & $\begin{array}{l}\text { Low-affinity amino acid permease with broad substrate } \\
\text { range }\end{array}$ & $6.08 \mathrm{E}-03$ & -2.44 & 128.21 \\
\hline YNR074C & AIF1 & Mitochondrial cell death effector & $7.04 \mathrm{E}-02$ & 1.81 & 80.00 \\
\hline YKR099W & BAS1 & Myb-related transcription factor & $8.70 \mathrm{E}-03$ & 2.62 & 77.52 \\
\hline YDL074C & BRE1 & E3 ubiquitin ligase & $9.45 \mathrm{E}-03$ & -2.60 & 129.87 \\
\hline YOR026W & BUB3 & Kinetochore checkpoint WD40 repeat protein & $7.25 \mathrm{E}-05$ & 3.27 & 67.57 \\
\hline YBR131W & CCZ1 & $\begin{array}{l}\text { Subunit of a heterodimeric guanine nucleotide exchange } \\
\text { factor (GEF) }\end{array}$ & $1.70 \mathrm{E}-04$ & -1.83 & 128.21 \\
\hline YLR394W & CST9 & SUMO E3 ligase & $3.00 \mathrm{E}-02$ & 1.86 & 79.37 \\
\hline YOR037W & CYC2 & Mitochondrial peripheral inner membrane protein & $2.55 \mathrm{E}-02$ & 1.77 & 80.00 \\
\hline YBL101C & ECM21 & $\begin{array}{l}\text { Protein involved in regulating endocytosis of plasma } \\
\text { membrane proteins }\end{array}$ & $3.54 \mathrm{E}-03$ & 2.92 & 75.76 \\
\hline YCR034W & ELO2 & Fatty acid elongase, involved in sphingolipid biosynthesis & $2.74 \mathrm{E}-03$ & -3.00 & 135.14 \\
\hline YBR026C & ETR1 & 2-enoyl thioester reductase & $1.21 \mathrm{E}-02$ & 2.51 & 70.42 \\
\hline YER145C & FTR1 & High affinity iron permease & $2.22 \mathrm{E}-03$ & -3.06 & 138.89 \\
\hline YER083C & GET2 & Subunit of the GET complex & $4.90 \mathrm{E}-02$ & -1.97 & 131.58 \\
\hline YJR090C & GRR1 & $\begin{array}{l}\text { F-box protein component of an SCF ubiquitin-ligase } \\
\text { complex }\end{array}$ & $2.55 \mathrm{E}-02$ & 2.23 & 77.52 \\
\hline YOR038C & HIR2 & Subunit of HIR nucleosome assembly complex & $1.86 \mathrm{E}-04$ & 3.74 & 64.10 \\
\hline
\end{tabular}




\begin{tabular}{|c|c|c|c|c|c|}
\hline YJR140C & HIR3 & Subunit of the HIR complex & $4.92 \mathrm{E}-04$ & 3.48 & 69.44 \\
\hline YOL068C & HST1 & NAD(+)-dependent histone deacetylase & 7.97E-04 & 3.35 & 67.11 \\
\hline YOR025W & HST3 & $\begin{array}{l}\text { Member of the Sir2 family of NAD(+)-dependent protein } \\
\text { deacetylases }\end{array}$ & 1.87E-03 & 3.11 & 68.97 \\
\hline YOL012C & HTZ1 & Histone variant $\mathrm{H} 2 \mathrm{AZ}$ & 5.99E-03 & 1.95 & 78.74 \\
\hline YKR019C & IRS4 & EH domain-containing protein & $9.80 \mathrm{E}-03$ & 2.58 & 78.13 \\
\hline YLL007C & LMO1 & $\begin{array}{l}\text { Homolog of mammalian ELMO (Engulfment and celL } \\
\text { MOtility) }\end{array}$ & $1.79 \mathrm{E}-02$ & 2.37 & 80.00 \\
\hline YKR007W & MEH1 & Component of the EGO and GSE complexes & $1.93 \mathrm{E}-02$ & 2.34 & 80.00 \\
\hline YPR118W & MRI1 & 5'-methylthioribose-1-phosphate isomerase & $2.25 \mathrm{E}-02$ & 2.28 & 75.76 \\
\hline YOR209C & NPT1 & Nicotinate phosphoribosyltransferase & $2.76 \mathrm{E}-02$ & 2.20 & 76.34 \\
\hline YCR077C & PAT1 & Deadenylation-dependent mRNA-decapping factor & $6.78 \mathrm{E}-03$ & -2.71 & 131.58 \\
\hline YLR044C & PDC1 & Major of three pyruvate decarboxylase isozymes & $6.12 \mathrm{E}-02$ & 1.87 & 79.37 \\
\hline YGL059W & PKP2 & Mitochondrial protein kinase & $4.72 \mathrm{E}-02$ & 1.98 & 78.13 \\
\hline YML017W & PSP2 & Asn rich cytoplasmic protein that contains RGG motifs & $5.01 \mathrm{E}-02$ & 1.96 & 78.74 \\
\hline YOR279C & RFM1 & Component of the Sum1p-Rfm1p-Hst1p complex & 9.87E-04 & 3.29 & 72.99 \\
\hline $\begin{array}{l}\text { YCR028C- } \\
\text { A }\end{array}$ & RIM1 & $\begin{array}{l}\text { ssDNA-binding protein essential for mitochondrial genome } \\
\text { maintenance }\end{array}$ & $3.08 \mathrm{E}-04$ & -3.61 & 144.93 \\
\hline YJL121C & RPE1 & D-ribulose-5-phosphate 3-epimerase & $\mathrm{E}-03$ & 2.86 & 75.76 \\
\hline YDR388W & RVS167 & Calmodulin-binding actin-associated protein & $5.98 \mathrm{E}-05$ & 2.20 & 76.34 \\
\hline YMR016C & SOK2 & $\begin{array}{l}\text { Nuclear protein that negatively regulates pseudohyphal } \\
\text { differentiation }\end{array}$ & 5.41E-03 & 2.78 & 68.03 \\
\hline YCL037C & SRO9 & Cytoplasmic RNA-binding protein & $4.68 \mathrm{E}-02$ & -1.99 & 123.46 \\
\hline YDR310C & SUM1 & $\begin{array}{l}\text { Transcriptional repressor that regulates middle-sporulation } \\
\text { genes }\end{array}$ & 2.77E-02 & 2.20 & 73.53 \\
\hline YLR426W & TDA5 & Putative protein of unknown function & $2.55 \mathrm{E}-03$ & 3.02 & 74.63 \\
\hline YCR053W & THR4 & Threonine synthase & $1.65 \mathrm{E}-03$ & -3.15 & 138.89 \\
\hline YFR010W & UBP6 & Ubiquitin-specific protease & $4.13 \mathrm{E}-02$ & -2.04 & 131.58 \\
\hline YEL038W & UTR4 & Protein with sequence similarity to acireductone synthases & $1.33 \mathrm{E}-02$ & -2.48 & 129.87 \\
\hline YAL002W & VPS8 & Membrane-binding component of the CORVET complex & $4.42 \mathrm{E}-02$ & -2.01 & 123.46 \\
\hline YDR290W & YDR290W & Dubious open reading frame; overlaps RTT103 & 2.17E-04 & 3.70 & 64.52 \\
\hline YJL120W & YJL120W & Dubious open reading frame; overlaps the RPE1 & $2.55 \mathrm{E}-02$ & 2.23 & 76.34 \\
\hline YLR236C & YLR236C & Dubious open reading frame; overlaps ORF YLR235C & $2.56 \mathrm{E}-05$ & 4.21 & 55.87 \\
\hline YNR025C & YNR025C & Dubious open reading frame; overlaps MPP6 & $4.03 \mathrm{E}-02$ & 2.05 & 77.52 \\
\hline YOR024W & YOR024W & Dubious open reading frame; Overlaps 5'UTR of HST3 & 4.56E-02 & 2.00 & 78.13 \\
\hline YOR072W & YOR072W & $\begin{array}{l}\text { Dubious open reading frame; overlaps the dubious gene } \\
\text { YOR072W-A }\end{array}$ & 1.59E-02 & 2.41 & 74.63 \\
\hline YPR195C & YPR195C & $\begin{array}{l}\text { Dubious open reading frame; at the } 5 \text { ' end of OPT2 (Oligo } \\
\text { peptide transporter 2) }\end{array}$ & 5.19E-02 & 1.94 & 78.74 \\
\hline
\end{tabular}

Table 7.2.3a - 52 putative drug-gene interactions with 3nM MTDIA. 4310 gene deletion strains

( $x x x \Delta+h E N T 1)$ were screened against 3 nM MTDIA or Vehicle control in SD-MAUC+MTA+RG media in agar in triplicate experiments. Deletion strains with a growth defect or growth improvement (80\%>growth $>120 \%, P$-value $<0.05)$ in at least two out of three SGAs were considered as hypersensitive or resistant to MTDIA and are shown. 


\begin{tabular}{|c|c|c|c|c|c|}
\hline \begin{tabular}{|l|} 
ORF \\
name
\end{tabular} & $\begin{array}{l}\text { Gene } \\
\text { name }\end{array}$ & Function & $\begin{array}{l}\text { \% } \\
\text { Growth }\end{array}$ & $\begin{array}{l}\text { Standard } \\
\text { deviation }\end{array}$ & $p$-value \\
\hline YAR015W & ADE1 & $\begin{array}{l}\text { N-succinyl-5-aminoimidazole-4-carboxamide ribotide } \\
\text { synthetase }\end{array}$ & 64.78 & 6.55 & $1.07 \mathrm{E}-04$ \\
\hline YMR300C & ADE4 & $\begin{array}{l}\text { Phosphoribosylpyrophosphate amidotransferase } \\
\text { (PRPPAT) }\end{array}$ & 69.80 & 3.05 & $2.16 \mathrm{E}-05$ \\
\hline YGL234W & ADE5,7 & & 62.03 & 2.45 & 2.05E-07 \\
\hline YGR061C & ADE6 & Formylglycinamidine-ribonucleotide (FGAM)-synthetase & 72.67 & 3.73 & $5.62 \mathrm{E}-05$ \\
\hline YDR408C & ADE8 & Phosphoribosyl-glycinamide transformylase & 69.67 & 1.45 & $2.14 \mathrm{E}-05$ \\
\hline YDR127W & ARO1 & Pentafunctional AROM protein & 69.01 & 1.93 & $1.74 \mathrm{E}-05$ \\
\hline YDL074C & BRE1 & E3 ubiquitin ligase & 68.16 & 4.25 & $2.39 \mathrm{E}-05$ \\
\hline YOR061W & CKA2 & Alpha' catalytic subunit of casein kinase 2 (CK2) & 53.31 & 1.69 & $8.82 \mathrm{E}-07$ \\
\hline YDR151C & CTH1 & Member of the $\mathrm{CCCH}$ zinc finger family & 72.27 & 10.13 & $7.53 \mathrm{E}-03$ \\
\hline YOR030W & DFG16 & Probable multiple transmembrane protein & 69.09 & 11.02 & $1.12 \mathrm{E}-02$ \\
\hline YDL160C & $\mathrm{DHH} 1$ & $\begin{array}{l}\text { Cytoplasmic DEAD-box helicase, stimulates mRNA } \\
\text { decapping }\end{array}$ & 65.57 & 9.96 & 2.09E-03 \\
\hline YDR121W & DPB4 & $\begin{array}{l}\text { Subunit of DNA pol epsilon and of ISW2 chromatin } \\
\text { accessibility complex }\end{array}$ & 72.37 & 0.73 & 4.77E-05 \\
\hline YGL043W & DST1 & General transcription elongation factor TFIIS & 71.23 & 0.00 & $3.89 \mathrm{E}-05$ \\
\hline YBL101C & ECM21 & $\begin{array}{l}\text { Protein involved in regulating endocytosis of plasma } \\
\text { membrane proteins }\end{array}$ & 19.33 & 2.28 & $7.26 \mathrm{E}-09$ \\
\hline YBR076W & ECM8 & Non-essential protein of unknown function next to SLM4 & 39.46 & 23.52 & $3.35 \mathrm{E}-02$ \\
\hline YLR246W & ERF2 & Subunit of a palmitoyltransferase & 67.54 & 4.98 & $5.38 \mathrm{E}-05$ \\
\hline YBR026C & ETR1 & 2-enoyl thioester reductase & 69.53 & 7.42 & $1.37 \mathrm{E}-03$ \\
\hline YDR221W & GTB1 & $\begin{array}{l}\text { Glucosidase II beta subunit, forms a complex with alpha } \\
\text { subunit Rot } 2 p\end{array}$ & 71.92 & 5.85 & $1.34 \mathrm{E}-04$ \\
\hline YGR163W & GTR2 & Subunit of a TORC1-stimulating GTPase complex & 74.99 & 7.83 & $1.30 \mathrm{E}-03$ \\
\hline YPR179C & HDA3 & Subunit of the HDA1 histone deacetylase complex & 40.89 & 16.43 & $1.15 \mathrm{E}-02$ \\
\hline YOR038C & HIR2 & Subunit of HIR nucleosome assembly complex & 33.48 & 14.19 & $4.96 \mathrm{E}-03$ \\
\hline YJR140C & HIR3 & Subunit of the HIR nucleosome assembly complex & 74.74 & 4.50 & $2.14 \mathrm{E}-04$ \\
\hline YIL112W & HOS4 & Subunit of the Set3 complex & 72.26 & 12.21 & $2.91 \mathrm{E}-02$ \\
\hline YDR533C & HSP31 & $\begin{array}{l}\text { Methylglyoxalase that converts methylglyoxal to D- } \\
\text { lactate }\end{array}$ & 60.20 & 9.66 & $1.69 \mathrm{E}-03$ \\
\hline YOL068C & HST1 & NAD(+)-dependent histone deacetylase & 41.48 & 5.46 & $1.03 \mathrm{E}-06$ \\
\hline YOR025W & HST3 & $\begin{array}{l}\text { Member of the Sir2 family of NAD(+)-dependent protein } \\
\text { deacetylases }\end{array}$ & 70.76 & 10.64 & $1.18 \mathrm{E}-02$ \\
\hline YIL090W & ICE2 & $\begin{array}{l}\text { Integral ER membrane protein with type-III } \\
\text { transmembrane domains }\end{array}$ & 55.40 & 5.56 & 8.49E-06 \\
\hline YLL019C & KNS1 & $\begin{array}{l}\text { Protein kinase involved in negative regulation of PollII } \\
\text { transcription }\end{array}$ & 72.25 & 1.25 & $3.98 \mathrm{E}-05$ \\
\hline YDR532C & KRE28 & Subunit of a kinetochore-microtubule binding complex & 73.47 & 9.32 & 4.27E-03 \\
\hline YDL056W & MBP1 & $\begin{array}{l}\text { Transcription factor involved in regulation of cell cycle } \\
\text { progression }\end{array}$ & 72.75 & 9.77 & $1.26 \mathrm{E}-02$ \\
\hline YDR128W & MTC5 & Subunit of SEACAT, a subcomplex of the SEA complex & 66.33 & 9.22 & $5.15 \mathrm{E}-03$ \\
\hline YOR209C & NPT1 & Nicotinate phosphoribosyltransferase & 58.34 & 3.85 & $2.13 \mathrm{E}-06$ \\
\hline
\end{tabular}




\begin{tabular}{|c|c|c|c|c|c|}
\hline YER178W & PDA1 & $\begin{array}{l}\text { E1 alpha subunit of the pyruvate dehydrogenase (PDH) } \\
\text { complex }\end{array}$ & 73.78 & 3.66 & 7.63E-05 \\
\hline YML017W & PSP2 & Asn rich cytoplasmic protein that contains RGG motifs & 48.04 & 10.95 & $2.21 \mathrm{E}-03$ \\
\hline YOR279C & RFM1 & Component of the Sum1p-Rfm1p-Hst1p complex & 46.33 & 4.52 & $3.38 \mathrm{E}-07$ \\
\hline YOR275C & RIM20 & Protein involved in proteolytic activation of Rim101p & 67.67 & 1.69 & $1.06 \mathrm{E}-05$ \\
\hline YNL294C & RIM21 & pH sensor molecule, component of the RIM101 pathway & 74.40 & 15.10 & 4.64E-02 \\
\hline YER117W & RPL23B & Ribosomal 60 S subunit protein L23B & 67.52 & 11.52 & $1.29 \mathrm{E}-02$ \\
\hline YDL020C & RPN4 & $\begin{array}{l}\text { Transcription factor that stimulates expression of } \\
\text { proteasome genes }\end{array}$ & 44.62 & 7.84 & $7.84 \mathrm{E}-05$ \\
\hline YOR293W & RPS10A & Protein component of the small (40S) ribosomal subunit & 58.46 & 6.53 & $8.32 \mathrm{E}-05$ \\
\hline YDL194W & SNF3 & Plasma membrane low glucose sensor, & 74.44 & 7.57 & $1.46 \mathrm{E}-03$ \\
\hline YBR289W & SNF5 & Subunit of the SWI/SNF chromatin remodelling complex & 72.20 & 4.79 & $4.92 \mathrm{E}-05$ \\
\hline YMR016C & SOK2 & $\begin{array}{l}\text { Nuclear protein that negatively regulates pseudohyphal } \\
\text { differentiation }\end{array}$ & 4.26 & 1.36 & 5.07E-09 \\
\hline YCR081W & SRB8 & Subunit of the RNA polymerase II mediator complex & 32.35 & 6.67 & 5.00E-04 \\
\hline YDR310C & SUM1 & $\begin{array}{l}\text { Transcriptional repressor that regulates middle- } \\
\text { sporulation genes }\end{array}$ & 36.77 & 3.49 & 5.16E-08 \\
\hline YLR426W & TDA5 & Putative protein of unknown function & 43.22 & 4.64 & 2.95E-07 \\
\hline YGR260W & TNA1 & High affinity nicotinic acid plasma membrane permease & 67.56 & 3.67 & $1.56 \mathrm{E}-04$ \\
\hline YJR066W & TOR1 & PIK-related protein kinase and rapamycin target & 74.48 & 7.11 & 2.00E-03 \\
\hline YLR425W & TUS1 & $\begin{array}{l}\text { Guanine nucleotide exchange factor (GEF) that } \\
\text { modulates Rho1p activity }\end{array}$ & 65.50 & 10.22 & 4.00E-03 \\
\hline YOR043W & WHI2 & $\begin{array}{l}\text { Protein required for full activation of the general stress } \\
\text { response }\end{array}$ & 48.58 & 5.44 & 2.86E-06 \\
\hline YDL224C & WHI4 & Putative RNA binding protein & 73.89 & 3.09 & 5.25E-05 \\
\hline YHR161C & YAP1801 & $\begin{array}{l}\text { Protein of the AP180 family, involved in clathrin cage } \\
\text { assembly }\end{array}$ & 74.44 & 7.81 & $1.28 \mathrm{E}-03$ \\
\hline YCR099C & YCR099C & $\begin{array}{l}\text { Putative protein overlaps another putative protein } \\
\text { YCR100C }\end{array}$ & 73.99 & 12.95 & $3.68 \mathrm{E}-02$ \\
\hline YDL242W & YDL242W & Dubious open reading frame & 74.56 & 6.07 & 8.06E-04 \\
\hline YGR054W & YGR054W & Eukaryotic initiation factor elF2A & 69.50 & 3.23 & $2.23 \mathrm{E}-05$ \\
\hline YHL008C & YHL008C & Putative protein of unknown function & 70.58 & 8.35 & $2.23 \mathrm{E}-03$ \\
\hline YLR235C & YLR235C & $\begin{array}{l}\text { Dubious open reading frame; overlaps gene TOP3 and } \\
\text { YLR236C }\end{array}$ & 30.87 & 9.11 & $1.08 \mathrm{E}-04$ \\
\hline YLR236C & YLR236C & Dubious open reading frame; overlaps gene TOP3 & 7.77 & 3.16 & 1.54E-09 \\
\hline YNL179C & YNL179C & $\begin{array}{l}\text { Dubious open reading frame; deletion in cyr1 mutant } \\
\text { results in loss of stress resistance }\end{array}$ & 52.79 & 9.31 & 4.15E-04 \\
\hline YOR024W & YOR024W & Dubious open reading frame adjacent to HST3 & 64.34 & 6.03 & $1.04 \mathrm{E}-04$ \\
\hline YOR072W & YOR072W & $\begin{array}{l}\text { Dubious open reading frame; overlaps gene YOROT2W-A } \\
\text { and adjacent to NRT1 }\end{array}$ & 19.98 & 1.66 & 1.67E-08 \\
\hline YGL255W & ZRT1 & High-affinity zinc transporter of the plasma membrane & 62.82 & 2.56 & 3.60E-06 \\
\hline
\end{tabular}

Table 7.2.3b - 62 validated gene deletions hypertensive to $3 \mathrm{nM}$ MTDIA. The growth of the single gene deletion mutants treated with MTDIA relative to WT treated with MTDIA ( $x x x \Delta+$ MTDIA) $x x x \Delta+$ carrier)/ (WT+MTDIA/ WT+carrier) $\times 100$ was determined in liquid media at $24 \mathrm{~h}$ and $\mathrm{OD}_{600}$. For slow growing deletion strains, $\mathrm{OD}_{600}$ was measured at later time points where $\mathrm{OD}_{600}$ units in the vehicle 
control was between $0.3-0.5$. The his $3 \Delta$ deletion strain was used as the WT control. The single mutants with average growth $<75 \%$, P-value $<0.05$ from three replicates compared to the WT control were considered hypersensitive to MTDIA. These were assigned to simplified GO categories using GO slim mapping tool on SGD and were involved in amino acid biosynthesis, multivesicular body formation, Sterol biosynthesis, ion homeostasis, ribosome biogenesis, glucose metabolism, gene expression, NAD+ biosynthesis, cell cycle progression. (61 without SRB8)

\begin{tabular}{|c|c|c|c|c|c|}
\hline ORF name & $\begin{array}{l}\text { Gene } \\
\text { name }\end{array}$ & Function & $\begin{array}{l}\text { \% } \\
\text { Growth }\end{array}$ & $\begin{array}{l}\text { Standard } \\
\text { deviation }\end{array}$ & $p$-value \\
\hline YBR036C & CSG2 & Endoplasmic reticulum membrane protein & 132.93 & 9.55 & $1.20 \mathrm{E}-02$ \\
\hline YOR037W & CYC2 & Mitochondrial peripheral inner membrane protein & 131.28 & 8.49 & $4.83 \mathrm{E}-03$ \\
\hline YIR023W & DAL81 & $\begin{array}{l}\text { Positive regulator of genes in multiple nitrogen } \\
\text { degradation pathways }\end{array}$ & 148.57 & 14.70 & 3.47E-02 \\
\hline YCR034W & ELO2 & $\begin{array}{l}\text { Fatty acid elongase, involved in sphingolipid } \\
\text { biosynthesis }\end{array}$ & 138.73 & 12.58 & $1.71 \mathrm{E}-02$ \\
\hline YAL048C & GEM1 & $\begin{array}{l}\text { Outer mitochondrial membrane GTPase, subunit of the } \\
\text { ERMES complex }\end{array}$ & 131.29 & 9.08 & $2.91 \mathrm{E}-02$ \\
\hline YDL035C & GPR1 & $\begin{array}{l}\text { Plasma membrane G protein coupled receptor (GPCR), } \\
\text { gpr1 } 1 \Delta \text { inhibits pexophagy }\end{array}$ & 132.76 & 10.12 & -02 \\
\hline YNL164C & IBD2 & $\begin{array}{l}\text { Component of the BUB2-dependent spindle checkpoint } \\
\text { pathway }\end{array}$ & 137.60 & 17.76 & $3.29 \mathrm{E}-02$ \\
\hline YFL013C & IES1 & Subunit of the INO80 chromatin remodelling complex & 131.32 & 11.59 & $3.55 \mathrm{E}-02$ \\
\hline YOR235W & IRC13 & Dubious open reading frame; Overlaps SNR17A & 134.45 & 2.45 & $1.52 \mathrm{E}-04$ \\
\hline YGL124C & MON1 & $\begin{array}{l}\text { Subunit of a heterodimeric guanine nucleotide } \\
\text { exchange factor (GEF) }\end{array}$ & 136.65 & 6.91 & $1.63 \mathrm{E}-03$ \\
\hline YEL062W & NPR2 & Subunit of the Iml1p/SEACIT complex & 135.99 & 5.92 & $4.26 \mathrm{E}-04$ \\
\hline YCR077C & PAT1 & Deadenylation-dependent mRNA-decapping factor & 152.76 & 1.10 & $3.23 \mathrm{E}-06$ \\
\hline YJR034W & PET191 & Protein required for assembly of cytochrome c oxidase & 135.98 & 8.40 & $6.18 \mathrm{E}-03$ \\
\hline YDR244W & PEX5 & $\begin{array}{l}\text { Peroxisomal membrane signal receptor for peroxisomal } \\
\text { matrix proteins }\end{array}$ & 135.14 & 9.37 & $9.90 \mathrm{E}-03$ \\
\hline YDL006W & PTC1 & Type $2 \mathrm{C}$ protein phosphatase (PP2C), cell wall integrity & 159.48 & 3.28 & $6.28 \mathrm{E}-07$ \\
\hline YOR001W & RRP6 & Nuclear exosome exonuclease component & 158.32 & 11.63 & 5.37E-03 \\
\hline YBR095C & RXT2 & Component of the histone deacetylase Rpd3L complex & 154.26 & 3.38 & $9.62 \mathrm{E}-07$ \\
\hline YDR227W & SIR4 & $\begin{array}{l}\text { SIR protein involved in assembly of silent chromatin } \\
\text { domains }\end{array}$ & 131.87 & 9.75 & $2.13 \mathrm{E}-02$ \\
\hline YEL031W & SPF1 & P-type ATPase, ion transporter of the ER membrane & 179.63 & 9.00 & $1.23 \mathrm{E}-04$ \\
\hline YKL081W & TEF4 & Gamma subunit of translational elongation factor eEF1B & 132.93 & 13.40 & $1.62 \mathrm{E}-02$ \\
\hline YDR074W & TPS2 & $\begin{array}{l}\text { Phosphatase subunit of the trehalose-6-P } \\
\text { synthase/phosphatase complex }\end{array}$ & 161.10 & 12.45 & 3.67E-03 \\
\hline YOR068C & VAM10 & Protein involved in vacuole morphogenesis & 144.82 & 15.63 & $2.01 \mathrm{E}-02$ \\
\hline YOR132W & VPS17 & Subunit of the membrane-associated retromer complex & 133.06 & 5.58 & $4.49 \mathrm{E}-04$ \\
\hline YPR173C & VPS4 & $\begin{array}{l}\text { AAA-ATPase involved in multivesicular body (MVB) } \\
\text { protein sorting }\end{array}$ & 181.28 & 26.75 & 2.80E-02 \\
\hline YOR069W & VPS5 & Nexin-1 homolog & 156.87 & 13.40 & 7.05E-03 \\
\hline
\end{tabular}




\begin{tabular}{|l|l|l|l|l|l|}
\hline YJL029C & VPS53 & $\begin{array}{l}\text { Component of the GARP (Golgi-associated retrograde } \\
\text { protein) complex }\end{array}$ & 162.27 & 7.87 & $1.40 \mathrm{E}-04$ \\
\hline YAL002W & VPS8 & Membrane-binding component of the CORVET complex & 142.09 & 7.35 & $7.80 \mathrm{E}-04$ \\
\hline YBR016W & YBR016W & $\begin{array}{l}\text { Tail-anchored plasma membrane protein with a } \\
\text { conserved CYSTM module }\end{array}$ & 144.15 & 7.08 & $6.21 \mathrm{E}-04$ \\
\hline YBR209W & YBR209W & Dubious open reading frame & 140.61 & 10.01 & $4.42 \mathrm{E}-03$ \\
\hline YDR455C & YDR455C & Dubious open reading frame; overlaps gene YDR456W & 164.06 & 21.16 & $4.51 \mathrm{E}-02$ \\
\hline
\end{tabular}

Table 7.2.3c-30 validated gene deletion strains were resistant to MTDIA. The growth of the single gene deletion mutants treated with MTDIA relative to WT treated with MTDIA ( $x x x \Delta+$ MTDIA) $x x x \Delta+$ carrier)/ (WT+MTDIA/ WT+carrier) $x 100$ was determined in liquid media at $24 \mathrm{~h}$ and OD600. For slow growing deletion strains, $\mathrm{OD}_{600}$ was measured at later time points where $\mathrm{OD}_{600}$ units in the vehicle control was between $0.3-0.5$. The his $3 \Delta$ deletion strain was used as the WT control. The single mutants with average growth $>130 \%, P$-value $<0.05$ from three replicates compared to the WT control were considered resistant to MTDIA. These were assigned to simplified GO categories using GO slim mapping tool on SGD and were involved in endosomal transport, vacuole fusion, mitochondrion organization, translation, chromatin organisation (29 without dal81).

\begin{tabular}{|l|l|}
\hline Gene ontology: Biological process & $\begin{array}{l}\text { Corrected } \\
\text { P-values }\end{array}$ \\
\hline Negative regulation of gene expression & $1.18 \mathrm{E}-04$ \\
\hline Negative regulation of macromolecule biosynthetic process & $1.31 \mathrm{E}-04$ \\
\hline IMP biosynthetic process & $1.46 \mathrm{E}-04$ \\
\hline Regulation of gene expression & $5.97 \mathrm{E}-04$ \\
\hline Negative regulation of transcription, DNA-templated & $6.15 \mathrm{E}-04$ \\
\hline Gene silencing & $1.98 \mathrm{E}-02$ \\
\hline Chromatin organisation & $2.80 \mathrm{E}-02$ \\
\hline Ribose phosphate biosynthetic process & $3.08 \mathrm{E}-02$ \\
\hline Transcription from RNA polymerase III promoter & $3.81 \mathrm{E}-02$ \\
\hline Histone deacetylation & $4.45 \mathrm{E}-02$ \\
\hline Negative regulation of transcription from RNA polymerase II promoter during mitotic cell cycle & $4.67 \mathrm{E}-02$ \\
\hline
\end{tabular}

Table 7.2.4 - GO enrichment of validated MTDIA hypersensitive strains. The validated genes were assigned to the three major hierarchical gene ontology $(\mathrm{GO})$ categories: biological process, molecular function and cellular component using the haploid non-essential gene deletion library as a reference set on YeastMine (Gene Ontology Consortium, 2004; Ashburner et al., 2000; Balakrishnan et al., 2012) with $p$-values corrected for multiple testing using the Benjamin and Hochberg false discovery rate of 0.05 (Benjamini \& Hochberg, 1995). 


\begin{tabular}{|c|c|c|c|}
\hline ORF & Gene & $\begin{array}{l}\mathrm{OD}_{600} \\
\text { at } 32 \mathrm{~h}\end{array}$ & Function \\
\hline YMR009W & ADI1 & 0.019 & $\begin{array}{l}\text { Acireductone dioxygenease involved in methionine salvage } \\
\text { pathway }\end{array}$ \\
\hline YBR131W & CCZ1 & 0.034 & $\begin{array}{l}\text { Subunit of a heterodimeric guanine nucleotide exchange factor } \\
\text { (GEF) }\end{array}$ \\
\hline YMR058W & FET3 & 0.012 & Ferro-O2-oxidoreductase \\
\hline YER145C & FTR1 & 0.011 & High affinity iron permease \\
\hline YBR020W & GAL1 & 0.013 & Galactokinase \\
\hline YBR019C & GAL10 & 0.001 & UDP-glucose-4-epimerase \\
\hline YDR009W & GAL3 & 0.022 & Transcriptional regulator \\
\hline YJR024C & MDE1 & 0.011 & 5'-methylthioribulose-1-phosphate dehydratase \\
\hline YLR017W & MEU1 & 0.03 & Methylthioadenosine phosphorylase (MTAP) \\
\hline YPR118W & MRI1 & 0.007 & 5'-methylthioribose-1-phosphate isomerase \\
\hline YJL121C & RPE1 & 0.013 & D-ribulose-5-phosphate 3-epimerase \\
\hline YLR357W & $\mathrm{RSC2}$ & -0.003 & Component of the RSC chromatin remodelling complex \\
\hline YCR009C & RVS161 & 0.019 & Amphiphysin-like lipid raft protein \\
\hline YDR388W & RVS167 & 0.016 & Calmodulin-binding actin-associated protein \\
\hline YGL026C & TRP5 & 0.001 & Tryptophan synthase \\
\hline YEL038W & UTR4 & 0.005 & Protein with sequence similarity to acireductone synthases \\
\hline YDR290W & $\begin{array}{l}\text { YDR290W/ } \\
\text { overlaps } \\
\text { RRT103 }\end{array}$ & 0.006 & $\begin{array}{l}\text { Protein involved in transcription termination by RNA } \\
\text { polymerase II }\end{array}$ \\
\hline YJL022W & $\begin{array}{l}\text { YJL022W/ } \\
\text { overlaps } \\
\text { PET130 }\end{array}$ & 0.01 & Protein required for respiratory growth \\
\hline
\end{tabular}

Table 7.2.5 - Genes that were unviable in MTA as the only sulphur source.

7.2.5 MTDIA treatment induces autophagy

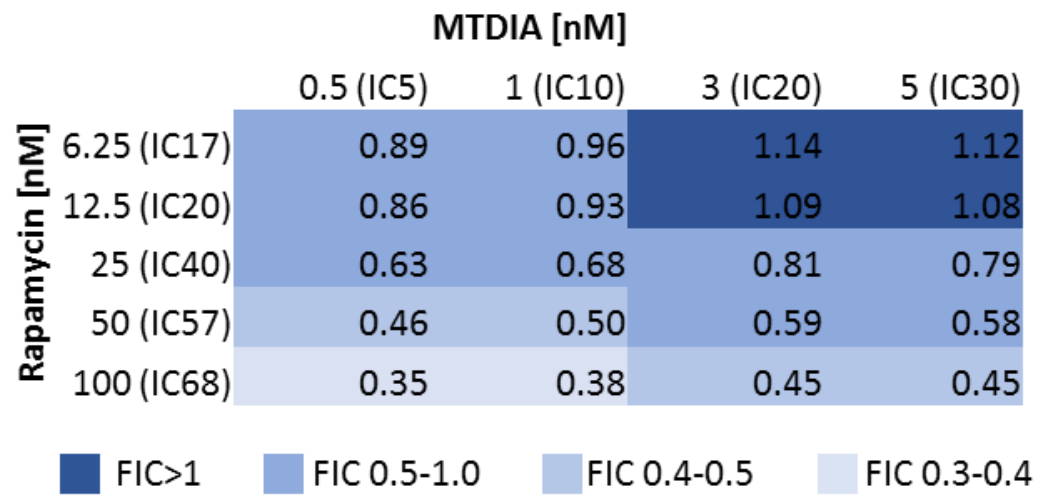

Table 7.2.6 - FIC index for rapamycin and MTDIA in combination. FIC index was calculated to confirm drug interactions where $\mathrm{FIC}$ is defined as (drug $\mathrm{A}$ in combination/drug $\mathrm{A}$ alone) + (drug $\mathrm{B}$ in 
combination/ drug B alone) (Hall et al., 1983; Odds, 2003; McElhaney-Feser et al., 1998). Synergistic ( $\mathrm{FIC} \leq 0.5), 0.5<\mathrm{FICl}<1$ additive, $1<\mathrm{FICl}>4$ indifferent and $\mathrm{FICl} \geq 4$ is antagonistic.

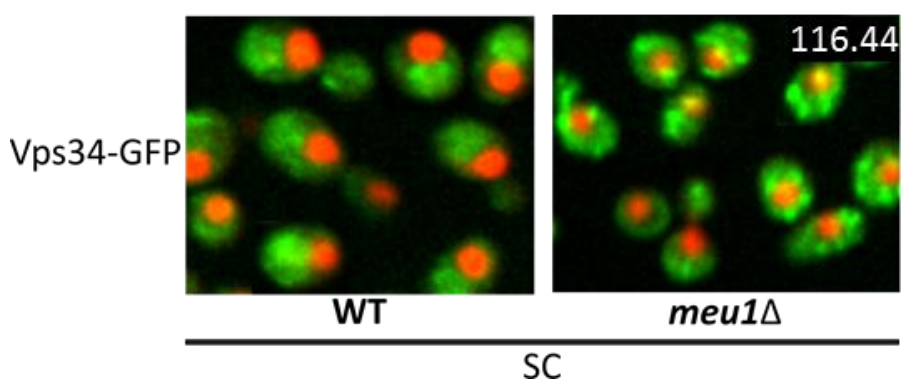

Figure 7.2.3 - Vps34-GFP in WT and meu1s in methionine replete complete minimal media. Florescent signal was detected at $488 \mathrm{~nm}$ (GFP) and 561 (nuclear localisation signal fused to RS2 and cytosolic mCherry) using the high-throughput spinning disk confocal microscope (Evo Tec OPERA, Perkin Elmer, Massachusetts, United States) using the 60x water immersion lens (NA 1.2). The GFP abundance and localisation was quantified by automated image analysis (Acapella, Perkin Elmer, Massachusetts, United States)(Bircham et al., 2011). Numbers inset in each image are the percent changes in GFP abundance in meu1 $\Delta$ relative to WT untreated.

\subsection{1 yCG640 and yCG643 construction}

a
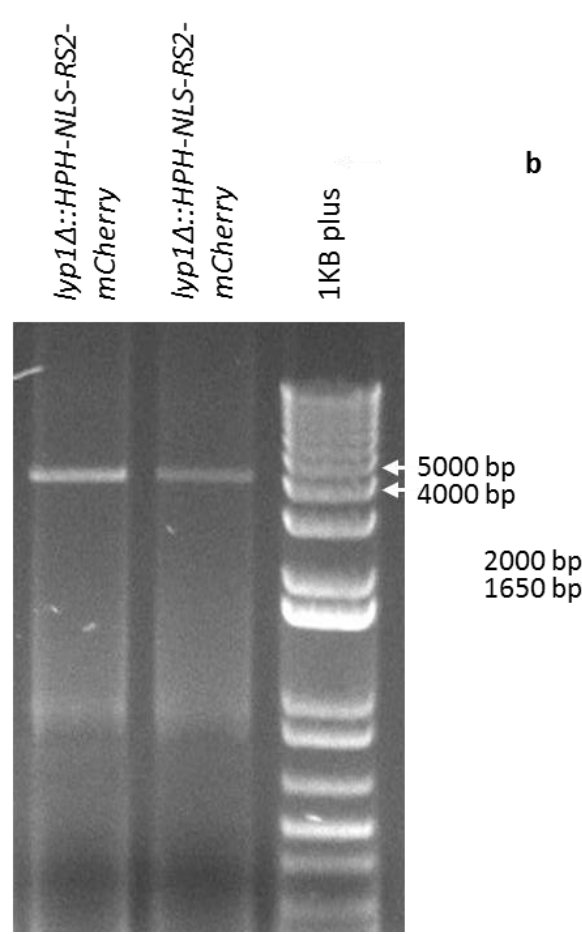
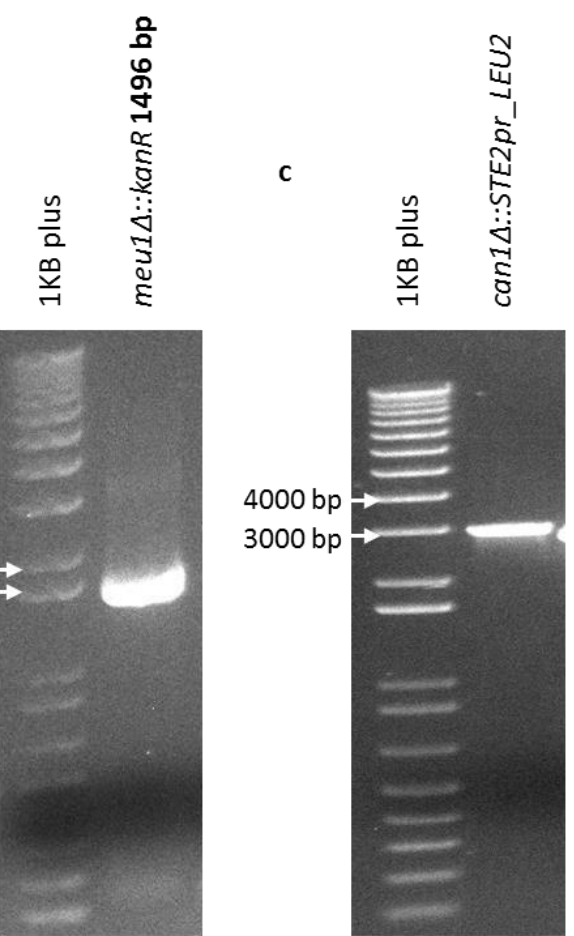

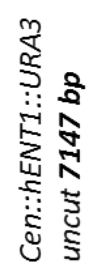

$\frac{\stackrel{n}{0}}{\frac{0}{2}}$

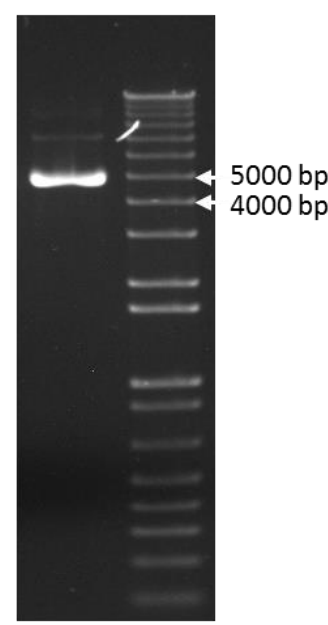

191 
Figure 7.3.1 - YCG640 and YCG643 construction. PCR products were run on a 1\% agarose gel with 1KB plus ladder at $100 \mathrm{mV}$. (a) lyp1 $1 \Delta:$ HPH-NLS-RS2 construction PCR. (b) can1 $1 \Delta:$ STE2pr-Sp_LEU2 construction PCR. (c) meu1A::kanR construction PCR. (d) Cen::hENT1::URA3.

\begin{tabular}{|c|c|}
\hline Primer & Sequence \\
\hline $\begin{array}{l}\text { lyp1 } 1:: H P H-N L S-R S 2- \\
\text { mCherry Fw (\#224) }\end{array}$ & $\begin{array}{l}\text { AATTGCATTGTCTATAACGATAACAAAAGACATCGTATATATATATATATCGTAC } \\
\text { GCTGCAGGTCGAC }\end{array}$ \\
\hline $\begin{array}{l}\text { lyp1 } 1:: H P H-N L S-R S 2- \\
\text { mCherry Rev (\#252) }\end{array}$ & $\begin{array}{l}\text { TCTATTTTTTTATTTTTTTTCTATTTTGAAGGCATGCAAGAGGTCTGTGAACTATAG } \\
\text { GGAGACCGGCAGA }\end{array}$ \\
\hline $\begin{array}{l}\text { meu1A::kanR Fw } \\
(\# 831)\end{array}$ & $\begin{array}{l}\text { AGATGAACGTGTGAATGAAAAAAAACTATTTCGAGATGAGATGAGATTAACGC } \\
\text { CGGGTCACCCGGCCAGC }\end{array}$ \\
\hline 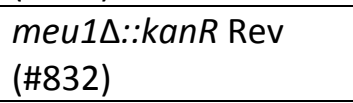 & $\begin{array}{l}\text { ATATATGTTTTCTCTCCTATTTATATTTTACATATGATTAGCGGCACCCACGTTAG } \\
\text { TATCGAATCGACAG }\end{array}$ \\
\hline $\begin{array}{l}\text { can1 } 1:: \text { STE2pr_LEU2 } \\
\text { Fw (\#749) }\end{array}$ & CGGCTTTTTTGAATTTCTTTT \\
\hline $\begin{array}{l}\text { can1 } 1 \text { ::STE2pr_LEU2 } \\
\operatorname{Rev}(\# 754)\end{array}$ & CTTTTCGGTGTATGACTTATG \\
\hline
\end{tabular}

Table 7.3.1 - yCG640 and yCG643 construction primers. Primers were designed according to Janke et al. (Janke et al., 2004). 
7.3.2 GFP proteins localised to subcellular structures.

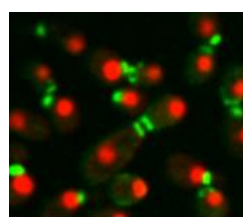

Bud neck

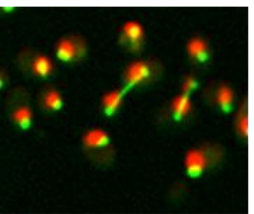

Spindle

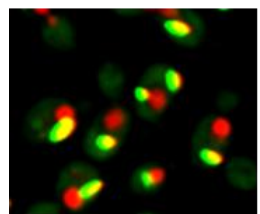

Septin

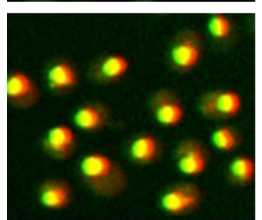

Nucleus

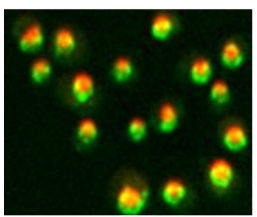

Nuclear

membrane

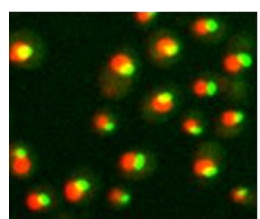

Nuclear foci

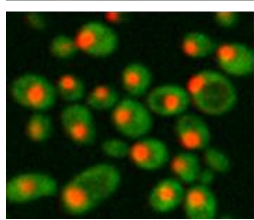

Cytoplasm

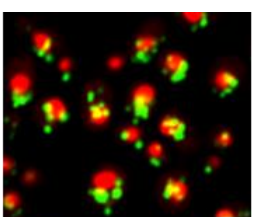

Cytoplasmic foci

(Lipid droplet)

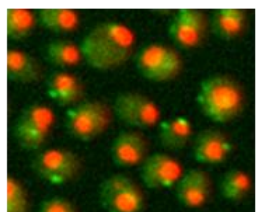

Cytoplasmic foci

(peroxisome)

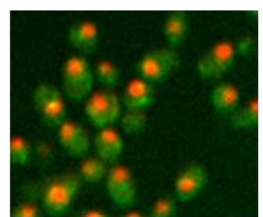

Cytoplasmic foci (endosomes and Golgi)

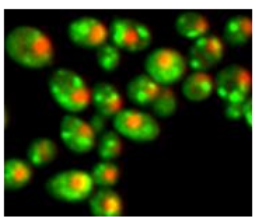

Cytoplasmic foci (Golgi and coated vesicles)

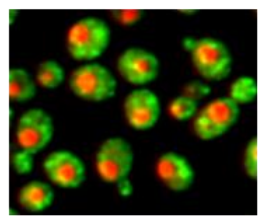

Cytoplasmic foci (Golgi)

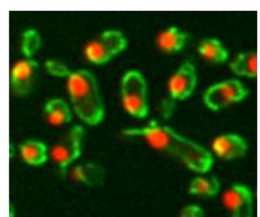

Mitochondrion

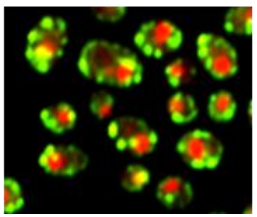

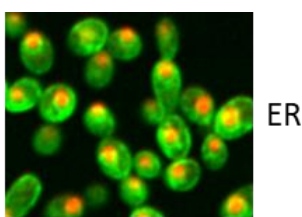

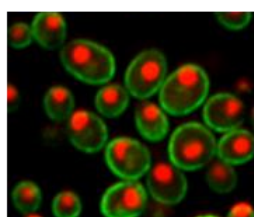

Plasma membrane

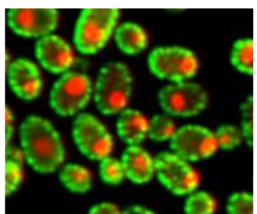

Plasma membrane foci

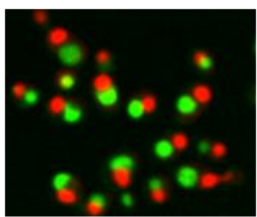

Vacuole lumen

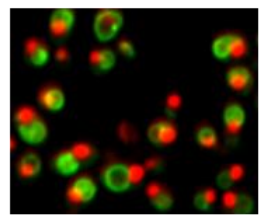

Vacuolar

membrane

Figure 7.3.2 - GFP proteins localised to subcellular structures. 
7.3.3 Changes in either protein localisation or abundance with $100 \mathrm{nM}$ MTDIA or in meu1 $\Delta$ and Go enrichment

\begin{tabular}{|c|c|c|c|c|c|c|c|c|c|c|}
\hline $\begin{array}{l}\text { ORF } \\
\text { name }\end{array}$ & $\begin{array}{l}\text { Gene } \\
\text { name }\end{array}$ & Function & \begin{tabular}{|l|} 
Median \\
WT GFP \\
intensity \\
(RFU)
\end{tabular} & \begin{tabular}{|l|}
$\%$ GFP \\
intensity \\
relative \\
to WT \\
(WT + \\
MTDIA \\
/WT) \\
\end{tabular} & \begin{tabular}{|l|} 
Median \\
WT + \\
MTDIA \\
GFP \\
intensity \\
(RFU)
\end{tabular} & \begin{tabular}{|l}
$\%$ GFP \\
intensity \\
relative \\
to WT \\
(meu1L/ \\
WT)
\end{tabular} & \begin{tabular}{|l|} 
Median \\
meu1s \\
GFP \\
intensity \\
(RFU) \\
\end{tabular} & $\begin{array}{l}\text { Localisation } \\
\text { with WT }\end{array}$ & $\begin{array}{l}\text { Localisation } \\
\text { with } 100 \mathrm{nM} \\
\text { MTDIA }\end{array}$ & $\begin{array}{l}\text { Localisation } \\
\text { with meu1s }\end{array}$ \\
\hline YNR016C & ACC1 & $\begin{array}{l}\text { Acetyl-CoA carboxylase, biotin } \\
\text { containing enzyme }\end{array}$ & 176.04 & 87.39 & 153.85 & 123.70 & 217.76 & Cytoplasm & $\begin{array}{l}\text { Lipid droplets, } \\
\text { cytoplasm }\end{array}$ & $\begin{array}{l}\text { Lipid droplets, } \\
\text { cytoplasm }\end{array}$ \\
\hline YBL015W & $\mathrm{ACH} 1$ & Protein with CoA transferase activity & 548.31 & 117.41 & 643.78 & 52.57 & 288.27 & Mitochondrion & Mitochondrion & Mitochondrion \\
\hline YAL054C & ACS1 & Acetyl-coA synthetase isoform & 299.41 & 97.20 & 291.04 & 93.53 & 280.03 & $\begin{array}{l}\text { Cytoplasm, } \\
\text { nucleus }\end{array}$ & $\begin{array}{l}\text { Cytoplasm, } \\
\text { nucleus }\end{array}$ & Cytoplasm \\
\hline YLR153C & ACS2 & Acetyl-coA synthetase isoform & 402.41 & 95.98 & 386.21 & 85.46 & 343.88 & $\begin{array}{l}\text { Cytoplasm, } \\
\text { nucleus }\end{array}$ & $\begin{array}{l}\text { Cytoplasm, } \\
\text { nucleus }\end{array}$ & $\begin{array}{l}\text { Cytoplasm, } \\
\text { nucleus }\end{array}$ \\
\hline YMR120C & ADE17 & Enzyme of 'de novo' purine biosynthesis & 79.72 & 107.58 & 85.77 & 121.27 & 96.68 & $\begin{array}{l}\text { Cytoplasm, } \\
\text { nucleus }\end{array}$ & $\begin{array}{l}\text { Cytoplasm, } \\
\text { nucleus }\end{array}$ & $\begin{array}{l}\text { Cytoplasm, } \\
\text { nucleus }\end{array}$ \\
\hline YMR303C & $\mathrm{ADH} 2$ & $\begin{array}{l}\text { Glucose-repressible alcohol } \\
\text { dehydrogenase II }\end{array}$ & 352.70 & 111.55 & 393.42 & 152.45 & 537.69 & $\begin{array}{l}\text { Cytoplasm, } \\
\text { nucleus }\end{array}$ & $\begin{array}{l}\text { Cytoplasm, } \\
\text { nucleus }\end{array}$ & $\begin{array}{l}\text { Cytoplasm, } \\
\text { nucleus }\end{array}$ \\
\hline $\begin{array}{l}\text { YMR083 } \\
\text { W }\end{array}$ & ADH3 & $\begin{array}{l}\text { Mitochondrial alcohol dehydrogenase } \\
\text { isozyme III }\end{array}$ & 137.93 & 100.70 & 138.89 & 81.21 & 112.01 & Mitochondrion & Mitochondrion & Mitochondrion \\
\hline YGL256W & $\mathrm{ADH} 4$ & $\begin{array}{l}\text { Alcohol dehydrogenase isoenzyme type } \\
\text { IV }\end{array}$ & 43.70 & 97.02 & 42.40 & 79.64 & 34.80 & Cytoplasm & Cytoplasm & Cytoplasm \\
\hline YMR318C & ADH6 & $\begin{array}{l}\text { NADPH-dependent medium chain } \\
\text { alcohol dehydrogenase }\end{array}$ & 206.76 & 79.48 & 164.33 & 61.44 & 127.03 & $\begin{array}{l}\text { Cytoplasm, } \\
\text { nucleus }\end{array}$ & $\begin{array}{l}\text { Cytoplasm, } \\
\text { nucleus }\end{array}$ & $\begin{array}{l}\text { Cytoplasm, } \\
\text { nucleus }\end{array}$ \\
\hline YDR226W & ADK1 & $\begin{array}{l}\text { Adenylate kinase, required for purine } \\
\text { metabolism }\end{array}$ & 481.02 & 105.44 & 507.21 & 52.80 & 253.96 & $\begin{array}{l}\text { Cytoplasm, } \\
\text { nucleus }\end{array}$ & $\begin{array}{l}\text { Cytoplasm, } \\
\text { nucleus }\end{array}$ & $\begin{array}{l}\text { Cytoplasm, } \\
\text { nucleus }\end{array}$ \\
\hline YCL025C & AGP1 & $\begin{array}{l}\text { Low-affinity amino acid permease with } \\
\text { broad substrate range }\end{array}$ & 180.39 & 82.05 & 148.00 & 69.10 & 124.64 & ER & ER & ER \\
\hline
\end{tabular}




\begin{tabular}{|c|c|c|c|c|c|c|c|c|c|c|}
\hline YBR132C & AGP2 & $\begin{array}{l}\text { Plasma membrane regulator of } \\
\text { polyamine and carnitine transport }\end{array}$ & 68.76 & 97.79 & 67.24 & 93.73 & 64.45 & \begin{tabular}{|l|} 
Plasma \\
membrane, \\
vacuolar lumen
\end{tabular} & $\begin{array}{l}\text { Plasma } \\
\text { membrane, } \\
\text { vacuolar lumen }\end{array}$ & $\begin{array}{l}\text { Plasma } \\
\text { membrane, } \\
\text { vacuolar lumen }\end{array}$ \\
\hline YDR214W & AHA1 & $\begin{array}{l}\text { Co-chaperone that binds Hsp82p and } \\
\text { activates its ATPase activity }\end{array}$ & 118.59 & 148.01 & 175.53 & 100.29 & 118.94 & Cytoplasm & $\begin{array}{l}\text { Cytoplasm, } \\
\text { nucleus }\end{array}$ & $\begin{array}{l}\text { Cytoplasm, } \\
\text { nucleus }\end{array}$ \\
\hline YLR109W & AHP1 & Thiol-specific peroxiredoxin & 884.41 & 117.98 & 1043.43 & 205.54 & 1817.80 & $\begin{array}{l}\text { Cytoplasm, } \\
\text { nucleus }\end{array}$ & $\begin{array}{l}\text { Cytoplasm, } \\
\text { nucleus }\end{array}$ & $\begin{array}{l}\text { Cytoplasm, } \\
\text { nucleus }\end{array}$ \\
\hline YJL122W & ALB1 & Shuttling pre-60S factor & 65.06 & 66.48 & 43.26 & 76.31 & 49.65 & Nucleus & Nuclear foci & Nuclear foci \\
\hline YMR169C & ALD3 & Cytoplasmic aldehyde dehydrogenase & 32.62 & 108.90 & 35.52 & 256.40 & 83.64 & Cytoplasm & Cytoplasm & $\begin{array}{l}\text { Cytoplasm, } \\
\text { nucleus }\end{array}$ \\
\hline YPL061W & ALD6 & Cytosolic aldehyde dehydrogenase & 751.09 & 77.96 & 585.57 & 75.28 & 565.40 & Cytoplasm & Cytoplasm & Cytoplasm \\
\hline YNL219C & ALG9 & $\begin{array}{l}\text { Mannosyltransferase, involved in N- } \\
\text { linked glycosylation }\end{array}$ & 57.93 & 88.50 & 51.26 & 81.26 & 47.07 & ER & ER & ER \\
\hline YKR021W & ALY1 & Alpha arrestin, substrate of calcineurin & 36.89 & 101.87 & 37.59 & 709.88 & 261.91 & Cytoplasm & Cytoplasm & Cytoplasm \\
\hline YCL050C & APA1 & AP4A phosphorylase & 36.00 & 103.57 & 37.28 & 291.04 & 104.77 & Mitochondrion & Mitochondrion & Cytoplasm \\
\hline YKL103C & APE1 & Vacuolar aminopeptidase yscl & 75.27 & 105.80 & 79.64 & 89.19 & 67.14 & $\begin{array}{l}\text { Cytoplasm, } \\
\text { cytoplasmic foci }\end{array}$ & $\begin{array}{l}\text { Cytoplasm, } \\
\text { cytoplasmic foci }\end{array}$ & $\begin{array}{l}\text { Cytoplasm, } \\
\text { cytoplasmic foci }\end{array}$ \\
\hline $\begin{array}{l}\text { YML022 } \\
\text { W }\end{array}$ & APT1 & Adenine phosphoribosyltransferase & 71.32 & 75.92 & 54.15 & 79.60 & 71.32 & Cytoplasm & Cytoplasm & Cytoplasm \\
\hline YBR149W & ARA1 & $\begin{array}{l}\text { NADP+ dependent arabinose } \\
\text { dehydrogenase }\end{array}$ & 86.88 & 111.76 & 97.10 & 218.83 & 190.13 & $\begin{array}{l}\text { Cytoplasm, } \\
\text { nucleus }\end{array}$ & $\begin{array}{l}\text { Cytoplasm, } \\
\text { nucleus }\end{array}$ & $\begin{array}{l}\text { Cytoplasm, } \\
\text { nucleus }\end{array}$ \\
\hline YDL192W & ARF1 & ADP-ribosylation factor & 304.19 & 93.83 & 285.43 & 120.87 & 367.69 & Cytoplasmic foci & Cytoplasmic foci & Cytoplasmic foci \\
\hline YHL040C & ARN1 & $\begin{array}{l}\text { ARN family transporter for siderophore- } \\
\text { iron chelates }\end{array}$ & 42.62 & 81.57 & 34.76 & 117.80 & 50.21 & $\begin{array}{l}\text { Plasma } \\
\text { membrane, } \\
\text { cytoplasmic foci }\end{array}$ & Vacuolar lumen & Vacuolar lumen \\
\hline YHR137W & ARO9 & Aromatic aminotransferase II & 90.56 & 101.87 & 92.25 & 147.11 & 133.22 & $\begin{array}{l}\text { Cytoplasm, } \\
\text { nucleus }\end{array}$ & $\begin{array}{l}\text { Cytoplasm, } \\
\text { nucleus }\end{array}$ & $\begin{array}{l}\text { Cytoplasm, } \\
\text { nucleus }\end{array}$ \\
\hline YDR101C & ARX1 & $\begin{array}{l}\text { Nuclear export factor for the ribosomal } \\
\text { pre-60S subunit }\end{array}$ & 75.58 & 78.37 & 59.23 & 82.94 & 62.69 & Nucleus & Nucleus & $\begin{array}{l}\text { Cytoplasm, } \\
\text { nucleus }\end{array}$ \\
\hline YOR058C & ASE1 & $\begin{array}{l}\text { Mitotic spindle midzone-localized } \\
\text { microtubule bundling protein }\end{array}$ & 28.62 & 106.95 & 30.61 & 117.85 & 33.73 & Cytoplasm & Cytoplasm & $\begin{array}{l}\text { Cytoplasm, } \\
\text { vacuolar lumen }\end{array}$ \\
\hline
\end{tabular}




\begin{tabular}{|c|c|c|c|c|c|c|c|c|c|c|}
\hline YKL052C & ASK1 & $\begin{array}{l}\text { Essential subunit of the Dam1 complex } \\
\text { (aka DASH complex) }\end{array}$ & 54.33 & 85.56 & 46.49 & 82.28 & 44.70 & $\begin{array}{l}\text { Nuclear foci, } \\
\text { spindle }\end{array}$ & $\begin{array}{l}\text { Nuclear foci, } \\
\text { spindle }\end{array}$ & Nuclear foci \\
\hline YGL180W & ATG1 & Protein serine/threonine kinase & 44.25 & 106.35 & 47.06 & 104.21 & 46.12 & $\begin{array}{l}\text { Cytoplasm, } \\
\text { cytoplasmic foci }\end{array}$ & $\begin{array}{l}\text { Cytoplasm, } \\
\text { vacuolar lumen }\end{array}$ & $\begin{array}{l}\text { Cytoplasm, } \\
\text { vacuolar lumen }\end{array}$ \\
\hline YJL178C & ATG27 & $\begin{array}{l}\text { Type I membrane protein involved in } \\
\text { autophagy and the Cvt pathway }\end{array}$ & 51.36 & 80.99 & 41.60 & 94.77 & 48.68 & Cytoplasmic foci & Vacuolar lumen & Cytoplasmic foci \\
\hline YDR384C & ATO3 & $\begin{array}{l}\text { Plasma membrane protein, putative } \\
\text { ammonium transporter }\end{array}$ & 62.26 & 93.37 & 58.13 & 80.48 & 50.11 & \begin{tabular}{|l|} 
Plasma \\
membrane, \\
vacuolar lumen
\end{tabular} & $\begin{array}{l}\text { Plasma } \\
\text { membrane, } \\
\text { vacuolar lumen }\end{array}$ & Vacuolar lumen \\
\hline YPL271W & ATP15 & $\begin{array}{l}\text { Epsilon subunit of the F1 sector of } \\
\text { mitochondrial F1F0 ATP synthase }\end{array}$ & 263.26 & 77.41 & 203.80 & 63.45 & 167.04 & Mitochondrion & Mitochondrion & Mitochondrion \\
\hline YKL016C & ATP7 & $\begin{array}{l}\text { Subunit d of the stator stalk of } \\
\text { mitochondrial F1F0 ATP synthase }\end{array}$ & 196.97 & 105.36 & 207.53 & 55.95 & 110.21 & Mitochondrion & Mitochondrion & Mitochondrion \\
\hline YIL124W & AYR1 & $\begin{array}{l}\text { Bifunctional triacylglycerol lipase and 1- } \\
\text { acyl DHAP reductase }\end{array}$ & 146.17 & 114.67 & 167.61 & 78.49 & 114.73 & ER, lipid droplet & ER, lipid droplet & ER \\
\hline YBR068C & BAP2 & High-affinity leucine permease & 84.47 & 83.35 & 70.40 & 83.53 & 70.55 & $\begin{array}{l}\text { Plasma } \\
\text { membrane, } \\
\text { vacuolar lumen }\end{array}$ & Vacuolar lumen & Vacuolar lumen \\
\hline YDR046C & BAP3 & Amino acid permease & 72.86 & 75.95 & 55.34 & 87.62 & 63.83 & \begin{tabular}{|l|} 
Plasma \\
membrane, \\
vacuolar lumen
\end{tabular} & Vacuolar lumen & Vacuolar lumen \\
\hline YHR040W & BCD1 & $\begin{array}{l}\text { Essential protein required for the } \\
\text { accumulation of box C/D snoRNA }\end{array}$ & 38.77 & 102.86 & 39.88 & 92.53 & 35.88 & Nucleus & Nucleus & $\begin{array}{l}\text { Cytoplasm, } \\
\text { nucleus }\end{array}$ \\
\hline YAL061W & $\mathrm{BDH} 2$ & $\begin{array}{l}\text { Putative medium-chain alcohol } \\
\text { dehydrogenase with similarity to } \mathrm{BDH} 1\end{array}$ & 50.73 & 123.51 & 62.66 & 121.17 & 61.47 & Cytoplasm & Cytoplasm & Cytoplasm \\
\hline $\begin{array}{l}\text { YOR304C- } \\
\text { A }\end{array}$ & BIL1 & $\begin{array}{l}\text { Protein that binds Bud } 6 p \text { and has a role } \\
\text { in actin cable assembly }\end{array}$ & 42.33 & 96.00 & 40.64 & 115.92 & 49.07 & Bud neck & Bud neck & Bud neck \\
\hline YNR056C & $\mathrm{BIO5}$ & $\begin{array}{l}\text { Putative transmembrane protein } \\
\text { involved in the biotin biosynthesis }\end{array}$ & 59.34 & 164.25 & 97.47 & 160.39 & 95.18 & $\begin{array}{l}\text { Plasma } \\
\text { membrane, } \\
\text { vacuolar lumen }\end{array}$ & $\begin{array}{l}\text { Plasma } \\
\text { membrane, } \\
\text { vacuolar lumen }\end{array}$ & $\begin{array}{l}\text { Plasma } \\
\text { membrane, } \\
\text { vacuolar lumen }\end{array}$ \\
\hline
\end{tabular}




\begin{tabular}{|c|c|c|c|c|c|c|c|c|c|c|}
\hline YNL166C & BNI5 & $\begin{array}{l}\text { Linker protein responsible for } \\
\text { recruitment of myosin to the bud neck }\end{array}$ & 48.13 & 97.63 & 46.99 & 96.14 & 46.27 & Bud neck & Bud neck & Bud neck \\
\hline YLR074C & BUD20 & $\begin{array}{l}\text { C2H2-type zinc finger protein required } \\
\text { for ribosome assembly }\end{array}$ & 59.83 & 68.69 & 41.10 & 72.65 & 43.46 & Nucleus & Nucleus & Nucleus \\
\hline YOR078W & BUD21 & $\begin{array}{l}\text { Component of small ribosomal subunit } \\
\text { (SSU) processosome }\end{array}$ & 47.51 & 72.00 & 34.21 & 82.58 & 39.24 & Nuclear foci & Nuclear foci & Nuclear foci \\
\hline YJR092W & BUD4 & $\begin{array}{l}\text { Anillin-like protein involved in bud-site } \\
\text { selection }\end{array}$ & 34.33 & 99.25 & 34.07 & 101.08 & 34.70 & Bud neck & Bud neck & Bud neck \\
\hline YLR438W & CAR2 & L-ornithine transaminase (OTAse) & 134.29 & 81.40 & 109.31 & 57.27 & 76.91 & $\begin{array}{l}\text { Cytoplasm, } \\
\text { nucleus }\end{array}$ & $\begin{array}{l}\text { Cytoplasm, } \\
\text { nucleus }\end{array}$ & $\begin{array}{l}\text { Cytoplasm, } \\
\text { nucleus }\end{array}$ \\
\hline $\begin{array}{l}\text { YLR390W } \\
\text {-A }\end{array}$ & CCW14 & Covalently linked cell wall glycoprotein & 121.94 & 75.29 & 91.81 & 71.08 & 86.68 & ER & ER & ER \\
\hline YCR002C & CDC10 & $\begin{array}{l}\text { Component of the septin ring, required } \\
\text { for cytokinesis }\end{array}$ & 66.39 & 96.21 & 63.87 & 107.80 & 71.57 & Bud neck & Bud neck & $\begin{array}{l}\text { Bud neck, } \\
\text { plasma } \\
\text { membrane }\end{array}$ \\
\hline YJR076C & CDC11 & $\begin{array}{l}\text { Component of the septin ring that is } \\
\text { required for cytokinesis }\end{array}$ & 63.61 & 98.18 & 62.45 & 98.11 & 62.41 & Bud neck & Bud neck & $\begin{array}{l}\text { Bud neck, } \\
\text { plasma } \\
\text { membrane }\end{array}$ \\
\hline YAL038W & CDC19 & Pyruvate kinase & 741.87 & 94.83 & 703.49 & 72.38 & 536.94 & $\begin{array}{l}\text { Cytoplasm, } \\
\text { nucleus }\end{array}$ & $\begin{array}{l}\text { Cytoplasm, } \\
\text { nucleus }\end{array}$ & $\begin{array}{l}\text { Cytoplasm, } \\
\text { nucleus }\end{array}$ \\
\hline YGR157W & $\mathrm{CHO} 2$ & $\begin{array}{l}\text { Phosphatidylethanolamine } \\
\text { methyltransferase (PEMT) }\end{array}$ & 87.73 & 77.04 & 67.59 & 73.36 & 64.36 & ER & Cytoplasmic foci & Cytoplasmic foci \\
\hline YNR001C & CIT1 & Citrate synthase & 241.03 & 153.05 & 368.89 & 185.02 & 445.96 & Mitochondrion & Mitochondrion & Mitochondrion \\
\hline YCR005C & $\mathrm{CIT} 2$ & $\begin{array}{l}\text { Citrate synthase, peroxisomal isozyme } \\
\text { involved in glyoxylate cycle }\end{array}$ & 38.64 & 106.13 & 41.01 & 121.89 & 47.10 & Cytoplasm & Cytoplasm & Cytoplasm \\
\hline YPR001W & CIT3 & $\begin{array}{l}\text { Dual specificity mitochondrial citrate } \\
\text { and methylcitrate synthase }\end{array}$ & 56.66 & 82.36 & 46.66 & 71.22 & 40.35 & Mitochondrion & Mitochondrion & Mitochondrion \\
\hline YGL187C & COX4 & Subunit IV of cytochrome c oxidase & 464.40 & 100.83 & 468.24 & 54.10 & 251.23 & Mitochondrion & Mitochondrion & Mitochondrion \\
\hline YDR155C & CPR1 & $\begin{array}{l}\text { Cytoplasmic peptidyl-prolyl cis-trans } \\
\text { isomerase (cyclophilin) }\end{array}$ & 395.38 & 100.54 & 397.53 & 153.34 & 606.28 & $\begin{array}{l}\text { Cytoplasm, } \\
\text { nucleus }\end{array}$ & $\begin{array}{l}\text { Cytoplasm, } \\
\text { nucleus }\end{array}$ & $\begin{array}{l}\text { Cytoplasm, } \\
\text { nucleus }\end{array}$ \\
\hline YOL007C & $\mathrm{CSI} 2$ & Protein of unknown function & 37.49 & 93.11 & 34.91 & 97.83 & 36.68 & Vacuolar lumen & Vacuolar lumen & Vacuolar lumen \\
\hline
\end{tabular}




\begin{tabular}{|c|c|c|c|c|c|c|c|c|c|c|}
\hline YBR291C & CTP1 & $\begin{array}{l}\text { Mitochondrial inner membrane citrate } \\
\text { transporter }\end{array}$ & 49.06 & 69.65 & 34.17 & 77.09 & 37.82 & Mitochondrion & Mitochondrion & Cytoplasmic foci \\
\hline YPR124W & CTR1 & $\begin{array}{l}\text { High-affinity copper transporter of } \\
\text { plasma membrane }\end{array}$ & 47.73 & 96.08 & 45.86 & 130.79 & 62.42 & $\begin{array}{l}\text { Plasma } \\
\text { membrane }\end{array}$ & $\begin{array}{l}\text { Plasma } \\
\text { membrane, } \\
\text { vacuolar lumen }\end{array}$ & $\begin{array}{l}\text { Plasma } \\
\text { membrane, } \\
\text { vacuolar lumen }\end{array}$ \\
\hline YGR088W & CTT1 & Cytosolic catalase T & 34.22 & 101.80 & 34.84 & 143.82 & 49.22 & $\begin{array}{l}\text { Cytoplasm, } \\
\text { nucleus }\end{array}$ & $\begin{array}{l}\text { Cytoplasm, } \\
\text { nucleus }\end{array}$ & $\begin{array}{l}\text { Cytoplasm, } \\
\text { nucleus }\end{array}$ \\
\hline $\begin{array}{l}\text { YKL096W } \\
-A\end{array}$ & CWP2 & $\begin{array}{l}\text { Covalently linked cell wall } \\
\text { mannoprotein }\end{array}$ & 786.37 & 85.31 & 670.85 & 57.16 & 449.46 & ER & ER & ER \\
\hline YKR083C & DAD2 & $\begin{array}{l}\text { Essential subunit of the Dam1 complex } \\
\text { (aka DASH complex) }\end{array}$ & 52.01 & 95.15 & 49.49 & 82.49 & 42.90 & $\begin{array}{l}\text { Spindle, nuclear } \\
\text { foci }\end{array}$ & $\begin{array}{l}\text { Spindle, nuclear } \\
\text { foci }\end{array}$ & Nuclear foci \\
\hline YNL112W & DBP2 & $\begin{array}{l}\text { ATP-dependent RNA helicase of the } \\
\text { DEAD-box protein family }\end{array}$ & 38.62 & 97.26 & 37.56 & 318.01 & 122.81 & Bud neck & Bud neck & Nucleus \\
\hline YGL078C & DBP3 & $\begin{array}{l}\text { RNA-Dependent ATPase, member of } \\
\text { DExD/H-box family }\end{array}$ & 73.43 & 85.40 & 62.71 & 76.32 & 56.04 & Nuclear foci & Nuclear foci & Nuclear foci \\
\hline YLR270W & DCS1 & $\begin{array}{l}\text { Non-essential hydrolase involved in } \\
\text { mRNA decapping }\end{array}$ & 62.07 & 144.21 & 89.51 & 132.58 & 82.29 & $\begin{array}{l}\text { Cytoplasm, } \\
\text { nucleus }\end{array}$ & $\begin{array}{l}\text { Cytoplasm, } \\
\text { nucleus }\end{array}$ & $\begin{array}{l}\text { Cytoplasm, } \\
\text { nucleus }\end{array}$ \\
\hline $\begin{array}{l}\text { YOL052C- } \\
\text { A }\end{array}$ & DDR2 & Multi-stress response protein & 87.57 & 121.09 & 106.04 & 63.96 & 56.00 & ER & ER & Vacuolar lumen \\
\hline YLR348C & DIC1 & Mitochondrial dicarboxylate carrier & 44.77 & 68.65 & 30.74 & 89.63 & 40.13 & Mitochondrion & Mitochondrion & Mitochondrion \\
\hline YEL071W & DLD3 & $\begin{array}{l}\text { 2-hydroxyglutarate transhydrogenase, } \\
\text { and minor D-lactate dehydrogenase }\end{array}$ & 178.77 & 74.64 & 133.43 & 69.12 & 123.57 & $\begin{array}{l}\text { Cytoplasm, } \\
\text { nucleus }\end{array}$ & $\begin{array}{l}\text { Cytoplasm, } \\
\text { nucleus }\end{array}$ & $\begin{array}{l}\text { Cytoplasm, } \\
\text { nucleus }\end{array}$ \\
\hline YGL043W & DST1 & $\begin{array}{l}\text { General transcription elongation factor } \\
\text { TFIIS }\end{array}$ & 73.49 & 100.14 & 73.59 & 66.28 & 48.70 & Nucleus & Nucleus & Nucleus \\
\hline YGL061C & DUO1 & $\begin{array}{l}\text { Essential subunit of the Dam1 complex } \\
\text { (aka DASH complex) }\end{array}$ & 41.43 & 99.71 & 41.31 & 85.42 & 35.39 & $\begin{array}{l}\text { Spindles, } \\
\text { nuclear foci, } \\
\text { cytoplasm }\end{array}$ & $\begin{array}{l}\text { Spindles, } \\
\text { nuclear foci, } \\
\text { cytoplasm }\end{array}$ & $\begin{array}{l}\text { Nuclear foci, } \\
\text { cytoplasm }\end{array}$ \\
\hline YAL059W & ECM1 & $\begin{array}{l}\text { Pre-ribosomal factor involved in } 605 \\
\text { ribosomal protein subunit export }\end{array}$ & 51.12 & 74.20 & 37.93 & 73.32 & 37.48 & Nucleus & Nucleus & $\begin{array}{l}\text { Cytoplasm, } \\
\text { nucleus }\end{array}$ \\
\hline YER035W & EDC2 & $\begin{array}{l}\text { RNA-binding protein that directly } \\
\text { activates mRNA decapping }\end{array}$ & 79.76 & 106.50 & 84.95 & 73.68 & 58.77 & $\begin{array}{l}\text { Cytoplasm, } \\
\text { nucleus }\end{array}$ & $\begin{array}{l}\text { Cytoplasm, } \\
\text { nucleus }\end{array}$ & $\begin{array}{l}\text { Cytoplasm, } \\
\text { nucleus }\end{array}$ \\
\hline
\end{tabular}




\begin{tabular}{|c|c|c|c|c|c|c|c|c|c|c|}
\hline YOR133W & EFT1 & $\begin{array}{l}\text { Elongation factor } 2 \text { (EF-2), also encoded } \\
\text { by EFT2 }\end{array}$ & 106.44 & 53.00 & 56.41 & 39.15 & 41.67 & Cytoplasm & Cytoplasm & Cytoplasm \\
\hline YDR385W & EFT2 & $\begin{array}{l}\text { Elongation factor } 2 \text { (EF-2), also encoded } \\
\text { by EFT1 }\end{array}$ & 256.47 & 63.31 & 162.38 & 24.35 & 62.44 & Cytoplasm & Cytoplasm & Cytoplasm \\
\hline YGR254W & ENO1 & Enolase I, a phosphopyruvate hydratase & 1063.48 & 166.33 & 1768.88 & 263.92 & 2806.70 & $\begin{array}{l}\text { Cytoplasm, } \\
\text { nucleus }\end{array}$ & $\begin{array}{l}\text { Cytoplasm, } \\
\text { nucleus }\end{array}$ & $\begin{array}{l}\text { Cytoplasm, } \\
\text { nucleus }\end{array}$ \\
\hline YGR175C & ERG1 & Squalene epoxidase & 143.62 & 77.87 & 111.83 & 87.48 & 125.64 & ER & ER & ER \\
\hline YNL280C & ERG24 & C-14 sterol reductase & 58.14 & 77.56 & 45.10 & 78.47 & 45.63 & Lipid droplets & Lipid droplets & Vacuolar lumen \\
\hline YLR056W & ERG3 & C-5 sterol desaturase & 91.28 & 75.82 & 69.21 & 72.81 & 66.46 & ER & ER & Vacuolar lumen \\
\hline YML008C & ERG6 & Delta(24)-sterol C-methyltransferase & 122.33 & 86.79 & 106.18 & 92.65 & 113.35 & ER, lipid droplet & ER, lipid droplet & Lipid droplet \\
\hline $\begin{array}{l}\text { YAR002C- } \\
\text { A }\end{array}$ & ERP1 & $\begin{array}{l}\text { Member of the p24 family involved in } \\
\text { ER to Golgi transport }\end{array}$ & 108.83 & 66.69 & 72.58 & 63.25 & 68.84 & Cytoplasmic foci & $\begin{array}{l}\text { Cytoplasmic } \\
\text { foci, vacuolar } \\
\text { lumen }\end{array}$ & Vacuolar lumen \\
\hline $\begin{array}{l}\text { YMR246 } \\
\text { W } \\
\end{array}$ & FAA4 & Long chain fatty acyl-CoA synthetase & 133.13 & 89.46 & 119.10 & 101.54 & 135.17 & ER & Lipid droplets & Lipid droplets \\
\hline YKL187C & FAT3 & Protein required for fatty acid uptake & 42.04 & 120.92 & 50.83 & 112.99 & 47.50 & $\begin{array}{l}\text { Plasma } \\
\text { membrane, } \\
\text { vacuolar lumen }\end{array}$ & $\begin{array}{l}\text { Plasma } \\
\text { membrane, } \\
\text { vacuolar lumen }\end{array}$ & $\begin{array}{l}\text { Plasma } \\
\text { membrane }\end{array}$ \\
\hline YLR051C & FCF2 & $\begin{array}{l}\text { Nucleolar protein involved in the early } \\
\text { steps of 35S rRNA processing }\end{array}$ & 48.02 & 78.17 & 37.54 & 88.56 & 42.53 & Nuclear foci & Nuclear foci & Nuclear foci \\
\hline YER056C & FCY2 & Purine-cytosine permease & 82.39 & 70.00 & 57.67 & 78.76 & 64.88 & $\begin{array}{l}\text { Plasma } \\
\text { membrane, } \\
\text { vacuolar lumen }\end{array}$ & Vacuolar lumen & Vacuolar lumen \\
\hline $\begin{array}{l}\text { YMR144 } \\
\text { W }\end{array}$ & FDO1 & $\begin{array}{l}\text { Protein involved in directionality of } \\
\text { mating type switching }\end{array}$ & 35.39 & 95.38 & 33.75 & 100.55 & 35.58 & Nucleus & Nucleus & Cytoplasm \\
\hline $\begin{array}{l}\text { YMR058 } \\
\text { W }\end{array}$ & FET3 & Ferro-O2-oxidoreductase & 128.31 & 70.20 & 90.08 & 73.35 & 94.11 & $\begin{array}{l}\text { Plasma } \\
\text { membrane, } \\
\text { vacuolar lumen }\end{array}$ & $\begin{array}{l}\text { Plasma } \\
\text { membrane, } \\
\text { vacuolar lumen }\end{array}$ & Vacuolar lumen \\
\hline YDR070C & FMP16 & Protein of unknown function & 206.80 & 223.70 & 462.60 & 241.06 & 498.51 & Mitochondrion & Mitochondrion & Mitochondrion \\
\hline YDL222C & FMP45 & $\begin{array}{l}\text { Integral membrane protein localized to } \\
\text { mitochondria }\end{array}$ & 50.47 & 150.37 & 75.89 & 174.55 & 88.10 & $\begin{array}{l}\text { Plasma } \\
\text { membrane }\end{array}$ & $\begin{array}{l}\text { Plasma } \\
\text { membrane }\end{array}$ & $\begin{array}{l}\text { Plasma } \\
\text { membrane }\end{array}$ \\
\hline
\end{tabular}




\begin{tabular}{|c|c|c|c|c|c|c|c|c|c|c|}
\hline YER145C & FTR1 & High affinity iron permease & 82.76 & 88.15 & 72.95 & 100.00 & 82.76 & $\begin{array}{l}\text { Plasma } \\
\text { membrane }\end{array}$ & $\begin{array}{l}\text { Plasma } \\
\text { membrane, } \\
\text { vacuolar lumen }\end{array}$ & $\begin{array}{l}\text { Plasma } \\
\text { membrane, } \\
\text { vacuolar lumen }\end{array}$ \\
\hline YBL042C & FUI1 & $\begin{array}{l}\text { High affinity uridine permease, localizes } \\
\text { to the plasma membrane }\end{array}$ & 62.04 & 76.90 & 47.71 & 88.15 & 54.69 & $\begin{array}{l}\text { Plasma } \\
\text { membrane }\end{array}$ & $\begin{array}{l}\text { Plasma } \\
\text { membrane, } \\
\text { vacuolar lumen }\end{array}$ & $\begin{array}{l}\text { Plasma } \\
\text { membrane, } \\
\text { vacuolar lumen }\end{array}$ \\
\hline YPL262W & FUM1 & Fumarase & 210.60 & 128.94 & 271.54 & 93.38 & 196.65 & Mitochondrion & Mitochondrion & Mitochondrion \\
\hline YDR009W & GAL3 & Transcriptional regulator & 91.65 & 82.19 & 75.33 & 71.61 & 65.64 & $\begin{array}{l}\text { Cytoplasm, } \\
\text { nucleus }\end{array}$ & $\begin{array}{l}\text { Cytoplasm, } \\
\text { nucleus }\end{array}$ & $\begin{array}{l}\text { Cytoplasm, } \\
\text { nucleus }\end{array}$ \\
\hline YHR089C & GAR1 & $\begin{array}{l}\text { Protein component of the H/ACA } \\
\text { snoRNP pseudouridylase complex }\end{array}$ & 42.47 & 73.59 & 31.25 & 88.66 & 37.65 & $\begin{array}{l}\text { Nuclear foci, } \\
\text { cytoplasm }\end{array}$ & $\begin{array}{l}\text { Nuclear foci, } \\
\text { cytoplasm }\end{array}$ & Vacuolar lumen \\
\hline $\begin{array}{l}\text { YMR307 } \\
\text { W }\end{array}$ & GAS1 & Beta-1,3-glucanosyltransferase & 239.05 & 52.53 & 125.58 & 43.41 & 103.77 & Mitochondrion & Mitochondrion & Mitochondrion \\
\hline YDR019C & GCV1 & $\begin{array}{l}\text { T subunit of the mitochondrial glycine } \\
\text { decarboxylase complex }\end{array}$ & 55.77 & 140.37 & 78.29 & 165.09 & 92.07 & Mitochondrion & Mitochondrion & Mitochondrion \\
\hline $\begin{array}{l}\text { YMR189 } \\
\text { W }\end{array}$ & GCV2 & $\begin{array}{l}\text { P subunit of the mitochondrial glycine } \\
\text { decarboxylase complex }\end{array}$ & 68.59 & 119.93 & 82.27 & 134.95 & 92.57 & Mitochondrion & Mitochondrion & Mitochondrion \\
\hline YAL044C & GCV3 & $\begin{array}{l}\text { H subunit of the mitochondrial glycine } \\
\text { decarboxylase complex }\end{array}$ & 71.08 & 115.34 & 81.98 & 194.01 & 137.90 & Mitochondrion & Mitochondrion & Mitochondrion \\
\hline YOR375C & GDH1 & $\begin{array}{l}\text { NADP(+)-dependent glutamate } \\
\text { dehydrogenase }\end{array}$ & 634.56 & 156.74 & 994.62 & 142.24 & 902.62 & $\begin{array}{l}\text { Cytoplasm, } \\
\text { vacuolar } \\
\text { membrane foci }\end{array}$ & $\begin{array}{l}\text { Cytoplasm, } \\
\text { vacuolar } \\
\text { membrane foci }\end{array}$ & $\begin{array}{l}\text { Cytoplasm, } \\
\text { vacuolar } \\
\text { membrane foci }\end{array}$ \\
\hline YDL215C & GDH2 & $\begin{array}{l}\mathrm{NAD}(+) \text {-dependent glutamate } \\
\text { dehydrogenase }\end{array}$ & 124.83 & 112.08 & 139.91 & 91.27 & 113.93 & $\begin{array}{l}\text { Cytoplasm, } \\
\text { cytoplasmic foci }\end{array}$ & $\begin{array}{l}\text { Cytoplasm, } \\
\text { cytoplasmic foci }\end{array}$ & Cytoplasm \\
\hline YOR355W & GDS1 & Protein of unknown function & 37.44 & 88.23 & 33.03 & 96.68 & 36.19 & $\begin{array}{l}\text { Nuclear foci, } \\
\text { cytoplasm }\end{array}$ & Cytoplasm & $\begin{array}{l}\text { Cytoplasm, } \\
\text { vacuolar lumen }\end{array}$ \\
\hline YNL255C & GIS2 & $\begin{array}{l}\text { Translational activator for mRNAs with } \\
\text { internal ribosome entry sites }\end{array}$ & 226.78 & 77.84 & 176.52 & 78.64 & 178.33 & $\begin{array}{l}\text { Cytoplasm, } \\
\text { nucleus }\end{array}$ & $\begin{array}{l}\text { Cytoplasm, } \\
\text { nucleus }\end{array}$ & $\begin{array}{l}\text { Cytoplasm, } \\
\text { nucleus }\end{array}$ \\
\hline YCL040W & GLK1 & Glucokinase & 47.67 & 108.61 & 51.78 & 123.50 & 58.88 & $\begin{array}{l}\text { Cytoplasm, } \\
\text { cytoplasmic foci }\end{array}$ & $\begin{array}{l}\text { Cytoplasm, } \\
\text { cytoplasmic foci }\end{array}$ & $\begin{array}{l}\text { Cytoplasm, } \\
\text { cytoplasmic foci }\end{array}$ \\
\hline
\end{tabular}




\begin{tabular}{|c|c|c|c|c|c|c|c|c|c|c|}
\hline YPR035W & GLN1 & Glutamine synthetase (GS) & 310.36 & 98.82 & 306.69 & 89.98 & 279.26 & $\begin{array}{l}\text { Cytoplasm, } \\
\text { nucleus }\end{array}$ & $\begin{array}{l}\text { Cytoplasm, } \\
\text { nucleus }\end{array}$ & $\begin{array}{l}\text { Cytoplasm, } \\
\text { nucleus }\end{array}$ \\
\hline YOR168W & GLN4 & Glutamine tRNA synthetase & 165.32 & 98.26 & 162.44 & 70.72 & 116.91 & Cytoplasm & Cytoplasm & Cytoplasm \\
\hline YDR508C & GNP1 & High-affinity glutamine permease & 164.99 & 75.23 & 124.12 & 78.93 & 130.23 & ER & Vacuolar lumen & Vacuolar lumen \\
\hline YNL274C & GOR1 & Glyoxylate reductase & 64.51 & 121.42 & 78.33 & 158.60 & 102.32 & $\begin{array}{l}\text { Cytoplasm, } \\
\text { nucleus }\end{array}$ & $\begin{array}{l}\text { Cytoplasm, } \\
\text { nucleus }\end{array}$ & $\begin{array}{l}\text { Cytoplasm, } \\
\text { nucleus }\end{array}$ \\
\hline YDL022W & GPD1 & $\begin{array}{l}\text { NAD-dependent glycerol-3-phosphate } \\
\text { dehydrogenase }\end{array}$ & 200.86 & 114.78 & 230.55 & 126.11 & 253.31 & Cytoplasmic foci & Cytoplasmic foci & Cytoplasmic foci \\
\hline YOL059W & GPD2 & $\begin{array}{l}\text { NAD-dependent glycerol 3-phosphate } \\
\text { dehydrogenase }\end{array}$ & 111.04 & 122.00 & 135.48 & 99.13 & 110.08 & Cytoplasm & Cytoplasm & Cytoplasm \\
\hline YPR160W & GPH1 & $\begin{array}{l}\text { Glycogen phosphorylase required for } \\
\text { the mobilization of glycogen }\end{array}$ & 135.75 & 110.67 & 150.24 & 54.04 & 73.36 & Cytoplasm & Cytoplasm & Cytoplasm \\
\hline YKL152C & GPM1 & Tetrameric phosphoglycerate mutase & 851.45 & 116.33 & 990.49 & 138.16 & 1176.37 & $\begin{array}{l}\text { Cytoplasm, } \\
\text { nucleus }\end{array}$ & $\begin{array}{l}\text { Cytoplasm, } \\
\text { nucleus }\end{array}$ & $\begin{array}{l}\text { Cytoplasm, } \\
\text { nucleus }\end{array}$ \\
\hline YDL021W & GPM2 & $\begin{array}{l}\text { Homolog of Gpm1p phosphoglycerate } \\
\text { mutase }\end{array}$ & 44.08 & 115.79 & 51.04 & 137.82 & 60.75 & $\begin{array}{l}\text { Cytoplasm, } \\
\text { nucleus }\end{array}$ & $\begin{array}{l}\text { Cytoplasm, } \\
\text { nucleus }\end{array}$ & $\begin{array}{l}\text { Cytoplasm, } \\
\text { nucleus }\end{array}$ \\
\hline YER062C & GPP2 & $\begin{array}{l}\text { DL-glycerol-3-phosphate phosphatase } \\
\text { involved in glycerol biosynthesis }\end{array}$ & 169.54 & 81.36 & 137.95 & 46.13 & 78.21 & $\begin{array}{l}\text { Cytoplasm, } \\
\text { nucleus }\end{array}$ & $\begin{array}{l}\text { Cytoplasm, } \\
\text { nucleus }\end{array}$ & $\begin{array}{l}\text { Cytoplasm, } \\
\text { nucleus }\end{array}$ \\
\hline YPL223C & GRE1 & $\begin{array}{l}\text { Hydrophilin essential in desiccation- } \\
\text { rehydration process }\end{array}$ & 36.19 & 77.03 & 27.87 & 233.70 & 84.57 & Cytoplasm & Cytoplasm & $\begin{array}{l}\text { Cytoplasm, } \\
\text { nucleus }\end{array}$ \\
\hline YOL151W & GRE2 & $\begin{array}{l}\text { 3-methylbutanal reductase and NADPH- } \\
\text { dependent methylglyoxal reductase }\end{array}$ & 51.12 & 106.53 & 54.46 & 132.87 & 67.92 & $\begin{array}{l}\text { Cytoplasm, } \\
\text { nucleus }\end{array}$ & $\begin{array}{l}\text { Cytoplasm, } \\
\text { nucleus }\end{array}$ & $\begin{array}{l}\text { Cytoplasm, } \\
\text { nucleus }\end{array}$ \\
\hline YLR364W & GRX8 & $\begin{array}{l}\text { Glutaredoxin that employs a dithiol } \\
\text { mechanism of catalysis }\end{array}$ & 83.31 & 113.01 & 94.15 & 165.89 & 138.20 & $\begin{array}{l}\text { Cytoplasm, } \\
\text { nucleus }\end{array}$ & $\begin{array}{l}\text { Cytoplasm, } \\
\text { nucleus }\end{array}$ & $\begin{array}{l}\text { Cytoplasm, } \\
\text { nucleus }\end{array}$ \\
\hline YLR258W & GSY2 & Glycogen synthase & 88.24 & 112.35 & 99.14 & 118.44 & 104.52 & Cytoplasm & Cytoplasm & Cytoplasm \\
\hline YIL155C & GUT2 & $\begin{array}{l}\text { Mitochondrial glycerol-3-phosphate } \\
\text { dehydrogenase }\end{array}$ & 40.28 & 107.56 & 43.33 & 68.20 & 27.47 & Mitochondrion & Mitochondrion & Mitochondrion \\
\hline YIL041W & GVP36 & $\begin{array}{l}\text { BAR domain protein that localizes to } \\
\text { early and late Golgi vesicles }\end{array}$ & 232.29 & 97.85 & 227.29 & 73.55 & 170.84 & Cytoplasmic foci & Cytoplasmic foci & Cytoplasmic foci \\
\hline YDL223C & HBT1 & $\begin{array}{l}\text { Shmoo tip protein, substrate of Hub1p } \\
\text { ubiquitin-like protein }\end{array}$ & 54.24 & 78.22 & 42.42 & 67.02 & 36.35 & $\begin{array}{l}\text { Cytoplasm, } \\
\text { nucleus }\end{array}$ & $\begin{array}{l}\text { Cytoplasm, } \\
\text { nucleus }\end{array}$ & $\begin{array}{l}\text { Cytoplasm, } \\
\text { nucleus }\end{array}$ \\
\hline
\end{tabular}




\begin{tabular}{|c|c|c|c|c|c|c|c|c|c|c|}
\hline YGR191W & HIP1 & High-affinity histidine permease & 54.48 & 74.12 & 40.39 & 88.68 & 48.32 & $\begin{array}{l}\text { Cytoplasm, } \\
\text { vacuolar lumen }\end{array}$ & Vacuolar lumen & Vacuolar lumen \\
\hline YGL077C & HNM1 & $\begin{array}{l}\text { Plasma membrane transporter for } \\
\text { choline, ethanolamine, and carnitine }\end{array}$ & 90.23 & 76.91 & 69.40 & 92.63 & 83.59 & $\begin{array}{l}\text { Plasma } \\
\text { membrane, } \\
\text { vacuolar lumen }\end{array}$ & $\begin{array}{l}\text { Plasma } \\
\text { membrane, } \\
\text { vacuolar lumen }\end{array}$ & Vacuolar lumen \\
\hline YNR055C & HOL1 & $\begin{array}{l}\text { Putative transporter in the major } \\
\text { facilitator superfamily }\end{array}$ & 38.70 & 94.54 & 36.58 & 97.28 & 37.64 & $\begin{array}{l}\text { Plasma } \\
\text { membrane }\end{array}$ & $\begin{array}{l}\text { Plasma } \\
\text { membrane }\end{array}$ & $\begin{array}{l}\text { Plasma } \\
\text { membrane, } \\
\text { vacuolar lumen }\end{array}$ \\
\hline $\begin{array}{l}\text { YMR251 } \\
\text { W-A }\end{array}$ & HOR7 & Protein of unknown function & 297.99 & 92.39 & 275.32 & 119.76 & 356.88 & ER & ER & ER \\
\hline YPL204W & HRR25 & Conserved casein kinase & 57.45 & 95.01 & 54.58 & 90.09 & 51.75 & Cytoplasm & Cytoplasmic foci & Cytoplasmic foci \\
\hline $\begin{array}{l}\text { YMR186 } \\
\text { W }\end{array}$ & HSC82 & $\begin{array}{l}\text { Cytoplasmic chaperone of the Hsp90 } \\
\text { family }\end{array}$ & 1189.26 & 99.20 & 1179.76 & 127.46 & 1515.89 & $\begin{array}{l}\text { Cytoplasm, } \\
\text { nucleus }\end{array}$ & $\begin{array}{l}\text { Cytoplasm, } \\
\text { nucleus }\end{array}$ & $\begin{array}{l}\text { Cytoplasm, } \\
\text { nucleus }\end{array}$ \\
\hline YFL014W & HSP12 & $\begin{array}{l}\text { Plasma membrane protein involved in } \\
\text { maintaining membrane organization }\end{array}$ & 860.19 & 167.27 & 1438.82 & 138.52 & 1191.55 & $\begin{array}{l}\text { Cytoplasm, } \\
\text { nucleus }\end{array}$ & $\begin{array}{l}\text { Cytoplasm, } \\
\text { nucleus }\end{array}$ & $\begin{array}{l}\text { Cytoplasm, } \\
\text { nucleus }\end{array}$ \\
\hline YBR072W & HSP26 & $\begin{array}{l}\text { Small heat shock protein (sHSP) with } \\
\text { chaperone activity }\end{array}$ & 39.81 & 130.19 & 51.83 & 204.26 & 81.32 & $\begin{array}{l}\text { Cytoplasm, } \\
\text { cytoplasmic foci }\end{array}$ & $\begin{array}{l}\text { Cytoplasm, } \\
\text { cytoplasmic foci }\end{array}$ & $\begin{array}{l}\text { Cytoplasm, } \\
\text { cytoplasmic foci }\end{array}$ \\
\hline YCR021C & HSP30 & $\begin{array}{l}\text { Negative regulator of the } \mathrm{H}(+) \text {-ATPase } \\
\text { Pma1p }\end{array}$ & 43.20 & 81.48 & 35.20 & 85.82 & 37.08 & $\begin{array}{l}\text { Plasma } \\
\text { membrane, } \\
\text { vacuolar lumen }\end{array}$ & $\begin{array}{l}\text { Plasma } \\
\text { membrane, } \\
\text { vacuolar lumen }\end{array}$ & $\begin{array}{l}\text { Plasma } \\
\text { membrane, } \\
\text { vacuolar lumen }\end{array}$ \\
\hline YDR533C & HSP31 & $\begin{array}{l}\text { Methylglyoxalase that converts } \\
\text { methylglyoxal to D-lactate }\end{array}$ & 77.91 & 91.76 & 71.49 & 155.19 & 120.91 & $\begin{array}{l}\text { Cytoplasm, } \\
\text { nucleus }\end{array}$ & $\begin{array}{l}\text { Cytoplasm, } \\
\text { nucleus }\end{array}$ & $\begin{array}{l}\text { Cytoplasm, } \\
\text { nucleus }\end{array}$ \\
\hline YDR258C & HSP78 & $\begin{array}{l}\text { Oligomeric mitochondrial matrix } \\
\text { chaperone }\end{array}$ & 118.17 & 103.47 & 122.27 & 128.35 & 151.67 & Mitochondrion & Mitochondrion & Mitochondrion \\
\hline YDR224C & HTB1 & Histone H2B & 1862.32 & 103.93 & 1935.47 & 63.32 & 1179.22 & Nucleus & Nucleus & Nucleus \\
\hline YFR053C & HXK1 & Hexokinase isoenzyme 1 & 1815.01 & 139.93 & 2539.72 & 84.02 & 1525.05 & $\begin{array}{l}\text { Cytoplasm, } \\
\text { nucleus }\end{array}$ & $\begin{array}{l}\text { Cytoplasm, } \\
\text { nucleus }\end{array}$ & $\begin{array}{l}\text { Cytoplasm, } \\
\text { nucleus }\end{array}$ \\
\hline YGL253W & HXK2 & Hexokinase isoenzyme 2 & 1304.57 & 119.75 & 1562.22 & 135.76 & 1771.07 & $\begin{array}{l}\text { Cytoplasm, } \\
\text { nucleus }\end{array}$ & $\begin{array}{l}\text { Cytoplasm, } \\
\text { nucleus }\end{array}$ & $\begin{array}{l}\text { Cytoplasm, } \\
\text { nucleus }\end{array}$ \\
\hline YOR136W & IDH2 & $\begin{array}{l}\text { Subunit of mitochondrial NAD(+)- } \\
\text { dependent isocitrate dehydrogenase }\end{array}$ & 189.73 & 105.68 & 200.51 & 159.27 & 302.17 & Mitochondrion & Mitochondrion & Mitochondrion \\
\hline
\end{tabular}




\begin{tabular}{|c|c|c|c|c|c|c|c|c|c|c|}
\hline YNL157W & IGO1 & $\begin{array}{l}\text { Protein required for initiation of } \mathrm{G0} \\
\text { program }\end{array}$ & 59.28 & 77.57 & 45.98 & 129.84 & 76.97 & $\begin{array}{l}\text { Cytoplasm, } \\
\text { nucleus }\end{array}$ & $\begin{array}{l}\text { Cytoplasm, } \\
\text { nucleus }\end{array}$ & $\begin{array}{l}\text { Cytoplasm, } \\
\text { nucleus }\end{array}$ \\
\hline YLR413W & INA1 & Protein of unknown function & 105.52 & 71.21 & 75.13 & 58.91 & 62.16 & $\begin{array}{l}\text { Plasma } \\
\text { membrane, } \\
\text { vacuolar lumen }\end{array}$ & $\begin{array}{l}\text { Plasma } \\
\text { membrane, } \\
\text { vacuolar lumen }\end{array}$ & Vacuolar lumen \\
\hline YIR036C & IRC24 & Putative benzil reductase & 62.49 & 127.93 & 79.94 & 89.77 & 56.09 & $\begin{array}{l}\text { Cytoplasm, } \\
\text { nucleus }\end{array}$ & $\begin{array}{l}\text { Cytoplasm, } \\
\text { nucleus }\end{array}$ & $\begin{array}{l}\text { Cytoplasm, } \\
\text { nucleus }\end{array}$ \\
\hline YIL026C & IRR1 & Subunit of the cohesin complex & 41.79 & 99.52 & 41.59 & 97.11 & 40.58 & Nucleus & Nucleus & Nucleus \\
\hline YDR497C & ITR1 & Myo-inositol transporter & 217.37 & 99.87 & 217.09 & 102.45 & 222.70 & $\begin{array}{l}\text { Plasma } \\
\text { membrane, } \\
\text { vacuolar lumen }\end{array}$ & Vacuolar lumen & Vacuolar lumen \\
\hline YOL103W & ITR2 & Myo-inositol transporter & 42.93 & 88.58 & 38.03 & 92.31 & 39.63 & $\begin{array}{l}\text { Plasma } \\
\text { membrane }\end{array}$ & $\begin{array}{l}\text { Plasma } \\
\text { membrane, } \\
\text { vacuolar lumen }\end{array}$ & $\begin{array}{l}\text { Plasma } \\
\text { membrane, } \\
\text { vacuolar lumen }\end{array}$ \\
\hline YHR158C & KEL1 & $\begin{array}{l}\text { Protein required for proper cell fusion } \\
\text { and cell morphology }\end{array}$ & 41.95 & 102.92 & 43.18 & 116.29 & 48.79 & Bud neck & Bud neck & $\begin{array}{l}\text { Plasma } \\
\text { membrane }\end{array}$ \\
\hline YDR148C & KGD2 & Dihydrolipoyl transsuccinylase & 77.51 & 114.16 & 88.48 & 122.74 & 95.14 & Mitochondrion & Mitochondrion & Mitochondrion \\
\hline YPR159W & KRE6 & Type II integral membrane protein & 71.67 & 90.61 & 64.95 & 84.13 & 60.30 & ER & ER & ER \\
\hline YNL071W & LAT1 & $\begin{array}{l}\text { Dihydrolipoamide acetyltransferase } \\
\text { component (E2) of the PDC }\end{array}$ & 84.39 & 107.24 & 90.51 & 75.01 & 63.30 & Mitochondrion & Mitochondrion & Mitochondrion \\
\hline YFL018C & LPD1 & Dihydrolipoamide dehydrogenase & 396.21 & 124.26 & 492.32 & 71.31 & 282.54 & Mitochondrion & Mitochondrion & Mitochondrion \\
\hline YKL029C & MAE1 & Mitochondrial malic enzyme & 47.94 & 89.20 & 42.77 & 82.07 & 39.35 & Mitochondrion & Mitochondrion & Mitochondrion \\
\hline YKL021C & MAK11 & $\begin{array}{l}\text { Protein involved in an early step of } 605 \\
\text { ribosomal subunit biogenesis }\end{array}$ & 52.21 & 101.61 & 53.05 & 108.59 & 56.69 & $\begin{array}{l}\text { Nuclear } \\
\text { membrane }\end{array}$ & $\begin{array}{l}\text { Nuclear } \\
\text { membrane }\end{array}$ & Nuclear foci \\
\hline YNL307C & MCK1 & $\begin{array}{l}\text { Dual-specificity ser/thr and tyrosine } \\
\text { protein kinase }\end{array}$ & 91.60 & 133.47 & 122.25 & 100.94 & 92.46 & $\begin{array}{l}\text { Cytoplasm, } \\
\text { nucleus }\end{array}$ & $\begin{array}{l}\text { Cytoplasm, } \\
\text { nucleus }\end{array}$ & $\begin{array}{l}\text { Cytoplasm, } \\
\text { nucleus }\end{array}$ \\
\hline YOL126C & $\mathrm{MDH} 2$ & Cytoplasmic malate dehydrogenase & 262.38 & 142.11 & 372.86 & 47.64 & 124.99 & $\begin{array}{l}\text { Cytoplasm, } \\
\text { cytoplasmic } \\
\text { foci, nucleus }\end{array}$ & $\begin{array}{l}\text { Cytoplasm, } \\
\text { cytoplasmic } \\
\text { foci, nucleus }\end{array}$ & $\begin{array}{l}\text { Cytoplasm, } \\
\text { cytoplasmic } \\
\text { foci, nucleus }\end{array}$ \\
\hline YLR188W & MDL1 & $\begin{array}{l}\text { Mitochondrial inner membrane half- } \\
\text { type } A B C \text { transporter }\end{array}$ & 43.60 & 64.71 & 28.21 & 94.06 & 41.01 & Mitochondrion & Mitochondrion & Mitochondrion \\
\hline
\end{tabular}




\begin{tabular}{|c|c|c|c|c|c|c|c|c|c|c|}
\hline YGR264C & MES1 & Methionyl-tRNA synthetase & 199.31 & 87.68 & 174.77 & 119.02 & 237.22 & Cytoplasm & Cytoplasm & Cytoplasm \\
\hline YKL001C & MET14 & Adenylylsulfate kinase & 135.83 & 95.07 & 129.13 & 142.68 & 193.81 & \begin{tabular}{|l|} 
Cytoplasm, \\
nucleus
\end{tabular} & $\begin{array}{l}\text { Cytoplasm, } \\
\text { nucleus }\end{array}$ & $\begin{array}{l}\text { Cytoplasm, } \\
\text { nucleus }\end{array}$ \\
\hline YOL064C & MET22 & Bisphosphate-3'-nucleotidase & 128.80 & 133.73 & 172.25 & 103.03 & 132.70 & $\begin{array}{l}\text { Cytoplasm, } \\
\text { nucleus }\end{array}$ & $\begin{array}{l}\text { Cytoplasm, } \\
\text { nucleus }\end{array}$ & $\begin{array}{l}\text { Cytoplasm, } \\
\text { nucleus }\end{array}$ \\
\hline YJR137C & MET5 & Sulfite reductase beta subunit & 194.90 & 92.35 & 179.98 & 64.20 & 125.12 & Cytoplasm & Cytoplasm & Cytoplasm \\
\hline YJR077C & MIR1 & Mitochondrial phosphate carrier & 225.79 & 69.77 & 157.55 & 56.59 & 127.77 & Mitochondrion & Mitochondrion & Mitochondrion \\
\hline YLL006W & MMM1 & $\begin{array}{l}\text { ER integral membrane protein, ERMES } \\
\text { complex subunit }\end{array}$ & 140.48 & 105.76 & 148.57 & 28.06 & 39.42 & Cytoplasm & Cytoplasm & Cytoplasmic foci \\
\hline YMR224C & MRE11 & $\begin{array}{l}\text { Nuclease subunit of the MRX complex } \\
\text { with Rad50p and Xrs2p }\end{array}$ & 36.18 & 100.24 & 36.27 & 96.67 & 34.98 & $\begin{array}{l}\text { Cytoplasm, } \\
\text { nucleus }\end{array}$ & $\begin{array}{l}\text { Cytoplasm, } \\
\text { nucleus }\end{array}$ & $\begin{array}{l}\text { Cytoplasm, } \\
\text { nucleus }\end{array}$ \\
\hline YDR033W & MRH1 & $\begin{array}{l}\text { Protein that localizes primarily to the } \\
\text { plasma membrane }\end{array}$ & 137.91 & 100.06 & 138.00 & 71.06 & 98.00 & $\begin{array}{l}\text { Plasma } \\
\text { membrane }\end{array}$ & $\begin{array}{l}\text { Plasma } \\
\text { membrane }\end{array}$ & $\begin{array}{l}\text { Plasma } \\
\text { membrane }\end{array}$ \\
\hline $\begin{array}{l}\text { YDL045W } \\
-A\end{array}$ & MRP10 & $\begin{array}{l}\text { Mitochondrial ribosomal protein of the } \\
\text { small subunit }\end{array}$ & 72.74 & 81.19 & 59.06 & 131.61 & 95.73 & Mitochondrion & Mitochondrion & Mitochondrion \\
\hline $\begin{array}{l}\text { YAL034W } \\
\text {-A }\end{array}$ & MTW1 & $\begin{array}{l}\text { Essential component of the MIND } \\
\text { kinetochore complex }\end{array}$ & 42.96 & 80.30 & 34.50 & 82.50 & 35.44 & Nuclear foci & Nuclear foci & Nuclear foci \\
\hline YGR055W & MUP1 & High affinity methionine permease & 384.45 & 111.90 & 430.20 & 88.99 & 342.12 & $\begin{array}{l}\text { Plasma } \\
\text { membrane, } \\
\text { vacuolar lumen }\end{array}$ & $\begin{array}{l}\text { Plasma } \\
\text { membrane, } \\
\text { vacuolar lumen }\end{array}$ & $\begin{array}{l}\text { Plasma } \\
\text { membrane, } \\
\text { vacuolar lumen }\end{array}$ \\
\hline YER042W & MXR1 & Methionine-S-sulfoxide reductase & 67.42 & 89.17 & 60.12 & 160.45 & 108.18 & $\begin{array}{l}\text { Cytoplasm, } \\
\text { nucleus }\end{array}$ & $\begin{array}{l}\text { Cytoplasm, } \\
\text { nucleus }\end{array}$ & $\begin{array}{l}\text { Cytoplasm, } \\
\text { nucleus }\end{array}$ \\
\hline YPR149W & NCE102 & Protein of unknown function & 281.90 & 128.42 & 362.03 & 108.15 & 304.88 & $\begin{array}{l}\text { Plasma } \\
\text { membrane }\end{array}$ & $\begin{array}{l}\text { Plasma } \\
\text { membrane }\end{array}$ & $\begin{array}{l}\text { Plasma } \\
\text { membrane }\end{array}$ \\
\hline YIL048W & NEO1 & $\begin{array}{l}\text { Phospholipid translocase (flippase), role } \\
\text { in phospholipid asymmetry o }\end{array}$ & 48.68 & 80.23 & 39.05 & 102.02 & 49.66 & Cytoplasm & Cytoplasm & Vacuolar lumen \\
\hline YDL208W & NHP2 & $\begin{array}{l}\text { Protein related to mammalian high } \\
\text { mobility group (HMG) proteins }\end{array}$ & 214.32 & 91.32 & 195.73 & 70.31 & 150.69 & Nucleus & Nucleus & Nucleus \\
\hline $\begin{array}{l}\text { YBR089C- } \\
\text { A }\end{array}$ & NHP6B & High-mobility group (HMG) protein & 128.17 & 97.07 & 124.42 & 145.47 & 186.45 & Nucleus & Nucleus & Nucleus \\
\hline YKL151C & NNR2 & Widely-conserved NADHX dehydratase & 39.82 & 118.96 & 47.37 & 131.86 & 52.51 & Cytoplasm & Cytoplasm & Cytoplasm \\
\hline
\end{tabular}




\begin{tabular}{|c|c|c|c|c|c|c|c|c|c|c|}
\hline YOR056C & NOB1 & $\begin{array}{l}\text { Protein involved in proteasomal and } 40 \mathrm{~S} \\
\text { ribosomal subunit biogenesis }\end{array}$ & 55.61 & 111.94 & 62.25 & 101.18 & 56.27 & Cytoplasm & Cytoplasm & Nuclear foci \\
\hline YPL093W & NOG1 & Putative GTPase & 97.44 & 82.99 & 80.87 & 76.58 & 74.62 & Nucleus & Nucleus & Nuclear foci \\
\hline YNR053C & NOG2 & Putative GTPase & 46.19 & 85.63 & 39.55 & 90.44 & 41.77 & Nucleus & $\begin{array}{l}\text { Cytoplasm, } \\
\text { nucleus }\end{array}$ & $\begin{array}{l}\text { Cytoplasm, } \\
\text { nucleus }\end{array}$ \\
\hline YDL213C & NOP6 & $\begin{array}{l}\text { rRNA-binding protein required for } 40 \mathrm{~S} \\
\text { ribosomal subunit biogenesis }\end{array}$ & 98.66 & 83.04 & 81.93 & 57.77 & 57.00 & Nucleus & Nucleus & Nucleus \\
\hline YGR103W & NOP7 & $\begin{array}{l}\text { Component of several different pre- } \\
\text { ribosomal particles }\end{array}$ & 31.98 & 102.10 & 32.65 & 153.25 & 49.01 & Cytoplasm & Cytoplasm & Nucleus \\
\hline YGR043C & NQM1 & Transaldolase of unknown function & 56.72 & 111.10 & 63.02 & 135.34 & 76.77 & Nucleus & Nucleus & Nucleus \\
\hline YDR001C & NTH1 & Neutral trehalase, degrades trehalose & 61.53 & 105.55 & 64.94 & 151.34 & 93.12 & Cytoplasm & Cytoplasm & Cytoplasm \\
\hline YJR073C & OPI3 & $\begin{array}{l}\text { Methylene-fatty-acyl-phospholipid } \\
\text { synthase }\end{array}$ & 163.76 & 71.96 & 117.85 & 112.21 & 183.75 & ER & $\begin{array}{l}\text { ER, vacuolar } \\
\text { lumen }\end{array}$ & $\begin{array}{l}\text { ER, vacuolar } \\
\text { lumen }\end{array}$ \\
\hline YJL002C & OST1 & $\begin{array}{l}\text { Alpha subunit of the } \\
\text { oligosaccharyltransferase complex of } \\
\text { the ER lumen }\end{array}$ & 124.27 & 92.27 & 114.67 & 78.43 & 97.47 & ER & ER & ER \\
\hline \multicolumn{2}{|c|}{ YHR179W OYE2 } & \multicolumn{9}{|l|}{ Conservededservedn mon-2( )n1 0cleo1 C } \\
\hline
\end{tabular}




\begin{tabular}{|c|c|c|c|c|c|c|c|c|c|c|}
\hline YLR044C & PDC1 & $\begin{array}{l}\text { Major of three pyruvate decarboxylase } \\
\text { isozymes }\end{array}$ & 2120.15 & 111.25 & 2358.72 & 148.68 & 3152.25 & $\begin{array}{l}\text { Cytoplasm, } \\
\text { nucleus }\end{array}$ & $\begin{array}{l}\text { Cytoplasm, } \\
\text { nucleus }\end{array}$ & $\begin{array}{l}\text { Cytoplasm, } \\
\text { nucleus }\end{array}$ \\
\hline YGR087C & PDC6 & $\begin{array}{l}\text { Minor isoform of pyruvate } \\
\text { decarboxylase }\end{array}$ & 95.33 & 151.71 & 144.62 & 273.38 & 260.61 & $\begin{array}{l}\text { Cytoplasm, } \\
\text { nucleus }\end{array}$ & $\begin{array}{l}\text { Cytoplasm, } \\
\text { nucleus }\end{array}$ & $\begin{array}{l}\text { Cytoplasm, } \\
\text { nucleus }\end{array}$ \\
\hline YPL058C & PDR12 & $\begin{array}{l}\text { Plasma membrane ATP-binding cassette } \\
(\mathrm{ABC}) \text { transporter }\end{array}$ & 51.76 & 114.50 & 59.27 & 125.52 & 64.97 & Vacuolar lumen & Vacuolar lumen & Vacuolar lumen \\
\hline YOR153W & PDR5 & $\begin{array}{l}\text { Plasma membrane ATP-binding cassette } \\
(A B C) \text { transporter }\end{array}$ & 66.46 & 85.81 & 57.02 & 98.88 & 65.71 & $\begin{array}{l}\text { Plasma } \\
\text { membrane, } \\
\text { vacuolar lumen }\end{array}$ & Vacuolar lumen & Vacuolar lumen \\
\hline YKR046C & PET10 & $\begin{array}{l}\text { Protein of unknown function that } \\
\text { localizes to lipid particles }\end{array}$ & 124.97 & 162.26 & 202.77 & 67.23 & 84.02 & Lipid droplets & Lipid droplets & Lipid droplets \\
\hline YDR244W & PEX5 & $\begin{array}{l}\text { Peroxisomal membrane signal receptor } \\
\text { for peroxisomal matrix proteins }\end{array}$ & 36.23 & 100.03 & 36.24 & 92.17 & 33.39 & Peroxisomes & Peroxisomes & Peroxisomes \\
\hline YMR205C & PFK2 & $\begin{array}{l}\text { Beta subunit of heterooctameric } \\
\text { phosphofructokinase }\end{array}$ & 505.42 & 103.38 & 522.49 & 137.87 & 696.81 & Cytoplasm & Cytoplasm & Cytoplasm \\
\hline YNL149C & PGA2 & $\begin{array}{l}\text { Essential protein required for } \\
\text { maturation of Gas1p and Pho8p }\end{array}$ & 56.16 & 93.26 & 52.37 & 96.88 & 54.40 & $\begin{array}{l}\text { ER, lipid } \\
\text { droplets }\end{array}$ & $\begin{array}{l}\text { ER, lipid } \\
\text { droplets }\end{array}$ & ER \\
\hline YBR196C & PGI1 & $\begin{array}{l}\text { Glycolytic enzyme phosphoglucose } \\
\text { isomerase }\end{array}$ & 635.08 & 109.81 & 697.40 & 78.80 & 500.44 & $\begin{array}{l}\text { Cytoplasm, } \\
\text { nucleus }\end{array}$ & Nucleus & Nucleus \\
\hline YCR012W & PGK1 & 3-phosphoglycerate kinase & 2328.94 & 126.40 & 2943.72 & 139.66 & 3252.51 & $\begin{array}{l}\text { Cytoplasm, } \\
\text { nucleus }\end{array}$ & $\begin{array}{l}\text { Cytoplasm, } \\
\text { nucleus }\end{array}$ & $\begin{array}{l}\text { Cytoplasm, } \\
\text { nucleus }\end{array}$ \\
\hline YMR105C & PGM2 & Phosphoglucomutase & 465.15 & 107.24 & 498.80 & 62.43 & 290.39 & $\begin{array}{l}\text { Cytoplasm, } \\
\text { nucleus }\end{array}$ & $\begin{array}{l}\text { Cytoplasm, } \\
\text { nucleus }\end{array}$ & $\begin{array}{l}\text { Cytoplasm, } \\
\text { nucleus }\end{array}$ \\
\hline YJL097W & PHS1 & $\begin{array}{l}\text { Essential 3-hydroxyacyl-CoA } \\
\text { dehydratase of the ER membrane }\end{array}$ & 73.21 & 85.11 & 62.31 & 86.99 & 63.69 & ER & $\begin{array}{l}\text { ER, vacuolar } \\
\text { lumen }\end{array}$ & Vacuolar lumen \\
\hline YGR086C & PIL1 & Eisosome core component & 243.97 & 140.82 & 343.55 & 168.16 & 410.25 & $\begin{array}{l}\text { Plasma } \\
\text { membrane }\end{array}$ & $\begin{array}{l}\text { Plasma } \\
\text { membrane }\end{array}$ & $\begin{array}{l}\text { Plasma } \\
\text { membrane }\end{array}$ \\
\hline YLR016C & PML1 & Subunit of the RES complex & 39.01 & 77.92 & 30.40 & 98.12 & 38.28 & Nuclear foci, & Nuclear foci & Cytoplasm \\
\hline $\begin{array}{l}\text { YEL017C- } \\
\text { A }\end{array}$ & PMP2 & $\begin{array}{l}\text { Proteolipid associated with plasma } \\
\text { membrane } \mathrm{H}(+) \text {-ATPase (Pma1p) }\end{array}$ & 403.09 & 99.44 & 400.84 & 67.10 & 270.46 & $\begin{array}{l}\text { Plasma } \\
\text { membrane, } \\
\text { vacuolar lumen }\end{array}$ & $\begin{array}{l}\text { Plasma } \\
\text { membrane, } \\
\text { vacuolar lumen }\end{array}$ & $\begin{array}{l}\text { Plasma } \\
\text { membrane, } \\
\text { vacuolar lumen }\end{array}$ \\
\hline
\end{tabular}




\begin{tabular}{|c|c|c|c|c|c|c|c|c|c|c|}
\hline YGL167C & PMR1 & High affinity $\mathrm{Ca} 2+/ \mathrm{Mn} 2+$ P-type ATPase & 39.06 & 69.20 & 27.03 & 213.36 & 83.33 & Cytoplasm & Cytoplasm & ER \\
\hline YNR032W & PPG1 & $\begin{array}{l}\text { Putative serine/threonine protein } \\
\text { phosphatase }\end{array}$ & 32.61 & 99.02 & 32.29 & 132.90 & 43.34 & Cytoplasm & Cytoplasm & Cytoplasm \\
\hline YCL057W & PRD1 & Zinc metalloendopeptidase & 113.23 & 102.21 & 115.73 & 80.36 & 90.99 & $\begin{array}{l}\text { Cytoplasm, } \\
\text { nucleus }\end{array}$ & $\begin{array}{l}\text { Cytoplasm, } \\
\text { nucleus }\end{array}$ & $\begin{array}{l}\text { Cytoplasm, } \\
\text { nucleus }\end{array}$ \\
\hline \begin{tabular}{|l|} 
YMR314 \\
W
\end{tabular} & PRE5 & Alpha 6 subunit of the $20 \mathrm{~S}$ proteasome & 199.69 & 94.82 & 189.35 & 65.61 & 131.02 & $\begin{array}{l}\text { Cytoplasm, } \\
\text { nucleus }\end{array}$ & $\begin{array}{l}\text { Cytoplasm, } \\
\text { nucleus }\end{array}$ & $\begin{array}{l}\text { Cytoplasm, } \\
\text { nucleus }\end{array}$ \\
\hline YGR135W & PRE9 & Alpha 3 subunit of the $20 \mathrm{~S}$ proteasome & 183.90 & 98.98 & 182.02 & 69.06 & 127.00 & $\begin{array}{l}\text { Cytoplasm, } \\
\text { nucleus }\end{array}$ & $\begin{array}{l}\text { Cytoplasm, } \\
\text { nucleus }\end{array}$ & $\begin{array}{l}\text { Cytoplasm, } \\
\text { nucleus }\end{array}$ \\
\hline YJR059W & PTK2 & Serine/threonine protein kinase & 55.28 & 99.60 & 55.06 & 131.45 & 72.66 & Cytoplasm & Cytoplasm & Cytoplasm \\
\hline YLR414C & PUN1 & $\begin{array}{l}\text { Plasma membrane protein with a role in } \\
\text { cell wall integrity }\end{array}$ & 75.96 & 91.26 & 69.32 & 110.45 & 83.90 & $\begin{array}{l}\text { Plasma } \\
\text { membrane }\end{array}$ & $\begin{array}{l}\text { Plasma } \\
\text { membrane }\end{array}$ & $\begin{array}{l}\text { Plasma } \\
\text { membrane, } \\
\text { vacuolar lumen }\end{array}$ \\
\hline YELO20C & PXP1 & Peroxisomal matrix protein & 62.18 & 124.07 & 77.14 & 138.98 & 86.41 & Cytoplasm & Cytoplasm & Cytoplasm \\
\hline YBR218C & PYC2 & Pyruvate carboxylase isoform & 129.60 & 98.64 & 127.85 & 95.87 & 124.25 & Cytoplasm & Cytoplasm & Cytoplasm \\
\hline YFR033C & QCR6 & $\begin{array}{l}\text { Subunit } 6 \text { of the ubiquinol cytochrome-c } \\
\text { reductase complex }\end{array}$ & 301.87 & 109.09 & 329.31 & 83.80 & 252.96 & $\begin{array}{l}\text { Cytoplasm, } \\
\text { nucleus }\end{array}$ & $\begin{array}{l}\text { Cytoplasm, } \\
\text { nucleus }\end{array}$ & $\begin{array}{l}\text { Cytoplasm, } \\
\text { nucleus }\end{array}$ \\
\hline YGL058W & RAD6 & Ubiquitin-conjugating enzyme (E2) & 94.64 & 83.27 & 78.80 & 43.71 & 41.37 & $\begin{array}{l}\text { Cytoplasm, } \\
\text { nucleus }\end{array}$ & $\begin{array}{l}\text { Cytoplasm, } \\
\text { nucleus }\end{array}$ & $\begin{array}{l}\text { Cytoplasm, } \\
\text { nucleus }\end{array}$ \\
\hline YDL090C & RAM1 & \begin{tabular}{|l} 
Beta subunit of the CAAX \\
farnesyltransferase (FTase)
\end{tabular} & 42.62 & 102.66 & 43.75 & 93.98 & 40.05 & $\begin{array}{l}\text { Cytoplasm, } \\
\text { nucleus }\end{array}$ & $\begin{array}{l}\text { Cytoplasm, } \\
\text { nucleus }\end{array}$ & Cytoplasm \\
\hline YDR003W & RCR2 & Vacuolar protein & 49.46 & 87.88 & 43.46 & 106.25 & 52.55 & Cytoplasm & Cytoplasm & $\begin{array}{l}\text { Cytoplasm, } \\
\text { vacuolar lumen }\end{array}$ \\
\hline YBR073W & RDH54 & DNA-dependent ATPase & 36.32 & 91.06 & 33.08 & 99.45 & 36.12 & Nucleus & Nucleus & Cytoplasm \\
\hline YOR285W & RDL1 & Thiosulfate sulfurtransferase & 236.78 & 102.10 & 241.76 & 61.05 & 144.55 & Cytoplasmic foci & Cytoplasmic foci & Cytoplasmic foci \\
\hline YIL057C & RGI2 & Protein of unknown function & 107.71 & 129.55 & 139.53 & 103.39 & 111.35 & $\begin{array}{l}\text { Cytoplasm, } \\
\text { nucleus }\end{array}$ & $\begin{array}{l}\text { Cytoplasm, } \\
\text { nucleus }\end{array}$ & $\begin{array}{l}\text { Cytoplasm, } \\
\text { nucleus }\end{array}$ \\
\hline $\begin{array}{l}\text { YMR139 } \\
\text { W }\end{array}$ & RIM11 & Protein kinase & 49.09 & 106.88 & 52.47 & 131.28 & 64.44 & Cytoplasm & Cytoplasm & Cytoplasm \\
\hline YBR030W & RKM3 & Ribosomal lysine methyltra & 37.38 & 104.07 & 38.90 & 95.03 & 35.52 & Nucleus & Nucleus & $\begin{array}{l}\text { Cytoplasm, } \\
\text { nucleus }\end{array}$ \\
\hline
\end{tabular}




\begin{tabular}{|c|c|c|c|c|c|c|c|c|c|c|}
\hline YDL001W & RMD1 & $\begin{array}{l}\text { Cytoplasmic protein required for } \\
\text { sporulation }\end{array}$ & 49.07 & 97.87 & 48.02 & 79.36 & 38.95 & $\begin{array}{l}\text { Cytoplasm, } \\
\text { nucleus }\end{array}$ & $\begin{array}{l}\text { Cytoplasm, } \\
\text { nucleus }\end{array}$ & Cytoplasm \\
\hline YER070W & RNR1 & $\begin{array}{l}\text { Major isoform of large subunit of } \\
\text { ribonucleotide-diphosphate reductase }\end{array}$ & 731.72 & 103.99 & 760.93 & 124.61 & 911.83 & Septins & Septins & $\begin{array}{l}\text { Septins, } \\
\text { cytoplasm }\end{array}$ \\
\hline YJR063W & RPA12 & RNA Polymerase A & 109.81 & 71.43 & 78.44 & 86.87 & 109.81 & Nucleus & Nucleus & Nucleus \\
\hline YPR010C & RPA135 & $\begin{array}{l}\text { RNA polymerase I second largest } \\
\text { subunit A135 }\end{array}$ & 103.41 & 96.61 & 99.91 & 66.92 & 69.20 & Nucleus & Nucleus & Nucleus \\
\hline YOR340C & RPA43 & RNA polymerase I subunit A43 & 72.93 & 87.81 & 64.04 & 71.38 & 52.06 & Nucleus & Nucleus & Nucleus \\
\hline $\begin{array}{l}\text { YHR143W } \\
-A\end{array}$ & RPC10 & $\begin{array}{l}\text { RNA polymerase subunit ABC10-alpha, } \\
\text { found in RNA pol I, II, and III }\end{array}$ & 112.31 & 80.64 & 90.57 & 56.79 & 63.78 & $\begin{array}{l}\text { Cytoplasm, } \\
\text { nucleus }\end{array}$ & $\begin{array}{l}\text { Cytoplasm, } \\
\text { nucleus }\end{array}$ & $\begin{array}{l}\text { Cytoplasm, } \\
\text { nucleus }\end{array}$ \\
\hline YNL330C & RPD3 & $\begin{array}{l}\text { Histone deacetylase, component of } \\
\text { both the Rpd3S and Rpd3L complexes }\end{array}$ & 49.33 & 100.13 & 49.39 & 90.68 & 44.73 & Cytoplasm & Cytoplasm & Nucleus \\
\hline YPR102C & RPL11A & Ribosomal 60S subunit protein L11A & 90.50 & 83.28 & 75.37 & 61.66 & 55.80 & $\begin{array}{l}\text { Cytoplasm, } \\
\text { nucleus }\end{array}$ & $\begin{array}{l}\text { Cytoplasm, } \\
\text { nucleus }\end{array}$ & $\begin{array}{l}\text { Cytoplasm, } \\
\text { nucleus }\end{array}$ \\
\hline YDR418W & RPL12B & Ribosomal 60S subunit protein L12B & 484.89 & 94.51 & 458.25 & 67.95 & 329.49 & Cytoplasm & Cytoplasm & Cytoplasm \\
\hline YMR121C & RPL15B & Ribosomal 60S subunit protein L15B & 41.92 & 69.59 & 29.17 & 93.88 & 39.35 & $\begin{array}{l}\text { Cytoplasm, } \\
\text { nuclear foci }\end{array}$ & $\begin{array}{l}\text { Cytoplasm, } \\
\text { nuclear foci }\end{array}$ & $\begin{array}{l}\text { Cytoplasm, } \\
\text { nuclear foci }\end{array}$ \\
\hline YIL133C & RPL16A & Ribosomal 60S subunit protein L16A & 201.42 & 76.55 & 154.19 & 22.14 & 44.60 & Cytoplasm & Cytoplasm & Cytoplasm \\
\hline YKL180W & RPL17A & Ribosomal 60S subunit protein L17A & 109.89 & 90.20 & 99.12 & 66.55 & 73.13 & $\begin{array}{l}\text { Plasma } \\
\text { membrane }\end{array}$ & $\begin{array}{l}\text { Plasma } \\
\text { membrane }\end{array}$ & $\begin{array}{l}\text { Plasma } \\
\text { membrane }\end{array}$ \\
\hline YPL220W & RPL1A & Ribosomal 60S subunit protein L1A & 110.39 & 75.41 & 83.24 & 50.36 & 55.59 & Cytoplasm & Cytoplasm & Cytoplasm \\
\hline YGL135W & RPL1B & Ribosomal 60S subunit protein L1B & 585.16 & 74.51 & 436.02 & 57.93 & 339.01 & Cytoplasm & Cytoplasm & Cytoplasm \\
\hline YBR191W & RPL21A & Ribosomal 60S subunit protein L21A & 140.20 & 85.92 & 120.46 & 66.11 & 92.69 & Cytoplasm & Cytoplasm & Cytoplasm \\
\hline YPL079W & RPL21B & Ribosomal 60S subunit protein L21B & 283.79 & 84.17 & 238.87 & 50.71 & 143.92 & Cytoplasm & Cytoplasm & Cytoplasm \\
\hline YLR061W & RPL22A & Ribosomal 60S subunit protein L22A & 1141.26 & 104.89 & 1197.12 & 62.63 & 714.73 & Cytoplasm & Cytoplasm & Cytoplasm \\
\hline YBL087C & RPL23A & Ribosomal 60S subunit protein L23A & 64.25 & 53.71 & 34.51 & 64.93 & 41.71 & Nucleus & Nucleus & Nucleus \\
\hline YGR148C & RPL24B & Ribosomal 60S subunit protein L24B & 353.74 & 71.68 & 253.57 & 68.43 & 242.07 & Cytoplasm & Cytoplasm & Cytoplasm \\
\hline YOR063W & RPL3 & Ribosomal 60S subunit protein L3 & 418.92 & 104.22 & 436.60 & 70.26 & 294.32 & $\begin{array}{l}\text { Cytoplasm, } \\
\text { nuclear foci }\end{array}$ & $\begin{array}{l}\text { Cytoplasm, } \\
\text { nuclear foci }\end{array}$ & $\begin{array}{l}\text { Cytoplasm, } \\
\text { nuclear foci }\end{array}$ \\
\hline YOR234C & RPL33B & Ribosomal 60S subunit protein L33B & 175.82 & 59.48 & 104.58 & 73.07 & 128.48 & Cytoplasm & Cytoplasm & Cytoplasm \\
\hline
\end{tabular}




\begin{tabular}{|c|c|c|c|c|c|c|c|c|c|c|}
\hline $\begin{array}{l}\text { YER056C- } \\
\text { A }\end{array}$ & RPL34A & Ribosomal 60S subunit protein L34A & 334.68 & 78.00 & 261.05 & 57.19 & 191.40 & Cytoplasm & Cytoplasm & Cytoplasm \\
\hline $\begin{array}{l}\text { YMR194 } \\
\text { W }\end{array}$ & RPL36A & Ribosomal 60S subunit protein L36A & 207.02 & 71.13 & 147.24 & 57.08 & 118.16 & Cytoplasm & Cytoplasm & Cytoplasm \\
\hline $\begin{array}{l}\text { YPL249C- } \\
\text { A }\end{array}$ & RPL36B & Ribosomal 60S subunit protein L36B & 223.06 & 86.89 & 193.81 & 62.75 & 139.97 & Cytoplasm & Cytoplasm & Cytoplasm \\
\hline YIL148W & RPL40A & $\begin{array}{l}\text { Ubiquitin-ribosomal } 60 \text { s subunit protein } \\
\text { L40A fusion protein }\end{array}$ & 51.11 & 60.29 & 30.81 & 72.67 & 37.14 & $\begin{array}{l}\text { Cytoplasm, } \\
\text { nucleus }\end{array}$ & Cytoplasm & Cytoplasm \\
\hline YKR094C & RPL40B & $\begin{array}{l}\text { Ubiquitin-ribosomal } 605 \text { subunit protein } \\
\text { L40B fusion protein }\end{array}$ & 163.57 & 38.61 & 63.15 & 22.21 & 36.32 & Cytoplasm & Cytoplasm & Cytoplasm \\
\hline YLR448W & RPL6B & Ribosomal 60S subunit protein L6B & 303.44 & 79.41 & 240.98 & 46.61 & 141.42 & Cytoplasm & Cytoplasm & Cytoplasm \\
\hline YPL198W & RPL7B & Ribosomal 60 S subunit protein L7B & 148.57 & 94.15 & 139.88 & 70.31 & 104.47 & $\begin{array}{l}\text { Cytoplasm, } \\
\text { nuclear foci }\end{array}$ & $\begin{array}{l}\text { Cytoplasm, } \\
\text { nuclear foci }\end{array}$ & $\begin{array}{l}\text { Cytoplasm, } \\
\text { nuclear foci }\end{array}$ \\
\hline YHL033C & RPL8A & Ribosomal 60S subunit protein L8A & 905.39 & 87.74 & 794.42 & 52.19 & 472.53 & Cytoplasm & Cytoplasm & Cytoplasm \\
\hline YLL045C & RPL8B & Ribosomal 60S subunit protein L8B & 502.72 & 87.99 & 442.33 & 59.70 & 300.11 & Cytoplasm & Cytoplasm & Cytoplasm \\
\hline YGL147C & RPL9A & Ribosomal 60S subunit protein L9A & 201.33 & 68.52 & 137.95 & 62.79 & 126.42 & Cytoplasm & Cytoplasm & Cytoplasm \\
\hline YNL067W & RPL9B & Ribosomal 60S subunit protein L9B & 1137.98 & 98.87 & 1125.17 & 56.14 & 638.81 & $\begin{array}{l}\text { Cytoplasm, } \\
\text { nucleus }\end{array}$ & $\begin{array}{l}\text { Cytoplasm, } \\
\text { nucleus }\end{array}$ & $\begin{array}{l}\text { Cytoplasm, } \\
\text { nucleus }\end{array}$ \\
\hline YFR052W & RPN12 & $\begin{array}{l}\text { Subunit of the } 19 \mathrm{~S} \text { regulatory particle of } \\
\text { the } 26 \mathrm{~S} \text { proteasome lid }\end{array}$ & 154.66 & 66.88 & 103.44 & 76.58 & 118.45 & Nucleus & Nucleus & Nucleus \\
\hline YPR108W & RPN7 & $\begin{array}{l}\text { Essential non-ATPase regulatory subunit } \\
\text { of the } 26 \mathrm{~S} \text { proteasome }\end{array}$ & 34.32 & 99.45 & 34.13 & 147.38 & 50.58 & Vacuolar lumen & Vacuolar lumen & Nucleus \\
\hline YDL081C & RPP1A & Ribosomal stalk protein P1 alpha & 808.19 & 106.79 & 863.03 & 52.95 & 427.95 & Cytoplasm & Cytoplasm & Cytoplasm \\
\hline YOR293W & RPS10A & $\begin{array}{l}\text { Protein component of the small (40S) } \\
\text { ribosomal subunit }\end{array}$ & 43.88 & 71.49 & 31.37 & 82.68 & 36.28 & Nuclear foci & Nuclear foci & Nuclear foci \\
\hline YJL191W & RPS14B & $\begin{array}{l}\text { Protein component of the small (40S) } \\
\text { ribosomal subunit }\end{array}$ & 59.37 & 70.39 & 41.79 & 73.29 & 43.52 & $\begin{array}{l}\text { Cytoplasm, } \\
\text { nucleus }\end{array}$ & $\begin{array}{l}\text { Cytoplasm, } \\
\text { nucleus }\end{array}$ & $\begin{array}{l}\text { Cytoplasm, } \\
\text { nucleus }\end{array}$ \\
\hline YML026C & RPS18B & $\begin{array}{l}\text { Protein component of the small (40S) } \\
\text { ribosomal subunit }\end{array}$ & 65.32 & 68.08 & 44.47 & 64.45 & 42.10 & Cytoplasm & Cytoplasm & Cytoplasm \\
\hline YLR367W & RPS22B & $\begin{array}{l}\text { Protein component of the small (40S) } \\
\text { ribosomal subunit }\end{array}$ & 234.68 & 57.32 & 134.52 & 46.92 & 110.11 & Nucleus & Nucleus & Nucleus \\
\hline
\end{tabular}




\begin{tabular}{|c|c|c|c|c|c|c|c|c|c|c|}
\hline YPR132W & RPS23B & $\begin{array}{l}\text { Ribosomal protein } 28 \text { (rp28) of the small } \\
(40 S) \text { ribosomal subunit }\end{array}$ & 131.72 & 70.72 & 93.16 & 54.83 & 72.23 & $\begin{array}{l}\text { Cytoplasm, } \\
\text { nucleus }\end{array}$ & $\begin{array}{l}\text { Cytoplasm, } \\
\text { nucleus }\end{array}$ & $\begin{array}{l}\text { Cytoplasm, } \\
\text { nucleus }\end{array}$ \\
\hline YER074W & RPS24A & $\begin{array}{l}\text { Protein component of the small (40S) } \\
\text { ribosomal subunit }\end{array}$ & 233.52 & 87.07 & 203.33 & 79.23 & 185.01 & Cytoplasm & Cytoplasm & Cytoplasm \\
\hline YIL069C & RPS24B & $\begin{array}{l}\text { Protein component of the small (40S) } \\
\text { ribosomal subunit }\end{array}$ & 107.37 & 90.14 & 96.78 & 53.28 & 57.20 & Cytoplasm & Cytoplasm & Cytoplasm \\
\hline \begin{tabular}{|l|} 
YER131W \\
\end{tabular} & RPS26B & $\begin{array}{l}\text { Protein component of the small (40S) } \\
\text { ribosomal subunit }\end{array}$ & 383.18 & 90.13 & 345.38 & 58.52 & 224.22 & Cytoplasm & Cytoplasm & Cytoplasm \\
\hline $\begin{array}{l}\text { YLR287C- } \\
\text { A }\end{array}$ & RPS30A & $\begin{array}{l}\text { Protein component of the small (40S) } \\
\text { ribosomal subunit }\end{array}$ & 294.68 & 82.18 & 242.17 & 68.97 & 203.23 & Cytoplasm & Cytoplasm & Cytoplasm \\
\hline YPL090C & RPS6A & $\begin{array}{l}\text { Protein component of the small (40S) } \\
\text { ribosomal subunit }\end{array}$ & 525.86 & 104.67 & 550.42 & 67.50 & 354.98 & Cytoplasm & Cytoplasm & Cytoplasm \\
\hline YPL012W & RRP12 & $\begin{array}{l}\text { Protein required for export of the } \\
\text { ribosomal subunits }\end{array}$ & 64.49 & 83.96 & 54.15 & 79.66 & 51.38 & $\begin{array}{l}\text { Cytoplasm, } \\
\text { nuclear foci }\end{array}$ & $\begin{array}{l}\text { Cytoplasm, } \\
\text { nuclear foci }\end{array}$ & Nuclear foci \\
\hline YIL127C & RRT14 & Putative protein of unknown function & 50.59 & 75.57 & 38.23 & 82.18 & 41.57 & Nuclear foci & Nuclear foci & Nuclear foci \\
\hline YHR087W & RTC3 & $\begin{array}{l}\text { Protein of unknown function involved in } \\
\text { RNA metabolism }\end{array}$ & 390.72 & 217.41 & 849.46 & 39.76 & 155.34 & $\begin{array}{l}\text { Cytoplasm, } \\
\text { nucleus }\end{array}$ & $\begin{array}{l}\text { Cytoplasm, } \\
\text { nucleus }\end{array}$ & $\begin{array}{l}\text { Cytoplasm, } \\
\text { nucleus }\end{array}$ \\
\hline YDL204W & RTN2 & Reticulon protein & 40.78 & 114.59 & 46.73 & 147.14 & 60.01 & $\begin{array}{l}\text { Plasma } \\
\text { membrane, } \\
\text { cytoplasm }\end{array}$ & $\begin{array}{l}\text { Plasma } \\
\text { membrane, } \\
\text { cytoplasm }\end{array}$ & $\begin{array}{l}\text { Plasma } \\
\text { membrane, } \\
\text { cytoplasm }\end{array}$ \\
\hline YOR077W & RTS2 & Basic zinc-finger protein & 46.40 & 81.63 & 37.88 & 87.57 & 40.64 & Nucleus & Nucleus & Nucleus \\
\hline YKL212W & SAC1 & $\begin{array}{l}\text { Phosphatidylinositol phosphate } \\
\text { (PtdlnsP) phosphatase }\end{array}$ & 90.23 & 81.71 & 73.72 & 76.03 & 68.60 & ER & $\begin{array}{l}\text { ER, vacuolar } \\
\text { lumen }\end{array}$ & $\begin{array}{l}\text { ER, vacuolar } \\
\text { lumen }\end{array}$ \\
\hline YKL117W & SBA1 & $\begin{array}{l}\text { Co-chaperone that binds and regulates } \\
\text { Hsp90 family chaperones }\end{array}$ & 295.67 & 99.60 & 294.50 & 74.14 & 219.22 & $\begin{array}{l}\text { Cytoplasm, } \\
\text { nucleus }\end{array}$ & $\begin{array}{l}\text { Cytoplasm, } \\
\text { nucleus }\end{array}$ & $\begin{array}{l}\text { Cytoplasm, } \\
\text { nucleus }\end{array}$ \\
\hline YER120W & SCS2 & $\begin{array}{l}\text { Integral ER membrane protein, } \\
\text { regulates phospholipid metabolism }\end{array}$ & 52.90 & 78.61 & 41.58 & 90.17 & 47.70 & ER & $\begin{array}{l}\text { Plasma } \\
\text { membrane }\end{array}$ & $\begin{array}{l}\text { Plasma } \\
\text { membrane }\end{array}$ \\
\hline YBL011W & SCT1 & $\begin{array}{l}\text { Glycerol 3-phosphate/dihydroxyacetone } \\
\text { phosphate sn-1 acyltransferase }\end{array}$ & 50.90 & 102.87 & 52.36 & 82.72 & 42.10 & ER & ER & ER \\
\hline YDR178W & SDH4 & $\begin{array}{l}\text { Membrane anchor subunit of succinate } \\
\text { dehydrogenase (SDH) }\end{array}$ & 169.21 & 107.46 & 181.84 & 59.70 & 101.02 & Mitochondrion & Mitochondrion & Mitochondrion \\
\hline
\end{tabular}




\begin{tabular}{|c|c|c|c|c|c|c|c|c|c|c|}
\hline YNL272C & SEC2 & $\begin{array}{l}\text { Guanyl-nucleotide exchange factor for } \\
\text { the small G-protein Sec4p }\end{array}$ & 51.54 & 100.28 & 51.69 & 86.46 & 44.56 & $\begin{array}{l}\text { Cytoplasm, bud } \\
\text { neck }\end{array}$ & $\begin{array}{l}\text { Cytoplasm, bud } \\
\text { neck }\end{array}$ & Cytoplasm \\
\hline YPR055W & SEC8 & $\begin{array}{l}\text { Essential } 121 \mathrm{kDa} \text { subunit of the exocyst } \\
\text { complex }\end{array}$ & 47.10 & 81.27 & 38.28 & 86.94 & 40.95 & $\begin{array}{l}\text { Bud neck, } \\
\text { plasma } \\
\text { membrane foci }\end{array}$ & $\begin{array}{l}\text { Bud neck, } \\
\text { plasma } \\
\text { membrane foci }\end{array}$ & $\begin{array}{l}\text { Bud neck, } \\
\text { plasma } \\
\text { membrane foci }\end{array}$ \\
\hline YDR077W & SED1 & Suppression of Exponential Defect & 244.84 & 121.45 & 297.37 & 85.36 & 244.84 & ER & ER & $\begin{array}{l}\text { ER, vacuolar } \\
\text { lumen }\end{array}$ \\
\hline YOR007C & SGT2 & $\begin{array}{l}\text { Glutamine-rich cytoplasmic } \\
\text { cochaperone }\end{array}$ & 119.81 & 100.36 & 120.25 & 78.69 & 94.28 & $\begin{array}{l}\text { Cytoplasm, } \\
\text { cytoplasmic foci }\end{array}$ & $\begin{array}{l}\text { Cytoplasm, } \\
\text { cytoplasmic foci }\end{array}$ & $\begin{array}{l}\text { Cytoplasm, } \\
\text { cytoplasmic foci }\end{array}$ \\
\hline YLR058C & SHM2 & $\begin{array}{l}\text { Cytosolic serine } \\
\text { hydroxymethyltransferase }\end{array}$ & 176.15 & 108.51 & 191.14 & 147.33 & 259.53 & $\begin{array}{l}\text { Cytoplasm, } \\
\text { cytoplasmic foci }\end{array}$ & $\begin{array}{l}\text { Cytoplasm, } \\
\text { cytoplasmic foci }\end{array}$ & $\begin{array}{l}\text { Cytoplasm, } \\
\text { cytoplasmic foci }\end{array}$ \\
\hline YDL225W & SHS1 & $\begin{array}{l}\text { Component of the septin ring that is } \\
\text { required for cytokinesis }\end{array}$ & 50.52 & 97.33 & 49.17 & 115.85 & 58.53 & Bud neck & Bud neck & $\begin{array}{l}\text { Bud neck, } \\
\text { plasma } \\
\text { membrane }\end{array}$ \\
\hline YGR081C & SLX9 & $\begin{array}{l}\text { Protein required for pre-rRNA } \\
\text { processing }\end{array}$ & 59.58 & 75.47 & 44.96 & 79.30 & 47.25 & Nucleus & Nuclear foci & Nuclear foci \\
\hline $\begin{array}{l}\text { YML058 } \\
\text { W }\end{array}$ & SML1 & Ribonucleotide reductase inhibitor & 224.77 & 59.11 & 132.86 & 54.65 & 122.83 & $\begin{array}{l}\text { Cytoplasm, } \\
\text { nucleus }\end{array}$ & $\begin{array}{l}\text { Cytoplasm, } \\
\text { nucleus }\end{array}$ & $\begin{array}{l}\text { Cytoplasm, } \\
\text { nucleus }\end{array}$ \\
\hline YDR011W & SNQ2 & $\begin{array}{l}\text { Plasma membrane ATP-binding cassette } \\
(A B C) \text { transporter }\end{array}$ & 68.22 & 96.57 & 65.88 & 98.65 & 67.30 & $\begin{array}{l}\text { Plasma } \\
\text { membrane }\end{array}$ & $\begin{array}{l}\text { Plasma } \\
\text { membrane, } \\
\text { vacuolar lumen }\end{array}$ & $\begin{array}{l}\text { Plasma } \\
\text { membrane, } \\
\text { vacuolar lumen } \\
\end{array}$ \\
\hline YJR104C & SOD1 & $\begin{array}{l}\text { Cytosolic copper-zinc superoxide } \\
\text { dismutase }\end{array}$ & 907.13 & 113.60 & 1030.47 & 57.42 & 520.85 & $\begin{array}{l}\text { Cytoplasm, } \\
\text { nucleus }\end{array}$ & $\begin{array}{l}\text { Cytoplasm, } \\
\text { nucleus }\end{array}$ & $\begin{array}{l}\text { Cytoplasm, } \\
\text { nucleus }\end{array}$ \\
\hline YNR034W & SOL1 & $\begin{array}{l}\text { Protein with a possible role in tRNA } \\
\text { export }\end{array}$ & 57.60 & 102.98 & 59.31 & 125.61 & 72.35 & $\begin{array}{l}\text { Cytoplasm, } \\
\text { nucleus }\end{array}$ & $\begin{array}{l}\text { Cytoplasm, } \\
\text { nucleus }\end{array}$ & $\begin{array}{l}\text { Cytoplasm, } \\
\text { nucleus }\end{array}$ \\
\hline YPR069C & SPE3 & Spermidine synthase & 105.40 & 84.22 & 88.77 & 68.59 & 72.30 & $\begin{array}{l}\text { Cytoplasm, } \\
\text { nucleus }\end{array}$ & $\begin{array}{l}\text { Cytoplasm, } \\
\text { nucleus }\end{array}$ & $\begin{array}{l}\text { Cytoplasm, } \\
\text { nucleus }\end{array}$ \\
\hline YGR104C & SRB5 & $\begin{array}{l}\text { Subunit of the RNA polymerase II } \\
\text { mediator complex }\end{array}$ & 57.65 & 94.67 & 54.58 & 66.30 & 38.22 & Mitochondrion & Mitochondrion & Mitochondrion \\
\hline YCL037C & SRO9 & Cytoplasmic RNA-binding protein & 157.03 & 86.89 & 136.44 & 71.30 & 111.97 & $\begin{array}{l}\text { Cytoplasm, } \\
\text { nucleus }\end{array}$ & $\begin{array}{l}\text { Cytoplasm, } \\
\text { nucleus }\end{array}$ & $\begin{array}{l}\text { Cytoplasm, } \\
\text { nucleus }\end{array}$ \\
\hline
\end{tabular}




\begin{tabular}{|c|c|c|c|c|c|c|c|c|c|c|}
\hline YKR092C & SRP40 & Nucleolar serine-rich protein & 124.38 & 82.97 & 103.20 & 60.47 & 75.21 & Nuclear foci & Nuclear foci & Nuclear foci \\
\hline YKL086W & SRX1 & Sulfiredoxin & 42.11 & 98.86 & 41.63 & 131.68 & 55.45 & Cytoplasm & Cytoplasm & $\begin{array}{l}\text { Cytoplasm, } \\
\text { nucleus }\end{array}$ \\
\hline YPL106C & SSE1 & $\begin{array}{l}\text { ATPase component of heat shock } \\
\text { protein Hsp90 chaperone complex }\end{array}$ & 536.49 & 76.10 & 408.28 & 61.60 & 330.47 & $\begin{array}{l}\text { Cytoplasm, } \\
\text { nucleus }\end{array}$ & $\begin{array}{l}\text { Cytoplasm, } \\
\text { nucleus }\end{array}$ & $\begin{array}{l}\text { Cytoplasm, } \\
\text { nucleus }\end{array}$ \\
\hline YBR283C & SSH1 & Subunit of the Ssh1 translocon complex & 39.41 & 70.82 & 27.91 & 229.60 & 90.48 & Cytoplasm & Cytoplasm & ER \\
\hline YMR183C & $\mathrm{SSO} 2$ & Plasma membrane t-SNARE & 38.45 & 70.60 & 27.14 & 105.44 & 40.54 & Cytoplasm & Cytoplasm & $\begin{array}{l}\text { Cytoplasm, } \\
\text { vacuolar lumen }\end{array}$ \\
\hline YOR212W & STE4 & G protein beta subunit & 54.17 & 83.59 & 45.28 & 71.59 & 38.78 & ER & ER & Cytoplasm \\
\hline $\begin{array}{l}\text { YDL130W } \\
-A\end{array}$ & STF1 & $\begin{array}{l}\text { Protein involved in regulation of the } \\
\text { mitochondrial F1F0-ATP synthase }\end{array}$ & 174.55 & 123.68 & 215.89 & 139.60 & 243.67 & Mitochondrion & Mitochondrion & Mitochondrion \\
\hline YJR130C & STR2 & $\begin{array}{l}\text { Cystathionine gamma-synthase, } \\
\text { converts cysteine into cystathionine }\end{array}$ & 39.54 & 102.40 & 40.49 & 106.84 & 42.24 & Cytoplasm & Cytoplasm & $\begin{array}{l}\text { Cytoplasm, } \\
\text { nucleus }\end{array}$ \\
\hline YDR167W & TAF10 & $\begin{array}{l}\text { Subunit (145 kDa) of TFIID and SAGA } \\
\text { complexes }\end{array}$ & 70.30 & 75.75 & 53.26 & 61.09 & 42.95 & Nucleus & Nucleus & Nucleus \\
\hline YMR227C & TAF7 & TFIID subunit (67 kDa) & 32.47 & 101.23 & 32.87 & 116.60 & 37.86 & Cytoplasm & Cytoplasm & Nucleus \\
\hline YOL020W & TAT2 & $\begin{array}{l}\text { High affinity tryptophan and tyrosine } \\
\text { permease }\end{array}$ & 47.45 & 89.91 & 42.66 & 97.15 & 46.10 & Vacuolar lumen & Vacuolar lumen & Vacuolar lumen \\
\hline YJL052W & TDH1 & $\begin{array}{l}\text { Glyceraldehyde-3-phosphate } \\
\text { dehydrogenase (GAPDH), isozyme } 1\end{array}$ & 285.70 & 142.31 & 406.59 & 212.55 & 607.26 & $\begin{array}{l}\text { Cytoplasm, } \\
\text { nucleus }\end{array}$ & $\begin{array}{l}\text { Cytoplasm, } \\
\text { nucleus }\end{array}$ & $\begin{array}{l}\text { Cytoplasm, } \\
\text { nucleus }\end{array}$ \\
\hline YJR009C & $\mathrm{TDH} 2$ & $\begin{array}{l}\text { Glyceraldehyde-3-phosphate } \\
\text { dehydrogenase (GAPDH), isozyme } 2\end{array}$ & 2051.60 & 103.20 & 2117.26 & 43.82 & 898.93 & $\begin{array}{l}\text { Cytoplasm, } \\
\text { nucleus }\end{array}$ & $\begin{array}{l}\text { Cytoplasm, } \\
\text { nucleus }\end{array}$ & $\begin{array}{l}\text { Cytoplasm, } \\
\text { nucleus }\end{array}$ \\
\hline YGR192C & TDH3 & $\begin{array}{l}\text { Glyceraldehyde-3-phosphate } \\
\text { dehydrogenase (GAPDH), isozyme } 3\end{array}$ & 2315.14 & 149.08 & 3451.40 & 175.44 & 4061.58 & $\begin{array}{l}\text { Cytoplasm, } \\
\text { nucleus }\end{array}$ & $\begin{array}{l}\text { Cytoplasm, } \\
\text { nucleus }\end{array}$ & $\begin{array}{l}\text { Cytoplasm, } \\
\text { nucleus }\end{array}$ \\
\hline YDR311W & TFB1 & $\begin{array}{l}\text { Subunit of TFIIH and nucleotide excision } \\
\text { repair factor } 3 \text { complexes }\end{array}$ & 47.65 & 95.64 & 45.57 & 79.50 & 37.88 & $\begin{array}{l}\text { Cytoplasm, } \\
\text { nucleus }\end{array}$ & $\begin{array}{l}\text { Cytoplasm, } \\
\text { nucleus }\end{array}$ & $\begin{array}{l}\text { Cytoplasm, } \\
\text { nucleus }\end{array}$ \\
\hline YLR178C & TFS1 & $\begin{array}{l}\text { Inhibitor of carboxypeptidase Y (Prc1p), } \\
\text { and Ras GAP (Ira2p) }\end{array}$ & 54.47 & 80.55 & 43.88 & 151.95 & 82.77 & $\begin{array}{l}\text { Cytoplasm, } \\
\text { nucleus }\end{array}$ & $\begin{array}{l}\text { Cytoplasm, } \\
\text { nucleus }\end{array}$ & $\begin{array}{l}\text { Cytoplasm, } \\
\text { nucleus }\end{array}$ \\
\hline YBR117C & TKL2 & Transketolase & 33.72 & 103.23 & 34.81 & 142.48 & 48.04 & $\begin{array}{l}\text { Cytoplasm, } \\
\text { nucleus }\end{array}$ & $\begin{array}{l}\text { Cytoplasm, } \\
\text { nucleus }\end{array}$ & $\begin{array}{l}\text { Cytoplasm, } \\
\text { nucleus }\end{array}$ \\
\hline
\end{tabular}




\begin{tabular}{|c|c|c|c|c|c|c|c|c|c|c|}
\hline YLR327C & TMA10 & $\begin{array}{l}\text { Protein of unknown function that } \\
\text { associates with ribosomes }\end{array}$ & 767.19 & 106.31 & 815.58 & 6.86 & 52.62 & $\begin{array}{l}\text { Cytoplasm, } \\
\text { nucleus }\end{array}$ & $\begin{array}{l}\text { Cytoplasm, } \\
\text { nucleus }\end{array}$ & $\begin{array}{l}\text { Cytoplasm, } \\
\text { nucleus }\end{array}$ \\
\hline YJR014W & TMA22 & Protein of unknown function & 99.49 & 87.07 & 86.62 & 65.81 & 65.47 & Cytoplasm & Cytoplasm & Cytoplasm \\
\hline $\begin{array}{l}\text { YLR262C- } \\
\text { A }\end{array}$ & TMA7 & $\begin{array}{l}\text { Protein of unknown that associates with } \\
\text { ribosomes }\end{array}$ & 116.81 & 82.79 & 96.70 & 67.72 & 79.11 & $\begin{array}{l}\text { Cytoplasm, } \\
\text { nucleus }\end{array}$ & $\begin{array}{l}\text { Cytoplasm, } \\
\text { nucleus }\end{array}$ & $\begin{array}{l}\text { Cytoplasm, } \\
\text { nucleus }\end{array}$ \\
\hline YNL070W & TOM7 & $\begin{array}{l}\text { Component of the TOM (translocase of } \\
\text { outer membrane) complex }\end{array}$ & 46.79 & 65.38 & 30.59 & 75.93 & 35.53 & Mitochondrion & Cytoplasmic foci & Cytoplasm \\
\hline YNL300W & TOS6 & & 70.37 & 75.43 & 53.08 & 85.45 & 70.37 & ER & ER & $\begin{array}{l}\text { ER, vacuolar } \\
\text { lumen }\end{array}$ \\
\hline YDR050C & TPI1 & $\begin{array}{l}\text { Triose phosphate isomerase, abundant } \\
\text { glycolytic enzyme }\end{array}$ & 354.11 & 98.25 & 347.90 & 165.00 & 584.30 & $\begin{array}{l}\text { Cytoplasm, } \\
\text { nucleus }\end{array}$ & $\begin{array}{l}\text { Cytoplasm, } \\
\text { nucleus }\end{array}$ & $\begin{array}{l}\text { Cytoplasm, } \\
\text { nucleus }\end{array}$ \\
\hline YOR273C & TPO4 & $\begin{array}{l}\text { Polyamine transporter of the major } \\
\text { facilitator superfamily }\end{array}$ & 74.99 & 112.90 & 84.67 & 108.33 & 81.24 & $\begin{array}{l}\text { Plasma } \\
\text { membrane, } \\
\text { vacuolar lumen }\end{array}$ & $\begin{array}{l}\text { Plasma } \\
\text { membrane, } \\
\text { vacuolar lumen }\end{array}$ & $\begin{array}{l}\text { Plasma } \\
\text { membrane, } \\
\text { vacuolar lumen }\end{array}$ \\
\hline YBR126C & TPS1 & $\begin{array}{l}\text { Synthase subunit of trehalose-6-P } \\
\text { synthase/phosphatase complex }\end{array}$ & 219.30 & 132.70 & 291.01 & 162.86 & 357.15 & Cytoplasm & Cytoplasm & Cytoplasm \\
\hline YDR074W & TPS2 & $\begin{array}{l}\text { Phosphatase subunit of the trehalose-6- } \\
\text { P synthase/phosphatase complex }\end{array}$ & 306.90 & 96.14 & 295.04 & 47.87 & 146.92 & Cytoplasm & Cytoplasm & Cytoplasm \\
\hline YNL299W & TRF5 & Non-canonical poly $(\mathrm{A})$ polymerase & 33.15 & 94.61 & 31.37 & 99.15 & 32.87 & Nuclear foci & Nuclear foci & Cytoplasm \\
\hline $\begin{array}{l}\text { YML028 } \\
\text { W } \\
\end{array}$ & TSA1 & Thioredoxin peroxidase & 1157.80 & 121.49 & 1406.58 & 90.44 & 1047.10 & $\begin{array}{l}\text { Cytoplasm, } \\
\text { cytoplasmic foci }\end{array}$ & $\begin{array}{l}\text { Cytoplasm, } \\
\text { cytoplasmic foci }\end{array}$ & $\begin{array}{l}\text { Cytoplasm, } \\
\text { cytoplasmic foci }\end{array}$ \\
\hline YKR088C & TVP38 & Integral membrane protein & 60.82 & 99.14 & 60.30 & 98.31 & 59.79 & Cytoplasmic foci & Vacuolar lumen & Vacuolar lumen \\
\hline YBR082C & UBC4 & Ubiquitin-conjugating enzyme (E2) & 104.84 & 47.05 & 49.33 & 51.31 & 53.79 & $\begin{array}{l}\text { Cytoplasm, } \\
\text { nucleus }\end{array}$ & $\begin{array}{l}\text { Cytoplasm, } \\
\text { nucleus }\end{array}$ & $\begin{array}{l}\text { Cytoplasm, } \\
\text { nucleus }\end{array}$ \\
\hline YGR019W & UGA1 & $\begin{array}{l}\text { Gamma-aminobutyrate (GABA) } \\
\text { transaminase }\end{array}$ & 81.02 & 115.14 & 93.29 & 144.69 & 117.23 & Cytoplasm & Cytoplasm & Cytoplasm \\
\hline YKL035W & UGP1 & $\begin{array}{l}\text { UDP-glucose pyrophosphorylase } \\
\text { (UGPase) }\end{array}$ & 106.46 & 104.93 & 111.71 & 125.07 & 133.14 & Cytoplasm & Cytoplasm & $\begin{array}{l}\text { Cytoplasm, } \\
\text { nucleus }\end{array}$ \\
\hline YBL039C & URA7 & $\begin{array}{l}\text { Major CTP synthase isozyme (see also } \\
\text { URA8) }\end{array}$ & 167.86 & 98.34 & 165.08 & 55.56 & 93.26 & $\begin{array}{l}\text { Cytoplasm, } \\
\text { cytoplasmic foci }\end{array}$ & $\begin{array}{l}\text { Cytoplasm, } \\
\text { cytoplasmic foci }\end{array}$ & $\begin{array}{l}\text { Cytoplasm, } \\
\text { cytoplasmic foci }\end{array}$ \\
\hline
\end{tabular}




\begin{tabular}{|c|c|c|c|c|c|c|c|c|c|c|}
\hline YDR400W & URH1 & $\begin{array}{l}\text { Uridine nucleosidase (uridine-cytidine } \\
\text { N-ribohydrolase) }\end{array}$ & 35.33 & 121.53 & 42.94 & 117.48 & 41.50 & Cytoplasm & Cytoplasm & Cytoplasm \\
\hline YGL212W & VAM7 & Vacuolar SNARE protein & 58.59 & 105.05 & 61.54 & 82.14 & 48.12 & Vacuolar lumen & Vacuolar lumen & $\begin{array}{l}\text { Cytoplasm, } \\
\text { vacuolar lumen }\end{array}$ \\
\hline YGR065C & VHT1 & $\begin{array}{l}\text { High-affinity plasma membrane } \mathrm{H+-} \\
\text { biotin (vitamin } \mathrm{H} \text { ) symporter }\end{array}$ & 79.34 & 170.21 & 135.05 & 111.25 & 88.27 & $\begin{array}{l}\text { Plasma } \\
\text { membrane, } \\
\text { vacuolar lumen }\end{array}$ & Vacuolar lumen & $\begin{array}{l}\text { Plasma } \\
\text { membrane, } \\
\text { vacuolar lumen }\end{array}$ \\
\hline YDL185W & VMA1 & $\begin{array}{l}\text { Subunit A of the V1 peripheral } \\
\text { membrane domain of V-ATPase }\end{array}$ & 573.31 & 112.70 & 646.12 & 53.56 & 307.06 & Vacuolar lumen & Vacuolar lumen & Vacuolar lumen \\
\hline YEL027W & VMA3 & $\begin{array}{l}\text { Proteolipid subunit c of the V0 domain } \\
\text { of vacuolar } \mathrm{H}(+) \text {-ATPase }\end{array}$ & 37.06 & 92.95 & 34.45 & 1612.18 & 597.49 & $\begin{array}{l}\text { Cytoplasm, } \\
\text { cytoplasmic foci }\end{array}$ & $\begin{array}{l}\text { Cytoplasm, } \\
\text { cytoplasmic foci }\end{array}$ & Nucleus \\
\hline YER072W & VTC1 & $\begin{array}{l}\text { Regulatory subunit of the vacuolar } \\
\text { transporter chaperone (VTC) complex }\end{array}$ & 45.87 & 94.17 & 43.19 & 77.44 & 35.52 & $\begin{array}{l}\text { ER, vacuolar } \\
\text { lumen }\end{array}$ & $\begin{array}{l}\text { ER, vacuolar } \\
\text { lumen }\end{array}$ & Vacuolar lumen \\
\hline YOR230W & WTM1 & Transcriptional modulator & 181.54 & 100.79 & 182.97 & 117.07 & 212.53 & Nucleus & Nucleus & Nucleus \\
\hline YBL055C & YBL055C & $\begin{array}{l}3^{\prime-->5} \text { exonuclease and endonuclease } \\
\text { with a possible role in apoptosis }\end{array}$ & 47.22 & 98.15 & 46.35 & 81.97 & 38.71 & Mitochondrion & Mitochondrion & Cytoplasm \\
\hline $\begin{array}{l}\text { YCR087C- } \\
\text { A }\end{array}$ & $\begin{array}{l}\text { YCR087C- } \\
\text { A }\end{array}$ & Putative protein of unknown function & 47.15 & 67.23 & 31.70 & 83.22 & 39.24 & Nuclear foci & Nuclear foci & Nuclear foci \\
\hline YLL055W & YCT1 & $\begin{array}{l}\text { High-affinity cysteine-specific } \\
\text { transporter }\end{array}$ & 55.86 & 140.31 & 78.37 & 266.87 & 149.07 & $\begin{array}{l}\text { Cytoplasm, } \\
\text { nucleus }\end{array}$ & $\begin{array}{l}\text { Cytoplasm, } \\
\text { nucleus }\end{array}$ & $\begin{array}{l}\text { Cytoplasm, } \\
\text { nucleus }\end{array}$ \\
\hline YDL124W & YDL124W & $\begin{array}{l}\text { NADPH-dependent alpha-keto amide } \\
\text { reductase }\end{array}$ & 155.31 & 135.27 & 210.10 & 173.29 & 269.15 & $\begin{array}{l}\text { Cytoplasm, } \\
\text { nucleus }\end{array}$ & $\begin{array}{l}\text { Cytoplasm, } \\
\text { nucleus }\end{array}$ & $\begin{array}{l}\text { Cytoplasm, } \\
\text { nucleus }\end{array}$ \\
\hline YDL129W & YDL129W & Protein of unknown function & 36.47 & 77.80 & 28.38 & 101.88 & 37.16 & Cytoplasm & Cytoplasm & $\begin{array}{l}\text { Cytoplasm, } \\
\text { nucleus }\end{array}$ \\
\hline YDR262W & YDR262W & Putative protein of unknown function & 36.43 & 98.96 & 36.05 & 131.88 & 48.04 & Vacuolar lumen & Vacuolar lumen & Vacuolar lumen \\
\hline YLR249W & YEF3 & Translation elongation factor 3 & 1100.43 & 87.53 & 963.23 & 70.36 & 774.21 & Cytoplasm & Cytoplasm & Cytoplasm \\
\hline $\begin{array}{l}\text { YER053C- } \\
A\end{array}$ & $\begin{array}{l}\text { YER053C- } \\
\text { A }\end{array}$ & Protein of unknown function & 43.33 & 96.47 & 41.81 & 169.94 & 73.64 & ER & ER & ER \\
\hline $\begin{array}{l}\text { YMR241 } \\
\text { W }\end{array}$ & YHM2 & Citrate and oxoglutarate carrier protein & 149.58 & 80.29 & 120.10 & 64.13 & 95.93 & Mitochondrion & Mitochondrion & Mitochondrion \\
\hline YKL071W & YKL071W & Putative protein of unknown function & 502.12 & 82.43 & 413.92 & 69.46 & 348.75 & Cytoplasm & Cytoplasm & Cytoplasm \\
\hline
\end{tabular}




\begin{tabular}{|c|c|c|c|c|c|c|c|c|c|c|}
\hline YKR018C & YKR018C & Protein of unknown function & 74.16 & 94.41 & 70.02 & 133.67 & 99.13 & $\begin{array}{l}\text { Cytoplasm, } \\
\text { nucleus }\end{array}$ & $\begin{array}{l}\text { Cytoplasm, } \\
\text { nucleus }\end{array}$ & $\begin{array}{l}\text { Cytoplasm, } \\
\text { nucleus }\end{array}$ \\
\hline YLR297W & YLR297W & Protein of unknown function & 37.96 & 79.31 & 30.11 & 124.11 & 47.12 & $\begin{array}{l}\text { Cytoplasm, } \\
\text { vacuolar lumen }\end{array}$ & $\begin{array}{l}\text { Cytoplasm, } \\
\text { vacuolar lumen }\end{array}$ & Vacuolar lumen \\
\hline YMR099C & YMR099C & $\begin{array}{l}\text { Glucose-6-phosphate 1-epimerase } \\
\text { (hexose-6-phosphate mutarotase) }\end{array}$ & 95.47 & 106.19 & 101.38 & 70.73 & 67.53 & $\begin{array}{l}\text { Cytoplasm, } \\
\text { nucleus }\end{array}$ & $\begin{array}{l}\text { Cytoplasm, } \\
\text { nucleus }\end{array}$ & $\begin{array}{l}\text { Cytoplasm, } \\
\text { nucleus }\end{array}$ \\
\hline \begin{tabular}{|l|} 
YMR178 \\
W \\
\end{tabular} & $\begin{array}{l}\text { YMR178 } \\
\text { W }\end{array}$ & Protein of unknown function & 51.29 & 86.80 & 44.52 & 127.40 & 65.34 & $\begin{array}{l}\text { Cytoplasm, } \\
\text { nucleus }\end{array}$ & $\begin{array}{l}\text { Cytoplasm, } \\
\text { nucleus }\end{array}$ & $\begin{array}{l}\text { Cytoplasm, } \\
\text { nucleus }\end{array}$ \\
\hline YNL194C & YNL194C & Integral membrane protein & 44.31 & 105.64 & 46.82 & 144.02 & 63.82 & $\begin{array}{l}\text { Plasma } \\
\text { membrane }\end{array}$ & $\begin{array}{l}\text { Plasma } \\
\text { membrane }\end{array}$ & $\begin{array}{l}\text { Plasma } \\
\text { membrane }\end{array}$ \\
\hline YGR281W & YOR1 & $\begin{array}{l}\text { Plasma membrane ATP-binding cassette } \\
(\mathrm{ABC}) \text { transporter }\end{array}$ & 39.66 & 99.25 & 39.37 & 106.73 & 42.33 & $\begin{array}{l}\text { Cytoplasm, } \\
\text { vacuolar lumen }\end{array}$ & $\begin{array}{l}\text { Cytoplasm, } \\
\text { vacuolar lumen }\end{array}$ & $\begin{array}{l}\text { Cytoplasm, } \\
\text { vacuolar lumen }\end{array}$ \\
\hline YOR292C & YOR292C & Putative protein of unknown function & 39.01 & 74.61 & 29.11 & 102.27 & 39.90 & Cytoplasm & $\begin{array}{l}\text { Cytoplasm, } \\
\text { vacuolar lumen }\end{array}$ & $\begin{array}{l}\text { Cytoplasm, } \\
\text { vacuolar lumen }\end{array}$ \\
\hline YPR127W & YPR127W & Putative pyridoxine 4-dehydrogenase & 33.20 & 105.76 & 35.11 & 118.29 & 39.28 & $\begin{array}{l}\text { Cytoplasm, } \\
\text { nucleus }\end{array}$ & $\begin{array}{l}\text { Cytoplasm, } \\
\text { nucleus }\end{array}$ & $\begin{array}{l}\text { Cytoplasm, } \\
\text { nucleus }\end{array}$ \\
\hline YPR172W & YPR172W & Protein of unknown function & 64.03 & 92.97 & 59.53 & 67.50 & 43.22 & $\begin{array}{l}\text { Cytoplasm, } \\
\text { nucleus }\end{array}$ & $\begin{array}{l}\text { Cytoplasm, } \\
\text { nucleus }\end{array}$ & $\begin{array}{l}\text { Cytoplasm, } \\
\text { nucleus }\end{array}$ \\
\hline YBR054W & YRO2 & $\begin{array}{l}\text { Protein with a putative role in response } \\
\text { to acid stress }\end{array}$ & 54.76 & 158.37 & 86.73 & 178.73 & 97.88 & $\begin{array}{l}\text { Plasma } \\
\text { membrane, } \\
\text { vacuolar lumen }\end{array}$ & $\begin{array}{l}\text { Plasma } \\
\text { membrane, } \\
\text { vacuolar lumen }\end{array}$ & $\begin{array}{l}\text { Plasma } \\
\text { membrane, } \\
\text { vacuolar lumen }\end{array}$ \\
\hline YNL310C & ZIM17 & $\begin{array}{l}\text { Protein co-chaperone with a zinc finger } \\
\text { motif }\end{array}$ & 40.13 & 72.53 & 29.11 & 109.77 & 44.05 & Nuclear foci & Nuclear foci & Nucleus \\
\hline YMR243C & ZRC1 & Vacuolar membrane zinc transporter & 50.57 & 74.58 & 37.71 & 145.48 & 73.57 & Cytoplasmic foci & $\begin{array}{l}\text { Vacuolar } \\
\text { membrane }\end{array}$ & $\begin{array}{l}\text { Vacuolar } \\
\text { membrane }\end{array}$ \\
\hline
\end{tabular}

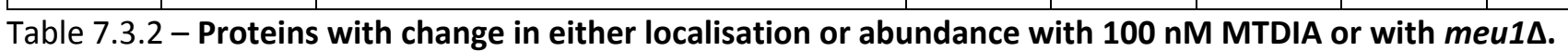




\begin{tabular}{|l|l|}
\hline Go: biological process & $\begin{array}{l}\text { Corrected } \\
\text { enrichment }\end{array}$ \\
\hline Transmembrane transport & $4.39 \mathrm{E}-07$ \\
\hline Organic acid transport & $5.67 \mathrm{E}-07$ \\
\hline Carboxylic acid transport & $1.38 \mathrm{E}-06$ \\
\hline Establishment of localization & $3.63 \mathrm{E}-05$ \\
\hline Ion transport & $1.48 \mathrm{E}-04$ \\
\hline Amino acid transmembrane transport & $3.06 \mathrm{E}-04$ \\
\hline
\end{tabular}

Table 7.3.3 - GO Enrichment for proteins affected by MTDIA treatment. Presented are the nonoverlapping GO categories for biological processes following correction for multiple testing using Benjamini Hochberg false discovery rate of 0.05 .

\begin{tabular}{|l|l|}
\hline Go: biological process & $\begin{array}{l}\text { Corrected } \\
\text { enrichment }\end{array}$ \\
\hline Pyruvate metabolic process & $1.70 \mathrm{E}-10$ \\
\hline Pyridine nucleotide metabolic process & $2.02 \mathrm{E}-09$ \\
\hline Nicotinamide nucleotide metabolic process & $2.02 \mathrm{E}-09$ \\
\hline Cytoplasmic translation & $4.34 \mathrm{E}-09$ \\
\hline Glycolytic process & $5.62 \mathrm{E}-08$ \\
\hline Acyl-CoA metabolic process & $5.12 \mathrm{E}-06$ \\
\hline Acetyl-CoA metabolic process & $1.34 \mathrm{E}-05$ \\
\hline Ribosomal large subunit biogenesis & $2.05 \mathrm{E}-04$ \\
\hline Transmembrane transport & $7.07 \mathrm{E}-04$ \\
\hline Glycine catabolic process & $7.58 \mathrm{E}-04$ \\
\hline Gluconeogenesis & $9.58 \mathrm{E}-04$ \\
\hline Glyceraldehyde-3-phosphate metabolic process & $7.90 \mathrm{E}-03$ \\
\hline Glutamate metabolic process & $1.08 \mathrm{E}-02$ \\
\hline lon transport & $1.45 \mathrm{E}-02$ \\
\hline Acetate metabolic process & $2.61 \mathrm{E}-02$ \\
\hline Amino acid transport & $4.31 \mathrm{E}-02$ \\
\hline Tricarboxylic acid cycle & $4.36 \mathrm{E}-02$ \\
\hline Citrate metabolic process & $4.36 \mathrm{E}-02$ \\
\hline
\end{tabular}

Table 7.3.4 - GO enrichment for proteins affected by meu1 $\Delta$. Presented are the non-overlapping GO categories for biological processes following correction for multiple testing using Benjamini Hochberg false discovery rate of 0.05 .

\begin{tabular}{|l|l|l|}
\hline Pathway & $\begin{array}{l}\text { Corrected } \\
\text { enrichment }\end{array}$ & Genes \\
\hline Glucose fermentation & $1.71 \mathrm{E}-07$ & $\begin{array}{l}A D H 2, A D H 3, A D H 4, A L D 6, C D C 19, \text { ENO1, GLK1, GPM1, HXK2, } \\
P D C 1, P D C 6, \text { PFK2, PGI1, PGK1, TDH1, TDH2, TDH3, TPI1 }\end{array}$ \\
\hline Glycolysis & $1.83 \mathrm{E}-04$ & $C D C 19$, ENO1, GPM1, PFK2, PGI1, PGK1, TDH1, TDH2, TDH3, TPI1 \\
\hline Gluconeogenesis & $3.21 \mathrm{E}-04$ & ENO1, GPM1, MAE1, MDH2, PGI1, PGK1, PYC2, TDH1, TDH2, TDH3 \\
\hline
\end{tabular}




\begin{tabular}{|l|l|l|}
\hline Glycine cleavage complex & $2.11 \mathrm{E}-02$ & GCV1, GCV2, GCV3, LPD1 \\
\hline $\begin{array}{l}\text { Pyruvate dehydrogenase } \\
\text { complex }\end{array}$ & $2.11 \mathrm{E}-02$ & GCV1, GCV2, GCV3, LPD1 \\
\hline
\end{tabular}

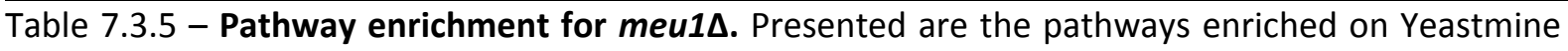
following correction for multiple testing using Benjamini Hochberg false discovery rate of 0.05 .

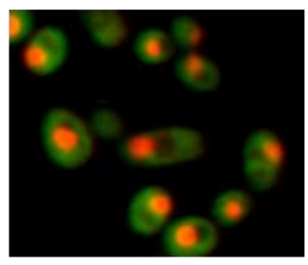

O nM MTDIA

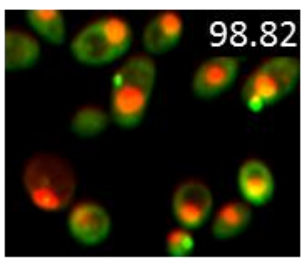

100 nM MTDIA

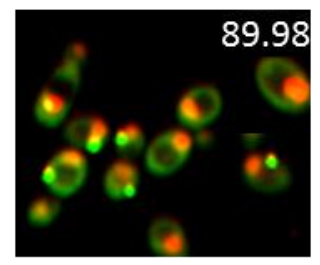

meu1s

GIn1-GFP

WT

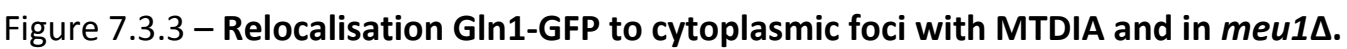

7.3.4 Go analysis of >2-fold upregulated or downregulated gene transcripts from Basu et al. 2011.

\begin{tabular}{|l|l|l|l|l|}
\hline GO biological process complete & $\begin{array}{l}\text { Homo } \\
\text { sapiens } \\
\text { (reference } \\
\text { list of } \\
\text { proteins } \\
\mathbf{2 0 9 7 2 )}\end{array}$ & $\begin{array}{l}\text { Basu et al. } \\
\mathbf{2 0 1 1} \\
\text { (proteins } \\
\text { with } \\
\text { annotations } \\
\text { - 690) }\end{array}$ & $\begin{array}{l}\text { Fold } \\
\text { enrichment } \\
\text { (proteins in } \\
\text { Basu et al. } \\
\text { /reference } \\
\text { list) }\end{array}$ & $\begin{array}{l}\text { Go } \\
\text { enrichment } \\
\text { (p-value) }\end{array}$ \\
\hline Negative regulation of wound healing (GO:0061045) & 63 & 13 & 6.27 & $2.42 \mathrm{E}-03$ \\
\hline Regulation of steroid biosynthetic process (GO:0050810) & 81 & 16 & 6 & $1.92 \mathrm{E}-04$ \\
\hline Negative regulation of response to wounding (GO:1903035) & 74 & 13 & 5.34 & $1.41 \mathrm{E}-02$ \\
\hline $\begin{array}{l}\text { Negative regulation of cysteine-type endopeptidase activity } \\
\text { involved in apoptotic process (GO:0043154) }\end{array}$ & 84 & 14 & 5.07 & $1.04 \mathrm{E}-02$ \\
\hline $\begin{array}{l}\text { Negative regulation of cysteine-type endopeptidase activity } \\
\text { (GO:2000117) }\end{array}$ & 89 & 14 & 4.78 & $2.02 \mathrm{E}-02$ \\
\hline Regulation of steroid metabolic process (GO:0019218) & 106 & 16 & 4.59 & $6.69 \mathrm{E}-03$ \\
\hline Regulation of mitotic nuclear division (GO:0007088) & 149 & 18 & 3.67 & $3.13 \mathrm{E}-02$ \\
\hline $\begin{array}{l}\text { Regulation of cysteine-type endopeptidase activity involved in } \\
\text { apoptotic process (GO:0043281) }\end{array}$ & 202 & 21 & 3.16 & $4.99 \mathrm{E}-02$ \\
\hline Mitotic cell cycle phase transition (GO:0044772) & 265 & 25 & 2.87 & $3.59 \mathrm{E}-02$ \\
\hline Response to drug (GO:0042493) & 411 & 37 & 2.74 & $6.01 \mathrm{E}-04$ \\
\hline Negative regulation of hydrolase activity (GO:0051346) & 407 & 36 & 2.69 & $1.37 \mathrm{E}-03$ \\
\hline Small molecule biosynthetic process (GO:0044283) & 436 & 38 & 2.65 & $8.96 \mathrm{E}-04$ \\
\hline Positive regulation of transferase activity (GO:0051347) & 657 & 57 & 2.64 & $6.41 \mathrm{E}-07$ \\
\hline Positive regulation of protein kinase activity (GO:0045860) & 481 & 41 & 2.59 & $5.06 \mathrm{E}-04$ \\
\hline Positive regulation of kinase activity (GO:0033674) & 519 & 43 & 2.52 & $5.22 \mathrm{E}-04$ \\
\hline
\end{tabular}




\begin{tabular}{|c|c|c|c|c|}
\hline $\begin{array}{l}\text { Regulation of protein serine/threonine kinase activity } \\
\text { (GO:0071900) }\end{array}$ & 475 & 38 & 2.43 & 7.07E-03 \\
\hline Organic hydroxy compound metabolic process (GO:1901615) & 412 & 33 & 2.43 & $3.62 \mathrm{E}-02$ \\
\hline Response to growth factor (GO:0070848) & 495 & 39 & 2.39 & $7.32 \mathrm{E}-03$ \\
\hline Mitotic cell cycle (GO:0000278) & 693 & 54 & 2.37 & $8.00 \mathrm{E}-05$ \\
\hline Response to wounding (GO:0009611) & 554 & 43 & 2.36 & 2.97E-03 \\
\hline Wound healing (GO:0042060) & 466 & 36 & 2.35 & 2.97E-02 \\
\hline Cellular response to growth factor stimulus (GO:0071363) & 468 & 36 & 2.34 & $3.26 \mathrm{E}-02$ \\
\hline Mitotic cell cycle process (GO:1903047) & 635 & 48 & 2.3 & $1.32 \mathrm{E}-03$ \\
\hline Myeloid leukocyte mediated immunity (GO:0002444) & 518 & 39 & 2.29 & $2.13 \mathrm{E}-02$ \\
\hline $\begin{array}{l}\text { Negative regulation of cellular protein metabolic process } \\
\text { (GO:0032269) }\end{array}$ & 1023 & 77 & 2.29 & $2.22 \mathrm{E}-07$ \\
\hline Regulation of protein kinase activity (GO:0045859) & 750 & 56 & 2.27 & 1.77E-04 \\
\hline Regulation of mitotic cell cycle (GO:0007346) & 523 & 39 & 2.27 & $2.66 \mathrm{E}-02$ \\
\hline Neutrophil mediated immunity (GO:0002446) & 497 & 37 & 2.26 & $4.93 \mathrm{E}-02$ \\
\hline Positive regulation of protein phosphorylation (GO:0001934) & 886 & 66 & 2.26 & $9.85 \mathrm{E}-06$ \\
\hline $\begin{array}{l}\text { Myeloid cell activation involved in immune response } \\
\text { (GO:0002275) }\end{array}$ & 514 & 38 & 2.25 & $4.32 \mathrm{E}-02$ \\
\hline $\begin{array}{l}\text { Symbiosis, encompassing mutualism through parasitism } \\
\text { (GO:0044403) }\end{array}$ & 789 & 58 & 2.23 & 1.67E-04 \\
\hline Interspecies interaction between organisms (GO:0044419) & 791 & 58 & 2.23 & $1.82 \mathrm{E}-04$ \\
\hline Response to organonitrogen compound (GO:0010243) & 880 & 64 & 2.21 & 4.41E-05 \\
\hline Positive regulation of cell death (GO:0010942) & 632 & 46 & 2.21 & $6.68 \mathrm{E}-03$ \\
\hline Positive regulation of apoptotic process (GO:0043065) & 591 & 43 & 2.21 & $1.55 \mathrm{E}-02$ \\
\hline Negative regulation of programmed cell death (GO:0043069) & 845 & 61 & 2.19 & $1.34 \mathrm{E}-04$ \\
\hline Negative regulation of apoptotic process (GO:0043066) & 832 & 60 & 2.19 & $1.83 \mathrm{E}-04$ \\
\hline Positive regulation of programmed cell death (GO:0043068) & 597 & 43 & 2.19 & 1.99E-02 \\
\hline $\begin{array}{l}\text { Negative regulation of protein metabolic process } \\
\text { (GO:0051248) }\end{array}$ & 1087 & 78 & 2.18 & 1.47E-06 \\
\hline Regulation of kinase activity (GO:0043549) & 810 & 58 & 2.18 & $4.04 \mathrm{E}-04$ \\
\hline Regulated exocytosis (GO:0045055) & 686 & 49 & 2.17 & 4.94E-03 \\
\hline Regulation of cellular response to stress (GO:0080135) & 615 & 44 & 2.17 & $1.80 \mathrm{E}-02$ \\
\hline Regulation of transferase activity (GO:0051338) & 984 & 70 & 2.16 & $1.96 \mathrm{E}-05$ \\
\hline Positive regulation of phosphorylation (GO:0042327) & 929 & 66 & 2.16 & $6.11 \mathrm{E}-05$ \\
\hline Response to organic cyclic compound (GO:0014070) & 885 & 63 & 2.16 & $1.28 \mathrm{E}-04$ \\
\hline Regulation of protein phosphorylation (GO:0001932) & 1300 & 92 & 2.15 & $5.74 \mathrm{E}-08$ \\
\hline Regulation of cell motility (GO:2000145) & 748 & 53 & 2.15 & 2.16E-03 \\
\hline Response to nitrogen compound (GO:1901698) & 981 & 69 & 2.14 & $4.02 \mathrm{E}-05$ \\
\hline $\begin{array}{l}\text { Positive regulation of phosphorus metabolic process } \\
\text { (GO:0010562) }\end{array}$ & 1055 & 74 & 2.13 & 1.19E-05 \\
\hline $\begin{array}{l}\text { Positive regulation of phosphate metabolic process } \\
\text { (GO:0045937) }\end{array}$ & 1055 & 74 & 2.13 & 1.19E-05 \\
\hline $\begin{array}{l}\text { Positive regulation of protein modification process } \\
\text { (GO:0031401) }\end{array}$ & 1146 & 80 & 2.12 & $2.98 \mathrm{E}-06$ \\
\hline $\begin{array}{l}\text { Positive regulation of intracellular signal transduction } \\
\text { (GO:1902533) }\end{array}$ & 905 & 63 & 2.12 & 2.84E-04 \\
\hline Regulation of phosphorylation (GO:0042325) & 1395 & 97 & 2.11 & 3.77E-08 \\
\hline
\end{tabular}




\begin{tabular}{|c|c|c|c|c|}
\hline Regulation of cell migration (GO:0030334) & 695 & 48 & 2.1 & $1.61 \mathrm{E}-02$ \\
\hline Regulation of MAPK cascade (GO:0043408) & 670 & 46 & 2.09 & 3.07E-02 \\
\hline Response to oxygen-containing compound (GO:1901700) & 1410 & 97 & 2.09 & $6.81 \mathrm{E}-08$ \\
\hline Negative regulation of cell death (GO:0060548) & 917 & 63 & 2.09 & $4.53 \mathrm{E}-04$ \\
\hline Exocytosis (GO:0006887) & 774 & 53 & 2.08 & 5.97E-03 \\
\hline Regulation of proteolysis (GO:0030162) & 803 & 55 & 2.08 & $3.63 \mathrm{E}-03$ \\
\hline Response to hormone (GO:0009725) & 777 & 53 & 2.07 & 6.69E-03 \\
\hline Regulation of cell cycle (GO:0051726) & 1019 & 69 & 2.06 & $1.75 \mathrm{E}-04$ \\
\hline Response to lipid (GO:0033993) & 831 & 56 & 2.05 & $4.69 \mathrm{E}-03$ \\
\hline Response to endogenous stimulus (GO:0009719) & 1264 & 85 & 2.04 & 4.67E-06 \\
\hline Regulation of locomotion (GO:0040012) & 818 & 55 & 2.04 & $6.36 \mathrm{E}-03$ \\
\hline Regulation of cellular component movement (GO:0051270) & 820 & 55 & 2.04 & $6.84 \mathrm{E}-03$ \\
\hline Secretion by cell (GO:0032940) & 963 & 64 & 2.02 & 1.14E-03 \\
\hline $\begin{array}{l}\text { Cellular response to oxygen-containing compound } \\
(\text { GO:1901701) }\end{array}$ & 874 & 58 & 2.02 & $4.74 \mathrm{E}-03$ \\
\hline Leukocyte mediated immunity (GO:0002443) & 725 & 48 & 2.01 & 4.89E-02 \\
\hline $\begin{array}{l}\text { Positive regulation of protein metabolic process } \\
\text { (GO:0051247) }\end{array}$ & 1543 & 102 & 2.01 & 1.76E-07 \\
\hline $\begin{array}{l}\text { Positive regulation of cellular protein metabolic process } \\
(\text { GO:0032270) }\end{array}$ & 1449 & 96 & 2.01 & 6.69E-07 \\
\hline $\begin{array}{l}\text { Positive regulation of cellular component organization } \\
\text { (GO:0051130) }\end{array}$ & 1180 & 78 & 2.01 & 5.39E-05 \\
\hline Negative regulation of molecular function (GO:0044092) & 1136 & 75 & 2.01 & $1.14 \mathrm{E}-04$ \\
\hline Immune effector process (GO:0002252) & 1003 & 66 & 2 & $1.02 \mathrm{E}-03$ \\
\hline Negative regulation of catalytic activity (GO:0043086) & 866 & 57 & 2 & 7.67E-03 \\
\hline Carboxylic acid metabolic process (GO:0019752) & 868 & 57 & 2 & 8.23E-03 \\
\hline Regulation of protein modification process (GO:0031399) & 1680 & 110 & 1.99 & 4.39E-08 \\
\hline Regulation of phosphate metabolic process (GO:0019220) & 1633 & 107 & 1.99 & $8.68 \mathrm{E}-08$ \\
\hline Regulation of organelle organization (GO:0033043) & 1190 & 78 & 1.99 & 7.70E-05 \\
\hline Cell cycle process (GO:0022402) & 1006 & 66 & 1.99 & $1.13 \mathrm{E}-03$ \\
\hline Apoptotic process (GO:0006915) & 903 & 59 & 1.99 & $6.18 \mathrm{E}-03$ \\
\hline Regulation of response to stress (GO:0080134) & 1382 & 90 & 1.98 & 6.59E-06 \\
\hline Cell death (GO:0008219) & 1061 & 69 & 1.98 & 7.96E-04 \\
\hline Programmed cell death (GO:0012501) & 1026 & 67 & 1.98 & 1.07E-03 \\
\hline Regulation of phosphorus metabolic process (GO:0051174) & 1647 & 107 & 1.97 & $1.43 \mathrm{E}-07$ \\
\hline Regulation of programmed cell death (GO:0043067) & 1437 & 93 & 1.97 & 4.51E-06 \\
\hline Regulation of apoptotic process (GO:0042981) & 1423 & 92 & 1.97 & $5.95 \mathrm{E}-06$ \\
\hline Regulation of intracellular signal transduction (GO:1902531) & 1740 & 112 & 1.96 & 7.68E-08 \\
\hline Cellular response to endogenous stimulus (GO:0071495) & 1053 & 68 & 1.96 & $1.28 \mathrm{E}-03$ \\
\hline Positive regulation of cell proliferation (GO:0008284) & 851 & 55 & 1.96 & $2.05 \mathrm{E}-02$ \\
\hline Regulation of cellular component biogenesis (GO:0044087) & 825 & 53 & 1.95 & $3.71 \mathrm{E}-02$ \\
\hline Regulation of cell death (GO:0010941) & 1537 & 98 & 1.94 & $3.14 \mathrm{E}-06$ \\
\hline Cell cycle (GO:0007049) & 1333 & 85 & 1.94 & 5.37E-05 \\
\hline Secretion (GO:0046903) & 1068 & 68 & 1.94 & $2.12 \mathrm{E}-03$ \\
\hline $\begin{array}{l}\text { Regulation of cellular protein metabolic process } \\
\text { (GO:0032268) }\end{array}$ & 2436 & 155 & 1.93 & 5.95E-12 \\
\hline
\end{tabular}




\begin{tabular}{|c|c|c|c|c|}
\hline $\begin{array}{l}\text { Regulation of anatomical structure morphogenesis } \\
(\mathrm{GO}: 0022603)\end{array}$ & 951 & 60 & 1.92 & 1.49E-02 \\
\hline Positive regulation of signal transduction (GO:0009967) & 1443 & 90 & 1.9 & $5.15 E-05$ \\
\hline Oxoacid metabolic process (GO:0043436) & 976 & 61 & 1.9 & $1.64 \mathrm{E}-02$ \\
\hline Regulation of protein metabolic process (GO:0051246) & 2684 & 167 & 1.89 & $2.32 \mathrm{E}-12$ \\
\hline Negative regulation of signal transduction (GO:0009968) & 1134 & 70 & 1.88 & 4.27E-03 \\
\hline Regulation of cellular component organization (GO:0051128) & 2321 & 143 & 1.87 & $1.33 \mathrm{E}-09$ \\
\hline Organic acid metabolic process (GO:0006082) & 993 & 61 & 1.87 & $2.80 \mathrm{E}-02$ \\
\hline Response to organic substance (GO:0010033) & 2612 & 159 & 1.85 & $1.01 \mathrm{E}-10$ \\
\hline Negative regulation of response to stimulus (GO:0048585) & 1411 & 86 & 1.85 & $3.22 \mathrm{E}-04$ \\
\hline Cellular response to organic substance (GO:0071310) & 1967 & 119 & 1.84 & 7.33E-07 \\
\hline Regulation of cell differentiation (GO:0045595) & 1544 & 93 & 1.83 & $1.45 \mathrm{E}-04$ \\
\hline Positive regulation of molecular function (GO:0044093) & 1916 & 115 & 1.82 & $2.59 \mathrm{E}-06$ \\
\hline Negative regulation of signalling (GO:0023057) & 1236 & 74 & 1.82 & 6.10E-03 \\
\hline Nitrogen compound transport (GO:0071705) & 1692 & 100 & 1.8 & $9.90 \mathrm{E}-05$ \\
\hline Regulation of hydrolase activity (GO:0051336) & 1386 & 82 & 1.8 & $2.33 \mathrm{E}-03$ \\
\hline Negative regulation of cell communication (GO:0010648) & 1232 & 73 & 1.8 & $1.06 \mathrm{E}-02$ \\
\hline Response to stress (GO:0006950) & 3263 & 192 & 1.79 & $1.63 \mathrm{E}-12$ \\
\hline Regulation of catalytic activity (GO:0050790) & 2447 & 144 & 1.79 & $3.30 \mathrm{E}-08$ \\
\hline Positive regulation of catalytic activity (GO:0043085) & 1612 & 95 & 1.79 & $2.78 \mathrm{E}-04$ \\
\hline Regulation of cell proliferation (GO:0042127) & 1558 & 92 & 1.79 & $4.32 \mathrm{E}-04$ \\
\hline Positive regulation of cell communication (GO:0010647) & 1570 & 92 & 1.78 & 6.10E-04 \\
\hline $\begin{array}{l}\text { Positive regulation of macromolecule metabolic process } \\
\text { (GO:0010604) }\end{array}$ & 2892 & 168 & 1.77 & 8.09E-10 \\
\hline Multi-organism process (GO:0051704) & 2398 & 140 & 1.77 & $1.26 \mathrm{E}-07$ \\
\hline Organic substance transport (GO:0071702) & 2048 & 119 & 1.77 & 8.13E-06 \\
\hline Positive regulation of signalling (GO:0023056) & 1578 & 92 & 1.77 & 7.65E-04 \\
\hline $\begin{array}{l}\text { Positive regulation of nitrogen compound metabolic process } \\
(\text { GO:0051173) }\end{array}$ & 2814 & 163 & 1.76 & 2.76E-09 \\
\hline Positive regulation of developmental process (GO:0051094) & 1207 & 70 & 1.76 & $3.66 \mathrm{E}-02$ \\
\hline Regulation of molecular function (GO:0065009) & 3032 & 174 & 1.74 & 7.14E-10 \\
\hline Cellular protein localization (GO:0034613) & 1331 & 76 & 1.74 & $2.41 \mathrm{E}-02$ \\
\hline Positive regulation of metabolic process (GO:0009893) & 3129 & 178 & 1.73 & 7.17E-10 \\
\hline $\begin{array}{l}\text { Positive regulation of cellular metabolic process } \\
\text { (GO:0031325) }\end{array}$ & 2917 & 165 & 1.72 & $1.32 \mathrm{E}-08$ \\
\hline Cellular localization (GO:0051641) & 2104 & 119 & 1.72 & 3.83E-05 \\
\hline Positive regulation of response to stimulus (GO:0048584) & 2088 & 118 & 1.72 & $4.72 \mathrm{E}-05$ \\
\hline Cellular macromolecule localization (GO:0070727) & 1341 & 76 & 1.72 & $3.14 \mathrm{E}-02$ \\
\hline Protein localization (GO:0008104) & 1920 & 108 & 1.71 & $3.03 \mathrm{E}-04$ \\
\hline Cellular response to chemical stimulus (GO:0070887) & 2416 & 135 & 1.7 & $5.75 \mathrm{E}-06$ \\
\hline Regulation of developmental process (GO:0050793) & 2284 & 128 & 1.7 & $1.50 \mathrm{E}-05$ \\
\hline Vesicle-mediated transport (GO:0016192) & 1748 & 98 & 1.7 & $1.68 \mathrm{E}-03$ \\
\hline Tissue development (GO:0009888) & 1643 & 92 & 1.7 & $4.42 \mathrm{E}-03$ \\
\hline Establishment of localization in cell (GO:0051649) & 1647 & 92 & 1.7 & 4.90E-03 \\
\hline Establishment of protein localization (GO:0045184) & 1498 & 84 & 1.7 & 1.39E-02 \\
\hline
\end{tabular}




\begin{tabular}{|c|c|c|c|c|}
\hline $\begin{array}{l}\text { Positive regulation of multicellular organismal process } \\
\text { (GO:0051240) }\end{array}$ & 1464 & 82 & 1.7 & 1.95E-02 \\
\hline Cellular response to stress (GO:0033554) & 1561 & 87 & 1.69 & $1.13 \mathrm{E}-02$ \\
\hline $\begin{array}{l}\text { Negative regulation of nitrogen compound metabolic process } \\
\text { (GO:0051172) }\end{array}$ & 2249 & 124 & 1.68 & 7.39E-05 \\
\hline $\begin{array}{l}\text { Regulation of multicellular organismal development } \\
\text { (GO:2000026) }\end{array}$ & 1738 & 96 & 1.68 & 4.40E-03 \\
\hline Single-organism localization (GO:1902578) & 2646 & 145 & 1.67 & $4.16 \mathrm{E}-06$ \\
\hline Regulation of signal transduction (GO:0009966) & 2783 & 152 & 1.66 & $1.74 \mathrm{E}-06$ \\
\hline Macromolecule localization (GO:0033036) & 2214 & 121 & 1.66 & $1.90 \mathrm{E}-04$ \\
\hline Regulation of multicellular organismal process (GO:0051239) & 2685 & 146 & 1.65 & 6.01E-06 \\
\hline $\begin{array}{l}\text { Negative regulation of cellular metabolic process } \\
\text { (GO:0031324) }\end{array}$ & 2405 & 130 & 1.64 & 9.54E-05 \\
\hline Phosphorus metabolic process (GO:0006793) & 2185 & 118 & 1.64 & $5.58 \mathrm{E}-04$ \\
\hline Small molecule metabolic process (GO:0044281) & 1801 & 97 & 1.64 & $1.14 \mathrm{E}-02$ \\
\hline $\begin{array}{l}\text { Positive regulation of macromolecule biosynthetic process } \\
(\text { GO:0010557) }\end{array}$ & 1628 & 88 & 1.64 & 3.26E-02 \\
\hline Positive regulation of biological process (GO:0048518) & 5400 & 288 & 1.62 & $4.20 \mathrm{E}-16$ \\
\hline $\begin{array}{l}\text { Negative regulation of macromolecule metabolic process } \\
\text { (GO:0010605) }\end{array}$ & 2444 & 130 & 1.62 & 2.43E-04 \\
\hline Positive regulation of cellular process (GO:0048522) & 4808 & 254 & 1.61 & $1.29 \mathrm{E}-12$ \\
\hline Regulation of signalling (GO:0023051) & 3139 & 166 & 1.61 & $2.18 \mathrm{E}-06$ \\
\hline Regulation of cell communication (GO:0010646) & 3086 & 163 & 1.61 & $3.70 \mathrm{E}-06$ \\
\hline Response to external stimulus (GO:0009605) & 1808 & 96 & 1.61 & 2.35E-02 \\
\hline Positive regulation of biosynthetic process (GO:0009891) & 1791 & 95 & 1.61 & 2.79E-02 \\
\hline Immune system process (GO:0002376) & 2512 & 132 & 1.6 & 3.74E-04 \\
\hline Localization (GO:0051179) & 5375 & 283 & 1.6 & 7.77E-15 \\
\hline Single-organism transport (GO:0044765) & 2521 & 133 & 1.6 & 2.60E-04 \\
\hline Cellular component assembly (GO:0022607) & 2254 & 119 & 1.6 & $1.59 \mathrm{E}-03$ \\
\hline $\begin{array}{l}\text { Phosphate-containing compound metabolic process } \\
\text { (GO:0006796) }\end{array}$ & 2092 & 110 & 1.6 & 6.17E-03 \\
\hline Establishment of localization (GO:0051234) & 4499 & 235 & 1.59 & $1.41 \mathrm{E}-10$ \\
\hline Regulation of response to stimulus (GO:0048583) & 3761 & 196 & 1.58 & 8.62E-08 \\
\hline Regulation of biological quality (GO:0065008) & 3487 & 181 & 1.58 & $1.08 \mathrm{E}-06$ \\
\hline Regulation of localization (GO:0032879) & 2553 & 132 & 1.57 & 9.39E-04 \\
\hline Response to chemical (GO:0042221) & 3897 & 199 & 1.55 & $3.41 \mathrm{E}-07$ \\
\hline Negative regulation of metabolic process (GO:0009892) & 2688 & 137 & 1.55 & 1.15E-03 \\
\hline Cell surface receptor signalling pathway (GO:0007166) & 2217 & 113 & 1.55 & $1.85 \mathrm{E}-02$ \\
\hline Cellular component biogenesis (GO:0044085) & 2508 & 127 & 1.54 & $5.25 \mathrm{E}-03$ \\
\hline Negative regulation of cellular process (GO:0048523) & 4291 & 214 & 1.52 & 3.77E-07 \\
\hline Transport (GO:0006810) & 4368 & 217 & 1.51 & $3.64 \mathrm{E}-07$ \\
\hline Animal organ development (GO:0048513) & 2961 & 145 & 1.49 & 4.69E-03 \\
\hline $\begin{array}{l}\text { Regulation of macromolecule metabolic process } \\
\text { (GO:0060255) }\end{array}$ & 6010 & 293 & 1.48 & $6.25 \mathrm{E}-11$ \\
\hline Negative regulation of biological process (GO:0048519) & 4778 & 233 & 1.48 & $2.76 \mathrm{E}-07$ \\
\hline Organelle organization (GO:0006996) & 3097 & 151 & 1.48 & $3.27 \mathrm{E}-03$ \\
\hline Regulation of metabolic process (GO:0019222) & 6533 & 316 & 1.47 & $5.05 \mathrm{E}-12$ \\
\hline
\end{tabular}




\begin{tabular}{|c|c|c|c|c|}
\hline Regulation of primary metabolic process (GO:0080090) & 5976 & 289 & 1.47 & $3.34 \mathrm{E}-10$ \\
\hline Single-multicellular organism process (GO:0044707) & 5539 & 267 & 1.47 & 1.11E-08 \\
\hline Cellular component organization (GO:0016043) & 5242 & 253 & 1.47 & $6.17 \mathrm{E}-08$ \\
\hline $\begin{array}{l}\text { Regulation of nitrogen compound metabolic process } \\
\text { (GO:0051171) }\end{array}$ & 5811 & 279 & 1.46 & 3.48E-09 \\
\hline Single-organism metabolic process (GO:0044710) & 3572 & 171 & 1.46 & $1.38 \mathrm{E}-03$ \\
\hline Cellular component organization or biogenesis (GO:0071840) & 5457 & 258 & 1.44 & 3.09E-07 \\
\hline Regulation of gene expression (GO:0010468) & 4544 & 213 & 1.42 & 9.99E-05 \\
\hline Response to stimulus (GO:0050896) & 7844 & 367 & 1.42 & $2.37 \mathrm{E}-13$ \\
\hline Regulation of cellular metabolic process (GO:0031323) & 6029 & 282 & 1.42 & 5.59E-08 \\
\hline Multicellular organism development (GO:0007275) & 4745 & 221 & 1.42 & $7.68 \mathrm{E}-05$ \\
\hline Developmental process (GO:0032502) & 5436 & 252 & 1.41 & 4.77E-06 \\
\hline Single-organism developmental process (GO:0044767) & 5350 & 247 & 1.4 & $1.21 \mathrm{E}-05$ \\
\hline Anatomical structure development (GO:0048856) & 5073 & 234 & 1.4 & $4.94 \mathrm{E}-05$ \\
\hline System development (GO:0048731) & 4156 & 191 & 1.4 & 3.50E-03 \\
\hline Regulation of biosynthetic process (GO:0009889) & 4432 & 202 & 1.39 & $2.48 \mathrm{E}-03$ \\
\hline Organonitrogen compound metabolic process (GO:1901564) & 5458 & 248 & 1.38 & $5.08 \mathrm{E}-05$ \\
\hline Organic cyclic compound metabolic process (GO:1901360) & 4945 & 224 & 1.38 & $6.02 \mathrm{E}-04$ \\
\hline Cellular response to stimulus (GO:0051716) & 6204 & 278 & 1.36 & $1.12 \mathrm{E}-05$ \\
\hline $\begin{array}{l}\text { Regulation of macromolecule biosynthetic process } \\
\text { (GO:0010556) }\end{array}$ & 4169 & 186 & 1.36 & $3.73 E-02$ \\
\hline Organic substance biosynthetic process (GO:1901576) & 4742 & 210 & 1.35 & 1.07E-02 \\
\hline Cell communication (GO:0007154) & 5341 & 236 & 1.34 & $1.74 \mathrm{E}-03$ \\
\hline Biosynthetic process (GO:0009058) & 4809 & 211 & 1.33 & $1.93 \mathrm{E}-02$ \\
\hline Cellular metabolic process (GO:0044237) & 8936 & 388 & 1.32 & $3.78 \mathrm{E}-09$ \\
\hline Multicellular organismal process (GO:0032501) & 6633 & 289 & 1.32 & 8.04E-05 \\
\hline Signalling (GO:0023052) & 5254 & 229 & 1.32 & $8.82 \mathrm{E}-03$ \\
\hline Single organism signalling (GO:0044700) & 5250 & 228 & 1.32 & $1.25 \mathrm{E}-02$ \\
\hline Single-organism cellular process (GO:0044763) & 9841 & 424 & 1.31 & $1.12 \mathrm{E}-10$ \\
\hline Organic substance metabolic process (GO:0071704) & 9416 & 404 & 1.3 & $3.68 \mathrm{E}-09$ \\
\hline
\end{tabular}

Table 7.3.6 - GO analysis of >2-fold upregulated or downregulated gene transcripts from Basu et al.

2011. GO enrichment for biological processes were determined using Panter (Mi et al., 2017) with human genome as a reference list with Bonferroni (Simes, 1986) false discovery rate of 0.05 . Highlighted in red are biological processes relevant to this decertation. 
7.4.1 pnp1 $\Delta$ mutant construction

a

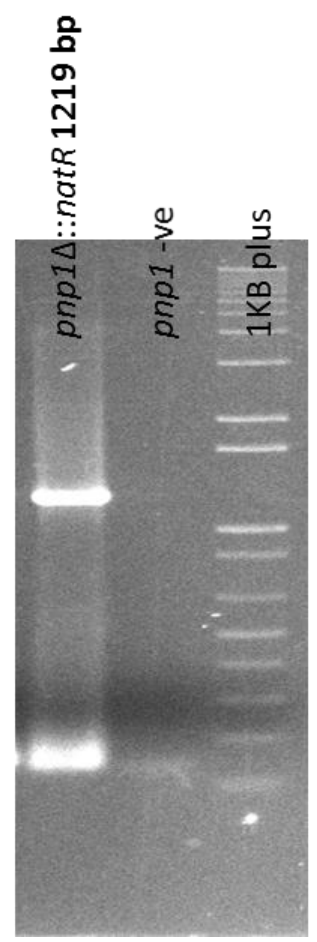

b

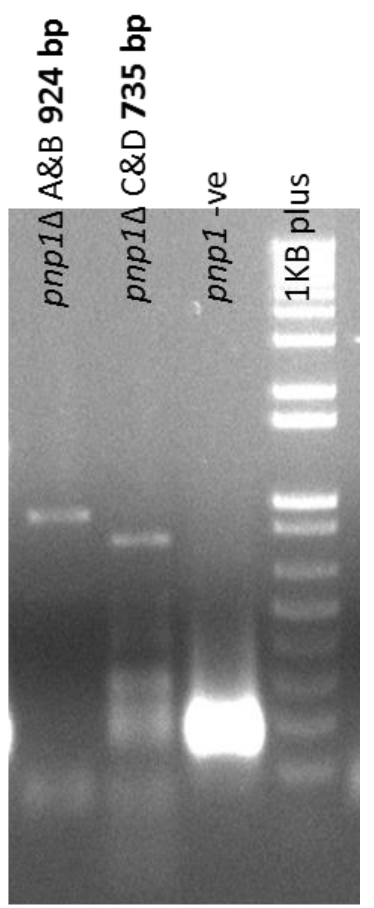

Figure 7.4.1 - pnp1 $1 \Delta$ construction and validation PCRs. PCR products were run on a $1 \%$ agarose gel with $1 \mathrm{~KB}$ plus ladder at $100 \mathrm{mV}$. (a) pnp1 $1:$ :natR deletion construction PCR. (b) PCR validation of PNP1 knockout.

\begin{tabular}{|l|l|}
\hline Primer & Sequence \\
\hline $\begin{array}{l}\text { Fw primer } \\
(\# 381)\end{array}$ & ATCTTGCTAATCTTCTAGAACACATGAGAAACTCCGAATAAACTTAAAAGACATGGAGGCCC \\
\hline $\begin{array}{l}\text { Rev primer } \\
(\# 382)\end{array}$ & AGGTACATCTTCCCGCTATGTAATAAATAGAGGTATTTAAGTTATAACAACAGTATAGCGAC \\
\hline $\begin{array}{l}\text { Conformation } \\
\text { primer A (\#3) }\end{array}$ & TCAAGCATCATCTGTCAA \\
\hline $\begin{array}{l}\text { Conformation } \\
\text { primer B } \\
(\# 385)\end{array}$ & TACGAGACGACCACGAAGC \\
\hline $\begin{array}{l}\text { Conformation } \\
\text { primer C } \\
(\# 386)\end{array}$ & TGGAACCGCCGGCTGACC \\
\hline $\begin{array}{l}\text { Conformation } \\
\text { primer D (\#4) }\end{array}$ & TTATACGTTACTGTACAT \\
\hline
\end{tabular}

Table 7.4.1 - pnp1 construction and conformation primers. Primers were designed as previously described (Janke et al., 2004). 
7.4.2 Genetic interactions with PNP1 in complete and minimal media

\begin{tabular}{|c|c|c|c|c|c|}
\hline \begin{tabular}{|l|} 
ID \\
Column
\end{tabular} & Gene & Function & $p$-value & $\begin{array}{l}\text { Z- } \\
\text { Score }\end{array}$ & $\begin{array}{l}\text { \% } \\
\text { Growth }\end{array}$ \\
\hline YNL141W & $\mathrm{AAH} 1$ & Adenine deaminase (adenine aminohydrolase) & $1.64 \mathrm{E}-02$ & 2.40 & 53.76 \\
\hline YGL105W & ARC1 & $\begin{array}{l}\text { Protein that binds tRNA and methionyl- and glutamyl-tRNA } \\
\text { synthetases }\end{array}$ & $1.12 \mathrm{E}-11$ & 6.79 & 16.67 \\
\hline YNL242W & ATG2 & $\begin{array}{l}\text { Peripheral membrane protein required for autophagic } \\
\text { vesicle formation }\end{array}$ & $1.37 \mathrm{E}-02$ & 2.46 & 52.91 \\
\hline YGR157W & $\mathrm{CHO} 2$ & Phosphatidylethanolamine methyltransferase (PEMT) & $2.17 \mathrm{E}-02$ & 2.29 & 54.95 \\
\hline YBR036C & CSG2 & Endoplasmic reticulum membrane protein & $1.38 \mathrm{E}-03$ & 3.20 & 43.29 \\
\hline YIR023W & DAL81 & $\begin{array}{l}\text { Positive regulator of genes in multiple nitrogen degradation } \\
\text { pathways }\end{array}$ & 8.03E-03 & 2.65 & 50.51 \\
\hline YHL031C & GOS1 & V-SNARE protein involved in Golgi transport & 3.99E-02 & 2.06 & 59.17 \\
\hline YJR090C & GRR1 & $\begin{array}{l}\text { F-box protein component of an SCF ubiquitin-ligase } \\
\text { complex }\end{array}$ & $3.14 \mathrm{E}-18$ & 8.71 & 9.99 \\
\hline YNL021W & HDA1 & $\begin{array}{l}\text { Putative catalytic subunit of a class II histone deacetylase } \\
\text { complex }\end{array}$ & $4.31 \mathrm{E}-02$ & 2.02 & 59.17 \\
\hline YPR179C & HDA3 & Subunit of the HDA1 histone deacetylase complex & 5.09E-03 & 2.80 & 48.08 \\
\hline YIL090W & ICE2 & $\begin{array}{l}\text { Integral ER membrane protein with type-III transmembrane } \\
\text { domains }\end{array}$ & 1.49E-03 & 3.18 & 43.86 \\
\hline YDR123C & INO2 & Transcription factor & 1.69E-03 & 3.14 & 44.25 \\
\hline YIL125W & KGD1 & $\begin{array}{l}\text { Subunit of the mitochondrial alpha-ketoglutarate } \\
\text { dehydrogenase complex }\end{array}$ & $2.56 \mathrm{E}-15$ & 8.09 & 11.81 \\
\hline YDR034C & LYS14 & $\begin{array}{l}\text { Transcriptional activator involved in regulating lysine } \\
\text { biosynthesis }\end{array}$ & -12 & 7.08 & 15.55 \\
\hline YHR194W & MDM31 & $\begin{array}{l}\text { Mitochondrial protein that may have a role in phospholipid } \\
\text { metabolism }\end{array}$ & $1.43 \mathrm{E}-10$ & 6.41 & 18.45 \\
\hline YGL124C & MON1 & $\begin{array}{l}\text { Subunit of a heterodimeric guanine nucleotide exchange } \\
\text { factor (GEF) }\end{array}$ & $3.72 \mathrm{E}-02$ & 2.08 & 58.48 \\
\hline YJR073C & OPI3 & Methylene-fatty-acyl-phospholif & 3.77E-02 & 2.08 & 58.48 \\
\hline YBR093C & PHO5 & Repressible acid phosphatase & $1.75 \mathrm{E}-04$ & 3.75 & 37.45 \\
\hline YBR106W & PHO88 & $\begin{array}{l}\text { Protein involved in SRP-independent targeting of substrates } \\
\text { to the ER }\end{array}$ & $5.21 \mathrm{E}-12$ & 6.90 & 16.10 \\
\hline YER162C & RAD4 & $\begin{array}{l}\text { Protein that recognizes and binds damaged DNA (with } \\
\text { Rad23p) during NER }\end{array}$ & $2.80 \mathrm{E}-02$ & 2.20 & 57.14 \\
\hline YJL204C & RCY1 & F-box protein involved in recycling endocytosed proteins & 1.37E-05 & 4.35 & 31.85 \\
\hline \begin{tabular}{|l|} 
YLR371W \\
\end{tabular} & ROM2 & $\begin{array}{l}\text { Guanine nucleotide exchange factor (GEF) for Rho1p and } \\
\text { Rho2p }\end{array}$ & $4.08 \mathrm{E}-02$ & 2.05 & 59.17 \\
\hline YLR357W & RSC2 & Component of the RSC chromatin remodelling complex & $1.44 \mathrm{E}-04$ & 5.16 & 38.91 \\
\hline YBR289W & SNF5 & Subunit of the SWI/SNF chromatin remodelling complex & $4.22 \mathrm{E}-08$ & 5.48 & 23.53 \\
\hline YCL032W & STE50 & Adaptor protein for various signalling pathways & $1.84 \mathrm{E}-02$ & 2.36 & 54.35 \\
\hline YIL047C & SYG1 & Plasma membrane protein of unknown function & $3.14 \mathrm{E}-12$ & 7.37 & 16.21 \\
\hline YKL081W & TEF4 & Gamma subunit of translational elongation factor eEF1B & $2.38 \mathrm{E}-05$ & 4.23 & 32.89 \\
\hline YIL011W & TIR3 & Cell wall mannoprotein & 8.77E-16 & 8.04 & 11.93 \\
\hline YDR074W & TPS2 & $\begin{array}{l}\text { Phosphatase subunit of the } t \\
\text { synthase/phosphatase comp }\end{array}$ & $2.24 \mathrm{E}-02$ & 2.28 & 55.56 \\
\hline YGL026C & TRP5 & Tryptophan synthase & $2.59 \mathrm{E}-12$ & 7.00 & 15.77 \\
\hline
\end{tabular}




\begin{tabular}{|l|l|l|l|l|l|}
\hline YDR359C & VID21 & Component of the NuA4 histone acetyltransferase complex & $1.67 \mathrm{E}-09$ & 6.03 & 20.33 \\
\hline YOR270C & VPH1 & Subunit a of vacuolar-ATPase V0 domain & $1.73 \mathrm{E}-02$ & 2.38 & 53.76 \\
\hline YHR012W & VPS29 & Subunit of the membrane-associated retromer complex & $2.19 \mathrm{E}-02$ & 2.29 & 55.56 \\
\hline YDL041W & YDL041W & Dubious open reading frame overlaps SIR2 & $4.24 \mathrm{E}-18$ & 8.67 & 10.09 \\
\hline YDR128W & YDR128W & Subunit of SEACAT, a subcomplex of the SEA complex & $3.16 \mathrm{E}-02$ & 2.15 & 57.47 \\
\hline $\begin{array}{l}\text { YFL013W- } \\
\text { A }\end{array}$ & YFL013W- & Dubious open reading frame overlaps IES1 & $4.32 \mathrm{E}-02$ & 2.02 & 59.52 \\
\hline YIL012W & YIL012W & Putative protein of unknown function & $1.84 \mathrm{E}-10$ & 6.37 & 57.14 \\
\hline YLR184W & YLR184W & Dubious open reading frame adjacent to TOP4 & $2.08 \mathrm{E}-03$ & 3.08 & 44.84 \\
\hline YPR024W & YME1 & Catalytic subunit of i-AAA protease complex & $3.10 \mathrm{E}-05$ & 4.17 & 33.56 \\
\hline YNL080C & YNL080C & Protein involved in N-glycosylation & $1.04 \mathrm{E}-02$ & 2.56 & 51.55 \\
\hline YGL255W & ZRT1 & High-affinity zinc transporter of the plasma membrane & $7.32 \mathrm{E}-03$ & 2.68 & 49.75 \\
\hline YNL241C & ZWF1 & Glucose-6-phosphate dehydrogenase (G6PD) & $2.45 \mathrm{E}-02$ & 2.25 & 56.18 \\
\hline
\end{tabular}

Table 7.4.2a - 42 putative genetic interactions with PNP1 gene in synthetic complete media. The haploid single gene deletion library was mated en masse against the pnp1s query strain on agar according to SGA methodology using the Singer RoToR HAD (Singer Instrument Co) (Tong et al., 2001). The haploid pnp1 $1 \Delta x x x \Delta$ double mutant meiotic progeny were assessed for epistatic colony size relative to the haploid parental mutants using Gitter on RStudio and ScreenMill. The putative interactions with an epistatic growth defect $(p n p 1 \Delta x x x \Delta / p n p 1 \Delta)$ in colony size with a $p<0.05$ in at least two out of three independent SGAs are presented.

\begin{tabular}{|c|c|c|c|c|c|}
\hline \begin{tabular}{|l} 
ORF \\
name
\end{tabular} & $\begin{array}{l}\begin{array}{l}\text { Gene } \\
\text { name }\end{array} \\
\end{array}$ & Function & $p$-value & $\begin{array}{l}\text { Standard } \\
\text { deviation }\end{array}$ & $\begin{array}{l}\text { \% } \\
\text { Growth }\end{array}$ \\
\hline YNL141W & AAH1 & Adenine deaminase (adenine aminohydrolase) & $3.53 E-26$ & 1.59 & 25.99 \\
\hline YGL234W & ADE5,7 & $\begin{array}{l}\text { Enzyme of the 'de novo' purine nucleotide biosynthetic } \\
\text { pathway }\end{array}$ & 5.31E-05 & 7.28 & 46.48 \\
\hline YML035C & AMD1 & AMP deaminase & $1.50 \mathrm{E}-02$ & 19.15 & 19.39 \\
\hline YPR185W & ATG13 & Regulatory subunit of the Atg1p signalling complex & $7.85 \mathrm{E}-04$ & 8.78 & 48.89 \\
\hline YKR099W & BAS1 & Myb-related transcription factor & 4.75E-03 & 10.64 & 54.11 \\
\hline YOR078W & BUD21 & $\begin{array}{l}\text { Component of small ribosomal subunit (SSU) } \\
\text { processosome }\end{array}$ & $1.52 \mathrm{E}-24$ & 1.41 & 30.86 \\
\hline YBR131W & CCZ1 & $\begin{array}{l}\text { Subunit of a heterodimeric guanine nucleotide exchange } \\
\text { factor (GEF) }\end{array}$ & 3.15E-02 & 20.39 & 38.69 \\
\hline YER061C & CEM1 & Mitochondrial beta-keto-acyl synthase & 2.59E-03 & 12.67 & 30.69 \\
\hline YLR394W & CST9 & SUMO E3 ligase & 4.16E-06 & 7.49 & 20.42 \\
\hline YIR023W & DAL81 & $\begin{array}{l}\text { Positive regulator of genes in multiple nitrogen } \\
\text { degradation pathways }\end{array}$ & $6.22 \mathrm{E}-08$ & 5.91 & 30.18 \\
\hline YDR359C & EAF1 & $\begin{array}{l}\text { Component of the NuA4 histone acetyltransferase } \\
\text { complex }\end{array}$ & $1.45 \mathrm{E}-43$ & 0.90 & 2.45 \\
\hline YLR206W & ENT2 & $\begin{array}{l}\text { Epsin-like protein required for endocytosis and actin } \\
\text { patch assembly }\end{array}$ & $1.38 \mathrm{E}-06$ & 7.99 & -1.00 \\
\hline YNL080C & EOS1 & Protein involved in N-glycosylation & $2.02 \mathrm{E}-13$ & 4.41 & 22.58 \\
\hline YBR026C & ETR1 & 2-enoyl thioester reductase & $1.06 \mathrm{E}-13$ & 4.55 & 16.64 \\
\hline
\end{tabular}




\begin{tabular}{|c|c|c|c|c|c|}
\hline YOR038C & HIR2 & Subunit of HIR nucleosome assembly complex & $1.24 \mathrm{E}-07$ & 6.48 & 23.39 \\
\hline YOR025W & HST3 & $\begin{array}{l}\text { Member of the Sir2 family of NAD(+)-dependent protein } \\
\text { deacetylases }\end{array}$ & 3.30E-02 & 51.91 & 239.38 \\
\hline YOL012C & HTZ1 & Histone variant $\mathrm{H} 2 \mathrm{AZ}$ & $1.06 \mathrm{E}-17$ & 3.13 & 33.41 \\
\hline YDR123C & INO2 & Transcription factor & 2.67E-07 & 5.97 & 13.90 \\
\hline YKR019C & IRS4 & EH domain-containing protein & 2.80E-09 & 4.78 & 41.32 \\
\hline YLL019C & KNS1 & $\begin{array}{l}\text { Protein kinase involved in negative regulation of PollII } \\
\text { transcription }\end{array}$ & $2.84 \mathrm{E}-05$ & 6.50 & 56.73 \\
\hline YPR070W & MED1 & Subunit of the RNA polymerase II mediator complex & $2.62 \mathrm{E}-04$ & 11.62 & -1.02 \\
\hline YGL124C & MON1 & $\begin{array}{l}\text { Subunit of a heterodimeric guanine nucleotide exchange } \\
\text { factor (GEF) }\end{array}$ & $5.44 \mathrm{E}-05$ & 12.00 & 34.97 \\
\hline YDR128W & MTC5 & Subunit of SEACAT, a subcomplex of the SEA complex & 2.19E-02 & 15.59 & 43.05 \\
\hline YJR073C & OPI3 & Methylene-fatty-acyl-phospholipid synthase & 2.69E-05 & 7.98 & 3.65 \\
\hline YCR077C & PAT1 & Deadenylation-dependent mRNA-decapping factor & $1.84 \mathrm{E}-07$ & 5.74 & 38.15 \\
\hline YLR199C & PBA1 & Protein involved in $20 \mathrm{~S}$ proteasome assembly & $7.68 \mathrm{E}-21$ & 3.66 & 2.34 \\
\hline YLR134W & PDC5 & Minor isoform of pyruvate decarboxylase & 7.91E-07 & 7.10 & 20.50 \\
\hline \multirow[t]{2}{*}{ YBR093C } & $\mathrm{PHO5}$ & Repressible acid phosphatase & $1.78 \mathrm{E}-03$ & 11.67 & 19.52 \\
\hline & PHO88 & & & & \\
\hline YPL036W & PMA2 & Plasma membrane H+-ATPase & $1.46 \mathrm{E}-13$ & 3.79 & 39.87 \\
\hline YDL006W & PTC1 & Type 2C protein phosphatase (PP2C) & $2.24 \mathrm{E}-30$ & 1.03 & 36.91 \\
\hline YER162C & RAD4 & $\begin{array}{l}\text { Protein that recognizes and binds damaged DNA (with } \\
\text { Rad23p) during NER }\end{array}$ & $1.02 \mathrm{E}-19$ & 3.17 & 25.75 \\
\hline \begin{tabular}{|l|} 
YCR028C- \\
A
\end{tabular} & RIM1 & $\begin{array}{l}\text { ssDNA-binding protein essential for mitochondrial } \\
\text { genome maintenance }\end{array}$ & $4.74 \mathrm{E}-03$ & 11.00 & 58.95 \\
\hline YMR154C & RIM13 & Calpain-like cysteine protease & $2.75 \mathrm{E}-09$ & 5.27 & 159.25 \\
\hline \begin{tabular}{|l|} 
YLR061W \\
\end{tabular} & RPL22A & Ribosomal 60S subunit protein L22A & $4.20 \mathrm{E}-10$ & 5.41 & 17.62 \\
\hline YGL147C & RPL9A & Ribosomal 60S subunit protein L9A & $3.60 \mathrm{E}-13$ & 4.20 & 28.68 \\
\hline YOL121C & RPS19A & Protein component of the small (40S) ribosomal subunit & 4.93E-10 & 5.52 & 14.68 \\
\hline YPL090C & RPS6A & Protein component of the small (40S) ribosomal subunit & 8.60E-07 & 7.27 & 18.22 \\
\hline YNL257C & SIP3 & Putative sterol transfer protein & $5.75 \mathrm{E}-11$ & 5.36 & 10.55 \\
\hline YBR289W & SNF5 & Subunit of the SWI/SNF chromatin remodelling complex & 4.99E-04 & 7.93 & 48.47 \\
\hline YMR016C & SOK2 & $\begin{array}{l}\text { Nuclear protein that negatively regulates pseudohyphal } \\
\text { differentiation }\end{array}$ & $6.56 \mathrm{E}-06$ & 6.92 & 37.26 \\
\hline YOR190W & SPR1 & Sporulation-specific exo-1,3-beta-glucanase & $1.70 \mathrm{E}-06$ & 6.73 & 31.69 \\
\hline YIL047C & SYG1 & Plasma membrane protein of unknown function & $1.57 \mathrm{E}-27$ & 2.76 & 2.76 \\
\hline YKL081W & TEF4 & Gamma subunit of translational elongation factor eEF1B & 7.49E-04 & 9.14 & 50.20 \\
\hline YDR074W & TPS2 & $\begin{array}{l}\text { Phosphatase subunit of the trehalose-6-P } \\
\text { synthase/phosphatase complex }\end{array}$ & 8.87E-03 & 12.19 & 57.53 \\
\hline YDR207C & UME6 & Rpd3L histone deacetylase complex subunit & $2.61 \mathrm{E}-06$ & 7.30 & 21.83 \\
\hline YOR106W & VAM3 & Syntaxin-like vacuolar t-SNARE & $4.68 \mathrm{E}-07$ & 6.32 & 3.82 \\
\hline YOR270C & VPH1 & Subunit a of vacuolar-ATPase V0 domain & $2.00 \mathrm{E}-02$ & 19.02 & 35.25 \\
\hline YHR012W & VPS29 & Subunit of the membrane-associated retromer complex & $5.45 \mathrm{E}-10$ & 5.18 & 27.26 \\
\hline YDR080W & VPS41 & Subunit of the HOPS endocytic tethering complex & 4.43E-03 & 17.15 & 23.05 \\
\hline YDR290W & YDR290W & Dubious open reading frame overlaps RTT103 & $2.95 \mathrm{E}-03$ & 11.27 & 47.45 \\
\hline YLR200W & YKE2 & $\begin{array}{l}\text { Subunit of the heterohexameric Gim/prefoldin protein } \\
\text { complex }\end{array}$ & $7.10 \mathrm{E}-21$ & 3.66 & -0.75 \\
\hline
\end{tabular}




\begin{tabular}{|l|l|l|l|l|l|}
\hline YLR184W & YLR184W & Dubious open reading frame adjacent to TOS4 & $4.05 \mathrm{E}-03$ & 14.53 & 18.69 \\
\hline $\begin{array}{l}\text { YMR085 } \\
\text { W }\end{array}$ & $\begin{array}{l}\text { YMR085 } \\
\text { W }\end{array}$ & $\begin{array}{l}\text { Putative protein adjacent YMR084W and are merged in } \\
\text { related strains, and together forms a paralogous to GFA1 }\end{array}$ & $3.31 \mathrm{E}-04$ & 9.27 & 152.12 \\
\hline YNL198C & YNL198C & Dubious open reading frame overlaps GCR2 & $3.17 \mathrm{E}-02$ & 19.29 & 42.44 \\
\hline YNL296W & YNL296W & Dubious open reading frame overlaps MON2 & $1.07 \mathrm{E}-03$ & 13.23 & 189.83 \\
\hline YOR082C & YOR082C & Dubious open reading frame overlaps WHI5 & $9.44 \mathrm{E}-04$ & 13.85 & 193.95 \\
\hline YOR097C & YOR097C & Putative protein of unknown function & $2.43 \mathrm{E}-03$ & 11.78 & 33.27 \\
\hline
\end{tabular}

Table 7.4.2b - 53 negative and 5 positive genetic interactions with PNP1. The growth of the double mutants relative to the single haploid parental strains $(p n p 1 \Delta x x x \Delta / p n p 1 \Delta) /(x x x \Delta /$ WT) $\times 100$ was determined in liquid media at $16 \mathrm{~h}$ and $O D^{600}$ for all cultures with the exception $x x x \Delta$ slow growers where the OD600 was measured at later time points. The growth of the double mutants was normalised to $p n p 1 \Delta h i s 3 \Delta$ control strain and the double deletion mutants with an average growth $> \pm$ $40 \%, p<0.05$ of the pnp1 $1 \Delta$ his $3 \Delta$ strain were considered a growth defect or a growth improvement.

\begin{tabular}{|l|l|l|l|l|l|}
\hline ORF name & $\begin{array}{l}\text { Gene } \\
\text { name }\end{array}$ & Function & $\begin{array}{l}\% \\
\text { Growth }\end{array}$ & $\boldsymbol{p}$-value & $\begin{array}{l}\text { Z- } \\
\text { Score }\end{array}$ \\
\hline YLR131C & ACE2 & $\begin{array}{l}\text { Transcription factor required for septum destruction after } \\
\text { cytokinesis }\end{array}$ & 53.76 & $2.99 \mathrm{E}-04$ & 3.62 \\
\hline YOR128C & ADE2 & Phosphoribosylaminoimidazole carboxylase & 32.15 & $1.93 \mathrm{E}-11$ & 6.71 \\
\hline YMR300C & ADE4 & $\begin{array}{l}\text { Phosphoribosylpyrophosphate amidotransferase } \\
\text { (PRPPAT) }\end{array}$ & 256.41 & $9.94 \mathrm{E}-09$ & -5.73 \\
\hline YGR061C & ADE6 & Formylglycinamidine-ribonucleotide (FGAM)-synthetase & 153.85 & $7.70 \mathrm{E}-03$ & -2.66 \\
\hline YDR408C & ADE8 & Phosphoribosyl-glycinamide transformylase & 222.22 & $1.05 \mathrm{E}-06$ & -4.88 \\
\hline YMR282C & AEP2 & Mitochondrial protein & 60.61 & $3.42 \mathrm{E}-03$ & 2.93 \\
\hline YPR060C & ARO7 & Chorismate mutase & 178.57 & $3.13 \mathrm{E}-04$ & -3.60 \\
\hline YNL315C & ATP11 & Molecular chaperone & 67.57 & $2.48 \mathrm{E}-02$ & 2.24 \\
\hline YDR375C & BCS1 & $\begin{array}{l}\text { Protein translocase and chaperone required for Complex } \\
\text { III assembly }\end{array}$ & 61.35 & $4.42 \mathrm{E}-03$ & 2.85 \\
\hline YDR254W & CHL4 & $\begin{array}{l}\text { Outer kinetochore protein required for chromosome } \\
\text { stability }\end{array}$ & 138.89 & $4.07 \mathrm{E}-02$ & -2.05 \\
\hline YGR157W & CHO2 & Phosphatidylethanolamine methyltransferase (PEMT) & 166.67 & $1.86 \mathrm{E}-03$ & -3.11 \\
\hline YOL008W & COQ10 & Coenzyme Q (ubiquinone) binding protein & 67.57 & $2.36 \mathrm{E}-02$ & 2.26 \\
\hline YIR023W & DAL81 & $\begin{array}{l}\text { Positive regulator of genes in multiple nitrogen } \\
\text { degradation pathways }\end{array}$ & 250.00 & $3.81 \mathrm{E}-08$ & -5.50 \\
\hline YCR076C & FUB1 & Proteasome-binding protein & 69.93 & $4.14 \mathrm{E}-02$ & 2.04 \\
\hline YJL165C & HAL5 & Putative protein kinase & 52.36 & $1.55 \mathrm{E}-04$ & 3.78 \\
\hline YER057C & HMF1 & Member of the p14.5 protein family & 144.93 & $2.05 \mathrm{E}-02$ & -2.32 \\
\hline YGL168W & HUR1 & Protein of unknown function & 151.52 & $1.00 \mathrm{E}-02$ & -2.57 \\
\hline YOR135C & IRC14 & Dubious open reading frame & 65.36 & $1.45 \mathrm{E}-02$ & 2.45 \\
\hline YDR475C & JIP4 & Protein of unknown function & 60.61 & $3.65 \mathrm{E}-03$ & 2.91 \\
\hline YDR318W & MCM21 & Component of the kinetochore sub-complex COMA & 144.93 & $2.35 \mathrm{E}-02$ & -2.27 \\
\hline YOL126C & MDH2 & Cytoplasmic malate dehydrogenase & $3.83 \mathrm{E}-02$ & 2.07 \\
\hline
\end{tabular}




\begin{tabular}{|c|c|c|c|c|c|}
\hline YDR336W & MRX8 & Protein that associates with mitochondrial ribosome & 136.99 & $4.89 \mathrm{E}-02$ & 1.97 \\
\hline YEL033W & MTC7 & Protein of unknown function & 136.99 & $4.96 \mathrm{E}-02$ & -1.96 \\
\hline YNL316C & PHA2 & Prephenate dehydratase & 196.08 & $3.28 \mathrm{E}-05$ & -4.15 \\
\hline YJL198W & PHO90 & Low-affinity phosphate transporter & 68.97 & $3.29 \mathrm{E}-02$ & 2.13 \\
\hline YCR028C-A & RIM1 & $\begin{array}{l}\text { ssDNA-binding protein essential for mitochondrial } \\
\text { genome maintenance }\end{array}$ & 35.97 & $1.48 \mathrm{E}-09$ & 6.05 \\
\hline YLR357W & RSC2 & Component of the RSC chromatin remodelling complex & 13.33 & $4.43 E-33$ & 11.98 \\
\hline YNL254C & RTC4 & Protein of unknown function & 149.25 & $1.20 \mathrm{E}-02$ & -2.51 \\
\hline YCR008W & SAT4 & Ser/Thr protein kinase involved in salt tolerance & 49.02 & $2.79 \mathrm{E}-05$ & 4.19 \\
\hline YDR104C & SPO71 & $\begin{array}{l}\text { Meiosis-specific protein required for prospore membrane } \\
\text { morphogenesis }\end{array}$ & 138.89 & $4.33 \mathrm{E}-02$ & -2.02 \\
\hline YDR410C & STE14 & Farnesyl cysteine-carboxyl methyltransferase & 10.62 & $1.21 \mathrm{E}-40$ & 13.35 \\
\hline YDR463W & STP1 & Transcription factor & 147.06 & $1.88 \mathrm{E}-02$ & -2.35 \\
\hline YKL081W & TEF4 & Gamr & 149.25 & $1.42 \mathrm{E}-02$ & -2.45 \\
\hline YHR025W & THR1 & Homoserine kinase & 12.14 & $4.41 \mathrm{E}-36$ & 12.54 \\
\hline YDR074W & TPS2 & $\begin{array}{l}\text { Phosphatase subunit o } \\
\text { synthase/phosphatase }\end{array}$ & 140.85 & $3.00 \mathrm{E}-02$ & -2.17 \\
\hline YDR007W & TRP1 & Phosphoribosylanthranil & 37.74 & $8.76 \mathrm{E}-09$ & 5.75 \\
\hline YER090W & TRP2 & Anthranilate synthase & 35.71 & 1.19E-09 & 6.08 \\
\hline YDR092W & UBC13 & E2 ubiquitin-conjugating enzyme & 138.89 & $3.96 \mathrm{E}-02$ & -2.06 \\
\hline YFR010W & UBP6 & Ubiquitin-s & 43.10 & $6.92 \mathrm{E}-07$ & 4.96 \\
\hline YOR106W & VAM3 & Syntaxin-like vacuolar t-SNARE & 45.66 & $3.79 \mathrm{E}-06$ & 4.62 \\
\hline YDL077C & VAM6 & Guanine nucleotide exchange factor for the GTPase Gtr1p & 58.82 & $2.03 \mathrm{E}-03$ & 3.09 \\
\hline YEL014C & YEL014C & Putative protein of unknown function & 144.93 & $1.91 \mathrm{E}-02$ & -2.34 \\
\hline YER067C-A & YER067C-A & Dubious open reading frame overlaps $R$ & 136.99 & $4.97 \mathrm{E}-02$ & -1.96 \\
\hline YGL218W & YGL218W & Dubious open reading frame & 44.64 & $2.12 \mathrm{E}-06$ & 4.74 \\
\hline YMR244W & YMR244W & Putative protein of unknown function & 144.93 & $2.33 \mathrm{E}-02$ & -2.27 \\
\hline YOR139C & YOR139C & $\begin{array}{l}\text { Dubious open reading frame overlaps IDH2, subunit of } \\
\text { mitochondrial } N A D(+) \text {-dependent isocitrate } \\
\text { dehydrogenase }\end{array}$ & 144.93 & $2.24 \mathrm{E}-02$ & -2.28 \\
\hline YJL139C & YUR1 & Mannosyltransferase involved in protein $\mathrm{N}$-glycosylation & 69.93 & $4.06 \mathrm{E}-02$ & 2.05 \\
\hline
\end{tabular}

Table 7.4.3 - 47 putative genetic interactions with PNP1 gene in minimal media. The pnp1 $\Delta x x x \Delta$

double mutants and the $x x x \Delta$ parental haploid mutants were pinned onto minimal media supplemented with the essential amino acids methionine, leucine and uracil using the RoToR HAD (Singer Instrument Co. Ltd) and were incubated for $5 \mathrm{~d}$ at $30^{\circ} \mathrm{C}$. The putative interactions with an epistatic growth defect $((x x x \Delta p n p 1 \Delta / x x x \Delta \times 100)<30 \%, p<0.05)$ or improvement $((x x x \Delta p n p 1 \Delta / x x x \Delta$ $x$ 100) $>130 \%, p<0.05)$ in the pnp1 $\Delta x x x \Delta$ double mutants relative to the single mutants $x x x \Delta$ were determined using the Gitter package Gitter tools (Wagih \& Parts, 2014) in RStudio v0.99.896 (Racine, 2012) and ScreenMill (Dittmar et al., 2010). 